LBL --33328

DE93 007669

\title{
Bimolecular Reaction Dynamics from Photoelectron Spectroscopy of Negative Ions
}

\author{
Stephen Edmund Bradforth \\ Ph.D. Thesis \\ Department of Chemistry \\ University of California \\ and \\ Chemical Sciences Division \\ Lawrence Berkeley Laboratory \\ University of California \\ Berkeley, CA 94720
}

November 1992

This work was supported in part by the Director, Office of Energy Research, Office of Basic Energy Sciences, Chemical Sciences Division of the U.S. Department of Energy under contract No. DE-AC03-76SF00098, and in part by the Air Force Office for Scientific Research Contract No. AFOSR-91-0084. 


\section{Bimolecular Reaction Dynamics from Photoelectron Spectroscopy of Negative Ions}

Copyright $\odot 1992$

by

Stephen Edmund Bradforth

The U.S. Department of Energy has the right to use this thesis for any purpose whatsoever including the right to reproduce all or any part thereof 


\title{
Binolecular Reaction Dynamics from Photoelectron \\ Spectroscopy of Negative Ions
}

by

\author{
Stephen Edmund Bradforth
}

\begin{abstract}
The transition state region of a neutral bimolecular reaction may be experimentally investigated by photoelectron spectroscopy of an appropriate negative ion. The photoelectron spectrum provides information on the spectroscopy and dynamics of the short lived transition state and may be used to develop model potential energy surfaces that are semi-quantitative in this important region.

The principles of bound $\rightarrow$ bound negative ion photoelectron $:_{4}$ ectroscopy are illustrated by way of an example: a full analysis of the photoelectron bands of $\mathrm{CN}^{-}$, $\mathrm{NCO}^{-}$and $\mathrm{NCS}^{-}$. Transition state photoelectron spectra are presented for the following systems $\mathrm{Br}+\mathrm{HI}, \mathrm{Cl}+\mathrm{HI}, \mathrm{F}+\mathrm{HI}, \mathrm{F}+\mathrm{CH}_{3} \mathrm{OH}, \mathrm{F}+\mathrm{C}_{2} \mathrm{H}_{5} \mathrm{OH}, \mathrm{F}+\mathrm{OH}$ and $\mathrm{F}$ $+\mathrm{H}_{2}$. A time dependent framework for the simulation and interpretation of the bound $\rightarrow$ free transition state photoelectron spectra is subsequently developed and applied to the hydrogen transfer reactions $\mathrm{Br}+\mathrm{HI}, \mathrm{F}+\mathrm{OH} \rightarrow \mathrm{O}\left({ }^{3} \mathrm{P},{ }^{1} \mathrm{D}\right)+\mathrm{HF}$ and $\mathrm{F}+\mathrm{H}_{2}$. The theoretical approach for the simulations is a fully quantum-mechanical wave packet propagation on a collinear model reaction potential surface. The connection between the wavepacket time evolution and the photoelectron spectrum is given by the time autocorrelation function. For the benchmark $\mathrm{F}+\mathrm{H}_{2}$ system, comparisons with three-dimensional quantum calculations are made.
\end{abstract}


Each system investigated also reveals information on electronically excited potential energy surfaces in addition to the ground reaction surface. Transitions to different electronic surfaces may be distinguished and assigned by photoelectron anisotropy measurements. Upper potential energy surfaces are evaluated for the excited state interactions correlating to $\mathrm{O}\left({ }^{1} \mathrm{D}\right)+\mathrm{HF}$ and $\mathrm{F}\left({ }^{2} \mathrm{P}_{1 / 2}\right)+\mathrm{H}_{2}$ with reference to the $\mathrm{OHF}^{-}$and $\mathrm{FH}_{2}^{-}$experimental photoelectron spectra. 
Dedicated to my parents

Jackie and Philip Bradforth 


\section{Table of Contents}

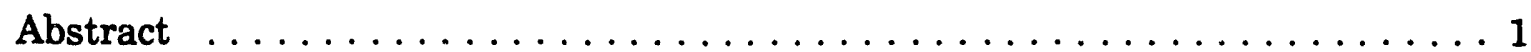

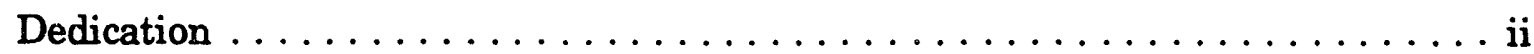

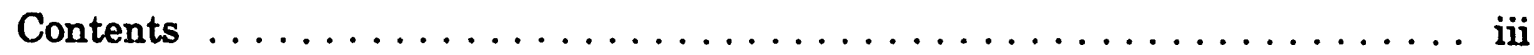

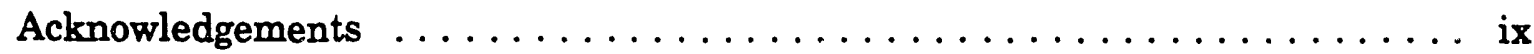

\section{Chapter 1. Introduction.}

1. Spectroscopy of the Transition State $\ldots \ldots \ldots \ldots \ldots \ldots \ldots 1$

2. Principles of Photoelectron Spectroscopy $\ldots \ldots \ldots \ldots \ldots \ldots 5$

3. Relationship of the photoelectron spectrum to short time chemical dynamics $\ldots \ldots \ldots \ldots \ldots \ldots \ldots \ldots \ldots$

4. Photoelectron Angular Distribution $\ldots \ldots \ldots \ldots \ldots \ldots$

5. Transition state spectroscopy by negative ion photodetachment $\ldots \ldots \ldots \ldots \ldots \ldots \ldots \ldots \ldots \ldots \ldots$

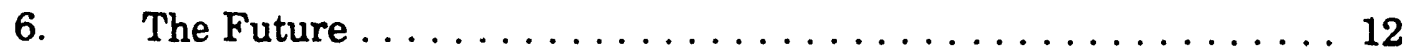

Chapter 2. Experimental: modifications and improvements to the negative ion photoelectron spectrometer.

1. Enhancements to the apparatus $\ldots \ldots \ldots \ldots \ldots \ldots \ldots 22$

$1.1 \quad$ Electron detection $\ldots \ldots \ldots \ldots \ldots \ldots \ldots \ldots \ldots 22$

$1.2 \quad$ Ion Source $\ldots \ldots \ldots \ldots \ldots \ldots \ldots \ldots \ldots \ldots$

2. Procedures for improved data collection $\ldots \ldots \ldots \ldots \ldots \ldots 29$

$2.1 \quad$ Calibration description $\ldots \ldots \ldots \ldots \ldots \ldots \ldots \ldots 29$ 
2.1.1 Calibration at $213 \mathrm{~nm} \ldots \ldots \ldots \ldots 33$

$2.2 \quad$ Background Subtraction $\ldots \ldots \ldots \ldots \ldots$

\section{Chapter 3. Photoelectron spectroscopy of $\mathrm{CN}^{-}, \mathrm{NCO}^{-}$, and NCS-}

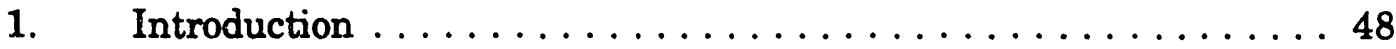

2. Experimental $\ldots \ldots \ldots \ldots \ldots \ldots \ldots \ldots \ldots \ldots \ldots \ldots \ldots \ldots \ldots$

3. $\quad$ Results $\ldots \ldots \ldots \ldots \ldots \ldots \ldots \ldots \ldots \ldots \ldots \ldots$

4. Analysis and Discussion $\ldots \ldots \ldots \ldots \ldots 6$

$4.1 \quad$ Electron Affinities $\ldots \ldots \ldots \ldots \ldots$

4.2 Derived Thermochemical quantities ...........61

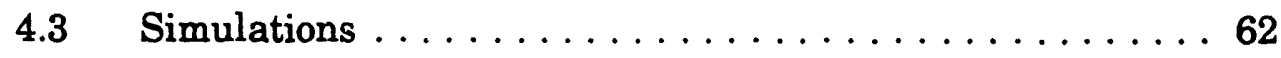

5. Conclusions $\ldots \ldots \ldots \ldots \ldots \ldots \ldots \ldots \ldots \ldots \ldots$

6. Acknowledgements ..................... 75

Chapter 4. Theoretical: Time dependent framework for analyzing dissociative photoelectron spectra.

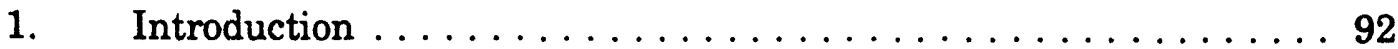

2. The photoelectron spectrum from the wave packet time

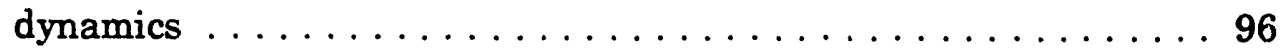

2.1 The Autocorrelation function from the photoelectron spectrum $\ldots \ldots \ldots \ldots \ldots \ldots \ldots \ldots$

3. Wave packet propagation $\ldots \ldots \ldots \ldots \ldots \ldots 10 \ldots \ldots$

$3.1 \quad$ Method $\ldots \ldots \ldots \ldots \ldots 10 \ldots \ldots \ldots$

3.2 Stability criteria and propagation errors ........ 105 
3.3 Implementation of wave packet propagation to the simulation of photoelectron spectra $\ldots \ldots \ldots \ldots 107$

3.3.1 Grid size, time step and potential shelves . . . . . 108

3.3.2 Absorbing boundaries . . . . . . . . . 110

3.3.3 Windowing and convolution of the autocorrelation ............... 111

4. Conclusions $\ldots \ldots \ldots \ldots \ldots \ldots \ldots \ldots \ldots \ldots \ldots$

Chapter 5. Examination of the $\mathrm{Br}+\mathrm{HI}, \mathrm{Cl}+\mathrm{HI}$, and $\mathrm{F}+\mathrm{HI}$ Hydrogen Abstraction Reactions by Photoelectron Spectroscopy of $\mathrm{BrHI}^{-}, \mathrm{CLHI}^{-}$, and FHI-

1. Introduction $\ldots \ldots \ldots \ldots \ldots \ldots \ldots \ldots \ldots \ldots \ldots$

2. Experimental $\ldots \ldots \ldots \ldots \ldots \ldots \ldots \ldots \ldots \ldots \ldots$

3. Results ........................... 124

4. Analysis and Discussion . . . . . . . . . . . 128

$4.1 \quad$ Preliminary considerations $\ldots \ldots \ldots \ldots \ldots$

4.2 One dimensional analysis of $\mathrm{XHY}^{-}$spectra $\ldots \ldots 130$

4.2.1 $\mathrm{BrHI}^{-}$and $\mathrm{BrDI}^{-} \ldots \ldots \ldots \ldots \ldots \ldots$

$4.2 .2 \mathrm{ClHI}^{-}$and $\mathrm{FHI}^{-} \ldots \ldots \ldots \ldots \ldots$

$4.3 \quad$ Electronic structure in $\mathrm{XHY}^{-}$spectra $\ldots \ldots \ldots \ldots$

4.4 Time dependent simulation . . . . . . . . . . . 139

4.4.1 One dimensional time-dependent treatment of the $\mathrm{BrHI}^{-}$spectrum $\ldots \ldots \ldots \ldots \ldots \ldots 14$ 
4.4.2 Two dimensional analysis of the $\mathrm{BrHI}^{-}$and $\mathrm{BrDI}^{-}$photoelectron spectra $\ldots \ldots \ldots \ldots \ldots 143$

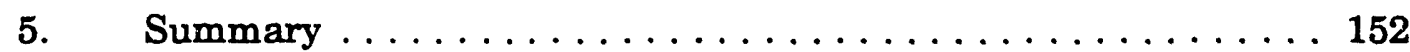

6. Acknowledgements ..................... 153

7. Postscript: new experimental results on $\mathrm{BrHI}^{-} \ldots \ldots \ldots \ldots 184$

Chapter 6. Spectroscopy of the Transition State: Hydrogen Abstraction Reactions of Fluorine

1. Introduction $\ldots \ldots \ldots \ldots \ldots \ldots \ldots \ldots \ldots$

2. Experimental ........................ 193

3. Results \& Analysis . . . . . . . . . . . . . . . . . . . . 194

$3.1 \quad$ Experimental Results . . . . . . . . . . . . . . 194

3.2 Ab initio calculations: $\operatorname{method}$ and results . . . . . . . 199

3.3 Simulation of the $\mathrm{OHF}^{-}$photoelectron spectrum .....206

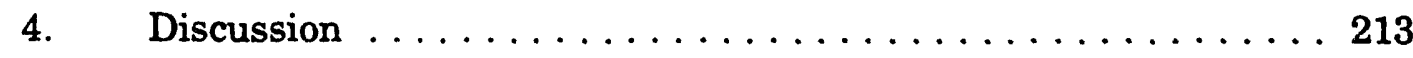

$4.1 \mathrm{OHF}^{-}$photoelectron spectrum $\ldots \ldots \ldots 213$

$4.2 \mathrm{CH}_{3} \mathrm{OHF}^{-}$and $\mathrm{C}_{2} \mathrm{H}_{5} \mathrm{OHF}^{-}$photoelectron spectra $\ldots \ldots 218$

$4.3 \quad$ Electronic effects $\ldots \ldots \ldots \ldots \ldots \ldots \ldots \ldots \ldots$

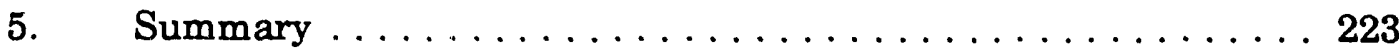

6. Acknowledgements . . . . . . . . . . . . . . . . 224

7. Postscript: new experimental results for $\mathrm{OHF}^{-} \ldots \ldots \ldots 253$ 
Chapter 7. Photoelectron spectroscopy of $\mathrm{FH}_{2}^{-}$: Results for the $\mathrm{F}+$ para $\mathrm{H}_{2}$ reaction and analysis of the ${ }^{2} \Pi$ electronic bands.

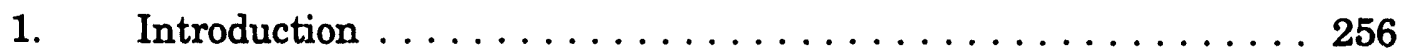

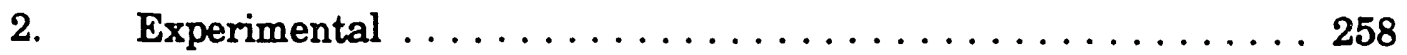

3. Results $\ldots \ldots \ldots \ldots \ldots \ldots \ldots \ldots \ldots \ldots \ldots \ldots \ldots \ldots$

4. Analysis and Discussion $\ldots \ldots \ldots \ldots \ldots \ldots \ldots \ldots 262$

4.1 Nuclear spin statistics in the anion and its effect on the photoelectron spectrum $\ldots \ldots \ldots \ldots \ldots \ldots 262$

4.2 New work on the $\mathrm{F}+\mathrm{H}_{2}$ reaction using the 5SEC surface $\ldots \ldots \ldots \ldots \ldots \ldots \ldots \ldots \ldots \ldots 267$

$4.3 \quad$ Electronic effects $\ldots \ldots \ldots \ldots \ldots \ldots \ldots \ldots \ldots 269$

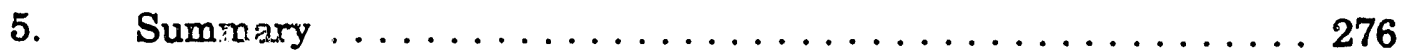

6. Acknoviledgements . . . . . . . . . . . . . . . 278

Appendix A. Propensities in Photoelectron Angular Distributions for linear molecular anions. 295

Appendix B. Calibration and Background Subtraction Subroutines for experimental code TENURE.

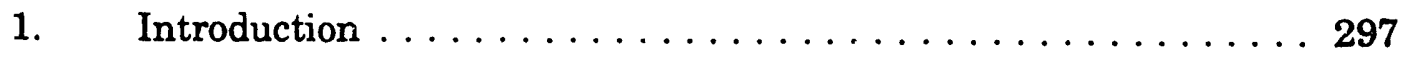

2. Source code listing $\ldots \ldots \ldots \ldots \ldots \ldots \ldots \ldots \ldots \ldots$

Appendix C. Time Dependent Wave packet Propagation Codes.

1. Introduction $\ldots \ldots \ldots \ldots \ldots \ldots \ldots \ldots \ldots \ldots \ldots \ldots \ldots$ 
2. One-dimensional Propagation Code - prop10 . . . . . . . 316

3. Two-dimensional Propagation Code - prop2d22 ... . . . 321

4. How the prop10 and prop2d22 codes work $\ldots \ldots \ldots \ldots \ldots 28$

$4.1 \quad$ Source code listing $\ldots \ldots \ldots \ldots \ldots \ldots \ldots \ldots$

4.1.1 One-dimensional code prop10 .......... 331

4.1.2 Two-dimensional code prop $2 \mathrm{~d} 22 \ldots \ldots \ldots \ldots 55$

Appendix D. Publications from graduate work $\ldots \ldots \ldots \ldots \ldots$. . . . 407 


\section{Acknowledgements}

I would like to thank Dan Neumark for presenting me with a challenging and exciting research project, and giving me the opportunity to be involved in such rewarding science. When I joined the group Dan promised that I would learn a great deal - how right he was. His guidance and suggestions over matters experimental, theoretical, conceptual, and postdoctoral has always been forthcoming and (usually!) very valuable. I have appreciated his generosity in carefully attributing credit to his students in talks and at conferences and encouraging me to attend and present work at conferences. I am also grateful to him for his efforts in helping me through the bureaucracy to (hopefully) secure a pos tribute to Dan's enthusiasm and scientific vision that these have been the most rewarding, and, despite the long hours, some of the most enjoyable, years of my life.

An essential ingredient to the project has been the teamwork and the strong supporting environment of the talented group of graduate students and postdocs. I cannot overemphasize the importance to me of those interactions and the amount I have learned from my fellow coworkers. Of my fellow "Stonehengers", Alex Weaver and Ricardo Metz were responsible for my initiation in the art of photoelectron spectroscopy, vacuum hardware, lasers, American idioms, you name it - it was all new

to me. I was most fortunate to arrive in the group at a time of great excitement: a machine up and running with a first communication about to appear in the literature. Alex turned me into a "trained photoelectron spectroscopist" and Ricky inspired me into adventures in quantum land. Don Arnold arrived a year after myself and brought a great deal of enthusiasm, machining talent, soot and bad jokes to the project. Don has been a terrific colleague a good friend: I doubt I would have enjoyed things so 
much without his good natured presence around the place. Eun $\mathrm{Ha} \mathrm{Kim}$, while carrying on the torch for transition sta spectroscopy, is still trying to figure out what Don and I are up to.

To provide the answers to the questions we could not answer, a second, higher resolution, machine was built. This ZEKE spectrometer was put together by Theo Kitsopoulos and Irene Waller. Theo, not only the other "European" in the group but also the only person more stubborn than I (I believe it was that way round Theo), showed me how fast a Fiat SuperBrava can go. Theo's hair brained ideas continue to provoke some memorable arguments. Irene taught us all the value of photodiodes. Caroline Arnold has ve -y impressively taken over the controls of the ZEKE machine and proved that ZEKE works for non-linear ions. Yuexing Zhao deserves special mention for buying all my furniture off me! Moving onto the third, and most grossly oversized, machine, Doug Cyr anỏ Bob Continetti, along with Ricky Metz, have put together a remarkable experiment. Doug, who, even after four years, continues with the same annoying British accent impersonation, has not quite got to the end of his hoard of paper towels from the stockroom that he purchased when the project began. Professor Bob, now a surfing instructor down south, is a tremendous role model for an experimental scientist. He brought much experimental know-how into the group and was always challenging others by his example. Dave Osborn has the dubious delight of inheriting the job of computer guru and sorting out the mess I leave behind. Dave also managed to sell the University of Chicago to me. Over the past few months a new crew has come on board, David Leahy, Georg Reiser, Cangshan Xu and Ivan Yourshaw; I wish them all the best. 
Much of the nuts and bolts of research could not take place without the tremendous support of many other members of the department. I would particularly like to thank George Weber and Ron Dal Porto in the machine shop, Harry Chiladakis, Tim Robinson for help with color graphics and Marcia Bogart in Purchasing who put an unreasonable number of rush orders through for me. Cheryn Gliebe, Dan's secretary, has been wonderful. As a foreign student at the Berkeley, I feel particularly grateful to the University of California for generous fellowship support over a large part of my time here. I thank also the Fulbright Commission, London for a scholarship in my first year.

I have been fortunate to have interacted with a number of other scientists in the course of this research project. I would like to acknowledge the help and advice of the following: Professor Rich Saykally, Dr. Soo-Y. Lee, Dr. David Manolopoulos and Professor George Schatz. I would like to thank Professor Y. T. Lee for the loan of a $\mathrm{CO}_{2}$ laser, and the Saykally and Lee groups for the loan of many other pieces of equipment and gases. Mr. Chris Jackson, my chemistry teacher at King's School, Canterbury and Mr. Chris Dashwood, my maths teacher at preparatory school, deserve much of the credit for inspiring in me an interest in science that has brought me this far.

I could not have survived graduate school without the friendship of a number of people inside and outside the laboratory. I had a wonderful first year at the International House; many of those friendships have lasted my entire stay here and hopefully will continue for many years to come. Special mention to former roommates Gary, Hugh, and Nikki and to Rachel, Rich, Sarah, Mimi, Ian, Neil, Matteo, Joe, Anne, Ken and of course Susan. Susan has shared much of the ups and downs of 
graduate school with me over the last three and a half years, and I thank her for her understanding, companionship, patience and love.

Finally, none of this could have been possible without the constant love and support my parents have given nie. They have given me the most wonderful opportwaitiss in life through their constant commitment to my education. For this I am immensely grateful and proud. Their support and understanding has been ever present even when I chose to go away to graduate school 5,000 miles from home. This thesis is dedicated to them.

This research was supported in part by the Director, Office of Energy Research, Office of Basic Energy Sciences, Chemical Sciences Division of the U. S. Department of Energy under contract No. DE-AC03-76SF00098, and in part by the Air Force Office for Scientific Research Contract No. AFOSR-91-0084. 


\section{Chapter 1. Introduction}

\section{Spectroscopy of the Transition State}

The fundamental question we wish to address in this research is the detailed description of a chemical reaction, and, in particular, of the chemical transition state. We undertake this from a microscopic, or molecular, viewpoint trying to uncover the forces and interactions experienced by the individual atoms involved in the transformation from reactants to products. This research builds upon the wealth of work in the field of reaction dynamics, and is essentially a pursuit of a complete understanding of chemical mechanism. ${ }^{1}$

The key to the description of a chemical reaction lies in the transition state. For a bimolecular reaction, e.g. $\mathrm{A}+\mathrm{HB} \rightarrow \mathrm{AH}+\mathrm{B}$, this is where bonds are forming and being broken at the same time: $[A . . H . . B]^{\ddagger}$. This was recognized by Arrhenius and elaborated by Eyring; it has been the linchpin to many theories of chemical reactivity. The transition state is the geometrical configuration of the reaction partners where there is a bottleneck on the free energy surface describing the reaction. Therefore, the intermolecular forces at play at or around the transition state are the most important in the dynamics of the reactive encounter.

The goal of reaction dynamics is then to characterize precisely the potential energy surface(s) governing a reaction particularly in the region of the transition state. This should, in turn, lead to a complete understanding of the electronic interactions determining the microscopic mechanism for branching and energy disposal in a reaction. Unfortunately, the transition state is the hardest part of the potential energy surface to characterize because of its very nature as an extremely 
short lived transient. A kinetics experiment, which determines the reaction rate as a function of temperature, provides some information in this respect: the derived activation energy gives a crude estimate of the barrier height at the transition state. However, much more discriminating experiments are required to uncover quantitative information about the potential energy surface.

This thesis describes the application of photoelectron spectroscopy of negative ions to the investigation of the transition state in a chemical reaction. By using appropriate negative ions as precursors for our experiment we have developed a technique that is a direct spectroscopic probe which is uniquely sensitive to this extremely short lived reaction transient species. Thus this work is termed, along with others in a new generation of reaction dynamics experiments, "transition state spectroscopy".2,3

The chemical reactions for which this technique proves useful are the so-called "elementary" bimolecular rua individual steps in a chemical process. As such, these are ubiquitous in chemistry, but their study is particularly important in atmospheric and combustion processes. Historically, these reactions have been experimentally investigated in the gas phase by measuring energy disposal and/or angular distributions among the reaction products with various degrees of control over reactant initial conditions. ${ }^{1,4,5,6}$ Under single collision conditions these experiments can provide fairly detailed evidence for the shapes of the potential surfaces in the interaction region. However, their handle on the dynamics is firmly connected to the asymptotic distribution of products. More ambitious scattering experiments where rovibrational state resolved differential cross sections are measured, either in crossed beam instruments, ${ }^{7}$ or via 
laser basec vector correlation methods, ${ }^{8}$ will provide more rigorous tests of the potential energy surfaces through comparisons with quantum scattering calculations. Van der Waals cluster initiated reactions have more recently introduced a new flavor to full collision dynamics experiments, ${ }^{9,10}$ as have aligned and oriented molecular beam experiments. ${ }^{11}$

The results of these experiments provide different and complementary pieces of information about the important transition state region for the reaction. Although the differential cross sections and detailed microscopic state-to-state cross sections are sensitive to the entire reaction potential surface, there is not a straightforward or intuitive connection between the data and the potential at the transition state. In contrast, our technique, being a half collision rather than a full collision experiment, probes the transition state region directly. By preparing a negative ion, $\mathrm{AHB}^{-}$, which is geometrically similar to the supposed neutral transition state structure $[\mathrm{AHB}]^{\ddagger}$, and removing an electron from the ion, we access the transition state region of the potential energy surface describing the reaction $A+H B \rightarrow A H+B$. The electron energy spectrum is a probe of the quantum states at the transition state rather than a mapping onto the asymptotic product states.

As we shall see, photoelectron spectroscopy provides an experimental route from which the spectrum of an internally cold, mass-selected species yields detailed information on the ground state dynamics of a neutral reaction. The experiment is described in Chapter 2. Mass selection is an important feature of our experiment as we can be sure that there is no background signal duo to other chemical pathways; such secondary reactions often make the results of full collision experiments hard to interpret. In addition, because the anion precursor is rotationally cold, the dynamics 
of the half collision are restricted to only a few angular momentum states. This facilitates comparison with theory.

In many respects our half collision technique is conceptually similar to molecular beam photodissociation experiments, particularly those that monitor the photodissociation cross section as a function of excitation wavelength (action spectroscopy). ${ }^{10,12}$ However, these latter experiments normally measure the photodissociation cross section by detecting products. The closest analog to our experimental "transition state" spectrum is the absorption spectrum of a molecule to a dissociative upper state, such as the first absorption band of $\mathrm{H}_{2} \mathrm{O}^{13}$ Such absorption experiments have not, to date, been recorded in a molecular beam environment. The use of negative ions as precursors in our experiments is important as this half collision technique is directly applicable to reactions that normally occur via bimolecular gas phase collisions (i.e. thermal rather than photochemical processes), in contrast to photodissociation studies on neutrals. Thus half collision data (the negative ion photoelectron spectrum) and complementary full collision kinetic, cross-beam, and product state data may be compared for a single reaction system. This allows, for the first time, something of a full picture for a bimolecular reaction to emerge. Many of the advantages of negative ion transition state spectroscopy as a probe of the chemical transition state are detailed in a recent review paper. $^{14}$

Although transition state dissociation occurs on a femtosecond time scale, the activated complex can vibrate one or more times before complete fragmentation: it is this motion that leads to structure in the observed photoelectron spectrum. In some special circumstances long lived states, called scattering resonances, which live for 
many tens of vibrational periods, may be observed in the photoelectron spectrum. Zewail has pioneered efforts to observe the femtosecond dynamics of the transition state in real time using femtosecond lasers. ${ }^{15}$ The clocking of the dissociation dynamics by an internal vibration, however, suggests a time domain analysis of our photoelectron spectra, and we have indeed pursued this route for an alternate understanding of the information revealed in the photoelectron spectra.

\section{Principles of Photoelectron Spectroscopy}

Photoelectron spectroscopy is a well developed branch of molecular spectroscopy. The interaction of light with a molecule to form a molecular ion and an electron (photoionization) is the most common form of photoelectron spectroscopy. If the precursor is instead a negative ion, the process is termed photodetachment and a neutral molecule and an electron result. Negative ion photoelectron spectroscopy, developed in the last 20 years and made possible by the appearance of high power visible and UV lasers, can be represented as follows:

$$
M^{-}\left(n^{\prime \prime}, v^{\prime \prime}\right)+h v \rightarrow M\left(n^{\prime}, v^{\prime}\right)+e^{-}\left(E_{k}\right)
$$

$\mathrm{hv}$ is the photon energy, and is constant in this experiment, i.e. the light source has a fixed wavelength. $E_{k}$, the kinetic energy of the electrons resulting from photodetachment, is the experimental observable. $n^{\prime \prime}, v^{\prime \prime}, n^{\prime}$ and $v^{\prime}$ describe the electronic and vibrational states of the anion and neutral molecule respectively. By the relationship 


$$
E_{k}=h v-E A(M)-E^{\prime}\left(n^{\prime}, v^{\prime}\right)+E^{\prime \prime}\left(n^{\prime \prime}, v^{\prime \prime}\right)
$$

the observed electron kinetic energy distribution, $\mathrm{I}\left(\mathrm{E}_{\mathrm{k}}\right)$, may be associated with the internal energies, vibrational and electronic, of the negative ion and neutral. As the energy resolution of current electron detection systems $(-5-10 \mathrm{meV}$, that is $-40-$ $\left.80 \mathrm{~cm}^{-1}\right)$ is inadequate to resolve the rotational spacings of most molecules, the rotational state labels are omitted and we shall seldom concern ourselves with the rotational behavior of the ion or neutral.

In our experiments, the negative ions are formed in a free jet expansion. This means that they are created with a restricted range of internal energies; typically the vast majority of the ions are present in their ground vibrational and electronic state. Hence, $E^{\prime \prime}$ in equation (2) is usually zero. Therefore, the photoelectron spectrum $I\left(E_{k}\right)$ is a direct measure of the energy levels of the neutral species $M$. The peaks in the spectrum at highest electron kinetic energy correspond to transitions to neutral states with lowest internal energy.

If we consider the distribution of electrons in a molecule in terms of a molecular orbital model, then Equation (1) implies the removal of an electron from a single m olecular orbital. This "one-electron" picture is often used in photoelectron spectroscopy and characterizes the only electronic selection rule: any transition between ion and neutral is allowed that occurs by removal of an electron from a single orbital without rearrangement of the electron occupation of the other orbitals. If several electronic states of the neutral are accessible energetically from the anion with the photon energy employed, those that are related to the anion electronic configuration by a one-electron process will be seen in the photoelectron spectrum. 
The photoelectron process for a diatomic negative ion is illustrated schematically in Figure 1-1. The potential energy curve for the anion and for the ground and first excited states of the neutral are shown. The expected photoelectron spectrum is shown on the right hand side. The spectrum shows two bands due to the two electronic states of the neutral; each band exhibits a vibrational progression. The spacings of peaks in the spectrum directly yields the separations of eigenstates in the neutral. The length of the progression and the exact intensities of the peaks in each band are governed by the Franck Condon Principle. This principle states that electronic transitions are fast compared to nuclear motion; $;^{16}$ for example, a photoelectron departs its parent negative ion in $-10^{-16} \mathrm{~s}$. Therefore, the neutral is born on the upper state potential surface in the configuration of the precursor anion. Quantum mechanically speaking the resulting distribution of neutral states is given by the projection of the anion wave function $\psi^{\prime \prime}$ over the neutral vibrational wave functions $\psi^{\prime} .{ }^{17}$ The intensities are given by the so called Franck Condon Factors (FCFs)

$$
F C F\left(v^{\prime}-v^{\prime \prime}\right)=\mid\left\langle\Psi_{v^{\prime}}^{\prime}\left|\Psi_{v^{\prime \prime}}^{\prime \prime}\right|^{2}\right.
$$

Therefore, if the anion and neutral have very different equilibrium geometries, there is a correspondingly long vibrational progression in the photoelectron spectrum, and vice versa. The theoretical evaluation of the Franck Condon factor, for the purposes of simulating the photoelectron spectra, is discussed in detail in Chapter 4.

The above description and schematic pertains to case where the upper neutral state has a bound potential. Chapter 3 , which reports the photoelectron spectra of 
$\mathrm{CN}^{-}, \mathrm{NCO}^{-}$and $\mathrm{NCS}^{-}$, provides a complete example of this bound $\rightarrow$ bound spectroscopy and shows the straightforward generalization of the above rules to polyatomics. This chapter lays much of the groundwork and language for the more complex transition state spectroscopy results that follows. The bound $\rightarrow$ free formalism required to describe the transition state experiments is detailed in Chapter 4.

\section{Relationship of the photoelectron spectrum to short time chemical dynamics.}

An alternative picture for the interpretation of a photoelectron spectrum is to consider the relationship between spectral structure and the short time dynamics of the neutral species formed by photodetachment. ${ }^{18}$ A simple Fourier transformation relates the photoelectron spectrum, $\mathrm{I}\left(\mathrm{E}_{\mathrm{k}}\right)$, with the time autocorrelation function, $\mathrm{C}(\mathrm{t})$. $\mathrm{C}(\mathrm{t})$ describes the time evolution of a wave packet prepared on the neutral potential surface. ${ }^{19}$ The time resolution of the autocorrelation function constructed in this way is given by the overall spectral bandwidth. Likewise, the window in time allowed from Fourier transformation of the experimental spectrum depends on the instrumental energy resolution. Assuming a typical bandwidth of $1-2 \mathrm{eV}$ and the energy resolution of our spectrometer $(c a .10 \mathrm{meV})$, the autocorrelation function could be constructed with a time resolution of 0.5 fs for a Lorentzian time window with a half-width at half-maximum (HWHM) of 66 femtoseconds.

This time dependent picture is developed in Chapter 4, where a derivation of the photoelectron spectrum couched in the time-dependent formulation is also given. We will, in the main, use the connection between the time dynamics, expressed by 
$C(t)$, and the spectrum in the opposite sense, i.e. wave packet propagation will be used to theoretically simulate the dissociation dynamics of the transition state species, and the Fourier tiansformation of the calculated autocorrelation is compared with the experimental photoelectron spectrum. The quantum wave packet method is also described in Chapter 4.

The theoretical simulations will prove invaluable in assigning and interpreting the structure in the transition state photoelectron spectra. Once qualitatively assigned, we may modify the potential energy surface on which the wave packet propagation takes place, to try and improve the fit between theoretical simulation and experiment. In most cases $a b$ initio calculations to estimate the properties of the anion are necessary as a precursor to the dynamics simulation because data of spectroscopic quality is scarce for negative ions. This, unfortunately, limits the extent to which the neutral reaction surface may be fit from the experimental spectrum and the simulation process.

\section{Photoelectron Angular Distribution}

The photoelectron departing the neutral molecule carries with it a weil defined, quantized, angular momentum. For atomic anion photoelectron spectroscopy the angular momentum of the leaving electron is restricted by selection rules relating to the orbital angular momentum of the parent ion and detached neutral. The nature of the allowed angular momentum states of the departing electron, and their mutual interference, along with the polarization of the detachment laser, determines the angular distribution of electrons in the laboratory frame. If the electron leaves the neutral complex as an "s-wave" ( $1=0)$ only, as for the threshold photodetachment of 
an electron from a $p$ atomic orbital of a halide ion, the angular distribution is isotropic. $^{20}$ For all other waves, and for a superposition of various waves, the distribution of electrons is anisotropic. To first order, the differential photodetachment cross section is given by

$$
\frac{d \sigma}{d \Omega}=\frac{\sigma^{2}}{4 \pi}\left(1+\beta\left(\frac{3}{2} \cos ^{2} \theta-\frac{1}{2}\right)\right)
$$

where $\sigma_{\text {total }}$ is the total photodetachment cross section and $\beta$ is the asymmetry parameter $(-1 \leq \beta \leq 2, \beta=0$ implies an isotropic distribution) and is a function of electron kinetic energy. $d \Omega$ is the solid angle subtended by an imaginary infinitesimal detector and $\theta$ is the angle the detector position vector makes with the laser polarization axis. Notice that the effect of the asymmetry parameter is removed if the direction of electron collection is chosen to be at the "magic angle", $\theta=54.7^{\circ}$.

For molecular photcelectron spectroscopy, $\beta$ parameters are often hard to rigorously relate to the symmetry of the molecular orbital in the ion from which the electron was removed. ${ }^{21}$ However, qualitative information is available from polarization measurements. Although $\beta$ may vary weakly over a vibrational progression in transitions to a single electronic state of the neutral, transitions to different electronic states of the neutral are expected to have quite different $\beta$ values, particularly if the neutral states arise by the removal of an electron from molecular orbitals of different symmetry in the anion. Therefore it is often useful, particularly in cases where the spectrum has overlapping electronic bands, to record photoelectron spectra at both extreme laser polarizations $\left(\theta=0^{\circ}\right.$ and $\left.\theta=90^{\circ}\right)$ to facilitate assignment of peaks to the different states. The actual value of $\beta$ for a photoelectron 
reaction, where to some extent the energy release for the reaction is decided. Photodetachment of $\mathrm{FH}_{2}^{-}$, on the other hand, lands to the entrance valley side of a very early barrier, and finally $\mathrm{OHF}^{-}$has an anion geometry which coincides with the anticipated saddle point geometry.

In all cases, the structure in the photoelectron spectra contains information about the ground reaction potential energy surface in the vicinity of the transition state, or equivalently the femtnsecond dynamics of the transition state complex. In each of the three chapters we make strong connections with theoretical simulations and explore possible electronically excited Born-Oppenheimer surfaces that may also contribute to the spectra. The assignment of spectral features to these excited electronic surfaces is supported by photoelectron anisotropy measurements.

\section{The Future}

The extension of the technique to reaction systems with much higher molecular complexity, e.g. $\mathrm{F}+\mathrm{ROH}$, and the success of transition state studies in solvated complexes $^{22}$ is very encouraging. This represents a significant new avenue for exploration. Diatom-diatom reactions remain an important target, especially as these present a challenge to full quantum scattering theory. For the more esoteric goal of using our spectroscopic technique to quantitatively determine a reaction's potential energy surface, much new work remains to be done but is achievable.

The negative ions, which provide such a useful springboard to learning about the neutral reaction, need to be much more quantitatively characterized. Although $a b$ initio calculations can provide helpful estimates of their properties, full spectroscopic investigation of their equilibrium geometries, vibrational energy levels 
band can often be useful in adding weight to the assignment of that band to a particular electronic state. ${ }^{21}$ This is explored in Appendix A.

\section{Transition state spectroscopy by negative ion photodetachment}

The remaining chapters of this thesis demonstrate the application of negative ion photodetachment as a transition state spectroscopy for a number of reaction systems. All are hydrogen transfer reactions of the type $\mathrm{A}+\mathrm{HB} \rightarrow \mathrm{AH}+\mathrm{B}$. This is, however, a very important class of reaction systems, ${ }^{1}$ in terms of both fundamental reaction dynainics interest and in applications such as chemical lasers and atmospheric processes. Chapter 5 reports the photoelectron spectra of $\mathrm{FHI}^{-}, \mathrm{ClHI}^{-}$ and $\mathrm{BrHI}^{-}$and describes their relationship to the bimolecular reactions $\mathrm{X}+\mathrm{HI} \rightarrow \mathrm{HX}$ $+\mathrm{I}(\mathrm{X}=\mathrm{F}, \mathrm{Cl}, \mathrm{Br})$. In Chapter 6 the series of reactions $\mathrm{F}+\mathrm{OH} \rightarrow \mathrm{HF}+\mathrm{O}, \mathrm{F}+\mathrm{CH}_{3} \mathrm{OH}$ $\rightarrow \mathrm{HF}+\mathrm{CH}_{3} \mathrm{O}$ and $\mathrm{F}+\mathrm{C}_{2} \mathrm{H}_{5} \mathrm{OH} \rightarrow \mathrm{HF}+\mathrm{C}_{2} \mathrm{H}_{5} \mathrm{O}$ are investigated. Finally, in Chapter 7 the benchmark $\mathrm{F}+\mathrm{H}_{2}$ reaction is studied by transition state spectroscopy of $\mathrm{FH}_{2}-$.

The systems featured in the three chapters turn out to illustrate three experimental scenarios. Photodetachment of $\mathrm{BrHI}^{-}, \mathrm{OHF}^{-}$and $\mathrm{FH}_{2}^{-}$accesses different parts of their respective ground neutral reaction surface. This is shown pictorially in Figure 1-2. Let us divide up the transition state (or interaction) region of the potential surface describing a direct reaction arbitrarily into three parts: the saddle and the entrance and exit valleys to either side of the saddle. The latter are not, however, the same as the asymptotic reactant or product regions. Further, we adopt the usual chemical dynamics convention and talk about a reaction proceeding from reactants (entrance) to products (exit) in the exothermic direction. In this language, we see that $\mathrm{BrHI}^{-}$photodetachment probes the exit valley for the $\mathrm{Br}+\mathrm{HI}$ 
and dissociation energies is urgently required. Using this information, experiments that control the region of the neutral potential energy surface probed in the anion photodetachment can be devised. In much the same way as collision based experiments can use rovibrational state selected reactants to probe higher detail in the reactive collision dynamics, ${ }^{6}$ so may we vibrationally pump the anion prior to photodetachment to choose the part of the reaction transition state region we wish to learn about. This is not unlike Crim's photodissociation excitation spectroscopy from O-H overtone pumped $\mathrm{H}_{2} \mathrm{O}^{23}$

Figure 1-3 shows this selectivity with a schematic of such an experiment using the negative ion $\mathrm{BrHI}^{-}$as an example. The vibrationally excited ion has substantially better overlap (lightly shaded) with the saddle point than the ground state ion (darker shading). Vibrational excitation of a single quantum in this hydrogen stretching mode $v_{3}$ requires around $920 \mathrm{~cm}^{-1}$ of energy to be resonantly deposited in the ion. ${ }^{24}$ Two possible optical excitation schemes to achieve this are shown in the lower half of Figure 1-3. Tunable infrared sources of radiation, required for scheme (i), in this range are few: difference frequency mixing of visible light in non-linear media or line tunable $\mathrm{CO}_{2}$ lasers are possibilities. Free-electron lasers, which can provide tunable, high power, $1 \mathrm{~cm}^{-1}$ bandwidth radiation in this region look particuiarly suitable, and if such a source becomes available ${ }^{25}$ this may well be the optimal photon source. Another attractive possibility is stimulated Raman pumping, as shown in scheme (ii), using two visible photons from a YAG/ dye or YAG/ Ti:sapphire laser system. ${ }^{26}$

The results of such an experiment are simulated in the closing section of Chapter 5. The simulations indicate that the restriction imposed by the anion 
equilibrium geometry may be lifted by the proposed vibrational pumping scheme. Preliminary experiments to investigate the feasibility of such an approach have demonstrated that a line tunable $\mathrm{CO}_{2}$ laser, despite its high fluence, is inappropriate for two reasons. It is not continuously tunable and the linewidth is too narrow to move a large population of rotational substates. Prior to a new effort in this direction, the exact gas phase anion fundamental frequency needs to be determined.

It is perhaps evident that spectroscopic approaches to the characterization of reaction transient species are becoming increasingly important in the experimental armory of the reaction dynamics field. The recent introduction of commercially available femtosecond laser systems will surely bring an explosion of time-domain measurements of reaction transients in the gas phase. ${ }^{15,27}$ The advance of theoretical methods to describe the quantum dynamics of a reaction, and the development of electronic structure methods that are accurate enough to compute reaction potential energy surfaces to chemical accuracy, are driven by the quality of experimental information available. Transition state spectroscopy experiments can provide the sort of high quality data on the part of a chemical transformation that is most important in this respect. 


\section{References for Chapter 1.}

1. R. D. Levine and R. B. Bernstein, Molecular Reaction Dynamics and Chemical Reactivity, Oxford University Press, New York (1987)

2. P. R. Brooks, Chem. Rev. 88, 407 (1988)

3. D. M. Neumark, Ann. Rev. Phys. Chem. 43, 153 (1992)

4. D. Brandt, L. W. Dickson, L. N. Y. Kwan and J. C. Polanyi, Chem. Phys. 39, 189 (1979)

5. D. M. Neumark, A. Wodtke, G. N. Robinson, C. C. Hayden and Y. T. Lee, J. Chem. Phys. 82, 3045 (1985)

6. for example R. Zhang, W. J. van der Zande, M. J. Bronikowski and R. N. Zare, J. Chem. Phys. 93, 4779 (1990)

7. L. Schneider, K. Seekamp-Rahn, F. Liedeker, H. Steuwe and K. H. Welge, Farad. Discuss. Chem. Soc. 91, 259 (1991); M. Vrakking, Ph. D. thesis, University of California, Berkeley (1992)

8. M. Brouard, S. P. Duxon, P. A. Enriquez, R. Sayos and J. P. Simons, Laser Chem. 11, 265 (1991)

9. E. Bohmer, S. K. Shin, Y. Chen and C. Wittig, J. Chem. Phys. 97, 2536 (1992); S. Buelow, G. Radhakrishnan, J. Catanzarite and C. Wittig, J. Chem. Phys. 83, 444 (1985)

10. B. Soep, C. J. Whitham, A. Keller and J. P. Visticot, Farad. Discuss. Chem. Soc. 91, 191 (1991)

11. H. J. Loesch and A. Remscheid, J. Chem. Phys 93, 4779 (1990); D. H. Parker, H. Jalink and S. Stolte, J. Phys. Chem. 91, 5427 (1987)

12. J. T. Brandon, S. A. Reid, D. C. Robie and H. Reisler, J. Chem. Phys. 97, 5246 (1992)

13. H-t. Wang, W. S. Felps and S. P. McGlynn, J. Chem. Phys. 67, 2614 (1977)

14. R. B. Metz, S. E. Bradforth and D. M. Neumark, Adv. Chem. Phys. 81, 1 (1992)

15. A. H. Zewail, Farad. Disc. Chem. Soc. 91, 207 (1991)

16. J. Franck, Trans. Faraday Soc. 21, 536 (1925); E. U. Condon, Physic. Rev. 32, 858 (1928)

17. G. Herzberg, Molecular Spectra and Molecular Structure Vol. I (2nd edition), pp. 194, Robert E. Krieger Publishing Company, Malabar, Florida (1989) 
18. E. J. Heller, Acc. Chem. Res. 14, 368 (1981)

19. A. J. Lorquet, J. C. Lorquet, J. Delwiche and M. J. Hubin-Franskin, J. Chem. Phys. 76, 4692 (1982); J. E. Pollard, D. J. Trevor, J. E. Reutt, Y. T. Lee and D. A. Shirley, J. Chem. Phys. 81, 5302 (1984)

20. J. Cooper and R. N. Zare, J. Chem. Phys. 48, 942 (1968)

21. K. M. Ervin and W. C. Lineberger in Advances in Gas Phase Ion Chemistry, Vol 1; N. G. Adams and L. M. Babcock, Eds., JAI Press, Greenwich, Conn. in press

22. D. M. Neumaik in Electronic and Atomic Collisions: Invited Papers of the XVI ICPEAC (AIP Conference proceedings 205) eds. A. Dalgarno, R. F. Freund, P. Cook, M. S. Luebell and T. B. Lucatorto (American Institute of Physics, New York, 1990); D. W. Arnold, unpublished work

23. R. L. Vander Wal, J. C. Scott and F. F. Crim, J. Chem. Phys. 94, 1859 (1991)

24. C. M. Ellison and B. S. Ault, J. Phys. Chem. 83, 832 (1979)

25. At the time of writing, a joint facility for Sandia National Laboratories and Lawrence Berkeley Laboratories, to be located at Berkeley, has been proposed, but awaits funding, that would include a free-electron laser.

26. B. F. Henson, G. V. Hartland, V. A. Venturo and P. M. Felker, J. Chem. Phys. 97, 2189 (1992)

27. Y. Chen, L. Hunziker, P. Ludowise and M. Morgen, J. Chem. Phys. 97, 2149 (1992) 


\section{Figure Captions for Chapter 1.}

Figure 1-1. Schematic of the photodetachment process for a diatomic negative ion. The photoelectron spectrum expected for the hypothetical potential energy curves is shown on the right hand side of the Figure.

Figure 1-2. Photodetachment of the anions (a) $\mathrm{BrHI}^{-}$, (b) $\mathrm{OHF}^{-}$and (c) $\mathrm{FH}_{2}^{-}$ access different regions on their respective neutral surface. Contours of the neutral potential and the approximate extent of the Franck Condon region (shaded) are shown. The region probed is, in all three cases, in the three atom interaction region of the potential energy surface, however each case is sensitive to a different segment of the transition state region. Assumptions for anion and neutral potential surfaces for each system detailed in chapters 5,6 and 7 .

Figure 1-3. Schematic of a vibrationally-pumped photodetachment experiment. (Top) $\mathrm{BrHI}^{-}$anion and $\mathrm{Br}+\mathrm{HI} \rightarrow \mathrm{HBr}+\mathrm{I}$ neutral potential curves along an idealized reaction coordinate. The anion $v_{3}=0$ and $v_{3}=1$ wave functions are shown, and the upward shaded regions indicate the section of neutral reaction curve detachment from each vibrational state would access. (Bottom) Excitation schemes with anion levels shown in solid lines and detachment continuum shaded; (i) direct infra-red excitation of negative ion with tunable IR laser, (ii) stimulated Raman pumping of negative ion with two-color scheme where $h v_{1}$ is "pump" and $\mathrm{hv}_{2}$ "Stokes" laser. 


$$
\mathrm{M}^{-} \stackrel{h v}{\longrightarrow} \mathrm{M}+\mathrm{e}^{-}
$$

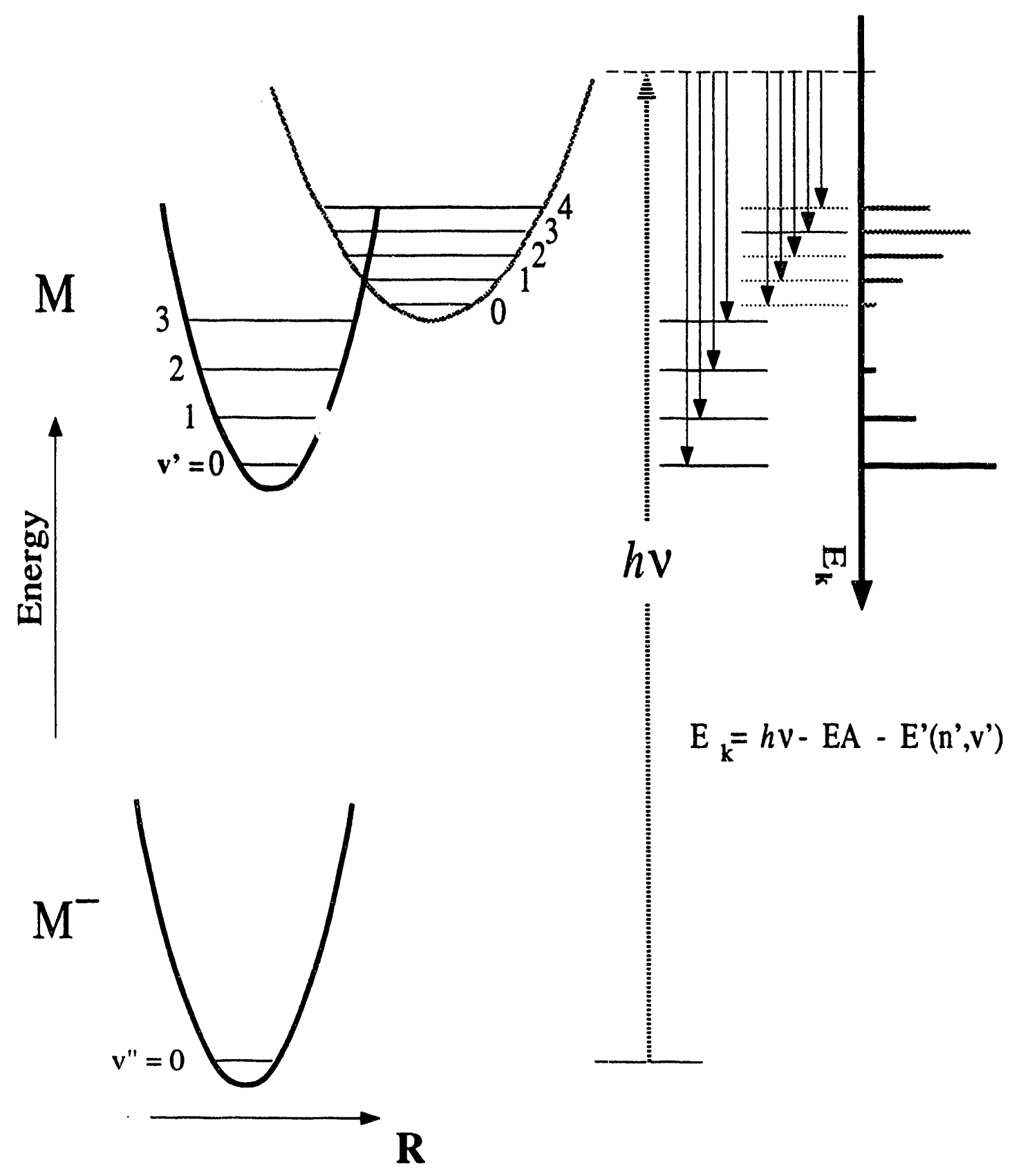

Figure 1.1 
(a) Exit Valley

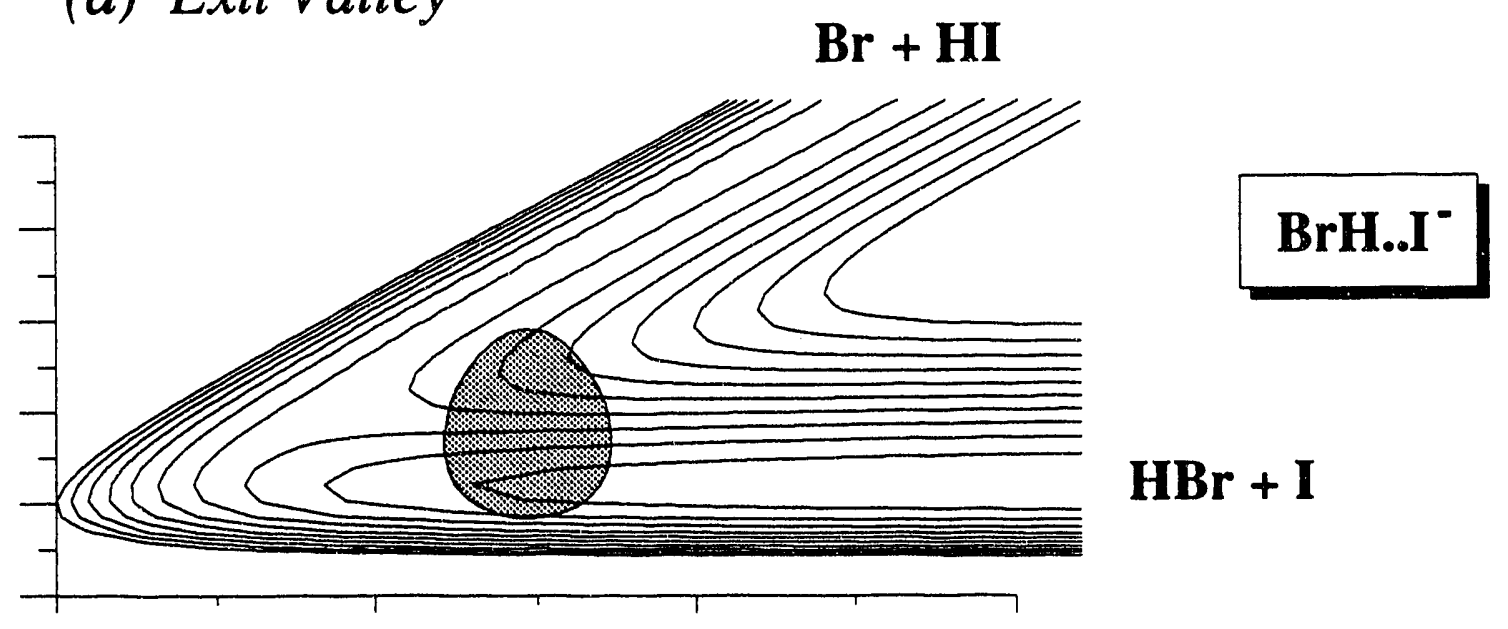

(b) Saddle to exit valley

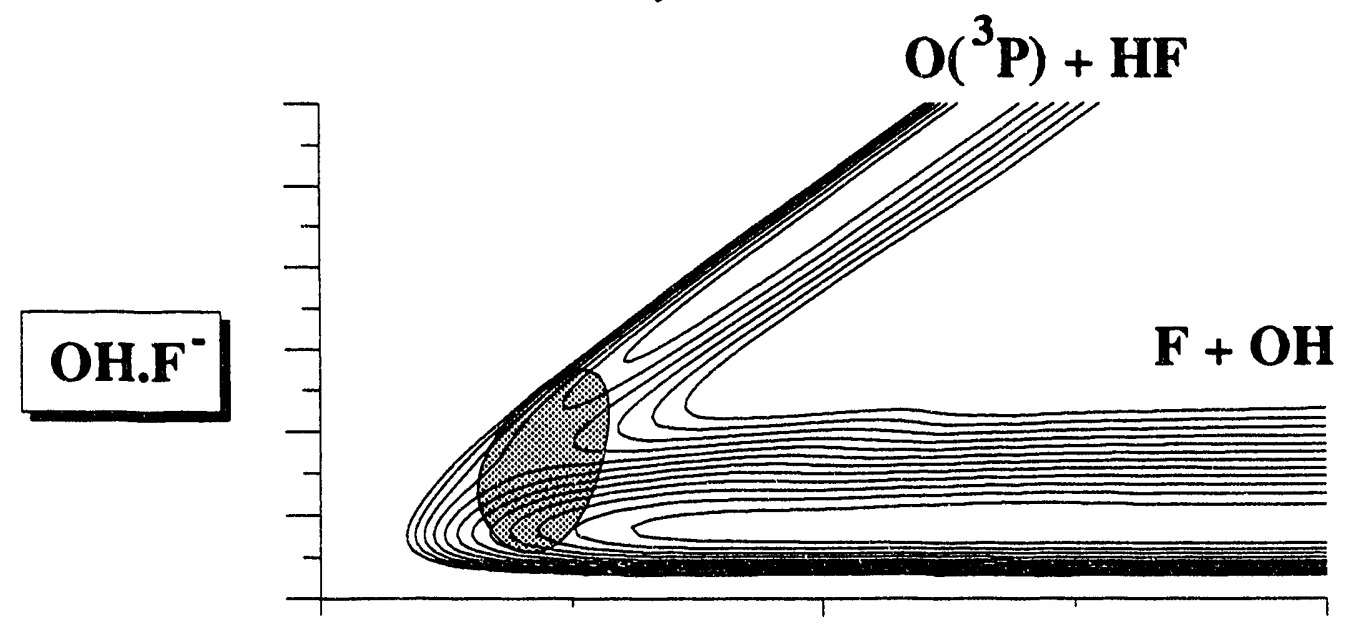

(c) Entrance valley to saddle

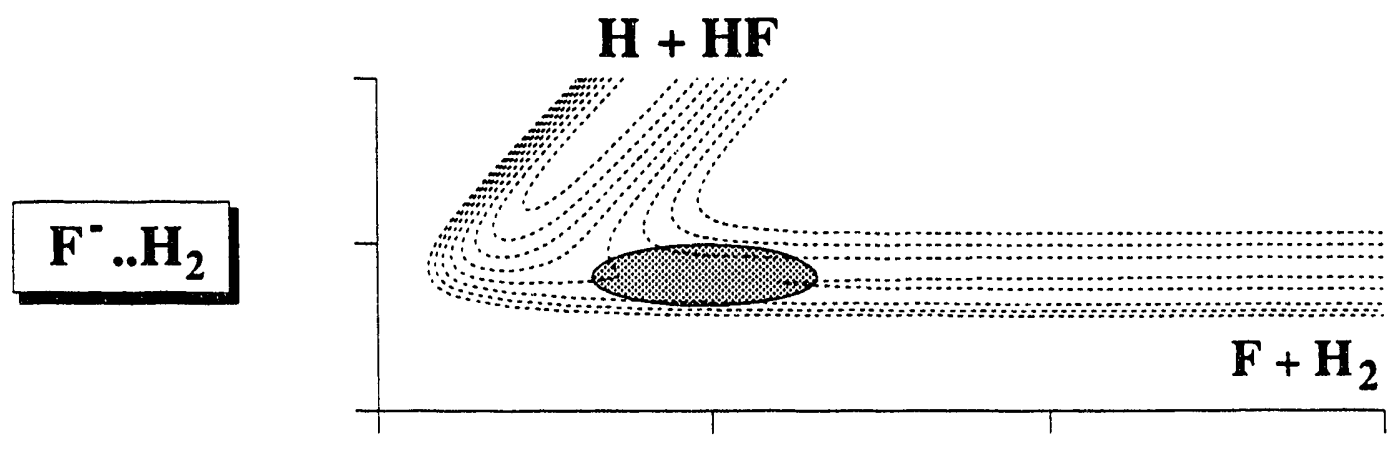

Figure 1-2 


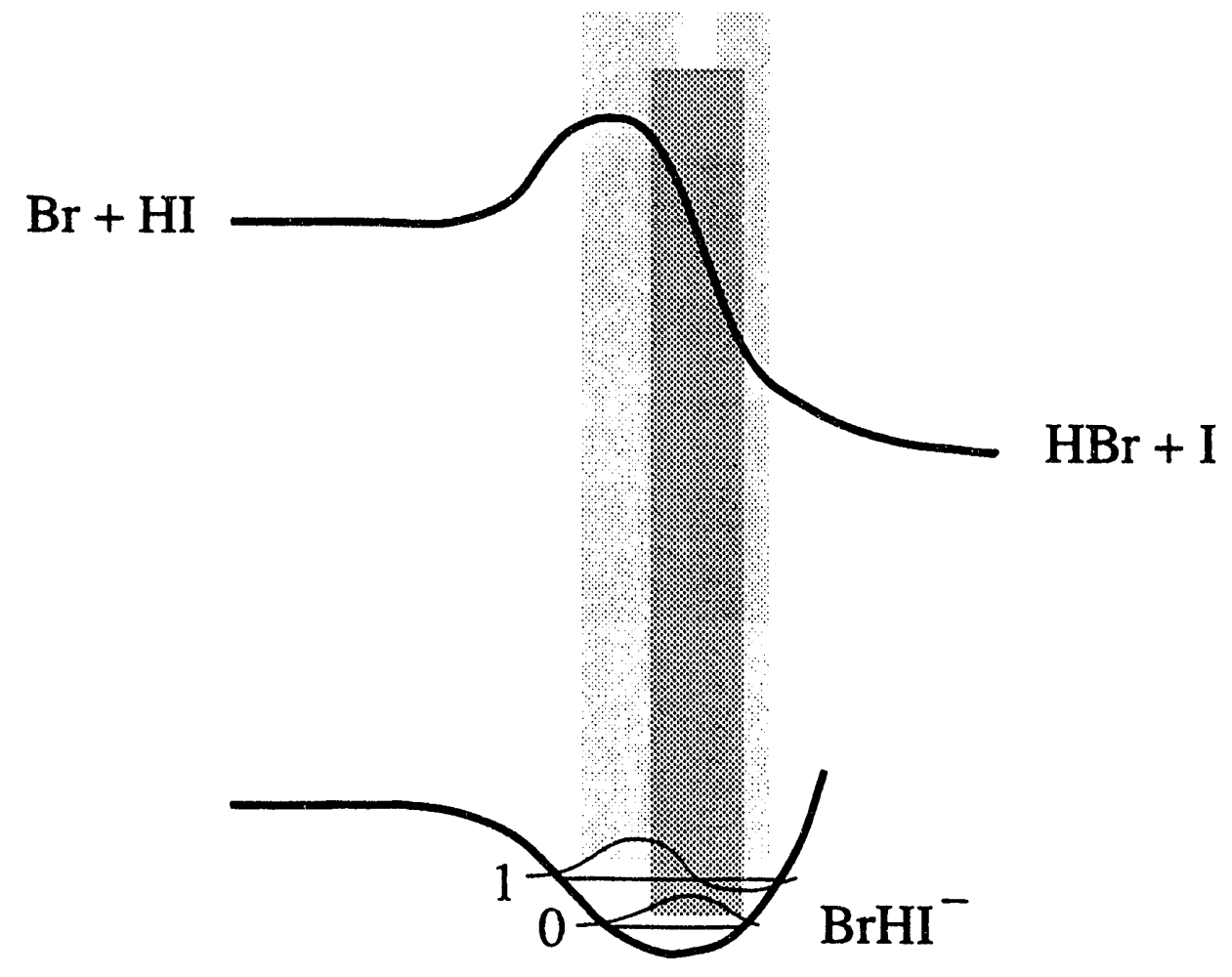

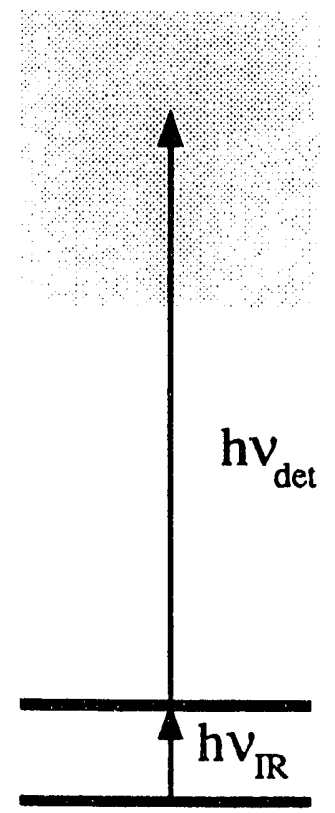

(i)

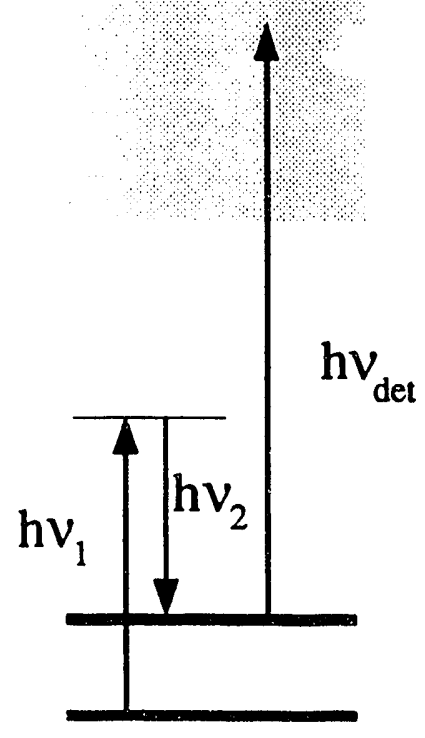

(ii)

Figure 1-3 
Chapter 2. Experimental: modifications and improvements to the negative ion photoelectron spectrometer

The experimental apparatus employed for all work presented in this thesis, a pulsed time-of-flight negative ion photoelectron spectrometer, was constructed by Theo Kitsopoulos, Ricardo Metz, Alex Weaver and Dan Neumark in 1986-7. The machine is described in detail in the $\mathrm{Ph}$. $\mathrm{D}$. thesis of Alex Weaver. ${ }^{1}$ The computer program used to control the apparatus and to perform some of the data manipulation is likewise described in the thesis of Ricardo Metz. ${ }^{2}$ A brief description of the apparatus appears here and also in each of chapters $3,5,6$ and 7 with emphasis on the particular features important to those individual studies.

In this chapter, I will describe in detail the major modifications to the apparatus hardware and enhancements to the data collection program, that have been made in the last few years. A full description of the calibration and background subtraction procedures applied to the raw data is given. As these two processes are routinely applied as a first step in analyzing virtually all data recorded on the photoelectron spectrometer, it seems appropriate to detail their correct usage.

The apparatus is a dual time-of-flight spectrometer. Ions are created in a freejet/ electron impact source of Johnson and Lineberger design. ${ }^{3}$ The ion for study is selected in a Wiley-McLaren type mass spectrometer, ${ }^{4}$ and the electrons liberated from the ion on irradiation with the photodetachment laser are energy analyzed by time-of-flight. A schematic of the experimental apparatus is shown in Figure 2-1. The experiment relies heavily on the advances in negative ion sources in recent years. The source region is shown on the left hand side. A pulsed molecular beam valve (1) 
introduces the reagent gases into the source chamber. A free jet expansion ensues which, when crossed by a fast electron beam (2), generates cold molecular ions suitable for spectroscopic study. The ion source is described in greater detail in section 1.2. The negative ions produced in the free jet are injected into a time-of-flight mass spectrometer (3) which separates them into packets according to their mass. The ion signal can be monitored at the microchannel plate detector (4). The timing of a pulsed fixed-frequency laser, which intersects the ion beam at (5), is chosen so that only the ion packet corresponding to the mass of interest is photodetached. The resulting neutrals can also be monitored at the detector (4) when a retardation field is applied to the front of the detector to block undetached ions.

The polarization of the laser radiation is varied by means of a half wave plate. The angle between the electric vector of the radiation field and the direction of electron detection, denoted by $\theta$, may therefore be controlled. Photodetachment produces electrons that travel away from the center of mass over all $4 \pi$ steradians. Only electrons that are released into the small solid angle subtended by the electron detector (6) at the end of a 1 meter flight tube are detected. The energy of each electron is determined by its flight time in the field-free flight tube (6). The resultant electron energy distribution gives information about the vibrational eigenstates, or scattering states, of the neutral.

\section{Enhancements to the apparatus}

\subsection{Electron Detection}

A simple but significant improvement to the apparatus was the installation, in July 1991, of a new electron detector with a set of larger diameter microchannel 
plates (at position (6) on Figure 2-1). The original $40 \mathrm{~mm}$ diameter plates (the quoted diameter refers to plate active area) were replaced by two new $75 \mathrm{~mm}$ diameter plates (Galileo), and a new detector mount was constructed. The channel plates are, as usual, mounted in chevron configuration. An identical circuit to that used previously is employed to bias each of the detector elements. ${ }^{1}$ The electron collection efficiency is improved by a factor of -3.5 because of the increased solid angle subtended by the electron detector at the laser interaction region. Now about $0.04 \%$ of the total photoelectrons are collected yielding a three- to four-fold signal-to-noise improvement in the photoelectron spectra. One would expect a small degradation in the electron energy resolution because of this change; there is now a greater uncertainty in the lab to center-of-mass correction term (see section 2.1 below) for the electron energy due to the increased acceptance angle of the detector. ${ }^{5}$ Calculations indicate an expected resolution of $11-12 \mathrm{meV}$ for a $0.65 \mathrm{eV}$ electron, photodetached from $\mathrm{I}^{-}$, with this new detector. Indeed, we experimentally observe the peak width of the $I\left({ }^{2} P_{1 / 2}\right) \leftarrow$ $\mathrm{I}^{-}$transition in the $266 \mathrm{~nm}$ photoelectron spectrum of $\mathrm{I}^{-}$, which occurs at this electron kinetic energy, broadens from $8-9 \mathrm{meV}$ with the $40 \mathrm{~mm}$ plates to $12-13 \mathrm{meV}$ with the new plates.

Despite the increased electron collection efficiency, the background electron signal, which is due to electrons released from metal surfaces in the detector chamber by scattered light, has been reduced. This has been achieved by new laser baffles, to reduce the amount of scattered light, and a realignment of the electron baffles (marked (7) on Figure 2-1) in the electron flight tube, ${ }^{1}$ which block many of the background electrons. Together, the improvements to the detector and to the baffling 
have increased the signal-to-background ratio for $213 \mathrm{~nm}$ photoelectron spectra by a factor of four.

These signal collection improvements make a significant difference to the experiment in several ways. Clearly, it takes less time to accumulate spectra of comparable quality to earlier work, but also it allows spectra to be recorded for systems that were impossible before the change. For example, spectra can now be recorded for systems at both parallel and perpendicular polarizations of the laser, where collecting data on one of the polarizations may have been impossible earlier due to very low signal. This is demonstrated in results presented by way of postscripts to chapters 5 and 6, and in all the new data in chapter 7. The most persuasive demonstration of the power of a "factor of four" is the recent successful observation of resonances in the $213 \mathrm{~nm}$ photoelectron spectrum of $\mathrm{ClHCl}^{-6}$, which had eluded us in many previous attempts!

\subsection{Ion Source}

The operation of our ion source is described in Weaver's thesis. ${ }^{1}$ Synthesis of a large variety of negative ions has been achieved in this source. The modifications to our basic source, necessary to make various ions, and some of the more unconventional modes of operation of the source, are described below. For example, a small mixing chamber can be added to the front of the main pulsed valve for the purpose of mixing a secondary gas into the expansion. A more complicated version of this arrangement involves two pulsed valves. To improve the cooling and clustering properties of the pulsed expansion, a piezo-electric valve has been incorporated into 
our source. An important piece of diagnostic equipment for this work is the Fast Ion Gauge $^{8}$ (FIG) for testing the gas pulses from each valve arrangement.

The two main components of the source are the pulsed valve, or the device that introduces the gaseous sample into the vacuum system, and the electron beam that crosses the free jet and induces various fragmentation, ionization and electron capture events which in turn generate the desired negative ions. The continuous electron beam, produced from an electron gun of a Tektronix scope, is easy to manipulate; consequently this component of our source is seldom altered. The pulsed valve in contrast has seen numerous changes. The 'basic' arrangement is a small pulsed commercial solenoid valve, a General Valve Series 9 (General Valve Corporation, Fairfield N.J.), which is backed by $2-5$ atmospheres of a dilute gas mixture. The operation of this valve and the pulsing circuits used to drive it are described in detail elsewhere. ${ }^{1,9}$ Synthesis of many of the negative ions described in this thesis have been achieved with this basic arrangement.

Often, a precursor required as a reagent in the jet exists in the liquid state under standard conditions. If the liquid is volatile at room temperature, the liquid may be entrained into a carrier gas by bubbling the carrier through the liquid and then pulsed successfully into the vacuum chamber. All valves will seize up after a period of time in this mode of operation, and need some serious maintenance work. An advantage of the simple solenoid valve, over a more advanced valve like the piezoelectric valve, is the General valve has only a few internal parts, which may be cleaned, or if necessary, cheaply replaced in a few minutes. Moreover, it is often hard to get sufficient liquid entrained into the carrier gas, i.e the liquid does not have a sufficiently large vapor pressure at room temperature. The General Valve is robust 
enough that the liquid sample may be physically dropped into the body of the valve, while it is hanging in vacuum, and then the valve pulsed a few times to flow some of the liquid $\cdots$ r the surfaces inside the valve. Once this initial cycling has been completed, a fairly stable beam of the precursor, entrained in the carrier at much higher density, can be achieved. This 'short cut' has been employed for the liquids benzyl isocyanate, benzyl thioisocyanate, formic acid and methanol to prepare the ions $\mathrm{NCO}^{-}, \mathrm{NCS}^{-}, \mathrm{HCO}_{2}^{-}$and $\mathrm{CH}_{3} \mathrm{OH} . \mathrm{F}^{-}$respectively.

Further experimentation with pulsed nozzle design however has been necessitated by the quest for other negative ions. A common requirement is for two different reagent gases to be present in an expansion in order to perform the desirsd ion-molecule chemistry in the electron beam interaction region. One gas mav be required to produce the seed ion, say $\mathrm{N}_{2} \mathrm{O}$ to prod ce $\mathrm{O}^{-}$, and the secund as the target molecule for reaction or clustering, e.g. methar 9 or hydrogen for the processes $\mathrm{O}^{-}+$ $\mathrm{CH}_{4} \rightarrow \mathrm{OH}^{-}+\mathrm{CH}_{3}$ or $\mathrm{O}^{-}+\mathrm{H}_{2} \rightarrow \mathrm{O}^{-}\left(\mathrm{H}_{2}\right)$. In many cases, the two gases can be premixed thoroughly in a stainless steel cylinder, for example $\mathrm{N}_{2} \mathrm{O}$ and $\mathrm{HCl}$ used in the source clustering reaction of $\mathrm{O}^{-}$with $\mathrm{HCl}$. However, in these two examples the reagent gases definitely cannot be premixed at high pressure before introduction to the pulsed valve. Other such combinations of active gases, e.g. oxidizing and reducing agents or acid/ base gaseous mixtures, would often be desirable for producing some interesting ions, but are impossible to premix and thus to use with the single valve inlet arrangement. Instead, each active gas needs to be introduced separately into the jet expansion. Another powerful use of such a double inlet arrangement would be for varying the ratio of two active gases at run time, rather than finding the optimal ratio 
for premixing by trial and error - each trial mix needs to stand for several hours. For some of the more exotic $\mathrm{AHB}^{-}$ions (where $\mathrm{A} \neq \mathrm{B}$ ) this would be extremely useful.

The basic General valve is ideal for designing hybrid gas inlet assemblies as it is small and it is easy to attach add-ons to its flat faceplate. We have experimented with both continuous and pulsed secondary gas inlets. The idea is to merge the secondary reagent gus with the main pulsed beam after the pulsed valve but prior to the free jet expansion. The gas through the main valve carries one reagent and the carrier gas fo the expansion, and the secondary reagent gas is introduced via a small mixing chamber (see Figure 2-2) attached to the exterior of the pulsed nozzle orifice. Ideally, the mixing is optimized by creating maximum turbulence in the main flow within the mixing chamber. This is how we arrived at the triple-injector design of Figure 2-2. A free jet expansion, albeit somewhat weaker than that from the unimpeded pulsed valve, then takes place from the front aperture of this mixing chamber. This approach has been successful for producing good denaities of $\mathrm{OH}^{-}$by the reaction of $\mathrm{O}^{-}\left(\right.$from $\left.\mathrm{N}_{2} \mathrm{O}\right)$ with $\mathrm{NH}_{3}$ introduced continuously through the "mixing chamber". The flow of ammonia is controlled by a leak valve outside the chamber. $\mathrm{OH}^{-}$is surprisingly difficult to make from any simple dissociative attachment process; weither $\mathrm{H}_{2} \mathrm{O}$ or $\mathrm{H}_{2} \mathrm{O}_{2}$ produces $\mathrm{OH}^{-}$in our source! Clusters based on $\mathrm{OH}^{-}$are in fact highly desirable targets for future transition state studies in our laboratory.

A pulsed design for the secondary gas inlet would clearly be advantageous over the above continuous scheme so as to reduce the gas load on the pumping system and to increase the density of the secondary gas present during the main valve pulse cycle. An in-line General Valve (Series 9, two way) has successfully been incorporated into the secondary gas supply line to the mixing chamber. Instead of the mix chamber 
design of Figure 2-2, we use a similar, but simpler, single injector (0.070" diameter) chamber; the central hole, for the main flow, has the same 0.080 " diameter as before. Unfortunately the secondary gas pulses are not limited by the in-line valve's open time, but rather by the small conductance of the $1 / 8$ " tubing (inner diameter is only 0.052 ") attaching the second valve to the mix chamber. Even so, the $-2 \mathrm{~ms}$ duration pulses reduce the pumping load by a factor of ca. 25 when running at $20 \mathrm{~Hz}$, allowing much higher concentrations of the secondary gas in the expansion at reasonable total source chamber pressures. The yield of $\mathrm{FHCl}^{-}$in the mass spectrometer, when running $\mathrm{NF}_{3}$ behind the main valve and $5 \% \mathrm{HCl} / \mathrm{He}$ behind the second in-line pulsed valve, can be varied over an order of magnitude by adjusting the pulsing delay and duration of the in-line valve driving circuit.

Despite the flexibility of the General valve, the fast ion gauge clearly shows that the gas pulses produced by this valve are not limited only by the flow through the faceplate aperture ('choke' flow). The internal armature of the valve, which withdraws the poppet sealing the valve when the solenoid is activated, does not respond very fast compared to the overall pulse duration. This problem is compounded by the wedge tip design of the poppet in the General Valve, and so choke flow is achieved only very late in the gas pulse. The properties of a free jet expansion that make its use attractive to spectroscopists are the high degree of internal cooling achieved in the jet and the high local molecular densities that accompany this dramatic cooling. Pulsed valves are used to reduce the overall load on the vacuum system. Thus a pulsed valve that delivers a high gas intensity in a short pulse, with choked flow during the large part of that pulse, is optimal. The above two problems of the General valve in this respect are mostly removed in the piezo-electric valve. ${ }^{8,10}$ This valve has a fast rise 
time - the motion of the piezo-electric crystal keeps up with the high voltage driving pulse - and uses a flat o-ring, rather than a wedge, seal against the faceplate aperture. We have therefore recently built ${ }^{11}$ and used a piezo-electric valve in our experiments on $\mathrm{FH}_{2}{ }^{-}$and other clusters. ${ }^{12}$

\section{Procedures for improved data collection}

\subsection{Calibration description}

The calibration procedure has been described briefly in the thesis of Alex Weaver. ${ }^{2}$ Here we outline the procedure in full, detailing several new aspects of this important part of the photoelectron experiment. The calibration of $213 \mathrm{~nm}$ photoelectron spectra is dealt with; hitherto there were no good calibrant ions to anchor the electron energy scale for this laser photon energy $(5.822 \mathrm{eV})$ in the region of principal use (0.5 - $1.5 \mathrm{eV})$. This work has indicated a more serious problem in the calibration process itself. The linear calibration procedure used by our group up until now proves inadequate to accurately calibrate over a $2 \mathrm{eV}$ range of electron energies. A quadratic scheme, similar to one used in the Zare group for their multiphoton ionization photoelectron spectrometer (MPI-PES) apparatus, ${ }^{13}$ has been implemented, and yields a much more useful and accurate energy scale. This is important for the precise determination of electron affinities and electronic state separations from spectra recorded on our apparatus.

Conversion of the electron flight time, $t$, measured on our apparatus, to the electron center-of-mass kinetic energy, $\mathrm{E}_{\mathrm{e}^{-}, \text {com }}$, is given, in the absence of any electric or magnetic fields, by 


$$
E_{a, 00 m}-\Delta E=\frac{1}{2} m_{e} \frac{l^{2}}{\left(t-t_{0}\right)^{2}}
$$

where $\Delta \mathrm{E}$ is the correction term to the electron energy from the laboratory to centerof-mass frame. For electron detection at right angles to the ion beam, $\Delta \mathrm{E}$ is given by

$$
\Delta E=\frac{m_{e}}{m_{\text {ben }}}\left(V_{f}+\frac{V_{\text {ext }}}{2}\right)
$$

and $m_{e}$ and $m_{i o n}$ are the mass of the electron and the mass of the ion from which the electron was photodetached. ${ }^{1} V_{f}$ and $V_{e x t}$ are the experimental float and extraction voltages, which have been set up in the experiment to yield a stable and focussed ion packet at the interaction region for the particular ion of mass $m_{i o n}$. The parameters 1 and $t_{0}$, respectively, describe the zero of time and the distance from the laser interaction region to microchannel plate detector. To find these parameters, which in fact do vary from day to day, it is necessary to record photoelectron spectra of several atomic ions, to calibrate the energy scale. The energies for the transitions of these calibrant ions are well known to fractions of $\mathrm{meV}$.

To perform this calibration we invert equation (1) to yield

$$
t=t_{0}+\sqrt{\frac{m_{e} l^{2}}{2}} \cdot \frac{1}{\sqrt{E-\Delta E}}+\frac{\gamma}{E-\Delta E}
$$

where we have dropped the $\mathrm{e}^{-}$, com label for the electron energy, and we have included an extra term, $\gamma /(E-\Delta E)$, for flexibility. For the moment, we shall assume $\gamma=0$, however later we will see it is necessary in some cases to include a non-zero $\gamma$ to yield a reasonable calibration fit. The introduction of this term physically corresponds to recognition that there is some acceleration of the electron over its flight 
path, and therefore not all fields have been eliminated. One important field that should not be neglected is the interaction of the electron with the residual charge cloud due to the undetached ions.

The calibration takes place as follows. A set of photoelectron spectra are recorded at the lowest possible ion levels, or at least below the "space charge limit" for that ion. The Coulomb repulsion of the residual ion cloud on the departing electron shifts the peaks in the ion's photoelectron spectrum to higher electron kinetic energy. The space charge limit is the highest ion density at which the peaks in the ion's photoelectron spectrum are unshifted due to this Coulomb repulsion. This varies for ion to ion, and to some extent on the nature of the ion's spectrum. For example, a molecular, rather than atomic, calibrant's photoelectron spectrum will have broader peaks and so the onset of Coulomb shifting will seem to appear at a higher ion level. For cases where it proves impossible to obtain spectra at the space charge limit (usually around $10 \mathrm{mV}$ ion level, with ion detector stack voltage set at $1650 \mathrm{~V}$, see Table 2-1), because of signal-to-noise considerations, extrapolation down to "zerospace-charge" will be necessary. This should occur only for $\mathrm{CN}^{-}$calibration at $213 \mathrm{~nm}$ because of the high background level for the low electron energy lines. In my experience this calibrant ion may be satisfactorily run at $80 \mathrm{mV}$ ion level, and a "zerospace-charge" extrapolation (of the order of $5 \mathrm{meV}$ for $80 \mathrm{mV} \mathrm{CN}^{-}$ion level), with care, can be carried out to good accuracy."1 These more tedious measures are

"1 This assumes that the space charge shift is constant for all peaks in the ion's photoelectron spectrum. Recent work shows that there is a dependence on the electron's kinetic energy, but that this is small for overall shifts less than 10 meV. 
unfortunately necessary as the calibration fit for $213 \mathrm{~nm}$ can be poor without lines at low electron kinetic energies.

Table 2-1. Estimated space charge shift (in $\mathrm{meV}$ ) for calibrant lines, as a function of ion level. Ion levels are height of ion peak measured on scope trace (in $\mathrm{mV}$ ) when ion detector stack voltage set at $1650 \mathrm{~V}$.

\begin{tabular}{cccccc}
\hline \hline & $\begin{array}{r}\text { Ion Level } \\
\text { Ion }\end{array}$ & $100 \mathrm{mV}$ & $50 \mathrm{mV}$ & $30 \mathrm{mV}$ & $10 \mathrm{mV}$ \\
\hline $\mathrm{F}^{-}$ & 19 & 4.5 & 3.0 & 1.5 & 0.0 \\
$\mathrm{CN}^{-}$ & 26 & 5.0 & 3.0 & 1.5 & 0.0 \\
$\mathrm{Cl}^{-}$ & 35 & 6.0 & 4.0 & 2.5 & 0.0 \\
$\mathrm{Br}^{-}$ & 79 & & & 4.5 & 1.0 \\
$\mathrm{I}^{-}$ & 127 & & 10.0 & 4.0 & 1.0 \\
\hline \hline
\end{tabular}

For each calibrant photoelectron spectrum, the time-of-flight for each spectral line and the extraction and float voltages set while obtaining that ion's spectrum, $\mathrm{V}_{\text {ext }}$ and $V_{f}$, are recorded. Note, in order to achieve zero-space-charge conditions for each calibrant ion the extraction voltage may vary widely. It is therefore important to record, to the nearest $10 \mathrm{~V}$, the extraction voltage used for each ion so as to compute accurately the center-of-mass correction, which may be as large as $35 \mathrm{meV}^{-\mathrm{F}^{-}}$) and depends on $V_{\text {ext }}$ according to Eqn. 2. This set of observed information, along with the expected electron energies (Table 2-2 and 2-3) are used to perform a weighted linear least squares fit to Eqn. 3. ${ }^{14}$ The uncertainties in the time-of-flight for each line are the weights input into the fit; for most lines this is the $2.5 \mathrm{~ns}$ uncertainty in estimating a peak center. This fitting procedure has been simply and conveniently 
automated $^{* 2}$ in the TENURE data acquisition computer program, ${ }^{2}$ and should be performed at the time of calibrant data collection. The goodness-of-fit, $\chi^{2}$, a quality factor (Q) and any disparities in the fit are displayed, along with an root-mean-square (rms) error, in meV. $Q$ is the probability of $\chi^{2}$, equal to or poorer (higher) than that in the fit, occurring by chance. ${ }^{14} Q$ factors higher than 0.50 (1.00 is perfect fit, indicative of a fudge!) and rms errors smaller than $2 \mathrm{meV}$ are usually obtainable, $\mathbf{Q}$ factors above 0.95 usually indicate that the uncertainties in the time-of-fiight datapoints have been over-estimated. Because this information is available at run time, the operator can go back and re-record specific calibration spectra immediately if this is deemed necessary. In this way highly accurate electron energies, with quantitative error bars, can be deduced from recorded spectra. This procedure has been adopted in the reporting of all spectra in this thesis, and particular attention has been paid to this issue in Chapter 3.

\subsubsection{Calibration at $213 \mathrm{~nm}$}

Whenever a spectrum has peaks over a broad range of electron energies, the electron energy scale needs to be calibrated over the entire range. This is generally the case for our $213 \mathrm{~nm}$ spectra, although it may be true at any other wavelength. This causes a particular problem for $213 \mathrm{~nm}$, as the shortest wavelength of our atomic calibrant lines $\left.\left(\mathrm{I}^{2} \mathrm{P}_{1 / 2}\right) \leftarrow \mathrm{I}^{-}\right)$yields electrons with $1.82 \mathrm{eV}$ kinetic energy. New calibrant lines to cover smaller electron kinetic energies at this laser wavelength are required. No suitable atomic ions have excited states in this region. The $\mathrm{CN}^{-}$

"2 The subroutine that performs the calibration in the TENURE program has been completely rewritten and is reproduced in Appendix A. 
photoelectron spectrum does have several lines spanning this electron kinetic energy range, ${ }^{15}$ and so we choose this diatomic as our calibrant of the $213 \mathrm{~nm}$ spectra, along with some of the halide ions for the high electron kinetic energy end of the scale. Using a diatomic ion has disadvantages; the peaks are broader and peak shapes temperature dependent, and so assignment of peak centers have larger uncertainties.

For $213 \mathrm{~nm}$ calibration, and for any other laser wavelength where a large energy range is being calibrated, a satisfactory linear calibration of the energy scale is impossible, and a quadratic fit is needed. Then the factor $\gamma$, called the quadratic scale compression factor, is non-zero in Eqn. 3. It is noted that $\gamma$ should always be negative and typically has a value in the range of -100 to $-130 \mathrm{eV} \cdot \mathrm{ns}$. Once the inclusion of this parameter becomes necessary, the conversion of observed flight times to electron kinetic energies no longer follows Eq. 1, but instead is given by

$$
E_{e-, 00 m}-\Delta E=\frac{b^{2}+2 \gamma\left(t-t_{0}\right)+\sqrt{b^{2}\left(b^{2}+4 \gamma\left(t-t_{0}\right)\right)}}{2\left(t-t_{0}\right)^{2}}
$$

where $b^{2}=1 / 2 m_{e} \ell^{2}$. Eq. 4 reduces to Eq. 1 in the limit of $\gamma \rightarrow 0$, as required. This reformulation of the time-of-flight to energy relationship has been included into the data acquisition program TENURE. 
Table 2-2 Calibration lines typically used for UV laser wavelengths. Electron kinetic energies for atomic and diatomic transitions at 213, 266, 299 and 355 nm."

\begin{tabular}{|c|c|c|c|c|c|c|}
\hline & Transition $^{b}$ & Mass & $\begin{array}{l}213 \mathrm{~nm} \\
5.822 \mathrm{eV} \\
\end{array}$ & $\begin{array}{c}266 \mathrm{~nm} \\
4.657 \mathrm{eV} \\
\end{array}$ & $\begin{array}{l}299 \mathrm{~nm}^{\circ} \\
4.141 \mathrm{eV} \\
\end{array}$ & $\begin{array}{c}355 \mathrm{~nm} \\
3.493 \mathrm{eV} \\
\end{array}$ \\
\hline $1 \%$ & $I\left(P{ }_{s / 2}\right)$ & 127 & 2.7624 & 1.5981 & 1.0821 & 0.4338 \\
\hline $\mathrm{I}^{-}$ & $\mathrm{I}\left({ }^{2} \mathrm{P}_{1 / 2}\right)$ & 127 & 1.8197 & 0.6554 & 0.1394 & \\
\hline $\mathrm{Br}^{-}$. & $\mathrm{Br}\left(\mathrm{C}^{2} \mathrm{P}_{\mathrm{B} / 2}\right)$ & 79 & 2.4579 & 1.2936 & 0.7776 & 0.1293 \\
\hline $\mathrm{Br}^{-}$ & $\mathrm{Br}\left({ }^{2} \mathrm{P}_{1 / 2}\right)$ & 79 & 2.0010 & 0.8367 & 0.3207 & \\
\hline $\mathrm{Cl}^{-}$ & $\mathrm{Cl}\left({ }^{2} \mathrm{P}_{3 / 2}\right)$ & 35 & 2.2088 & 1.0445 & 0.5285 & \\
\hline $\mathrm{Cl}^{-}$ & $\mathrm{Cl}\left({ }^{2} \mathrm{P}_{1 / 2}\right)$ & 35 & 2.0994 & 0.9351 & 0.4191 & \\
\hline $\mathrm{F}^{-}$ & $\mathrm{F}\left(\mathbf{P}_{3,2}\right)$ & 19 & 2.4203 & 1.2560 & 0.7400 & \\
\hline $\mathrm{F}^{-}$ & $F\left({ }^{2} P_{1 / 2}\right)$ & 19 & 2.3702 & 1.2059 & 0.6899 & \\
\hline $\mathrm{CN}^{-}$ & $\mathrm{CN}(\mathrm{X}, \mathrm{v}=0)$ & 26 & 1.961 & 0.796 & 0.280 & \\
\hline $\mathrm{CN}^{-}$ & $\mathrm{CN}(\mathrm{A}, \mathrm{v}=0)$ & 26 & 0.831 & & & \\
\hline $\mathrm{CN}^{-}$ & $\operatorname{CN}(A, v=1)$ & 26 & 0.609 & & & \\
\hline $\mathrm{CN}^{-}$ & $\mathrm{CN}(\mathrm{A}, \mathrm{v}=2)$ & 26 & 0.391 & & & \\
\hline
\end{tabular}

a) All electron kinetic energies correspond to the zero space charge limit.

b) $\mathrm{CN}^{-}$transitions labelled by neutral electronic $\left(\mathrm{X}^{2}\left({ }^{2} \Sigma^{+}\right), \mathrm{A}\left({ }^{2} \Pi\right)\right)$ and vibrational state. See Figures 3-1 and 3-2 for sample spectra.

c) First Stokes Raman line in $\mathrm{H}_{2}$ using Nd:YAG fourth harmonic (266 nm) as pump wavelength.

Data used to construct table: Nd:YAG fundamental $1064.8 \mathrm{~nm},\left(9391 \mathrm{~cm}^{-1}, 1.164 \mathrm{eV}\right)$. First Stokes Raman scattering in $\mathrm{H}_{2}, v=v_{\text {pump }}-0.516 \mathrm{eV} .{ }^{16} \mathrm{EA}(\mathrm{I})=3.0591 \mathrm{eV},{ }^{17}$ $\mathrm{EA}(\mathrm{Br})=3.363590 \mathrm{eV},{ }^{17} \mathrm{EA}(\mathrm{Cl})=3.61269 \mathrm{eV},{ }^{17} \mathrm{EA}(\mathrm{F})=3.401190 \mathrm{eV}^{17}$ and raw $\mathrm{EA}(\mathrm{CN})=3.861(3) \mathrm{eV} .{ }^{15}$ Atomic spin orbit splittings: $\left(\mathrm{F}, 0.05010 ;{ }^{18} \mathrm{Cl}, 0.10940 ;{ }^{18} \mathrm{Br}\right.$, $0.4569 ;{ }^{19}$ and $\left.0.94268 \mathrm{eV},{ }^{19}\right)$. CN electronic and vibrational levels. ${ }^{16}$ 
Table 2-3 Calibration lines typically used for near UV and visible laser wavelengths. Electron kinetic energies for atomic and diatomic transitions at $355,416,532 \mathrm{~nm}^{\mathrm{a}}$

\begin{tabular}{|c|c|c|c|c|c|}
\hline & Trissition & Mass & $\begin{array}{c}355 \mathrm{~nm} \\
3.493 \mathrm{eV} \\
\end{array}$ & $\begin{array}{c}416 \mathrm{~nm}^{\mathrm{c}} \\
2.977 \mathrm{eV}\end{array}$ & $\begin{array}{c}532 \mathrm{~nm} \\
2.329 \mathrm{eV} \\
\end{array}$ \\
\hline $\mathrm{O}_{2}-$. & $\mathrm{O}_{2}(\mathrm{X}, \mathrm{v}=0)$ & 32 & 3.039 & 2.523 & 1.875 \\
\hline $\mathrm{O}_{2}^{-}$ & $\mathrm{O}_{2}(\mathrm{X}, \mathrm{v}=1)$ & 32 & 2.846 & 2.330 & 1.682 \\
\hline $\mathrm{O}_{2}-$. & $\mathrm{O}_{2}(\mathrm{X}, \mathrm{v}=2)$ & 32 & 2.656 & 2.140 & 1.492 \\
\hline $\mathrm{O}_{2}^{-}$ & $\mathrm{O}_{2}(\mathrm{X}, \mathrm{v}=3)$ & 32 & 2.469 & 1.953 & 1.305 \\
\hline $\mathrm{O}_{2}$ & $\mathrm{O}_{2}(\mathrm{X}, \mathrm{v}=4)$ & 32 & 2.285 & 1.769 & 1.121 \\
\hline $\mathrm{O}_{2}^{-}$ & $\mathrm{O}_{2}(\mathrm{X}, \mathrm{v}=5)$ & 32 & - & 1.588 & 0.940 \\
\hline $\mathrm{O}_{2}-$ & $\mathrm{O}_{2}(\mathrm{a}, \mathrm{v}=0)$ & 32 & - & 1.546 & 0.897 \\
\hline $\mathrm{O}_{2}^{-}$ & $\mathrm{O}_{2}(\mathrm{a}, \mathrm{v}=1)$ & 32 & 1.881 & 1.365 & $\begin{array}{l}0.726 \\
0.706^{d}\end{array}$ \\
\hline $\mathrm{O}_{2}^{-}$ & $\mathrm{O}_{2}(\mathrm{a}, \mathrm{v}=2)$ & 32 & 1.703 & 1.187 & $\begin{array}{l}0.548 \\
0.528^{d}\end{array}$ \\
\hline $\mathrm{O}_{2}^{-}$ & $\mathrm{O}_{2}(\mathrm{a}, \mathrm{v}=3)$ & 32 & 1.528 & 1.012 & - \\
\hline $\mathrm{O}_{2}^{-}$ & $\mathrm{O}_{2}(\mathrm{a}, \mathrm{v}=4)$ & 32 & 1.356 & 0.840 & 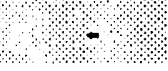 \\
\hline $\mathrm{O}_{2}^{-}$ & $\mathrm{O}_{2}(\mathrm{~b}, \mathrm{v}=1)$ & 32 & 1.238 & 0.722 & - \\
\hline $\mathrm{O}_{2}^{-}$ & $\mathrm{O}_{2}(\mathrm{a}, \mathrm{v}=5)$ & 32 & 1.187 & 0.671 & 2 \\
\hline $\mathrm{O}_{2}^{-}$ & $\mathrm{O}_{2}(\mathrm{~b}, \mathrm{v}=2)$ & 32 & 1.067 & 0.551 & - \\
\hline $\mathrm{O}_{2}^{-}$ & $\mathrm{O}_{2}(\mathrm{~b}, \mathrm{v}=3)$ & 32 & 0.900 & - & - \\
\hline $\mathrm{O}_{2}^{-}$ & $\mathrm{O}_{2}(\mathrm{~b}, \mathrm{v}=4)$ & 32 & 0.736 & - & - \\
\hline $\mathrm{I}^{-}$ & $\mathrm{I}\left({ }^{2} \mathrm{P}_{3 / 2}\right)$ & 127 & 0.434 & - & - \\
\hline
\end{tabular}

a) All electron kinetic energies correspond to the zero space charge limit. All $\mathrm{O}_{2}-$ transitions are to centers of unresolved spin orbit doublets.

b) $\mathrm{O}_{2}^{-}$transitions labeled by neutral electronic $\left(\mathrm{X}\left({ }^{3} \Sigma_{\mathrm{g}}^{-}\right), \mathrm{a}\left({ }^{1} \Delta_{\mathrm{g}}\right), \mathrm{b}\left({ }^{1} \Sigma_{\mathrm{g}}^{+}\right)\right)$and vibrational state. See sample spectra in Figure 2-3 and 2-4. 
Table 2-3 continued

c) First Stokes Raman line in $\mathrm{H}_{2}$ using Nd:YAG third harmonic (355 $\mathrm{nm}$ ) as pump wavelength.

d) Spin orbit splitting $(20 \mathrm{meV})$ of anion $\mathrm{O}_{2}^{-}$resolved in these transitions.

Data used to construct table: Nd:YAG fundamental $1064.8 \mathrm{~nm}\left(9391 \mathrm{~cm}^{-1}, 1.164 \mathrm{eV}\right)$.

First Stokes Raman scattering in $\mathrm{H}_{2}, v=v_{\text {pump }}-0.516 \mathrm{eV} \cdot{ }^{16} \mathrm{EA}(\mathrm{I})=3.0591 \mathrm{eV},{ }^{17} \mathrm{raw}$ $\operatorname{EA}\left(\mathrm{O}_{2}\right)=0.454(3),{ }^{20}$ and $\mathrm{O}_{2}$ electronic and vibrational levels from Ref. 16. 


\subsection{Background subtraction}

The background signal, despite improvements described in section 1.1, can still be signifirart for $213 \mathrm{~nm}$ photoelectron spectroscopy. We estimate that around one background electron is collected every shot at this laser wavelength. This signal must be removed to restore the correct intensity distribution in the molecular photoelectron spectrum. As the spectrum of background electrons is smooth and does not change from day to day, the background signal may be separately averaged, typically for at least 120,000 laser shots, and stored. This background spectrum may be filtered to remove any high frequency noise, and then scaled and subtracted from the photoelectron spectrum of the ion under study. This procedure is less time consuming than background subtraction at run time, where alternately 250 shots, say, of signal + background and then 250 shots of background are collected and subtracted. Additionally, as the signal-averaged background spectrum is filtered before subtraction, the overall signal-to-noise for the subtracted data is higher than in the "run time" method.

The method of filtering the background data deserves some discussion. The background time-of-flight dataset is fast Fourier transformed. ${ }^{14}$ A fast Fourier

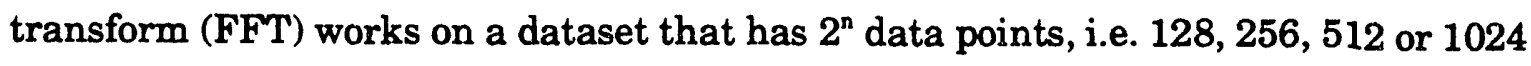
etc. points. Although padding of the time-of-flight dataset from the usual 800 channels ( 0 - $4000 \mathrm{~ns}$ time-of-flight) with zeroes up to 1024 channels works, in practice it is better to sample the background spectrum into 1000 channels (i.e. out to $5000 \mathrm{~ns}$ ) and pad with only 24 zeroes. The power spectrum of the dataset, that is the modulus squared of the complex frequency (Fourier) representation of the dataset, has approximately the form of a Lorentzian function centered about zero frequency, 
although it has a non-Lorentzian tail. This is no surprise; the infinitely averaged background spectrum varies smoothly, i.e. would have only low frequency components. The high frequency components of the power spectrum are due to noise in the incompletely averaged data. To remove the noise we use optimal (Wiener) filtering. The reader is referred to Ref. 14 for a complete discussion of this technique. Essentially, the Fourier spectrum is multiplied by a narrow Lorentzian filter function that has approximately the same form, i.e. the same FWHM, as the Fourier dataset itself. In this way the high frequency tail is damped out. The user can alter the precise value of the filter width so as to control the damping of the high frequency components. The product dataset is then reverse Fourier transformed back into the time domain by another FFT. The result is a smoothed background sime-of-flight dataset, which can be truncated to 800 channels $(0-4000 \mathrm{~ns})$ and saved to disk. Comparison of the filtered and original background datasets, by plotting the two files, is strongly recommended.

This Fourier filtering is preferable to simply fitting the background spectrum with a polynomial function for two reasons. Firstly, it does a better job at reproducing the shape of the background spectrum, and secondly it is a lot less arbitrary as the only variable is the Lorentzian filter width. The filtered background is subtracted off the summed photoelectron spectrum of the ion under study, after appropriate scaling of the background spectrum by the ratio of the total laser shots for which data was accumulated for the ion to the total shots for the background.

We have observed that the background spectrum is slightly different for different polarizations of the laser, mostly in the integrated number of counts, but there can also be small variations in the background distribution. Therefore, 
background spectra should be recorded for each polarization used in the ion photoelestron study. The reader is referred to Appendix A for details on the use, and source cole, of the relevant background filtering and subtraction subroutines in the program TENURE. 


\section{References for Chapter 2.}

1. A. Weaver, Ph. D. thesis, University of California, Berkeley (1991)

2. R. B. Metz, Ph. D. thesis, University of California, Berkeley (1991)

3. M. A. Johnson and W. C. Lineberger, in Techniques in Chemistry 20, 591 (1988), J. M. Farrar and W. H. Saunders, ed. (Wiley, New York, 1988)

4. W. C. Wiley and I. H. McLaren, Rev. Sci. Instrum. 26, 1150 (1955)

5. H. Hotop and W. C. Lineberger, J. Chem. Phys. Ref. Data 14, 731 (1985)

6. S. E. Bradforth, E. H. Kim and D. M. Neumark, to be published (1992)

7. R. B. Metz, T. N. Kitsopoulos, A. Weaver and D. M. Neumark, J. Chem. Phys. 88, 1463 (1988); R. B. Metz, A. Weaver, S. E. Bradforth, T. N. Kitsopoulos and D. M. Neumark, J. Phys. Chem. 94, 1377 (1990)

8. R. E. Continetti, Ph. D. thesis, University of California, Berkeley (1989)

9. T. N. Kitsopoulos, Ph. D. thesis, University of California, Berkeley (1991)

10. D. Proch and T. Trickl, Rev. Sci. Instrum. 60, 713 (1989)

11. D. W. Arnold, unpublished work (1991)

12. D. W. Arnold, S. E. Bradforth, E. H. Kim and D. M. Neumark, J. Chem. Phys. in press

13. S. L. Anderson, L. Goodman, K. Krogh-Jespersen, A. G. Ozkabak, R. N. Zare and C. Zheng, J. Chem. Phys. 82, 5329 (1985)

14. W. H. Press, B. P. Flannery, S. A. Teukolsky and V. T. Vetterling, Numerical Recipes; Cambridge University Press (1986)

15. See chapter 3.

16. K. P. Huber and G. Herzberg, Spectra of Diatomic Molecules, Vol IV, Van Nostrand Rheinhold Company, New York, 1979

17. T. M. Miller in CRC Handbook of Chemistry and Physics, 72nd edition, ed. D.

R. Lide, CRC Press (1991), p. 10-180

18. S. Baskin, J. O. Stoner, Jr., Atomic Energy Level and Grotian Diagrams Vol. 2, North Holland Publishing Company, New York (1978)

19. C. E. Moore, Atomic Energy Levels Vol. I, NSRDS-NBS 35 (1971) 
20. M. J. Travers, D. C. Cowles and G. B. Ellison, Chem. Phys. Lett. 164, 449 (1989) 


\section{Figure Captions for Chapter 2.}

Figure 2-1. Experimental apparatus schematic. The photoelectron spectrometer contains four differentially pumped chambers. Numbered components are described in Figure key and in the text.

Figure 2-2. Mixing chamber for the continuous introduction of secondary reagent gas into the pulsed expansion, to the vacuum side of the main pulsed valve. The Figure shows the two component parts of the chamber fronton and in cross section. The two pieces are assembled (the smaller piece fits tightly inside the larger donut), and bolted to the faceplate of the General Valve by the four bolt holes. The overall dimensions of the assembled chamber is 1.330 " diameter by $0.394 "$ thick.

Figure 2-3. Raw time-of-light photoelectron spectrum for $\mathrm{O}_{2}-$ recorded at $355 \mathrm{~nm}$. Peaks labelled according to transitions denoted in Table 2-3. The positions of unlabelled peaks $(x 5, a 0)$ are typically too poorly determined without lengthy signal averaging, and are not therefore used in the calibration.

Figure 2-4. Raw time-of-flight photoelectron spectrum for $\mathrm{O}_{2}-$ recorded at $532 \mathrm{~nm}$. Peaks labelled according to transitions denoted in Table 2-3. The spin orbit splitting in the $\mathrm{O}_{2}^{-}$ion $(20 \mathrm{meV})$ can often be resolved in the al and $a 2$ peaks. 


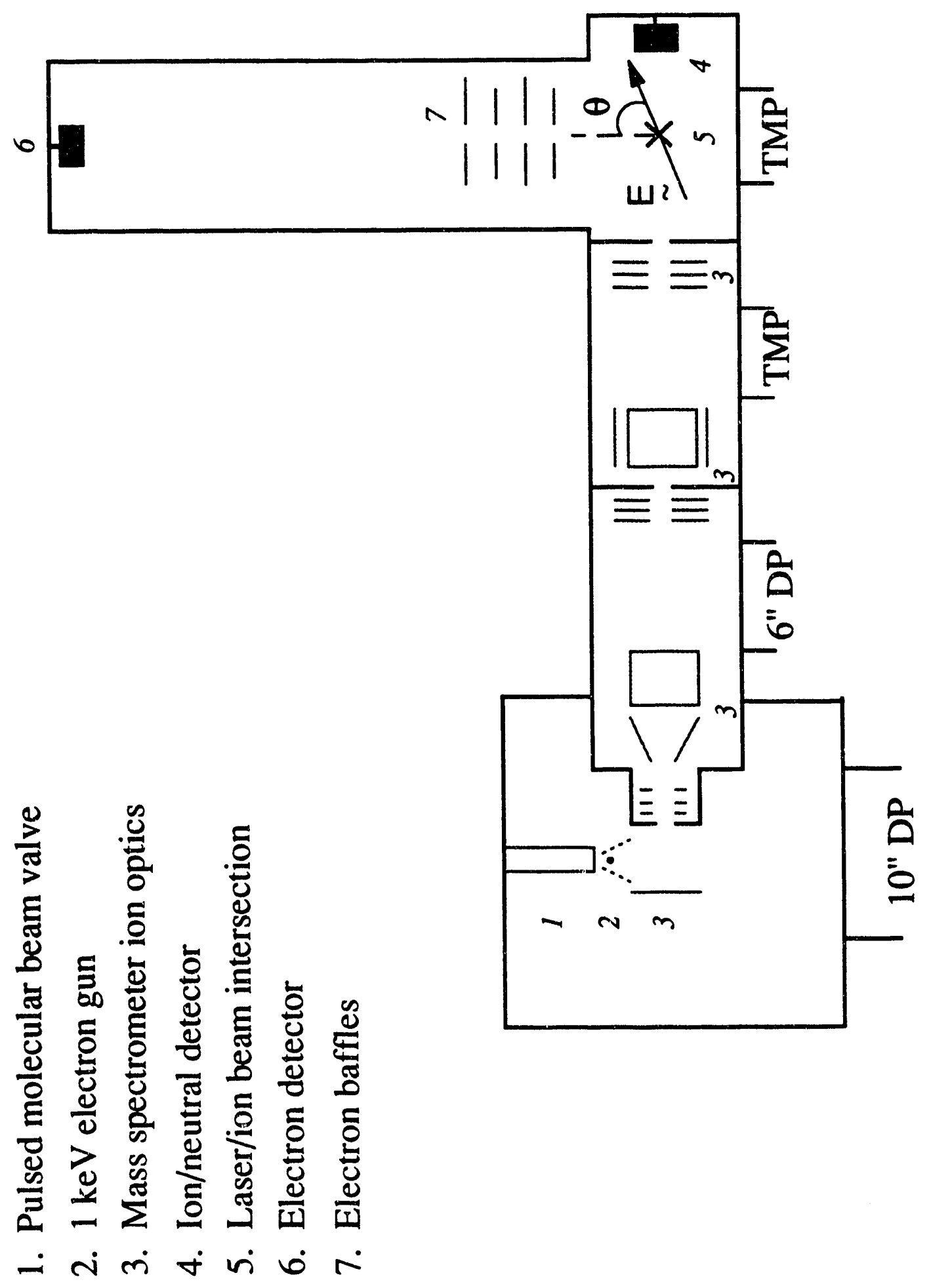

Figure 2-1 

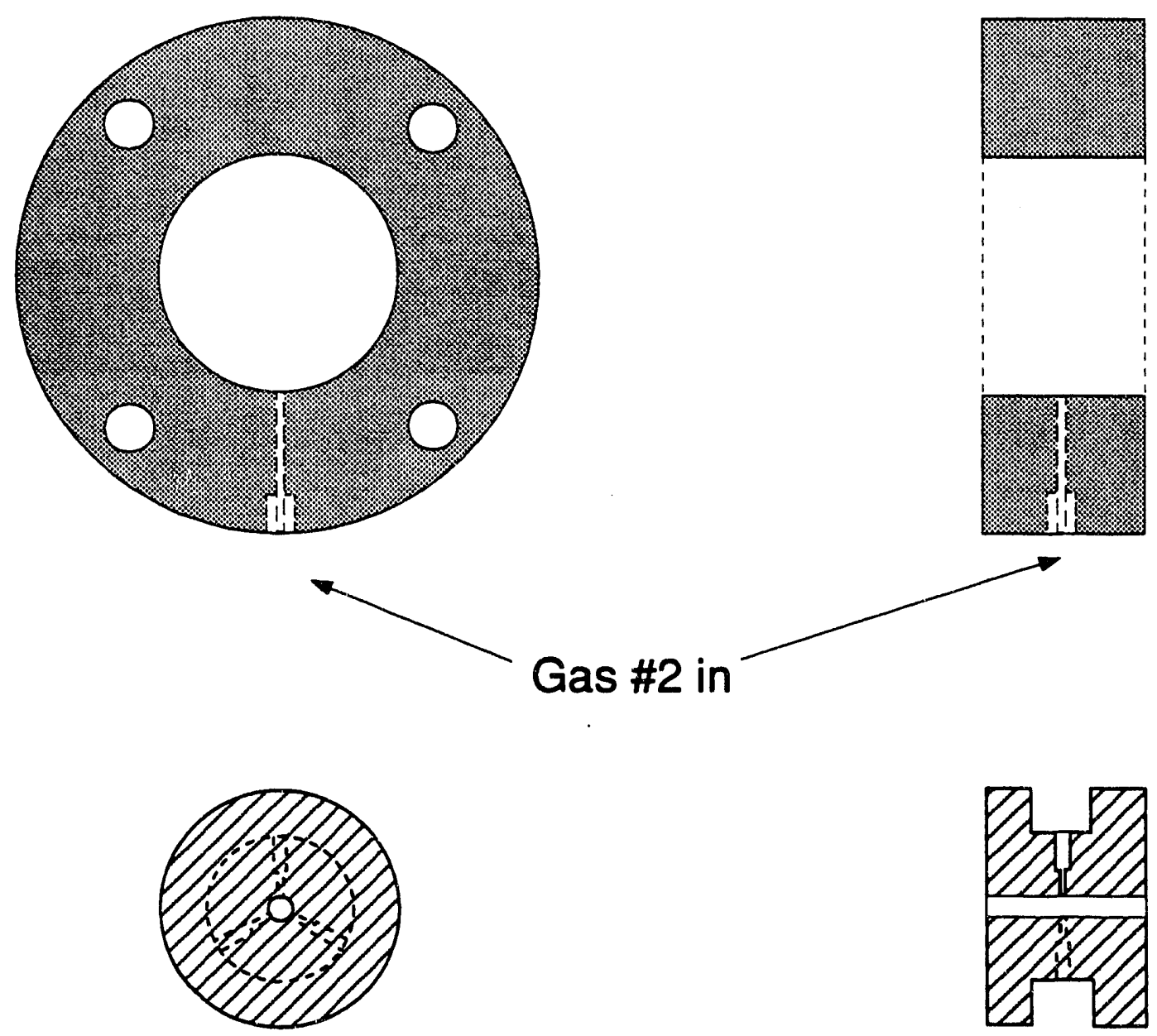

Front View

Side View

Notes: Center hole has $0.080^{\prime \prime}$ dia.

Three feeder holes for second gas have inner dia. of 0.010"

Material Aluminum

Chamber bolts to General Valve Faceplate

Second gas introduced through $0.0625^{\prime \prime}$ stainless steel tubing

which mates at indicated point by a $1-72 \mathrm{NF}$ fitting.

Figure 2-2 


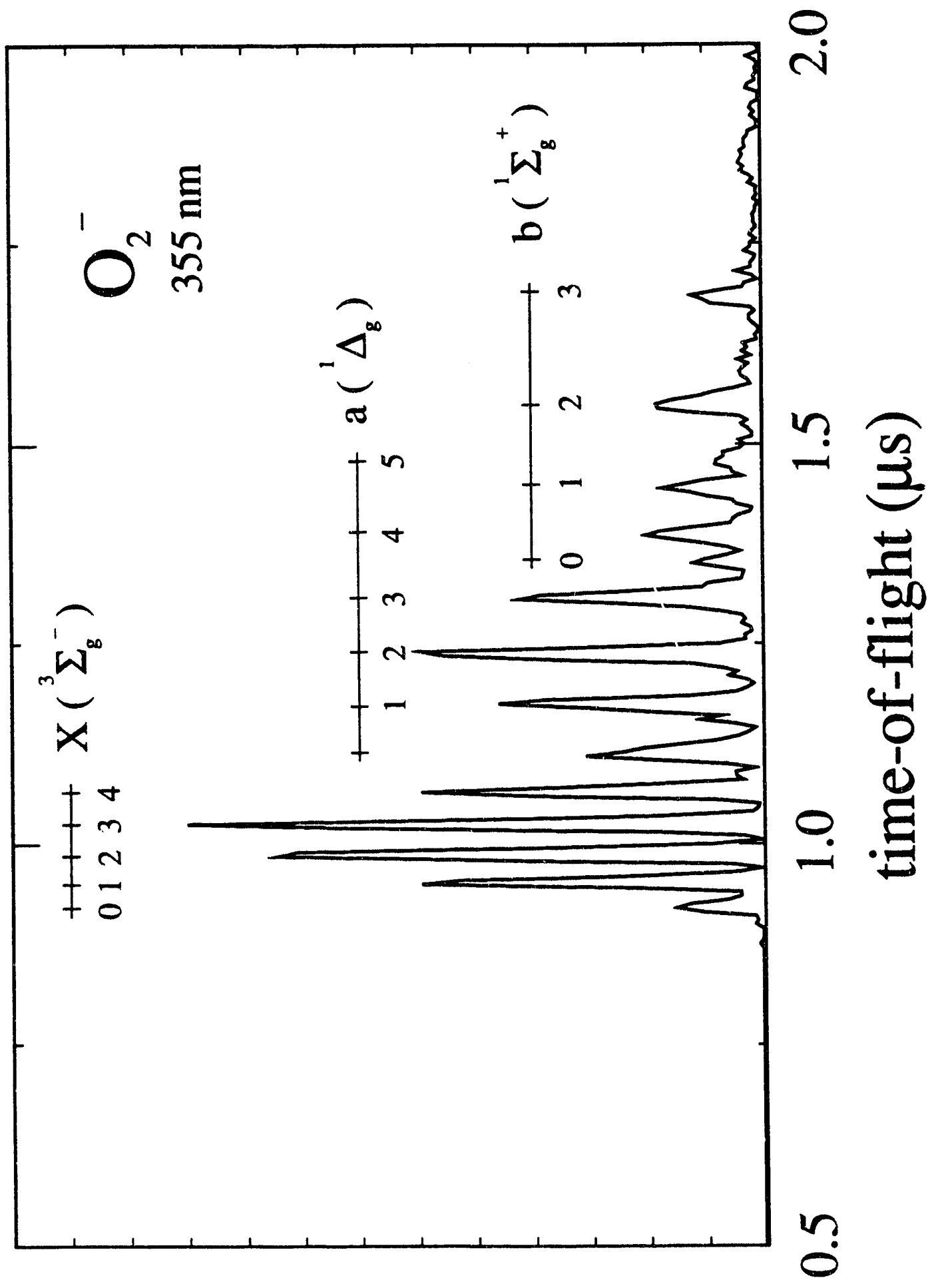

Figure 2-3 


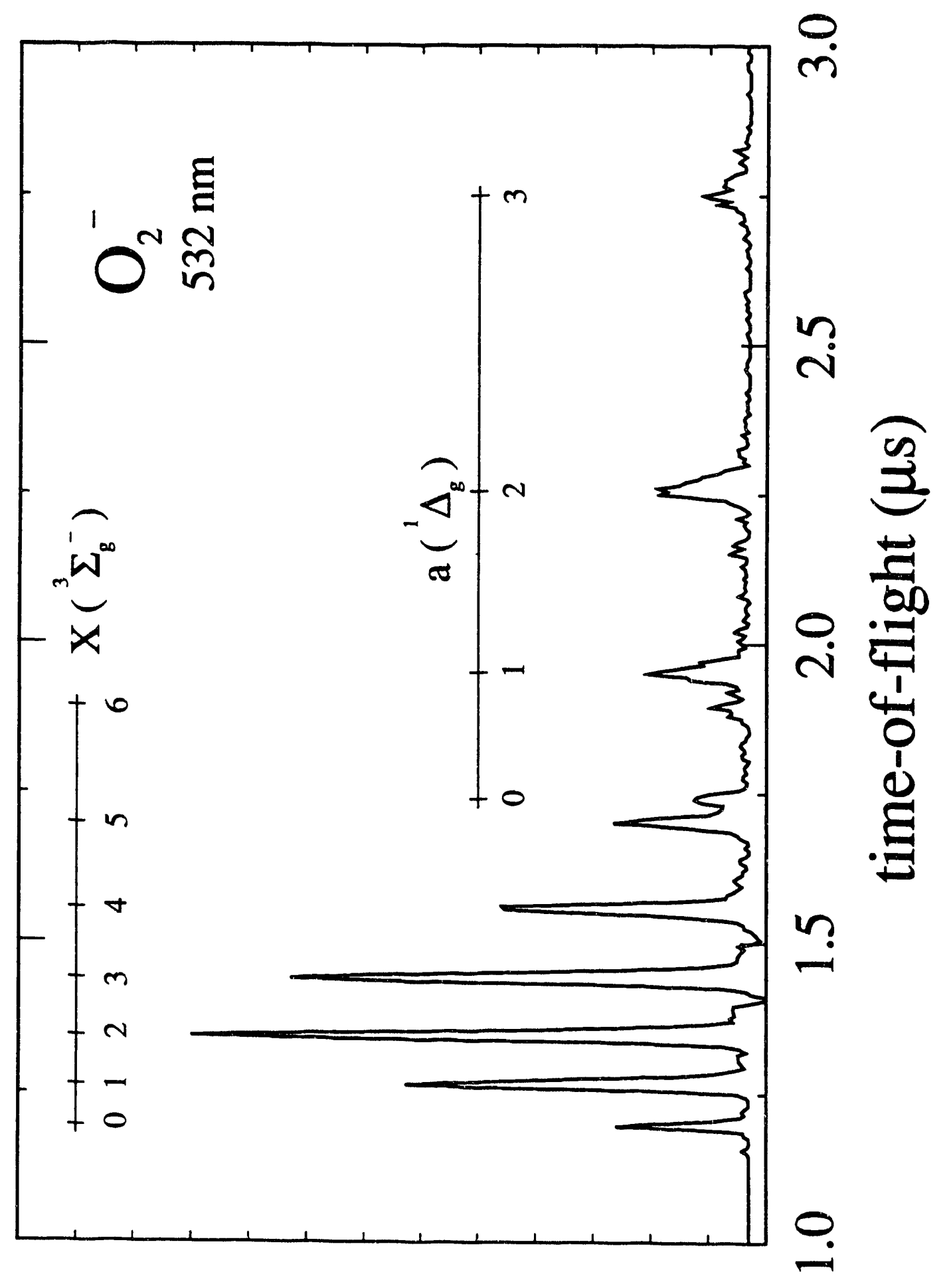

Figure 2-4 
Chapter 3. Photoelectron spectroscopy of $\mathrm{CN}^{-}, \mathrm{NCO}^{-}$, and $\mathrm{NCS}^{-*}$

\begin{abstract}
The $266 \mathrm{~nm}$ photoelectron spectra of $\mathrm{CN}^{-}, \mathrm{NCO}^{-}$and $\mathrm{NCS}^{-}$have been recorded with a pulsed time-of-flight photoelectron spectrometer. The photoelectron spectrum of $\mathrm{CN}^{-}$has also been recorded at $213 \mathrm{~nm}$ revealing transitions to the $\mathrm{A}^{2} \Pi$ state as well as the ground $\mathrm{X}^{2} \Sigma^{+}$state of the $\mathrm{CN}$ radical. The following adiabatic electron affinities (EAs) are determined: $\operatorname{EA}(\mathrm{CN})=3.862 \pm 0.004 \mathrm{eV}, \mathrm{EA}(\mathrm{NCO})=$ $3.609 \pm 0.005 \mathrm{eV}$ and $\mathrm{EA}(\mathrm{NCS})=3.537 \pm 0.005 \mathrm{eV}$. The adiabatic electron affinity of cyanide is in disagreement with the currently accepted literature value. Our measurement of the electron affinity of NCS confirms recent theoretical estimates that dispute the literature experimental value. By Franck Condon analysis of the vibrational progressions observed in each spectrum, the change in bond lengths between anion and neutral are also determined. For $\mathrm{NCO}^{-}$this yields $\mathrm{R}_{0}(\mathrm{C}-\mathrm{N})=1.17$ $\pm 0.01 \AA$ and $R_{0}(C-O)=1.26 \pm 0.01 \AA$, and for $\mathrm{CN}^{-}$the equilibrium bond length is found to be $R_{e}(C-N)=1.177 \pm 0.004 \AA$. The gas phase fundamental for $\mathrm{CN}^{-}$is determined for the first time: $v=2035 \pm 40 \mathrm{~cm}^{-1}$.
\end{abstract}

\title{
1. Introduction
}

The $\mathrm{CN}^{-}, \mathrm{NCO}^{-}$, and $\mathrm{NCS}^{-}$anions are of considerable interest in both solution phase and gas phase chemistry. The three anions are "pseudohalides" in that they are closed shell species with relatively high electron binding energies. On the other hand, there are important chemical differences in comparison to the halide ions; in

•J. Chem. Phys., in press (1993) 
transition metal complexes, for example, the halide and cyanide anions are at opposite ends of the spectrochemical series. A number of experimental and theoretical studies of the spectroscopy and thermochemistry of these anions have been performed in recent years. However, several quantities, particularly the electron affinities of the NCO and NCS radicals, are not well-determined. In order to address this, we have measured the ultraviolet photoelectron spectra of the three anions. The spectra yield accurate values of the radical electron affinities, as well as some anion vibrational frequencies and bond lengths.

The spectroscopy of the CN radical has been thoroughly studied, ${ }^{1}$ but, while $\mathrm{CN}^{-}$has been investigated in various condensed phase environments, ${ }^{2,3,4,5} \mathrm{CN}^{-}$has not been fully characterized in the gas phase. Neither the bond length nor the vibrational frequency for gas phase $\mathrm{CN}^{-}$have been experimentally determined, although there has been very high quality $a b$ initio theory performed to describe the ion. ${ }^{6,7}$ The CN electron affinity (EA) has been measured by Leone and coworkers, ${ }^{8}$ whose value of $3.821 \pm 0.004$ was in good agreement with the previous experimental measurement of Berkowitz $(3.82 \pm 0.02 \mathrm{eV}){ }^{9} \mathrm{CN}^{-}$has a higher reported electron binding energy than any atomic or other diatomic species, and is therefore a desirable calibration standard for our photoelectron spectrometer. However, our photoelectron spectra show the CN electron affinity to be slightly but significantly higher than the currently accepted value of Leone. In addition, we observe a hot band' from vibrationally excited $\mathrm{CN}^{-}$, yielding the gas phase vibrational frequency, and we obtain the $\mathrm{CN}^{-}$bond length from a Franck-Condon analysis.

Despite the well characterized spectroscopy of the radicals NCO and NCS, ${ }^{10}$ the electron affinities for these species have not been accurately determined; 
currently the EA's listed in the compilation of Lias et $a l^{11}$ are $3.59 \pm 0.36$ and 2.15 $\pm 0.02 \mathrm{eV}$ for NCO and NCS respectively. The value for the electron affinity of NCS, obtained from Page's magnetron experiments, ${ }^{12}$ is particularly suspect. Not only does it seem inconsistent with the other $\mathrm{CN}$ containing molecules, it is also considerably lower than theoretical estimates of this quantity. ${ }^{13}$ We note that Page's accompanying result for $\mathrm{EA}(\mathrm{CN}), 2.80 \mathrm{eV}$, is similarly too low. ${ }^{12}$ Dillard and Franklin derived $\mathrm{EA}(\mathrm{NCS})=3.51 \mathrm{eV}$ from measured heats of formation of NCS and NCS- in ion molecule reactions; ${ }^{14}$ the most recent theoretical work supports this value with a calculated EA of $3.45 \mathrm{eV} .^{13}$ We show that the true electron affinity is close to that derived from the thermochemical cycle and the theoretical value, and that the previous "direct" measurement of the EA is in error.

The vibrational spectroscopy of the $\mathrm{NCO}^{-}$and $\mathrm{NCS}^{-}$anions have been thoroughly investigated in various alkali halide matrices. ${ }^{15,16,17,18}$ In addition, high resolution infrared gas phase spectroscopy has been performed on both $\mathrm{NCO}^{-}$ and NCS- in Saykally's laboratory. ${ }^{19,20}$ This work yielded the $v_{3}$ fundamental frequency and the equilibrium rotational constant for each of these linear ions. However, as isotopically substituted spectra were not recorded, the rotational constant does not completely define the molecular structure, and therefore the two bond lengths remain unknown for each ion. In contrast, for the $\mathrm{NCO}$ radical at least, ${ }^{15} \mathrm{~N}$ isotopic substitution in the optical spectra, along with microwave data for ${ }^{14} \mathrm{NCO}$, yields the individual neutral bond lengths, $R_{0}(C-N)$ and $R_{0}(C-O){ }^{21}$ With this data for the neutral bond lengths, and a Franck Condon analysis of the $\mathrm{NCO}^{-}$photoelectron spectrum, we determine the individual bond lengths for the $\mathrm{NCO}^{-}$anion. 


\section{Experimental}

The photoelectron spectra of $\mathrm{NCO}^{-}, \mathrm{NCS}^{-}$, and $\mathrm{CN}^{-}$were recorded on our fixed-frequency negative ion photoelectron spectrometer. This apparatus has been described in detail elsewhere. ${ }^{22}$ Briefly, the instrument is a dual time-of-flight photoelectron spectrometer. A gas mixture at a stagnation pressure of $c a .3 \mathrm{~atm}$ is expanded through a pulsed molecular beam valve. Just below the orifice of the pulsed valve, a continuous $1 \mathrm{keV}$ electron beam intersects the gas beam at $90^{\circ}$. Ions are made in the continuum flow region of the jet. Subsequent collisions in the expansion cool the internal degrees of freedom of the ions. Varying degrees of cooling of the vibrational modes can be achieved by changing the carrier gas.

In these experiments, to make $\mathrm{CN}^{-}$, a mixture of $3 \% \mathrm{HCN}, 8 \% \mathrm{NF}_{3}$ seeded in $\mathrm{N}_{2}$ is expanded through the pulsed valve. $\mathrm{CN}^{-}$is produced in the jet by the following reactions:

$$
\begin{aligned}
\mathrm{NF}_{3}+\mathrm{e}^{-} & \rightarrow \mathrm{NF}_{2}+\mathrm{F}^{-} \\
\mathrm{F}^{-}+\mathrm{HCN} & \rightarrow \mathrm{HF}+\mathrm{CN}^{-}
\end{aligned}
$$

To produce $\mathrm{NCO}^{-}$and $\mathrm{NCS}^{-}$, a few drops of benzyl isocyanate or benzyl thioisocyanate respectively are dropped into the valve, and 3 atmospheres of He or a $40 \% \mathrm{CF}_{4} / \mathrm{He} \mathrm{mix}$, is expanded through the 'wet' valve. $\mathrm{NCO}^{-}$and $\mathrm{NCS}^{-}$are produced by dissociative attachment of an electron to $\mathrm{PhCH}_{2} \mathrm{NCO}$ or $\mathrm{PhCH}_{2} \mathrm{NCS}$. The $\mathrm{CF}_{4} / \mathrm{He}$ mix is found to give far superior cooling for vibrational modes of the NCSion.

The ions are perpendicularly extracted into a time-of-flight mass spectrometer of Wiley-McLaren design. ${ }^{23}$ The mass-selected ion of interest is photodetached using a pulsed Nd:YAG laser that propagates perpendicular to the ion beam. In these 
experiments, the fourth $(266 \mathrm{~nm}, 4.657 \mathrm{eV})$ or fifth $(213 \mathrm{~nm}, 5.822 \mathrm{eV})$ harmonic of the Nd:YAG laser is used. Photoelectrons ejected from the mass selected ion are detected at the end of a 1 meter field-free flight tube which is orthogonal to the laser and ion beams. The energy of the detached electrons is determined by time-of-flight. The energy resolution is $8 \mathrm{meV}$ for electrons with $0.65 \mathrm{eV}$ of kinetic energy and degrades as $\mathrm{E}^{3 / 2}$ for higher kinetic energies. The polarization of the laser can be adjusted by means of a half-wave plate such that the angle $\theta$ between the electric vector of the laser radiation and the direction of electron detection may be sampled. Adjustment of the laser polarization to the magic angle $\left(\theta=55^{\circ}\right)$ can be used to eliminate the photoelectron angular anisotropy term. ${ }^{24}$

Due to the high photon energy employed, any scattered light will release electrons from metal surfaces inside the chamber. This effect is minimized by electron and laser baffles but, even so, at $213 \mathrm{~nm}$ the background level is sufficiently high that the background signal must be subtracted from the data. As the kinetic energy distribution of the background photoelectrons is smooth and does not change from day to day, a smooth function is fitted to the background, scaled and subtracted from the experimental spectrum. This subtraction procedure has been followed for the $213 \mathrm{~nm}$ photoelectron spectrum of $\mathrm{CN}^{-}$here.

The calibration procedure used in these spectra is worthy of some discussion. In the photoelectron spectrum of $\mathrm{A}^{-}$at photon energy hv, the electron kinetic energy of each peak is determined by

$$
e K E=h v-E A(A)-E_{i}(A)+E_{i}\left(A^{-}\right)
$$

where $\mathrm{EA}(\mathrm{A})$ is the electron affinity of $\mathrm{A}$ (or, equivalently, the electron binding energy of $\left.A^{-}\right)$, and $E_{i}(A)$ and $E_{i}\left(A^{-}\right)$are the internal energies of $A$ and $A^{-}$for that transition. 
Ideally, for calibration purposes, the electron affinity and the internal energies of the calibrant species should be extremely well known. At $266 \mathrm{~nm}$ the electron kinetic energy scale is calibrated using photoelectron spectra of the atomic ions $\mathrm{F}^{-}, \mathrm{Cl}^{-}, \mathrm{Br}^{-}$, and $\mathrm{I}^{-}$, for which the corresponding neutral electron affinities $(3.401190,3.61269$, 3.363590 , and $3.0591 \mathrm{eV}$, respectivel ${ }^{25}$ ) and neutral atom spin-orbit splittings $\left(0.05010,{ }^{26} 0.10940,{ }^{27} 0.4569^{26}\right.$ and $0.94268 \mathrm{eV},{ }^{26}$ respectively) are known to good precision. Each calibrant ion gives two narrow peaks in the photoelectron spectrum, ${ }^{28}$ and these atomic lines cover the range of electron kinetic energies $(0.65$ $1.60 \mathrm{eV}$ ) appropriate for photodetachment at $266 \mathrm{~nm}$. At this wavelength, we can access the ground electronic states of $\mathrm{CN}, \mathrm{NCO}$, and NCS, and can therefore accurately determine their electron affinities.

The calibration proceeds as follows: the recorded flight times, $t$, for each calibrant line are fitted to the following form:

$$
t=t_{0}+\sqrt{\frac{m_{e} l^{2}}{2}} \cdot \frac{1}{\sqrt{E_{l a b}}}+\frac{\gamma}{E_{l a b}}
$$

where $m_{e}$ is the electronic mass and $E_{\text {lab }}$ is the expected electron kinetic energy in the laboratory frame-of-reference. Using the 8 or more calibrant lines, the offset $t_{0}$, the effective flight length $\ell$, and the quadratic correction factor $\gamma$ are determined by least squares. For $266 \mathrm{~nm}$, a linear calibration of the energy scale $(\gamma=0)$ is generally sufficient. Using these constants that define the electron energy scale, the flight times for the spectrum of interest are converted to electron kinetic energies; this conversion procedure includes a small center-of-mass correction to the energy. ${ }^{22}$

The situation at $213 \mathrm{~nm}$ is less auspicious. For this photon energy the halide lines are clustered near electron kinetic energies of $2 \mathrm{eV}$ or above. There are no other 
atomic negative ions with higher electron binding energies so it is necessary to go to a molecular calibrant ion. One of our motivations for studying $\mathrm{CN}^{-}$was to obtain calibration points for $213 \mathrm{~nm}$ at lower electron kinetic energies. This can be done using the transitions from $\mathrm{CN}^{-}$to the first excited electronic state of $\mathrm{CN}$, the $\mathrm{A}^{2} \Pi$ state, which lies $0.83 \mathrm{eV}$ above the $\mathrm{X}^{2} \Sigma^{+}$ground state. This, of course, requires the accurate value for $\mathrm{EA}(\mathrm{CN})$ determined at $266 \mathrm{~nm}$. Thus, the results presented in the next section will allow us to better calibrate future spectra at $213 \mathrm{~nm}$. We note here that to fit the electron kinetic energy scale at $213 \mathrm{~nm}$ over the entire range covered by the halide and cyanide transitions, the quadratic scale compression factor, $\gamma$, must be included. This calibration fit then reproduces all lines to within the measured time-of-flight uncertainties for all points. ${ }^{29}$ The inclusion of a quadratic term in the calibration is similar to that employed on negative ion photoelectron instruments in the Lineberger ${ }^{30}$ and Ellison ${ }^{31}$ groups. Our conversion scheme from time-of-flight to electron kinetic energy is analogous to that used on the multiphoton ionization photoelectron spectrometer described by Anderson et $a l .^{32}$

\section{Results}

The $266 \mathrm{~nm}$ photoelectron spectra of the three molecular ions are shown in Figure 3-1. The $\mathrm{NCO}^{-}$spectrum was recorded using the laser polarized at the magic angle $\left(\theta=55^{\circ}\right)$ because a change in relative intensities across the band was noticed as a function of laser polarization. For $\mathrm{NCS}^{-}$and $\mathrm{CN}^{-}$, although there is sizeable change in absolute counts recorded with the two extreme laser polarizations, the band profile did not change, so spectra were recorded with polarization chosen to maximize signal/noise. This was with $\theta=0^{\circ}$ for $\mathrm{CN}^{-}$and $\theta=90^{\circ}$ for NCS-. Figure 3-2 shows 
the $213 \mathrm{~nm}$ photoelectron spectrum of $\mathrm{CN}^{-}$with the polarization of the laser perpendicular $\left(\theta=90^{\circ}\right)$ to the electron collection direction.

The spectra in Figure 3-1 are all relatively simple. Photodetachment of each ion leads to very little vibrational excitation in the corresponding neutral. The $\mathrm{CN}^{-}$ $266 \mathrm{~nm}$ spectrum show's only a single peak, the 0-0 transition, indicating virtually no geometry change between $\mathrm{CN}^{-}$and the $\mathrm{X}^{2} \Sigma^{+}$ground state of $\mathrm{CN}$. In the $213 \mathrm{~nm}$ spectrum (Fig. 3-2), in addition to the $\mathrm{X}$ state transition, a more extended progression (peaks A0-A2) in the vibrational levels of the $A^{2} \Pi$ state is observed. Thus, according to the Franck-Condon principle, there is a somerwhat larger geometry change for this anion $\rightarrow$ neutral transition. Since the vibrational spacings and the energy of the electronic origin in the $\mathrm{CN} \mathrm{A}^{2} \Pi$ state are known, ${ }^{1}$ peaks $\mathrm{A} 1$ and $\mathrm{A} 2$ can be used in addition to AO to calibrate the electron energy scale at $213 \mathrm{~nm}$. The only new spectroscopic feature in Figure 3-2 is peak a0. This is a hot band originating from the $v=1$ level of the anion. This spectrum was recorded under source conditions which increased the vibrational temperature of the anions; in other spectra (not shown), this feature can be eliminated. Observation of the hot band is desirable since it represents the first gas phase measurement of the $\mathrm{CN}^{-}$vibrational fundamental frequency, 2035 $\pm 40 \mathrm{~cm}^{-1}$.

The $\mathrm{NCO}^{-}$photoelectron spectrum shows a short progression in the $\mathrm{v}_{3}$ 'antisymmetric stretch' mode of the NCO X $\Pi$ state (Fig. 3-1). Each peak consists of overlapping transitions to the two spin-orbit components of $\mathrm{NCO}\left(\mathrm{A}_{000}=-95.6 \mathrm{~cm}^{-1}\right)^{33}$ For the NCS $X^{2} \Pi$ state, the spin-orbit splitting is much larger $\left(A_{000}=-323.4 \mathrm{~cm}^{-1}\right){ }^{34}$ and transitions to the individual fine structure components are resolved. The spacing between the doublets is close to the $v_{3}$ (C-S stretch) fundamental in NCS. However, 
a more detailed comparison of the observed peak positions and the term values derived from extensive optical measurements ${ }^{33}$ reveals some discrepancies. This is discussed in more detail when we attempt to simulate the $\mathrm{NCS}^{-}$photoelectron spectrum.

The widths of the observed peaks $\left(-28 \mathrm{meV}\right.$ for $\mathrm{NCO}^{-},-21 \mathrm{meV}$ for $\mathrm{NCS}^{-}$ and - $16 \mathrm{meV}$ for $\mathrm{CN}^{-}, 266 \mathrm{~nm}$ ) are larger than that due the instrumental resolution alone. In the case of NCO, the extra width is due to the unresolved spin-orbit splitting. For the remainder, the peak width is due to sequence bands and the underlying rotational contour for the transition.

\section{Analysis and Discussion}

\subsection{Electron Affinities}

The electron affinities for the three radicals can be determined from the $\mathbf{2 6 6}$ nm spectra shown in Figure 3-1. The adiabatic electron affinity is estimated as follows. The electron kinetic energy at the peak center of the assigned origin $\left(E_{i}(A)\right.$ $=E_{i}\left(A^{-}\right)=0.0$, see Eq. 1) yields the raw electron affinity via

$$
E A=h v-e K E
$$

where $\mathrm{h} v$ is $4.657 \mathrm{eV}$ for $266 \mathrm{~nm}$. Corrections are made for the spin-orbit splitting (if appropriate), sequence bands, and any shift between the center of the rotational contour and the rotationless origin. Another factor to be considered in deriving accurate electron affinities is the effect of Coulomb repulsion of the remaining ion charge cloud on the ejected electrons. The interaction of the undetached ion packet with the electron causes the kinetic energy of the departing electron to be increased slightly. We correct for this effect by determining the magnitude of this shift for an 
atomic ion of similar mass, for the same ion density. Values for all of these corrections are listed in Table 3-I. As can be seen, the magnitude of this latter 'space charge' effect is fairly small.

The rotational correction is calculated by simulating the expected rotational contour ${ }^{35,36}$ using the known rotational constants for each molecule and assuming a simple s-wave detachment model developed by Fano $^{37}$ and Walker ${ }^{38}$. This is an approximation; an s-wave model is not strictly appropriate here, since the electron kinetic energies are on the order of $1 \mathrm{eV}$. Using an anion rotational temperature of $200 \pm 50 \mathrm{~K}$, the rotational contour, when convoluted with the instrumental resolution function, matches the experimental lineshape, and the (small) correction between peak maximum and rotationless origin for the transition may be estimated. It turns out for all these systems that the correction is smaller than the error bars for the correction process, which nevertheless are propagated into the final uncertainty in the electron affinity. The rotational contour simulation for the photoelectron band to the $\mathrm{CN}$ ground state is shown in the inset of Figure 3-1. The possibility of broadening of the origin peak in the $\mathrm{CN}^{-} 266 \mathrm{~nm}$ photoelectron spectrum due to the $1 \leftarrow 1$ sequence band has been checked for; the simulated profile does not change even for vibrational temperatures as large as $1400 \mathrm{~K}$. The final electron affinities derived from this work appear in the final column of Table 3-I.

Let us compare our electron affinity determinations with those currently in the literature. The reported $\mathrm{CN}$ electron affinity of Klein et al. ${ }^{8}$ obtained via laser optogalvanic spectroscopy is slightly lower than our measurement, and both measurements lie outside the range of their mutual error bars. Klein reports $\mathrm{EA}(\mathrm{CN})$ $=3.821 \pm 0.004 \mathrm{eV}^{8}$ whereas we deduce $\mathrm{EA}(\mathrm{CN})=3.862 \pm 0.004 \mathrm{eV}$ from the $266 \mathrm{~nm}$ 
spectrum. We have repeated this measurement several times with independent calibrations, and are therefore confident in our value. To resolve the discrepancy between these two measurements we attempted to measure the total photodetachment cross section on a different apparatus, ${ }^{39}$ with a tunable dye laser. Such an experiment is much more akin to the optogalvanic experiment, and should provide an independent test. However, in contrast to Klein's experiment, we mass-select the $\mathrm{CN}^{-}$ before irradiation. This experiment confirmed that the threshold for $\mathrm{CN}^{-}$ photodetachment occurs to the blue of Klein's reported threshold; our total detachment cross section rises at $321.1 \pm 0.3 \mathrm{~nm}(3.862 \mathrm{eV})$ compared to $324.4 \mathrm{~nm}(3.821 \mathrm{eV})$. One possible explanation of this discrepancy is that Klein et al., who only observed the $324.4 \mathrm{~nm}$ threshold when using $\mathrm{BrCN}$ as their source of ions, were actually observing the threshold for the channel $\left.\mathrm{Br}^{2}{ }^{2} \mathrm{P}_{1 / 2}\right) \leftarrow \mathrm{Br}^{-}$, which occurs at $3.8205 \mathrm{eV} .^{25,26}$ Indeed the authors noted a strong slowly rising background due to the $B r^{2}\left(\mathrm{P}_{3 / 2}\right) \leftarrow \mathrm{Br}^{-}$ transition throughout the wavelength region they investigated.

Our reported vaiues for the electron affinity of NCO and NCS are in excellent agreement with some recent theoretical determinations, but in varying agreement with experimental estimates. For NCO, there have been numerous experimental determinations of the electron affinity. Brauman and coworkers ${ }^{40}$ observed that NCO has a higher EA than fluorine $\left(3.401 \mathrm{eV}^{25}\right)$. Oster and Illenberger estimate $\mathrm{EA}(\mathrm{NCO})=3.8 \pm 0.2 \mathrm{eV}$ based their observed $0 \mathrm{eV}$ appearance potential of $\mathrm{NCO}^{-}$and $\mathrm{SF}_{5}$ - from low energy electron attachment to $\mathrm{SF}_{5} \mathrm{NCO}^{41}$ although this is only an upper limit if the appearance energies are less than $0 \mathrm{eV}$. Dillard and Franklin calculated $\mathrm{EA}(\mathrm{NCO})=1.56 \mathrm{eV}$ from he heats of formation of $\mathrm{NCO}$ and $\mathrm{NCO}^{-}$ measured in their ion-molecule experiments, ${ }^{14}$ but the heats of formation found in this 
work appear to be inconsistent with currently accepted values. Wight and Beauchamp calculated a value of $3.62 \pm 0.2 \mathrm{eV}$ from their measured $\mathrm{NCO}^{-}$proton affinity using literature heats of formation for $\Delta \mathrm{H}_{\Gamma}^{\circ}(\mathrm{HNCO})$ and $\Delta \mathrm{H}_{\Gamma}^{\circ}(\mathrm{NCO}){ }^{42} \mathrm{Our}$ direct measurement of the adiabatic electron affinity is consistent with the measurements of both Brauman and Oster, and also with Wight and Beauchamp's derived value. As discussed in the Introduction, previous 'direct' measurements of the NCS electron affinity, ${ }^{12}$ in contrast, appear to be incorrect. Our value of $3.537 \pm 0.005$ $\mathrm{eV}$, however, is in agreement with Dillard's derived value of $3.51 \mathrm{eV} .{ }^{14}$

The theoretical values for the adiabatic electron affinities of Koch and Frenking, $3.71 \mathrm{eV}$ for $\mathrm{NCO}$ and $3.45 \mathrm{eV}$ for $\mathrm{NCS},{ }^{13}$ are in good agreement (better than $0.1 \mathrm{eV}$ ) with our observed values. These calculated electron affinities are zero-point corrected MP2/6-31+G* energy differences between the optimized ion and neutral structures. Baker et al. have presented an exhaustive comparison of $a b$ initio estimates of electron affinities for several molecular systems including NCO. ${ }^{43 \mathrm{a}} \mathrm{An}$ interesting conclusion of that study was that the use of a simple MP2/6-31+G* scheme for calculating the energy difference between ion and neutral was among the most effective methods for estimating the adiabatic EA, with the caveat that spin contamination in the unrestricted (UHF) radical wavefunction should be small (as is the case for NCO). This explains the success of Koch's calculations, and we have ourselves found that a MP2/6-31++G** model reliably yields excellent EA's in our own calculations when we compare to other experimental measurements in our laboratory. This is certainly an encouraging result for $a b$ initio theory which has traditionally viewed negative ions as one of the hardest classes of molecules to describe correctly. 
It appears that all three ions studied here have very similar electron binding energies. This would lead one to suspect that the 'extra' electron would be closely associated with the $\mathrm{CN}$ part of the molecule. The considerably larger electron affinities of $\mathrm{NCO}$ and NCS relative to $\mathrm{OH}$ and $\mathrm{SH}\left(1.828,2.314 \mathrm{eV}\right.$ respectively $\left.{ }^{25}\right)$ seem to support this, since, in $\mathrm{OH}^{-}$and $\mathrm{SH}^{-}$, the electron is localized on the oxygen and sulfur atoms. However, the HOMO (of $\pi$ symmetry) for $\mathrm{NCO}^{-}$has amplitude over all three atoms, not just the $\mathrm{CN}$ group, as is shown in Figure 3-3. This suggest that the 'extra' electron is actually somewhat delocalized just as for $\mathrm{N}_{3}{ }^{-}$(E.A. $\left(\mathrm{N}_{3}\right)=2.68$ $\mathrm{eV}^{39}$ ). The HOMO in $\mathrm{NCS}^{-}$is also shown in Figure 3-3. It appears more localized than the $\mathrm{HOMO}$ in $\mathrm{NCO}^{-}$, but actually has more amplitude on the sulfur end of the molecule than on the CN group. This is consistent with Ramsay's explanation for the large spin-orbit splitting in $\mathrm{X}^{2} \Pi \mathrm{NCS}$ radical $\left(323 \mathrm{~cm}^{-1}\right)$ which he attributed to the valence structure that has the unpaired electron localized on the sulfur atom dominating the electronic description of this radical. ${ }^{44}$ The replacement of an oxygen atom by a sulfur atom usually raises the electron affinity of a molecule, but the observation that NCO and NCS have similar electron affinities suggests this effect is approximately canceled by the more extensive electron delocalization in $\mathrm{NCO}^{-}$than in NCS$^{-}$. Overall, the electron affinities appear to be determined by the interaction of electron delocalization and electronegativities of various chromophores in the molecule. 


\subsection{Derived Thermochemical quantities}

With a precise estimate of the NCO and NCS electron affinities, we may critically review some related thermochemical quantities. Shobatake $e^{45}$ has recently reported the threshold for photodissociation of HNCO

$$
\mathrm{HNCO}+\mathrm{hv} \rightarrow \mathrm{H}+\mathrm{NCO}\left(\overline{\mathrm{A}}^{2} \Sigma^{+}\right)
$$

to be $\lambda=162 \pm 1 \mathrm{~nm}$. This implies the bond dissociation energy to the $\tilde{\mathrm{A}}$ state of $\mathrm{NCO}, \mathrm{D}^{\mathrm{A}}(\mathrm{H}-\mathrm{NCO})=7.65 \mathrm{eV} .{ }^{45}$ As the $\mathrm{T}_{0}$ for the $\tilde{\mathrm{A}}$ state is well known at $2.82 \mathrm{eV},{ }^{34}$ these data together yield a bond dissociation energy, $\mathrm{D}_{\mathrm{o}}(\mathrm{H}-\mathrm{NCO}) \leq 111.4 \pm 1 \mathrm{kcal} / \mathrm{mol}$. Using the ionization potential of hydrogen and our electron affinity for NCO, we calculate $\mathrm{D}_{0}\left(\mathrm{H}^{+}-\mathrm{NCO}^{-}\right) \leq 341.9 \pm 1 \mathrm{kcal} / \mathrm{mol}$, and a bond enthalpy $\mathrm{DH}_{298}\left(\mathrm{H}^{+}-\mathrm{NCO}^{-}\right)=$ $343.2 \pm 1 \mathrm{kcal} / \mathrm{mol}$. Wight and Beauchamp observed $\mathrm{DH}_{298}\left(\mathrm{H}^{+}-\mathrm{NCO}^{-}\right)=344.7 \pm 2$ $\mathrm{kcal} / \mathrm{mol}$ by the proton abstraction reaction of $\mathrm{HCO}_{2}{ }^{-}$with $\mathrm{HNCO}$ in an ion cyclotron resonance (ICR) machine. Their heterolytic bond dissociation enthalpy is evaluated by comparison to that for formic acid, $\mathrm{HCO}_{2} \mathrm{H}$. The homolytic and heterolytic bond dissociation thermochemistry therefore appears to be consistent. $\Delta \mathrm{H}_{\mathrm{f}}^{\circ}\left(\mathrm{NCO}^{-}\right)$may also be re-evaluated from the recent direct determination of the heat of formation of neutral NCO in our laboratory, ${ }^{46} \Delta \mathrm{H}_{\mathrm{f}}(\mathrm{NCO}, \mathrm{g}, 0 \mathrm{~K})=+30.4 \pm 1 \mathrm{kcal} / \mathrm{mol}$, and the electron affinity reported here. Adopting the "ion convention" for ionic heats of formation, ${ }^{11} \Delta \mathrm{H}_{\mathrm{r}}\left(\mathrm{NCO}^{-}, \mathrm{g}, \mathrm{OK}\right)=-52.8 \pm 1 \mathrm{kcal} / \mathrm{mol}$. Using the frequencies in Table 3-II, we estimate $\Delta \mathrm{H}_{\mathrm{f}}^{\circ}\left(\mathrm{NCO}^{-}\right)$at $298 \mathrm{~K}$ to be unchanged at $-52.8 \mathrm{kcal} / \mathrm{mol}$.

For the thermochemical cycles involving NCS-, the energy for the HNCS homolytic bond dissociation is less well defined than the heterolytic bond dissociation. We may thus use our electron affinity with the $\mathrm{DH}_{298}\left(\mathrm{H}^{+}-\mathrm{NCS}^{-}\right)$of Bierbaum et al. ${ }^{47}$ and the ionization potential of the $\mathrm{H}$ atom to deduce $\mathrm{DH}_{298}(\mathrm{H}-\mathrm{NCS})=96 \pm 6 \mathrm{kcal} / \mathrm{mol}$. 
The temperature dependence of the electron affinity and ionization potential have been ignored here. The homolytic bond dissociation energy calculated here is considerably lower than the $111 \pm 1 \mathrm{kcal} / \mathrm{mol}$ literature value listed in Lias. ${ }^{11}$ However this value for $\mathrm{DH}_{298}(\mathrm{H}-\mathrm{NCS})$ was derived from Page's comparison of the 'apparent' electron affinities of NCS measured with the magnetron technique using HNCS and (NCS) ${ }_{2}$ as precursors. ${ }^{12}$ It is becoming fairly clear that this technique relies on rather too many other thermochemical assumptions to be trusted. Surprisingly, Lias' compilation neglected the more recent work of D'Amario ${ }^{48}$ from which $\mathrm{DH}_{298}(\mathrm{H}-\mathrm{NCS})$ could be calculated. D'Amario's derived $\Delta \mathrm{H}_{\mathrm{f}}^{\circ}(\mathrm{NCS})=76.4 \pm 1$ $\mathrm{kcal} / \mathrm{mol}$ from the photodissociation thresholds of $\mathrm{CH}_{3} \mathrm{SCN}$ and $\mathrm{CH}_{3} \mathrm{NCS}$ to produce NCS. Using

$$
\mathrm{DH}_{298}(\mathrm{H}-\mathrm{NCS})=\Delta \mathrm{H}_{\mathrm{f}}^{\circ}(\mathrm{NCS})-\Delta \mathrm{H}_{\mathrm{f}}^{\circ}(\mathrm{HNCS})+\Delta \mathrm{H}_{\mathrm{f}}^{\circ}(\mathrm{H})
$$

$\Delta \mathrm{H}_{\mathrm{f}}^{\circ}(\mathrm{HNCS})=+30.6 \pm 0.5 \mathrm{kcal} / \mathrm{mol},{ }^{11}$ and $\Delta \mathrm{H}_{\mathrm{f}}^{\circ}(\mathrm{H})=+52.1 \mathrm{kcal} / \mathrm{mol}^{49} \mathrm{DH}_{298}(\mathrm{H}-\mathrm{NCS})$ $=98.0 \pm 1 \mathrm{kcal} / \mathrm{mol}$ is derived..$^{50}$ Our unambiguous result for the electron affinity of NCS, coupled with Bierbaum's gas phase acidity for HNCS confirms this lower value for $\mathrm{DH}_{298}$ (H-NCS).

\subsection{Simulations}

Our goal in this section is to use a simple Franck-Condon model to simulate the photoelectron spectra, allowing us to derive some structural and vibrational parameters for the negative ions. The method employed for Franck-Condon modelling is due to Hutchisson, ${ }^{51}$ and treats each mode as an independent Morse or harmonic oscillator within the normal mode approximation. In addition, we assume that the form of the normal coordiuate for a mode changes little between anion and neutral; 
this is known as the parallel mode approximation. Anion state populations are determined by Boltzmann factors characterized by one (or more) vibrational temperatures.

\section{$\mathrm{CN}^{-}$}

The $266 \mathrm{~nm}$ spectrum (Fig. 3-1) consists of a single peak: CN $\left(X^{2} \Sigma^{+}\right)\left(v^{\prime}=0\right) \leftarrow$ $\mathrm{CN}^{-}\left(\mathrm{v}^{\prime \prime}=0\right)$. The absence of $\mathrm{a}\left(\mathrm{v}^{\prime}=1\right) \leftarrow\left(\mathrm{v}^{\prime \prime}=0\right)$ peak indicates that $\mathrm{CN}^{-}$has a bond length similar to ground state of CN: $1.1718 \AA^{1}$. The $213 \mathrm{~nm}$ spectrum (Fig. 3-2) shows a single peak due to a transition to the $\mathrm{X}$ state, as well as a progression due to transitions to the A state of $\mathrm{CN}$. The A state bond length is $1.2223 \AA{ }^{1}$ By simulating the Franck Condon Factors for transitions to the two states we may bracket the value of $R_{e}$ in $\mathrm{CN}^{-}$.

In the simulations we use the known equilibrium bond lengths, harmonic frequencies and anharmonicities for the $\mathrm{X}$ and $\mathrm{A}$ states of $\mathrm{CN}$, as well as the spin orbit coupling parameter of $-52.6 \mathrm{~cm}^{-1}$ for $\mathrm{CN} \mathrm{A}\left({ }^{2} \Pi\right) .^{1}$ There is no spin orbit splitting in the CN ground $X\left({ }^{2} \Sigma^{+}\right)$state. The anion vibration is also treated as a Morse oscillator; the anharmonicity used is that calculated for $\mathrm{CN}^{-}$by Peterson and Woods, ${ }^{6}$ and the harmonic frequency is derived from this anharmonicity and the value of the fundamental observed in our spectrum. We vary only the anion equilibrium bond length and the vibrational temperature until a satisfactory fit to the experimental spectrum is obtained. Franck-Condon factors for transitions to the two electronic states of the neutral are calculated separately. For transitions to the $\mathrm{X}\left({ }^{2} \Sigma^{+}\right)$state, values of $R_{e}^{\text {anion }}$ in the range $1.162 \AA<R_{e}^{\text {anion }}<1.182 \AA$ were acceptable in predicting intensity $<2 \%$ in the $v=1 \leftarrow 0$ transition, in accord with the absence of this feature in 
the experimental spectrum; this range is centered on the value of $R_{e}$ in the ground state of the neutral (1.1718 $\AA$ ). This result also confirms that the anion bond length is shorter than that in the $\mathrm{CN} \mathrm{A}\left({ }^{2} \Pi\right)$ state (1.2333 $\AA$ ). Figure $3-4$ shows our best fit to the photoelectron band due to transitions to the $A$ state, with $R_{e}{ }^{\text {anion }}(C-N)=1.1765$ $\AA$. Values of $R_{e}$ ranging from 1.173 to $1.182 \AA$ gave acceptable fits to the observed intensity distribution, allowing for the uncertainties in experimental peak heights determined from Poisson counting statistics. The experimental observation of two photoelectron bands allows independent determinations of $R_{e}^{\text {anion }}(C-N)$. The results are completely consistent; our final estimate of the anion equilibrium bond length is $R_{e}=1.177 \pm 0.004 \AA$. This result is in excellent agreement with the Peterson and Wood's MP4(SDQ) prediction of $R_{e}=1.1772 \AA$ and Botschwina's value of $1.1768 \pm$ $0.001 \AA$ calculated with the Coupled Electron Pair Approximation (CEPA-1) method. ${ }^{6,7}$

Let us compare our observed gas phase value of $2035 \pm 40 \mathrm{~cm}^{-1}$ for the $\mathrm{CN}^{-}$ fundamental frequency with other reported values. The vibrational frequency of the anion is $2080 \mathrm{~cm}^{-1}$ in aqueous solution and $2076 \mathrm{~cm}^{-1}$ in $\mathrm{KCN}$ crystal. ${ }^{2}$ Frequencies ranging from 2068 to $2106 \mathrm{~cm}^{-1}$ were observed in various $\mathrm{CN}^{-}$doped alkali-metal halides. ${ }^{3}$ Mendenhall et al gave $\omega_{\mathrm{e}}=2125 \pm 6 \mathrm{~cm}^{-1}$ and $\omega_{\mathrm{e}} \mathrm{x}_{\mathrm{e}}=14.2 \pm 0.7 \mathrm{~cm}^{-1}$ for $\mathrm{CN}^{-}$ in $\mathrm{KCl}$, and similar values for $\mathrm{CN}^{-}$in $\mathrm{NaCl}$ and $\mathrm{NaBr}{ }^{3}$ Sherman and Wilkinson plotted the observed $\mathrm{CN}^{-}$frequency in these various alkali halides (over 250 measurements) versus the estimated shift due to the matrix, calculated from a lattice perturbation model, and predicted a free space vibrational frequency for $\mathrm{CN}^{-}$of 2038 $\pm 3 \mathrm{~cm}^{-1}, 4$ in excellent agreement with our measurement. Very recently, Forney et al. ${ }^{5}$ isolated $\mathrm{CN}^{-}$in a neon matrix. The observed fundamental frequency in this inert and non-polar environment was $2053.1 \mathrm{~cm}^{-1}$. The $a b$ initio calculation of Peterson and 
Woods predicted $\omega_{\mathrm{e}}=2081.7 \mathrm{~cm}^{-1}$ (and $\omega_{\mathrm{e}} \mathrm{x}_{\mathrm{e}}=13.58 \mathrm{~cm}^{-1}$ which we have used in our fit), giving a fundamental of $2055 \pm 6 \mathrm{~cm}^{-1.6}$ Botschwina similarly computed $2052 \pm$ $6 \mathrm{~cm}^{-1}$ for the anion fundamental. ${ }^{7}$ It appears that the observed value is in reasonable accord with the $a b$ initio work, and is, as expected, lower than the reported frequencies in condensed media. This trend is also reported for the C-N stretching frequency in $\mathrm{NCO}^{-}$and $\mathrm{NCS}^{-4,52}$ For comparison, the vibrational fundamentals for $\mathrm{CN}$ radical in the $\mathrm{X}\left({ }^{2} \Sigma^{+}\right)$and $\mathrm{A}\left({ }^{2} \Pi\right)$ states are 2042.4 and $1787.3 \mathrm{~cm}^{-1}$ respectively. ${ }^{1}$

A simple molecular orbital picture of bonding in the $\mathrm{CN}$ species would suggest that the neutral has a bond order of $2 \frac{1}{2}$ while the negative ion has a bond order of 3 ; we thus expect the bond length in the ion to be shorter than that of the radical (1.1718 $\AA$ ) and the harmonic frequency to be higher. The A state of CN also has a bond order of $2 \frac{1}{2}$, but it has a longer bond length than the ground state $(1.2333 \AA$ ). In fact we find, in agreement with the $a b$ initio calculations, that the negative ion has a slightly longer bond length than the ground state of the neutral and a similar or slightly smaller vibrational frequency. Thus, in contradiction to the bond order arguments, the bond in $\mathrm{CN}^{-}$is the same strength or slightly weaker than that in $\mathrm{CN}$ radical. It appears that the $\sigma$ electron removed from the negative ion is only very weakly bonding and has part lone pair character (see Fig. 3-3), whereas the $\pi$ electron removed to form the $A\left({ }^{2} \Pi\right)$ state is strongly bonding. This result is consistent with the photoelectron spectra of $\mathrm{N}_{2},{ }^{53}$ where ionization to $\mathrm{N}_{2}^{+} \mathrm{X}\left({ }^{2} \Sigma_{\mathrm{g}}^{+}\right)$gives a very small lengthening of the $\mathrm{N}-\mathrm{N}$ bond, and a photoelectron band dominated by the 0-0 transition, but ionization to $\mathrm{N}_{2}^{+} \mathrm{A}\left({ }^{2} \Pi_{u}\right)$ gives a much larger bond length change and consequently a longer progression. One additional effect comes into play for negative ions: the extra charge on the negative ion weakens, in the absence of any other 
effects, the bonding overall because all valence electrons are held less tightly by the nuclear charge relative to the corresponding neutral. These arguments go some way in explaining why $\mathrm{CN}^{-}$has a longer equilibrium bond length than $\mathrm{CN}$, and the relative vibrational frequencies of the anion and radical $\mathrm{X}$ and $\mathrm{A}$ states.

$\mathrm{NCO}^{-}$

Both NCO and NCS are known to be linear in neutral and anion ground states. Therefore photodetachment is expected to excite only the bond stretching normal modes. In fact, only the $v_{3}$ mode is appreciably excited in the $\mathrm{NCO}^{-}$and $\mathrm{NCS}^{-}$ photoelectron spectrum. The intensity distribution in the $v_{3}$ progression allows us to evaluate the normal coordinate displacement between anion and neutral. From these displacements and the force constant matrix, the individual bond length changes between the neutral and anionic species can be determined. We will employ ab initio predictions to guide this process. The literature $a b$ initio values have been supplemented by our own computations where appropriate; we have used the Gaussian 90 package for all our calculations. ${ }^{54}$ Because of the larger data set available for $\mathrm{NCO}$, we will describe our simulation for the $\mathrm{NCO}^{-}$photoelectron spectrum in detail. For NCO, the individual neutral bond lengths have been experimentally determined by comparing the ground state rotational constant for ${ }^{15} \mathrm{NCO}$, observed in the spectroscopy of the $\tilde{\mathrm{A}}^{2} \Sigma \leftarrow \overline{\mathrm{X}}^{2} \Pi$ electronic band, ${ }^{21}$ and the rotational constant for ${ }^{14} \mathrm{NCO}$ determined (to higher precision) in the microwave spectrum. ${ }^{55,56}$ This means that we can then use the bond length changes derived from our photoelectron data to extract the two anion bond lengths. These values are 
then checked for consistency with the anion rotational constant as evaluated by vibration-rotation spectroscopy. ${ }^{19}$

The $a b$ initio data in Table $3-\mathrm{III}^{13,43,57,58}$ suggest that the difference in equilibrium structure between anion and neutral is that the $\mathrm{C}-\mathrm{N}$ bond lengthens and the $\mathrm{C}-\mathrm{O}$ bond contracts on removal of an electron. This is because the HOMO of the anion (from which the electron is detached to form ground state NCO) is C-N bonding and C-O anti-bonding in character (Fig. 3-3). Further, the $a b$ initio data suggests that the change in equilibrium structure involves very little change in the overall end-toend length $\left(R_{e}(N-O)\right)$. As the normal modes for this molecule are very close to the symmetric and antisymmetric stretch of $\mathrm{CO}_{2}$, these described geometry changes map almost exclusively onto a displacement along the 'antisymmetric' normal coordinate, $Q_{3}$, and little change along the 'symmetric' stretch, $Q_{1}$. This qualitative description is clearly in good agreement with the progression seen in the $v_{3}$ mode in the experimental spectrum in Figure 3-1. Using the data for vibrations of anion and neutral in Table 3-II, a Franck-Condon simulation is performed to fit the observed photoelectron band. The data shown in the table comes entirely from experimental determinations for $\mathrm{NCO}$ in the gas phase, ${ }^{34,59,60,61}$ and for $\mathrm{NCO}^{-}$mainly from extensive measurements of vibrational frequencies in several alkali halide matrices..$^{15,16,17}$ All three vibrational modes are included in the simulation to model all sequence and hot bands arising from excited anion states. For both the anion and neutral, Morse potentials are used to describe the $v_{1}$ and $v_{3}$ modes and the bending mode is treated as a degenerate harmonic oscillator; the Renner-Teller effect in the neutral radical is neglected. This level of treatment for the bending mode should be sufficient to describe the peak broadening due to sequence bands. In our one- 
dimensional model there can be no provision for cross anharmonicity terms $\chi_{i j}$. Consequently, the 'harmonic' frequencies, $\omega_{\mathrm{i}}^{\text {, }}$ quoted in Table 3-II are effective values given the independent and diagonal treatment of anharmonicity. Thus $\omega_{1}^{\prime}=\omega_{1}+$ $1 / 2 \chi_{13}+\chi_{12}$, and $\omega_{3}^{\prime}=\omega_{3}+1 / 2 \chi_{13}+\chi_{23}{ }^{1} \cdot$ The simulation yields a stick spectrum which is then convoluted with our instrumental resolution function, ${ }^{22}$ and with an $8 \mathrm{meV}$ Gaussian to approximately include the rotational band contour for the transition.

In the fit, all vibrational parameters (anion and neutral) and the spin-orbit coupling constant are treated as fixed; transitions to each of the spin-orbit components of NCO are weighted equally. The $Q_{3}$ displacement is the most important variable parameter in the fit. The $Q_{1}$ displacement and the temperatures, $T_{1}, T_{2}$ and $T_{3}$ describing the Boltzmann distribution of anion vibrational states, are also varied. Varying the temperatures will essentially fit the width and lineshape of each peak. Finally, ihe electrun kinetic energy for the progression origin is allowed to vary; this essentially allows improved estimation of the electron affinity and the effect of sequence bands. We use this to evaluate the sequence band correction to the electron affinity (See Table 3-I).

The overall best fit is shown in Figure 3-5. The variable parameters are determined as $\left|\Delta Q_{3}\right|=0.128 \pm 0.008 \mathrm{amu}^{\mathrm{h}} \cdot \dot{\mathrm{A}}, \mathrm{T}_{3}=\mathrm{T}_{1}=775 \pm 50 \mathrm{~K}, \mathrm{~T}_{2}=600 \pm 50$ $K$. The change in the 'symmetric' stretch coordinate is limited to be $\left|\Delta Q_{1}\right| \leq 0.04$ $a m u^{3} \cdot \dot{A}$. The position of the $3_{1}^{0}$ hot band at $1.32 \mathrm{eV}$ is well fit by the $v_{3}$ fundamental from the gas phase work of Saykally et al. ${ }^{19}$ The intensity of this hnt band determines the vibrational temperature, $T_{3}$, describing this $v_{3}$ anion mode; we obtain an improved fit by assuming a higher temperature for the stretching vibrational modes relative to the lower frequency $v_{2}$ bend mode. This is presumably justifiable 
because the stretching modes are expected to be more strongly excited initially in the dissociative electron attachment reaction used to generate the $\mathrm{NCO}^{-}$ions. Moreover, the lower frequency bend mode should be cooled more effectively by collisions in the free jet expansion. Overall, the high vibrational temperatures needed in the fit show that vibrational cooling is fairly poor in the helium expansion.

Jacox has constructed a force constant matrix from infrared observations of all vibrational frequencies of various isotopically substituted forms of NCO in an argon matrix..$^{62}$ From these force constants, we have calculated the bond stretching normal coordinates. When combined with the values of $\left|\Delta Q_{3}\right|$ and $\left|\Delta Q_{1}\right|$ derived from the fit, we may calculate $\Delta R_{C N}$ and $\Delta R_{c o}$ from anion to neutral.

$$
\left(\begin{array}{l}
\Delta R_{C N} \\
\Delta R_{C O}
\end{array}\right)=\left(\begin{array}{rr}
0.210 & -0.332 \\
0.155 & 0.349
\end{array}\right)\left(\begin{array}{l}
\Delta Q_{1} \\
\Delta Q_{3}
\end{array}\right)
$$

As we expect the C-O bond to shorten in the neutral and the C-N bond to lengthen, the sign of $\Delta Q_{3}$ must be negative, but it is not possible to determine the sign of the small $\Delta Q_{1}$. Let us then consider 3 values for $\Delta Q_{1}:+0.04,0.0$ and -0.04 . Using $\Delta Q_{1}=$ +0.04 and $\Delta Q_{3}=-0.128$, Equation 5 yields $\Delta R_{\mathrm{CN}}=0.050 \dot{\mathrm{A}}$ and $\Delta R_{\mathrm{co}}=-0.039 \dot{\mathrm{A}}$. If we take the $\mathrm{R}_{0}$ structure for NCO neutral from Misra et al. (Table 3-III), ${ }^{21}$ then these displacements yield $R_{0}(C-N)=1.15 \AA$ and $R_{0}(C-O)=1.25 \AA$ for NCO-. For $\Delta Q_{1}=0.0$ and $\Delta Q_{3}=-0.128$, then $\Delta R_{C N}=0.042 \AA$ and $\Delta R_{C O}=-0.045 \AA$; resulting in $R_{0}(C-N)=$ $1.16 \AA$ and $R_{0}(C-O)=1.25 \AA$. Finally if $\Delta Q_{1}=-0.04$ and $\Delta Q_{3}=-0.128$, then $\Delta R_{C N}=$ $0.034 \AA$ and $\Delta R_{\text {co }}=-0.051 \dot{A}$; resulting in $R_{0}(C-N)=1.17 \dot{A}$ and $R_{0}(C-O)=1.26 \AA$ for $\mathrm{NCO}^{-}$. If we use these values to calculate the rotational constant $\mathrm{B}_{0}$ for $\mathrm{NCO}^{-}$, then, by comparing with the high resolution experimental rotational constant, we can determine the sign for $\Delta Q_{1}$. For $\Delta Q_{1}=+0.04,0.0$ and -0.04 the calculated rotational 
constants are $B_{0}=0.3925,0.3878$ and $0.3834 \mathrm{~cm}^{-1}$ respectively. The last of these is closest to the observed $B_{0}, 0.3841 \mathrm{~cm}^{-1},{ }^{19}$ indicating that the sign of $\Delta Q_{1}$ is negative. The values that best fit the data and agree with Saykally's rotational constant are then $\Delta Q_{1}=-0.035 \pm 0.01, \Delta Q_{3}=-0.128 \pm 0.008$.

The final result for the anion $R_{0}$ bond lengths are shown in Table 3-III. The quoted error bars of $\pm 0.01 \AA$ include the uncertainties in the normal coordinate changes in our fit and the error bars in Misra's neutral bond lengths, but not the error in assuming the parallel mode approximation (i.e. the neglect of Duchinsky rotation ${ }^{35}$ ) or in Jacox's force constants. Comparing our result to the $a b$ initio values shows that the $\mathrm{C}-\mathrm{N}$ bond length in $\mathrm{NCO}^{-}$is considerably overestimated at the highest level of theory (MP2). In fact, it appears that all levels of $a b$ initio theory shown do not correctly describe the relative bond lengths $R(C-N)$ or $R(C-O)$ in either anion or neutral. It is well known that multiply bonded systems are difficult to describe theoretically and it appears that this system, which has somewhere between a single and double bond between $\mathrm{C}$ and $\mathrm{O}$ atoms, and between a double and a triple bond between $\mathrm{C}$ and $\mathrm{N}$ atoms, is certainly a strong test case.

NCS-

The NCS- photoelectron spectrum has four major peaks; as already noted, these are due to a short progression in the C-S stretch $\left(v_{3}\right)$ in each of the two spinorbit components of the NCS $\tilde{\mathrm{X}}^{2} \Pi$ state. According to this assignment, the four peaks correspond to transitions to the ${ }^{2} \Pi_{3 / 2}(000),{ }^{2} \Pi_{1 / 2}(000),{ }^{2} \Pi_{3 / 2}(001)$ and ${ }^{2} \Pi_{1 / 2}(001)$ levels of the neutral. However, the spacing of the peak centers from the origin, $0.040,0.091$ and $0.130 \mathrm{eV}$, differ for the two $3_{0}^{1}$ transitions from the corresponding term values 
given by Northrup and $\operatorname{Sears}^{33}(0.040,0.094$ and $0.137 \mathrm{eV})$ in their laser induced fluorescence/stimulated emission pumping study of NCS. Although these discrepancies are small relative to our resolution, a simulation using Northrup's observed 001 term values cannot fit the observed photoelectron band.

The apparent shift in our peak spacings is probably from Fermi resonances in NCS; Northrup showed these are responsible for extensive mixing of the 020 and 001 states with $\Pi$ vibronic symmetry. The separation between the ${ }^{2} \Pi_{3 / 2}(001)$ and $\mu^{2} \Pi_{3 / 2}(020)$ levels is less than $10 \mathrm{meV}\left(80 \mathrm{~cm}^{-1}\right)-$ the ${ }^{2} \Pi_{3 / 2}(001)$ is higher - as is the separation between the ${ }^{2} \Pi_{122}(001)$ and $\kappa^{2} \Pi_{12}(020)$ levels. $^{33}$ (The subscript here refers to $P$, the projection of the electronic, vibrational, and spin angular momentum along the internuclear axis. ${ }^{63}$ ) Close-lying levels with the same value of $P$ can interact via Fermi resonance. In the absence of this effect, the unperturbed 020 levels are expected to have poor Franck-Condon overlap with the $\mathrm{NCO}^{-}$ground vibrational level. However, because of the Fermi resonance, transitions to the 020 levels can occur with appreciable intensity in the photoelectron spectrum via intensity borrowing from the nearby 001 levels. Thus, we would expect to observe two unresolved doublets in the photoelectron spectrum for the " $3_{0}^{1 "}$ peaks. The spacing from the origin of the center of each unresolved doublet would be expected to be the weighted average of the two mixed states making up the doublet. While our resolution is insufficient to resolve these doublets, each " $3_{0}^{1 "}$ peak in the photoelectron spectrum does lie approximately at the average of Northrup's term values for the strongly interacting $\mu^{2} \Pi_{3 / 2}(020)$ and ${ }^{2} \Pi_{3 / 2}(001)$ levels, for the $P=3 / 2$ component and at the average of $\mathrm{K}^{2} \Pi_{1 / 2}(020)$ and ${ }^{2} \Pi_{1 / 2}(001)$ levels for the $P=1 / 2$ component. Additional evidence for the hypothesis that the " 30 l" peaks are unresolved doublets is provided by the observation that these peaks 
around $1.0 \mathrm{eV}$ are broader $(25-28 \mathrm{meV})$ than their respective origin peaks $(21 \mathrm{meV})$ at around $1.11 \mathrm{eV}$. The contribution to the peak width from instrumental resolution, in contrast, is smaller for peaks at lower electron kinetic energy.

An analogous Franck-Condon simulation can still be performed for the NCSspectrum as for $\mathrm{NCO}^{-}$. However, because of the complications due to the Fermi resonance, which we ignore, and because the observed vibrational progression is very short, we use a simpler, purely harmonic model in this simulation. Because the bending mode is included only for simulation of sequence bands, the $v_{2}$ mode is treated as a degenerate harmonic oscillator for the neutral as well as the ion, without account for the Renner-Teller effect. Northrup and Sears' vibronically deperturbed harmonic frequencies are used for the $v_{2}$ and $v_{3}$ modes (Table 3-II). The deperturbed $\omega_{3}$ frequency actually matches the observed " $v_{3}$ " peak spacing in the photoelectron spectrum. The calculated intensities, however, average the complicated state mixing taking place in the " $3_{0}^{1 "}$ peaks. For the anion, we also use an entirely harmonic treatment despite the existence of a thorough anharmonic force field derived from alkali halide matrix spectroscopy of $\mathrm{NCS}^{-18}$ This is reasonable because the anion vibrational temperature turns out to be far lower than in $\mathrm{NCO}^{-}$, so that anion states higher than $v=1$ are not significantly populated. Further, a more complicated treatment does not seem warranted given the simple treatment of the neutral vibrations. The anion $v_{1}$ (C-N stretch) frequency is fixed at the gas phase fundamental observed by Polak et. al., ${ }^{20}$ whereas the $v_{2}$ and $v_{3}$ frequencies are taken from CsI matrix work. ${ }^{18}$ Of all the alkali halide matrices, CsI is expected to have the least perturbation on the NCS- vibrational frequencies, as compared to the gas phase, because it has the largest vacancy sites. Even so, the free ion value for the C-S 
stretching frequency $\left(v_{3}\right)$ has been the subject of considerable discussion; it has been suggested that there is still some perturbation caused by the CsI matrix on this 'soft' vibration. ${ }^{18,52}$ The position of the $3_{1}^{0}$ hot band in our photoelectron spectra, particularly where this feature is enhanced in spectra recorded from ions which are formed in a hotter pure helium expansion, is consistent with the $v_{3}$ fundamental observed in cesium iodide. Our resolution precludes determining the free-ion value with any greater precision.

The variable parameters in the simulation are $\left|\Delta Q_{3}\right|$, the position of the origin, and the vibrational temperature $T_{\text {vib. }}$. Here, we can adequately fit the spectrum assuming the same temperature for each vibrational degree of freedom. $\left|\Delta Q_{1}\right|$ is constrained to be less than $0.03 \mathrm{amu}^{3 / 2} \cdot \AA$, because little signal is observed at $0.24 \mathrm{eV}$ to lower kinetic energy of the origin, where the $1_{0}^{1}$ transition is expected, and $\left|\Delta Q_{2}\right|$ must be zero by symmetry. The best fit is shown in Figure 3-6, where $T_{\text {vib }}=350 \mathrm{~K}$ and $\left|\Delta Q_{3}\right|=0.13 \mathrm{amu}^{1 / 3} . \AA$. Unlike the NCO radical, only the overall rotational constant for the neutral is known, ${ }^{64}$ and thus the two individual bond lengths are unknown. While there is no force constant matrix available for the radical, one has been constructed for the ion from the alkali halide matrix work. ${ }^{18}$ Calculating the normal coordinates for the stretching modes from this force constant matrix, we may again translate our observed $\Delta Q_{3}$ value into equilibrium bond length changes. In using the anion normal coordinates for this purpose we are once again invoking the parallel mode approximation. By noting that $\mathrm{Q}_{3}$ corresponds to almost purely $\mathrm{C}-\mathrm{S}$ shortening/lengthening, that there is no change in $Q_{1}$, and that the rotational constant increases (therefore the overall molecule contracts) from anion to neutral, we can determine that the change in equilibrium bond length between anion and neutral is 
$\Delta \mathrm{R}(\mathrm{C}-\mathrm{N})=0.00 \pm 0.01 \AA$ and $\Delta \mathrm{R}(\mathrm{C}-\mathrm{S})=-0.03 \pm 0.01 \AA$. Comparing these geometry changes with the $a b$ initio data in Table $3-\mathrm{IV}^{13,65}$ the HF/6-31+G* and HF/6-31G* results are consistent with the changes derived from the Franck Condon analysis. However the MP2 results are surprisingly poor; they predict the opposite result, $\triangle R(C$ S) > 0 and a substantial shortening in the C-N bond, which is clearly not consistent with the absence of the $1_{0}^{1}$ peak in the photoelectron spectrum.

\section{Conclusions}

We have presented the photoelectron spectra of three pseudohalogen anions. The relatively simple spectra have yielded the electron affinity of $\mathrm{CN}, \mathrm{NCO}$, and NCS to a precision of about $5 \mathrm{meV}$. The electron affinities for all three radicals are now clearly established. Various related thermochemical quantities, including the bond dissociation enthalpy of HNCS, have been derived. The first gas phase determinations of the equilibrium bond length and vibrational frequency for the cyanide ion have also been reported. These data compare very well with high level ab initio theory. The results for the $213 \mathrm{~nm}$ photoelectron spectrum of $\mathrm{CN}^{-}$provide some useful calibrant lines for negative ion photoelectron spectroscopy at this and shorter laser wayngths where there have been none hitherto available. A Franck-Condon analysis has jrielded the bond lengths in $\mathrm{NCO}^{-}$and the change in geometry for $\mathrm{NCS}^{-}$to NCS. These have been compared to $a b$ initio results. 


\section{Acknowledgements}

This work has been sponsored by the United States Air Force Office of Scientific Research under contract number AFOSR-91-0084. We thank Dr. R. B. Metz for early work on these systems and for performing the total cross section measurements on $\mathrm{CN}^{-}$. We would like to thank Professor S. R. Leone for useful discussions on the cyanide results. 


\section{References for Chapter 3.}

1. K. P. Huber and G. Herzberg, Spectra of Diatomic Molecules Vol. IV, Van Nostrand Rheinhold Company, New York, 1979

2. B. M. Chadwick and H. G. M. Edwards, in: Specialist Periodical Report. Molecular Spectroscopy, Vol. 1, eds. R. F. Barrow, D. A. Long and D. J. Millen (Chem. Soc., London, 1973) p. 446.

3. M. Mendenhall, A. Barnes, P. Bunton, R. Hıaglund, L. Hudson, R. Rosenberg, D. Russell, J. Sarnthein, P. Savundararaj, N. Tolk and J. Tellinghuisen, Chem. Phys. Lett. 147, 59 (1988)

4. W. F. Sherman and G. R. Wilkinson, in Vibrational Spectroscopy of Trapped Species, ed . H. E. Hallam (Wiley, New York, 1973)

5. D. Forney, W. E. Thompson and M. E. Jacox, J. Chem. Phys. 97, 1664 (1992)

6. K. A. Peterson and R. C. Woods, J. Chem. Phys. 87, 4409 (1987)

7. P. Botschwina, Chem. Phys. Lett. 114, 58 (1985)

8. R. Klein, R. P. McGinnis and S. R. Leone, Chem. Phys. Lett. 100, 475 (1983)

9. J. Berkowitz, W. A. Chupka, T. A. Walter, J. Chem. Phys. 50, 1497 (1969)

10. M. E. Jacox, J. Chem. Phys. Ref. Data, 17, 269 (1988); M. E. Jacox, J. Chem. Phys. Ref. Data 19, 1387 (1990)

11. S. G. Lias, J. E. Bartmess, J. F. Liebman, J. L. Holmes, R. D. Levin and W. G. Mallard, Gas phase Ion and Neutral Thermochemistry, J. Phys. Chem. Ref. Data 17, Supp. 1 (1988)

12. R. Napper and F. M. Page, Trans. Farad. Soc. 59, 1086 (1963); F. M. Page, Adv. Chem. Ser. 36, 68 (1972)

13. W. Koch and G. Frenking, J. Phys. Chem. 91, 49 (1987)

14. J. G. Dillard and J. L. Franklin, J. Chem. Phys. 48, 2353 (1968)

15. W. C. Price, W. F. Sherman and G. R. Wilkinson, Proc. Roy. Soc. (London) A255, 5 (1960)

16. V. Schettino and I. C. Hisatsune, J. Chem. Phys. 52, 9 (1970)

17. D. F. Smith, J. Jverend, J. C. Decius, and D. J. Gordon, J. Chem. Phys. 58, 1636 (1973).

18. D. F. Smith, Jr., J. Mol Spectros. 57, 447 (1975) 
19. M. Grubele, M. Polak and R. J. Saykally, J. Chem. Phys. 86, 6631 (1987)

20. M. Polak, M. Grubele and R. J. Saykally, J. Chem. Phys. 87, 3352 (1987)

21. P. Misra, C. W. Mathews and D. A. Ramsay, J. Mol. Spec. 130, 419 (1988)

22. R. B. Metz, A. Weaver, S. E. Bradforth, T. N. Kitsopoulos and D. M. Neumark, J. Phys. Chem. 94, 1377 (1990)

23. W. C. Wiley and I. H. McLaren, Rev. Sci. Instrum. 26, 1150 (1955)

24. A. Weaver, D. W. Arnold, S. E. Bradforth and D. M. Neumark, J. Chem. Phys. 94, $1740(1991)$

25. T. M. Miller in CRC Handbook of Chemistry and Physics, 72nd edition, ed. D. R. Lide, CRC Press (1991), p. 10-180

26. C. E. Moore, Atomic Energy Levels Vol. I, NSRDS-NBS 35 (1971)

27. S. Baskin and J. O. Stoner, Jr., Atomic Energy Level and Grotian Diagrams Vol. 2, North Holland Publishing Company, New, York (1978)

28. A. Weaver, Ph. D. Thesis, University of California, Berkeley, 1991

29. The fit is then a 3 parameter fit to 12 calibrant data points.

30. D. Leopold, K. K. Murray, A. Stevens Miller and W. C. Lineberger, J. Chem. Phys. 83, 4849 (1985)

31. D. C. Cowles, M. J. Travers, J. L. Frueh and G. B. Ellison, J. Chem. Phys. 94, 3517 (1991)

32. S. L. Anderson, L. Goodman, K. Krogh-Jespersen, A. G. Ozkabak, R. N. Zare and C. Zheng, J. Chem. Phys. 82, 5329 (1985)

33. F. J. Northrup and T. J. Sears, J. Chem. Phys. 91, 762 (1989); F. J. Northrup and T. J. Sears, Mol. Phys. 71, 45 (1990)

34. F. J. Northrup, M. Wu and T. J. Sears, J. Chem. Phys. 96, 7218 (1992)

35. K. Ervin and W. C. Lineberger in Advances in Gas Phase Ion Chemistry, Vol. $1 ;$ N. G. Adams and L. M. Babcock, Eds., JAI Press, Greenwich, Conn. in press

36. P. C. Engelking, J. Phys. Chem. 90, 4544 (1986)

37. A. R. P. Rau and U. Fano, Phys. Rev. A, 4, 1751 (1971)

38. T. E. H. Walker, Chem. Phys. Lett. 19, 493 (1973) 
39. R. E. Continetti, D. R. Cyr, R. B. Metz and D. M. Neumark, Chem. Phys. Lett. 182, 406 (1991)

40. R. L. Jackson, M. J. Pellerite and J. I. Brauman, J. Am. Chem. Soc. 103, 1802 (1981)

41. T. Oster and E. Illenberger, Intl. J. Mass Spectrom. Ion Processes 85, 125 (1988)

42. C. A. Wight and J. L. Beauchamp, J. Phys. Chem. 84, 2503 (1980)

43. (a) J. Baker, R. H. Nobes and L. Radom, J. Comp. Chem. 7, 349 (1986);

(b) W-K Li, J. Baker and L. Radom, Aust. J. Chem. 39, 913 (1986)

44. R. N. Dixon and D. A. Ramsay, Can. J. Phys. 46, 2619 (1968)

45. K. Uno, T. Hikida, A. Hiraya and K. Shobatake, Chem. Phys. Lett. 186, 475 (1990)

46. D. R. Cyr, R. E. Continetti, R. B. Metz, D. L. Osborn and D. M. Neumark J. Chem. Phys. 97, 4937 (1992)

47. We use the $\Delta \mathrm{H}_{\text {acid }}^{\mathrm{o}}$ re-evaluated in Ref. 11 from the original data of $\mathrm{V}$. $M$. Bierbaum, J. J. Grabowski and C. H. DePuy, J. Phys. Chem. 88, 1389 (1984)

48. P. D'Amario, G. di Stefano, M. Lenzi and A. Mele, J. Chem. Soc. Faraday Trans. I 68, 940 (1972)

49. M. W. Chase, Jr., C. A. Davies, J. R. Downey, Jr., D. J. Frurip, R. A. McDonald and A. N. Syverud, JANAF Thermochemical Tables, 3rd Edition (American Chemical Society and American Institute of Physics, New York, 1986)

50. M. Lenzi, A. Mele and M. Paci, Gazz. Chim. Ital. 103, 977 (1973)

51. E. Hutchisson, Phys. Rev. 36, 410 (1930); E. Hutchisson, ibid 37, 45 (1931)

52. M. L. Polak, Ph. D. Thesis, University of California, Berkeley, 1990

53. D. W. Turner, C. Baker, A. D. Baker and C. R. Brundle, Molecular Photoelectron Spectroscopy, Wiley, London, p. 46 (1970)

54. M. J. Frisch, M. Head-Gordon, H. B. Schlegel, K. Ragavachari, J. S. Binkley, C. Gonzales, D. J. Defrees, D. J. Fox, R. A. Whiteside, R. Seeger, C. F. Melius, J. Baker, R. L. Martin, L. R. Kahn, J. J. P. Stewart, E. M. Fleuder, S. Topiol and J. A. Pople; Gaussian Inc., Pittsburgh, PA (1990)

55. S. Saito and T. Amano, J. Mol. Spectros. 34, 383 (1970)

56. K. Kawaguchi, S. Saito and E. Hirota, Mol. Phys. 55, 341 (1985) 
57. M. Peric, B. A. Hess and R. J. Buenker, Mol. Phys. 58, 1001 (1986)

58. M. H. Alexander and A. Werner, private communication

59. J. Werner, W. Seebass, K. Koch, R. F. Curl, W. Urban and J. M. Brown, Mol. Phys. 56, 453 (1985)

60. S. Mrozowski, Phys. Rev. 72, 682 (1947)

61. P. S. H. Bolman, J. M. Brown, A. Carrington, I. Kopp, and D. A. Ramsay, Proc. Roy. Soc. (London) A343, 17 (1975)

62. D. E. Milligan and M. E. Jacox, J. Chem. Phys. 47, 5157 (1967)

63. $P$ is the only good quantum number here. It is defined by $P=I \pm \Omega \pm \ell$, where $\Omega$ is the projection of the sum of orbital and spin angular momentum on the internuclear axis and $\ell$ is the vibrational angular momentum.

64. T. Amano and T. Amano, J. Chem. Phys. 952275 (1991)

65. I. Tokue, K. Kobayashi, T. Honda and Y. Ito, J. Phys. Chem. 94, 3485 (1990) 


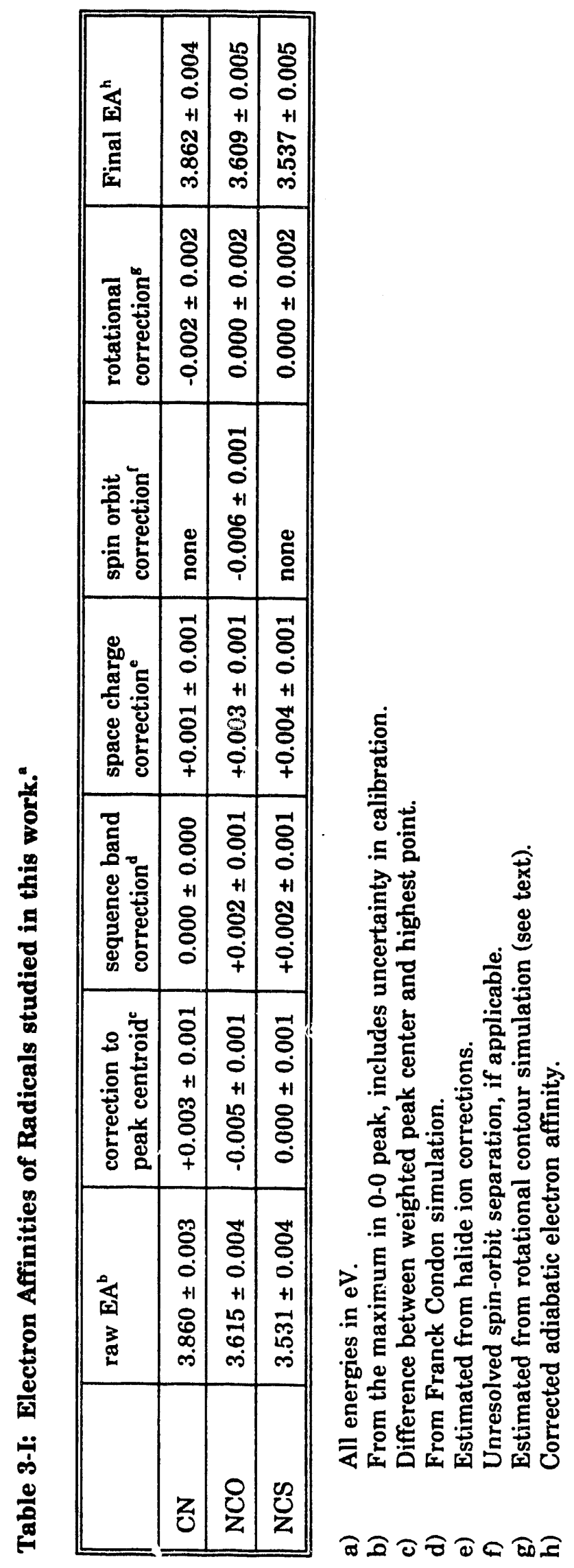




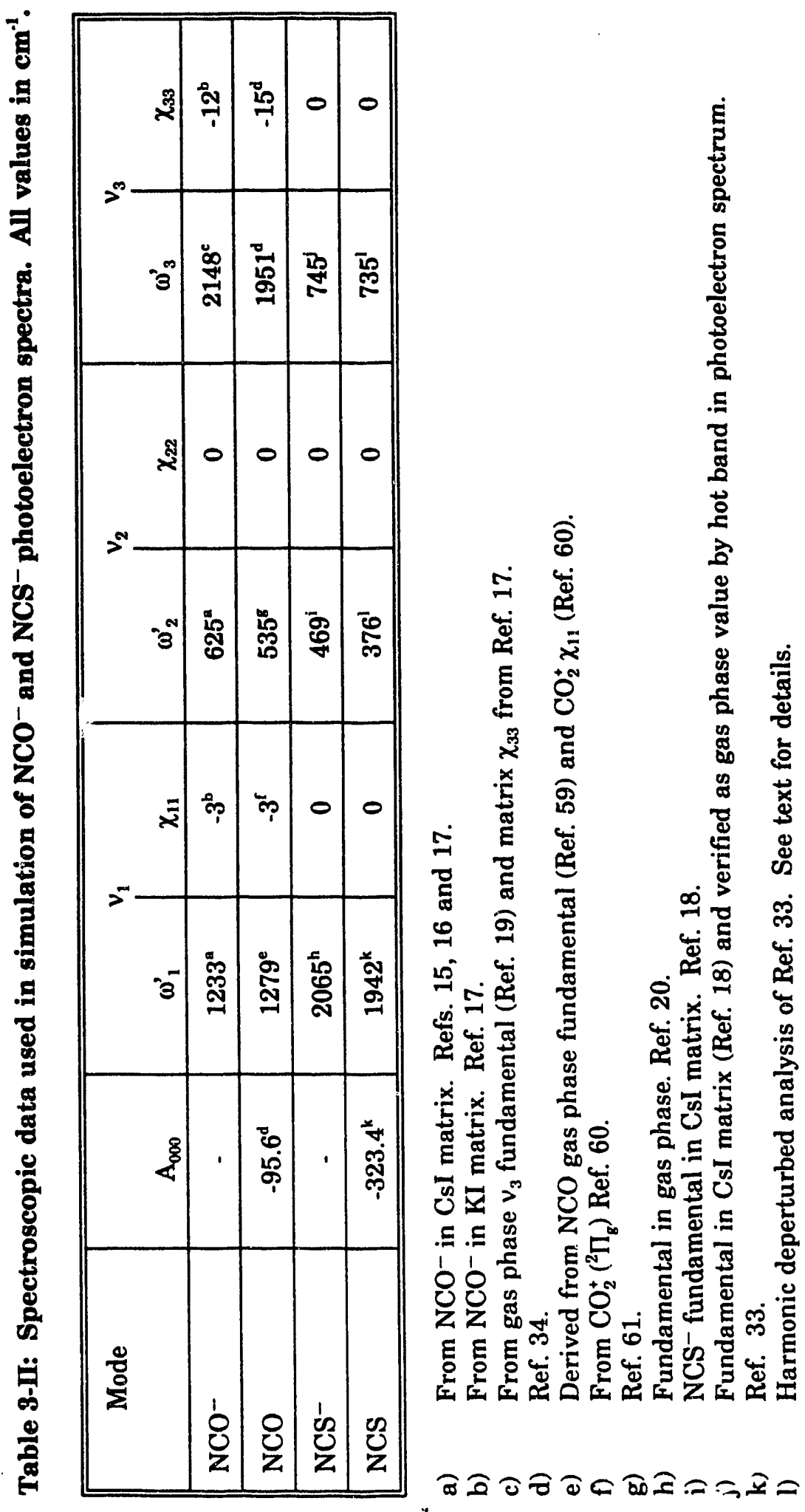




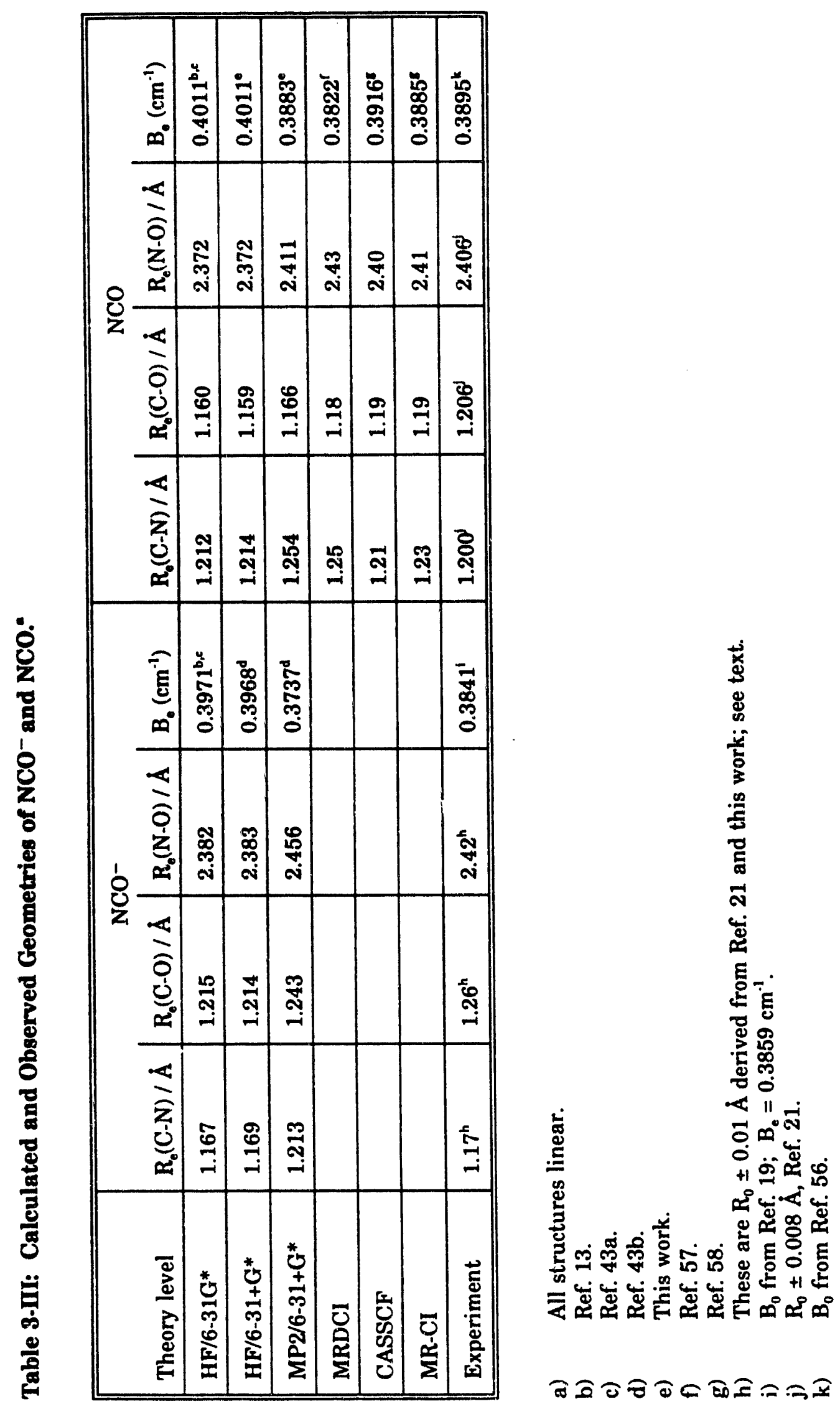




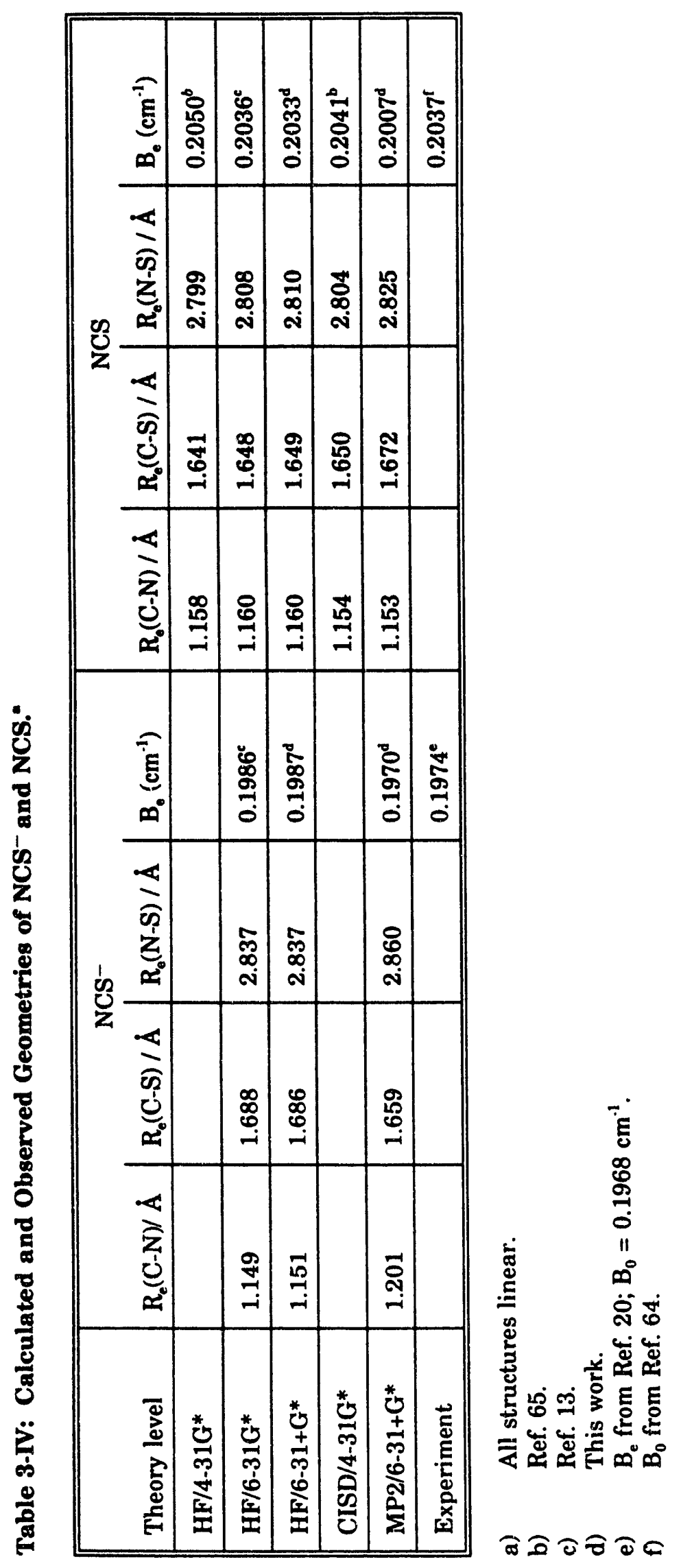


Figure Captions for Chapter 3.

Figure 3-1. Photoelectron spectra of (top) $\mathrm{CN}^{-}$, (middle) $\mathrm{NCO}^{-}$, and (bottom) $\mathrm{NCS}^{-}$ with $266 \mathrm{~nm}$ (4.657 eV) laser radiation. The polarization of laser with respect to the electron collection direction is parallel $\left(\theta=0^{\circ}\right)$ for $\mathrm{CN}^{-}$, 'magic angle' $\left(\theta=55^{\circ}\right)$ for $\mathrm{NCO}^{-}$, and perpendicular $\left(\theta=90^{\circ}\right)$ for NCS-. The experimental spectra have been smoothed by convolution with a $5 \mathrm{meV}$ full-width at half-maximum Gaussian. The inset of the $\mathrm{CN}^{-}$ photoelectron spectrum shows a rotational contour simulation of the CN $\mathrm{X}\left({ }^{2} \Sigma^{+}\right) \leftarrow \mathrm{CN}^{-} \mathrm{X}\left({ }^{1} \Sigma^{+}\right)$transition assuming an ion rotational temperature of $200 \mathrm{~K}$ (solid line); the experimental data points are indicated by circles. Rotational constants used in the simulation : $\mathrm{B}^{\prime \prime}=1.875 \mathrm{~cm}^{-1}$, $\mathrm{B}_{0}^{\prime}=1.891 \mathrm{~cm}^{-1}, \mathrm{D}_{0}^{\prime \prime}=-6.202 \times 10^{-6} \mathrm{~cm}^{-1}, \mathrm{D}_{0}^{\prime}=-6.73 \times 10^{-6} \mathrm{~cm}^{-1}$. Refs. 1,7

Figure 3-2. Photoelectron spectrum of $\mathrm{CN}^{-}$with $213 \mathrm{~nm}(5.822 \mathrm{eV})$ laser radiation. The polarization of the laser is perpendicular $\left(\theta=90^{\circ}\right)$ to electron detection direction.

Figure 3-3. The highest $\sigma$ and $\pi$ molecular orbitals for $\mathrm{CN}^{-}$, and the highest occupied molecular orbital (HOMO) for each of the $\mathrm{NCO}^{-}$and $\mathrm{NCS}^{-}$ anions. These are the SCF molecular orbitals computed at the MP2/6-31+G* optimized geometry for each ion. Photodetachment (removal) of an electron from each of these orbitals yields the ground electronic state of the respective neutral radical, except photodetachment from the $\mathrm{CN}^{-} \pi$ orbital which yields the $\mathrm{CN}\left(\mathrm{A}^{2} \Pi\right)$ excited state. 
Figure 3-4. Franck-Condon simulation for the $\left.\mathrm{CN} A\left({ }^{2} \Pi\right) \leftarrow \mathrm{CN}^{-} \mathrm{X}^{(1} \Sigma^{+}\right)$band. Experimental $213 \mathrm{~nm}$ data plotted as points. $R_{e}^{\text {anion }}=1.1765 \AA$, $\mathrm{T}_{\text {vib }}=1400 \mathrm{~K}$.

Figure 3-5. Simulated photoelectron band for $\mathrm{NCO}^{-}$photodetachment assuming spectroscopic parameters in Table 3-II. Simulation has anion vibrational temperature set at $\mathrm{T}=775 \mathrm{~K}$ for the two stretching modes and $\mathrm{T}=600 \mathrm{~K}$ for the bend mode. Experimental data plotted in points.

Figure 3-6. Simulated photoelectron band for NCS$^{-}$photodetachment with anion vibrational temperature set at $350 \mathrm{~K}$, assuming spectroscopic parameters in Table 3-II. Experimental data plotted in points. 


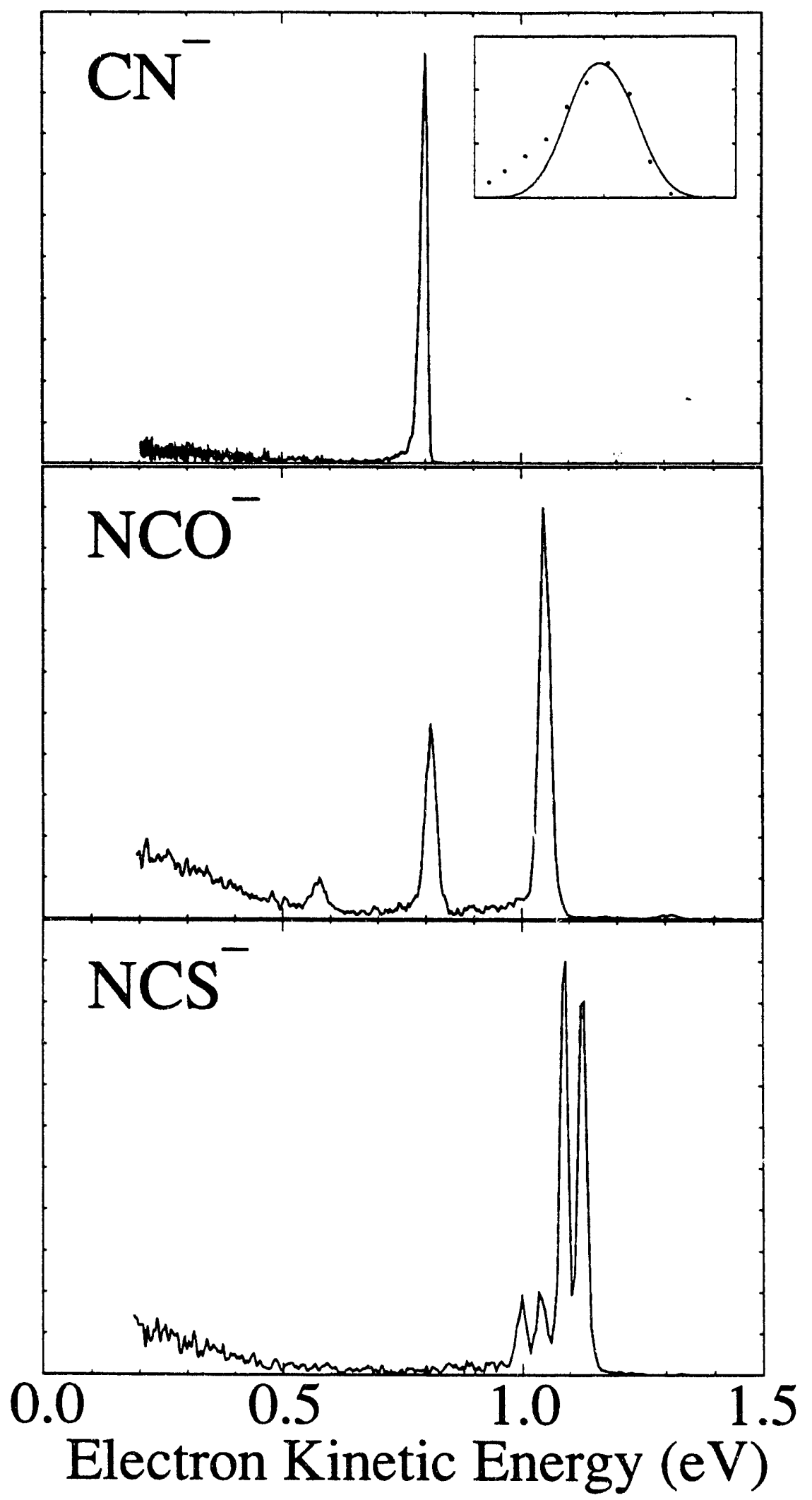

Figure 3-1 


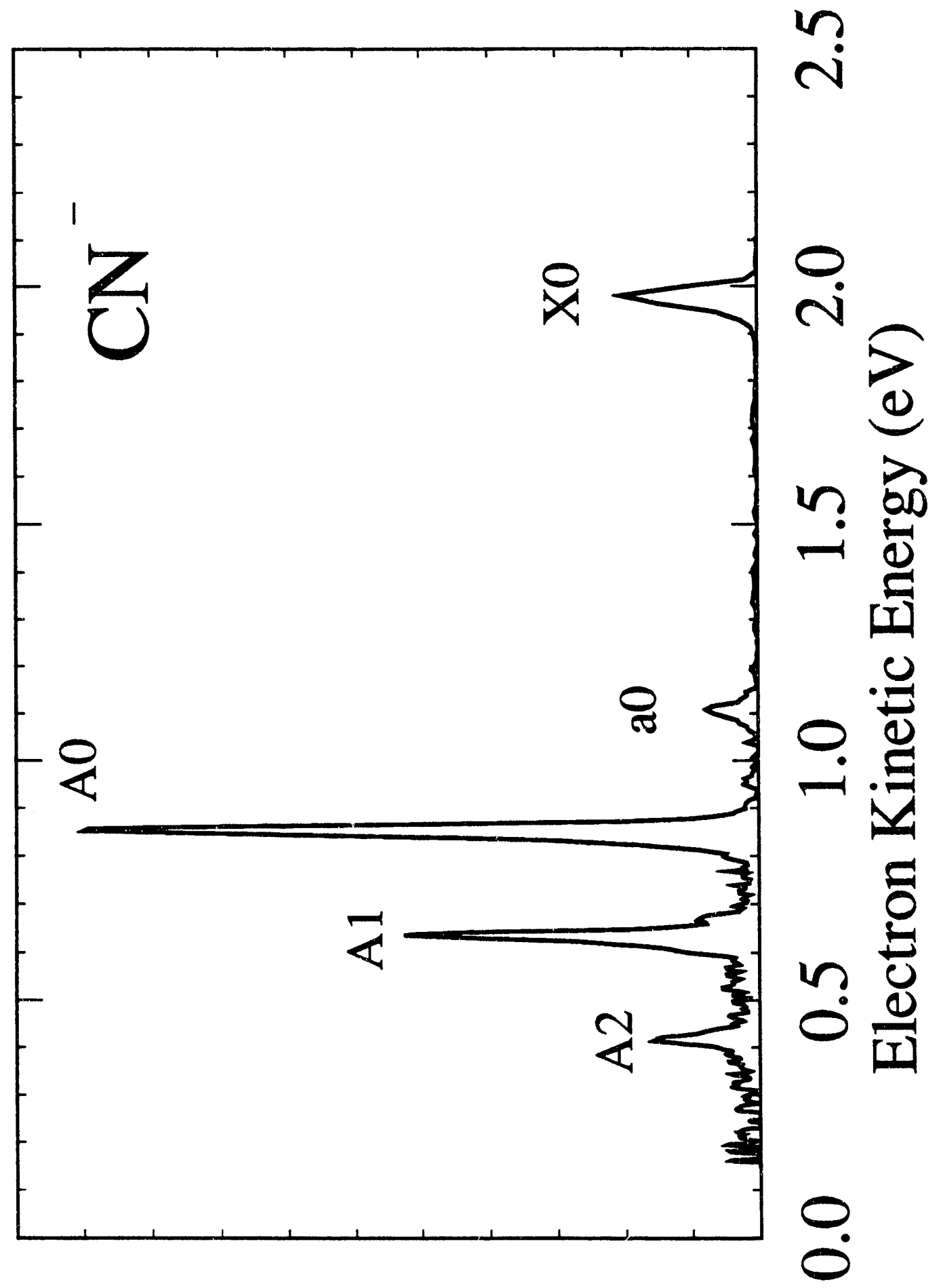

Figure 3-2 


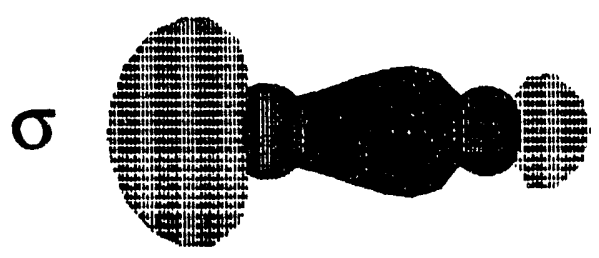

C N

$\pi$

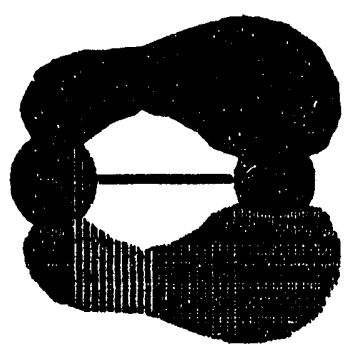

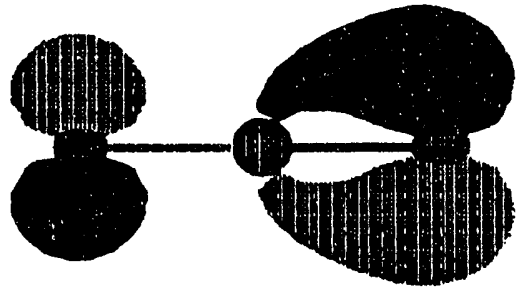

O $\mathrm{C} \quad \mathrm{N}$

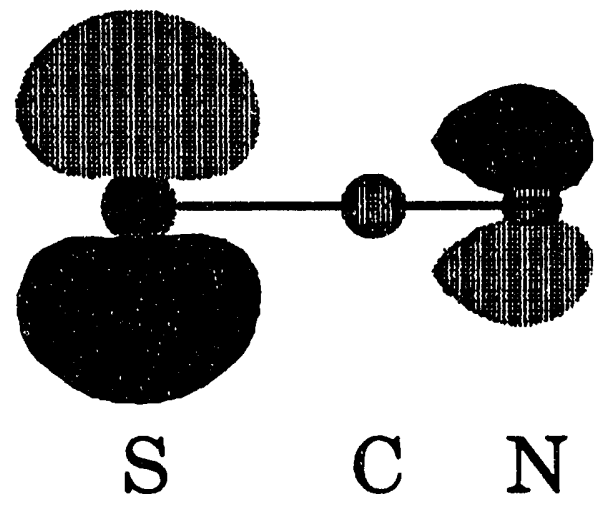

Figure 3-3 


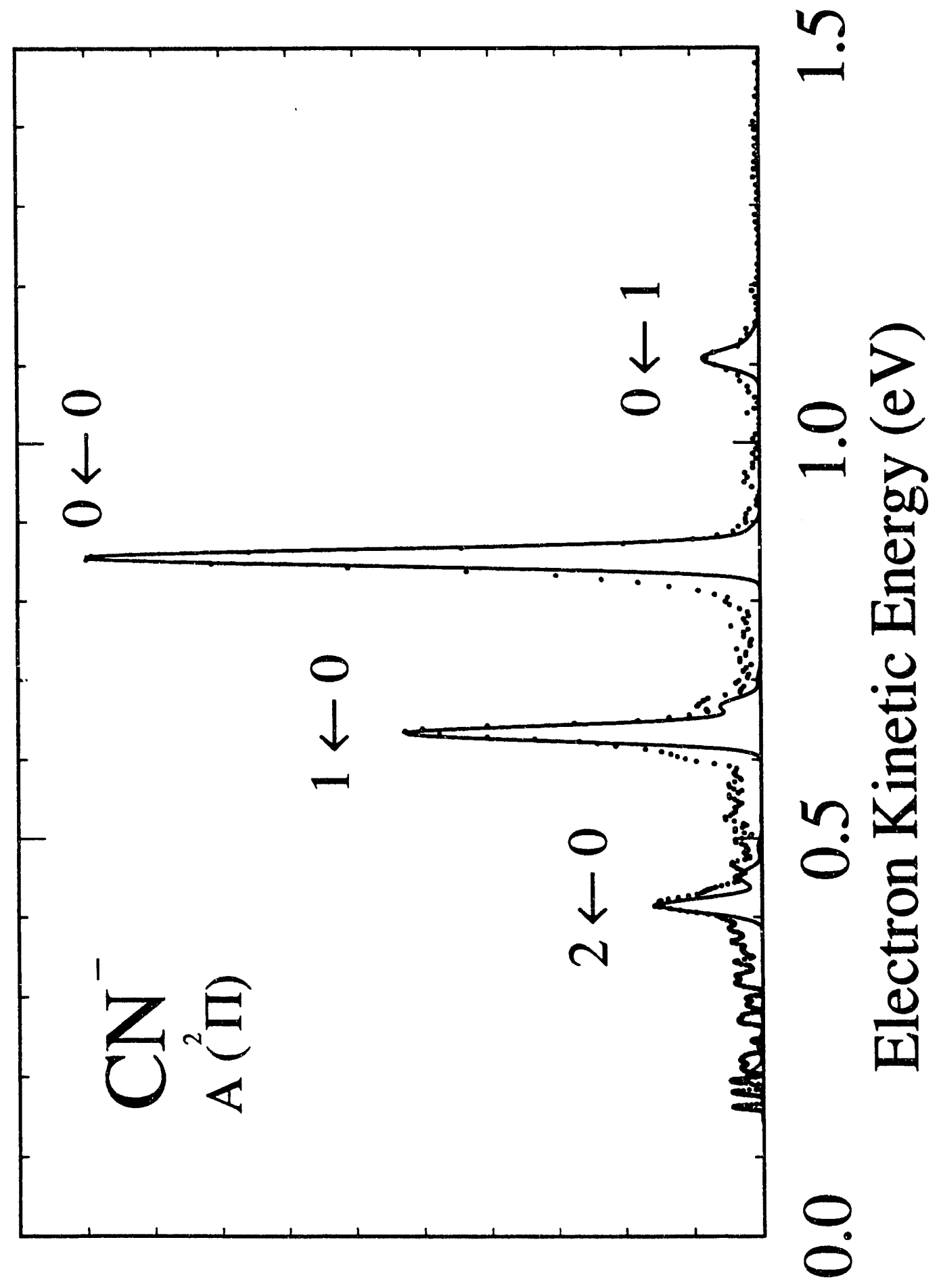

Figure 3-4 


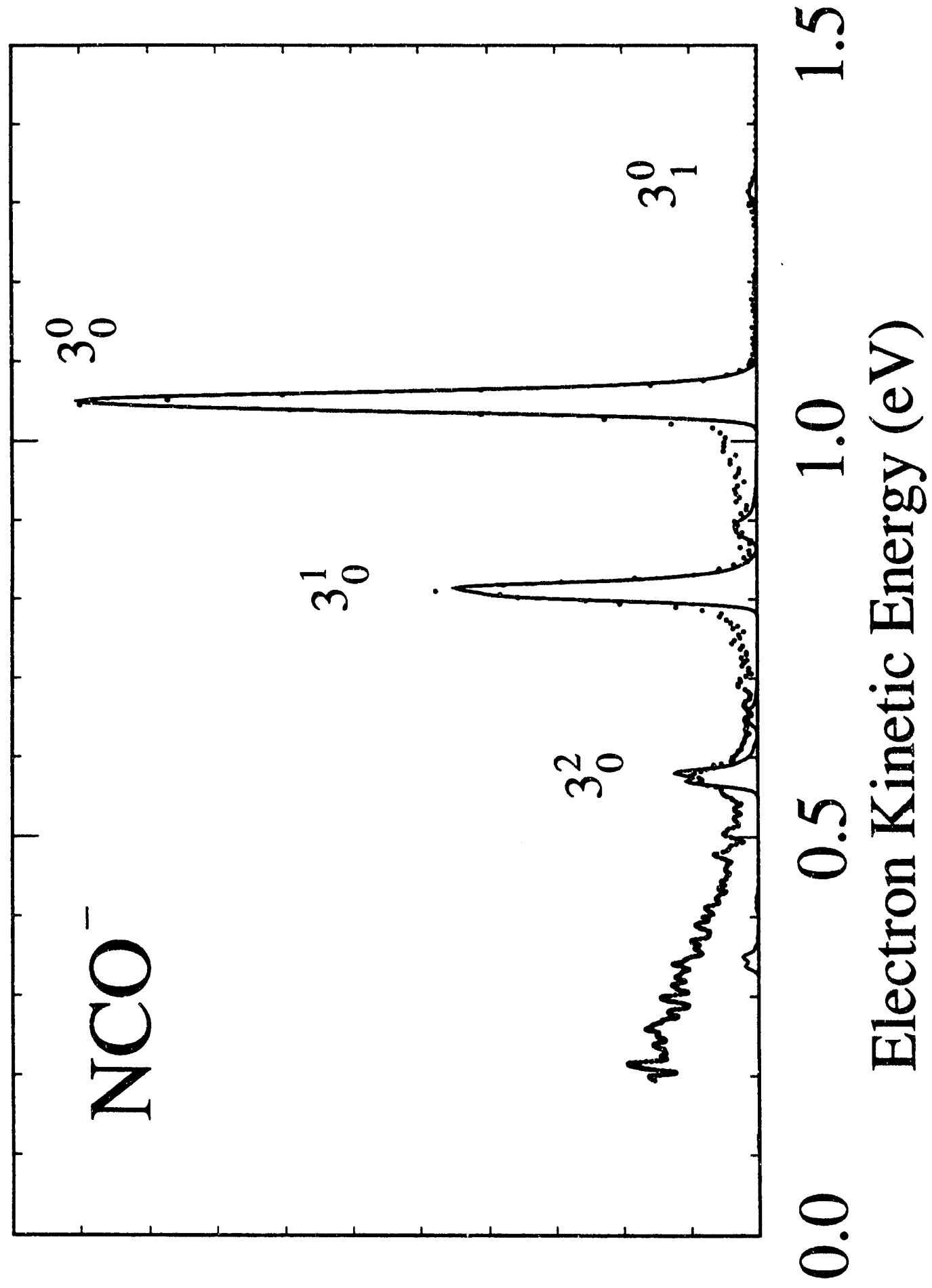

Figure 3-5 


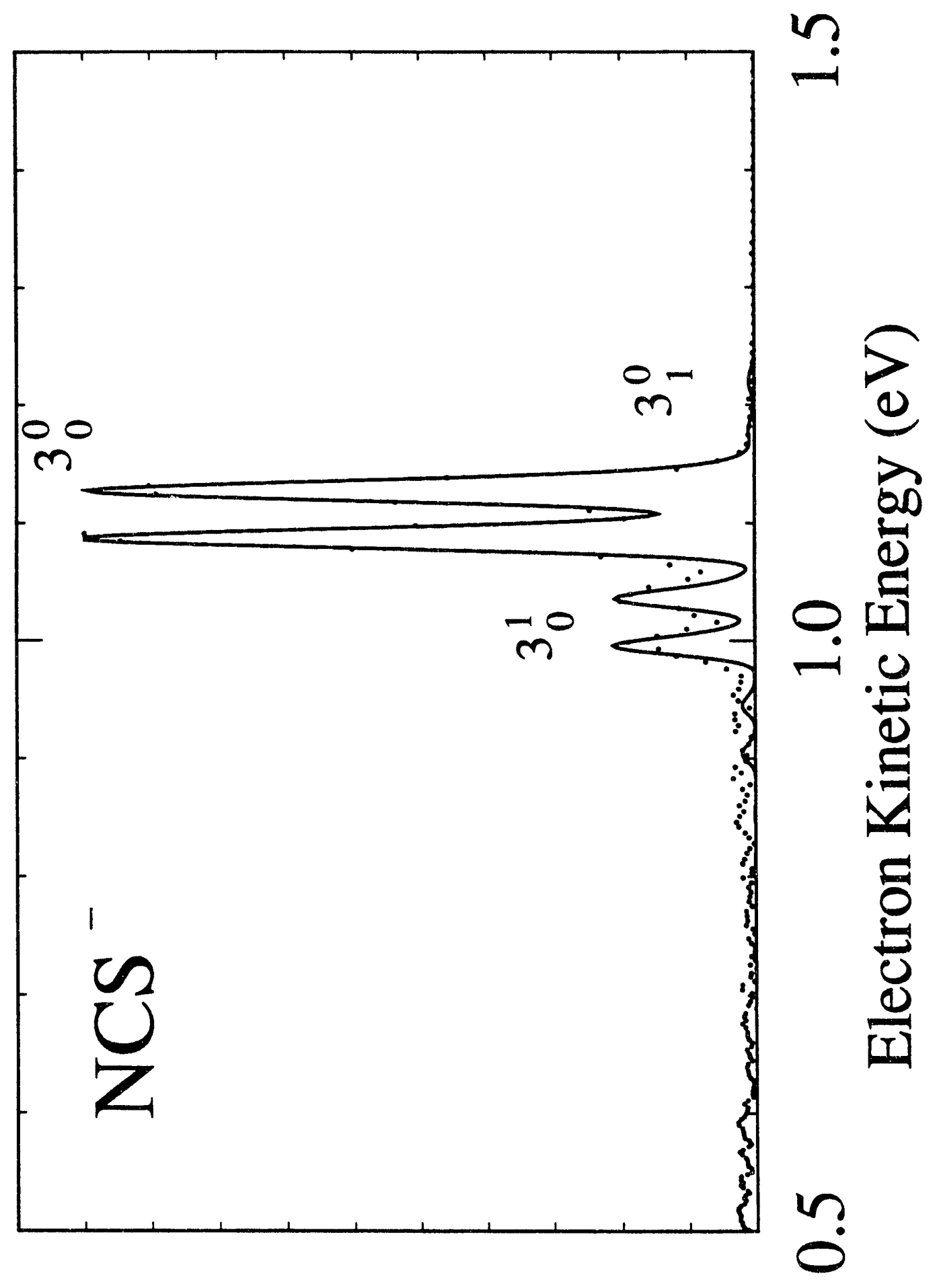

Figure 3-6 


\section{Chapter 4. Theoretical: Time-dependent framework for analyzing dissociative photoelectron spectra.}

\section{Introduction}

In the interpretation of our transition state photoelectron spectra we have to relate the observed structure to the neutral reaction potential energy surface(s). This will mean simulating each photoelectron band with a fairly rigorous quantum mechanical procedure. However, we should choose a model that is not overly complicated, as we would like to be able to vary the neutral potential energy surface several times to try to improve the fit with the observed spectrum. This chapter describes the methods we have chosen to perform this operation, and some of the underlying theory and the philosophy behind the model.

The photoelectron spectrum of a cold negative ion involves transitions from the ground vibrational state of the ion to many possible states supported by the upper neutral surface. If there is more than one neutral electronic state accessible from the ion with the photon energy used, and transitions to that surface are allowed, ${ }^{1}$ then bands due to each surface will be observed. Let us assume for the moment that there is only one neutral surface contributing to the photoelectron spectrum. The structure in the spectrum, i.e. the peak positions, widths and spacings, is due to the neutral states, and the peak intensities are due to the overlap of each neutral state with the anion ground state wave function. The problem then typically breaks into two parts, characterizing the upper neutral states, and calculating the overlap of these neutral state wave functions with the simple anion wave function. 
Expressing the above mathematically, the photoelectron spectrum as a function of energy is, within the Franck-Condon approximation,

$$
\sigma(E) \propto\left|\left\langle\psi_{0}^{\prime \prime} \mid \psi^{\prime}(E)\right\rangle\right|^{2}
$$

where $\psi_{0}^{\prime \prime}$ is the anion $v=0$ wave function and $\psi^{\prime}(E)$ is the wave function on the neutral potential energy surface with energy $E$. If the neutral is bound, i.e. is not unstable with respect to dissociation, then $\psi^{\prime}$ is a discrete function of energy. $\psi^{\prime}$ is non-zero only at eigenvalues of the neutral potential surface. If the neutral is unbound, then $\psi^{\prime}$ is a continuous function of energy. $E$ is defined with respect to some (arbitrary) zero of energy ualiy the bottom of the lowest well on the neutral surface. The relationship between the electron kinetic energy, $E_{k}$, of the photoelectron spectrum and $E$, often described as the neutral or scattering energy, is

$$
E_{k}=h v-\Delta_{0}-E
$$

where $h v$ is the photon energy and $\Delta_{0}$ is a constant energy relating the ground state level of the anion to the neutral's zero of energy. For example in a simple $\mathrm{M}^{-} \rightarrow \mathbf{M}$ photoelectron process where both anion $\mathrm{M}^{-}$and neutral $\mathrm{M}$ are bound molecules, $\Delta_{0}$ would be the adiabatic electron affinity less the zero point energy of the M neutral.

For the less interesting case when the neutral potential surface is bound, equation (1) is routinely solved by invoking the normal mode approximation, and separating out all the nuclear degrees of freedom. ${ }^{2}$ Usually the parallel mode approximation is also assumed, i.e. the normal coordinate decomposition of nuclear motion is identical for both the anion and neutral. The eigenvalues and eigenfunctions for motion in each separable mode are found, or are known analytically 
in the case of a Morse or harmonic oscillator, and the spectrum is calculated by performing the one dimensiona verlaps of each eigenfunction with $\psi_{0}^{\prime \prime}{ }^{3}$ This was the method used in Chapter 3 to simulate the photoelectron spectra of $\mathrm{CN}^{-}, \mathrm{NCO}^{-}$ and NCS-.

In cases where a mode is not modeled by a harmonic or Morse potential function, numerical solution of the Schrödinger equation is required, and this is can be accomplished by a variety of numerical algorithms. One of the most efficient algorithms is the DVR method of Light and coworkers, ${ }^{4}$ which has been described by Metz. ${ }^{2}$ I note here that it is also possible, and simple, to use time-dependent methods to solve for bound levels, and although not as efficient as DVR for this problem, a time-dependent solution has been used to find the eigenstates of the "one-dimensional cut" in Chapter 5.

The nature of our transition state experiments, where the species formed by photodetachment lives only on a femtosecond time scale, Jictates that the neutral state wave functions are not bound. The potential energy surface is repulsive and the $\psi^{\prime}$ are scattering states. In general the full Schrödinger equation must be solved numerically, and separation of nuclear degrees of freedom along the lines of the normal mode approximation has only limited success. An alternative formulation to the problem, and along with it an alternative languige from that of scattering states, is given by Heller. ${ }^{5}$ This is set up in time-dependent quantum mechanics, and involves motion of wave packets to describe molecular spectra. Although this formulation is mathematically equivalent to the above time-independent method, and the solutions via the two approaches are therefore identical, the time-dependent formalism gives rise to an entirely different conceptual framework to extract the 
dynamics from the photoelectron spectrum. In fact that framework is in many senses more appealing, as it carries with it a classical feel, but does not suffer from the inadequacies of classical mechanics in describing light particle motion. The inherent principle of dynamical processes occurring along a time axis is restored. This framework has become increasingly popular over the last few years, and the language of scattering and photodissociation is now decidedly mixed between time-independent and time-dependent. These developments have been spurred, in part, by the appearance of experiments that cibserve chemical dynamics explicitly in real time, such as those of Zewail and coworkers. ${ }^{6}$

The numerical inplementation of time-dependent solutions of the Schrödinger equation has been driven by the work of Kosloff. ${ }^{7,8}$ In section 3 , we will outline the Kosloff method for solving the time-dependent Schrödinger equation, and describe the practical issues involved in a successful wave packet propagation calculation. Several other groups have used the time-dependent approach to simulate frequency domain spectra, ${ }^{9,} 10$ as well as to interpret Zewail's time-domain experiments. The application of a time-deperdent analysis to a frequency-domain experiment, such as photoelectron spectroscopy, is not contradictory. Although a wave packet is not created in our "long pulse""1 photodetachment experiment, but rather a well defined neutral scattering state $\psi^{\prime}(E)$ with a transition probability given by Equation (1), the photoelectron spectrum can nonetheless be interpreted in terms of the dynamics of a wave packet prepared in a hypothetical "short-pulse" experiment. First let us

" Our experiments employ a nanosecond laser. Thus the pulse length is infinitely long compared to the molecular dynamics. 
establish the relationship between the time-dependent wave packet and the energy spectrum.

\section{The photoelectron spectrum from the wave packet time dynamics}

The ground state wave function of the lower (anion) surface, $\psi_{0}^{\prime \prime}(x)$, is transferred "up" to the neutral surface by the laser: an electronic transition dipole moment links the two surfaces. The vertical transition gives birth to a wave packet $\phi(x, 0)$ on the apper surface given by

$$
\phi(x, 0)=\mu(x) \Psi_{0}^{\prime \prime}(x) .
$$

$\mu(x)$, the electronic transition moment, is usually taken to be a constant over the range of coordinates where $\psi_{0}^{\prime \prime}(x)$ has any amplitude, as in the Franck Condon formula (1). $\phi(x, 0)$ is not an eigenfunction, or stationary state, of the neutral surface but evolves in time. $\phi(x, 0)$ of Equation (3) is then the wave packet at the "zero" of time. From now on we do not refer to the explicit coordinate dependence of the wavepacket: the initial wavepacket is thus denoted $\phi(0)$. The wave packet motion is governed by the ti.ne-dependent Schrödinger Equation

$$
i \hbar \frac{\partial \phi(t)}{\partial t}=H \phi(t)
$$

where $\boldsymbol{H}$ is the Hamiltonian for the neutral surface. The solution of (4) is formally expressed as 


$$
\phi(t)=e^{-t H t / \hbar} \phi(0)
$$

where $e^{\text {iHt/n }}$ is the time evolution operator. This gives the dynamics of the wave packet for all times $t$. The autocorrelation function, $C(t)$, is defined by

$$
C(t)=\langle\phi(0) \mid \phi(t)\rangle
$$

and monitors the time development of the overlap of the moving wave packet with the initial wave packet at $t=0$. In other words, the behavior of the wave packet with respect to the Franck-Condon region is mapped by $C(t)$. Once the wave packet has completely disappeared from the Franck-Condon region, $C(t) \rightarrow 0$.

The photoelectron spectrum is related to the autocorrelation function by the Fourier transformation ${ }^{5}$

$$
\sigma(E) \propto \int_{-\infty}^{+\infty} e^{i E t / \$} C(t) d t
$$

This can readily be shown to be rigorously equivalent to Equation (1) by the following steps. Substituting (5) and (6) into (7) yields

$$
\sigma(E) \propto \int_{-\infty}^{+\infty} e^{t E \pi / M}\left\langle\phi(0)\left|e^{-t H t / \phi}\right| \phi(0)\right\rangle d t
$$

Inserting the completeness relationship for the set of wave functions, $\psi^{\prime}$, of the neutral Hamiltonian $\mathbf{H}$,

$$
\int d E^{\prime}\left|\Psi^{\prime}\left(E^{\prime}\right)\right\rangle\left\langle\Psi^{\prime}\left(E^{\prime}\right)\right|=1
$$


into (8) gives

$$
\sigma(E) \propto \int_{-\infty}^{+\infty} e^{i E t / \hbar}\left\langle\phi(0)\left|e^{-i H t / \hbar} \int d E^{\prime}\right| \Psi^{\prime}\left(E^{\prime}\right)\right\rangle\left\langle\Psi^{\prime}\left(E^{\prime}\right) \mid \phi(0)\right\rangle d t
$$

As $\psi^{\prime}\left(E^{\prime}\right)$ are eigenfunctions of $\mathbf{H}$, then

$$
\begin{gathered}
\sigma(E) \propto \iint_{-\infty}^{+\infty} e^{i E t / \uparrow}\left\langle\phi(0)\left|e^{-i E^{\prime} t / \uparrow}\right| \Psi^{\prime}\left(E^{\prime}\right)\right\rangle\left\langle\Psi^{\prime}\left(E^{\prime}\right) \mid \phi(0)\right\rangle d E^{\prime} d t \\
\Rightarrow \sigma(E) \propto \int d E^{\prime} \int_{-\infty}^{+\infty} d t e^{i\left(E-E^{\prime}\right) t / \uparrow}\left|\left\langle\phi(0) \mid \Psi^{\prime}\left(E^{\prime}\right)\right\rangle\right|^{2} \\
-\sigma(E) \propto \int d E^{\prime} 2 \pi \hbar \delta\left(E-E^{\prime}\right)\left|\left\langle\phi(0) \mid \Psi^{\prime}\left(E^{\prime}\right)\right\rangle\right|^{2} \\
-\sigma(E) \propto\left|\left\langle\phi(0) \mid \Psi^{\prime}(E)\right\rangle\right|^{2}
\end{gathered}
$$

As we have assumed $3 x)$ is a constant over $x$ in Equation (3) then expression (14) is equivalent to the Franck-Condon relationship (1).

The major approximation in both expressions (1) and (7) is that the photodetachment electronic transition moment is taken to be a constant, and is an average over not only the nuclear coordinates but also over all final electron kinetic energies. Reutt has considered these approximations and given a similar, but more rigorous, derivation of (7) to that appearing here. ${ }^{11}$

We have established through (7) that the photoelectron spectrum is simply related to the autocorrelation function by a Fourier transform. This is a powerful result. If we calculate $\mathrm{C}(\mathrm{t})$ from a theoretical wave packet propagation, we can immediately simulate the photoelectron spectrum. Let us first make a few 
observations concerning the autocorrelation function. The wave packet is a complex function of the nuclear coordinates, i.e. it has real and imaginary parts. Even if $\phi(x ; t)$ is a stationery state, the wave function has a time evolution that involves a constantly changing phase. We usually define the phase to be zero at $t=0$. It follows that $C(t)$ is also complex, and even for a stationery state, where $|C(t)|=1$ for all $t$, the real and imaginary parts of $C(t)$ vary sinusoidally in time. Usually when the autocorrelation function is plotted to gain insight into the wave packet dynamics, only the absolute value, $|C(t)|$, is shown. However, numerically, the phase time dependence cannot be ignored.

The Fourier integral limits in Equation (7) indicate evaluation over the time interval $[-\infty, \infty]$. Two points concern us with the physical implementation of this Fourier transform (FT). Firstly, time reversal symmetry dictates that $C(t)$ is a Hermitian function, i.e. $C(-t)=C^{*}(t)$. Thus we need only run the dynamics from $t=$ $O$ onwards! Secondly, how long in time must $C(t)$ be computed so that the photoelectron spectrum may be evaluated? Clearly only a finite time of dynamics is required to yield the spectrum to a resolution equivalent to the experiment. We will return to this point later.

Some examples of the dynamical signatures one may expect in the autocorrelation are given in the papers of Heller, ${ }^{5}$ Imre, ${ }^{9}$ Reutt, ${ }^{11}$ and Lorquet ${ }^{12}$ as well as in chapters 5 and 6 of this thesis. Heller discusses some of the general relationships between peak spacings, homogeneous peak widths, as well as the overall Franck Condon envelope width in the energy spectrum and their characteristic time periods in $|C(t)|$. Some simple examples are illustrated in Figure 4-1. The reappearance of the wave packet in the Franck Condon region, signalled by a peak in 
the autocorrelation subsequent to $t=0$, is termed a recurrence. Recurrence features, are shown in Fig. 4-1(ii, iii). These are significant as they yield vibrational structure in the energy spectrum. In fact one single recurrence in $C(t)$ is necessary, but sufficient, to see undulations, or diffuse structure, in the energy spectrum (see Fig. 4-1(iii)). ${ }^{9}$ Correlation loss over several vibrational periods is possible from a number of processes other than direct dissociation. Anharmonicity in a bound vibrational mode or non-adiabatic effects are possible mechanisms for losses at each recurrence in $|C(t)|$.

\subsection{The Autocorrelation function from the photoelectron spectrum}

Equation (7) may be inverted

$$
C(t) \propto \int_{-\infty}^{+\infty} \sigma(E) e^{-t E t / h} d E
$$

yielding the autocorrelation from the (experimental) spectrum. This has been the approach adopted by other groups in all prior photoelectron studies where the spectrum was interpreted, via $\mathrm{C}(\mathrm{t})$, in terms of the short time molecular dynamics. ${ }^{11}$ 12, 13 iorcuet has demonstrated how the reverse Fourier transform procedure may also be used for deconvolution purposes. The instrumental response function, any spin orbit splitting and rotational peak broadening can be removed to uncover the bare vibrational dynamics. ${ }^{12}$

We make a much stronger connection to the dynamics by simulating the wave packet dynamics that determine $C(t)$. From this simulation we may then compare either the theoretical $|C(t)|$ with the Fourier transformed experimental data or the 
simulated photoelectron spectrum, from Equation (7), with the raw experimental photoelectron spectrum.

\section{Wave packet propagation}

\subsection{Method}

To compute the motion of a wave packet on a model potential energy surface we must find a numerical solution to Equation (4). $H$ is the Hamiltonian for the neutral state and is given by

$$
\boldsymbol{H}=\boldsymbol{T}+\boldsymbol{V}=-\frac{\hbar^{2}}{2 \mu} \nabla^{2}+\boldsymbol{V}
$$

where $\nabla$ and $V$ are the $\mathrm{N}$-dimensional Laplacian and potential respectively. Throughout we shall use atomic units (i.e. $\hbar=m_{e}=1$ ). Note that the potential energy, and therefore the Hamiltonian, is time independent. The actual physical problems we will address in this work involve one or two dimensions, although the approach is completely general. For sake of illustration, we will consider the twodimensional case where the Cartesian coordinates, $x$ and $y$, are the mass-scaled Jacobi coordinates for $\mathrm{A}+\mathrm{BC}$ collinear reactive scattering. The coordinates are defined by $x=\left(\mu_{C, A B} / \mu_{A B}\right)^{1 / 2} R_{C, A B}$ and $y=R_{A B} . \quad \mu_{C, A B}$ is the reduced mass of the system $\mathrm{C}$ and $\mathrm{AB}$, likewise $\mu_{A B}$ is the reduced mass of $\mathrm{A}-\mathrm{B}$. The kinetic energy operator for this case is separable 


$$
T=-\frac{1}{2 \mu_{A B}}\left[\frac{\partial^{2}}{\partial x^{2}}+\frac{\partial^{2}}{\partial y^{2}}\right] .
$$

The numerical solution of (4) thus involves both spatial and temporal derivatives. Following Kosloff's formulation, ${ }^{7,8}$ this differential equation is solved by discretizing both time and space on a uniform grid. Accordingly, let $\phi^{n}\left(i_{x}, i_{y}\right)$ represent the wave packet at time $t=(n-1) \Delta t$ at the location $x=\left(i_{x}-1\right) \Delta x, \quad y=$ $\left(i_{y}-1\right) \Delta y . \quad \Delta t, \Delta x$ and $\Delta y$ are the time step and the spacings of the Cartesian grid points respectively. Rewriting (4) in this discrete representation gives

$$
i \frac{\partial \phi^{n}\left(i_{x}, i_{y}\right)}{\partial t}=H \phi^{n}\left(i_{x}, i_{y}\right)
$$

This expression suggests an iterative (marching) scheme in time where the next wave packet is calculated from the previous packet(s) by evaluating the right hand side of Equation (18). Approximating the time derivative with a second order differencing formula

$$
\frac{\partial \phi^{n}\left(i_{x}, i_{y}\right)}{\partial t}=\frac{\phi^{n+1}\left(i_{x}, i_{y}\right)-\phi^{n-1}\left(i_{x}, i_{y}\right)}{2 \Delta t}
$$

then

$$
\phi^{n+1}=\phi^{n-1}-2 i \Delta t H \phi^{n}
$$

Thus the $(n+1)$-th wave packet at $t=n \Delta t$ may be calculated from the two preceding packets once we have established how to compute the $\mathbf{H}$ operator. This second order differencing propagation scheme is a stable iterative solution of the timedependent Schrödinger equation, whereas the slightly simpler first order analog is 
not. ${ }^{7}$ This is, therefore, the simplest finite difference scheme for solution of (18). Several more sophisticated propagators have been proposed that may be more efficient. $^{14}$

Equation (20) gives an iterative scheme that allows us, in principle, to propagate the wave packet for any desired time length. To initialize the solution, however, we require both $\phi^{1}$, the initial wave packet (which we are setting equal to the anion ground state wave function), and $\phi^{2}$. To find $\phi^{2}$ we use second order RungeKutta ${ }^{15}$ :

$$
\phi^{2}=\phi^{1}-i \Delta t H \phi^{I}
$$

where the intermediate packet $\phi^{I}$ is given by

$$
\phi^{I}=\phi^{\perp}-\frac{i \Delta t}{2} \boldsymbol{H} \phi^{1}
$$

To compute the spatial derivatives involved in the operation of the Hamiltonian a pseudo-spectral (or Fourier) method is adopted, in contrast to the standard finite difference approach employed for the time derivative. The advantage of using a Fourier method is that it is extremely efficient and it requires substantially fewer grid points than finite difference schemes of similar accuracy. ${ }^{16}$ The conceptual appeal of the method is that it allows calculation of both the kinetic and potential operators locally. The operation of $\mathbf{V}$ on the wave packet $\phi^{n}$ is simply to multiply together the value of the potential and the wave packet at each grid point - thus $\mathrm{V}$ is local in the position (Cartesian) representation. The Laplacian, however, is not local in the position representation - it involves spatial derivatives of the wave packet. The Fourier method uses the property of a Fourier transform that a derivative in the 
spatial domain becomes a multiplication in the Fourier (momentum) domain. Thus the Laplacian operator is local in the momentum representation. ${ }^{8}$

If the spatial grid is set up in a rectangular set of coordinates, i.e. the kinetic energy can be written in the form of Equation (17), then the kinetic energy operator in momentum space is also separable:

$$
T\left(k_{x}, k_{y}\right)=\frac{1}{2 \mu}\left(k_{x}^{2}+k_{y}^{2}\right)
$$

where $k_{x}$ and $k_{y}$ are the wavenumbers along the $x$ and $y$ spatial directions. Operating with $\mathbf{T}$ on $\Phi^{n}\left(k_{x}, k_{y}\right)$, the Fourier transformed wave packet, is as simple as multiplying $\Phi^{n}\left(k_{x}, k_{y}\right)$ by $\left(k_{x}^{2}+k_{y}^{2}\right) / 2 \mu$. In the discretized form

$$
T \Phi^{n}\left(i_{k_{x}}, i_{k_{y}}\right)=\frac{1}{2 \mu}\left[\left(\left(i_{k_{x}}-1\right) \Delta k_{x}\right)^{2}+\left(\left(i_{k_{y}}-1\right) \Delta k_{y}\right)^{2}\right] \Phi^{n}\left(i_{k_{x}}, i_{k_{y}}\right)
$$

From the properties of the discrete Fourier transform, $\Delta k_{x}$ is given by $\Delta k_{x}=2 \pi /\left(x_{\max }\right.$ " $\left.x_{\text {min }}\right)$ with a similar expression for $\Delta k_{y}{ }^{15}$ The resulting $T \Phi^{n}\left(i_{k_{x}}, i_{k_{y}}\right)$ is reverse Fourier transformed back to the spatial domain and added to $V \phi^{n}\left(i_{x} i_{y}\right)$ to form $H \phi^{n}$. Because the grid points are equally spaced, an two-dimensional fast Fourier transform (FFT) algorithm may be used to compute both Fourier transforms. Library routines for the extremely efficient evaluation of 1 and 2 dimensional FFTs are available. ${ }^{17}$

Numerical solution of the problem then boils down to the following recipe., 16

(a) Specify the initial wave packet on a chosen grid: $\phi^{1}=\psi_{0}^{\prime \prime}$, the anion ground state wave function.

(b) Calculate the time evolution of $\phi(t)$ by obtaining $\phi^{n}$ for successively higher $n$ by relation (20); each time step involves: 
(i) compute $T \phi^{n}$ by an N-dimensional FFT on $\phi^{n}$ followed by a multiplication by $\left(k_{x}^{2}+k_{y}^{2}\right) / 2 \mu$ and a reverse FFT.

(ii) calculate $V \phi^{n}$ by multiplication of the potential function evaluated at each grid point by the wave packet at the same grid point.

(iii) after summing (i) and (ii) to give $\boldsymbol{H} \phi^{n}$, form $\phi^{n+1}$ from $\phi^{n \cdot 1}$ and $\boldsymbol{H} \phi^{n}$.

(c) At intervals of $\Delta \tau_{\text {ovip }}$, compute the self-overlap of the wave packet $\left\langle\phi^{1} \mid \phi^{n}\right\rangle$ by integration on the spatial grid and store.

(d) At the end of the time propagation, Fourier transform the stored autocorrelation function $\mathrm{C}(\mathrm{t})$ according to (7) to yield the photoelectron spectrum.

The method is relatively easy to implement as most of the numerical work is done in the library FFT routine. Appendix C describes the wave packet propagation codes developed for use in this work.

\subsection{Stability criteria and propagation errors.}

Kosloff and Kosloff show that there is a numerical dispersion in the Fourier method, which must be considered when choosing the time step for a propagation.? For time steps larger than a certain value, known as $\Delta t_{\text {crit }}$, the propagation will become unstable and exponentially increasing solutions will take over from the desired wave-like solutions. $\Delta \mathrm{t}_{\mathrm{crit}}$ is given by the following relationship 


$$
\left|\Delta t_{c r k}\left[\frac{\pi^{2}}{2 \mu}\left(\frac{1}{\Delta x^{2}}+\frac{1}{\Delta y^{2}}\right)\right]\right|=1
$$

for a two dimensional spatial grid and zero potential. The term inside square brackets represents the maximum kinetic energy (in atomic units) for the wave packet allowed by the grid. This is derived from the range of momenta supported by the choice of grid; in the $x$ direction, for example, this is $-k_{x}{ }^{\text {max }}$ to $k_{x}{ }^{\text {max }}$ where $k_{x}{ }^{\text {max }}=\pi / \Delta x$. Relation (25) is merely a statement of the energy-time uncertainty principle: the time step cannot be larger than $1 / \Delta E$, where $\Delta E$ represents the total range of eigenvalues possible in the Hamiltonian. ${ }^{8}$ For a real system where the potential is non-zero, $\Delta E$ is the sum of the complete range of kinetic and potential energies. Therefore, Equation (25) is an overestimate for $\Delta t_{\text {crit }}$ and the range of potential energies expressed on the grid should be monitored carefully (see below). In order to achieve a converged solution of the time-dependent Schrödinger equation, values of the time step should be chosen such that $\Delta t-0.2 \Delta t_{\text {crit }}$. Kosloff and Kosloff show the numerical dispersion in this regime is almost identical to the intrinsic dispersion in the time-dependent Schrödinger equation.?

Kosloff has also shown that the Fourier/ second order differencing method necessarily preserves the norm and the energy of the wave packet. ${ }^{7}$ The error in a propagation thus accumulates in ine phase; this limitation essentially determines the maximum propagation times possible with this discrete propagator approximation. ${ }^{8}$ Convergence of a propagation solution is checked for by reducing the time step and/or decreasing the spatial grid spacings. 


\subsection{Implementation of wave packet propagation to simulation of photoelectron spectra.}

In later chapters, we apply the wave packet propagation method to simulate the photoelectron spectra for several transition state systems. The ions AHB- are in general linear, so photodetachment will access a linear configuration of the atoms in the transition state region of the neutral reaction surface. If the saddle point for the $A+H B$ reactions is collinear, ${ }^{* 2}$ a simulation that computes only the motion of the atoms on a collinear potential surface should yield a reasonable approximation to the true photoelectron spectrum. To construct the initial wave packet, i.e. the anion ground state wave function, we require information concerning the equilibrium bond lengths and fundamental frequencies of $\mathrm{AHB}^{-}$. If appropriate, the form of the two stretching normal modes and anharmonicity data should be included. At best, some vibrational frequencies may be available from matrix isolation work. In most cases the remaining information has to come from ab initio calculations, or even empirical guesses in the worst case scenario.

Next, a trial potential function for the neutral reaction is chosen; this should be an analytic function of the nuclear coordinates. A grid is set up in mass scaled coordinates subject to some of the criteria outlined below. The grid covers the reaction interaction region and the entrance and exit valleys. The number of grid points in each dimension should be a power of two for compatibility with the FFT algorithm. The grid may be fairly sparse: often a grid as small as $64 \times 32$ points is sufficient for

*2 Most potential surfaces for the systems we are studying indeed have collinear saddle geometries. However, recent $a b$ initio calculations for both the $\mathrm{O}+\mathrm{HF}$ and $\mathrm{F}+\mathrm{H}_{2}$ reaction suggest non-linear saddle point geometries. 
a converged propagation. Rarely is it necessary to exceed a grid size of $128 \times 64$ points. Finally a time step and total propagation time is chosen. The time step is chosen to meet the stability and convergence criteria outlined in section 3.2 ; the total propagation time is chosen to reflect the resolution required in the simulated energy spectrum. Propagation for $1 \mathrm{psec}$ is sufficient to yield features in the simulated photoelectron spectrum as narrow as $4 \mathrm{meV}$. Usually $300-400 \mathrm{fs}$ of dynamics is adequate to produce a simulation of resolution comparable to the experimental spectra (ca. $12 \mathrm{meV}$ ).

\subsubsection{Grid sizes, time steps and potential shelves.}

These three aspects of the numerical wave packet propagation are linked together. The denser the grid, i.e. the larger the number of spatial grid points used, the smaller the spatial grid intervals, $\Delta x$ and $\Delta y$, become. As the range of momenta that can be described in the related discrete $k$ space representation is inversely proportional to the spatial grid intervals, the smaller the gird spacings becomes the larger the range of kinetic energies that can be represented in the propagation. This may be physically necessary in some cases. However in cases where it is unnecessary, it leads to dramatically increased computation time for two reasons: (a) the more grid points used the slower the spatial fast Fourier transforms and (b) the larger the possible range of kinetic energies, according to relation (25) the smaller the time step becomes for a stable propagation. Thus, as a preliminary to a production run propagation it is useful to establish the range of kinetic energies that are physically reasonable for wave packet motion in each spatial dimension and plan the grid spacings accordingly. ${ }^{7}$ Then the overall range in coordinate space (i.e. $x_{\min }, x_{\max }$ etc.) 
should be decided such that the number of grid points in each direction is a power of two and that the grid includes the Franck Condon region and enough of the entrance/ exit valleys to accommodate an absorbing boundary (see 3.3.2 below). Finally, as mentioned earlier, for a converged propagation the time step must be around 5 times smaller than the time step that satisfies the stability criterion (25).

If the grid contains points where the potential becomes very high, as is usually the case for small interatomic separations (the potential "walls"), the range of potential energy may become very large and force a restrictively small time step for stable propagation. ${ }^{18}$ The fact that the potential is huge in those regions of configuration space is actually irrelevant to the propagation; as long as the potential at these "no go" regions is much higher than the energy available in the wave packet then the propagation will not "know" the difference. Thus an arbitrary shelf is routinely established for the potential energy: for any grid points where the potential energy would be higher than that value, the potential is set equal to the shelf value. ${ }^{18}$ The truncated range of potential energy is now contained at some reasonable value, and the time step necessary to converge tie propagation becomes manageable again. A typical time step for a two dimensional propagation (involving hydrogen motion) is 1 atomic time unit (1 a.t.u. $=0.024 \mathrm{fs})$. 


\subsubsection{Absorbing boundaries}

For the dissociative systems we are interested in studying, the wave packet will finally leave the grid, via either the entrance or exit valleys, or both. (It is not unusual for the wave packet to split up into fragments - this is a reflection of its quantum nature!) At the grid boundaries what happens to the wave packet? Discrete Fourier transforms, which are used in the numerical method to evaluate the motion of the packet, assume periodic boundary conditions. In other words, without a potential, the wave packet would leave one side of the grid and appear again at the far side. This is not acceptable behavior as far as the physics of our problem is concerned. As we are only interested in any parts of the wave packet that end up returning into the Franck Condon region, and parts of the wave packet that are leaving the grid are not expected physically to return (if the grid has been chosen sensibly), we may damp out all flux that approaches the edge of the grid, so that we do not encounter the "wrap around" effect described above.

We follow the simple scheme of Bisseling et al. ${ }^{18}$ The wave packet is multiplied by a one-sided gaussian absorbing function $\mathrm{f}_{\mathrm{abs}}$ at each propagation step. For a two dimensional grid $f_{a b s}$ is given by

$$
f_{a b s}(x, y)=f_{a b s}(x) f_{a b s}(y)
$$

where

$$
f_{a b s}(x)= \begin{cases}1 & \text { for } x \leq x_{a b s} \\ \exp \left(-c_{a b s}\left(x-x_{a b s}\right)^{2}\right) & \text { for } x \geq x_{a b s}\end{cases}
$$

and likewise for $\mathrm{f}_{\mathrm{abs}}(\mathrm{y})$. The situation is illustrated pictorially in Figure 4-2; the shaded regions of the grid are used for the absorbing boundary and the effect of the 
damping function can be seen on a wave packet leaving along the valley parallel to the $x$ axis. $\mathrm{x}_{\mathrm{abs}}$ and $\mathrm{y}_{\mathrm{abs}}$ are usually chosen so that a region 10 grid points wide is used for the absorbing boundary; $c_{a b s, x}$ and $c_{a b s, y}$ are empirically chosen to minimize both reflection and transmission of the wave packet through the boundary. This can require careful attention to plots of the wave packet evolution at the boundary and artifacts in the autocorrelation. If the wave packet is reflected from the boundary and makes it back to the Franck Condon region before the end of the propagation, false resonances will appear in the simulated photoelectron spectrum. A more detailed discussion of the absorbing boundary problem has been given by Kosloff. ${ }^{19}$

\subsubsection{Windowing and convolution of the autocorrelation.}

Once the propagation has been carried out to $t=t_{\max }$ and the autocorrelation has been stored at $\left(t_{\max } / \Delta \tau_{\text {oulp }}\right)+1$ values, the photoelectron spectrum $\sigma(E)$ is obtained by a one-dimensional fast Fourier transform of the discrete $C(t)$. The finite propagation of the wavepacket leads to a finite resolution in the simulated energy spectrum. In principle this is given, in atomic units, by $\Delta E=\pi / t_{\max } \cdot{ }^{15}$ However, in practice, if $C(t)$ has not decayed to zero by $t_{\max }$ then its Fourier transform will show artificial high frequency oscillations; this "leakage" problem is rectified by applying a windowing function before the Fourier transform. ${ }^{15}$

Choice of a Gaussian window function is equivalent to convoluting the theoretical spectrum with a Gaussian energy resolution function. ${ }^{* 3}$ Specifically, $C(t)$

*3 We are making use of the Convolution theorem (Ref. 15, p. 383) and the fact that the Fourier transform of a Gaussian is another Gaussian. 
is multiplied by a time window function, $\Gamma(t)=\exp \left(-\gamma t^{2}\right)$, where $\gamma$ is chosen so that the product $\Gamma(t) C(t)$ has fallen to zero by $t_{\max }$. The transform, $I(E)$ given by

$$
I(E)=\int_{-\infty}^{+\infty} e^{t E t / \AA} \Gamma(t) C(t) d t
$$

is the photoelectron spectrum convoluted with the energy resolution function. The energy resolution $\Delta E$, in atomic units, in the simulation is related to $\gamma$ by

$$
\Delta E=4 \sqrt{\log _{e} 2 \gamma} .
$$

Finally, a change of variable from $E$, the scattering energy, to $E_{k}$, the electron kinetic energy, using relation (2) allows us to compare the simulation with the spectrum measured in the laboratory.

\section{Conclusions}

The theoretical framework behind the numerical simulations and the qualitative time-dependent picture have been described. The mathematical approach is completely equivalent to a Franck-Condon time-independent approach. We apply the wave packet method in two dimensions which allows us to solve for the collinear dissociation dynamics of a transition state species. The wave packet methodology is suitable for spectra that manifest both long-lived resonance states and fast dissociating direct scattering states. The calculation is an exact quantum solution of the collinear dynamics. This can provide a useful test of more approximate adiabatic methods. $^{2}$ In the next chapters we use wave packet propagation to simulate photoelectron bands for $\mathrm{BrHI}^{-}, \mathrm{OHF}^{-}$and $\mathrm{FH}_{2}^{-}$. 
The most significant computational advance to be made in terms of simulating transition state spectra would be to extend the above technique to three dimensions so that a triatomic dissociation could be treated in full. This would allow routine simulations of the quality of the time-independent methods of Schatz, ${ }^{20}$ Zhang and Miller, ${ }^{21}$ and Manolopoulos, ${ }^{22}$ and would once again provide a useful comparison to Metz' approximate three-dimensional adiabatic simulations. ${ }^{2}$ Importantly, however, the results would retain the useful time-dependent perspective that allows insight into the mechanism behind features in the transition state spectrum. In the last few years several groups have achieved 3D wave packet codes; the methods used by each varies somewhat but most are a synthesis of basis set and FFT based solutions to the timedependent Schrödinger equation. ${ }^{23,24,25,26,27}$ 


\section{References for Chapter 4.}

1. See Chapter 1, section 2.

2. R. B. Metz, Ph. D. thesis, University of California, Berkeley (1991)

3. Rather complete computer programs have been written to explicitly handle combination bands, hot bands, multiple neutral states, and hot bands for such bound-bound spectra. For example the mstfit3 code of Don W. Arnold, unpublished work (1991)

4. J. C. Light, I. P. Hamilton and J. V. Lill, J. Chem. Phys. 82, 1400 (1985)

5. E. J. Heller, Acc. Chem. Res. 14, 368 (1981)

6. A. H. Zewail, Farad. Discuss. Chem. Soc. 91, 207 (1991)

7. D. Kosloff and R. Kosloff, J. Comput. Phys. 52, 35 (1983); D. Kosloff and R. Kosloff, J. Chem. Phys. 79, 1823 (1983)

8. R. Kosloff, J. Phys. Chem. 92, 2087 (1988)

9. N. Henriksen, J. Zhang and D. G. Imre, J. Chem. Phys. 89, 5607 (1988)

10. R. Schinke, K. Weide, B. Heumann and V. Engel, Farad. Discuss. Chem. Soc. 91, 31 (1991)

11. J. E. Reutt, Ph. D. thesis, University of California, Berkeley (1986)

12. A. J. Lorquet, J. C. Lorquet, J. Delwiche and M. J. Hubin-Franskin, J. Chem. Phys. 76, 4692 (1982)

13. J. E. Pollard, D. J. Trevor, J. E. Reutt, Y. T. Lee and D. A. Shirley, J. Chem. Phys. 81, 5302 (1984)

14. U. Manthe and H. Koppel, J. Chem. Phys. 93, 345 (1990); H. Tal-Ezer and R. Kosloff, J. Chem. Phys. 81, 3967 (1984)

15. W. H. Press, B. P. Flannery, S. A. Teukolsky and W. T. Vetterling, Numerical Recipes, Cambridge University Press, Cambridge (1986)

16. V. Mohan and N. Sathyamurthy, Comput. Phys. Rep. 7, 213 (1988)

17. See, for example, NAG Library Mark 14, Numerical Algorithms Group, Oxford, England.

18. R. H. Bisseling, R. Kosloff and J. Manz, J. Chem. Phys. 83, 993 (1985)

19. R. Kosloff and D. Kosloff, J. Comput. Phys. 63, 363 (1986) 
20. G. C. Schatz, J. Phys. Chem. 94, 6157 (1990); G. C. Schatz, J. Chem. Phys. 90, 4847 (1989)

21. J. Z. H. Zhang and W. H. Miller, J. Chem. Phys. 92, 1811 (1990)

22. D. E. Manolopoulos, private communication; see Chapter 7

23. S. K. Gray and C. E. Wozny, J. Chem. Phys. 94, 2817

24. U. Manthe and H. Koppel, Chem. Phys. Lett. 178, 36 (1991)

25. F. Le Quere and C. Leforestier, J. Chem. Phys. 94, 1118 (1991)

26. D. Neuhauser, R. S. Judson, R. L. Jaffe, M. Baer and D. Kouri, Chem. Phys. Lett. 176, 546 (1991)

27. R. N. Dixon, J. Chem. Soc. Farad. Trans. 88, 2575 (1992) 
Figure Captions for Chapter 4.

Figure 4-1. Example wave packet evolutions on upper state surfaces. In each case, the schematic potentials involved in the transition, the autocorrelation function and photoelectron spectrum obtained by Fourier transformation are shown. (i) bound $\rightarrow$ bound, no Franck-Condon excitation; (ii) bound $\rightarrow$ bound, large Franck Condon excitation; (iii) bound $\rightarrow$ (bound + continuum) and (iv) bound $\rightarrow$ free.

Figure 4-2. Schematic showing the region of the grid used for absorbing wave packet as it leaves grid. The shaded area indicates the absorbing boundary region. Contours of the wave packet as it hits this boundary are superimposed on contours of the potential energy. Parameters for this propagation are given in the caption of Figure 6-9. Absorbing parameters are [refer to Equation (27)], $\mathrm{x}_{\mathrm{abs}}=14.17, \mathrm{y}_{\mathrm{abs}}=2.625$, $c_{\mathrm{abs}, \mathrm{x}}=0.001 / \Delta \mathrm{x}, \mathrm{c}_{\mathrm{abs}, \mathrm{y}}=0.001 / \Delta \mathrm{y}$. 

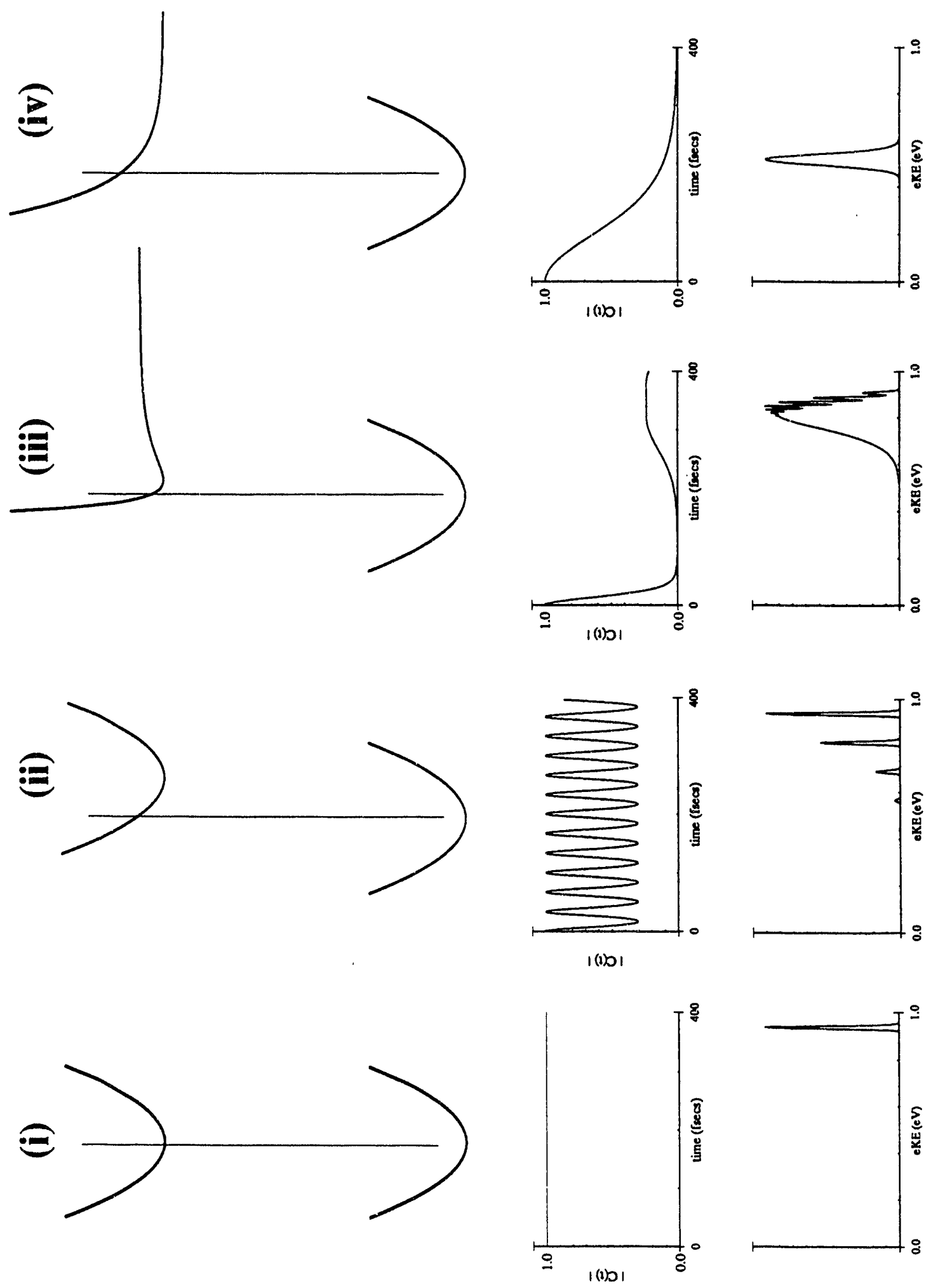

Figure 4-1 


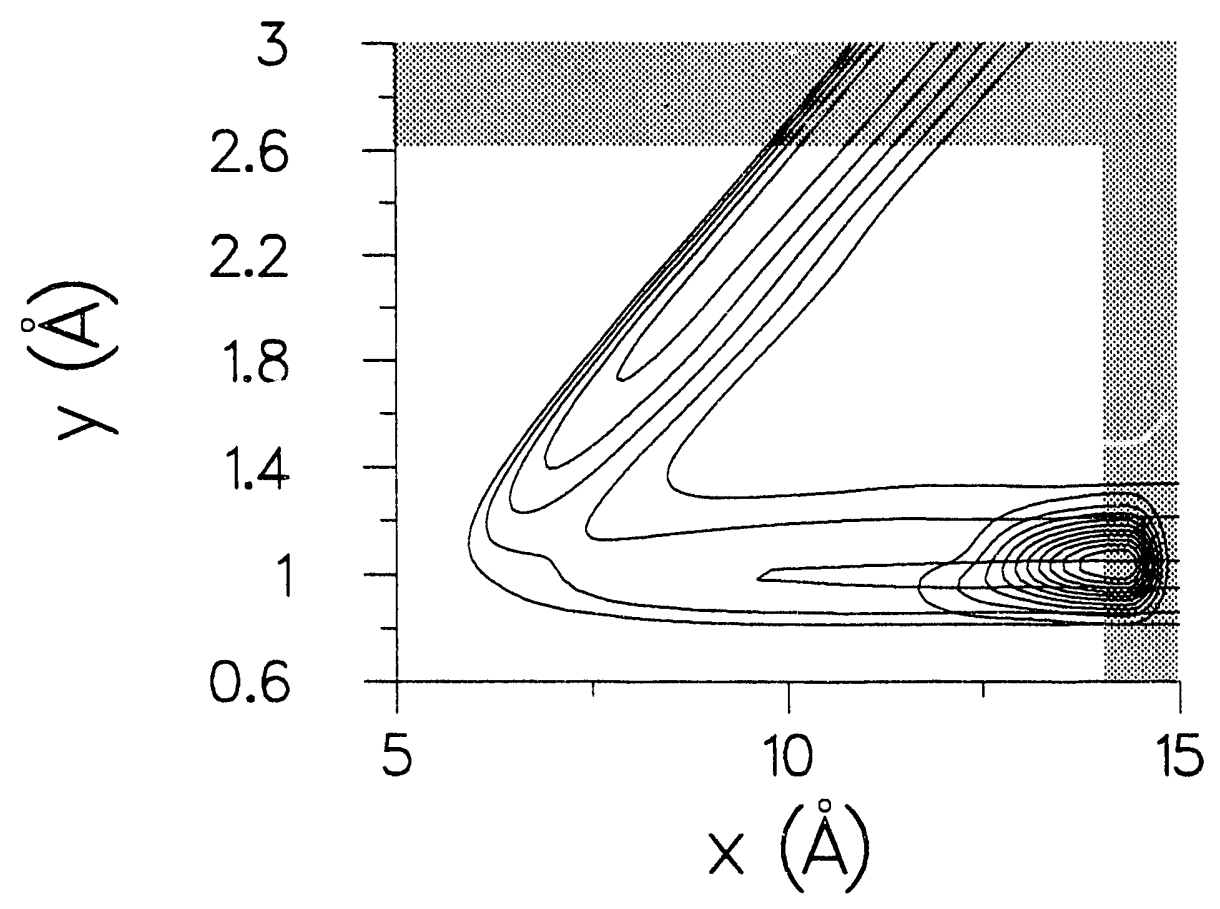

Figure 4-2 
Chapter 5. Examination of the $\mathrm{Br}+\mathrm{HI}, \mathrm{Cl}+\mathrm{HI}$, and $\mathrm{F}+\mathrm{HI}$ Hydrogen Abstraction Reactions by Photoelectron Spectroscopy of BrHI-, CHHI- and FHI-: $^{-}$

\begin{abstract}
The photoelectron spectra of the ions $\mathrm{BrHI}^{-}, \mathrm{ClHI}^{-}$and $\mathrm{FHI}^{-}$, along with their deuterated counterparts, are presented. These spectra provide information on the transition state region of the potential energy surfaces describing the exothermic neutral reactions $\mathrm{X}+\mathrm{HI} \rightarrow \mathrm{HX}+\mathrm{I}(\mathrm{X}=\mathrm{Br}, \mathrm{Cl}, \mathrm{F})$. Vibrational structure is observed in the $\mathrm{BrHI}^{-}$and $\mathrm{ClHI}^{-}$spectra that corresponds to hydrogen atom motion in the dissociating neutral complex. Transitions to electronically excited potential energy surfaces, that correlate to $\mathrm{HX}+\mathrm{I}\left({ }^{2} \mathrm{P}_{3 / 2},{ }^{2} \mathrm{P}_{1 / 2}\right)$ products, are also observed. $\mathrm{A}$ onedimensional analysis is used to understand the appearance of each spectrum and the $\mathrm{BrHI}^{-}$spectrum is compared to a two-dimensional simulation performed using timedependent wave packet propagation on a model $\mathrm{Br}+\mathrm{HI}$ potential energy surface.
\end{abstract}

\title{
1. Introduction
}

We have recently shown that negative ion photodetachment can be used to investigate the transition state region of a neutral bimolecular reaction. ${ }^{1 \cdot 3}$ In our experiments, the spectroscopy and dissociation dynamics of the short-lived [AHB] complex formed during the hydrogen transfer reaction $\mathrm{A}+\mathrm{HB} \rightarrow \mathrm{HA}+\mathrm{B}$ are studied via photoelectron spectroscopy of the stable, hydrogen-bonded anion AHB-. Thus far, results have been reported for the symmetric hydrogen transfer reactions $\mathrm{Cl}+\mathrm{HCl},{ }^{1}$

* $\quad$ Published in J. Chem. Phys. 92, 7205 (1990) 
$\mathrm{I}+\mathrm{HI},{ }^{2}$ and $\mathrm{Br}+\mathrm{HBr}^{3}$ which were investigated by photodetaching the negative ions $\mathrm{ClHCl}^{-}, \mathrm{IHI}^{-}$, and $\mathrm{BrHBr}^{-}$, respectively. The photoelectron spectra of these ions show resolved vibrational progressions assigned to the unstable neutral complex associated with the corresponding bimolecular reaction. Our analysis as well as simulations of these spectra by other investigators ${ }^{4,5,6}$ have shown that this vibrational structure provides a sensitive probe of the neutral potential energy surface near the transition state.

This paper describes the application of our method to asymmetric hydrogen transfer reactions. We have studied the entire series of reactions $\mathrm{X}+\mathrm{HY} \rightarrow \mathrm{HX}+\mathrm{Y}$, where $\mathrm{X}$ and $\mathrm{Y}$ are unlike halogen atoms, via photoelectron spectroscopy of the asymmetric bihalide ions $\mathrm{XHY}^{-}$. In the same fashion, we have also conducted experiments on the polyatomic reactions $\mathrm{F}+\mathrm{CH}_{3} \mathrm{OH} \rightarrow \mathrm{HF}+\mathrm{CH}_{3} \mathrm{O}$ and $\mathrm{F}+\mathrm{C}_{2} \mathrm{H}_{5} \mathrm{OH}$ $\rightarrow \mathrm{HF}+\mathrm{C}_{2} \mathrm{H}_{5} \mathrm{O} .{ }^{7}$ Results are presented here for the triatomic reactions $\mathrm{Br}+\mathrm{HI} \rightarrow$ $\mathrm{HBr}+\mathrm{I}, \mathrm{Cl}+\mathrm{HI} \rightarrow \mathrm{HCl}+\mathrm{I}$, and $\mathrm{F}+\mathrm{HI} \rightarrow \mathrm{HF}+\mathrm{I}$. The remaining XHY- spectra and the $\mathrm{ROHF}^{-}$results will be discussed in a future article. In each case, the photoelectron spectrum of the precursor negative ion yields resolved vibrational and/or electronic structure associated with the unstable neutral complex formed by photodetachment.

In contrast to the symmetric hydrogen transfer reactions, a vast body of experimental results exists concerning the kinetics and product state distributions for the asymmetric reactions. ${ }^{8}$ Experimental studies of the triatomic $\mathrm{X}+\mathrm{HY}$ reactions date back to the dawn of chemical reaction dynamics. This work has inspired the construction of model potential energy surfaces for these reactions which attempt to reproduce and explain the experimental results, using either classical trajectory ${ }^{9,10}$ 
or quantum scattering ${ }^{11}$ calculations. These model surfaces have provided the foundation of many fundamental ideas in our understanding of the relationship between the features of a potential energy surface and the experimentally measurable asymptotic properties of a chemical reaction. Our experiment provides a direct test of the validity of these proposed model $\mathrm{X}+\mathrm{HY}$ surfaces. Using such a surface, one can, in principle, simulate the $\mathrm{XHY}^{-}$photoelectron spectrum and compare the simulation to our experimental result.

The methods of analysis which have been developed to simulate the vibrational structure seen in the $\mathrm{XHX}^{-}$photoelectron spectra ${ }^{3-6}$ can also be applied to the $\mathrm{XHY}^{-}$ photoelectron spectra. These methods all involve calculating the Franck-Condon overlap between the initial vibrational level of the ion and the set of scattering wave functions supported by the neutral potential energy surface. A one-dimensional analysis, in which the scattering coordinate is ignored, can approximately predict the spacing and integrated intensities of the peaks in each vibrational progression. This type of analysis is applied to the spectra presented in this paper as a first step in understanding our results.

In addition to probing the ground electronic potential energy surfaces of the $\mathrm{X}+\mathrm{HY}$ reactions, photodetachment of $\mathrm{XHY}^{-}$anions can access electronically excited reactive surfaces. These excited states of the neutral complex are in most cases quite distinct in the spectra presented here and provide information on an aspect of these reactions largely inaccessible to scattering-based experiments. The electronic features in our spectra provide a more quantitative foundation for the electronic correlation diagrams proposed for these reactions ${ }^{12,13}$ and are discussed at length. 
Finally, the $\mathrm{BrHI}^{-}$photoelectron spectrum is simulated using time-dependent wave packet propagation in two dimensions. This approach simulates peak profiles and widths as well as peak separations, thus testing more stringently the accuracy of the potential surface used in this simulation, but differs from the time-independent treatments inentioned above in that the scattering wave functions are never calculated. The use and implementation of a time dependent formalism to describe spectral profiles due to a repulsive state draws on the ideas of Heller ${ }^{14}$ and Kosloff ${ }^{15}$. The time-dependent approach provides considerable insight into the experimental results and makes more concrete the relationship between our experimental spectra and the short time dynamics initiated on the naural reaction surface.

The systems chosen here illustrate both the promise and limitations of negative ion photodetachment as a probe of the neutral transition state region. The main concern is that the ion geometry must be similar to that of the neutral transition state. For symmetric $\mathrm{X}+\mathrm{HX}$ reactions, the precursor ion $\mathrm{XHX}^{-}$is most likely linear and centrosymmetric; ${ }^{16,17}$ the only issue is how close the equilibrium inierhalogen distance in the ion is to the saddle point geometry on the neutral surface. For an asymmetric $\mathrm{X}+\mathrm{HY}$ reaction, an additional fastor is the location of the hydrogen atom in $\mathrm{XHY}^{-}$. This is largely determined by the proton affinities of $\mathrm{X}^{-}$and $\mathrm{Y}^{-}$. In a related experiment, Brauman and co-workers observed substantial differences in the total photodetachment cross sections for the series of ions ROHF- depending on whether the $\mathrm{F}^{-}$or $\mathrm{RO}^{-}$proton affinity is higher. ${ }^{18}$ If $\mathrm{RO}^{-}$has the higher proton affinity, then photodetachment of the ion primarily accesses the $\mathrm{F}+\mathrm{ROH}$ entrance valley on the neutral reactive surface, whereas if the proton affinity of $\mathrm{F}^{-}$is higher, 
the ion is more accurately pictured as ( $\left.\mathrm{RO}^{-}\right) \mathrm{HF}$ and photodetachment accesses the RO + HF valley of the surface. We shall see that these considerations have a profound effect on our experiment.

\section{Experimental}

The experiments were performed on a negative ion time-of-flight photoelectron spectrometer which has been described in detail previously. ${ }^{3,19}$ Briefly, an internally cold, mass-selected negative ion beam is photodetached with a pulsed fixedfrequency laser. A small fraction of the ejected photoelectrons is collected and the electron kinetic energy distribution is analyzed by time-of-flight. The ion beam, based on the design of Lineberger and co-workers, ${ }^{20}$ is generated by expanding an appropriate mixture of neutral gases through a pulsed molecular beam valve and crossing the molecular beam with a $1 \mathrm{keV}$ electron beam just outside the valve orifice. Negative ions are formed through a variety of dissociative attachment and clustering procisses in the continuum flow region of the free-jet expansion and their internal degrees of freedom are cooled as the expansion progresses. $\mathrm{BrHI}^{-}$and $\mathrm{ClHI}^{-}$were generated from $5 \% \mathrm{HBr}(\mathrm{HCl}) / 1 \% \mathrm{HU} / \mathrm{Ar}$ mixture and $\mathrm{FHI}^{-}$was made from a $1 \% \mathrm{HF} /$ 1\% HUAr mixture. Similar mixtures were used to make the ions $\mathrm{BrDI}^{-}, \mathrm{ClOI}^{-}$and FDI-

Several centimeters downstream from the beam valve, the negative ions in the molecular beam are extracted at $90^{\circ}$ and injected into a time-of-flight mass spectrometer. ${ }^{21}$ The ions are accelerated to $1 \mathrm{keV}$ and spatially separate into bunches according to their masses as they pass through the mass spectrometer. The pulsed photodetachment laser crosses the ion beam at the spatial focus of the mass 
spectrometer, $140 \mathrm{~cm}$ downstream from the extraction region. Mass selection of the ions to be photodetached is achieved by timing the laser pulse to intersect the ion bunch of the desired mass. In the results presented here, either the fourth (266 nm, $4.66 \mathrm{eV})$ or fifth harmonic $(213 \mathrm{~nm}, 5.83 \mathrm{eV})$ of a Nd:YAG laser was used for photodetachment. A small fraction $(0.01 \%)$ of the photoelectrons produced are detected by a $40 \mathrm{~mm}$ diameter dual microchannel plate detector $100 \mathrm{~cm}$ from the laserfion beam interaction region. The electron time-of-flight distribution is recorded with a $200 \mathrm{MHz}$ transient digitizer. In all experiments reported here, the laser beam was plane polarized perpendicular to the direction of electron collection. The resolution of the spectrometer is $8 \mathrm{meV}$ for $0.65 \mathrm{eV}$ electrons and degrades as $\mathrm{E}^{3 / 2}$ at higher electron kinetic energies.

\section{Results}

The $\mathrm{BrHI}^{-}$and $\mathrm{BrDI}^{-}$photoelectron spectra at $213 \mathrm{~nm}$ are shown in Figure 5-1. Each spectrum shows two progressions of approximately evenly spaced peaks. The peak positions are listed in Table 5-1a. The peaks labelled A and A* occur at the same electron kinetic energy in both spectra and are taken to be band origins of the progressions. The peak spacing within each progression in Figure 5-1 is noticeably less in the $\mathrm{BrDI}^{-}$spectrum than in the $\mathrm{BrHI}^{-}$spectrum. The direction of this isotope shift shows we are observing progressions in the neutral [BrHI] complex in a vibrational mode primarily involving $H$ atom motion. This is assigned to the $v_{3}$ stretching mode of the $[\mathrm{BrHI}]$ complex. The A-A* separation in each spectrum is 0.90 $\pm 0.02 \mathrm{eV}\left(7300 \pm 200 \mathrm{~cm}^{-1}\right)$. This is slightly less than the spin-orbit splitting in atomic I $\left(7600 \mathrm{~cm}^{-1}\right)$ and suggests that the two progressions with band origins $A$ and 
$A^{*}$ correspond to two electronic states of the $[\mathrm{BrHI}]$ complex which asymptotically correlate to $\mathrm{HBr}+\mathrm{I}\left({ }^{2} \mathrm{P}_{3 / 2}\right)$ and $\mathrm{HBr}+\mathrm{I}^{*}\left({ }^{2} \mathrm{P}_{1 / 2}\right)$, respectively.

The peak widths in the progression at higher electron kinetic energy are -170 meV, somewhat wider than the peaks in the second progression $(-140 \mathrm{meV})$. All the peaks are substantially broader than our experimental resolution. Figure 5-2, the photoelectron spectrum of $\mathrm{BrHI}^{-}$at $266 \mathrm{~nm}$, shows only the first. two peaks (A and B), where their electron kinetic energies are $1.165 \mathrm{eV}$ lower than in Figure 5-1. Thus, for these peaks, the energy resolution of the spectrometer is considerably higher ( $8 \mathrm{meV}$ vs. $-37 \mathrm{meV}$ ). While the positions of these peak centers can be determined more precisely from Figure 5-2, the appearance of this region of the spectrum is essentially unchanged from Figure 5-1; no additional structure is observed at higher resolution. The $266 \mathrm{~nm}$ data are also tabulated in Table 5-1a.

The exothermicity of the $\mathrm{Br}+\mathrm{HI}$ reaction and relevant energetic quantities for the $\mathrm{BrHI}^{-}$anion are tabulated in Table 5-2, as are the same quantities for the other systems studied here. In Figure 5-1, the arrow at $2.07 \mathrm{eV}$ shows the electron kinetic energy that would result from forming $I+\operatorname{HBr}(v=0)$, which is the lowest energy asymptotic decay channel available to the [BrHI] complex. This energy is given by $E=h v-\mathrm{D}_{0}\left(\mathrm{BrHI}^{-}\right)-\mathrm{EA}(\mathrm{I})$. Here hv is the photon energy, $\mathrm{D}_{0}\left(\mathrm{BrHI}^{-}\right)=0.70 \pm 0.04 \mathrm{eV}$ is the dissociation energy of $\mathrm{BrHI}^{-}$to form $\mathrm{I}^{-}+\mathrm{HBr}(\mathrm{v}=0),{ }^{22}$ and $\mathrm{EA}(\mathrm{I})=3.059 \mathrm{eV}$ is the electron affinity of $I^{23}$ The electron energy corresponding to the higher energy $\mathrm{Br}+\mathrm{HI}(\mathrm{v}=0)$ asymptote is also indicated with the arrow at $1.36 \mathrm{eV}$. All of the peaks in Figure 5-1 occur at electron kinetic energies lower than $2.07 \mathrm{eV}$ and therefore correspond to states of the [BrHI] complex that are unstable with respect to 
dissociation to $\mathrm{I}+\mathrm{HBr}(\mathrm{v}=0)$. Recall that peaks at lower electron kinetic energy correspond to higher internal energy levels of the neutral species.

As discussed in previous work, ${ }^{3}$ the $v_{3}$ mode in the complex formed in a heavy + light-heavy reaction is essentially a bound degree of freedom; it is poorly coupled to the dissociation coordinate of the complex. This is why a progression in the $v_{3}$ mode can be observed in the $\mathrm{BrHI}^{-}$photoelectron spectrum. The $v_{3}$ progressions and multiple electronic states in the $\mathrm{BrHI}^{-}$and $\mathrm{BrDI}^{-}$spectra were also seen in the symmetric XHX- photoelectron spectra. An important difference between the symmetric and asymmetric systems becomes apparent, however, when the peak separations are compared to the asymptotic $\mathrm{HBr}$ and $\mathrm{DBr}$ vibrational energy level spacings (refer to Table 5-1a). In the $\mathrm{BrHBr}^{-}$spectrum, the peak separation was nearly $1000 \mathrm{~cm}^{-1}(0.13 \mathrm{eV})$ less than the $\mathrm{HBr}$ spacings. Much smaller shifts are seen in the $\mathrm{BrHI}^{-}$and $\mathrm{BrDI}^{-}$spectra. Table 5-1a shows that for the ground state progressions of both [BrHI] and [BrDI], the separation between peaks $A$ and $B$ is essentially equal to the $\mathrm{v}=0-\mathrm{v}=1$ spacing in $\mathrm{HBr}$ and $\mathrm{DBr}$. However, the $\mathrm{B}-\mathrm{C}$ separations in both spectra, and the C-D separation for the $\mathrm{BrDI}^{-}$spectrum, are smaller than the corresponding 1-2 and 2-3 vibrational spacings in the isolated diatomic. In the excited state progressions, a somewhat larger shift of the $v_{3}$ level spacing is observed.

The $\mathrm{ClHI}^{-}$and $\mathrm{ClDI}^{-}$spectra at $213 \mathrm{~nm}$ are shown in Figure 5-3. A comparison of the two spectra indicates that each consists of two vibrational progressions separated by $0.935 \pm 0.020 \mathrm{eV}\left(7540 \pm 160 \mathrm{~cm}^{-1}\right)$. As in the $\mathrm{BrHI}^{-} /$ $\mathrm{BrDI}^{-}$spectra, the two progressions are attributed to different electronic states of [ClHI], and once again all peaks correspond to states of the [ClHI] complex that are 
unstable with respect to dissociation into $\mathrm{I}+\mathrm{HCl}(\mathrm{v}=0) .{ }^{22,23}$ The most noticeable difference between the spectra in Figure 5-1 and Figure 5-3 is that the intensity of transitions to higher $\mathrm{v}_{3}$ levels of the neutral complex fall off more rapidly in the $\mathrm{CIHI}^{-}$ and $\mathrm{ClDI}^{-}$spectra than in $\mathrm{BrHI}^{-} / \mathrm{BrDI}^{-}$. The peak positions and widths are listed in Table 5-1b. In comparison to the $\mathrm{BrHI}^{-}$and $\mathrm{BrDI}^{-}$spectra the peak separations in $\mathrm{ClHI}^{-}$and $\mathrm{ClDI}^{-}$spectra are somewhat closer to the corresponding $\mathrm{HCl}$ and $\mathrm{DCl}$ vibrational spacings. In fact the A-B interval is just slightly larger than the diatomic 0-1 interval in both hydride and deuteride.

The $\mathrm{FHI}^{-}$and $\mathrm{FDI}^{-}$spectra at $213 \mathrm{~nm}$ are shown in Figure 5-4. The peak positions are listed in Table 5-1c. The two spectra are essentially identical. Each spectrum shows three peaks of comparable intensity. The splitting between the two highest energy peaks (labelled $\mathrm{X}$ and $\mathrm{Y}$ ) is shown to better resolution in Figure 5-5, the photoelectron spectrum of $\mathrm{FDI}^{-}$at $266 \mathrm{~nm}$. This splitting is $0.154 \pm 0.007 \mathrm{eV}$ $\left(1240 \pm 60 \mathrm{~cm}^{-1}\right)$. The large uncertainty in the dissociation energy ${ }^{24}$ of $\mathrm{FHI}^{-}$does not allow us to say whether or not the state that corresponds to peak $\mathrm{X}$ is stable with respect to dissociation into $\mathrm{I}+\mathrm{HF}(\mathrm{v}=0)$. The separation between peaks $\mathrm{X}$ and $\mathrm{Z}$ is $1.045 \pm 0.020 \mathrm{eV}\left(8430 \pm 160 \mathrm{~cm}^{-1}\right)$, which is larger than the separation between the two progressions in either the $\mathrm{BrHI}^{-}$or $\mathrm{ClHI}^{-}$spectrum. In contrast to the $\mathrm{BrHI}^{-} / \mathrm{BrDI}^{-}$and $\mathrm{ClHI}^{-} / \mathrm{ClDI}^{-}$spectra, no isotope shifts are observed. This implies that the two closely spaced peaks, $\mathrm{X}$ and $\mathrm{Y}$, do not represent a vibrational progression and that all the structure in the spectrum is due different electronic states of the [FHI] complex. We will show that this can be understood in terms of perturbations of the I atom electronic states by a neighboring $\mathrm{HF}$ molecule. 


\section{Analysis and discussion}

\subsection{Preliminary considerations}

As a prelude to understanding the peak positions and intensities in the $\mathrm{XHY}^{-}$ photoelectron spectra, one must consider what region of the $\mathrm{X}+\mathrm{HY}$ potential energy surface is probed when the ion is photodetached. Within the framework of the Franck-Condon approximation, this depends solely on the geometry of the ion. The available experimental ${ }^{16}$ and theoretical ${ }^{17}$ evidence indicates that the bihalide ions are linear. However, while $a b$ initio calculations on $\mathrm{FHCl}^{-}$have yielded both structural information ${ }^{25}$ and vibrational frequencies, ${ }^{26}$ there are no examples for which the equilibrium interhalogen distance and location of the hydrogen atom in an asymmetric $\mathrm{XHY}^{-}$ion have been experimentally determined. One can estimate the location of the hydrogen atom in $\mathrm{XHY}^{-}$from the proton affinities of $\mathrm{X}^{-}$and $\mathrm{Y}^{-}$. The zero-order structure of an asymmetric anion can be written as $\mathrm{XH}^{-.} \mathrm{Y}^{-}$or $\mathrm{X}^{-} \cdot . \mathrm{HY}$ depending on whether the proton affinity of $\mathrm{X}^{-}$or $\mathrm{Y}^{-}$is higher. The proton affinities of $\mathrm{F}^{-}, \mathrm{Cl}^{-}$, and $\mathrm{Br}^{-}$are $2.47 \mathrm{eV}, 0.82 \mathrm{eV}$, and $0.40 \mathrm{eV}$ higher, respectively, than the proton affinity of $\mathrm{I}^{-}{ }^{27}$ One therefore expects $\mathrm{BrHI}^{-}$to look like $\mathrm{I}^{-} . \mathrm{HBr}$, with the hydrogen atom considerably closer to the $\mathrm{Br}$ than to the I atom. This asymmetry should become progressively more pronounced in $\mathrm{ClHI}^{-}$and $\mathrm{FHI}^{-}$.

To understand the effects of the interhalogen distance and $\mathrm{H}$ atom location in $\mathrm{XHY}^{-}$on the photoelectron spectrum, the potential energy surface for the neutral reaction must be considered. Figure 5-6 shows ${ }^{28}$ a collinear section of the LondonEyring-Polanyi-Sato (LEPS) functional form proposed for the $\mathrm{Br}+\mathrm{HI}$ reaction by Broida and Persky (hereafter referred to as the BP surface). ${ }^{10}$ The three-dimensional surface has a collinear minimum energy path and a $0.21 \mathrm{kcal} / \mathrm{mol}$ barrier in the 
$\mathrm{Br}+\mathrm{HI}$ entrance valley. The surface is plotted using the mass-weighted coordinates defined in the figure caption. The acute skew angle and low entrance channel barrier in Figure 5-6 are characteristic of all $\mathrm{X}+\mathrm{HY}$ reactions. Note that the product valley is lower most in the figure.

The region of the surface in Figure 5-6 that has the best Franck-Condon overlap with $\mathrm{BrHI}^{-}$is in the neighborhood of the equilibrium geometry of the ion. In the figure, this geometry is given by the intersection of the dashed vertical line corresponding to the equilibrium interhalogen distance in the ion $\left(\mathrm{R}_{\mathrm{e}}(\mathrm{IBr})\right)$ and the dashed horizontal line corresponding to the location of the $H$ atom $\left(\mathrm{R}_{\mathrm{e}}(\mathrm{HBr})\right)$. The values for $R_{e}(\mathrm{IBr})$ and $R_{e}(\mathrm{HBr})$ used in the figure are obtained from the one dimensional fit discussed in Section 2, below. For the general $X+H Y$ case, if $R_{e}(X Y)$ is sufficiently small in $\mathrm{XHY}^{-}$, the corresponding vertical line in Figure 5-6 will pass through or near the barrier. Our experiment can then probe the transition state region on the $\mathrm{X}+\mathrm{HY}$ surface, where the vibrational and/or electronic properties of the [XHY] complex are distinct from separated reactants or products. On the other hand, $\mathrm{R}_{\mathrm{e}}(\mathrm{HX})$ in the ion determines if photodetachment primarily accesses the $\mathrm{X}+\mathrm{HY}$ reactant valley or the $\mathrm{Y}+\mathrm{HX}$ product valley.

In the case of $\mathrm{BrHI}^{-}$, since the ion can be pictured as $\mathrm{I}^{-} \cdot . \mathrm{HBr}$, better overlap with the $\mathrm{I}+\mathrm{HBr}$ product valley rather than the reactant valley is expected. The most intense peaks seen in the experimental spectrum lie well below the asymptote for $\mathrm{Br}+\mathrm{HI}(\mathrm{v}=0)$ confirming that the experiment accesses the product $\mathrm{I}+\mathrm{HBr}$ valley. However, the observation of a 'red shift' in the $v_{3}$ spacings of the [BrHI] and [BrDI] complexes, compared to the vibrational level spacings in $\mathrm{HBr}$ and $\mathrm{DBr}$, suggests that 
$\mathrm{Re}_{\mathrm{e}}(\mathrm{IBr})$ in $\mathrm{BrHI}^{-}$is sufficiently small so that the transition state region of the $\mathrm{Br}+\mathrm{HI}$ surface is accessed via photodetachment.

Similar considerations apply to the $\mathrm{ClHI}^{-}$and $\mathrm{FHI}^{-}$photoelectron spectra. The potential energy surfaces for the $\mathrm{Cl}+\mathrm{HI}$ and $\mathrm{F}+\mathrm{HI}$ reactions should resemble the surface in Figure 5-6, although earlier barriers might be expected due to the higher exothermicity of these reactions. However, the expected location of the $\mathrm{H}$ atom in $\mathrm{ClHI}^{-}$and $\mathrm{FHI}^{-}$means that photodetachment should result in progressively greater overlap with the I $+\mathrm{HX}$ product valley. This effect will be discussed in more detail below.

\subsection{One dimensional analysis of $\mathrm{XHY}^{-}$spectra}

In this section, the peak positions and intensities of the ground state vibrational progressions in the three $\mathrm{XHY}^{-}$(and $\mathrm{XDY}^{-}$) photoelectron spectra are analyzed using a one-dimensional model similar to that used in the analysis of the $\mathrm{BrHBr}^{-}$spectrum. ${ }^{3}$ The $\mathrm{BrHI}^{-}$and $\mathrm{BrDI}^{-}$spectra are simulated using this model, which then serves as a framework for discussing the $\mathrm{ClHI}^{-}$and $\mathrm{FHI}^{-}$spectra. In addition to explaining the observed spectra, the analysis yields an approximate equilibrium geometry for $\mathrm{BrHI}^{-}$which will be used in the time-dependent analysis in Section 4.

\subsection{1 $\mathrm{BrHI}^{-}$and $\mathrm{BrDI}^{-}$}

In order to simulate the peak positions and intensities in the $\mathrm{BrHI}^{-}$and $\mathrm{BrDI}^{-}$ photoelectron spectra, we need to calculate the Franck-Condon overlap between the $v_{3}=0$ level of the ion and the $v_{3}$ levels supported by the neutral potential energy surface. We assume the ions prepared in our experiment are in their vibrational ground states. This analysis requires approximate potential energy surfaces for 
$\mathrm{BrHI}^{-}$and the $\mathrm{Br}+\mathrm{HI}$ reaction. We will use the BP surface, shown in Figure $5-6$, for the $\mathrm{Br}+\mathrm{HI}$ reaction. The development of a model $v_{3}$ potential for $\mathrm{BrHI}^{-}$will now be discussed.

In our earlier analysis of the symmetric $\mathrm{XHX}^{-}$spectra, we assumed a harmonic potential for the $v_{3}$ vibration of the ion. This is likely to be a poor approximation for an asymmetric $\mathrm{XHY}^{-}$ion. In an $a b$ initio study on $\mathrm{FHCl}^{-}$, Sannigrahi and Peyerimhoff ${ }^{25}$ calculated the potential energy curves governing $\mathrm{H}$ atom motion for several fixed interhalogen distances. At the equilibrium F-Cl distance, they found a highly asymmetric, single minimum potential. Based on the $\mathrm{FHCl}^{-}$calculation, the analogous potential energy curve for $\mathrm{BrHI}^{-}$is expected to look like the solid curve in Figure 5-7. For the purposes of calculating the $v_{3}=0$ wave function which is localized near the minimum, this curve can be approximated by the Morse potential,

$$
U\left(R_{H-B r}\right)=D_{e}\left(1-\exp \left[-\beta\left(R_{H-B r}-R_{e}(H B r)\right)\right]\right)^{2},
$$

shown by the dashed curve in Figure 5-7. We use Eq. (1) as the $v_{3}$ stretching potential for $\mathrm{BrHI}^{-}$. The $v_{3}$ coordinate is $\mathrm{R}_{\mathrm{H}-\mathrm{Br}}$, and $\mu_{\mathrm{HBr}}$ is the appropriate reduced mass for determining the vibrational energy levels and wave functions. Here $R_{e}(H B r)$ is the $\mathrm{H}-\mathrm{Br}$ separation at the minimum of the potential; its determination is described below.

The parameters $D_{e}$ and $\beta$ in Eq. 1 are fixed using the matrix isolation values for the $v_{3}$ fundamental in $\mathrm{BrHI}^{-}$and $\mathrm{BrDI}^{-}{ }^{29}$ The choice of these values merits some discussion. Matrix studies by Ault and co-workers yielded two frequencies assignable to the $v_{3}$ mode for each asymmetric $\mathrm{XHY}^{-}$ion. ${ }^{29,30}$ This was attributed to the existence of two forms of the ion in a matrix: a highly 'asymmetric' structure (type I) with a relatively high $v_{3}$ frequency, and a more 'symmetric' structure (type II) 
with a considerably lower frequency. For $\mathrm{FHCl}^{-}$, the $\mathrm{v}_{3}$ fundamental is $2491 \mathrm{~cm}^{-1}$ for the type I structure and $933 \mathrm{~cm}^{-1}$ for the type II structure. ${ }^{30}$ The high degree of asymmetry in Peyerimhoff's potential ${ }^{25}$ and a recently calculated value by Botschwina ${ }^{26}$ of $2814 \mathrm{cn}^{-1}$ for the $v_{3}$ fundamental in $\mathrm{FHCl}$ - suggest that the more asymmetric type I structure is closer to the gas phase structure. We have therefore used the type I frequencies, ${ }^{29} 920 \mathrm{~cm}^{-1}$ and $728 \mathrm{~cm}^{-1}$, for the $v_{3}$ fundamental in $\mathrm{BrHI}^{-}$and $\mathrm{BrDI}^{-}$, respectively. $D_{e}$ and $\beta$ are then obtained analytically.

The BP potential energy surface in Figure 5-6 will be assumed for the $\mathrm{Br}+\mathrm{HI}$ reaction. This surface was devised on the basis of quasi-classical trajectory calculations which produce reasonable agreement with the experimental rate constants at several temperatures and the product $\mathrm{HBr} v=2 / \mathrm{v}=1$ ratio at $300 \mathrm{~K}$. 10 The $v_{3}$ coordinate is taken to be the same as in the ion, namely $y=R_{\mathrm{H} \cdot \mathrm{Br}}$. Thus, the effective potential for the hydrogen stretch in the neutral [BrHI] complex is found by taking a vertical cut through the surface at $x=x_{e}$, the value of $x$ at the equilibrium structure of the ion given by

$$
x_{e}=\left(\mu_{\mathrm{I}, \mathrm{HBr}} / \mu_{\mathrm{HBr}}\right)^{1 / 2}\left(\mathrm{R}_{\mathrm{e}}(\mathrm{IBr})-\left(\mathrm{m}_{\mathrm{H}} / \mathrm{m}_{\mathrm{HBr}}\right) \mathrm{R}_{\mathrm{e}}(\mathrm{HBr})\right)
$$

Note that $m_{\mathrm{H}} / \mathrm{m}_{\mathrm{HBr}} \approx 1 / 80$, so to a good approximation $\mathrm{x}_{\mathrm{e}} \approx 7.0 \mathrm{R}_{\mathrm{e}}(\mathrm{IBr})$. Here, as before, $R_{e}(\mathrm{IBr})$ is the equilibrium interhalogen distance in the ion. The energy levels and wave functions supported by this potential are then solved for numerically.

The peak spacings and intensities in the $\mathrm{BrHI}^{-}$photoelectron spectrum can now be simulated by calculating the Franck-Condon factors between the $v_{3}=0$ ion level supported by the Morse potential in Eq. (1) and the $v_{3}$ levels supported in the neutral surface cut at $x=x_{e}$. We assume the BP surface is correct and vary $R_{e}(I B r)$ and $R_{e}(\mathrm{HBr})$ in the ion until agreement with experiment is obtained. $R_{e}(\mathrm{IBr})$ largely 
determines the location of the cut on the neutral surface which in turn determines the peak spacings in the photoelectron spectra. The value of $R_{e}(\mathrm{HBr})$ determines the position of the minimum in the Morse potential for the ion along the $y$ axis of Figure 5-6 and therefore determines the intensity distribution of the simulated spectra. The best fit to both the $\mathrm{BrHI}^{-}$and $\mathrm{BrDI}^{-}$spectra is obtained with $\mathrm{Re}_{\mathrm{e}}(\mathrm{HBr})=1.55 \AA$ and $x_{e}=27.1 \AA$, so $R_{e}(I B r) \approx 3.88 \AA$. The hydrogen stretching $v_{3}$ potential for ion and neutral are shown in Figure 5-8 along with the energies of the ion $v_{3}=0$ and 1 levels and the first few neutral $v_{3}$ levels. The simulated stick spectra are superimposed on the experimental spectra in Figure 5-9.

This one-dimensional analysis provides a firmer foundation for some of the qualitative ideas discussed in the previous section. Although the line $x=x_{e}$ in Figure 5-6 passes very close to the barrier, the $\mathrm{v}_{3}=0$ level of $\mathrm{BrHI}^{-}$has the most overlap with the $v_{3}=0,1$ and 3 levels supported by the neutral potential. The wave functions for these levels are confined to the $\mathrm{I}+\mathrm{HBr}$ product valley of the potential energy surface and can be thought of as $\mathrm{HBr}$ vibrational levels perturbed by a neighboring I atom. This is why the peaks in the spectrum corresponding to transitions to these states are spaced by an interval only slightly less than the $\mathrm{HBr}$ fundamental. Note that the $\mathrm{v}_{3}=2$ wavefunction is localized in the $\mathrm{Br}+\mathrm{HI}$ valley. The anion wavefunction has very little overlap with this state but it does appear in the simulation as a small peak to the right of peak 3 in Figure 5-9 (top).

\subsection{2 $\mathrm{ClHI}^{-}$and $\mathrm{FHI}^{-}$}

The differences between the $\mathrm{ClHI}^{-}$and $\mathrm{BrHI}^{-}$photoelectron spectra can be understood by considering how the potential energy curves in Figure 5-8 should differ in the case of $\mathrm{ClHI}^{-}$photodetachment. The product valley well in the neutral $\mathrm{v}_{3}$ 
potential should be deeper because the $\mathrm{Cl}+\mathrm{HI}$ reaction is more exothermic (see Table 5-2). Thus the first few $v_{3}$ levels supported by this potential should look much like inslated $\mathrm{HCl}$ vibrational energy levels. This is confirmed by the peak spacings in Table 5-1b, which show little or no 'red shift' relative to the $\mathrm{HCl}$ and $\mathrm{DCl}$ vibrational energy levels. In addition, because of the larger difference in proton affinities between the halide ions in $\mathrm{ClHI}^{-}$compared to $\mathrm{BrHI}^{-}, \mathrm{ClHI}^{-}$will look more like $\mathrm{I}^{-}$clustered to a nearly unperturbed $\mathrm{HCl}$ molecule. We therefore expect the minima in the $v_{3}$ potentials for the anion and neutral to occur at a value of $R_{\mathrm{H}-\mathrm{Cl}}$ quite close to the equilibrium value for diatomic $\mathrm{HCl}$; the two minima should be much closer than the minima in the two potentials in Figure 5-8. Hence $\Delta v_{3}=0$ transitions to the neutral are expected to dominate more than in $\mathrm{BrHI}^{-}$photodetachment, in agreement with our observations.

The absence of a $v_{3}$ progression in the $\mathrm{FHI}^{-}$and $\mathrm{FDI}^{-}$photoelectron spectra can also be explained by considering the $v_{3}$ potentials for the ion and neutral. The $\mathrm{F}+\mathrm{HI}$ reaction is considerably more exothermic than either the $\mathrm{Br}+\mathrm{HI}$ or the $\mathrm{Cl}+\mathrm{HI}$ reactions, and the ion should be even more asymmetric than either $\mathrm{BrHI}^{-}$or $\mathrm{ClHI}^{-}$. Thus, we expect the $v_{3}$ potentials for the anion and neutral complex to look very much like the diatomic HF potential, at least near the bottom of the wells. With reference to Figure 5-8, in the case of $\mathrm{FHI}^{-}$photodetachment we expect that the wells in the ion and neutral potentials are very similar in shape and their minima essentially coincide (at $R_{e}$ for diatomic HF); this results in only $\Delta v_{3}=0$ transitions in the photoelectron spectrum. 


\subsection{Electronic structure in the $\mathrm{XHY}^{-}$spectra}

We now consider the electronic structure revealed in the $\mathrm{XHY}^{-}$photoelectron spectra. Photodetachment of $\mathrm{XHY}^{-}$provides a direct probe of the multiple electronic potential energy surfaces in the $\mathrm{HX}+\mathrm{Y}$ product valley (in the present case where $\mathrm{Y}=\mathrm{I})$. This is of considerable interest in light of past work on the role of electronically excited reactant and product states in these reactions. ${ }^{12,13,31,32,33}$

Let us first consider which neutral electronic states are accessible via photodetachment of $\mathrm{XHY}^{-}$, a closed shell ${ }^{1} \Sigma^{+}$species. Based on the simple molecular orbital picture proposed for $\mathrm{FHF}^{-}$by Pimentel, ${ }^{34}$ the two highest occupied molecular orbitals in $\mathrm{XHY}^{-}$are expected to be a $\sigma$ orbital which is a linear combination of the two halogen $2 p_{z}$ and $H 1 s$ orbitals, and a doubly degenerate $r$ orbital of the form $2 p_{x, y}(X)-\lambda 2 p_{x, y}(Y)$. Removal of an electron from the $\sigma$ orbital by photodetachment results in a neutral ${ }^{2} \Sigma$ state, whereas removal of an electron from the $\pi$ orbital yields $a^{2} \Pi$ state. If spin-orbit interactions are neglected in the collinear $X+H Y$ reaction, then when the ${ }^{2} \mathrm{P} \mathrm{X}$ atom begins interacting with the HY molecule, the lowest energy electronic state should be the $\Sigma$ state in which the unfilled p orbital on the $\mathrm{X}$ atom lies along the $\mathrm{XHY}$ internuclear axis. On the other hand, the $\Pi$ state, in which the unfilled orbital lies perpendicular to this axis, should result in a repulsive interaction. This is confirmed by DIM (diatomics-in-molecules) calculations by Duggan and Grice for the related systems $\mathrm{F}+\mathrm{HF}$ and $\mathrm{Cl}+\mathrm{HCl} .^{32}$

The inclusion of spin-orbit interactions results in a slightly more complicated picture of the electronic states involved in the reaction. A correlation diagram for the $\mathrm{Br}+\mathrm{HI}$ reaction including spin-orbit effects is shown in Figure 5-10. ${ }^{35}$ The Figure can be generalized to all $\mathrm{X}+\mathrm{HY}$ reactions ${ }^{13}$ and draws upon the DIM calculations on 
$\mathrm{F}+\mathrm{H}_{2}$ by Tully. ${ }^{33}$ Near the interaction region, we see that reaction on the ${ }^{2} \Sigma_{1 / 2}$ curve leads from ground state reactants to ground state products with only a small barrier, whereas reaction along the ${ }^{2} \Pi_{3 / 2}$ or ${ }^{2} \Pi_{1 / 2}$ curve passes through a much larger barrier resulting from an avoided crossing. Near either asymptote, the potential energy curves are similar to the well-studied interaction between a ${ }^{1} \mathrm{~S}$ and $a{ }^{2} \mathrm{P}$ atom. ${ }^{36}$ In this region, where the spin-orbit interaction in the ${ }^{2} \mathrm{P}$ atom is much larger than the intermolecular potential, it is more appropriate to label the three curves only with $\Omega$, the projection of the total electronic angular momentum on the internuclear axis, since $\Omega$ is a good quantum number but $\Lambda$ (projection of the orbital angular momentum only) is not. Thus, in the asymptotic region, Hund's case (c) applies. The three curves are typically labelled $X(1 / 2), I(3 / 2)$, and $I I(1 / 2)$, in order of increasing energy. The two $\Omega=1 / 2$ states are linear combinations of ${ }^{2} \Sigma_{1 / 2}$ and ${ }^{2} \Pi_{1 / 2}$ states, while the ${ }^{2} \Pi_{3 / 2}$ state is the only $\Omega=3 / 2$ state. The same notation is appropriate for the reactant and product valleys of collinear $\mathrm{X}+\mathrm{HY}$ reactions. In the $\mathrm{HX}+\mathrm{Y}$ product valley, the $\mathrm{X}(1 / 2)$ and $\mathrm{I}(3 / 2)$ curves eventually correlate to $\mathrm{Y}\left({ }^{2} \mathrm{P}_{3 / 2}\right)+\mathrm{HX}$, whereas the $\mathrm{I}(1 / 2)$ curve correlates to $\mathrm{Y}^{*}\left({ }^{2} \mathrm{P}_{1 / 2}\right)+\mathrm{HX}$. We therefore expect the photoelectron spectrum of $\mathrm{XHY}^{-}$to show transitions to a maximum of three low-lying electronic potential energy surfaces in the HX + Y product valley.

This is most likely the origin of the three peaks in the FHI-/FDIphotoelectron spectra. The correlation diagram in Figure 5-10 shows that as HF is brought up to an I atom, the degenerate ${ }^{2} \mathrm{P}_{3 / 2}$ state is split and the ${ }^{2} \mathrm{P}_{1 / 2}$ state experiences a repulsive interaction. In our spectra (Figures 5-4 and 5-5), the two peaks $\mathrm{X}$ and $\mathrm{Y}$ separated by $0.154 \mathrm{eV}$ are assigned transitions to the $\mathrm{X}(1 / 2)$ and $\mathrm{I}(3 / 2)$ states, respectively, which both asymptotically correlate to $I\left({ }^{2} P_{3 / 2}\right)+H F$. Peak $Z$ at the 
lowest electron energy lies $1.05 \mathrm{eV}$ from Peak $\mathrm{X}$. This is slightly larger than the I atom spin-orbit splitting and is assigned to a transition to the $\mathrm{II}(1 / 2)$ state which asymptotically correlates to $I^{*}\left({ }^{2} \mathrm{P}_{1 / 2}\right)+\mathrm{HF}$.

Haberland ${ }^{37}$ has shown that of the three potential energy curves resulting from the interaction between a ${ }^{1} \mathrm{~S}$ and a ${ }^{2} \mathrm{P}$ atom, one curve can be determined if the other two are known provided that the spin-orbit interaction is assumed to be independent of internuclear distance. In the Hund's case (c) limit, one obtains

$$
V_{I}(R)=2\left(V_{I I}(R)-\Delta\right),
$$

where $\Delta$ is the spin-orbit splitting in the ${ }^{2} \mathrm{P}$ atom, $\mathrm{R}$ is the internuclear distance, and $V_{1}$ and $V_{I I}$ are the potential energies of the upper two curves relative to the $X(1 / 2)$ curve. We can apply this formula to the three peaks in the FHI- photoelectron spectrum. In this case, $\Delta=0.943 \mathrm{eV}$ (the Iodine spin-orbit splitting) and the splitting between peaks $\mathrm{X}$ and $\mathrm{Z}$ is $\mathrm{V}_{\mathrm{II}}=1.045 \mathrm{eV}$. Equation (3) yields $\mathrm{V}_{\mathrm{I}}=0.205 \mathrm{eV}$, which should be compared to the experimental spacing of $0.154 \mathrm{eV}$ between peaks $\mathrm{X}$ and $\mathrm{Y}$. Somewhat better agreement with experiment is obtained using the more accurate equations from which (3) is derived ${ }^{37}$ that are appropriate for the intermediate region between the Hund's case (c) and (a) limits. In either case, the reasonable agreement with experiment supports our assignment of the three peaks to three electronic states in the I + HF product valley.

In the $\mathrm{BrHI}^{-}$and $\mathrm{ClHI}^{-}$photoelectron spectra, vibrational progressions from only two electronic states are apparent. The interval between the electronic states in the $\mathrm{ClHI}^{-}$spectrum is equal to the I atom spin-orbit splitting, whereas the interval in the $\mathrm{BrHI}^{-}$spectrum is slightly less. This suggests that in the region of the product valley probed by our experiment, the interaction between the $\mathrm{I}$ and $\mathrm{HCl}$ or $\mathrm{HBr}$ 
molecule is not strong enough to produce a resolvable splitting of the degenerate $\mathrm{I}\left({ }^{2} \mathrm{P}_{3 / 2}\right)$ state. However, in both spectra, the peaks in the progression from transitions to the lower electronic state are broader than in the excited-state progression. We suggest that the increased width in the former peak results from the splitting of the degenerate $\mathrm{I}\left({ }^{2} \mathrm{P}_{3 / 2}\right)+\mathrm{HX}$ state but the splitting is smaller than the widths of the individual transitions. This would mean that the peak observed is an envelope of two broad transitions, whose individual widths are probably comparable to the II(1/2) transition. The ground state peaks in the $\mathrm{ClHI}^{-}$spectrum are noticeably broader than in the $\mathrm{BrHI}^{-}$spectrum, indicating either a larger splitting of the I atom electronic degeneracy in the $[\mathrm{ClHI}]$ complex or a more repulsive interaction in the $\mathrm{I}+\mathrm{HCl}$ product valley (see next section).

In summary, as far as electronic effects are concerned, the interaction between $\mathrm{I}$ and $\mathrm{HF}$ in the region of the neutral surface probed by photodetachment of $\mathrm{FHI}^{-}$is stronger than the $\mathrm{I}+\mathrm{HBr}$ and $\mathrm{I}+\mathrm{HCl}$ interaction probed in the $\mathrm{BrHI}^{-}$and $\mathrm{ClHI}^{-}$ spectra. Two effects may contribute to this. The dipole moment of HF is considerably higher than for $\mathrm{HBr}$ or $\mathrm{HCl}(1.82 \mathrm{D}$ vs. $0.82 \mathrm{D}, 1.08 \mathrm{D}) .^{38}$ In addition, the bond length in $\mathrm{HF}$ is much less than in $\mathrm{HBr}$ or $\mathrm{HCl}\left(0.917 \AA\right.$ vs. $1.414 \AA$, $1.275 \AA$ ). ${ }^{39}$ We therefore might expect a shorter interhalogen distance in $\mathrm{FHI}^{-}$than in $\mathrm{BrHI}^{-}$or $\mathrm{ClHI}^{-}$. This means that subsequent to photodetachment, the spherical symmetry of the I atom will be most strongly perturbed by the neighboring $\mathrm{HX}$ molecule in the case of $\mathrm{FHI}^{-}$. The larger electronic effects seen in the $\mathrm{FHI}^{-}$spectrum are reasonable in light of these considerations.

A final point of interest is that the two vibrational progressions in the $\mathrm{BrHI}^{-}$ (and $\mathrm{BrDI}^{-}$) spectrum have similar intensity distributions. The peak spacings in each 
progression are also similar and are slightly less than the vibrational frequency in isolated $\mathrm{HBr}(\mathrm{DBr})$, although this difference is more noticeable in the excited state progression. This indicates that in the geometry probed by our experiment, the distortion of the $\mathrm{HBr}$ bond in the [BrHI] complex is approximately independent of the I atom electronic state, and suggests that the ground and excited electronic potential energy surfaces are not very different in the $\mathrm{I}+\mathrm{HBr}$ product valley. It would clearly be of great interest to probe the excited $\mathrm{Br}+\mathrm{HI}$ potential energy surfaces in the region of the barrier as this is where the largest differences among the various surfaces are expected. This possibility is discussed below.

\subsection{Time dependent simulation}

The discussion in section 2 presents a qualitative explanation for the structured spectrum observed in the $\mathrm{BrHI}^{-}$photodetachment experiment. The simple one-dimensional calculations provide Franck Condon stick spectra within a familiar bound - bound eigenstate framework. However, an essential aspect of this experiment is that the neutral [BrHI] complex dissociates rapidly. Our spectra offer a good deal of dynamical information concerning this process, largely through the peak widths. In order to extract this information we must include at least the dissociative degree of freedom in our simulations. As in our analysis of the $\mathrm{BrHBr}^{-}$and $\mathrm{IHI}^{-}$spectra, we assume that no bending excitation in the neutral complex results from photodetachment and we confine ourselves to a two-dimensional treatment in which only the $v_{1}$ and $v_{3}$ stretching motions are considered.

In our treatment of $\mathrm{BrHBr}^{-}$and $\mathrm{IHI}^{-}$photoelectron spectra we used an adiabatic approximation to separate the bound $\left(v_{3}\right)$ and dissociative $\left(v_{1}\right)$ degrees of freedom, justified because of the different time scales for the two motions in a heavy- 
light-heavy system. ${ }^{2,3}$ While the $\mathrm{BrHI}^{-}$and $\mathrm{BrDI}^{-}$spectra could be simulated in the same way, this approximation is not so straightforward for asymmetric systems. ${ }^{40}$ Alternatively one can exactly solve the two-dimensional problem with a coupled channel collinear scattering calculation. ${ }^{11 b, 41}$ However, we choose to use the timedependent wave packet propagation method which also yields an exact solution. The time-dependent picture reveals the relationship between our experimental spectrum and the short time dynamics of the half reaction initiated on the neutral reaction surface in a more intuitive manner than the time-independent analyses.

The practical difference between the two approaches is this. In timeindependent treatments, each of the many scattering states over a range of energies are solved for, and the simulated spectrum is described by the square of the overlap of the anion wave function with each of these neutral eigenfunctions. The timeCependent perspective is based on the fact that the photoelectron spectrum is equivalently expressed as the Fourier transform of a time autocorrelation function $C(t):$

$$
\sigma(E) \propto \quad \int_{-\infty}^{\infty} \exp (\mathrm{iEt} / \hbar) \mathrm{C}(\mathrm{t}) d t
$$

This complex function $C(t)$ monitors the overlap of a moving wave packet with the initial wave packet as a function of time:

$$
\mathrm{C}(\mathrm{t})=\langle\phi(0) \mid \phi(\mathrm{t})\rangle
$$

The initial wave packet, $\phi(0)$, in this case is defined as the ground state vibrational wavefunction of the anion, assuming the electronic dipole moment operator is a constant over the range of this wave function. The motion of the wave packet subsequently on the neutral surface is described by 


$$
|\phi(t)\rangle=e^{-i R t / \Delta}|\phi(0)\rangle
$$

where $e^{-i t^{i / h}}$ is the time propagation operator and $\hat{H}$ is the Hamiltonian for the upper (neutral) surface. In this way the spectrum is simulated by simply performing the wave packet propagation and transforming the resultant autocorrelation function. This dynamical approach to molecular spectroscopy has been applied by other workers to the analysis of absorption and emission, ${ }^{42,43}$ fluorescence, ${ }^{44}$ photoelectron ${ }^{45,46}$ and Raman ${ }^{47}$ experimental spectra.

The propagation scheme implemented here is due to Kosloff and Kosloff; ${ }^{48}$ we use the Fourier method for evaluation of the Hamiltonian and second order differencing to approximate the propagator. An advantage of the Fourier representation of the kinetic energy is that a relatively sparse spatial grid can be used. Convergence has been tested in each case by doubling the density of grid points along each dimension and halving the propagation time step. The parameters used for each calculation are shown in respective captions.

The concepts involved in spectral analysis based on the autocorrelation function have been described admirably elsewhere. ${ }^{14,43}$ The application of these concepts to our results will be undertaken in two steps. We first discuss the features that appear in the autocorrelation function when considering the bound $v_{3}$ degree of freedom alone. We then consider the extra features that result from a two dimensional analysis which includes the second $\left(v_{1}\right)$, dissociative degree of freedom.

\subsubsection{One-dimensional time-dependent treatment of the $\mathrm{BrHI}^{-}$spectrum}

The features of the ground state progression in the $\mathrm{BrHI}^{-}$photoelectron spectrum have been explained in terms of eigenvalues of a one dimensional double well potential in Section 2 . In a time dependent picture the key to understanding this 
structure lies in the autocorrelation function, $\langle\phi(0) \mid \phi(t)\rangle$, and its relationship to the motion of the wave packet. Figure 5-11 shows the modulus of the autocorrelation function calculated for the BrHI system, using the same anion ground state wave function and the same one dimensional cut of the neutral $\mathrm{Br}+\mathrm{HI}$ LEPS surface as was used in the time independent treatment. Figure 5-11 also shows the resulting photoelectron spectrum obtained by the Fourier transform of the complex $C(t)$ function. A comparison of this simulation and the time independent one in Figure 5-9 shows that they are identical, as we should expect because the two one-dimensional treatments are exact and equivalent.

Figure 5-11 (top) shows that the correlation between the initial wave packet, $\phi(0)$ and the wave packet at time $t, \phi(t)$, falls off rapidly after $t=0$. This indicates that the packet moves quickly away from the Franck Condon region, which in turn indicates that there is considerable excitation in this $v_{3}$ mode. In fact the faster the fall of $|\mathrm{C}(\mathrm{t})|$ from unity at $\mathrm{t}=0$, the longer the vibrational progression, or the larger the bandwidth in the photoelectron spectrum. The next feature is the recurrence, or oscillatory, structure in the autocorrelation. A recurrence occurs when the wave packet $\phi(t)$ returns to the Franck Condon region. The recurrence structure in $|C(t)|$ corresponds to the observation of discrete structure, rather than an unfeatured continuum, in the photoelectron spectrum. The $|C(t)|$ in Figure 5-11 has periodic structure out to infinite time; this serves only to make the peaks in the photoelectron spectrum infinitely narrow, which is to be expected for a treatment that includes only a single bound degree of freedom. As pointed out earlier, the peaks in the $\mathrm{BrHI}^{-}$ photoelectron spectrum are not equally spaced because the reaction potential surface cut does not have a single minimum. The motion of the wave packet in this potential 
cannot then be described as a coherent single frequency oscillation. The complex structure shown in Figure 5-11 and the fact that the height of the first recurrence is not unity are due to this effect which is comparable to dephasing of a wave packet moving in an anharmonic potential well. It is important to emphasize again that this peculiar double oscillatory feature is a result of the shape of the potential along the bound $v_{3}$ coordinate, and is not related to the dissociative degree of freedom.

The finite propagation of the wave packet in time, up until $t=t_{\max }$, leads to a finite resolution of the simulated photoelectron spectrum. In principle this is given, in atomic units, by $\Delta \mathrm{E}=\pi / \mathrm{t}_{\max }{ }^{49}$ However, in practice, if the autocorrelation has not fallen to zero by $t_{\max }$ then its Fourier transform will show artificial high frequency oscillations; this problem is rectified by convolution with a window function. ${ }^{50}$ Choice of a Gaussian window function is equivalent to convoluting the stick spectrum in energy with a Gaussian energy resolution function. This operation has been performed to the one dimensional autocorrelation Figure 5-11 (top) to yield the simulated spectrum (bottom) so that the sticks have FWHM of $10 \mathrm{meV}$.

\subsubsection{Two-dimensional analysis of the $\mathrm{BrHI}^{-}$and $\mathrm{BrDI}^{-}$photoelectron spectra \\ Method}

The extension to higher dimensions of the time dependent approach is conceptually simple. The propagator now allows for motion of the initial wave packet along two dimensions, namely the two stretching coordinates of the linear triatomic. The autocorrelation is calculated in the same manner and the transformation to a photoelectron spectrum simulation is identical to that described above. The two dimensional treatment allows us to assign physical meaning to the peak widths. In 
the following calculations we aim to simulate the full ground X(1/2) state progression for the photoelectron spectra of $\mathrm{BrHI}^{-}$and $\mathrm{BrDI}^{-}$, using the published potential energy surface. At present we neglect the effects of the nearby $I(3 / 2)$ surface, discussed in section 3 , on the $\mathrm{X}(1 / 2)$ progression in the photoelectron spectra. We shall discuss the agreement with the experimental spectra and comment on the interpretation of the peak widths.

The propagation is performed on the effective collinear reaction surface derived from the BP LEPS surface. To extract the effective collinear surface from the supplied LEPS function of all three internal coordinates, the bending angle is considered fixed at $180^{\circ}$, and the zero point energy due to bending motion is included at every grid point ${ }^{3}$ The zero point bend energy is calculated in an harmonic approximation. This approach is in the spirit of the reduced dimensionality model of Bowman. ${ }^{51}$ It is justified as long as little or no bending excitation is expected in the photoelectron spectrum. This is a reasonable assumption so long as the ion is linear and the minimum energy path on the neutral surface is collinear. The Broida and Persky LEPS parameters are not at any time adjusted to fit the experimental spectrum. The spatial grid used in the calculation is set up on the mass scaled coordinates, $\mathbf{x}$ and $\mathbf{y}$ defined in the caption of Figure 5-6, so that the kinetic energy operator is diagonalized. $\mu_{\mathrm{HBr}}$ is the appropriate reduced mass for describing motion on this surface. The anion potential surface is then the sum of the Morse potential (1) along $y$ and a harmonic oscillator with frequency $v_{1}$ along $x$. The equilibrium point of the anion potential is fixed at the best fit values found from the one dimensional analysis, namely $\mathrm{y}_{\mathrm{e}}=1.55 \AA$ and $\mathrm{x}_{\mathrm{e}}=27.1 \AA$. The initial wave packet is set equal to the ground state eigenfunction of this anion potential. Unfortunately there is no matrix isolation 
or calculated value for the $v_{1}$ fundamental; this frequency would indicate the extent of the initial wave packet along the dissociation coordinate $\mathbf{x}$. However combination bands have been seen in the matrix isolation spectra of $\mathrm{BrHBr}^{-}$and $\mathrm{IHI}^{-}$yielding $\mathrm{v}_{1}$ values for these ions of 164 and $121 \mathrm{~cm}^{-1}$ respectively. ${ }^{52}$ The calculated $\mathrm{FHCl}^{-} v_{1}$ is lower than the observed $v_{1}$ of both $\mathrm{FHF}^{-}$and $\mathrm{ClHCl}^{-} .{ }^{16,26}$ Furthermore the $v_{1}$ frequency should be approximately unchanged upon isotopic substitution of the hydrogen. We therefore set $v_{1}$ for $\mathrm{BrHI}^{-} / \mathrm{BrDI}^{-}$at $100 \mathrm{~cm}^{-1}$; only minor changes in the resulting simulation occur if we double this frequency.

This initial wave packet is propagated on the upper surface for 320 femtoseconds. The calculation is checked for convergence with respect to grid size and time step. The potential function has been 'shelved' at extremely high values ( $5 \mathrm{eV}$ above the $\mathrm{I}+\mathrm{HBr}$ energy zero), otherwise a prohibitively small time step is required for a stable propagation. ${ }^{53}$ For the two dimensional simulation shown here utilizing a $128 \times 64$ grid, the entire calculation took seven CPU hours on a VAX 8650 . As observed by Kosloff, ${ }^{15}$ the numerical method is particularly suitable for vectorization on a supercomputer; the same calculation required only 3.3 CPU minutes on a Cray $\mathrm{X} / \mathrm{MP}$ 14. Considerable reduction in run time can be achieved by employing absorbing grid boundaries which immediately allows use of a less extensive grid. ${ }^{53}$ By this device it was possible to perform propagations to a picosecond on a $64 \times 32$ grid and examine resonances to higher energy resolution; these computations required 2.5 CPU minutes total on the Cray.

\section{Results and Discussion}

The calculated autocorrelation functions for $\mathrm{BrHI}$ and $\mathrm{BrDI}$ wave packet dynamics are shown in Figure 5-12. The oscillatory structure out to $60 \mathrm{fs}$ is strongly 
reminiscent of the autocorrelation function calculated in one dimension (see Figure 5-11). However it is quite clear that the inclusion of motion along the dissociation coordinate leads to damping of the oscillations in the autocorrelation function over this time range. Each succeeding time that the wave packet bounces back along the $\mathrm{HBr}$ coordinate to the Franck Condon region, it has progressed further along the $\mathbf{x}$ coordinate and as such has diminishing overlap with $\phi(0)$. One single recurrence in the autocorrelation is necessary, but sufficient, to yield oscillatory structure in the energy spectrum, as shown by Imre for the photodissociation of $\mathrm{H}_{2} \mathrm{O}$. $^{43}$ If all $\mathrm{v}_{3}$ states supported by the one dimensional cut dissociated by the same direct mechanism one would expect essentially the one dimensional result convoluted with a single Gaussian envelope damping function to give the two dimensional autocorrelation function. The calculated function shown in Figure 5-12(a) clearly has a more complicated form; there is long time structure which has a qualitatively different form from the shorter time structure. Analysis of the wave packet dynamics and the Fourier transform of the time autocorrelation function show that the $v_{3}$ states have widely differing 'lifetimes'.

Figure 5-13 explicitly shows how the initial wave packet $\phi(0)$ evolves as a function of time on the $\mathrm{Br}+\mathrm{HI}$ surface. The modulus of $\phi(t)$ is plotted at several times ranging from $t=0$ to $t=966.4$ fs. The plots show the regions of the potential sampled by the wave packet, the mode or mechanism of dissociation, and the branching ratio between the arrangement and vibrational channels. The timedependent function $\phi(t)$ represents the evolution of a coherent superposition of scattering eigenfunctions $\psi_{E}$ weighted by $\left\langle\phi(0) \mid \psi_{E}\right\rangle$. Although $\phi(t)$ and the photoelectron spectrum are uniquely related through Equation (4), we point out that, 
in contrast to a 'short-pulse' laser absorption experiment, such a superposition is not created in our photoelectron spectroscopy experiment. Instead, each photodetachment event results in a well-defined neutral scattering state $\psi_{E}$ with probability $|<\phi(0)| \psi_{E}>\left.\right|^{2}$. However, the plots of $\phi(t)$ show what would occur if the initial wave packet $\phi(0)$ were created on the neutral potential energy surface and therefore provide considerable insight into the dissociation dynamics of the [BrHI] complex.

The first picture ( $t=0 \mathrm{fs}$ ) shows that the bulk of the initial wave packet's amplitude is in the $\mathrm{I}+\mathrm{HBr}$ exit valley, although $\phi(0)$ does have some amplitude at the saddle point region of the potential surface and therefore will have finite overlap with states localized in the HI valley. In the first few femtoseconds a small fraction of the wave packet breaks away upwards into the saddle point region while the rest moves downwards. The period of oscillation of this major component of the packet along the $\mathrm{y}$ coordinate is essentially that of diatomic $\mathrm{HBr}\left(\tau_{\mathrm{HBr}}=12.6 \mathrm{fs}\right)$. This is the dominant periodicity shown in the autocorrelation. The second frame shows the packet after $-1.5 \tau_{\mathrm{HBr}}$ where $|\mathrm{C}(\mathrm{t})|$ goes through a minimum. As commented on for the one dimensional autocorrelation, the anharmonicity of the potential governing this fast oscillation along the y coordinate leads to a more complicated periodic structure. On each of the first few occasions that the major component of the packet returus to the 'soft' wall, a fraction crosses the ridge between product and reactant valleys (seen in the pictures at $t=20.1$ and $40.3 \mathrm{fs}$ ).

Over the first $60 \mathrm{fs}$ the major part of the wave packet moves barely perceptibly along the dissociation coordinate. However at later times we see this motion becomes more dominant; the overlap of this component of $\phi(t)$ with $\phi(0)$ becomes much smaller, and thus its contribution to the shape of the autocorrelation is diminished. 
Therefore the autocorrelation at times later than 60 fs slowly loses resemblance to the earlier pattern and that seen in the one dimensional simulation. The pictures at longer times (e.g. at $t=201.3$ and $261.7 \mathrm{fs}$ ) show the component of the wave packet that exits through the product valley spreads along $x$ and from the leading edge (at higher $\mathbf{x})$ to the tail there is an increasing number of nodes along the $y$ direction. This suggests that states with higher $v_{3}$ excitation proceed more slowly along the dissociation coordinate. This is in accord with our simulations on $\mathrm{BrHBr}^{-},{ }^{3}$ and is manifested in the narrower linewidths of the $[\mathrm{BrHI}] \mathrm{v}_{3}=3$ and $\mathrm{v}_{3}=5$ peaks in the simulated photoelectron spectrum (Figure 5-14(a), Table 5-3). Moreover notice the series of later time pictures show some components of the wave packet that remain localized in coordinate space for extended periods of time (e.g. at $t=201.3,261.7$ and $966.4 \mathrm{fs})$. These correspond to a weighted superposition of quasi-stationary states or resonance states; the dominant resonance states seen have $v_{3}=4$. These are manifested in the autocorrelation at long time as oscillations modulated by a second frequency. In the simulated photoelectron spectrum the resonances appear as a series of closely spaced $\left(-18 \mathrm{meV}\right.$ or $\left.-150 \mathrm{~cm}^{-1}\right)$ peaks at $1.21 \mathrm{eV}$ electron energy. However they are not resolved individually when the simulation is convoluted with the experimental resolution function (Figure 5-14(a)). The positions of these resonances, which have not decayed by the end of a picosecond in this two dimensional simulation, are listed in Table 5-3. These states can be projected out and their individual mode of decay can be studied by time-dependent propagation as shown by Bisseling et al..$^{53}$

The differing lifetimes' of each vibrational state, and the resonance phenomenon supported by the potential energy surface used in this simulation, explain the complex decay of the autocorrelation function. The simulated 
photoelectron spectra for $\mathrm{BrHI}^{-}$and $\mathrm{BrDI}^{-}$have been convoluted with the true instrument resolution function (given in Ref. 3) and are shown in Figure 5-14 where they are superimposed on the experimental spectra. It is quite clear that the experimental spectra do not show many of the features discussed above, and the autocorrelation functions leading to these spectra should be much simpler than that shown in Figure 5-12. The spacing of the peaks and the qualitative trends in peak width, namely decreasing width with greater vibrational excitation, are in good agreement with the experimental spectra. However the discrepancy in the magnitudes of the widths is striking, and the variation of width is much less noticeable in the experimental spectrum as opposed to the halving of linewidth seen between the simulated $v_{3}=0$ and $v_{3}=3$ peaks. Moreover we have shown that in a two dimensional simulation the BP LEPS surface supports resonance states which should give rise to sharp peaks in the spectrum, although the intensity of these peaks is expected to be small. These are not evident in the experimental profiles. Thus, in time-independent language, the experimental peaks all appear to be from direct scattering states on the $\mathrm{Br}+\mathrm{HI}$ surface. Lastly the origins of the simulated photoelectron bands must be shifted to higher electron kinetic energy by approximately $60 \mathrm{meV}$ to match with the experimental ones. This shift is just larger than the cumulative error in the reported thermochemical and spectroscopic data used to link the energy zeros of ion and neutral.

We would like to be able to use the discrepancies between the simulated and experimental spectra, in particular the differences in the peak widths, to learn about the $\mathrm{Br}+\mathrm{HI}$ potential energy surface. One might argue that the broad experimental peaks result from the multiple electronic states of the neutral complex accessible via 
photodetachment. In section 3 , we have argued that each peak in the progression at higher electron kinetic energy could be split due to an electronic interaction between the dipole of the $\mathrm{HBr}$ and the open shell iodine atom. In the [FHI] system where the electronic interaction is largest and the components are resolved, some differences in peak width are seen for the three states (see Table 5-1c). In the case of [BrHI] less of a difference is expected in the peak shape and width among the three states and it is probable that the splitting is in fact a fairly small part of the width, the major part of the width being intrinsic to the transition to a dissociative state.

A similar discrepancy between the simulated and experimental peak widths was also seen in our analysis of the $\mathrm{BrHBr}^{-}$photoelectron spectrum when we assumed a LEPS potential energy surface for the $\mathrm{Br}+\mathrm{HBr}$ reaction. ${ }^{3}$ We therefore developed a more flexible functional form for an effective collinear $\mathrm{Br}+\mathrm{HBr}$ surface which allowed the construction of a surface with a steeper minimum energy path in the Franck-Condon region. Simulations on this surface did reproduce the broad peak widths observed in the $\mathrm{BrHBr}^{-}$spectrum. A similar modification may be required for the $\mathrm{BP} \mathrm{Br}+\mathrm{HI}$ surface; that is, the surface may not be steep enough in the $\mathrm{I}+\mathrm{HBr}$ exit valley. Another possibility is that the minimum energy path on the correct $\mathrm{Br}+\mathrm{HI}$ surface is not collinear, in which case the effective collinear approach is not appropriate and full three-dimensional simulations are required to accurately simulate the photoelectron spectrum. In any case, while it is somewhat risky to draw conclusions on the possible defects of a reactive potential energy surface based on an effective collinear analysis, this type of analysis provides an important first step in relating the features of the $\mathrm{BrHI}^{-}$photoelectron spectrum to the $\mathrm{Br}+\mathrm{HI}$ potential energy surface. 
Two variations on this experiment should provide considerably more information on the $\mathrm{Br}+\mathrm{HI}$ reaction. $\mathrm{BrHI}^{-}$in its ground vibrational state has good Franck-Condon overlap with the $\mathrm{I}+\mathrm{HBr}$ product valley. This is certainly an important region of the potential energy surface as it plays a major role in determining how the energy released in the reaction is partitioned among product degrees of freedom. However, we would also like to probe the reactant side of the potential energy surface, particularly the barrier. In Figure 5-8, note that the neutral levels with $v_{3} \geq 4$ span both valleys of the potential energy surface. While transitions to these levels from the $\mathrm{v}_{3}=0$ level of $\mathrm{BrHI}^{-}$are very weak, transitions originating from the $v_{3}=1$ level of the ion are considerably stronger. A simulated photoelectron spectrum assuming $\mathrm{BrHI}^{-}$with $\mathrm{v}_{3}=1$ is shown at the top of Figure 5-15. The appearance of this spectrum is quite different from the simulation in Figure 5-14 and shows intense peaks due to transitions to these higher neutral $v_{3}$ levels. Hence, vibrational excitation of the ion provides an elegant means of probing the reactant side of the $\mathrm{Br}+\mathrm{HI}$ surface. In general, vibrational excitation of various modes of the ion is akin to varying the distribution of reactant energy in a state-to-state experiment.

The simulated spectrum at the top of Figure 5-15 is convoluted with the experimental resolution of our photoelectron spectrometer. The spectrum at the bottom of Figure 5-15 assumes an constant experimental resolution of $4 \mathrm{meV}\left(35 \mathrm{~cm}^{-1}\right)$ and shows correspondingly more structure. For example, the $v_{3}=4$ peak splits into 4 closely spaced peaks which are actually resonance states quasi-bound along the $v_{1}$ coordinate. The appearance of these was discussed in the time dependent section above. Thus, a spectrometer with somewhat higher resolution has the capability to 
reveal considerably more detail concerning the $\mathrm{Br}+\mathrm{HI}$ potential energy surface. We currently have such an instrument: ${ }^{54}$ a threshold photodetachment spectrometer with a resolution of $3 \mathrm{~cm}^{-1}$. Recent results on $\mathrm{IHI}^{-}$have already shown vibrational features of the [IHI] complex that were obscured at lower resolution. ${ }^{65,56}$ Studies of asymmetric systems with this instrument will be undertaken in the near future.

\section{Summary}

Photoelectron spectra for the asymmetric bihalide anions $\mathrm{XHI}^{-}$and $\mathrm{XDI}^{-}(\mathrm{X}$ $=\mathrm{Br}, \mathrm{Cl}, \mathrm{F}$ ) have been obtained in order to learn about the transition state region on the neutral $\mathrm{X}+\mathrm{HI}$ potential energy surfaces. In the case of $\mathrm{BrHI}^{-}$and $\mathrm{ClHI}^{-}$, the spectra show resolved vibrational progressions assigned to the $v_{3}$ hydrogen stretching mode of the neutral [XHI] complex. In all the spectra, transitions are observed not only to the ground state reactive potential energy surface, but also to electronically excited surfaces which correlate asymptotically to $\left.\mathrm{HX}+\mathrm{I}^{2} \mathrm{P}_{3 / 2},{ }^{2} \mathrm{P}_{1 / 2}\right)$. The $\mathrm{BrHI}^{-}$and $\mathrm{BrDI}^{-}$spectra are analyzed in detail using an approximate geometry for the ion and a model potential energy surface for the $\mathrm{Br}+\mathrm{HI}$ reaction. A one-dimensional analysis is used both to simulate the peak positions and intensities of the $\mathrm{BrHI}^{-}$and $\mathrm{BrDI}^{-}$ spectra and to understand the appearance of the other $\mathrm{XHI}^{-}$spectra. We have also performed a two-dimensional quantum collinear simulation of the spectra of $\mathrm{BrHI}^{-}$ and $\mathrm{BrDI}^{-}$via the wave packet propagation technique. The results of this time dependent simulation provide further insight into the origin of the structure seen in our spectra. The simulated peaks are narrower than the experimental peaks; this is discussed in terms of properties of the model $\mathrm{Br}+\mathrm{HI}$ surface and approximations in the analysis. 


\section{Acknowledgements}

We would like to acknowledge Dr. Soo-Y. Lee for helping us start the time dependent simulation of the $\mathrm{BrHI}^{-}$spectrum by providing us with routines for the Kosloff algorithm in one dimension. We also thank Prof. M. Shapiro for useful discussions and Prof. P. Botschwina for communicating his results on $\mathrm{FHCl}^{-}$prior to publication. Support from the Air Force Office of Scientific Research under Contract No. AFOSR-87-0341 is gratefully acknowledged. D. M. N. thanks the Research Corporation and the Donors of the Petroleum Research Fund, administered by the American Chemical Society, for support. 


\section{References for Chapter 5.}

1. R. B. Metz, T. Kitsopoulos, A. Weaver, and D. M. Neumark, J. Chem. Phys. 88, 1463 (1988).

2. A. Weaver, R. B. Metz, S. E. Bradforth, and D. M. Neumark, J. Phys. Chem. 92, 5558 (1988).

3. R. B. Metz, A. Weaver, S. E. Bradforth, T. N. Kitsopoulos, and D. M. Neumark, J. Phys. Chem. 94, 1377 (1990).

4. G. C. Schatz, J. Chem. Phys. 90, 3582 (1989); G. C. Schatz, J. Chem. Phys. 90, 4847 (1989).

5. J. M. Bowman and B. Gazdy, J. Phys. Chem. 93, 5129 (1989).

6. B. Gazdy and J. M. Bowman, J. Chem. Phys. 91, 4615 (1989).

7. S. E. Bradforth, A. Weaver, R. B. Metz, and D. M. Neumark, Advances in Laser Science - IV Proceedings of the 1988 International Laser Science Conference, pp. 657, American Institute of Physics (1989).

8. N. Jonathon, C. M. Melliar-Smith, S. Okuda, D. H. Slater and D. Timlin, Mol. Phys. 22, 561 (1971); D. H. Maylotte, J. C. Polanyi, and K. B. Woodall, J. Chem. Phys. 57, 1547 (1972); J. R. Grover, C. R. Iden and H. V. Lilenfeld, J. Chem. Phys. 64, 4657 (1976); C.C. Mei and C. I. Moore, J. Chem. Phys. 67, 3936 (1977), J. Chem. Phys. 70, 1759 (1979); K. Tamagake, D. W. Setser, and J. P. Sung, J. Chem. Phys. 73, 2203 (1980); D. A. Dolson and S. R. Leone, J. Chem. Phys. 77, 4009 (1982).

9. C. A. Parr, J. C. Polanyi and W. H. Wong, J. Chem. Phys. 58, 5 (1973); D. J. Douglas, J. C. Polanyi, and J. J. Sloan, Chem. Phys. 13, 15 (1976); I. W. M. Smith, Chem. Phys. 20, 437 (1977); J. C. Brown, H. E. Bass, and D. L. Thompson, J. Phys. Chem. 81, 479 (1977); P. Beadle, M. R. Dunn, N. B. H. Jonathon, J. P. Liddy, and J. C. Naylor, J. Chem. Soc. Farad. Trans. II 74, 2170 (1978).

10. M. Broida and A. Persky, Chem. Phys. 133, 405 (1989).

11. M. Baer, J. Chem. Phys. 62, 305 (1975); J. A. Kaye and A. Kupperman, Chem. Phys. Lett. 92, 574 (1982); P. L. Gertitschke, J. Manz, J. Römelt, and H. H. R. Schor, J. Chem. Phys. 83, 208 (1985); J. Manz and H. H. R. Schor, Chem. Phys. Lett. 107, 549 (1984).

12. K. Bergmann, S. R. Leone and C. B. Moore, J. Chem. Phys. 63, 4161 (1975).

13. J. W. Hepburn, K. Liu, R. G. Macdonald, F. J. Nothrup, and J. C. Polanyi, J. Chem. Phys. 75, 3353 (1981). 
14. E. J. Heller, J. Chem. Phys. 68, 3891 (1978); Acc. Chem. Res. 14, 368 (1981).

15. R. Kosloff, J. Phys. Chem. 92, 2087 (1988).

16. K. Kawaguchi and E. Hirota, J. Chem. Phys. 87, 6838 (1987); K. Kawaguchi, J. Chem. Phys. 88, 4186 (1988).

17. P. Botschwina, P. Sebald and R. Durmeister, J. Chem. Phys. 88, 5246 (1988); A. B. Sannigrahi and S. D. Peyerimhoff, J. Molec. Struct. (Theochem.) 165, 55 (1988); S. Ikuta, T. Saitoh, and O. Nomura, Inorg. Chem. submitted.

18. C. R. Moylan, J. D. Dodd, C. Han, and J. I. Brauman, J. Chem. Phys. 86, 5350 (1987).

19. L. A. Posey, M. J. DeLuca, and M. A. Johnson, Chem. Phys. Lett. 131, 170 (1986).

20. M. L. Alexander, N. E. Levinger, M. A. Johnson, D. Ray, and W. C. Lineberger, J. Chem. Phys. 88, 6200 (1988).

21. W. C. Wiley and I. H. McLaren, Rev. Sci. Instrum. 26, 1150 (1955).

22. G. Caldwell and P. Kebarle, Can. J. Chem. 63, 1399 (1985).

23. H. Hotop and W. C. Lineberger, J. Phys. Chem. Ref. Data. 14, 731 (1985).

24. J. W. Larson and T. B. McMahon, Inorg. Chem. 23, 2029 (1984).

25. A. B. Sannigrahi and S. D. Peyerimhoff, Chem. Phys. Lett. 112, 267 (1984).

26. P. Sebald and P. Botschwina, to be published.

27. J. E. Bartmess and R. T. McIver in Gas Phase Ion Chemistry; M. T. Bowers ed.; Academic Press, New York (1979).

28. In fact the effective collinear surface, which has the zero point energy due to the doubly-degenerate bend added at every point (described in Section 4), is shown in Figure 6. The correction is small but the barrier on this effective surface is $0.84 \mathrm{kcal} / \mathrm{mol}$.

29. C. M. Ellsion and B. S. Ault, J. Phys. Chem. 83, 832 (1979).

30. B. S. Ault, J. Phys. Chem. 83, 837 (1979).

31. P. L. Houston, Chem. Phys. Lett. 47, 137 (1977).

32. J. J. Duggan and R. Grice, J. Chem. Soc. Faraday Trans. II 80, 739 (1983).

33. J. C. Tully, J. Chem. Phys. 60, 3042 (1974). 
34. G. C. Pimentel, J. Chem. Phys. 19, 446 (1951).

35. Figure adapted for collinear reaction from that appearing in Ref. 12.

36. see for example C. H. Becker, P. Casavecchia, Y. T. Lee, R. E. Olson, and W. A. Lester Jr., J. Chem. Phys. 70, 5477 (1979) and references therein.

37. H. Haberland, Z. Phys. A. 307, 35 (1982).

38. Nelson R. D., Lide D. R., and Maryott A. A., in CRC Handbook of Chemistry and Physics 69th edition, ed. Weast, CRC Press (1988), pp. E-58.

39. K. P. Huber and G. Herzberg, Molecular Spectra and Molecular Structure IV. Constants of Diatomic Molecules; Van Nostrand; New York (1979).

40. J. Römelt, Chem. Phys. 79, 197 (1983).

41. D. K. Bondi, J. N. L. Connor, J. Manz, J. Römelt, Mol. Phys. 50, 467 (1983).

42. S. O. Williams and D. G. Imre, J. Phys. Chem. 92, 3374 (1988).

43. N. E. Henriksen, J. Zhang, and D. G. Imre, J. Chem. Phys. 89, 5607 (1988); J. Zhang and D. G. Imre, J. Chem. Phys. 90, 1666 (1989).

44. D. G. Imre, J. L. Kinsey, R. W. Field and D. H. Katayama, J. Phys. Chem. 86, 2564 (1982).

45. A. J. Lorquet, J. C. Lorquet, J. Delwiche, and M. J. Hubin-Franskin, J. Chem. Phys. 76, 4692 (1982).

46. J. E. Pollard, D. J. Trevor, J. E. Reutt, Y. T. Lee, and D. A. Shirley, J. Chem. Phys. 81, 5302 (1984).

47. A. B. Myers, R. A. Mathies, D. J. Tannor and E. J. Heller, J. Chem. Phys. 77, 3857 (1982).

48. D. Kosloff and R. Kosloff, J. Comput. Phys. 52, 35 (1983); R. Kosloff and D. Kosloff, J. Chem. Phys. 79, 1823 (1983).

49. This is a result of the properties of a discrete Fourier transform (see Ref. 50). The transform is performed between $-t_{\max }$ and $+t_{\max }$.

50. W. H. Press, B. P. Flannery, S. A. Teukolsky, and V. T. Vetterling, Numerical Recipes; Cambridge University Press, Cambridge (1986).

51. J. M. Bowman, Adv. Chem. Phys. 61, 115 (1985).

52. M. E. Jacox, J. Phys. Chem. Ref. Data 13, 945 (1984). 
53. R. H. Bisseling, R. Kosloff, and J. Manz, J. Chem. Phys. 83, 993 (1985).

54. T. N. Kitsopoulos, I. M. Waller, J. G. Loeser, and D. M. Neumark, Chem. Phys. Lett. 159, 300 (1989).

55. D. M. Neumark in "Electronic and Atomic Collisions - Invited Papers of the XVI ICPEAC", in press.

56. I. M. Waller, T. N. Kitsopoulos and D. M. Neumark, J. Phys. Chem. in press. 
Table 5-1a: Experimental Results for Photoelectron Spectra of $\mathrm{BrHI}^{-}$and $\mathrm{BrDI}^{-}$. (All energies in $\mathrm{eV}$ )

\begin{tabular}{|c|c|c|c|c|c|c|}
\hline & $\begin{array}{l}\mathrm{eKE}^{\mathrm{a}} \\
213 \mathrm{~nm}\end{array}$ & $\begin{array}{l}\text { FWHM }^{b} \\
213 \mathrm{~nm}\end{array}$ & $\begin{array}{l}\mathrm{eKE}^{\mathrm{e}} \\
266 \mathrm{~nm}\end{array}$ & $\begin{array}{l}\mathrm{FWHM}^{\mathrm{b}} \\
266 \mathrm{~nm}\end{array}$ & Spacing & $\begin{array}{l}\text { Spacing in } \\
\mathrm{HBr} / \mathrm{DBr}^{\mathrm{c}}\end{array}$ \\
\hline \multicolumn{7}{|c|}{ BrHI $^{-}$} \\
\hline \multirow[t]{2}{*}{$\mathbf{A}$} & $1.970(17)$ & .175 & $0.783(4)$ & .170 & & \\
\hline & & & & & $0.313(5)^{d}$ & $0.317(0-1)$ \\
\hline \multirow[t]{2}{*}{ B } & $1.658(13)$ & .170 & $0.470(3)$ & .165 & & \\
\hline & & & & & $0.268(18)$ & $0.306(1-2)$ \\
\hline $\mathrm{C}$ & $1.390(10)$ & .160 & - & - & & \\
\hline \multirow[t]{2}{*}{$\mathrm{A}^{*}$} & $1.071(7)$ & .155 & - & - & & \\
\hline & & & & & $0.292(9)$ & $0.317(0-1)$ \\
\hline \multirow[t]{2}{*}{$\mathrm{B}^{*}$} & $0.779(5)$ & .140 & - & - & & \\
\hline & & & & & $0.234(10)$ & $0.306(1-2)$ \\
\hline $\mathrm{C}^{*}$ & $0.545(8)$ & -.120 & - & - & & \\
\hline \multicolumn{7}{|c|}{$\mathrm{BrDI}^{-}$} \\
\hline \multirow[t]{2}{*}{ A } & 1.980 (17) & .185 & & & & \\
\hline & & & & & $0.240(22)$ & $0.228(0-1)$ \\
\hline \multirow[t]{2}{*}{ B } & $1.740(14)$ & .170 & & & & \\
\hline & & & & & $0.203(18)$ & $0.222(1-2)$ \\
\hline \multirow[t]{2}{*}{$\mathrm{C}$} & $1.537(11)$ & .160 & & & & \\
\hline & & & & & $0.193(19)$ & $0.216(2-3)$ \\
\hline D & $1.344(15)$ & -.160 & & & & \\
\hline \multirow[t]{2}{*}{$A^{*}$} & $1.068(9)$ & .180 & & & & \\
\hline & & & & & $0.226(15)$ & $0.228(0-1)$ \\
\hline \multirow[t]{2}{*}{$\mathrm{B}^{*}$} & $0.842(11)$ & .170 & & & & \\
\hline & & & & & $0.192(17)$ & $0.22(1-2)$ \\
\hline \multirow[t]{2}{*}{$\mathrm{C}^{*}$} & $0.650(13)$ & -.150 & & & & \\
\hline & & & & & $0.170(20)$ & $0.216(2-3)$ \\
\hline $\mathrm{D}^{*}$ & $0.480(15)$ & -.170 & & & & \\
\hline
\end{tabular}


Table 5-1a continued.

a) Electron kinetic Energies (eKE) at each peak center found by fitting peaks to a set of Gaussians. Uncertainties, in parentheses, are approximate.

b) Uncertainties in widths are approximately $0.005 \mathrm{eV}$, except where indicated.

c) Spacings between vibrational levels indicated in parentheses; source Ref. 39.

d) A-B spacing from $266 \mathrm{~nm}$ data

$\mathrm{A} \rightarrow \mathrm{A}^{*}$ spacing in the $\mathrm{BrHI}^{-}$and $\mathrm{BrDI}^{-}$photoelectron spectra are $0.899 \pm 0.019 \mathrm{eV}$ and $0.912 \pm 0.020 \mathrm{eV}$ respectively. The free iodine atom spin-orbit splitting is 0.943 eV. 
Table 5-1b: Experimental results for Photoelectron spectra of $\mathrm{ClHI}^{-}$and $\mathrm{ClDI}^{-}$. (All energies in $\mathrm{eV}$ )

\begin{tabular}{|c|c|c|c|c|c|c|}
\hline & $\begin{array}{l}\mathrm{eKE}^{\mathrm{a}} \\
213 \mathrm{~nm}\end{array}$ & $\begin{array}{l}\text { FWHM' } \\
213 \mathrm{~nm}\end{array}$ & $\begin{array}{l}\mathrm{eKE}^{\mathrm{a}, \mathrm{c}} \\
266 \mathrm{~nm}\end{array}$ & $\begin{array}{l}F_{W H M}^{\mathrm{b}, c} \\
266 \mathrm{~nm}\end{array}$ & Spacing & $\begin{array}{l}\text { Spacing in } \\
\mathrm{HCV} / \mathrm{DCl}^{\mathrm{d}}\end{array}$ \\
\hline \multicolumn{7}{|c|}{$\mathrm{CHHI}^{-}$} \\
\hline \multirow[t]{2}{*}{ A } & $2.070(17)$ & .230 & $0.900(5)$ & .220 & & \\
\hline & & & & & $0.380(6)^{e}$ & $0.358(0-1)$ \\
\hline \multirow[t]{2}{*}{ B } & $1.678(13)$ & .220 & $0.520(3)$ & .220 & & \\
\hline & & & & & $0.323(18)$ & $0.345(1-2)$ \\
\hline C & 1.355 (13) & -.210 & - & - & & \\
\hline \multirow[t]{2}{*}{$A^{*}$} & $1.133(10)$ & .185 & - & - & & \\
\hline & & & & & $0.358(17)$ & $0.358(0-1)$ \\
\hline $\mathrm{B}^{*}$ & $0.775(5)$ & -.180 & - & - & & \\
\hline \multicolumn{7}{|c|}{$\mathrm{ClDI}^{-}$} \\
\hline \multirow[t]{2}{*}{ A } & 2.079 (17) & .205 & $0.900(5)$ & .190 & & \\
\hline & & & & & $0.279(7)^{e}$ & $0.259(0-1)$ \\
\hline \multirow[t]{2}{*}{ B } & $1.797(14)$ & .190 & $0.621(4)$ & .175 & & \\
\hline & & & & & $0.258(19)$ & $0.252(1-2)$ \\
\hline C & $1.539(13)$ & -.175 & & & & \\
\hline \multirow[t]{2}{*}{$A^{*}$} & $1.148(8)$ & .175 & & & & \\
\hline & & & & & $0.278(14)$ & $0.259(0-1)$ \\
\hline \multirow[t]{2}{*}{ B* } & $0.870(11)$ & .175 & & & & \\
\hline & & & & & $0.235(17)$ & $0.252(1-2)$ \\
\hline $\mathrm{C}^{*}$ & 0.635 (13) & -.170 & & & & \\
\hline
\end{tabular}


Table 5-1b continued

a) Electron kinetic Energies (eKE) at each peak center found by fitting peaks to a set of Gaussians. Uncertainties, in parentheses, are approximate.

b) Uncertainties in widths are approx. $0.005 \mathrm{eV}$, except where indicated.

c) $266 \mathrm{~nm}$ spectra not shown

d) Spacings between vibrational levels indicated in parentheses; source Ref. 39.

e) A-B spacing from $266 \mathrm{~nm}$ data

$\mathrm{A} \rightarrow \mathrm{A}^{*}$ spacing in the $\mathrm{ClHI}^{-}$and $\mathrm{ClDI}^{-}$photoelectron spectra are $0.937 \pm 0.020 \mathrm{eV}$ and $0.931 \pm 0.019 \mathrm{eV}$ respectively.

Table 5-1c: Experimental Results for Photoelectron Spectra of $\mathrm{FHI}^{-}$and FDI-. (All energies in $\mathrm{eV}$ )

\begin{tabular}{|c|c|c|c|c|c|}
\hline & $\begin{array}{l}\mathrm{eKE}^{\mathrm{a}} \\
213 \mathrm{~nm}\end{array}$ & $\begin{array}{l}\text { FWHM } \\
213 \mathrm{~nm}\end{array}$ & $\begin{array}{l}\mathrm{eKE}^{\mathrm{a}} \\
266 \mathrm{~nm}\end{array}$ & $\begin{array}{l}\text { FWHM }^{\mathrm{b}} \\
266 \mathrm{~nm}\end{array}$ & Spacing \\
\hline \multicolumn{6}{|c|}{ FHI- } \\
\hline \multirow[t]{2}{*}{$\mathbf{x}$} & 2.143 (19) & .100 & & & \\
\hline & & & & & $0.151(25)$ \\
\hline \multirow[t]{2}{*}{$\mathbf{Y}$} & $1.992(17)$ & .130 & & & \\
\hline & & & & & 0.894 (19) \\
\hline $\mathrm{Z}$ & $1.098(7)$ & .115 & & & \\
\hline \multicolumn{6}{|c|}{ FDI $^{-}$} \\
\hline \multirow[t]{2}{*}{$\mathbf{x}$} & $2.143(19)$ & .100 & $0.966(5)$ & .070 & \\
\hline & & & & & $0.154(7)^{e}$ \\
\hline \multirow[t]{2}{*}{$\mathbf{Y}$} & $1.992(17)$ & .130 & $0.812(4)$ & .110 & \\
\hline & & & & & 0.894 (19) \\
\hline $\mathbf{Z}$ & $1.098(7)$ & .115 & - & - & \\
\hline
\end{tabular}

a) Electron kinetic Energies (eKE) at each peak center found by fitting peaks to a set of Gaussians. Uncertainties, in parentheses, are approximate.

b) Uncertainties in widths are approximately $0.005 \mathrm{eV}$.

c) X-Y spacing from $266 \mathrm{~nm}$ data. 
Table 5-2: Available data for the anions and neutral reactions described in this work.

\begin{tabular}{lllllll}
\hline \hline Anion & $\mathrm{BrHI}^{-}$ & $\mathrm{BrDI}^{-}$ & $\mathrm{ClHI}^{-}$ & $\mathrm{ClDI}^{-}$ & $\mathrm{FHI}^{-}$ & $\mathrm{FDI}^{-}$ \\
\hline$\Delta \mathrm{H}_{300}^{\circ}(\mathrm{eV})^{\mathrm{a}}$ & 0.70 & & 0.62 & & $0.65^{\mathrm{b}}$ & \\
$v_{3}\left(\mathrm{~cm}^{-1}\right)^{\mathrm{c}}$ & $920^{\mathrm{d}}$ & $728^{\mathrm{d}}$ & $1560^{\mathrm{d}}$ & $1219^{\mathrm{d}}$ & $2955^{\mathrm{e}}$ & $2225^{\mathrm{e}}$ \\
E.B.E.(eV) & 3.88 & 3.85 & 3.76 & 3.76 & 3.68 & 3.70 \\
\hline
\end{tabular}

\begin{tabular}{lllllll}
\hline $\begin{array}{l}\text { Neutral } \\
\text { Reaction }\end{array}$ & $\mathrm{Br}+\mathrm{HI}$ & $\mathrm{Br}+\mathrm{DI}$ & $\mathrm{Cl}+\mathrm{HI}$ & $\mathrm{Cl}+\mathrm{DI}$ & $\mathrm{F}+\mathrm{HI}$ & $\mathrm{F}+\mathrm{DI}$ \\
\hline$\Delta \mathrm{H}_{0}^{\circ}(\mathrm{eV})^{\mathrm{g}}$ & 0.704 & 0.710 & 1.379 & 1.390 & 2.815 & 2.843 \\
\hline \hline
\end{tabular}

a) Hydrogen Bond Cleavage Enthalpy, i.e. the enthalpy change for the reaction $\mathrm{XH} . \mathrm{I}^{-} \rightarrow \mathrm{HX}+\mathrm{I}^{-}$at $300 \mathrm{~K}$, from Ref. 22. This value is used, in the absence of any other data, for $\mathrm{D}_{0}\left(\mathrm{XH} . \mathrm{I}^{-}\right)$in the text.

b) Estimated. See Ref. 24 for details.

c) Fundamental frequency for the Type I hydrogen stretching vibration, measured for ion prepared in an argon matrix. See text for discussion of choice of frequencies.

d) $\quad$ Ref. 29

e) $\quad$ Ref. 30

f) Approximate electron binding energy. This is estimated from center of 0-0 peak in photoelectron spectrum (this work)

g) Reaction exothermicity: $\Delta \mathrm{H}_{0}^{\circ}=\mathrm{D}_{0}^{\circ}(\mathrm{HX})-\mathrm{D}_{0}^{\circ}(\mathrm{HI})$. Data from Ref. 36 
Table 5-3: Results of an exact quantum collinear simulation of the photoelectron spectra of $\mathrm{BrHI}^{-}$and $\mathrm{BrDI}^{-}$using the BP LEPS surface with zero point bend included.

\begin{tabular}{|c|c|c|c|}
\hline & $\begin{array}{c}\text { Electron Kinetic } \\
\text { Energy }(\mathrm{eV})^{\mathbf{2}}\end{array}$ & $v_{3}$ assignment & Width (meV) \\
\hline \multirow[t]{13}{*}{ BrHI- $^{-}$} & 1.970 & 0 & 43 \\
\hline & 1.662 & 1 & 36 \\
\hline & 1.447 & 2 & $<4$ \\
\hline & 1.429 & 2 & $<4$ \\
\hline & 1.416 & 2 & $<4$ \\
\hline & 1.408 & 2 & $<4$ \\
\hline & 1.388 & 3 & 15 \\
\hline & 1.230 & 4 & $<4$ \\
\hline & 1.211 & 4 & $<4$ \\
\hline & 1.192 & 4 & $<4$ \\
\hline & 1.176 & 4 & $<4$ \\
\hline & 1.143 & 5 & 8 \\
\hline & 0.998 & 6 & $<4$ \\
\hline \multirow[t]{10}{*}{$\mathrm{BrDI}^{-}$} & 1.975 & 0 & 41 \\
\hline & 1.751 & 1 & 38 \\
\hline & 1.539 & 2 & 30 \\
\hline & 1.346 & 4 & 10 \\
\hline & 1.262 & 5 & $<4$ \\
\hline & 1.242 & 5 & $<4$ \\
\hline & 1.227 & 5 & $<4$ \\
\hline & 1.184 & 6 & $<4$ \\
\hline & 1.171 & 6 & $<4$ \\
\hline & 1.017 & 8 & $<4$ \\
\hline
\end{tabular}

a) Entire simulation has been shifted to higher electron energy by $0.062(0.058)$ $\mathrm{eV}$ for $\mathrm{BrHI}^{-}\left(\mathrm{BrDI}^{-}\right)$to line up with experimental bands. 
Figure Captions for Chapter 5

Figure 5-1. The photoelectron spectra of $\mathrm{BrHI}^{-}$and $\mathrm{BrDI}^{-}$recorded at $213 \mathrm{~nm}$. Arrows at 2.07, 1.36 and $1.12 \mathrm{eV}$ represent asymptotes for dissociation into $\mathrm{I}\left({ }^{2} \mathrm{P}_{3 / 2}\right)+\mathrm{HBr}(\mathrm{v}=0), \mathrm{Br}\left({ }^{2} \mathrm{P}_{3 / 2}\right)+\mathrm{HI}(\mathrm{v}=0)$ and $\mathrm{I}\left({ }^{2} \mathrm{P}_{1 / 2}\right)+\mathrm{HBr}$ $(v=0)$ respectively.

Figure 5-2. The photoelectron spectrum of $\mathrm{BrHI}^{-}$recorded at $266 \mathrm{~nm}$.

Figure 5-3. The photoelectron spectra of $\mathrm{ClHI}^{-}$and $\mathrm{ClDI}^{-}$recorded at $213 \mathrm{~nm}$.

Figure 5-4. The photoelectron spectra of $\mathrm{FHI}^{-}$and FDI- recorded at $213 \mathrm{~nm}$.

Figure 5-5. The photoelectron spectrum of $\mathrm{FDI}^{-}$recorded at $266 \mathrm{~nm}$.

Figure 5-6. The effective collinear LEPS surface for the $\mathrm{Br}+\mathrm{HI}$ reaction, derived from that of Broida and Persky, plotted in mass-weighted coordinates defined by:

$$
\begin{gathered}
\mathrm{x}=\left(\mu_{\mathrm{l}, \mathrm{HBr}} / \mu_{\mathrm{HBr}}\right)^{1 / 2}\left(\mathrm{R}_{\mathrm{I}}-\mathrm{R}_{\mathrm{cm}}(\mathrm{HBr})\right) \approx\left(\mu_{\mathrm{l}, \mathrm{HBr}} / \mu_{\mathrm{HBr}}\right)^{1 / 2} \mathrm{R}_{\mathrm{I}-\mathrm{Br}}-7.0 \mathrm{R}_{\mathrm{I}-\mathrm{Br}} \\
y=\mathrm{R}_{\mathrm{H}-\mathrm{Br}}
\end{gathered}
$$

Here $R_{1}$ and $R_{\mathrm{cm}}(\mathrm{HBr})$ are the position of the $I$ atom and the $\mathrm{HBr}$ center-of-mass respectively, and $\mu_{A B}$ signifies the reduced mass of system A-B. The skew angle $\theta$ is given by

$$
\tan \theta=\left(m_{H} M / m_{B} m_{1}\right)^{1 / 2}
$$

which for this system is $8.2^{\circ}$, noting that $M=m_{1}+m_{H}+m_{B r}$. Contours are plotted at $0.161,0.461,0.761,1.061,1.361,1.661$ and $1.961 \mathrm{eV}$ with respect to the $\mathrm{I}+\mathrm{HBr}$ asymptote. The assumed anion equilibrium geometry is marked by the intersection of the dashed vertical and 
horizontal lines, at $x_{e}=27.11$ and $y_{e}=1.55 \AA$, and the saddle point is marked with $\ddagger$.

Figure 5-7. Potential along hydrogen stretching coordinate $\left(v_{3}\right)$ in $\mathrm{BrHI}^{-}$anion: expected form (solid) based on ab initio potential for $\mathrm{FHCl}^{-}$(ref. 25) and the Morse potential (dashed) used to model this. The lowest vibrational eigenstate of the model potential is also shown. Morse parameters are $D_{e}=0.283 \mathrm{eV}$ and $\beta=3.243 \AA^{-1}$

Figure 5-8. Anion and neutral $v_{3}$ potentials used in the one-dimensional analysis of $\mathrm{BrHI}^{-}$spectrum. Calculated eigenstates are labelled by their $\mathrm{v}_{3}$ quantum number. Each tick mark on vertical axis represents $0.2 \mathrm{eV}$.

Figure 5-9. Franck-Condon stick spectra for (top) $\mathrm{BrHI}^{-}$and (bottom) $\mathrm{BrDI}^{-}$, simulated in one-dimensional time-independent analysis. Simulations superimposed on the respective experimental spectra (dashed). Simulated sticks are labelled by $v_{3}$ quantum numbers and for the $\mathrm{BrHI}^{-}$spectrum correspond to those snown on Figure 5-8.

Figure 5-10. Correlation diagram for the reaction $\mathrm{Br}+\mathrm{HI}$, assuming $\mathrm{C}_{w}$ symmetry. The relative spacing of asymptotic levels are approximately to scale. The region between the dotted lines is where Hund's case (a) is appropriate. Adapted from the correlation diagram in Ref. 12.

Figure 5-11. Absolute value of autocorrelation function, from one-dimensional propagation, for $\mathrm{BrHI}^{-}$(top) and the simulated photoelectron spectrum which results from the Fourier transform of this autocorrelation function (bottom). Propagation carried out for 16384 time steps, with 
$\Delta t=1.0$ a.u., and a 64 point spatial grid along $y\left(=R_{H \cdot B r}\right)$ between 0.79 and $3.4 \AA$.

Figure 5-12. Absolute value of autocorrelation function, from two-dimensional propagation, for (a) $\mathrm{BrHI}^{-}$and (b) $\mathrm{BrDI}^{-}$. Calculation parameters given with Figure 5-13.

Figure 5-13. Wave packet dynamics for the $[\mathrm{BrHI}]$ system. Equally spaced contours of $|\phi(t)|$ are shown at times indicated on each frame; the highest value contours are omitted for clarity. Also shown are contours of the effective collinear potential energy surface, shown in Figure 5-6. Figure plotted in mass scaled coordinates (see text and Figure 5-6); the $y$ axis has been expanded here. Propagation performed over 10240 time steps, with $\Delta t=1.3$ a.u., and a spatial grid with $128 \times 64$ points along $x$ and y respectively. The final wave packet shown ( $966.4 \mathrm{fs}$ ) was calculated in a separate propagation, using same grid and $\Delta t$, but with an absorbing function applied at grid boundaries (see text and Ref. 53). In this plot the contouring resolution has been increased.

Figure 5-14. Simulated photoelectron spectrum (solid) for (a) $\mathrm{BrHI}^{-}$and (b) $\mathrm{BrDI}^{-}$ resulting from two-dimensional calculation. The simulations have been shifted so $0-0$ bands line up (see text) and convoluted with the experimental resolution function. The experimental spectra are also shown (dashed).

Figure 5-15. Simulated photoelectron spectrum for $\mathrm{BrHI}^{-}$prepared in the $(0,0,1)$ state. Simulation has been convoluted with (top) our spectrometer's experimental resolution function and (bottom) with a constant energy 
resolution of $4.3 \mathrm{meV}$. Bands are labelled by their effective $v_{3}$ quantum numivers. Numerical parameters for the simulation are $\Delta t=1.3$ a.u., $t_{\max }=960$ fs, spatial grid $64 \times 32$ points over range $x=22-35 \AA$ and $y=0.95-3.1 \AA$. 

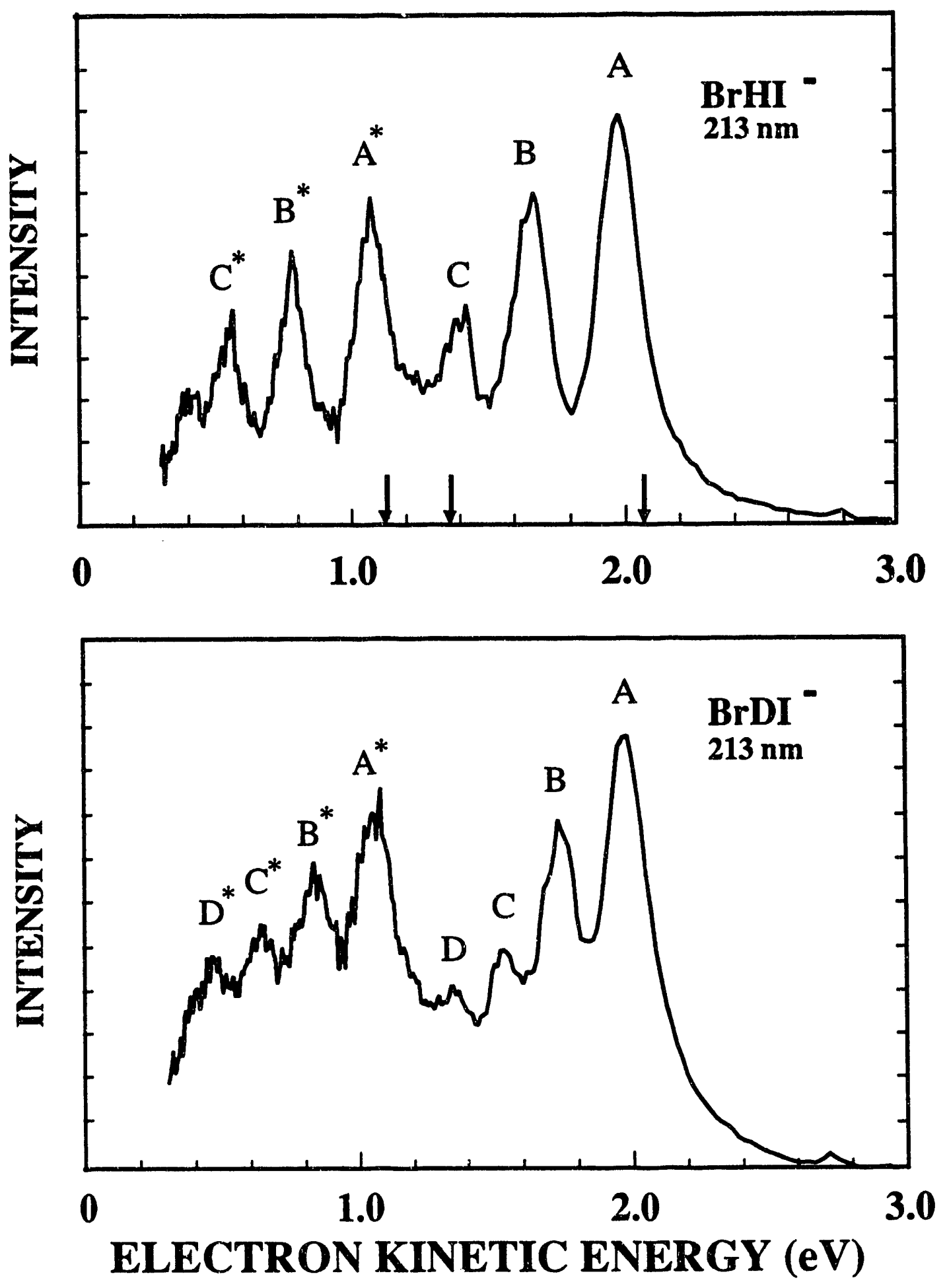

Figure 5-1 


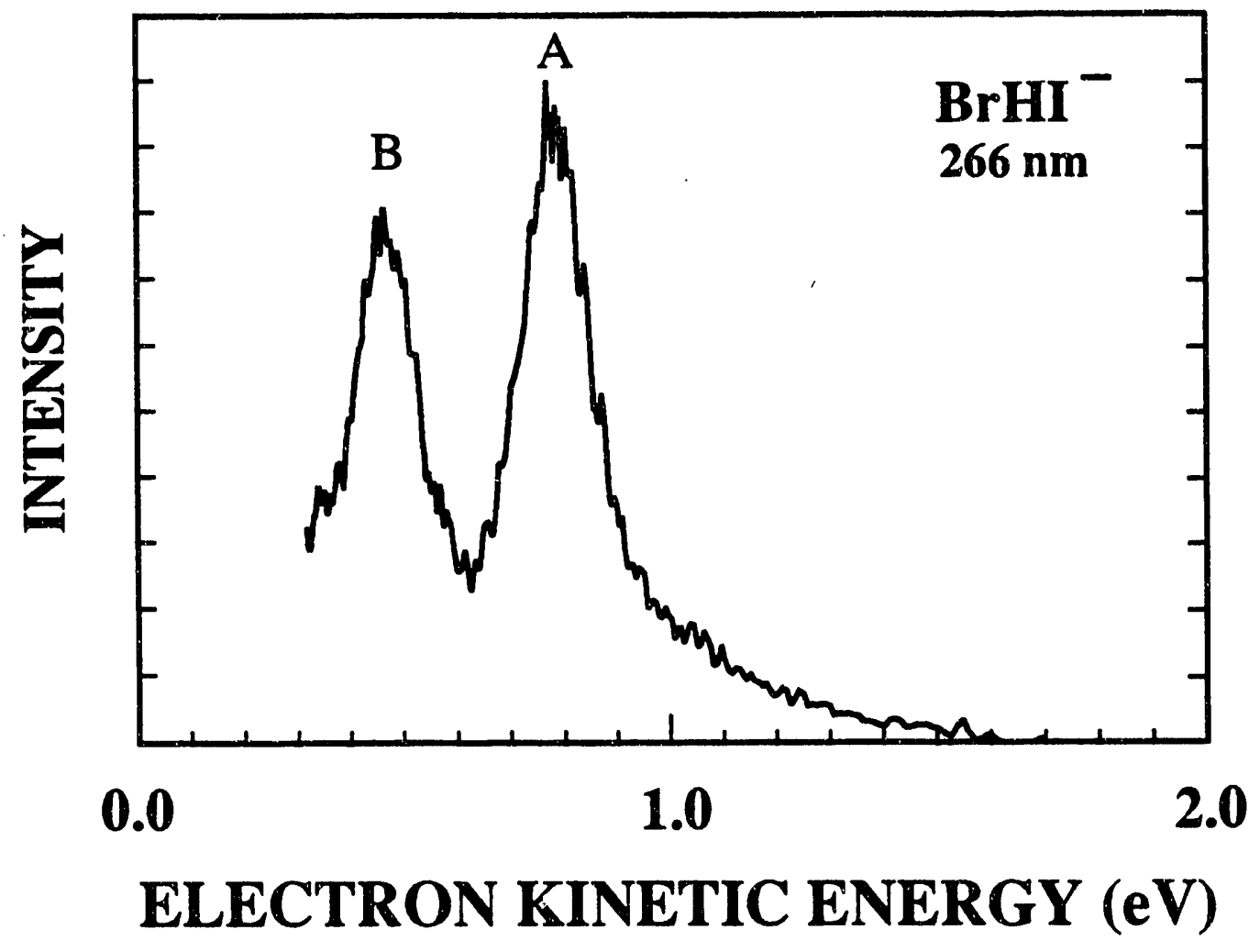

Figure 5-2 

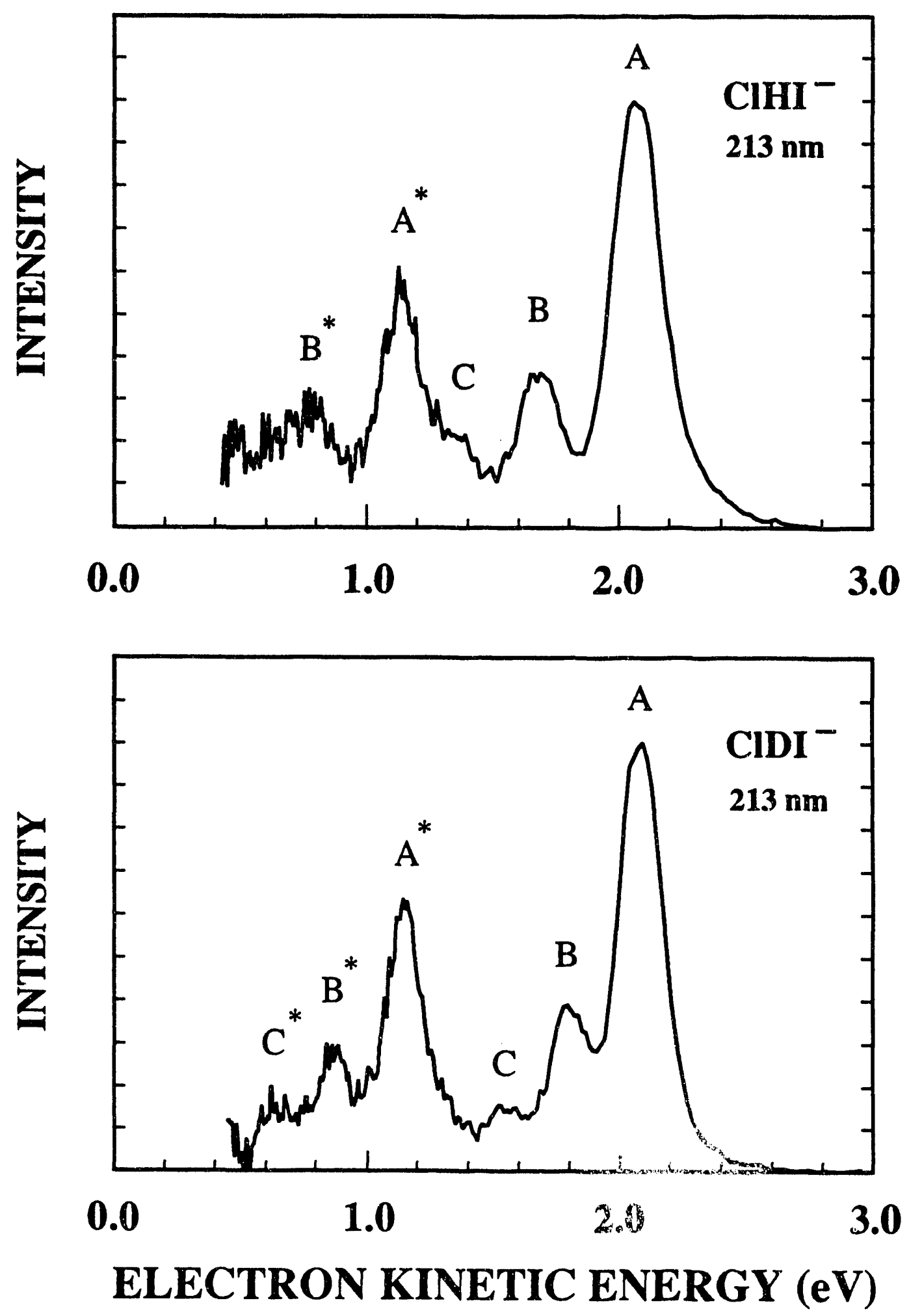

Figure 5-3 

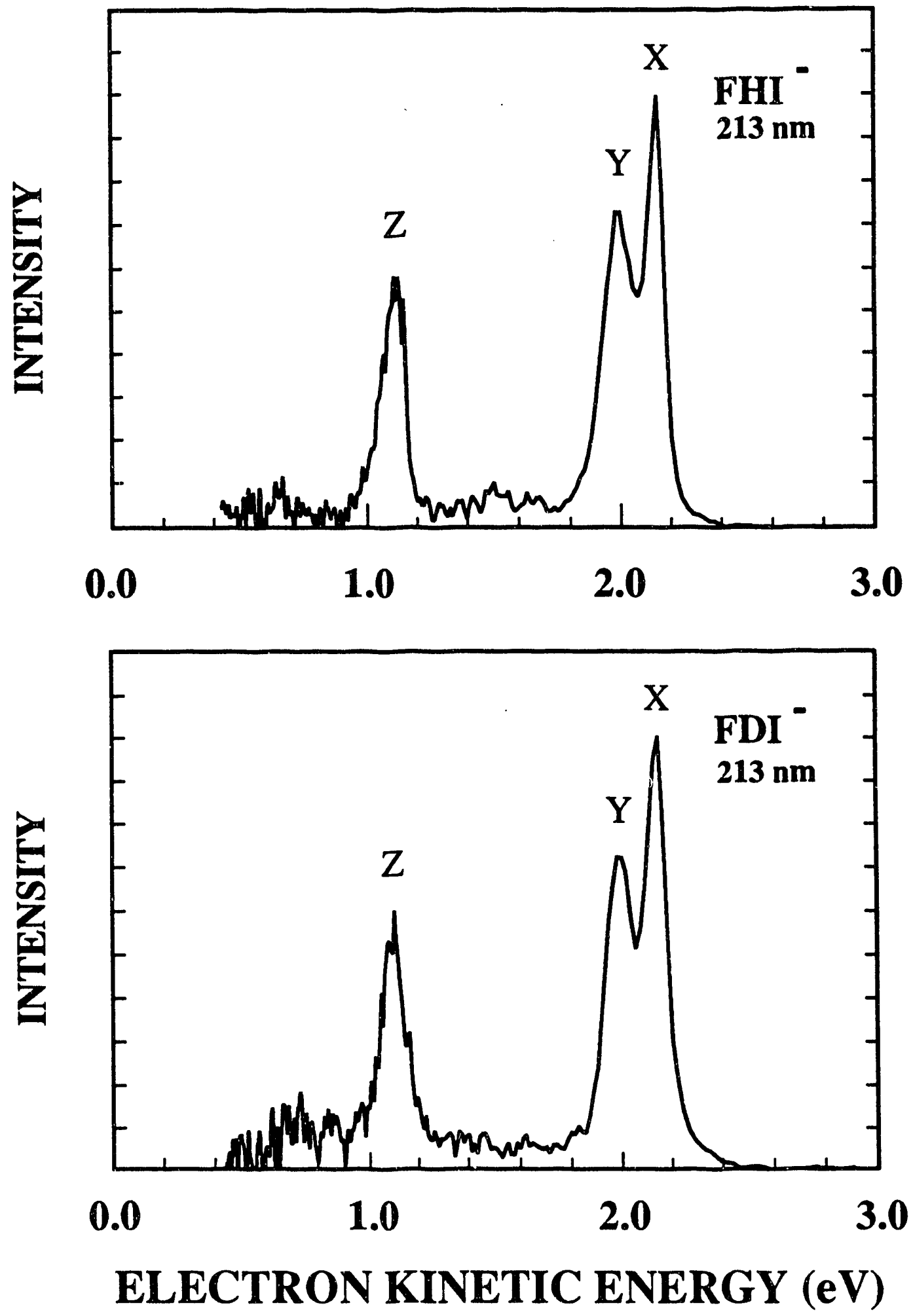

Figure 5-4 


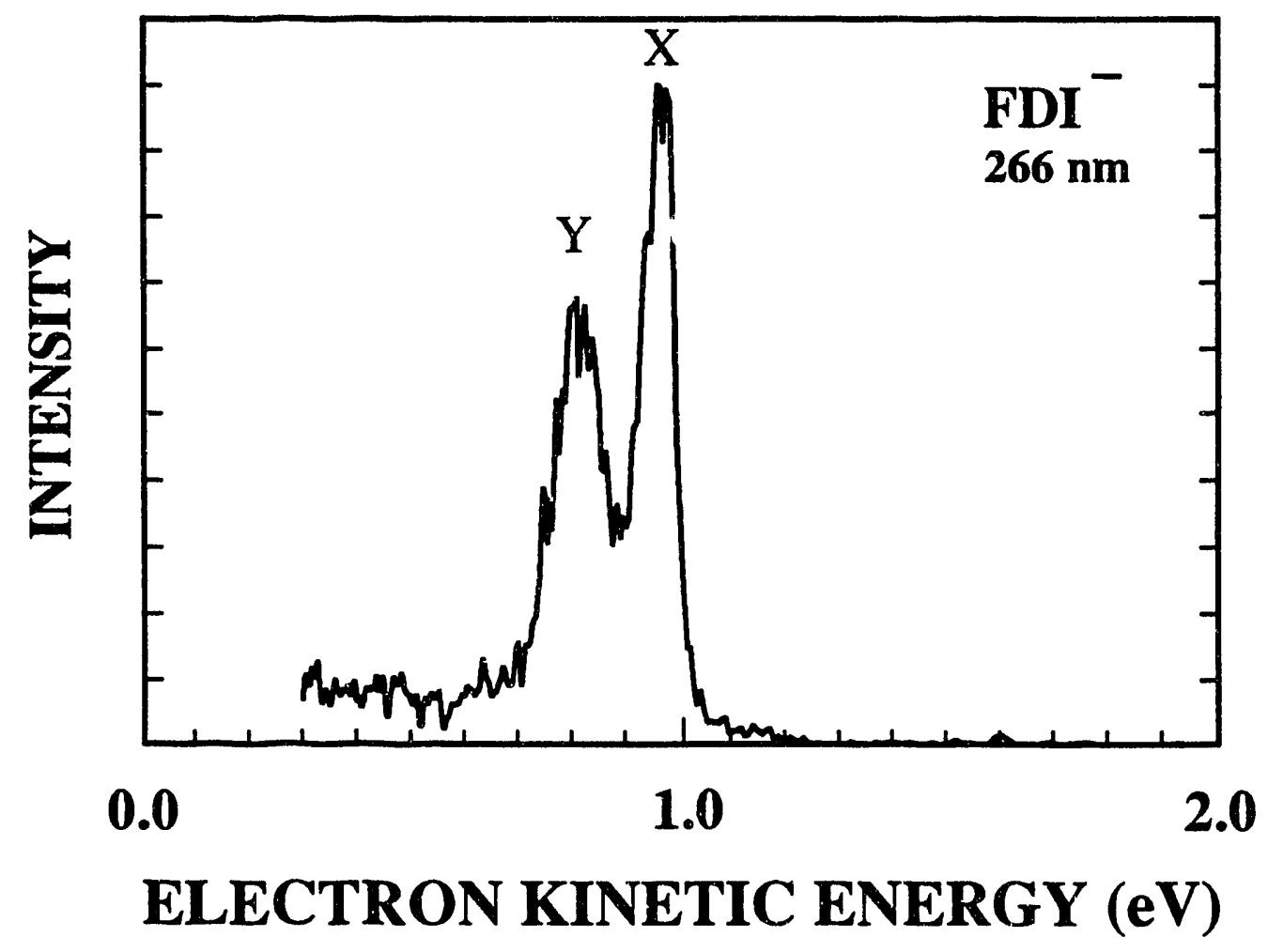

Figure 5-5 


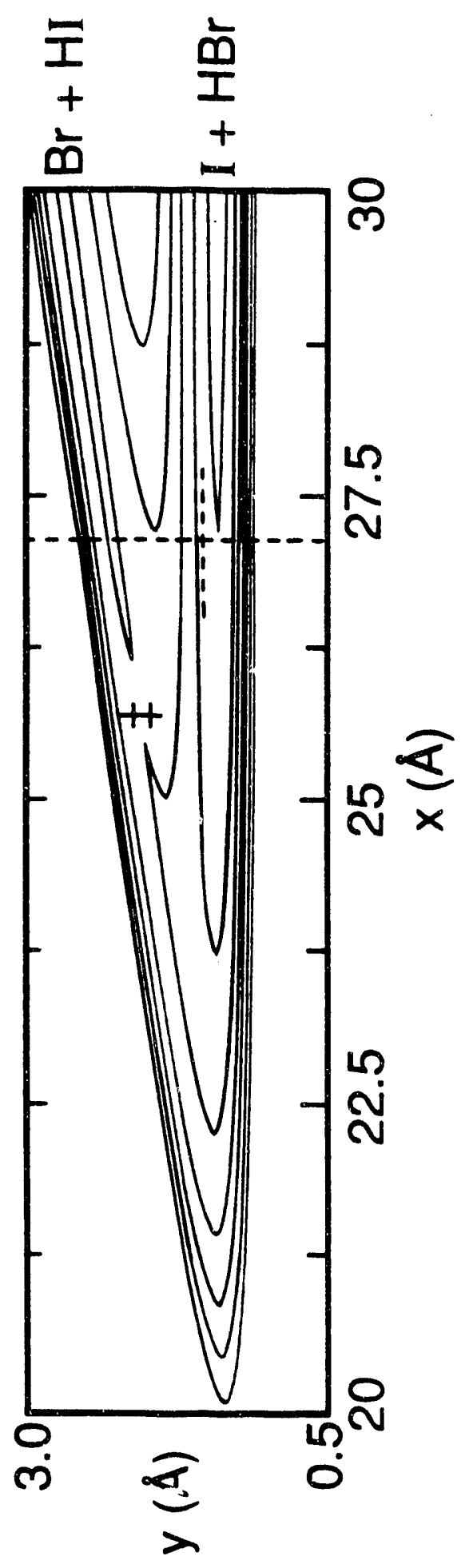

Figure 5-6 


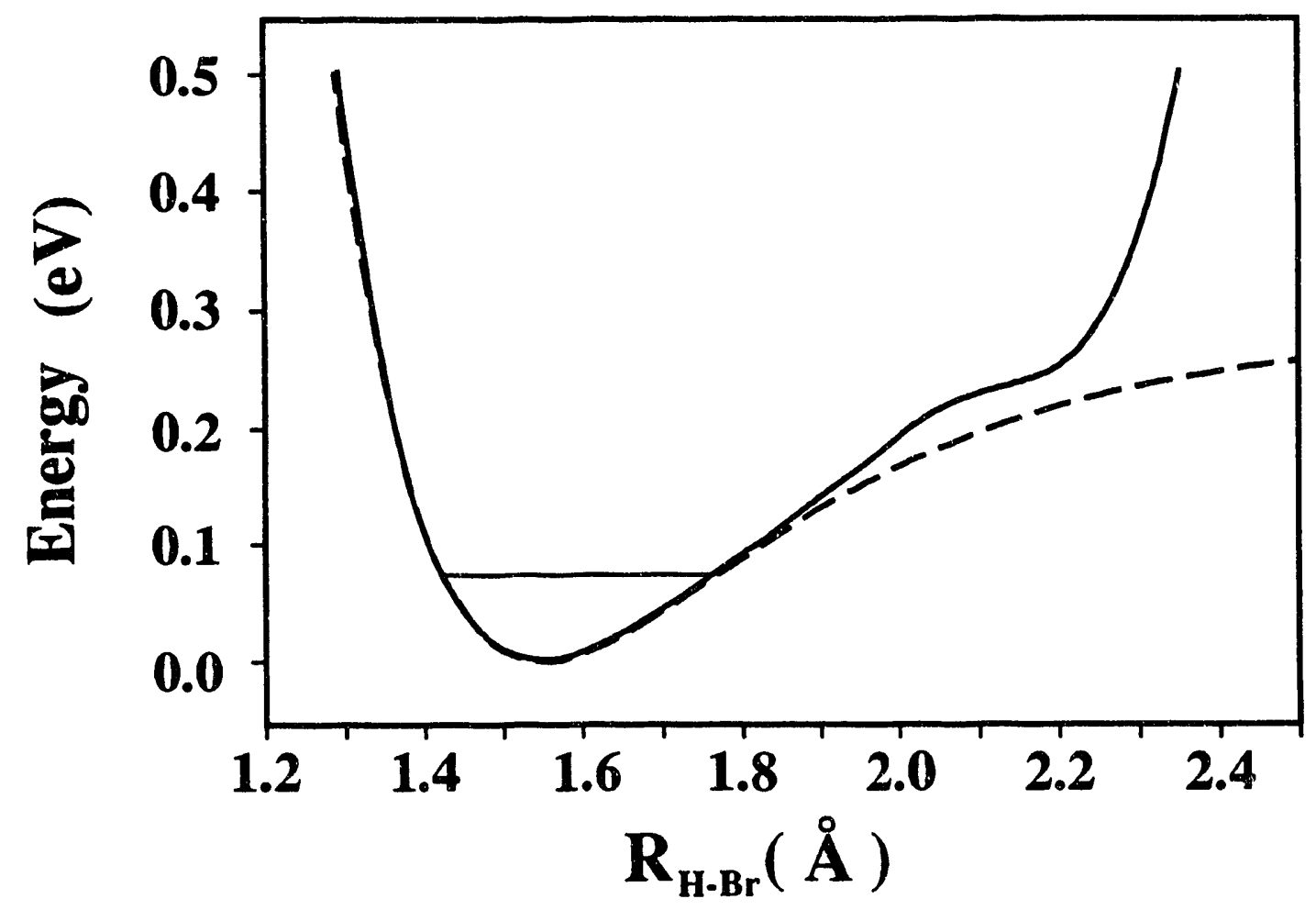

Figure 5-7 


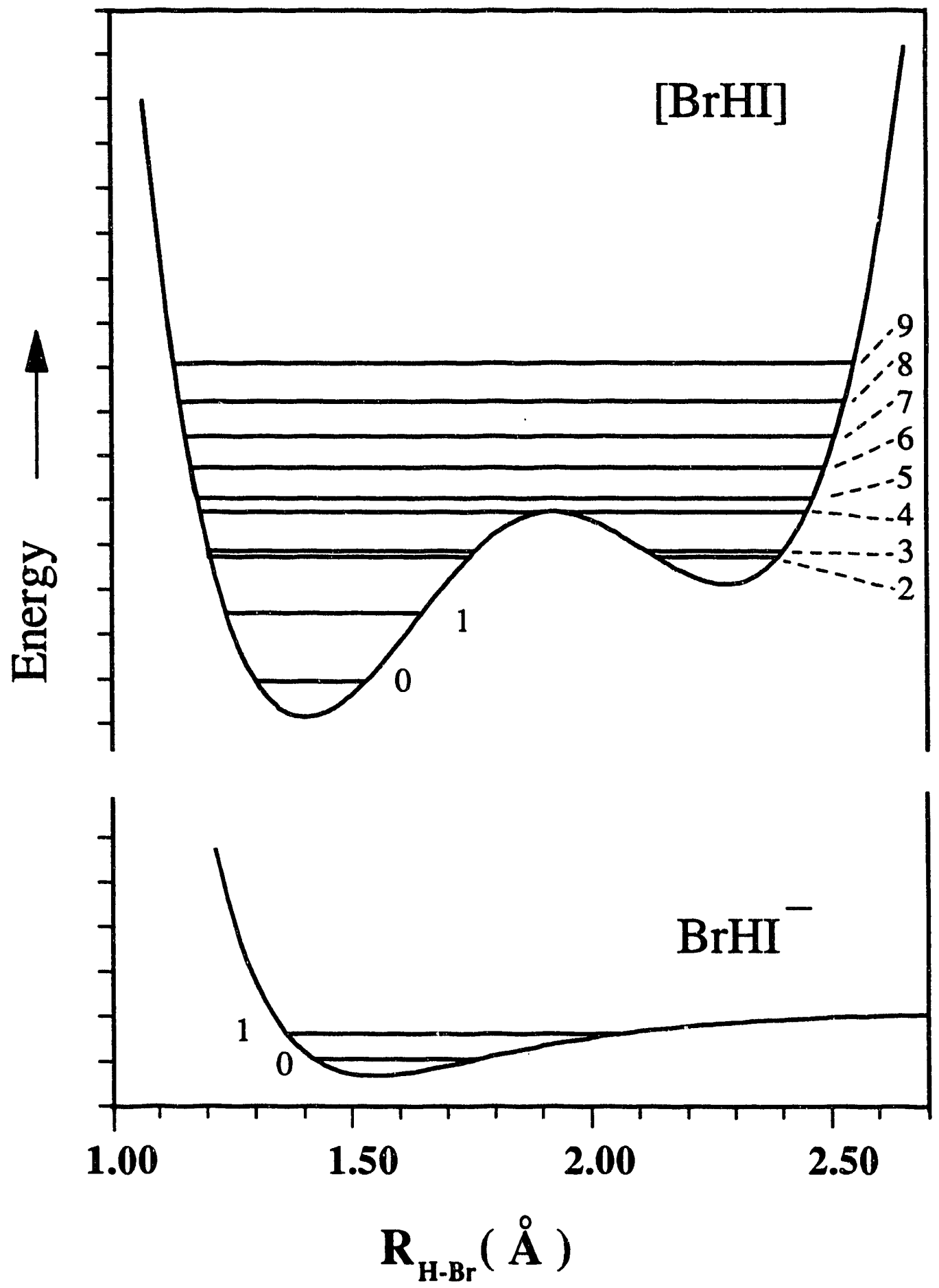

Figure 5-8 

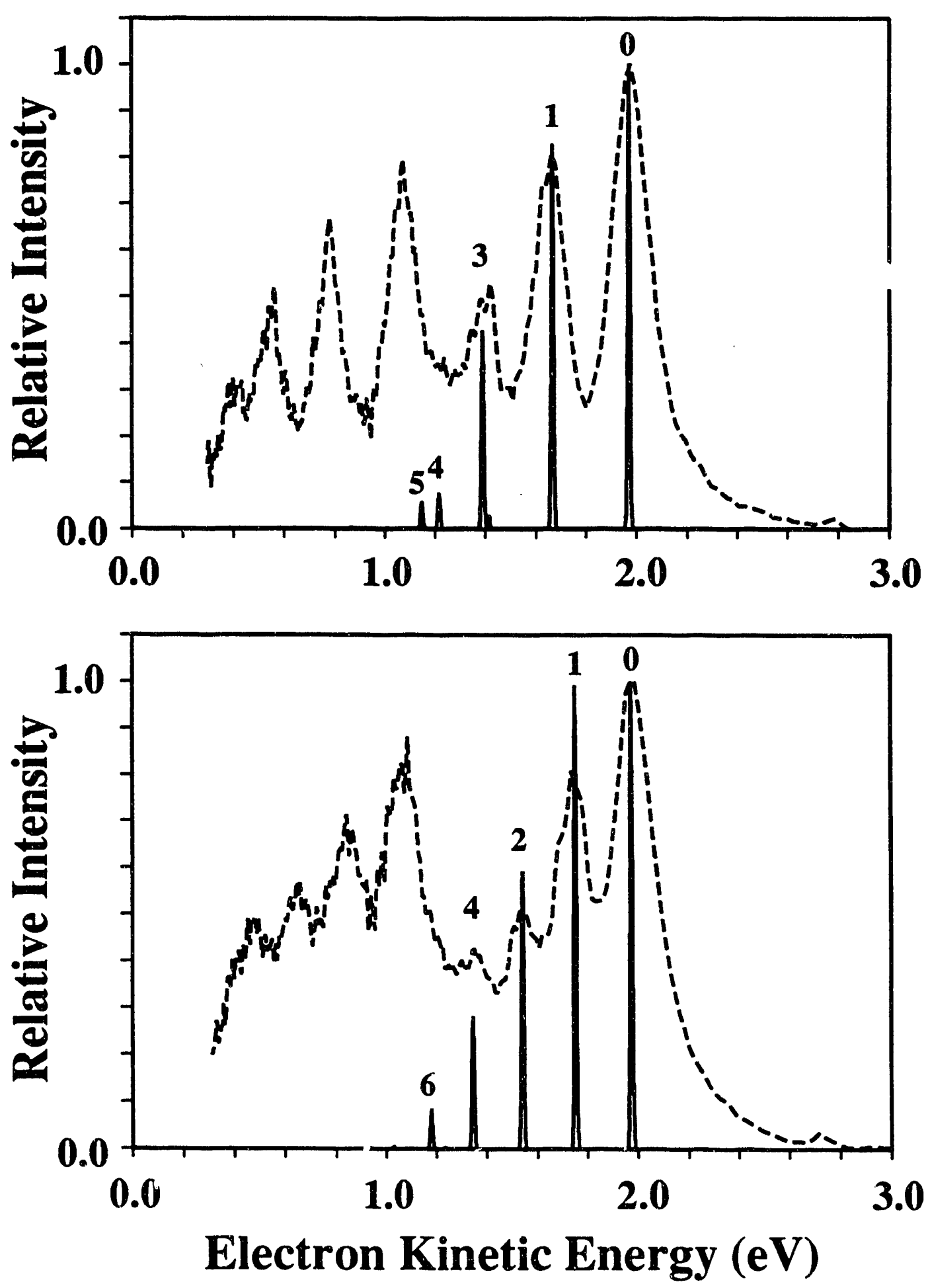

Figure 5-9 


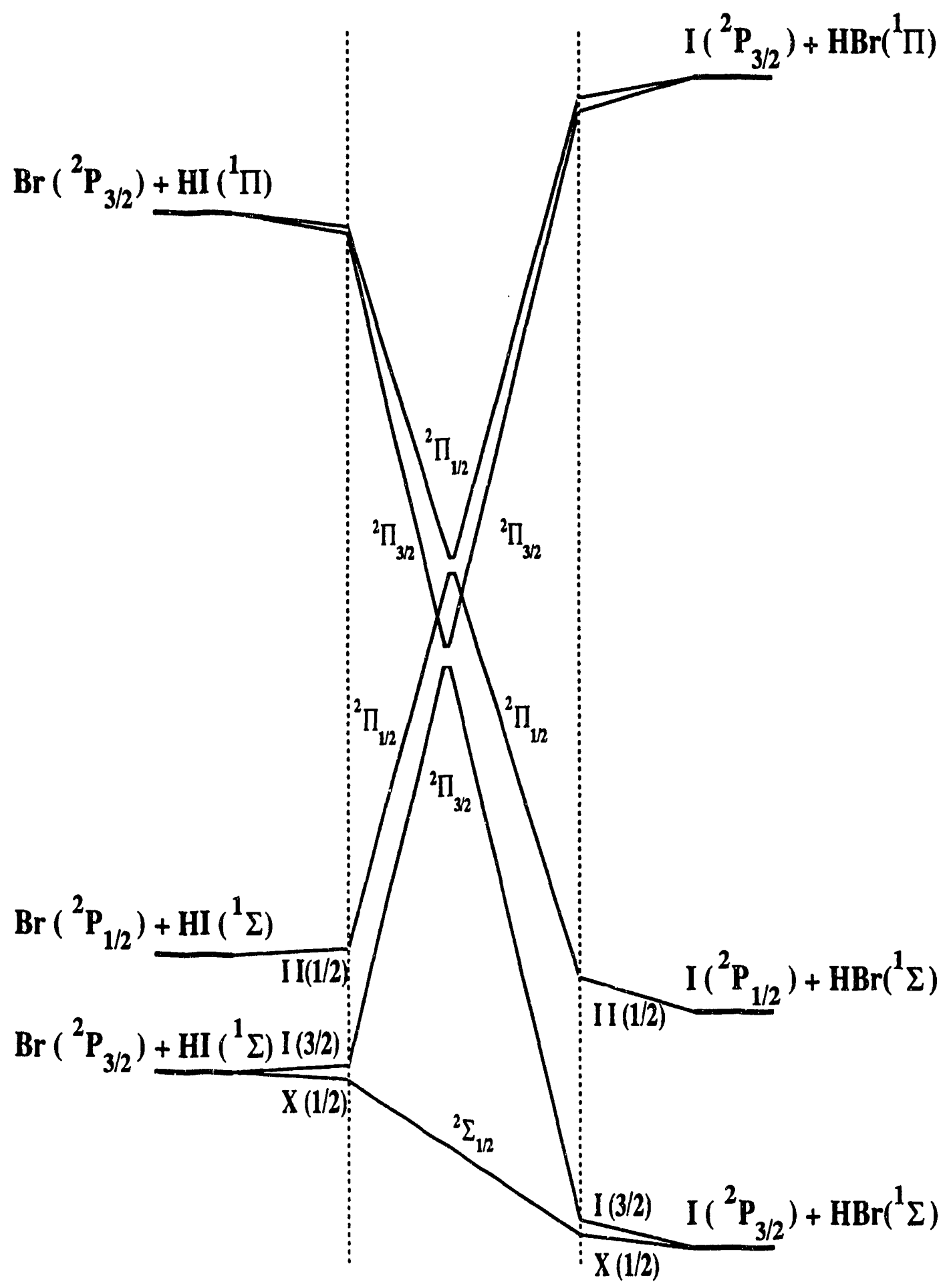

Figure 5-10 

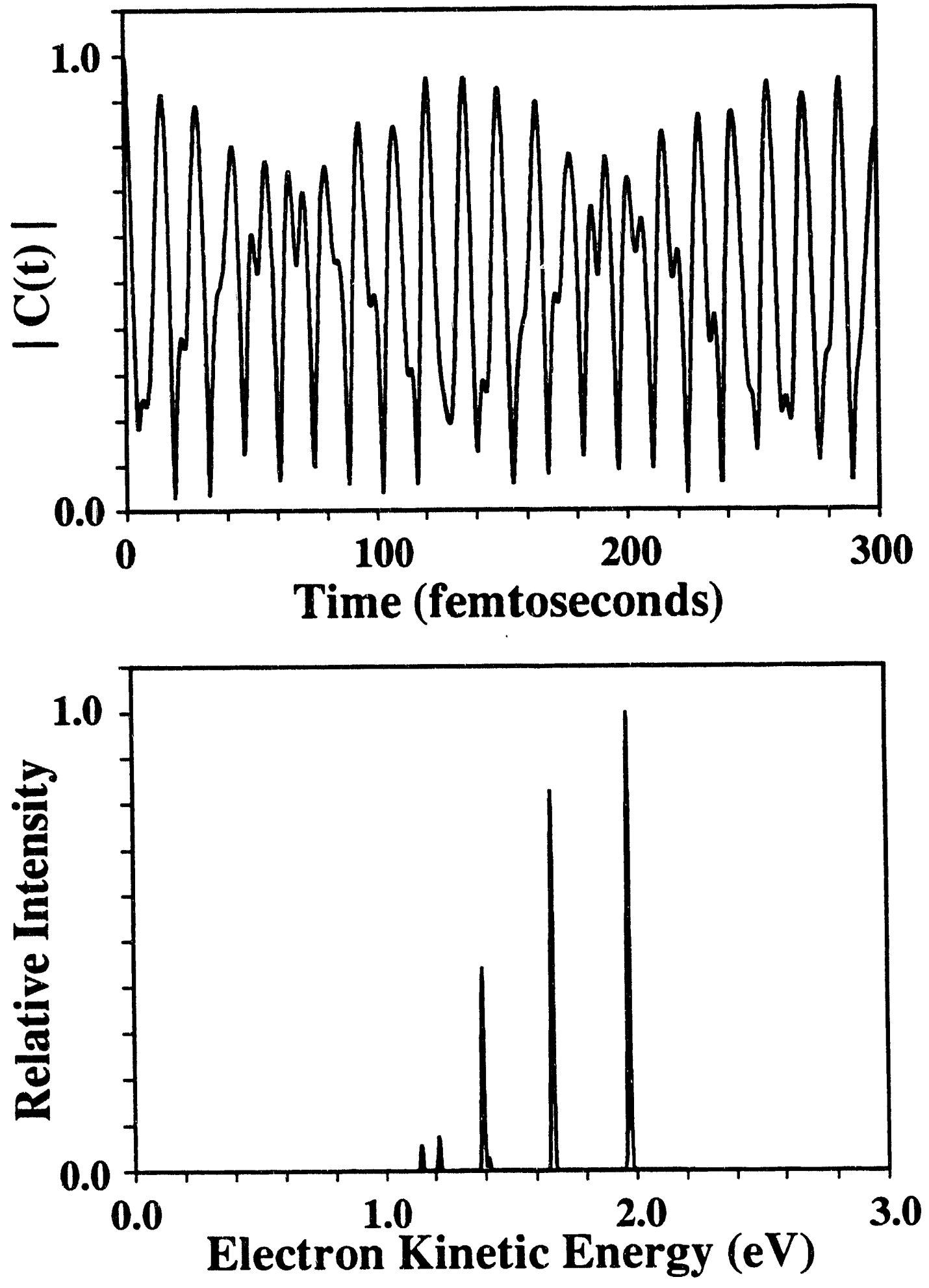

Figure 5-11 

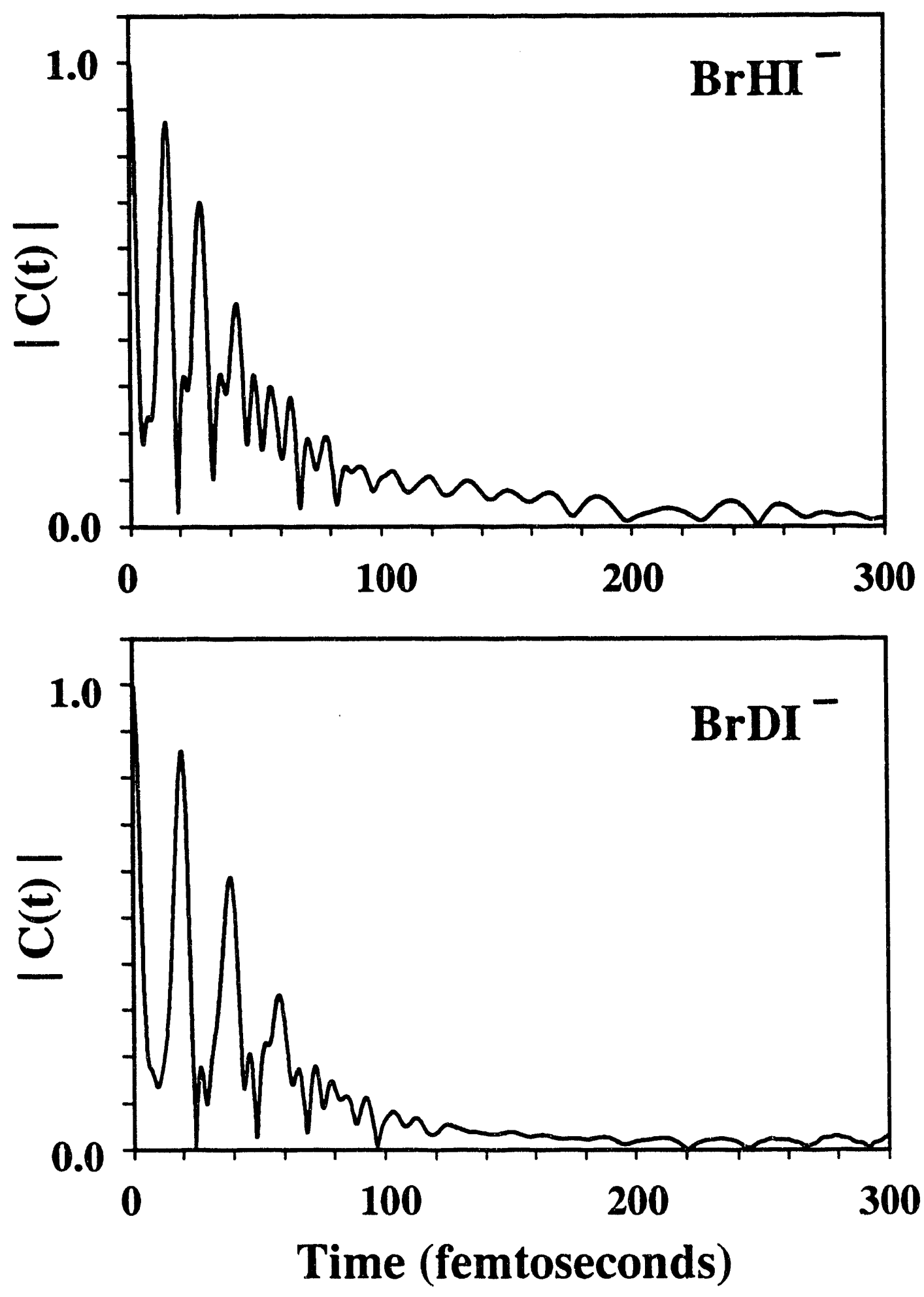

Figure 5-12 

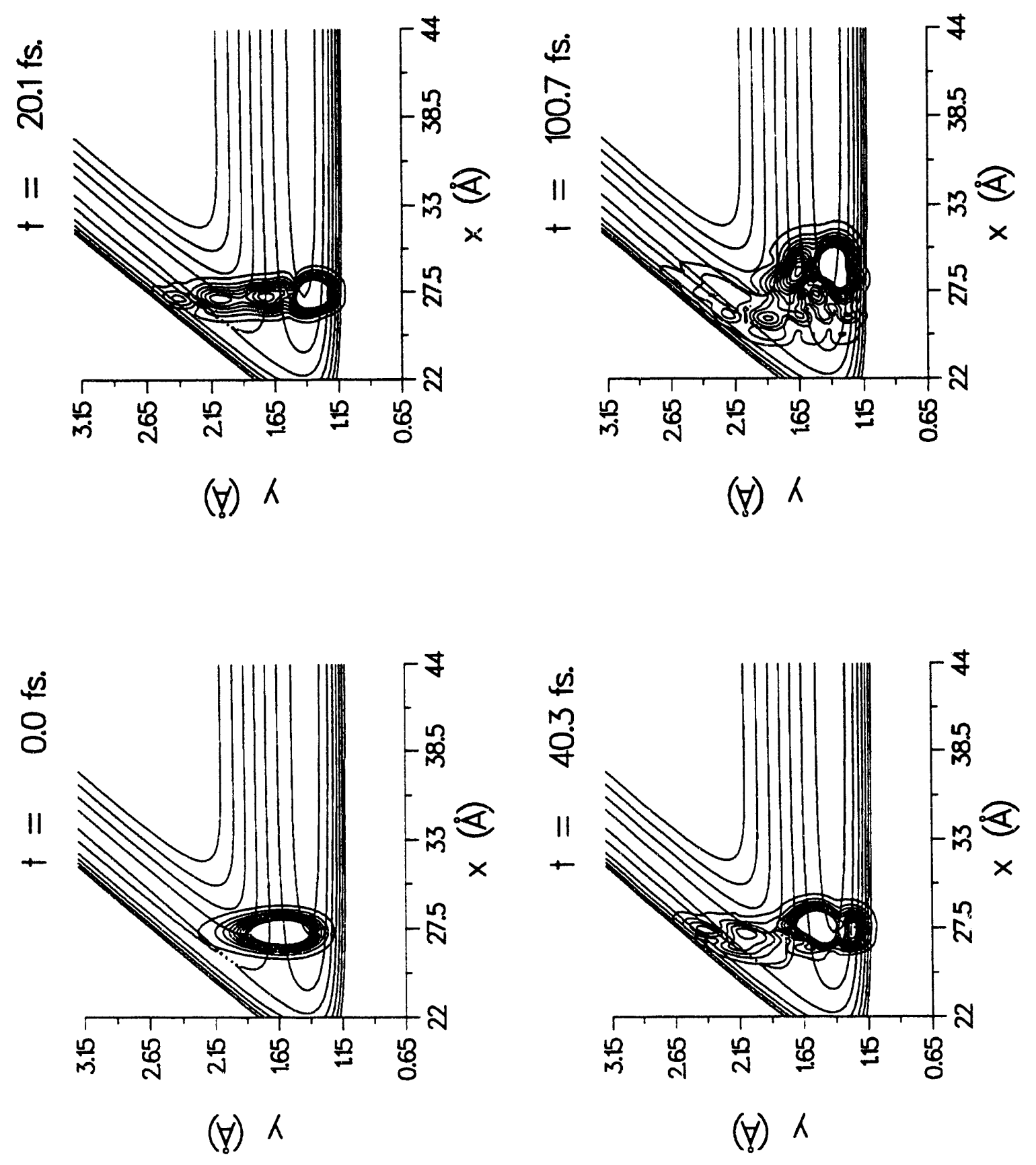

Figure 5-13 

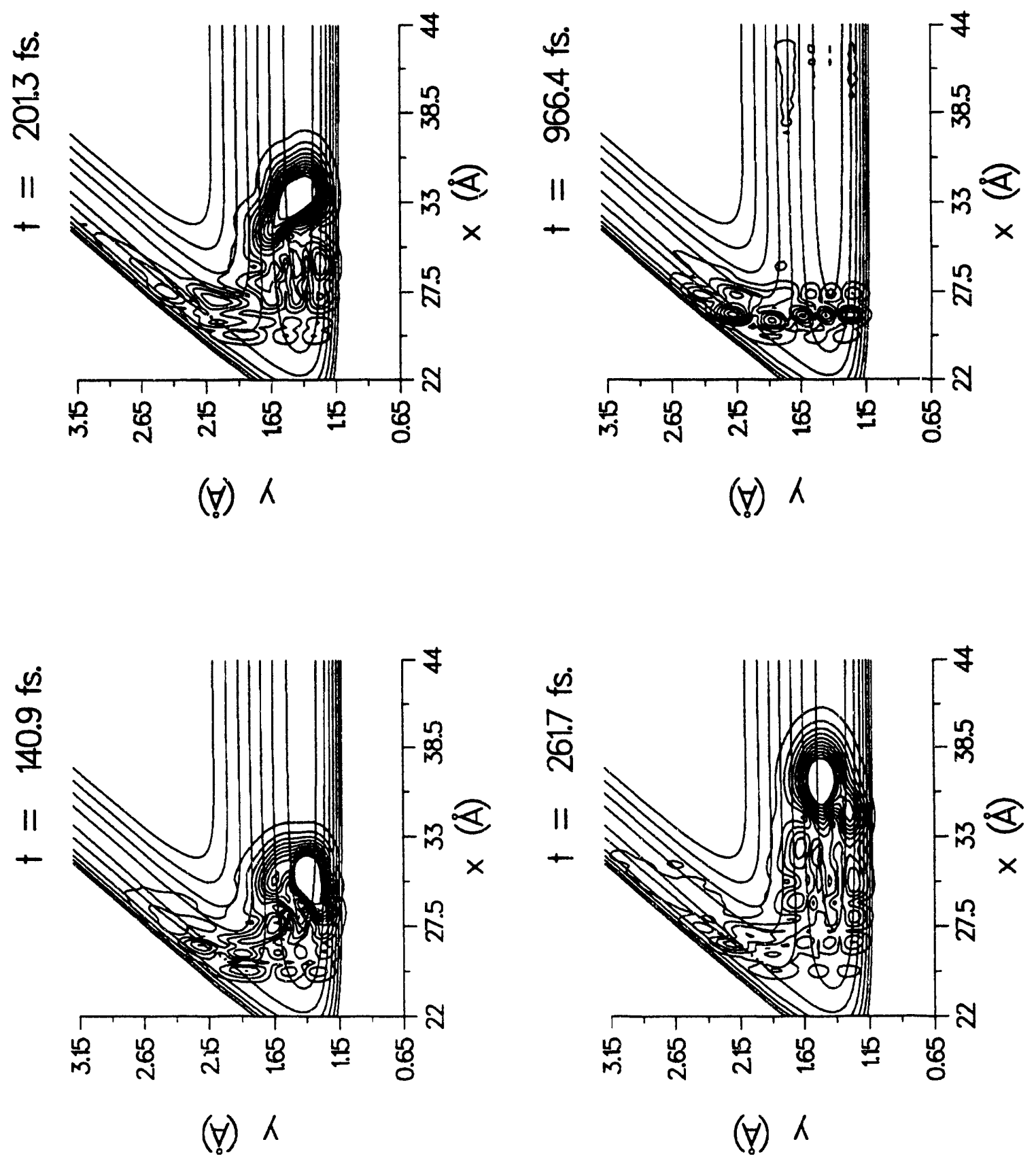

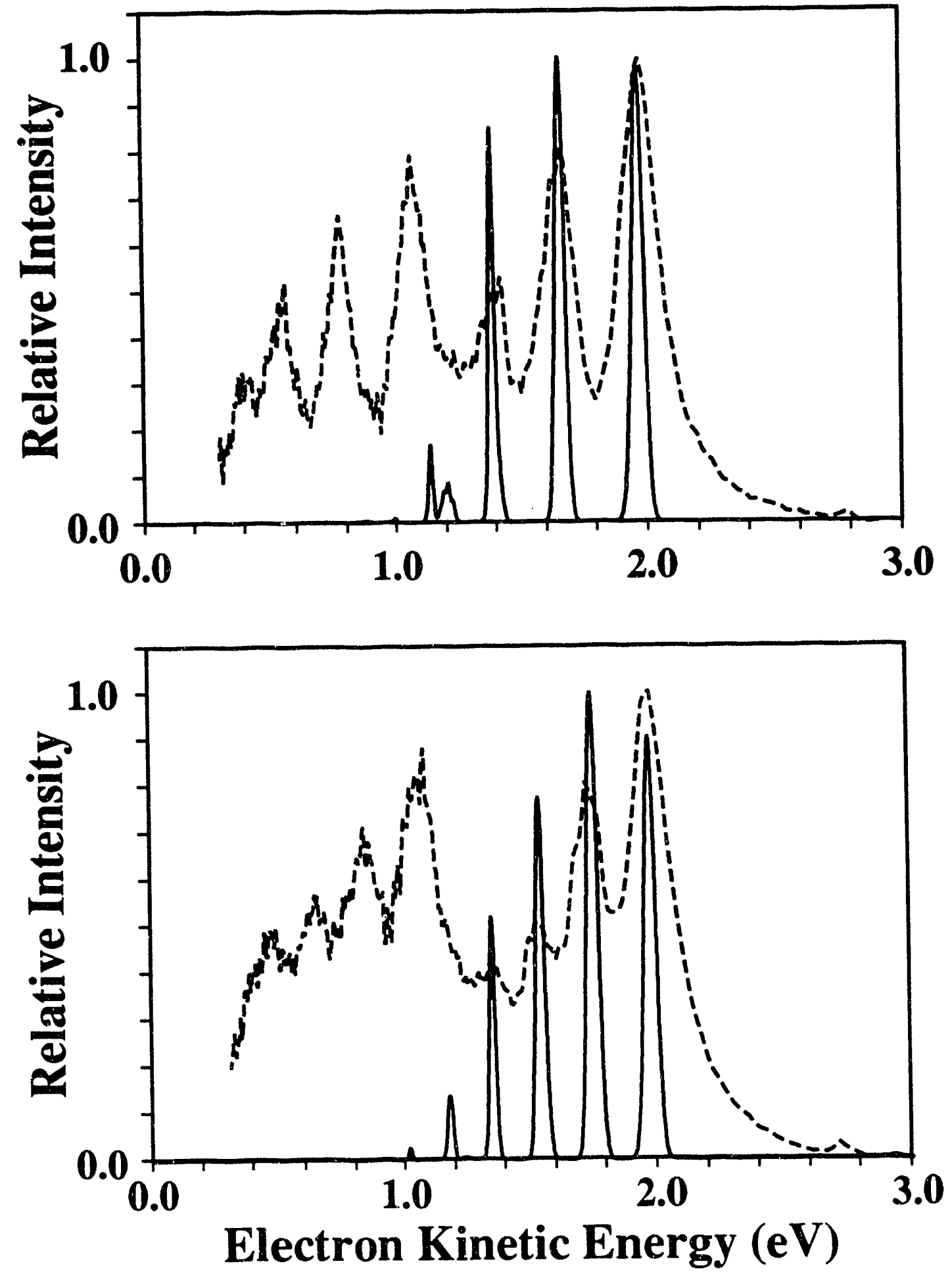

Figure 5-14 

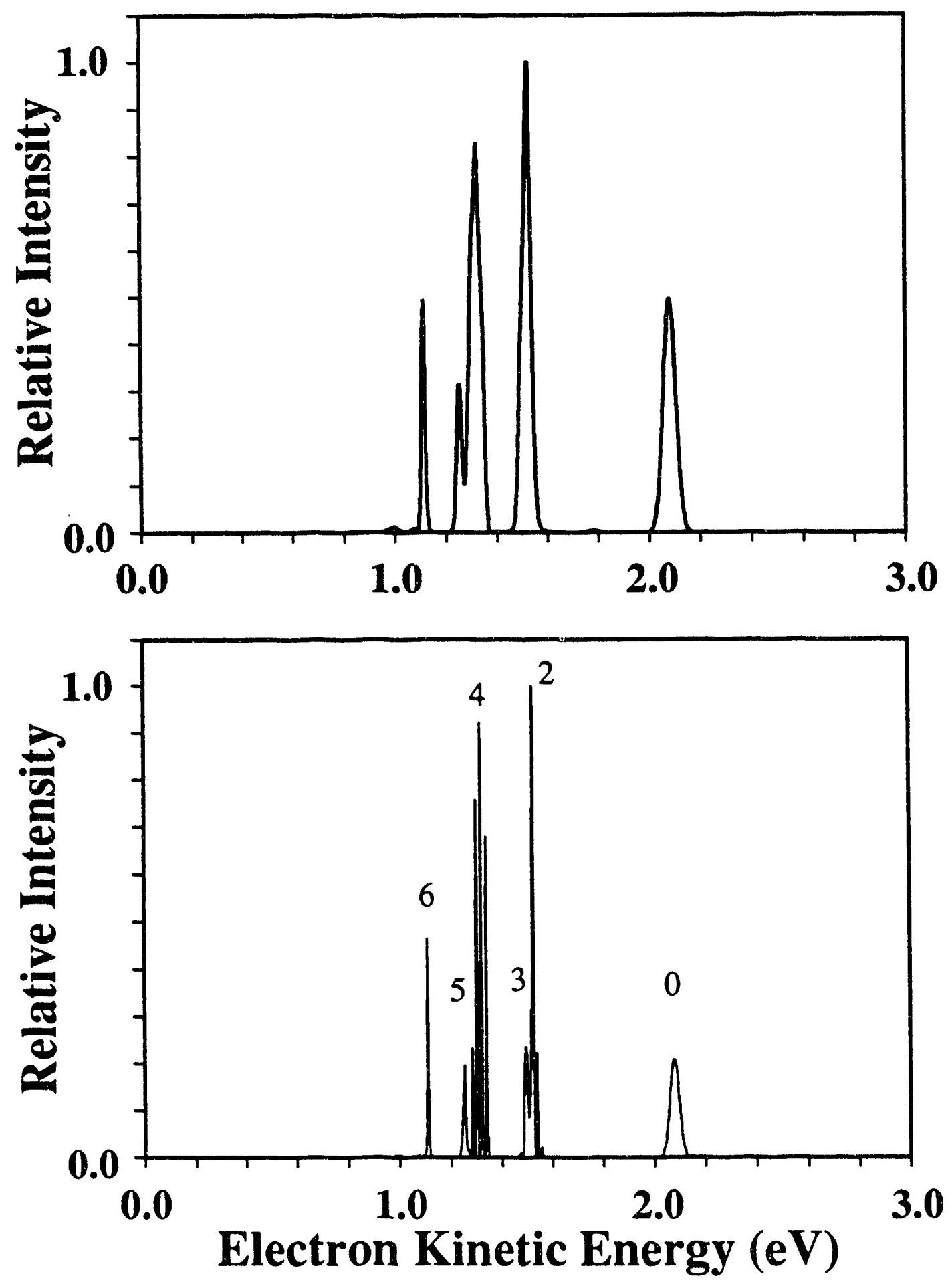

Figure 5-15 


\section{Postscript: new experimental results on BrHI-}

Since publication of this work, we have recorded new spectra of $\mathrm{BrHI}^{-}$in our laboratory. Our signal-to-noise has been much improved (see Chapter 2) and the background due to stray electrons has been significantly reduced in the meantime. Polarization studies have also been undertaken. Figure 5-16 shows our improved spectrum for $\mathrm{BrHI}^{-}$photodetachment at $213 \mathrm{~nm}$; the $\theta=90^{\circ}$ spectrum should be compared with Figure 5-1. Also shown is the $\mathrm{BrHI}^{-}$spectrum recorded with the laser polarization parallel to the electron collection direction, $\theta=0^{\circ}$. The peaks $A^{*}-C^{*}$, due to the excited ${ }^{2} \Pi_{1 / 2}$ state, have reduced relative intensity in the $\theta=0^{\circ}$ spectrum. This behavior confirms that transitions are occurring from the anion to different electronic states in the two observed progressions. In addition the new spectra indicate we have achieved more vibrational cooling in the free jet expansion; the tail to high electron kinetic energy (above $\mathrm{eKE}=2.1 \mathrm{eV}$ ) is less pronounced than in the earlier data (Figure 5-1).

Figure Caption for 5.7

Figure 5-16. $\mathrm{BrHI}^{-}$photoelectron spectrum recorded at $213 \mathrm{~nm}$. (top) $\theta=0^{\circ}$, and (bottom) $\theta=90^{\circ}$. 
BrHI' 213 nm
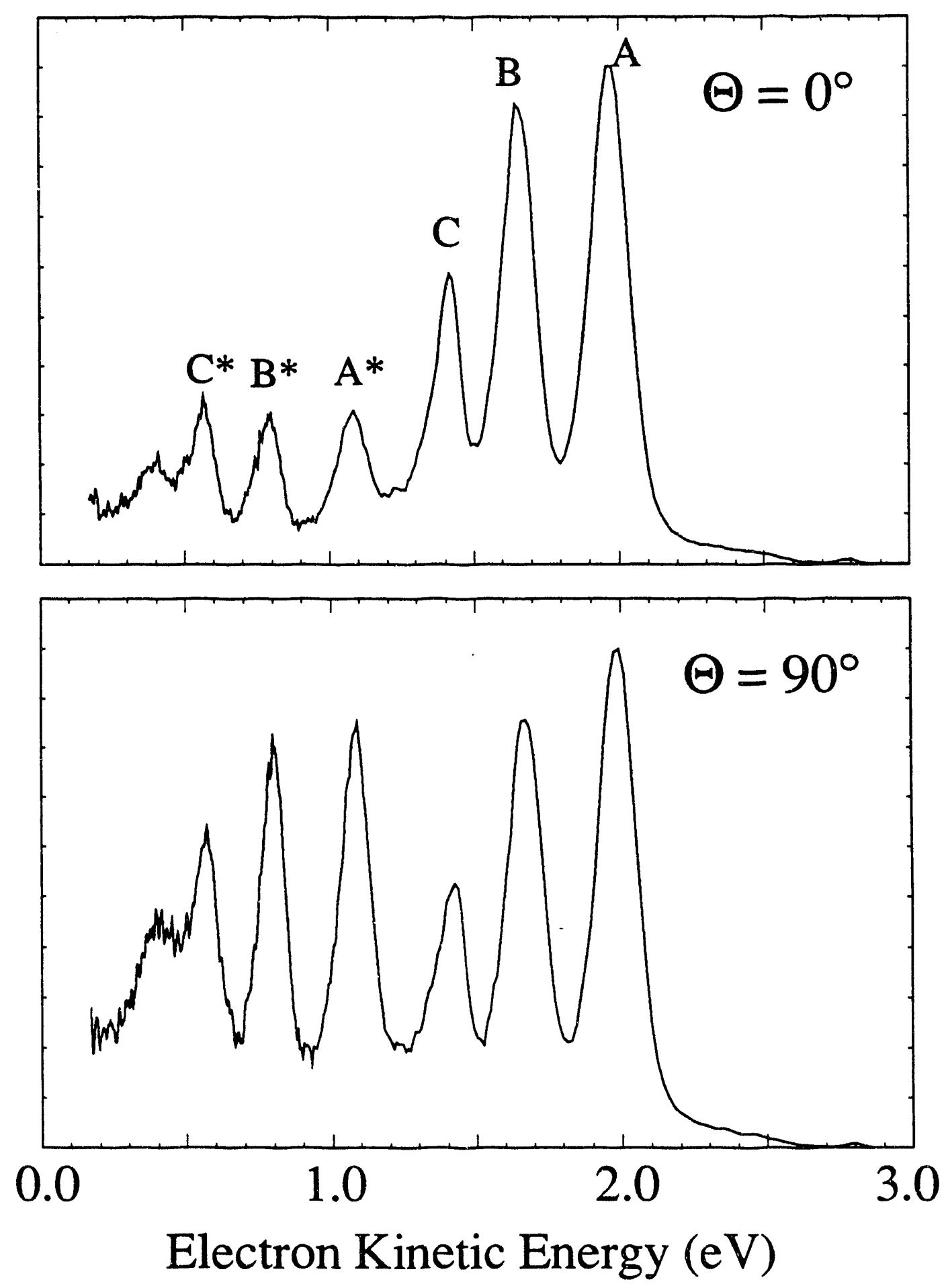

Figure 5.16 


\title{
Chapter 6. Spectroscopy of the Transition State: Hydrogen Abstraction Reactions of Fluorine*
}

\begin{abstract}
The reactions $\mathrm{F}+\mathrm{CH}_{3} \mathrm{OH} \rightarrow \mathrm{HF}+\mathrm{CH}_{3} \mathrm{O}, \mathrm{F}+\mathrm{C}_{2} \mathrm{H}_{5} \mathrm{OH} \rightarrow \mathrm{HF}+\mathrm{C}_{2} \mathrm{H}_{5} \mathrm{O}$, and $\mathrm{F}$ $+\mathrm{OH} \rightarrow \mathrm{HF}+\mathrm{O}\left({ }^{3} \mathrm{P},{ }^{1} \mathrm{D}\right)$ are studied by photoelectron spectroscopy of the negative ions $\mathrm{CH}_{3} \mathrm{OHF}^{-}, \mathrm{C}_{2} \mathrm{H}_{5} \mathrm{OHF}^{-}$, and $\mathrm{OHF}^{-}$. In each case, photodetachment accesses the transition state region for direct hydrogen abstraction. The photoelectron spectra exhibit resolved vibrational structure which is sensitive to details of the potential surface in the transition state region. To aid in the interpretation of the spectra, $a b$ initio equilibrium structures, harmonic frequencies, and hydrogen bond dissociation energies are calculated for the ions $\mathrm{CH}_{3} \mathrm{OHF}^{-}$and $\mathrm{OHF}^{-}$. The anharmonic hydroxyl hydrogen stretching potential is also calculated for the two ions. Using the calculated ion properties and the fitted $a b$ initio reaction surfaces of Sloan et al. (J. Chem. Phys. 1981, 75, 1190), a two-dimensional dynamical simulation of the photoelectron spectrum of $\mathrm{OHF}^{-}$is presented and modifications to the reaction surfaces are discussed. The spectra of the alcohol complexes are discussed in light of this simulation, and the role of the "bath" degrees of freedom in these spectra is considered.
\end{abstract}

- Published in J. Phys. Chem. 95, 8066 (1991) 


\section{Introduction}

During the last several years, considerable progress has been made towards the spectroscopic characterization of the transition state in simple chemical reactions. An array of frequency ${ }^{1}$ and time-resolved ${ }^{2}$ techniques has been developed which allow one to directly probe the transition state region of the potential energy surfaces describing these reactions. These experiments are aimed at achieving a better understanding of the microscopic forces which govern the dynamics of bimolecular and unimolecular reactions.

One of the more promising approaches to this problem has been to photodetach a stable negative ion in order to form an unstable neutral complex in the vicinity of the transition state for a chemical reaction. For example, in a study of a unimolecular transition state, Lineberger and co-workers ${ }^{3}$ used photoelectron spectroscopy of $\mathrm{C}_{2} \mathrm{H}_{2}^{-}$ to investigate the unstable vinylidene radical, which rapidly isomerizes to acetylene. In an experiment which serves as a precursor to the work described here, Brauman and co-workers ${ }^{4}$ examined total photodetachment cross sections in the visible and near ultraviolet $(\lambda>370 \mathrm{~nm}$ ) for several ions of the form ROHF-, thereby learning about the relationship of the ion geometry to the potential energy surface for the $\mathrm{F}+\mathrm{ROH} \rightarrow \mathrm{HF}+\mathrm{RO}$ reaction.

In our laboratory, negative ion photoelectron spectroscopy $\mathrm{y}^{5,6,7}$ and threshold photodetachment ${ }^{8}$ are used to study the transition state region of bimolecular hydrogen exchange reactions $\mathrm{A}+\mathrm{HB} \rightarrow \mathrm{HA}+\mathrm{B}$. By photodetaching the stable, hydrogen-bonded $\mathrm{AHB}^{-}$anion, one can form an unstable [AHB] complex located near the transition state for the bimolecular reaction. Most of our work to date has been on triatomic systems where $A$ and $B$ are like $e^{5,6,8}$ or unlike ${ }^{7}$ halogen atoms. The 
photoelectron spectra of the AHB-bihalide anions yield resolved vibrational structure characteristic of the unstable [AHB] complex. This structure is very sensitive to the nature of the $A+H B$ potential energy surface near the transition state.

In this paper, the extension of our method to reactions involving polyatomic reactants is described. We report studies ${ }^{9}$ of the reactions $\mathrm{F}+\mathrm{CH}_{3} \mathrm{OH} \rightarrow \mathrm{HF}+\mathrm{CH}_{3} \mathrm{O}$ and $\mathrm{F}+\mathrm{C}_{2} \mathrm{H}_{5} \mathrm{OH} \rightarrow \mathrm{HF}+\mathrm{C}_{2} \mathrm{H}_{5} \mathrm{O}$ via photoelectron spectroscopy of $\mathrm{CH}_{3} \mathrm{OHF}^{-}$and $\mathrm{C}_{2} \mathrm{H}_{5} \mathrm{OHF}^{-}$. In both cases, the photoelectron spectra reveal resolved vibrational features attributable to the $[R O H F]$ transition state region $\left(\mathrm{R}=\mathrm{CH}_{3}, \mathrm{C}_{2} \mathrm{H}_{5}\right)$. We also report a study of the related, but simpler, triatomic reaction, $\mathrm{F}+\mathrm{OH} \rightarrow \mathrm{HF}+\mathrm{O}$, from the photoelectron spectrum of the radical anion OHF-. The results on this last system are novel in their own right, but they also provide a model to compare and understand the struciure manifested in the spectra of the polyatomic systems.

There is a substantial body of experimental and theoretical work in the literature on the hydrogen abstraction reactions of fluorine. The $\mathrm{F}+\mathrm{CH}_{3} \mathrm{OH}$ and $\mathrm{F}+\mathrm{C}_{2} \mathrm{H}_{5} \mathrm{OH}$ reactions have been the subject of both kinetics and product-stateresolved experiments. For $\mathrm{F}+\mathrm{CH}_{3} \mathrm{OH}$, two channels are available:

$$
\begin{aligned}
\mathrm{F}+\mathrm{HOCH}_{3} & \rightarrow \mathrm{FH}+\mathrm{OCH}_{3} \\
\mathrm{~F}+\mathrm{HCH}_{2} \mathrm{OH} & \rightarrow \mathrm{FH}+\mathrm{CH}_{2} \mathrm{OH}
\end{aligned}
$$

The exothermicities for these reactions are given in Table 6-I. Both reactions (1) and (2) are very fast at room temperature: $k_{t o t}=1 \times 10^{-10}-2 \times 10^{-10} \mathrm{~cm}^{3} \mathrm{~s}^{-1} \cdot{ }^{10,11,12}$ There is a considerable range ${ }^{13,14}$ of reported branching ratios. ${ }^{11,12,13,14,15,16}$ However there is a consensus that reaction (1) accounts for more than the statistical $25 \%$ of total product at room temperature, and this fraction may be as high as $80 \% .^{13}$ For $\mathrm{F}+\mathrm{C}_{2} \mathrm{H}_{5} \mathrm{OH}$ there are three channels available. If the fluorine attack were 
completely non-site-specific, one would expect a branching ratio in $\mathrm{F}+\mathrm{C}_{2} \mathrm{H}_{5} \mathrm{OH}$ of $18 \%$ for $\mathrm{C}_{2} \mathrm{H}_{5} \mathrm{O}$ formation. As with methanol, the reported branching ratios ${ }^{17,18}$ indicate that fluorine abstracts at the hydroxyl group faster than at the methyl or methylene groups. Khatoon's work, ${ }^{12,17}$ using isotopically labelled reactants and a mass spectrometric detection scheme, indicates that the probability of abstraction of a particular hydrogen by fluorine is five times more likely at the hydroxyl site than at one of the alkyl sites, for both methanol and ethanol. This preference in both reactions is interestir since the $\mathrm{CH}_{3} \mathrm{O}$ and $\mathrm{C}_{2} \mathrm{H}_{5} \mathrm{O}$ product channels are less exoergic by $10 \mathrm{kcal} / \mathrm{mol}$ (see Table 6-I). As we shall see, our experimental data addresses the dynamics of the hydroxyl hydrogen abstraction channel only.

The energy disposal in both products of reaction (1) can be measured: the HF product by infrared chemiluminescence and the $\mathrm{CH}_{3} \mathrm{O}$ product by laser-induced fluorescence. This has been used in a series of experiments ${ }^{11,15,16,19}$ to determine, in detail, how the partitioning of product energy compares to results for the wellcharacterized triatomic $\mathrm{F}+\mathrm{HX}$ reactions $(\mathrm{X}=$ halogen $){ }^{20}$ The results indicate that for the $\mathrm{F}+\mathrm{CH}_{3} \mathrm{OH}$ reaction, the presence of a large number of product degrees of freedom has a small but noticeable effect on oirerall energy disposal, in comparison to the $\mathrm{F}+\mathrm{HX}$ reactions. The fraction of available energy appearing as $\mathrm{HF}$ vibrational excitation is slightly less than in the $\mathrm{F}+\mathrm{HX}$ reactions, and about $2 \%$ of the available energy appears as vibrational excitation of the $\mathrm{v}_{3}$ mode ( $\mathrm{C}-\mathrm{O}$ stretch) of the $\mathrm{CH}_{3} \mathrm{O}$ radical. The single chemiluminescence study of $\mathrm{F}+\mathrm{C}_{2} \mathrm{H}_{5} \mathrm{OH}$ suggests similar behavior for this system. ${ }^{21}$ Thus, one expects the potential energy surfaces for both the $\mathrm{F}+\mathrm{CH}_{3} \mathrm{OH}$ and $\mathrm{F}+\mathrm{C}_{2} \mathrm{H}_{5} \mathrm{OH}$ reactions to share important features with the $\mathrm{F}+\mathrm{HX}$ surfaces, particularly a small entrance channel barrier to reaction. 
The reaction of fluorine with hydroxyl has been less well studied. Possible reactions of $\mathrm{F}$ with $\mathrm{OH}$ are:

$$
\begin{gathered}
F+H O-H F+O\left({ }^{3} P\right) \\
F+O H-H O F^{*}-H F+O\left(^{3} P\right) \\
\longrightarrow \rightarrow H F+O\left({ }^{1} D\right) \\
F+H O-H F+O\left({ }^{1} D\right)
\end{gathered}
$$

In (3) and (5) fluorine directly attacks the hydrogen end of the hydroxyl; (3) proceeds on a triplet surface and is exothermic by $34 \mathrm{kcal} / \mathrm{mol}$, while (5), which is $11 \mathrm{kcal} / \mathrm{mol}$ endothermic, occurs on a singlet surface. This mechanism is akin to the reactions discussed so far with the alcohols. Reaction (4) involves the radical fluorine atom attacking the oxygen atom, the site of the unpaired electron on $\mathrm{OH}$, and forming vibrationally hot HOF $\left({ }^{1} A^{\prime}\right)$. This radical-radical recombination should proceed with no barrier. However, a spin-forbidden non-adiabatic transition is then required for $H F+O\left({ }^{3} P\right)$ production. The overall room temperature rate constant for reaction of $F$ $+\mathrm{OH}$ by all pathways has been measured to be $4.1 \times 10^{-11} \mathrm{~cm}^{3} \mathrm{~s}^{-1.22}$

Sloan and coworkers ${ }^{23}$ have measured product state distributions from the $\mathrm{F}+\mathrm{OH}$ reaction. They observed infrared chemiluminescence from product HF vibrational levels up to $v=3$ when reacting $F$ with $\mathrm{H}_{2} \mathrm{O}$. The $\mathrm{F}+\mathrm{H}_{2} \mathrm{O}$ reaction is exoergic enough to produce only $\mathrm{HF}(\mathrm{v}=0,1)$. The observation of population in higher HF vibrational levels was explained in terms of a secondary reaction, of $\mathrm{F}$ with $\mathrm{OH}$ produced by the $\mathrm{F}+\mathrm{H}_{2} \mathrm{O}$ reaction, taking place in their chamber. The product vibrational and rotational distributions from the secondary reaction appeared to be 
statistical. On the basis of this and an $a b$ initio calculation, which predicted a large barrier for (3), the authors concluded that the dominant reaction pathway is via attack of fluorine on the oxygen end of the OH molecule (4) and that HOF is a long- lived intermediate, which decays by a non-adiabatic transition. As we shall show below, the OHF- photoelectron spectrum is quite sensitive to the barrier for direct abstraction (3), allowing us to test Sloan's conclusions. This adiabatic pathway for $\mathrm{F}+\mathrm{OH}$ to yield $O\left({ }^{3} \mathrm{P}\right)+\mathrm{HF}$ by direct abstraction is entirely analogous to the alcohol reactions. Thus we will also use the results on the $\mathrm{F}+\mathrm{OH}$ system to serve as a model for understanding the $\mathrm{CH}_{3} \mathrm{OHF}^{-}$and $\mathrm{C}_{2} \mathrm{H}_{5} \mathrm{OHF}^{-}$photoelectron spectra.

Our experiment starts with the negative ion analog of the reaction transition state we wish to study. It is useful for that ion to be well-characterized. The $\mathrm{CH}_{3} \mathrm{OHF}$ - anion has been fairly well-studied and was first observed by Riveros in an ion cyclotron resonance (ICR) cell. ${ }^{24}$ In this anion, $\mathrm{F}^{-}$binds to the hydroxyl hydrogen of $\mathrm{CH}_{3} \mathrm{OH}$, since this hydrogen is considerably more acidic than the methyl hydrogens. Larson and $\mathrm{McMahon}$ determined the $\mathrm{CH}_{3} \mathrm{OH} \bullet \bullet \mathrm{F}^{-}$binding energy to be $29.6 \mathrm{kcal} / \mathrm{mol} .^{25}$ The proton affinity of $\mathrm{CH}_{3} \mathrm{O}^{-}$(or the gas phase acidity of $\mathrm{CH}_{3} \mathrm{OH}$ ) is slightly higher than that of $\mathrm{F}^{-}(381.2 \mathrm{kcal} / \mathrm{mol}$ versus $371.4 \mathrm{kcal} / \mathrm{mol}){ }^{26}$ This means that $\mathrm{F}^{-}+\mathrm{CH}_{3} \mathrm{OH}$ is the lowest dissociation channel for $\mathrm{CH}_{3} \mathrm{OHF}-$; Jasinski et $\mathrm{al}^{27}$ have experimentally confirmed this by infrared multiphoton dissociation of the ion in an ICR cell. In addition, the relative proton affinities lead one to expect the shared proton in $\mathrm{CH}_{3} \mathrm{OHF}^{-}$to lie closer to the $\mathrm{O}$ atom than to the $\mathrm{F}$ atom in the ion equilibrium structure.

The proton affinity of $\mathrm{C}_{2} \mathrm{H}_{5} \mathrm{O}^{-}(378.1 \mathrm{kcal} / \mathrm{mol})^{26}$ is nearer to, but still higher than, that of $\mathrm{F}^{-}$. Thus it would be expected that the shared proton in $\mathrm{C}_{2} \mathrm{H}_{5} \mathrm{OHF}^{-}$, 
though still closer to the $\mathrm{O}$ than the $\mathrm{F}$ atom, would be raore evenly shared than in $\mathrm{CH}_{3} \mathrm{OHF}^{-}$. In agreement with this, the measured binding energy of $\mathrm{C}_{2} \mathrm{H}_{5} \mathrm{OHF}^{-}, 31.5$ $\mathrm{kc}$ il $/ \mathrm{mol},{ }^{25}$ is higher than that of $\mathrm{CH}_{3} \mathrm{OHF}^{-}$. The OHF- ion has not been observed previously, but, since the proton affinity of $\mathrm{O}^{-}(382.2 \mathrm{kcal} / \mathrm{mol})^{26}$ is comparable to that of $\mathrm{CH}_{3} \mathrm{O}^{-}$, one expects binding and structure similar to that of the alcohol complexes, namely $\mathrm{OH} \bullet \mathrm{F}^{-}$.

The position of the shared proton in the anion is critical to our photoelectron spectroscopy experiment, since the region of the $A+H B$ potential energy surface accessible via photodetachment is determined by the ion geometry. For example, we have reported studies of the $\mathrm{Br}+\mathrm{HI}, \mathrm{Cl}+\mathrm{HI}$, and $\mathrm{F}+\mathrm{HI}$ reactions by photoelectron spectroscopy of $\mathrm{BrHI}^{-}, \mathrm{ClHI}^{-}$, and $\mathrm{FHI}^{-}$. The proton affinities of $\mathrm{Br}^{-}, \mathrm{Cl}^{-}$, and $\mathrm{F}^{-}$ are at least $10 \mathrm{kcal} / \mathrm{mol}$ greater than that of $\mathrm{I}^{-}$. We therefore expect $\mathrm{R}_{\mathrm{HX}}<\mathrm{R}_{\mathrm{HI}}$ in the $\mathrm{XHI}^{-}$anions. The ions consequently have better geometric overlap with the $\mathrm{I}+\mathrm{HX}$ product valleys on their respective neutral potential energy surfaces, and this is indeed the region that is probed in the reported photoelectron spectra. This turns out to be the case for all the asymmetric $\mathrm{X}+\mathrm{HY}$ reactions, where $\mathrm{X}$ and $\mathrm{Y}$ are halogen atoms.

On the other hand, photodetachment of $\mathrm{CH}_{3} \mathrm{OHF}^{-}, \mathrm{C}_{2} \mathrm{H}_{5} \mathrm{OHF}^{-}$and $\mathrm{OHF}^{-}$ should result in considerably better overlap with the $\mathrm{F}+\mathrm{CH}_{3} \mathrm{OH}, \mathrm{F}+\mathrm{C}_{2} \mathrm{H}_{5} \mathrm{OH}$ or $\mathrm{F}+\mathrm{OH}$ reactant valleys, respectively. This is arguably the most important region of the surface since it includes the barrier along the minimum energy path. Thus the significance of the results presented here is twofold. The photoelectron spectra of the $\mathrm{ROHF}^{-}$and $\mathrm{OHF}^{-}$anions represent an extension of our 'transition state spectroscopy' method to more complex reactions. Furthermore, the spectra of all three systems 
allow us to probe a more interesting part of the potential energy surface for the corresponding neutral reaction in comparison to the $\mathrm{XHY}^{-}$spectra.

In addition to the experimental results, we present $a b$ initio calculations that describe the equilibrium structure, harmonic frequencies and binding energies of the $\mathrm{CH}_{3} \mathrm{OHF}^{-}$and $\mathrm{OHF}^{-}$ions. A two-dimensional dynamical simulation of the $\mathrm{OHF}^{-}$ photoelectron spectrum is then described, which is used as a starting point for considering modifications to the $\mathrm{F}+\mathrm{OH}$ reaction potential surfaces. The spectra of the polyatomic systems are discussed, with reference to the OHF- simulation, and finally the role of the extra degrees of freedom is evaluated.

\section{Experimental}

The time-of-flight photoelectron spectrometer used in this study has been described in detail elsewhere. ${ }^{5}$ Briefly, negative ions are formed by crossing the expansion of a pulsed molecular beam valve with a $1 \mathrm{keV}$ continuous electron beam close to the valve orifice. For production of $\mathrm{OHF}^{-}$, the gas mixture expanded through the valve was $\mathrm{N}_{2} \mathrm{O}$ with a trace concentration of $\mathrm{HF}$. The $\mathrm{OHF}^{-}$formation mechanism is probably via dissociative attachment of $\mathrm{N}_{2} \mathrm{O}$ by slow secondary electrons, $\mathrm{N}_{2} \mathrm{O}+\mathrm{e}^{-}$ $\rightarrow \mathrm{O}^{-}+\mathrm{N}_{2}$, followed by the clustering process $\mathrm{O}^{-}+\mathrm{HF}^{+} \mathrm{M} \rightarrow \mathrm{OHF}^{-}+\mathrm{M}$. The best way we found to make a stable beam of $\mathrm{CH}_{3} \mathrm{OHF}^{-}$(or $\mathrm{C}_{2} \mathrm{H}_{5} \mathrm{OHF}^{-}$) was to put a few drops of $\mathrm{CH}_{3} \mathrm{OH}$ (or $\mathrm{C}_{2} \mathrm{H}_{5} \mathrm{OH}$ ) into the pulsed beam valve, which hangs vertically in the source chamber, and pass a $5 \% \mathrm{NF}_{3} / \mathrm{He}$ mixture through the valve. Dissociative attachment to $\mathrm{NF}_{3}$ produces $\mathrm{F}^{-}$, which then clusters to the alcohol. Under the same expansion conditions, we have obtained vibrational temperatures of $150-300 \mathrm{~K}$ for 
$\mathrm{CH}_{2} \mathrm{CN}^{-}$and rotational temperatures of less than $100 \mathrm{~K}$ for $\mathrm{SH}^{-}$prepared in a similar source. $^{28}$

The ions formed in the source chamber are extracted perpendicular to the molecular beam and injected into a Wiley-McLaren type time-of-flight mass spectrometer. ${ }^{29}$ Ions separate according to their masses and the ion of interest is photodetached by the fifth harmonic of a pulsed Nd:YAG laser (213 nm, $5.825 \mathrm{eV})$. The photoelectrons produced by this process are ejected into all solid angles and a small fraction $\left(10^{-4}\right)$ is collected by a detector at the end of a one meter field-free flight tube. The flight time of the electrons is recorded and converted into center-of-mass kinetic energy. The energy resolution function of the instrument is given in reference 5; essentially the apparatus' electron energy resolution is $8 \mathrm{meV}$ at $0.65 \mathrm{eV}$ and degrades a $a^{n-2}$ at higher electron kinetic energies.

\section{Results \& Analysis}

\subsection{Experimental Results}

The $\mathrm{CH}_{3} \mathrm{OHF}^{-}$and $\mathrm{C}_{2} \mathrm{H}_{5} \mathrm{OHF}^{-}$photoelectron spectra are shown in Figure 6-1. The spectra are considerably more complex than the spectra of the triatomic anions we have previously studied. The clearest structural pattern in both spectra are the four regularly spaced steps between 1.2 and $2.8 \mathrm{eV}$. The labelled arrows (A-D), at 2.69, 2.24, 1.85, and 1.54 eV in Figure 6-1a and 2.58, 2.18, 1.83, and $1.50 \mathrm{eV}$ in Figure 6-1b, mark the onset of each step. There are two sharper features, E at 1.15 $\mathrm{eV}$, and $\mathrm{F}$ at $1.03 \mathrm{eV}$, in the $\mathrm{CH}_{3} \mathrm{OHF}^{-}$spectrum. 
The peaks are labelled in order of increasing internal energy of the neutral complex. The electron kinetic energies (eKEs) are related to the internal energies of the neutral species $\left(E_{i}^{(0)}\right)$ by

$$
\mathrm{eKE}=\mathrm{h} v-\mathrm{D}_{0}^{\circ}(\mathrm{ROHF}-)-\mathrm{EA}(\mathrm{F})-\Delta \mathrm{E}_{0}^{0}-\mathrm{E}_{\mathrm{i}}^{(0)}+\mathrm{E}_{\mathrm{i}}^{(-)}
$$

Thus peaks with highest electron kinetic energy correspond to neutral states with lowest internal energy. In (6), hv is the laser photon energy, $5.825 \mathrm{eV}, \mathrm{EA}(\mathrm{F})$ is the electron affinity of fluorine, $3.399 \mathrm{eV}^{30}$ and $\mathrm{D}_{0}^{\circ}\left(\mathrm{ROHF}^{-}\right)$is the bond dissociation energy of ground state $\mathrm{ROHF}^{-}$to form ground state $\mathrm{ROH}$ and F-. For $\mathrm{D}_{0}^{\circ}\left(\mathrm{CH}_{3} \mathrm{OHF}-\right)$, we use the experimental ${ }^{25}$ bond dissociation enthalpy, $\Delta \mathrm{H}_{298}$, and correct to $0 \mathrm{~K}$ using a set of $a b$ initio calculated frequencies (see below); this yields $1.244 \mathrm{eV}$. For $\mathrm{D}_{0}^{\circ}\left(\mathrm{C}_{2} \mathrm{H}_{5} \mathrm{OHF}^{-}\right)$, we use the experimental ${ }^{25}$ bond enthalpy for this ion, and the same correction to $0 \mathrm{~K}$ as calculated for $\mathrm{CH}_{3} \mathrm{OHF}^{-}$, yielding $1.327 \mathrm{eV} . \Delta \mathrm{E}^{\circ}{ }_{0}$ is the exoergicity for the reaction $\mathrm{F}+\mathrm{ROH} \rightarrow \mathrm{RO}+\mathrm{HF}$ and is given in Table 6-I.I.,32,33,34,35,36 $E_{i}^{(0)}$ is the energy of the [ROHF] complex relative to ground state RO + HF. $\mathrm{E}_{\mathrm{i}}^{(-)}$is the internal energy of the precursor ion $\mathrm{ROHF}^{-}$above zero point. As all the anions studied here are expected to be prepared cold, this quantity is assumed throughout to be zero.

The eKE corresponding to $\mathrm{E}_{\mathrm{i}}^{(0)}=0$ is shown by arrows against the axis at 2.60 $\mathrm{eV}$ (Figure 6-1a) and 2.50 eV (Figure 6-1b). These arrows indicate the electron kinetic energy corresponding to photodetaching ground state $\mathrm{CH}_{3} \mathrm{OHF}^{-}\left(\mathrm{C}_{2} \mathrm{H}_{5} \mathrm{OHF}^{-}\right)$and forming ground state $\mathrm{HF}+\mathrm{CH}_{3} \mathrm{O}\left(\mathrm{C}_{2} \mathrm{H}_{5} \mathrm{O}\right)$. We refer to these energies henceforth as product asymptotes. The reactant asymptotes, indicated by arrows at $1.18 \mathrm{eV}$ (Figure 6-1a) and $1.10 \mathrm{eV}$ (Figure 6-1b), correspond to ground state $\mathrm{F}+\mathrm{CH}_{3} \mathrm{OH}$ $\left(\mathrm{C}_{2} \mathrm{H}_{5} \mathrm{OH}\right)$ formation for which $\mathrm{E}_{i}^{(0)}=-\Delta \mathrm{E}_{0}^{0}$. All the structure in each spectrum occurs 
at lower eKE than the product asymptote and is therefore due to states of the neutral complex that can dissociate to $\mathrm{HF}+\mathrm{CH}_{3} \mathrm{O}\left(\mathrm{C}_{2} \mathrm{H}_{5} \mathrm{O}\right)$ products. In addition, features in the spectra at lower eKE than the reactant asymptotes correspond to states of the complex which can also dissociate to $\mathrm{F}+\mathrm{CH}_{3} \mathrm{OH}\left(\mathrm{C}_{2} \mathrm{H}_{5} \mathrm{OH}\right)$ reactants.

The photoelectron spectrum of $\mathrm{CH}_{3} \mathrm{ODF}^{-}$(Figure 6-2) shows a clear isotope effect. The spectrum shows six steps more closely spaced than those in the $\mathrm{CH}_{3} \mathrm{OHF}^{-}$ spectrum. This isotope effect suggests that the step structure is due to a vibrational mode of the neutral complex analogous to the $v_{3}$ mode seen in the triatomic bihalide spectra; ${ }^{5,6,7}$ that is, the light hydrogen atom is vibrating between the much heavier $F$ atom and RO group in the complex. The spacing between the steps in Figures 6-1a and $6-1 \mathrm{~b}$ is less than that between the first few vibrational levels of $\mathrm{HF}^{37}$ and the spacing between the second, third and fourth steps is less than the corresponding levels of the $\mathrm{OH}$ stretch in $\mathrm{CH}_{3} \mathrm{OH}$. This 'red shift' occurs because the experiment probes the transition state region where the reacting species are interacting strongly; ${ }^{5,6}$ the $\mathrm{H}$ atom vibrates in a shallower potential than in isolated $\mathrm{HF}$ or $\mathrm{CH}_{3} \mathrm{OH}$.

The intensity in the $\mathrm{CH}_{3} \mathrm{OHF}^{-}$spectrum builds as one moves from the $\mathrm{HF}+\mathrm{CH}_{3} \mathrm{O}$ product asymptote to the $\mathrm{F}+\mathrm{CH}_{3} \mathrm{OH}$ reactant asymptote at lower electron kinetic energy. The intensity distribution in the $\mathrm{C}_{2} \mathrm{H}_{5} \mathrm{OHF}^{-}$spectrum is similar, although there is relatively more signal near the $\mathrm{HF}+\mathrm{C}_{2} \mathrm{H}_{5} \mathrm{O}$ asymptote. The breadth of all the features in the photoelectron spectra is considerably larger than the experimental resolution. Spectra recorded at $266 \mathrm{~nm}(4.66 \mathrm{eV}$, not shown) show the right-most steps in each of the $213 \mathrm{~nm}$ spectra with lower electron kinetic energy and 
therefore higher resolution. Despite this higher resolution, there is no additional structure observed; the step profiles are unchanged.

The photoelectron spectrum of $\mathrm{OHF}^{-}$is shown in Figure 6-3. The peak positions and widths are listed in Table 6-II. The electron kinetic energies corresponding to the dissociation asymptotes $\mathrm{O}\left({ }^{3} \mathrm{P}_{2}\right)+\mathrm{HF}(2.43 \mathrm{eV}), \mathrm{F}\left({ }^{2} \mathrm{P}_{3 / 2}\right)+\mathrm{OH}\left({ }^{2} \Pi_{3 / 2}\right)$ $(0.95 \mathrm{eV})$, and $\mathrm{O}\left({ }^{1} \mathrm{D}\right)+\mathrm{HF}(0.46 \mathrm{eV})$, are shown on Figure 6-3, as before, with arrows against the energy axis. These energies are calculated using a relationship similar to Equation (6), along with our best $a b$ initio ion binding energy estimate (see below), the exoergicity for reaction (3) in Table 6-I, and the experimental ${ }^{38}$ oxygen atomic energy level splittings. Again, all structure observed in the spectrum corresponds to states of the neutral unstable with respect to dissociation to the lowest energy product channel $\left(\mathrm{O}\left({ }^{3} \mathrm{P}\right)+\mathrm{HF}\right)$. Moreover peaks A-D correspond to neutral states which can only dissociate to $\mathrm{O}\left({ }^{3} \mathrm{P}\right)+$ HF products.

The overall appearance of the $\mathrm{OHF}^{-}$spectrum is clearly very similar to both of the $\mathrm{ROHF}^{-}$spectra. This is an interesting result considering the far fewer vibrational degrees of freedom in the [OHF] complex as well as its different electronic character (see below). However, there are several differences between the $\mathrm{OHF}^{-}$and $\mathrm{ROHF}^{-}$spectra. The onset of structure occurs at almost $0.3 \mathrm{eV}$ lower electron kinetic energy in the $\mathrm{OHF}^{-}$spectrum. Between the product and reactant asymptotes, the $\mathrm{OHF}^{-}$spectrum looks more like a set of broad peaks, in contrast to the step structure seen in the $\mathrm{CH}_{3} \mathrm{OHF}^{-}$and $\mathrm{C}_{2} \mathrm{H}_{5} \mathrm{OHF}^{-}$spectra. The feature below the reactant asymptote in the $\mathrm{OHF}^{-}$spectrum (labelled $\mathrm{E}$ ) is much more distinct than any corresponding structure in the other spectra. Finally, the intensity in the OHFspectrum builds in the same way as the other spectra but, in this spectrum, there is 
even less intensity at the $\mathrm{O}+\mathrm{HF}$ product asymptote than at the product asymptote in the $\mathrm{CH}_{3} \mathrm{OHF}^{-}$spectrum.

The photoelectron spectra of $\mathrm{OHF}^{-}, \mathrm{CH}_{3} \mathrm{OHF}^{-}$and $\mathrm{C}_{2} \mathrm{H}_{5} \mathrm{OHF}^{-}$all show maximum intensity near the asymptote for dissociation into reactants $\mathrm{F}+\mathrm{OH}$, $\mathrm{F}+\mathrm{CH}_{3} \mathrm{OH}$ and $\mathrm{F}+\mathrm{C}_{2} \mathrm{H}_{5} \mathrm{OH}$ rather than the energetically lower product asymptote. This is in sharp contrast to the asymmetric bihalide ions, ${ }^{7}$ all of which show maximum intensity at the product asymptote. We can understand the intensity distributions in terms of the qualitative discussion of the ion geometries in the Introduction. Because the proton affinities of $\mathrm{O}^{-}, \mathrm{CH}_{3} \mathrm{O}^{-}, \mathrm{C}_{2} \mathrm{H}_{5} \mathrm{O}^{-}$are $0.47,0.42$, and $0.29 \mathrm{eV}$ higher than that of $\mathrm{F}^{-}$, the hydrogen should lie closer to the $\mathrm{O}$ atom than the $\mathrm{F}$ atom in all three anions. We therefore expect good Franck-Condon overlap with $\mathrm{OH} \bullet \mathrm{F}$ or $\mathrm{ROH} \bullet \mathrm{F}$ configurations of the neutral complex localized in the entrance valley of the reactive potential energy surface. The overall intensity envelopes seen in the spectra are in accord with this reasoning. In particular, the observation that the $\mathrm{C}_{2} \mathrm{H}_{5} \mathrm{OHF}^{-}$ spectrum has more intensity at the product asymptote than the $\mathrm{CH}_{3} \mathrm{OHF}^{-}$spectrum is consistent with the higher proton affinity of $\mathrm{CH}_{3} \mathrm{O}^{-}$.

It is clear from this discussion that the anion geometry has a significant effect on the photoelectron spectrum. In contrast to the bihalide ions, no spectroscopic data exist for $\mathrm{ROHF}^{-}$and $\mathrm{OHF}^{-}$. Therefore, in order to obtain a more quantitative picture of the anions, we have performed $a b$ initio calculations on $\mathrm{OHF}^{-}$and $\mathrm{CH}_{3} \mathrm{OHF}^{-}$to determine their structure and properties. 


\subsection{Ab initio calculations: method and results}

Fully optimized geometries, frequencies and dissociation energies were calculated for the hydrogen-bonded anions $\mathrm{OHF}^{-}$and $\mathrm{CH}_{3} \mathrm{OHF}^{-}$at both the HartreeFock (HF) and second order Moller-Plesset (MP2) level of theory. In addition, MP4 level calculations were employed to estimate hydrogen bond dissociation energies. The calculations reported here were performed with the Gaussian $86,{ }^{39}$ Gaussian $88{ }^{40}$ and CADPAC $^{41} a b$ initio packages available at the San Diego Supercomputing Center. The standard $6-31++\mathrm{G}^{* *}$ basis set was used throughout; the incorporation of diffuse functions $(++)$ is mandatory for a proper description of these anions. For $\mathrm{OHF}^{-}$, a spin-unrestricted wave function was used to describe both the ${ }^{2} \Pi$ ground and ${ }^{2} \Sigma$ excited states.

The results of these calculations are summarized in Table 6-III - Table 6-VII. For the purposes of analyzing our photoelectron spectra, the most important results are the ion equilibrium geometries, the shape of their potential surfaces along the hydroxyl hydrogen stretching coordinate, and the $a b$ initio estimate of their dissociation energy into $\mathrm{F}^{-}$and $\mathrm{OH}$ or $\mathrm{CH}_{3} \mathrm{OH}$. For the last of these, an estimate of the zero point energy correction is required, so frequencies were calculated for the hydrogen-bonded ions and the product fragments at both the HF and MP2 level of theory. All energies quoted $\left(E_{0}\right)$ include this zero point energy correction, which is applied at the same level of theory as the calculated energy (except for the MP4 energies where the MP2 zero point energies are used). MP4 electronic energies include all single, double, triple and quadruple excitations while maintaining a frozen core (MP4SDTQ-FC); the MP2 energies have all electrons considered for correlation (MP2-FULL). The calculation of the ion force field, apart from providing zero point 
energies, was also useful for (a) obtaining a reasonable form for each anion's vibrational normal coordinates and (b) providing an estimate for the hydrogen bond stretching frequencies in the ions. Both of these will be used in the dynamical calculations described below.

The dissociation fragments of $\mathrm{OHF}^{-}$and $\mathrm{CH}_{3} \mathrm{OHF}^{-}$that we need to consider are $\mathrm{F}^{-}, \mathrm{CH}_{3} \mathrm{OH}, \mathrm{CH}_{3} \mathrm{O}^{-}, \mathrm{HF}, \mathrm{OH}$ and $\mathrm{O}^{-}$. Computations of fully optimized geometries and frequencies for these fragments compare well with literature values for calculations using similar sized basis sets. The MP2 geometries and frequencies agree well with experimental values for $\mathrm{CH}_{3} \mathrm{OH}, \mathrm{HF}$, and $\mathrm{OH}$, and the fluorine electron affinity is well reproduced at the MP2 level. The oxygen electron affinity, calculated at the same level of theory, $1.09 \mathrm{eV}$, is in poorer agreement with the experimental value of $1.46 \mathrm{eV} .{ }^{30}$ By calculating the MP2/6-31++G** equilibrium geometry of the Jahn-Teller distorted ground state of $\mathrm{CH}_{3} \mathrm{O},{ }^{42}$ and using scaled ${ }^{43}$ harmonic frequencies from the HF/6-31++ $\mathrm{G}^{* *}$ force fields for ion and neutral, a zero point corrected adiabatic electron affinity of $1.47 \mathrm{eV}$ for $\mathrm{CH}_{3} \mathrm{O}$ is computed. This is to be compared with the experimental value of $1.57 \mathrm{eV}^{44}$

Geometries \& Frequencies: Our qualitative expectations for the hydrogen-bonded ion geometries and dissociation energies are based on the proton affinity scale and have been outlined above. We expect that the closer the proton affinity of the bases $\mathrm{A}^{-}$and $\mathrm{B}^{-}$, the larger the degree of sharing of the proton and the stronger the hydrogen bond in $\mathrm{AHB}^{-25}$ From comparison of the proton affinities of the methoxide, hydroxide, and fluoride ions, we expect the hydrogen to be closer to the oxygen than fluorine in both $\mathrm{CH}_{3} \mathrm{OHF}^{-}$and $\mathrm{OHF}^{-}$. The quantitative ab initio results shown in Table 6-III and Table 6-VI show that indeed the hydrogen sits closer to the oxygen than the fluorine 
atom for both ions. In fact, the equilibrium parameters for the two ions are very similar; the difference in O-F separation is smaller than $0.01 \AA$ and the hydrogen position is different by only $0.02 \AA$. However, the $a b$ initio calculations suggest that $\mathrm{OHF}^{-}$has the hydrogen more centrally located. This is contrary to the proton affinity argument, but $\mathrm{CH}_{3} \mathrm{OH}$ is only slightly more acidic than $\mathrm{OH}$.

The equilibrium geometry for $\mathrm{CH}_{3} \mathrm{OHF}^{-}$, which has $\mathrm{C}_{8}$ symmetry, is shown in Figure 6-4. The $\mathrm{F}^{-} \cdot \circ \mathrm{H}-\mathrm{CH}_{2} \mathrm{OH}$ isomer is expected to lie considerably higher in energy than $\mathrm{CH}_{3} \mathrm{OH} \bullet \mathrm{F}^{-}$. The hydrogen bond is slightly bent, as found for $\mathrm{F}-\left(\mathrm{H}_{2} \mathrm{O}\right),{ }^{45}$ because of a weak interaction with the other nearby hydrogen atoms. With regard to the methanol frame, the staggered conformer is preferred to minimize steric repulsion, but the barrier to internal rotation about the $\mathrm{C}-\mathrm{O}$ bond is calculated to be even smaller than in methanol. ${ }^{46}$ The equilibrium configuration was found by optimization of all geometric parameters; in particular, the methoxy frame was also allowed to relax in response to the perturbation by the $F^{-}$. The changes in the frame with respect to the equilibrium $\mathrm{CH}_{3} \mathrm{OH}$ geometry described by the same theoretical model are modest (Table 6-III). The principal differences are a contraction in $r_{e}(C-O)$ by $0.03 \AA$ in $\mathrm{CH}_{3} \mathrm{OHF}^{-}$and some distortion of the bond angles around the tetrahedral carbon center. By comparison, the change at the active center is much larger: there is a $0.10 \AA$ lengthening of the O-H bond in the ion at the MP2 level. The framework relaxation will be considered when discussing possible "bath" mode excitations in the $\mathrm{CH}_{3} \mathrm{OHF}^{-}$photoelectron spectra. Previous calculations ${ }^{47}$ for this ion are in qualitative agreement with those shown here, but the earlier treatments were limited to small basis sets without a complete set of diffuse functions and neglected electron correlation. 
For $\mathrm{AHB}^{-}$species, a pertinent question to ask is whether there are two minima on the potential energy surface, corresponding to $A H \bullet \bullet B^{-}$and $A^{-} \bullet \bullet H B$, or whether the two coalesce to form one broad, fairly flat-bottomed minimum. In the case of the symmetric bihalide ions $\mathrm{AHA}^{-}$, this is extremely important as it determines whether the equilibrium structure is centrosymmetric or not. For example, calculations ${ }^{4,49,50}$ on $\mathrm{ClHCl}^{-}$and $\mathrm{BrHBr}^{-}$show that a Hartree-Fock description predicts a double minimum potential for these a.ions, whereas the inclusion of other electronic configurations in the ground state wave function leads to a significant lowering of the energy for centrosymmetric geometries compared to the AH॰A-geometries. Even using Møller-Plesset Perturbation Theory to second order (MP2) causes the double minimum potentials for the AHA- ions to coalesce into a single flat- bottomed minimum. ${ }^{50}$ High resolution spectroscopy studies indicate these anions do, in fact, have centrosymmetric equilibrium structures. ${ }^{51}$

For an asymmetric ion such as $\mathrm{CH}_{3} \mathrm{OHF}^{-}$, we might expect to find a local minimum, $\mathrm{CH}_{3} \mathrm{O}^{-\bullet \bullet H F}$, as well as the global one described by Figure 6-4 and Table 6-III. Figure 6-5 shows a plot of the potential energy as a function of the position of the hydrogen atom between the $\mathrm{O}$ and $\mathrm{F}$ atoms which are assumed, along with all other degrees of freedom, to be fixed. The plot shows that at the HartreeFock level there is evidence of a plateau in the potential corresponding to the $\mathrm{CH}_{3} \mathrm{O}-\bullet \cdot \mathrm{HF}$ structure, but that it disappears at the MP2 level. The MP2 correction clearly stabilizes geometries with the hydrogen more evenly shared between $\mathrm{F}$ and $\mathrm{O}$; the potential shape is distinctly different. This has a large effect on the vibrational level spacings for the 'O-H stretch' and the shape of the ground vibrational wave 
function along this coordinate. This will, in turn, have a profound effect on the intensity distribution seen in the photoelectron spectrum.

The calculated harmonic vibrational frequencies for the $\mathrm{CH}_{3} \mathrm{OHF}-$ ion are shown in Table 6-IV; $\omega_{3}$ is the O-H stretching mode which corresponds to the potential function we have been discussing. The harmonic frequency for this mode $\left(2215 \mathrm{~cm}^{-1}\right)$ is strongly perturbed from its value in $\mathrm{CH}_{3} \mathrm{OH}\left(\mathrm{MP2} / 6-31++\mathrm{G}^{* *}\right.$ value 3900 $\left.\mathrm{cm}^{-1}\right)$. The F--HOR stretching mode, $\omega_{9}$, is analogous to $\omega_{1}$ in the bihalide ions and $\mathrm{OHF}^{-}$(see below). These two stretching modes, the O-H stretch and the O-F stretch, are the two modes most important to understanding the photoelectron spectra.

The $a b$ initio results for the ground ${ }^{2} \Pi$ state of the $\mathrm{OHF}^{-}$anion are shown in Table 6-VI and Figure 6-4. It is clear from the similarity in the equilibrium geometry of this ion and the OHF moiety in $\mathrm{CH}_{3} \mathrm{OHF}^{-}$that the $\mathrm{CH}_{3}$ group has little effect on the hydrogen bond, except to slightly bend it. It is worth noting that, in contrast to $\mathrm{CH}_{3} \mathrm{OHF}^{-}, \mathrm{OHF}^{-}$is open shelled but the spin remains localized on the oxygen atom, as in $\mathrm{OH}$. The spin-orbit splitting in this state is expected to be smaller than the 139 $\mathrm{cm}^{-1}$ of $\mathrm{OH}\left({ }^{2} \Pi\right){ }^{37}$ The expectation values of the spin operator, $\left\langle\mathrm{S}^{2}\right\rangle$, listed in Table 6-VI indicate that spin contamination is not a problem in the unrestricted wave function; a pure doublet state has $\left\langle S^{2}\right\rangle=0.75$.

A schematic of the molecular orbitals for $\mathrm{OHF}^{-}$is shown in Figure 6-6. This qualitative figure will be used later to explain the different neutral electronic states and their relationship to anion photodetachment. The ${ }^{2} \Pi$ ground electronic state is the configuration shown in Figure 6-6. There is a low-lying ${ }^{2} \Sigma^{+}$state, which results on promoting a $6 \sigma$ electron to the $2 \pi$ orbital. This state has a single minimum at $\mathrm{O}^{-} \bullet \cdot \mathrm{HF}$; it does not correlate to ground state $\mathrm{OH}+\mathrm{F}^{-}$. Collinear potential cuts 
along the $R_{\mathrm{OH}}$ coordinate for the ${ }^{2} \Pi$ and ${ }^{2} \Sigma^{+}$states are shown in Figure 6-7. The ${ }^{2} \Pi$ curve is very similar to that shown for $\mathrm{CH}_{3} \mathrm{OHF}$ - in Figure 6-5. Table 6-VI contains the harmonic frequencies of the ground ${ }^{2} \Pi$ state. The O-H stietching frequency, 2015 $\mathrm{cm}^{-1}$, is significantly reduced from the value in free $\mathrm{OH}$ and is also lower than the same mode (also $\omega_{3}$ ) of $\mathrm{CH}_{3} \mathrm{OHF}$ - The O-F stretching frequency, $\omega_{1}$, at $433 \mathrm{~cm}^{-1}$ is higher than $\omega_{3}$ of $\mathrm{CH}_{3} \mathrm{OHF}$ - These two frequencies will be used to describe the anion wave function in the simulation below. The bending vibration has two components for the linear ground state of $\mathrm{OHF}^{-}$, as expected for a linear molecule in a degenerate electronic state. ${ }^{52}$

Dissociation energies: Accurate $a b$ initio estimates of bond dissociation energies are normally difficult to calculate. However, for dissociations that do not involve breaking of a bonding pair of electrons, as is the case here, correlation corrections are relatively small and $a b$ initio methods can yield reliable energies. ${ }^{53}$ As can be seen in Table 6-V, even the Hartree-Fock estimate for the hydrogen bond strength of $\mathrm{CH}_{3} \mathrm{OHF}$-, with respect to dissociation to $\mathrm{CH}_{3} \mathrm{OH}+\mathrm{F}^{-}$, is in fairly close agreement with the experimental value $\left(\mathrm{DH}_{298}=1.28 \mathrm{eV}\right)$. Handy et al. ${ }^{54}$ have shown that the Møller-Plesset Perturbation Theory treatment is convergent and reliable for interaction energies if bond breaking or curve crossing does not occur. Chalasinskik ${ }^{55}$ demonstrated that MP2 calculations with standard basis sets, when corrected for basis set superposition error (BSSE), yield quantitative estimates of hydrogen bonding energies. It is important to recognize that for estimating correlation corrections to the hydrogen bond dissociation energies, the size-consistent MP methods are to be preferred over configuration interaction (CI) methods. 
The calculated hydrogen bond dissociation energies $\mathrm{D}_{0}^{\circ}$, defined earlier, are shown in Table 6-V and Table 6-VII. $\mathrm{OHF}^{-}$is calculated to be more stable with respect to dissociation than $\mathrm{CH}_{3} \mathrm{OHF}$ - As mentioned earlier, the binding strength of $\mathrm{CH}_{3} \mathrm{OHF}$ - has been measured ${ }^{25}$ whereas that of $\mathrm{OHF}^{-}$has not. To compare the $a b$ initio value for $\mathrm{CH}_{3} \mathrm{OHF}^{-}$with the experimental value, we must convert $\mathrm{D}_{0}^{\circ}$ to a bond enthalpy at $298 \mathrm{~K}^{55}$ Using the MP2 harmonic frequencies of $\mathrm{CH}_{3} \mathrm{OHF}-$ (Table 6-IV) and $\mathrm{CH}_{3} \mathrm{OH}$, and assuming that the change in the average rotational energy on dissociation is zero, we calculate $\Delta \mathrm{H}_{298}^{\circ}\left(\mathrm{CH}_{3} \mathrm{OHF}^{-} \rightarrow \mathrm{F}^{-}+\mathrm{CH}_{3} \mathrm{OH}\right)=1.32 \mathrm{eV}(30.4$ $\mathrm{kcal} / \mathrm{mol})$ at MP2 and $1.29 \mathrm{eV}(29.8 \mathrm{kcal} / \mathrm{mol})$ at MP4 compared to the experimental value of $1.28 \pm 0.04 \mathrm{eV}(29.6 \pm 1 \mathrm{kcal} / \mathrm{mol}) .{ }^{25}$ It appears that the $a b$ initio estimate is in very good accord with the experimental data, placing confidence in the MP4 $\mathrm{D}_{0}^{\circ}$ estimate, $1.48 \mathrm{eV}(34.0 \mathrm{kcal} / \mathrm{mol})$, for $\mathrm{OHF}^{-}$.

It should be noted that no correction is made for basis set superposition error in these calculations. Further it appears that the correlation currection to fourth order for the $\mathrm{D}_{0}^{\circ}$ of $\mathrm{CH}_{3} \mathrm{OHF}^{-}$is more fully converged than that for $\mathrm{OHF}^{-}$. Thus it is probable that higher order corrections will be required for the latter ion. The fact remains that the $\mathrm{OHF}^{-}$anion is predicted to have a stronger hydrogen bond than $\mathrm{CH}_{3} \mathrm{OHF}^{-}$at every level of correlation treatment. This is shown not only in the dissociation energy but also in the stronger perturbation to the $\mathrm{O}-\mathrm{H}$ stretching frequency, the higher F-O stretching frequency and the more central positioning of the hydrogen between the end atoms. This result is also supported by the experimental observation that the photoelectron band origin for $\mathrm{OHF}^{-}$is shifted to lower electron kinetic energy by $0.3 \mathrm{eV}$ over $\mathrm{CH}_{3} \mathrm{OHF}^{-}$. 


\subsection{Simulation of the OHF- photoelectron spectrum}

In this section, we simulate the $\mathrm{OHF}^{-}$photoelectron spectrum using our $a b$ initio results for $\mathrm{OHF}^{-}$along with the $a b$ initio potential energy surfaces developed for the $\mathrm{F}+\mathrm{OH}$ reaction by Sloan and co-workers..$^{23}$ By comparing the simulated and experimental spectra, we can learn about deficiencies in the $\mathrm{F}+\mathrm{OH}$ potential energy surface. We have previously described a fully quantum dynamical method that will simulate the dissociative photoelectron spectrum of a linear triatomic $\mathrm{AHB}^{-} .^{7}$ This method, which is based on the wave packet propagation technique of Kosloff and Kosloff, ${ }^{56}$ treats dynamics along the two stretching coordinates exactly. It assumes all nuclear motion takes place on a collinear potential energy surface.

An initial wave packet $\phi(0)$, which, within the Franck-Condon approximation, is the anion ground vibrational wave function, is propagated on the neutral potential energy surface according to

$$
|\phi(t)\rangle=\mathrm{e}^{-i \mathrm{~A} t / \mathrm{h}}|\phi(0)\rangle
$$

Here $\mathrm{e}^{-i \mathrm{~A}_{\mathrm{L}} / \mathrm{t}}$ is the time evolution operator and $\hat{H}$ is the Hamiltonian for the neutral surface. The overlap of $\phi(t)$ with $\phi(0)$ defines the time autocorrelation function $C(t)$ :

$$
\mathrm{C}(\mathrm{t})=\langle\phi(0) \mid \phi(\mathrm{t})\rangle
$$

and the Fourier transform of this complex function yields the photoelectron spectrum: $: 37.58$

$$
\sigma(E) \propto \quad \int_{-\infty}^{\infty} \exp (i E t / \hbar) C(t) d t .
$$

The simulation makes use of the collinear $a b$ initio surfaces which have been developed for the lowest triplet and singlet channels of the F + OH system. ${ }^{23}$ Sloan 
and coworkers evaluated energy points across the two surfaces using a multi-reference configuration interaction (MRCI) scheme, employing the multi-reference analog of Davidson's correction ${ }^{69}$ to extrapolate energies to full configuration interaction (FCI). To calculate the entire collinear surfaces the authors were constrained to use of the relatively small 4-31G basis set. The surfaces were each fitted to a Rotated Morse Oscillator Spline (RMOS) function. ${ }^{60}$ The authors went on to show that the barrier height on the lowest triplet surface remained virtually unchanged when the basis set was improved to double zeta plus polarization (DZP) quality. We shall first consider only the lowest surface for the collinear reaction $\mathrm{F}\left({ }^{2} \mathrm{P}\right)+\mathrm{OH}\left({ }^{2} \Pi\right) \rightarrow \mathrm{HF}\left({ }^{1} \Sigma^{+}\right)+\mathrm{O}\left({ }^{3} \mathrm{P}\right)$ for our simulation. This surface has ${ }^{3} \Pi$ electronic symmetry, and a classical barrier of 12 $\mathrm{kcal} / \mathrm{mol}$ at $R_{\mathrm{OH}}=1.08 \AA$ and $R_{\mathrm{HF}}=1.32 \AA$.

Together with the neutral potential surface, we also require the wave function for the ground vibrational state of the anion. The wave function is assumed to be separable along the two normal coordinates, $Q_{1}$ and $Q_{3}$. To construct this vibrational wave function, we use our MP2 values for the anion equilibrium geometry $\left(R_{H F}^{e}\right.$ and $\left.\mathbf{R}_{\mathrm{OH}}{ }\right)$ and frequencies $\left(\omega_{1}\right.$ and $\left.\omega_{3}\right)$ for the two stretching vibrations. The following transformation from internal coordinates (in $\AA$ ) to normal coordinates (in $\mathrm{amu}^{1 / 2} \cdot \dot{\mathrm{A}}$ ) is derived from the $a b$ initio Cartesian force constant matrix:

$$
\begin{aligned}
& Q_{1}=2.989\left(R_{\mathrm{HF}}-R_{\mathrm{HF}}^{\mathrm{e}}\right)+2.747\left(\mathrm{R}_{\mathrm{OH}}-\mathbf{R}_{\mathrm{OH}}^{\mathrm{e}}\right) \\
& \mathrm{Q}_{3}=0.198\left(\mathrm{R}_{\mathrm{HF}}-\mathrm{R}_{\mathrm{HF}}^{\mathrm{e}}\right)+1.158\left(\mathrm{R}_{\mathrm{OH}}-\mathrm{R}_{\mathrm{OH}}^{\mathrm{e}}\right) .
\end{aligned}
$$

Note that $Q_{1} \approx 3\left(R_{O F}-R_{O F}^{e}\right)$ and $Q_{3} \approx\left(R_{O H}-R_{O H}^{e}\right)$.

In the simulations carried out for the $\mathrm{BrHI}^{-}$photoelectron spectrum, ${ }^{7}$ the experimental intensity distribution was impossible to model without including anharmonicity along the $Q_{3}$ coordinate for the anion. The anion potential surface was 
therefore described by the sum of a Morse potential for the $v_{3}$ mode and a harmonic potential for the heavy atom $v_{1}$ stretching mode. For OHF-, the MP2 potential energy cut along the $R_{O H}$ coordinate can be fit to a Morse function; this is shown in Figure 6-7. The $R_{O H}$ coordinate is an extremely good approximation to $Q_{3}$ if $R_{O F}$ is kept fixed, as seen in Equations (10) and (11), so the Morse potential in Figure 6-7 is assumed to be the potential energy along the $Q_{3}$ coordinate. The one-dimensional fit to this function yields $\omega_{e}=2015 \mathrm{~cm}^{-1}$ (as expected from Table 6-VI) and $\omega_{e} x_{e}=350$ $\mathrm{cm}^{-1}$. A harmonic potential is constructed along $Q_{1}$ using the MP2 frequency $\left(433 \mathrm{~cm}^{-1}\right)$ from Table 6-VI. The required initial wave packet, $\phi(0)$, is then set equal to the ground vibrational wave function of this anion potential surface.

The simulation is performed by propagating the initial wave packet on a grid in two dimensions, under the influence of the neutral collinear potential surface. The propagation is continued until all flux has left the grid. Absorbing boundaries are imposed at the edges of the grid; ${ }^{61}$ this device greatly reduces the configuration space the grid must span, and thus, the calculation time. All computational details of the method are contained in ref. 7 and the relevant parameters are listed in the figure captions for each simulation. Figure 6-8 shows the simulated photoelectron spectrum of $\mathrm{OHF}^{-}$resulting from wave packet propagation on the ${ }^{3} \Pi a b$ initio surface. The result is exact within the collinear approximation.

Figure 6-9 shows the wave packet dynamics that give rise to this simulation. The potential surface is plotted in the mass-scaled coordinates defined in ref. 7; essentially $x=3 \mathrm{R}_{\mathrm{OF}}$ and $y=\mathrm{R}_{\mathrm{OH}}$. These coordinates almost exactly parallel the normal coordinates of the ion $Q_{1}$ and $Q_{3}$. The first frame (Figure 6-9a) shows the initial wave packet, corresponding to the anion wave function. The asymmetric shape 
of the wave packet derives from the pronounced anharmonicity along $Q_{3}$. The wave packet sits right over the saddle point of the reaction surface (marked in the Figure with a cross), underlining the sensitivity of the photoelectron spectrum to features of the surface at the saddle point and to the magnitude of the barrier height. The initial wave packet also extends into both the reactant valley and around the reaction 'corner' into the product valley.

The following frames of Figure 6-9 show the bifurcation of the wave packet into both valleys; the subsequent disappearance of the wave packet out to fragments is very swift. The component of the wave packet exiting out to $O\left({ }^{3} \mathrm{P}\right)+\mathrm{HF}$ has a faster rate of disappearance from the Franck-Condon region than the component travelling down the entrance valley. The latter component, moving parallel to the $x$ axis and out to $\mathrm{F}+\mathrm{OH}$, shows little vibrational excitation as compared to that moving in the $\mathrm{O}\left({ }^{3} \mathrm{P}\right)$ + HF exit valley, where considerable nodal structure, corresponding to HF vibrational excitation, is seen perpendicular to the direction of motion. In Figure 6-9d the last component of the wave packet is seen leaving the grid; the shape of the wavepacket is distorted here because of the absorbing boundary.

In accord with the extremely fast movement of the wave packet away from its nascent position on the neutral potential energy surface, the autocorrelation function shows very fast decay and evidence of only a weak recurrence. The absolute value of the autocorrelation function, $|\mathrm{C}(\mathrm{t})|$, is plotted in Figure 6-10. The fast fall-time is in strong contrast with the long-lived recurrences in the autocorrelation functions calculated for bihalide photodetachment. ${ }^{7,62,63}$ The Fourier transform of this autocorrelation function yields a broad, but structured, simulated photoelectron spectrum and it is this that is shown with the dashed line in Figure 6-8. 
In this simulation, the two low intensity peaks between the product and reactant asymptotes are from transitions to states of the complex that correlate to different product $\mathrm{HF}$ vibrational levels. The single, more intense feature near the reactant asymptote is narrower than the other peaks (FWHM $<200 \mathrm{meV}$ versus $\approx 300$ $\mathrm{meV}$ ). This peak is most likely due to the component of the wave packet moving out along the entrance valley to $\mathrm{F}+\mathrm{OH}$, which, as noted above, leaves the Franck-Condon region more slowly than that departing along the exit valley.

To confirm these 'assignments', we have performed a one-dimensional simulation that treats only the potential along the $y$, or $\mathrm{R}_{\mathrm{OH}}$, coordinate with the mass weighted coordinate $x$ fixed at its value for the ion equilibrium geometry, $\mathbf{x}_{\mathrm{e}}$. This, to a good approximation, is the effective potential for the hydrogen stretching $v_{3}$ mode. In Figure 6-11 these one-dimensional potentials for the anion and neutral are shown along with the first few eigenvalues and eigenfunctions supported by each potential. The anion potential is the ${ }^{2} \Pi$ Morse potential shown in Figure 6-7, and the neutral potential results from taking a cut through the fitted ab initio $\mathrm{F}+\mathrm{OH}$ surface at $x=\mathrm{x}_{\mathrm{e}}$. By computing the Franck-Condon overlap between the ground state wave function supported by the anion potential with the first few neutral wave functions, a simulated stick spectrum, shown also in Figure 6-8, is generated.

The one-dimensional stick spectrum consists of a progression in the $v_{3}$ mode of the [OHF] complex; the peak labels in Figure 6-8 correspond to the energy levels in Figure 6-11. Peaks 0, 1 and 2 result from transitions to the $\mathrm{O}+\mathrm{HF}$ product (exit) valley, while an inspection of the one-dimensional neutral wave functions (Figure 6-11) shows that the $\mathrm{v}_{3}=3$ level is localized in the $\mathrm{F}+\mathrm{OH}$ reactant (entrance) valley. Peak 3 in the one-dimensional simulation lines up with the intense peak in 
the two-dimensional simulation, indicating that the latter is indeed due to a state of the complex localized in the reactant valley. Peaks 0 and 1 apparently correspond to the two peaks in the two-dimensional simulation between the reactant and product asymptotes, although the one-dimensional peaks occur at slightly higher electron kinetic energy. The comparison between the simulations suggests that peak 2 is hidden as a shoulder in the two-dimensional simulation. In fact, the intense peak in the two-dimensional simulation has a definite asymmetry and it can be readily confirmed that there is an intensity contribution, corresponding to the $v_{3}=2$ stick, on the high electron energy side of this peak. The one-dimensional treatment is approximate in that it does not consider overlap at different values of $x$, and the timescale separation of $x$ and $y$ is strictly only appropriate for extreme heavy-light-heavy systems. $^{5}$ It is these inadequacies that are presumably responsible for the onedimensional sticks not coinciding with the centers of the two-dimensional peaks. However this analysis does provide some insight into the appearance of the twodimensional simulation.

Let us now compare the simulated and experimental spectra (Figure 6-8). The two-dimensional peak widths and the spacings of the product progression agree reasonably well with experiment, and the intensity distribution is in qualitative agreement with the experimental profile. The overall agreement between experimental and simulated peak widths is particularly striking as it indicates that rapid dissociation on a collinear surface can explain the broad experimental peaks. On energetic grounds we assign peaks A-D to states of the [OHF] complex that dissociate to $\mathrm{O}+\mathrm{HF}$ products. Based on the one- and two-dimensional simulations, 
we assign the intense experimental peak at $0.80 \mathrm{eV}$ (E, Figure 6-3) as a reactant peak: a state of the $[\mathrm{OHF}]$ complex that dissociates to $\mathrm{F}+\mathrm{OH}$ reactants.

However, in Figure 6-8, the one- and two-dimensional simulations have been shifted by $0.22 \mathrm{eV}$ to higher electron kinetic energy so that the experimental peak $\mathrm{E}$ lines up with the most intense simulated peak. An even larger shift would he expected if the simulation had been performed on a comparable three-dimensional potential energy surface rather than the collinear surface used here; this additional shift will be approximately equal to the bending zero point energy near the saddle point on the three-dimensional surface. ${ }^{64,65}$ This bending zero point energy is estimated to be $\approx 0.05 \mathrm{eV}{ }^{66}$ Thus simulations on a comparable three-dimensional surface would need to be shifted by about $0.27 \mathrm{eV}$ to higher electron kinetic energy. The significance of this shift is discussed in the following section.

In our analysis of the bihalide spectra, ${ }^{5,7}$ we commented extensively on the role of excited electronic states of the neutral in our photoelectron spectra, and contributions from excited states are likely in the spectra presented here as well. For the $\mathrm{F}+\mathrm{OH}$ system, we can explicitly model the contribution of one excited state, the ${ }^{1} \Delta$ state, to the photoelectron spectrum, because a potential energy surface is available for this state. ${ }^{23}$ We shall defer a full discussion of the electronic structure of this and other excited states until later, but here we show the results of performing an identical simulation, using the $\operatorname{RMOS}^{1} \Delta$ surface of Sloan and coworkers, to that described above for the ${ }^{3} \Pi$ surface. The wave packet dynamics should be quite different on the ${ }^{1} \Delta$ surface, which adiabatically connects $\left.F+\mathrm{OH}^{2} \Pi\right)$ to $H F\left({ }^{1} \Sigma^{+}\right)+O\left({ }^{1} D\right)$. This is an endoergic process for which the barrier is expected to lie on the $O\left({ }^{1} \mathrm{D}\right)+\mathrm{HF}$ side of the potential energy surface, and this is borne out by the 
$a b$ initio calculated surface. Thus, photodetachment to the ${ }^{1} \Delta$ surface should result in less overlap with the saddle point region than photodetachment to the ${ }^{3} \Pi$ surface. Figure 6-12 shows the contribution of the ${ }^{1} \Delta$ simulation to the overall photoelectron spectrum. Both the ${ }^{3} \Pi$ and ${ }^{1} \Delta$ simulations havr been shifted to higher electron kinetic energy by the same $0.22 \mathrm{eV}$ discussed above. The electronic transition dipole moments for transition from ion ground state to the neutral ${ }^{3} \Pi$ and ${ }^{1} \Delta$ states have been set in the ratio 1:5 to best reproduce experiment, in the absence of any other data. By including the ${ }^{1} \Delta$ state, the simulation now reproduces the broad experimental peak $(\mathrm{F})$ at $0.45 \mathrm{eV}$. The overall agreement between the simulated and experimental spectra is quite remarkable considering that all potential parameters are the 'raw' $a b$ initio ones, including the energy separation between the two electronic state progressions. This would seem to be strong evidence for the overlapping contribution of excited states in the experimental photoelectron spectrum.

\section{Discussion}

\subsection{OHF- $^{-}$photoelectron spectrum}

Let us return to consider the neutral reaction surface that dominates the $\mathrm{OHF}^{-}$photoelectron spectrum, the ${ }^{3} \Pi$ surface. We wish to assess how realistic this surface is, and apply what we learn to the fluorine + alcohol reaction surfaces. Although there is qualitative agreement between the simulated and experimental profiles, there are some serious deficiencies. We shall discuss these discrepancies, and some possible causes, in the hope of obtaining a more detailed picture of the neutral potential energy surface in the transition state region. 
The three major areas in which the theoretical fit differs from the experimental spectrum are (i) the number of peaks in the product vibrational progression and their positions relative to the reactant peak, (ii) the large shift required for the entire simulation, and (iii) the intensity distribution of peaks corresponding to product states. These deviations are almost certainly due to discrepancies in the neutral potential energy surface and/or the equilibrium properties of the anion. Because $a b$ initio descriptions of potential minima are known to be predicted to a higher degree of accuracy than points on a global surface far away from minima, we shall make the assumption in the following discussion that the anion structure and dissociation energy have been correctly described, and that all changes need to be made to the neutral surface. This is not likely to be completely true, but merely reflects the likely relative error bars on the two calculations.

One obvious problem is that the reaction exoergicity on the RMOS ${ }^{3} \Pi$ surface is incorrect. The RMOS fit to the FCI/4-31G exoergicity is $1.198 \mathrm{eV}$ as compared to the experimental value, $1.502 \mathrm{eV}$ (these figures reflect bottom of the well energies for the diatomic molecules). Sloan et al. have shown that this problem stems mainly from the basis set used in the $a b$ initio calculation. ${ }^{23}$ This gross defect is certainly contributing to the absence of any structure in the simulation at electron kinetic energies higher than $2.0 \mathrm{eV}$ (Figure 6-8). With the surface as is, it is energetically impossible for a state to exist with an energy corresponding to the step at highest eKE in the experimental spectrum. Clearly, in any attempt to improve the simulation by changing the neutral potential energy surface, the exoergicity should be corrected.

The other feature of the $\mathrm{F}+\mathrm{OH}^{3} \Pi$ surface that calls for some adjustment is the barrier height. A major discrepancy between the simulation on the RMOS surface 
and the experimental spectrum was that the simulated band origins had to be displaced to higher electron kinetic energy to get the largest feature (assigned to the reactant channel) to match the experimental peak. For the ${ }^{3} \Pi$ surface, the saddle point lies in the center of the Franck-Condon region, so lowering the barrier height will shift the spectrum in the required direction. As the shift required, $0.27 \mathrm{eV}$, is considerably larger than the expected error in the ion binding energy $(<0.15 \mathrm{eV})$, it seems probable that the barrier on the reaction surface is indeed too high.

To show the effect of using a smaller barrier we have performed two further simulations. We first scaled ${ }^{67}$ the RMOS surface so as to reproduce the experimental exoergicity; this yielded a surface we shall call RMOS-A. A further round of scaling $^{68}$ was performed on RMOS-A in order to approximately halve the barrier to $0.24 \mathrm{eV}(5.5 \mathrm{kcal} / \mathrm{mol})$. This surface we denote RMOS-B. These surfaces are not suggested as optimized potential functions that reproduce our data, but they do demonstrate the result of some very simple improvements over the pure $a b$ initio surface. The simulated photoelectron spectra calculated on each of these surfaces, while ignoring the ${ }^{1} \Delta$ state, are shown in Figure 6-13. The changes are significant. The RMOS-A simulation shows three, rather than two, resolved members of the product vibrational progression. In particular a feature corresponding to experimental peak D now appears. However the entire simulated spectrum must still be shifted, again to higher electron kinetic energy, to align the simulated reactant peak with the experimental peak $\mathrm{E}$; for this surface the shift is $0.23 \mathrm{eV}$, or $0.28 \mathrm{eV}$ including the zero point bend correction.

In the RMOS-B simulation (Figure 6-13b), when including the zero point bend correction, no shift is required to line up experimental peak $\mathrm{E}$ with the intense peak 
in the simulation. Decreasing the barrier height has thus removed the discrepancy between the energetics in the simulated and experimental spectra. The simulated peaks are still quite broad, in agreement with experiment. However, only two product peaks are discernible in the RMOS-B simulation, just as in the first simulation in Figure 6-8. As in that simulation, the third peak in the product progression is hidden under the intense reactant peak. Essentially, in comparison to the RMOS-A simulation, lowering the barrier has shifted the intense reactant peak to higher electron kinetic energy while not affecting the product peaks, thereby obscuring the third product peak that appeared in the RMOS-A simulation.

One important feature we have not attempted to change is the location of the saddle point on the $\mathrm{F}+\mathrm{OH}$ surface. This is likely to have a major effect in a simulation. In particular, if the barrier occurred earlier in the $\mathrm{F}+\mathrm{OH}$ reactant valley, the anion would have better overlap with states of the [OHF] complex that dissociate to $\mathrm{O}+\mathrm{HF}$ products and these states would be shifted in energy closer toward the product asymptote.

In any case, the simulation on the RMOS-B surface has shown that a lower barrier can eliminate the need to shift the spectrum. Considering the errors in the calculated ion binding energy and the approximate treatment of the zero point bending energy we conclude that a realistic barrier height for the ${ }^{3} \Pi$ surface is in the range $0.09-0.39 \mathrm{eV}(2-9 \mathrm{kcal} / \mathrm{mol})$. This result is significant as it implies that even at room temperatures direct hydrogen abstraction may compete with the non-adiabatic pathway suggested by Sloan et al.

Is this proposed barrier height reasonable? By comparison to other reactions of fluorine, the original $a b$ initio barrier, $12 \mathrm{kcal} / \mathrm{mol}$, might be considered 
unreasonably large for an abstraction reaction of this type. In general, $a b$ initio reaction barriers reported are consistently too high, even with what must be considered current state-of-the-art methods. ${ }^{69,70}$ It is very likely that problems of incomplete basis set, and thus errors resulting from basis set superposition error, will be manifested in the raw fitted $a b$ initio surface used here.

The Evans-Polanyi relationship ${ }^{71}$ between reaction exoergicity and barrier height states that the larger the exoergicity, the lower and earlier the classical barrier is on the reaction potential surface. This postulate has been well tested for hydrogen abstraction reactions. From this postulate, it would be expected that the reactions studied here would occur on surfaces with barriers comparable with that for $\mathrm{F}+\mathrm{H}_{2}$ and smaller than that for $\mathrm{F}+\mathrm{H}_{2} \mathrm{O}$ (see exoergicities in Table 6-I). Stevens et al. ${ }^{72}$ have estimated a $4 \mathrm{kcal} / \mathrm{mol}$ barrie for $\mathrm{F}+\mathrm{H}_{2} \mathrm{O}$; the classical barrier height for $\mathrm{F}+\mathrm{H}_{2}$, although subject to some controversy, is currently considered to be $1.4-2.5$ $\mathrm{kcal} / \mathrm{mol} .^{69,70}$ The larger exoergicity for $\mathrm{F}+\mathrm{OH}$ and $\mathrm{F}+\mathrm{HOR}$ would seem to indicate that the barriers for direct abstraction for both reactions should be in the range $1-2$ $\mathrm{kcal} / \mathrm{mol}$. The result of our simulations, while in agreement with a substantially lower barrier than that calculated $a b$ initio for $\mathrm{F}+\mathrm{OH}$, seem to suggest a barrier somewhat higher than that predicted by the Evans-Polanyi relationship.

It is to be hoped that an ab initio reaction potential surface of improved quality can be generated which will confirm the lower barrier height for the direct abstraction process. In order to fully model the spectrum, it may be necessary to develop excited state poteritial energy surfaces as well. The role of low-lying excited surfaces is explored in part (c) below. 


\section{$4.2 \mathrm{CH}_{3} \mathrm{OHF}^{-}$and $\mathrm{C}_{2} \mathrm{H}_{5} \mathrm{OHF}^{-}$photoelectron spectra}

The above discussion and simulations on the $\mathrm{F}+\mathrm{OH}{ }^{3} \Pi$ surface serve as a model for understanding the photoelectron spectra of the alcohol complexes. The similarity of the OHF- spectrum to those of the polyatomic systems encourages one to think that the effect of the alkyl group is small, and that the ROHF- spectra can be largely explained by the analysis of the OHF- spectrum. In particular, the basic step structure and the peak widths observed can be understood in terms of the twodimensional model above, the differences in intensity distributions in the three sfectra are explained by the differing position of the bridging hydrog $2 n$ in the ion, and the missing low election energy, seak in the ROHF- spectra (peak F in the OHFspectrum, Figure 6-3) is attributable to the differing electronic structure in the [ROHF] and [OHF] complexes (see below).

However, as pointed out above, the features in the ROHF- spectra are, in general, broader than those in the OHF- spectrum. One could blithely attribute this to the presence of additional vibrational modes in the polyatomic systems. We would like to examine this more quantitatively to ascertain which, if any, of these vibrational modes play a major role in the appearance of the ROHF- photselectron spectra. We do this by attempting to determine which of the additional modes in the polyatomic ROH and RO fragments are excited subsequent to ROHF- photodetachment. These are referred to as 'bath' modes; in the case of $\mathrm{CH}_{3} \mathrm{OHF}$ - photodetachment, these include all the $\mathrm{CH}_{3} \mathrm{O}$ vibrations and all the $\mathrm{CH}_{3} \mathrm{OH}$ vibrations except the $\mathrm{O}-\mathrm{H}$ stretch.

We consider which fragment modes are excited solely on the basis of the differences between the geometry of the neutral complex created by photodetachment and the equilibrium geometries of the fragments. This is a variation of the 'Franck- 
Condon picture' which has been shown to provide a reasonable zero-order description of fragment excitation in photodissociation experiments. ${ }^{73}$ In the classical picture of photodetachment, the nuclear configuration of the neutral complex formed is the same as the equilibrium geometry of the ion. The displacement of the $\mathrm{C}, \mathrm{H}$ and $\mathrm{O}$ atoms in the $\left[\mathrm{CH}_{3} \mathrm{OHF}\right]$ complex from the equilibrium geometry of free $\mathrm{CH}_{3} \mathrm{OH}$ and $\mathrm{CH}_{3} \mathrm{O}$ will then lead to vibrations in the bath modes of these species. Mapping these displacements into changes along each of the normal coordinates of the free fragment, we can estimate the degree of vibrational excitation in each of the fragment modes. As a guide, we shall use the $a b$ initio changes in bond lengths and angles in the methoxy frame from $\mathrm{CH}_{3} \mathrm{OHF}-$ to $\mathrm{CH}_{3} \mathrm{OH}$ and $\mathrm{CH}_{3} \mathrm{O}$.

The $a b$ initio calculations (Table 6-III) show that the changes in geometry in the $\mathrm{CH}_{3} \mathrm{O}$ frame between $\mathrm{CH}_{3} \mathrm{OHF}^{-}$and $\mathrm{CH}_{3} \mathrm{OH}$, wile small, are principally in the $\mathrm{C}-\mathrm{O}$ bond length and the bond angles about the carbon atom; the $\angle \mathrm{COH}$ angle is not dramatically altered. Further the calculated MP2 force field of $\mathrm{CH}_{3} \mathrm{OH}$ gives us the normal coordinates for this molecule. By performing the transformation of the MP2 internal coordinate changes into $\mathrm{CH}_{3} \mathrm{OH}$ normal coordinates displacements, we determine which bath modes receive excitation in this simplified photodetachment process. The only mode appreciately excited is $\mathrm{v}_{7}\left(\mathrm{CH}_{3}\right.$ rock), whose observed fundamental frequency is $1060 \mathrm{~cm}^{-1} .^{35}$ The degree of vibrational excitation is, however, still small; if we compute Franck-Condon factors assuming two harmonic oscillators of the same frequency, for anion and neutral, displaced by the calculated value, a short progression is predicted with a $(v=1) /(v=0)$ peak intensity ratio of $14 \%$.

The changes in geometry from $\mathrm{CH}_{3} \mathrm{OHF}-$ to $\mathrm{CH}_{3} \mathrm{O}$ are also small; for the $\mathrm{CH}_{3} \mathrm{O}$ equilibrium geometry we use the UMP2/6-31++G** optimized structure calculated in 
C, symmetry, which describes the lower Jahn-Teller component ( $\left.A^{\prime}\right) .^{42}$ Performing the the internal coordinate transformation into displacements of $\left.\mathrm{CH}_{3} \mathrm{O}^{2} \mathrm{~A}^{\prime}\right)$ normal coordinates (this time derived from the UHF/6-31++ $\mathrm{G}^{* *}$ force field of $\mathrm{CH}_{3} \mathrm{O}$ ), we determine that no mode is significantly excited, i.e. no mode has $(v=1) /(v=0)$ intensity ratio greater than $5 \%$.

This 'Franck-Condon picture' therefore predicts relatively small effects in the $\mathrm{CH}_{3} \mathrm{OHF}$ - spectrum from the presence of additional vibrational modes. While this treatment is quite approximate in that it is largely independent of the details of the potential energy surface in the $\mathrm{F}+\mathrm{CH}_{3} \mathrm{OH}$ transition state region, we point out that the product state-resolved studies ${ }^{19}$ show that only $2 \%$ of the energy released in the $\mathrm{F}+\mathrm{CH}_{3} \mathrm{OH}$ reaction appears as vibrational excitation in the $\mathrm{v}_{3} \mathrm{C}-\mathrm{O}$ stretching mode of $\mathrm{CH}_{3} \mathrm{O}$. Although this was the only product vibrational mode investigated, these results suggest that the bath modes play only a minor role in the dynamics of the $F$ $+\mathrm{CH}_{3} \mathrm{OH}$ reaction.

We still are faced with the question of explaining the differences in widths in the $\mathrm{OHF}^{-}$and $\mathrm{CH}_{3} \mathrm{OHF}^{-}$photoelectron spectra. Our ab initio calculations indicate that the $\mathrm{COH}$ angle remains strongly bent in $\mathrm{CH}_{3} \mathrm{OHF}-$, as in $\mathrm{CH}_{3} \mathrm{OH}$. Therefore some of the energy released in the dissociation of the $\left[\mathrm{CH}_{3} \mathrm{OHF}\right]$ complex to $\mathrm{HF}+$ $\mathrm{CH}_{3} \mathrm{O}$ (by far the more exothermic channel) is likely to end up in rotational motion of both fragments. This rotational excitation may well provide a mechanism for adding width to the peaks that correspond to $\mathrm{HF}+\mathrm{CH}_{3} \mathrm{O}$ product states.

It is also worth noting that the precursor $\mathrm{OHF}^{-}$and $\mathrm{CH}_{3} \mathrm{OHF}^{-}$anions are most likely characterized by a non-zero temperature and some degree of vibrational excitation will be present. In $\mathrm{OHF}^{-}$, the O-F vibration is calculated to have the 
lowest frequency $\left(\omega_{1}=433 \mathrm{~cm}^{-1}\right)$, whereas in $\mathrm{CH}_{3} \mathrm{OHF}-$ there are three low frequency modes (Table 6-IV): $\omega_{9}=391 \mathrm{~cm}^{-1}$ (O-F stretch), $\omega_{10}=167 \mathrm{~cm}^{-1}$ (FOC bend), and $\omega_{15}$ $=77 \mathrm{~cm}^{-1}$ (hindered rotation). Assuming the two anions are formed at the same temperature, the excitation of the additional low frequency modes in $\mathrm{CH}_{3} \mathrm{OHF}-$ could lead to broader features in the photoelectron spectrum.

\subsection{Electronic effects}

In the analysis of the photoelectron spectra of $\mathrm{BrHI}^{-}, \mathrm{ClHI}^{-}$and $\mathrm{FHI}^{-}$, we presented $^{7}$ a full discussion of the role of multiple neutral electronic surfaces. For the $\mathrm{F}+\mathrm{CH}_{3} \mathrm{OH}$ and $\mathrm{F}+\mathrm{C}_{2} \mathrm{H}_{5} \mathrm{OH}$ systems the situation is similar to those $\mathrm{X}+\mathrm{HY}$ systems. In the entrance valley, the three-fold spatial degeneracy of the ${ }^{2} \mathrm{P} F$ atom (neglecting spin-orbit interactions) is broken by the approach of ROH, and all three resultant states may contribute to the photoelectron spectra. The states that correspond to approach of $\mathrm{F}$ on the $\mathrm{H}-\mathrm{O}$ axis of $\mathrm{HOR}$ with the unpaired electron in a $\pi$ type orbital will be much more repulsive than the ground state $\sigma$ type interaction. Morokuma et al. ${ }^{74}$ have shown from $a b$ initio work that there is a contribution from the first electronically excited neutral state in the photoelectron spectrum of $\mathrm{ClHCl}^{-}$ that closely overlaps the band to the ground state. Experimentally we have recently determined that transitions to excited $\mathrm{F}+\mathrm{H}_{2}$ potential energy surfaces overlap

transitions to the ground state surface in the photoelectron spectrum of $\mathrm{FH}_{2}-{ }^{-75}$ It is reasonable to expect this to occur in the $\mathrm{ROHF}^{-}$spectra also.

For $\mathrm{F}+\mathrm{OH}$ there are many more low lying electronic states than for $\mathrm{X}+\mathrm{HY}$, even when ignoring spin-orbit coupling and constraining the geometry to be linear. These can be considered either by constructing a full correlation diagram or by using 
the molecular orbitals of the anion (Figure 6-6) and depicting the various [OHF] states formed upon removal of an electron. The latter is simpler and more useful as it includes the selection rule for a photodetachment process, namely that only oneelectron processes are allowed. This reduces the number of neutral states to be considered. Sloan et al. considered four potential surfaces, ${ }^{3} \Pi,{ }^{3} \Sigma,{ }^{1} \Delta$, and ${ }^{1} \Pi$, all of which are accessible from the ${ }^{2} \Pi$ anion. ${ }^{23}$ We have already looked at two of these poter tial energy surfaces in the simulation section.

To estimate if the other states will play a role in the photoelectron spectrum we have performed an $a b$ initio calculation for the electronic energy of each state at the anion geometry. Table 6-VIII shows these energies, and the electronic configurations of each state with reference to anion molecular orbitals shown in Figure 6-6. These energies, which should be viewed as crude estimates only, indicate roughly where maximum intensity will occur in a photoelectron band to this state. They do not indicate where the onset of vibrational (or continuum) structure assignable to the respective surface would occur in the photoelectron spectrum. The energies in Table 6-VIII are calculated using single-reference wave functions that also suffer from spin contamination; the effects of the latter have been projected out by the standard method available in the Gaussian 88 program. $^{76}$ The above ab initio problems were not an issue for the anion calculations presented earlier. In comparison to the results in Table 6-VIII, the multi-reference calculation of Sloan et al. ${ }^{23}$ orders the states slightly differently: ${ }^{3} \Pi,{ }^{3} \Sigma,{ }^{1} \Delta,{ }^{1} \Pi$. Further the RMOS fitted surfaces yields a ${ }^{3} \Pi-{ }^{1} \Delta$ separation at the anion geometry of only $0.63 \mathrm{eV}$. Apparently all four surfaces should be considered before assigning all of the experimental 
spectrum's features. In addition, the inclusion of the spin-orbit interaction will result in even more potential energy surfaces to be considered.

\section{Summary}

We have shown that the photoelectron experiment successfully probes the transition state of an asymmetric triatomic hydrogen abstraction reaction, namely the $\mathrm{F}+\mathrm{OH}$ reaction. The nature of the system, where all atoms are first row and consequently few electrons are involved, makes it amenable to a high level $a b$ initio potential surface characterization. We hope the results presented here will stimulate such theoretical interest. The photoelectron spectra of $\mathrm{CH}_{3} \mathrm{OHF}^{-}$and $\mathrm{C}_{2} \mathrm{H}_{5} \mathrm{OHF}^{-}$ have demonstrated the extension of our method to polyatomic reactions, and have shown that vibrational structure at the transition state can still be resolved even when the transition species has ten atoms. The interpretation of our spectra is relatively simple at a qualitative level and mirrors the work on the bihalide systems. A simulation that explicitly treats the collinear dynamics of $\mathrm{F}+\mathrm{OH}$, using a multireference $a b$ initio potential surface, has been performed and yields reasonable agreement with the experimental result.

However a detailed understanding of the spectra is clouded by a number of difficult theoretical questions. The simulation for the $\mathrm{F}+\mathrm{OH}$ system assumes that the transition state is collinear, and ignores the effect of the bending degree of freedom on the dissociation dynamics and, thus, on the photoelectron spectra. Schatz ${ }^{77}$ has reviewed the theoretical formalism of photodetachment to the transition state of a bimolecular reaction in three dimensions. Schatz has also compared the results of exact collinear treatments, like this one, with three-dimensional $J=0$ 
Coupled Channel Hyperspherical (CCH) simulations for the $\mathrm{ClHCl}^{-}$and $\mathrm{IHI}^{-}$ photoelectron spectra. He finds good agreement in the qualitative features. For the bihalide systems, the assumption of a collinear transition state is more reasonable than it is here. There is considerable evidence ${ }^{78}$ that the $\left.\mathrm{O}^{3} \mathrm{P}\right)+\mathrm{HCl} \rightarrow \mathrm{OH}+\mathrm{Cl}$ reaction proceeds via a bent transition state; Gordon et al. $^{79}$ have calculated the saddle point geometry and find $\angle \mathrm{OHCl}=138^{\circ}$. The question then arises whether the collinear ${ }^{3} \Pi$ interaction $O\left({ }^{3} \mathrm{P}\right)+\mathrm{HF}$ is also unstable with respect to bending, and whether a bent transition state is preferred for this reaction also. However a major difference between the two reactions is that $\mathrm{O}+\mathrm{HCl}$ is approximately thermoneutral whereas $\mathrm{O}+\mathrm{HF}$ is endoergic by $34 \mathrm{kcal} / \mathrm{mol}$. The similarity in the $\mathrm{OHF}^{-}$, $\mathrm{CH}_{3} \mathrm{OHF}^{-}$, and $\mathrm{C}_{2} \mathrm{H}_{5} \mathrm{OHF}^{-}$spectra indicates that the pseudo-triatomic model fairly successfully describes the polyatomic systems' spectra. However development of theoretical methods of treating polyatomic reaction systems is clearly desirable. Further experiments from this laboratory will be forthcoming on transition state spectra for tetra-atomic systems, the results of which should be able to test theoretical methods for such systems.

\section{Acknowledgements}

Support from the Air Force Office of Scientific Research under contract No. AFOSR-91-0084 is gratefully acknowledged. We would like to thank Professor J. Wright for discussions on the rotated Morse potential surfaces. We thank the San Diego Supercomputer Center for a grant to undertake the $a b$ initio calculations. S.E.B. acknowledges fellowship support from the University of California during the course of the research presented here. 


\section{References for Chapter 6.}

1. Brooks, P. R. Chem. Rev. 1988, 88, 407; Arrowsmith, P.; Bartoszek, F. E.; Bly, S. H. P.; Carrington, T. Jr.; Charters, P. E.; Polanyi, J. C. J. Chem. Phys. 1980, 73, 5895; Grienseisen, H. P.; Xue-Jing, H.; Kompa, K. L. Chem. Phys. Lett. 1981, 82, 421; Imre, D. G.; Kinsey, J. L.; Field, R. W.; Katayama, D. H. J. Phys. Chem. 1982, 86, 2564; Buelow, S.; Radhakrishnan, G. Catanzarite, J.; Wittig, C. J. Chem. Phys. 1985, 83, 444; Kleiber, P. D.; Lyrra, A. M.; Sando, K. M.; Zafiropoulos, V.; Stwalley, W. C. J. Chem. Phys. 1986, 85, 5493; Benz, A.; Morgner, H. Mol. Phys. 1986, 57, 319; Breckenridge, W. H.; Jouvet, C.; Soep, B. J. Chem. Phys. 1986, 84, 1443; Lao, K. Q.; Person, M. D.; Xayaribon, P.; Butler, L. J. J. Chem. Phys. 1990, 92, 823

2. Bowman, R. M.; Dantus, M.; Zewail, A. H. Chem. Phys. Lett. 1989, 156, 131; Scherer, N. F.; Sipes, C.; Bernstein, R. B.; Zewail, A. H. J. Chem. Phys. 1990, 92, 5239; Dantus, M.; Bowman, R. M.; Gruebele, M.; Zewail, A. H. J. Chem. Phys. 1989, 91, 7437; Glownia, J. H.; Misewich, J. A.; Sorokin, P. P. J. Chem. Phys. 1990, 92, 3335

3. Ervin, K. M.; Ho, J.; Lineberger, W. C. J. Chem. Phys. 1989, 91, 5974

4. Moylan, C. R.; Dodd, J. D.; Han, C.; Brauman, J. I. J. Chem. Phys. 1987, 86, 5350

5. Metz, R. B.; Weaver, A.; Bradforth, S. E.; Kitsopoulos, T. N.; Neumark, D. M. J. Phys. Chem. 1990, 94, 1377

6. Weaver, A.; Metz, R. B.; Bradforth, S. E.; Neumark, D. M. J. Phys. Chem. $1988,92,5558$

7. Bradforth, S. E.; Weaver, A.; Arnold, D. W.; Metz, R. B.; Neumark, D. M. J. Chem. Phys. 1990, 92, 7205

8. Waller, I. M.; Kitsopoulos, T. N.; Neumark, D. M. J. Phys. Chem. 1990, 94, 2240

9. A communication of the $\mathrm{CH}_{3} \mathrm{OHF}^{-}$photoelectron spectrum has been published Bradforth, S. E.; Weaver, A.; Metz, R. B.; Neumark, D. M. Advances in Laser Science - IV Proceedings of the 1988 International Laser Science Conference (AIP conference proceedings 191) 1989, 657, American Institute of Physics, New York

10. Hoyermann, K.; Loftfield, N. L.; Sievert, R.; Wagner, H. Gg. Symp. (Int.) Combust. [Proc.], 18th 1981, 831

11. Wickramaaratchi, M. A.; Setser, D. W.; Hildebrandt, H.; Körbitzer, B.; Heydtmann, H. Chem. Phys. 1985, 94, 109 
12. Khatoon, T.; Hoyermann, K. Ber. Bunsenges. Phys. Chem. 1988, 92, 669

13. McCaulley, J. A.; Kelly, N.; Golde, M. F.; Kaufman, F. J. Phys. Chem. 1989, 93,1014

14. Bogan, D. J.; Kaufman, M.; Hand, C. W.; Sanders, W. A.; Brauer, B. E. J. Phys. Chem. 1990, 94, 8128

15. MacDonald, R. G.; Sloan, J. J.; Wassell, P. T. Chem. Phys. 1979, 41, 201

16. Dill, B.; Heydtmann, H. Chem. Phys. 1980, 54, 9

17. Khatoon, T.; Edelbüttel-Einhaus, J.; Hoyermann, K.; Wagner, H. Gg. Ber. Bunsenges. Phys. Chem. 1989, 93, 626

18. Meier, U.; Grotheer, H. H.; Riekert, G.; Just, Th. Chem. Phys. Lett. 1985, 115, 221

19. Agrawalla, B. S.; Setser, D. W. J. Phys. Chem. 1984, 88, 657; Agrawalla, B. S.; Setser, D. W. J. Phys. Chem. 1986, 90, 2450

20. Jonathan, N.; Melliar-Smith, S.; Okuda, S.; Slater, D. H.; Timlin, D. Mol. Phys. 1971, 22, 561; Maylotte, D. H.; Polanyi, J. C.; Woodall, K. B. J. Chem. Phys. 1972, 57, 1547; Brandt, D.; Dickson, L. W.; Kwan, L. N. Y.; Polanyi, J. C. Chem. Phys. 1979, 39, 189; Tamagake, K.; Setser, D. W.; Sung, J. P. J. Chem. Phys. 1980, 73, 2203

21. Dill, B.; Hildebrandt, B.; Vanni, H.; Heydtmann, H. Chem. Phys. 1981, 58, 163

22. Walther, C.-D.; Wagner, H. G. Ber. Bunsenges. Phy₹ Chem. 1983, 87, 403

23. Sloan, J. J.; Watson, D. G.; Williamson, J. M.; Wright, J. S. J. Chem. Phys. $1981,75,1190$

24. Faigle, J. F. G.; Isolani, P. C.; Riveros, J. M. J. Am. Chem. Soc. 1976, 98, 2049

25. Larson, J. W.; McMahon, T. B. J. Am. Chem. Soc. 1983, 105, 2944

26. Lias, S. G.; Bartmess, J. E.; Liebman, J. F.; Holmes, J. L.; Levin, R. D.; Mallard, W. G. J. Phys. Chem. Ref. Data 1988, 17, Supp. 1 pp. 666, 677, 768 and 791.

27. Jasinski, J. M.; Rosenfeld, R. N.; Meyer, F. K.; Brauman, J. I. Chem. Phys. Lett. 1982, 104, 652

28. Kitsopoulos, T. N.; Waller, I. M.; Loeser, J. G.; Neumark, D. M. Chem. Phys. Lett. 1989, 159, 300 
29. Wiley, W. C.; McLaren, I. H. Rev. Sci. Instrum. 1955, 26, 1150

30. Hotop, H.; Lineberger, W. C. J. Chem. Phys. Ref. Data 1985, 14, 731

31. Batt, L.; McCulloch, R. D. Int. J. Chem. Kinet. 1976, 8, 491

32. Batt, L.; Christie, K.; Milne, R. T.; Summers, A. J. Int. J. Chem. Kinet. 1974, 6,877

33. Kerr, J. A. Chem. Rev. 1966, 66, 465

34. McMillen, D. F.; Golden, D. M. Ann. Rev. Phys. Chem. 1982, 33, 493.

35. Shimanouchi, T. Tables of Molecular Vibrational Frequencies, Consolidated Vol. I, NSRDS-NBS 391979

36. Jacox, M. E. J. Chem. Phys. Ref. Data 1988, 17, 269

37. Huber, K. P.; Herzberg, G. Molecular Spectra and Molecular Structure IV. Constants of Diatomic Molecules, Van Nostrand: New York, 1979

38. Moore, C. E. Atomic Energy Levels Vol. I, NSRDS-NBS 351971

39. Frisch, M. J.; Binkley, J. S.; Schlegel, H. B.; Raghavachari, K.; Melius, C. F.; Martin, R. L.; Stewart, J. J. P.; Bobrowicz, F. W.; Rohlfing, C. M.; Kahn, L. R.; Defrees, D. J.; Seeger, R.; Whiteside, R. A.; Fox, D. J.; Fleuder, E. M.; Pople, J. A. Carnegie-Mellon Quantum Chemistry Publishing Unit, Pittsburgh, PA, 1984

40. Frisch, M. J.; Head-Gordon, M.; Schlegel, H.B.; Raghavachari, K.; Binkley, J. S.; Gonzalez, C.; Defrees, D. J.; Fox, D. J.; Whiteside, R. A.; Seeger, R.; Melius, C. F.; Baker, J.; Martin, R. L.; Kahn, L. R.; Stewart, J. J. P.; Fluder, E. M.; Topiol, S.; Pople, J. A. Gaussian Inc., Pittsburgh, PA.

41. Amos R. D.; Rice, J. E. Cambridge Analytic Derivatives Package, Cambridge, 1987.

42. $\quad r_{e}\left(C-H_{t r}\right)=1.096 \AA, r_{e}\left(C-H_{g}\right)=1.090 \AA, r_{e}(C-O)=1.388 \AA, \angle \mathrm{OCH}_{t r}=104.6^{\circ}$, $\angle \mathrm{OCH}_{\mathrm{g}}=112.1^{\circ}, \angle \mathrm{H}_{\mathrm{tr}} \mathrm{CH}_{\mathrm{g}}=108.1^{\circ}$; for a discussion of $\mathrm{CH}_{3} \mathrm{O}$ electronic structure calculations see Saebø, S.; Radom, L.; Schaefer, H. F. J. Chem. Phys. $1983,78,845$

43. 0.9 times the Hartree Fock harmonic frequency

44. Engelking, P. C.; Ellison, G. B.; Lineberger, W. C. J. Chem. Phys. 1978, 69, 1826

45. Yates, B. Y.; Schaefer, H. F.; Lee, T. J.; Rice, J. E. J. Am. Chem. Soc. 1988, 110,6327 
46. The difference between staggered and eclipsed at the RHF/6-31++g** level for $\mathrm{CH}_{3} \mathrm{OHF}$ is $165 \mathrm{~cm}^{-1}$, compared to $402 \mathrm{~cm}^{-1}$ for $\mathrm{CH}_{3} \mathrm{OH}$. (This work)

47. Sheldon, J.; Bowie, J. Aust. J. Chem. 1983, 36, 289; Emsley, J.; Parker, R. J.; Overhill, R. E. J. Chem. Soc. Farad. Trans. 21983 79, 1347

48. Botschwina, P.; Sebald, P.: Burmeister, R. J. Chem. Phys. 1988, 88, 5246

49. Sannigrahi, A. B.; Peyerimhoff, S. D. J. Mol. Struct.(Theochem) 1988, 165, 55

50. Ikuta, S.; Saitoh, T.; Nomura, O. J. Chem. Phys. 1989, 91, 3539; Ikuta, S.; Saitoh, T.; Nomura, O. J. Chem. Phys. 1990, 93, 2530

51. Kawaguchi, K; Hirota, E. J. Chem. Phys. 1987, 87, 6838; Kawaguchi, K J. Chem. Phys. 1988, 88, 4186

52. Herzberg, G. Molecular Spectra and Molecular Structure III - Electronic Spectra of Polyatomic Molecules, Van Nostrand Rheinhold Co., New York, 1966, p. 27

53. Hehre, W.; Radom, L.; Schleyer, P. v.R.; Pople, J. A. Ab initio Molecular Orbital Theory, John Wiley, New York, 1986, p. 270

54. Handy, N. C.; Knowles, P. J.; Somasundram, K. Theor. Chim. Acta. 1985, 68, 87; Knowles, P. J.; Somasundram, K.; Handy, N. C. Chem. Phys. Lett. 1986, 113,8

55. Chalasinśki, G.; Kendall, R. A.; Simons, J. J. Chem. Phys. 1987, 87, 2965

56. Kosloff, D.; Kosloff, R. J. Comput. Phys. 1983, 52, 35; Kosloff, R. J. Phys. Chem. 1988, 92, 2087

57. Heller, E. J. Acc. Chem. Res. 1981, 14, 368

58. Lorquet, A. J.; Lorquet, J. C.; Delwiche, J; Hubin-Franskin, J. J. Chem. Phys. 1982, 76, 4692; Pollard, J. E.; Trevor, D. J.; Reutt, J. E.; Lee, Y. T.; Shirley, D. A. J. Chem. Phys. 1984, 81, 5302

59. Davidson, E. R. in The World of Quantum Chemistry, ed. Daudel, R. and Pullman, D.; Reidel, Dordrecht, Holland, 1974, p. 17

60. Schatz, G. C. Rev. Mod. Phys. 1989, 61, 669

61. Bisseling, R. H.; Kosloff, R.; Manz, J. J. Chem. Phys. 1985, 83, 993

62. Metz, R. B.; Bradforth, S. E.; Neumark, D. M. Adv. Chem. Phys. in press

63. Engel, V. J. Chem. Phys. 1991, 94, 16 
64. Bowman, J. M. Adv. Chem. Phys. 1985, 61, 115

65. Schatz, G. C.; Kuppermann, A. Phys. Rev. Lett. 1975, 35, 1266

66. This estimate is based on the bending frequency calculated at the saddle point of several three-dimensional LEPS model surfaces that describe the F + HO system and have similar barrier heights to the collinear surface used in the present simulation.

67. The parameters defining the RMOS surface were changed such that $D(\theta)$ (describing the energy along the minimum energy path) was smoothly corrected with a function that varied as $\sin (\theta) . \beta(\theta)$ was adjusted to maintain the original harmonic frequency along each Morse ray. The $l_{e}(\theta)$ parameter was left unchanged. See ref. 23 for the definition of the RMOS parameters.

68. The $D(\theta)$ values were smoothly corrected with a function that varied as $\sin (2 \theta)$ and once again $\beta(\theta)$ was corrected to maintain original harmonic frequency along each ray while $l_{e}(\theta)$ was unchanged.

69. Schaefer, H. F. J. Phys. Chem. 1985, 89, 5336

70. Băuschlicher, C. W. Jr.; Walch, S. P.; Langhoff, S. R.; Taylor, P. R.; Jaffe, R. L. J. Chem. Phys. 1988, 88, 1743

71. Evans, M. G.; Polanyi, M. Trans. Faraday Soc. 1938, 34, 11

72. Stevens, P. S.; Brune, W. H.; Anderson, J. G. J. Phys. Chem. 1989, 93, 4068

73. Band, Y. B.; Freed, K. F. J. Chem. Phys. 1975, 63, 3382

74. Yamashita, K.; Morokuma, K. J. Chem. Phys. 1990, 93, 3716

75. Weaver, A.; Metz, R. B.; Bradforth, S. E. ; Neumark, D. M. J. Chem. Phys. 1990, 93, 5352; Weaver, A.; Neumark, D. M. Disc. Faraday Soc. in press

76. Schlegel, H. B. J. Chem. Phys. 1986, 84, 4530

77. Schatz, G. C. J. Phys. Chem. 1990, 94, 6157

78. Rakestraw, D. J.; McKendrick, K. G.; Zare, R. N. J. Chem. Phys. 1987, 87, 7341

79. Gordon, M. S.; Baldridge, K. K.; Bernholdt, D. E.; Bartlett, R. J. Chem. Phys. Lett. 1989, 158, 189 
Table 6-I: Hydrogen abstraction reactions of Fluorine

\begin{tabular}{|c|c|c|c|c|}
\hline Reaction & $\begin{array}{l}\Delta \mathrm{H}_{298}^{\circ} \\
\mathrm{kcal} / \mathrm{mol}\end{array}$ & $\begin{array}{c}\Delta \mathrm{E}_{0}^{\circ}{ }^{b} \\
\mathrm{kcal} / \mathrm{mol}\end{array}$ & $\begin{array}{c}k_{298} c \\
10^{11} \mathrm{~cm}^{3} \mathrm{~s}^{-1}\end{array}$ & $\begin{array}{c}\mathrm{E}_{\mathrm{b}}^{\mathrm{d}} \\
\mathrm{kcal} / \mathrm{mol}\end{array}$ \\
\hline $\mathrm{F}+\mathrm{H}-\mathrm{O}^{\mathrm{e}}$ & -34.0 & -34.0 & 4.1 & \\
\hline $\mathrm{F}+\mathrm{H}-\mathrm{OCH}_{3}$ & -32.2 & -32.6 & 7.7 & \\
\hline $\mathrm{F}+\mathrm{H}-\mathrm{OC}_{2} \mathrm{H}_{5}$ & -32.0 & -32.3 & 6.3 & \\
\hline $\mathrm{F}+\mathrm{H}-\mathrm{H}$ & -32.1 & -32.1 & 2.5 & -2 \\
\hline $\mathrm{F}+\mathrm{H}-\mathrm{OH}$ & -17.2 & -17.5 & 1.4 & 4 \\
\hline $\mathrm{F}+\mathrm{H}-\mathrm{CH}_{2} \mathrm{OH}$ & -42.2 & -42.9 & $1.7^{\mathrm{r}}$ & \\
\hline $\begin{array}{c}\mathrm{F}+\mathrm{H}-\mathrm{CH}\left(\mathrm{CH}_{3}\right) \mathrm{OH} \\
\text { and } \\
\mathrm{F}+\mathrm{H}-\mathrm{CH}_{2} \mathrm{CH}_{2} \mathrm{OH}\end{array}$ & -43.2 & -43.9 & $1.4^{\mathrm{f}}$ & \\
\hline
\end{tabular}

a) Exothermicity, $\Delta \mathrm{H}_{298}^{\circ}$, calculated from $\mathrm{DH}_{298}(\mathrm{H}-\mathrm{X})$ for $\mathrm{CH}_{3} \mathrm{OH}$ (ref. 31), $\mathrm{C}_{2} \mathrm{H}_{5} \mathrm{OH}$ (ref. 32), $\mathrm{H}_{2} \mathrm{O}$ (ref. 33), $\mathrm{H}-\mathrm{CH}_{2} \mathrm{OH}$ and $\mathrm{H}-\mathrm{CH}\left(\mathrm{CH}_{3}\right) \mathrm{OH}$ (ref. 34) and $\mathrm{D}_{0}^{\circ}$ for $\mathrm{OH}$, $\mathrm{H}_{2}$ and $\mathrm{HF}$ (ref. 37 ).

b) Exoergicity, $\Delta \mathrm{E}_{0}^{\circ}$, calculated by correction to $\Delta \mathrm{H}^{\circ}{ }_{298}$ using vibrational frequencies for $\mathrm{CH}_{3} \mathrm{OH}$ and $\mathrm{H}_{2} \mathrm{O}$ (ref. 35), $\mathrm{CH}_{3} \mathrm{O}$ and $\mathrm{CH}_{2} \mathrm{OH}$ (ref. 36); vibrational corrections for ethanol reactions assumed equal to those of methanol. All molecules are assumed to be ideal gases, and both hindered and full rotations are treated classically.

c) Kinetic data collected from refs. $12,17,22$, and 72 .

d) Estimated classical barriers from refs. 69, 70, and 72.

e) Products $\mathrm{O}\left({ }^{3} \mathrm{P}\right)+\mathrm{HF}$.

f) This is the measured rate divided by the number of available chemically equivalent hydrogen atoms for abstraction. 
Table 6-II: Estimated peak centers and widths from the photoelectron spectra of

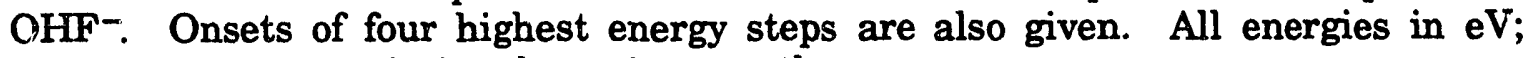
approximate uncertainties shown in parentheses.

\begin{tabular}{cccc}
\hline \multicolumn{4}{c}{ Electron kinetic energy } \\
Peak & Onset $^{\mathrm{a}}$ & Center $^{\mathrm{b}}$ & Width $^{\mathrm{b}}$ \\
\hline A & $2.46(0.01)$ & $2.25(0.02)$ & $0.35(0.03)$ \\
B & $1.95(0.02)$ & $1.82(0.02)$ & $0.31(0.03)$ \\
C & $1.51(0.02)$ & $1.38(0.04)$ & $0.30(0.04)$ \\
D & $1.16(0.02)$ & $1.01(0.02)$ & $0.30(0.04)$ \\
E & & $0.80(0.01)$ & $0.15(0.03)$ \\
F & & $0.45(0.03)$ & $\sim 0.4$ \\
\hline \hline
\end{tabular}

a) Step onsets are measured at $50 \%$ of rising edge.

b) Centers and widths are estimated by a six Gaussian fit to the photoelectron spectrum. Because bands overlap, the estimated uncertainties are large. 
Table 6-III: $A b$ initio geometries and zero point energies for $\mathrm{CH}_{3} \mathrm{OHF}^{-}$and $\mathrm{CH}_{3} \mathrm{OH}$

\begin{tabular}{|c|c|c|c|c|}
\hline & \multicolumn{2}{|c|}{$\mathrm{CH}_{3} \mathrm{OHF}^{-}$} & \multicolumn{2}{|c|}{$\mathrm{CH}_{3} \mathrm{OH}$} \\
\hline & $\begin{array}{c}\mathrm{RHF} / \\
6-31++\mathrm{G}^{* *}\end{array}$ & $\begin{array}{c}\mathrm{RMP2} / \\
6-31++\mathrm{G}^{* *}\end{array}$ & $\begin{array}{c}\mathrm{RHF} / \\
6-31++\mathrm{G}^{* *}\end{array}$ & $\begin{array}{c}\text { RMP2/ } \\
6-31++G^{* *}\end{array}$ \\
\hline$R_{e}(\mathrm{O}-\mathrm{H}) \AA$ & 1.004 & 1.059 & 0.942 & 0.964 \\
\hline$R_{e}(H-F) \AA$ & 1.462 & 1.373 & & \\
\hline$\angle \mathrm{OHF}$ & 173.8 & 175.3 & & \\
\hline$R_{e}(C-0) \dot{A}$ & 1.376 & 1.399 & 1.401 & 1.427 \\
\hline$\angle \mathrm{COH}$ & 108.4 & 106.4 & 110.5 & 108.6 \\
\hline$R_{e}\left(C-H_{t s}\right) \AA$ & 1.092 & 1.095 & 1.081 & 1.085 \\
\hline $\mathrm{R}_{\mathrm{e}}\left(\mathrm{C}-\mathrm{H}_{\mathrm{g}}\right) \AA$ & 1.094 & 1.099 & 1.087 & 1.091 \\
\hline$\angle \mathrm{OCH}_{\mathrm{tr}}$ & 109.4 & 109.3 & 107.1 & 106.2 \\
\hline$\angle \mathrm{H}_{\mathrm{sr}} \mathrm{CH}_{\mathrm{g}}$ & 107.5 & 107.6 & 108.6 & 108.9 \\
\hline$\angle \mathrm{H}_{8} \mathrm{CH}_{8}$ & 107.0 & 106.8 & 109.0 & 109.3 \\
\hline $\begin{array}{l}\text { Zero Point Energy } \\
(\mathrm{eV})\end{array}$ & 1.513 & 1.426 & 1.496 & 1.434 \\
\hline
\end{tabular}


Table 6-IV: Harmonic frequencies (in $\mathrm{cm}^{-1}$ ), calculated with $6-31++\mathrm{G}^{* *}$ basis set, for $\mathrm{CH}_{3} \mathrm{OHF}-$

\begin{tabular}{||c|c|c||c|c|c||c|c|c||}
\hline Mode & RHF & RMP2 & Mode & RHF & RMP2 & Mode & RHF & RMP2 \\
\hline \hline$\omega_{1}\left(a^{\prime}\right)$ & 3133 & 3102 & $\omega_{6}\left(a^{\prime}\right)$ & 1600 & 1498 & $\omega_{11}\left(a^{\prime}\right)$ & 3115 & 3060 \\
\hline$\omega_{2}\left(a^{\prime}\right)$ & 3106 & 3016 & $\omega_{7}\left(a^{\prime}\right)$ & 1253 & 1178 & $\omega_{12}\left(a^{\prime}\right)$ & 1610 & 1526 \\
\hline$\omega_{3}\left(a^{\prime}\right)$ & 2901 & 2215 & $\omega_{8}\left(a^{\prime}\right)$ & 1230 & 1132 & $\omega_{13}\left(a^{\prime}\right)$ & 1282 & 1248 \\
\hline$\omega_{4}\left(a^{\prime}\right)$ & 1731 & 1649 & $\omega_{9}\left(a^{\prime}\right)$ & 333 & 391 & $\omega_{14}\left(a^{\prime}\right)$ & 1214 & 1189 \\
\hline$\omega_{5}\left(a^{\prime}\right)$ & 1634 & 1556 & $\omega_{10}\left(a^{\prime}\right)$ & 168 & 167 & $\omega_{15}\left(a^{\prime}\right)$ & 94 & 77 \\
\hline
\end{tabular}

Table 6-V: $A b$ initio Energies for $\mathrm{CH}_{3} \mathrm{OHF}-$, including zero point energies.

\begin{tabular}{|c|c|c|c|c|c|c|}
\hline \multirow[t]{2}{*}{$\begin{array}{c}E_{0} \\
\text { Total Energy * }\end{array}$} & \multicolumn{2}{|c|}{$\begin{array}{l}\text { RHF/6-31++G**// } \\
\text { RHF/6-31++G**b }\end{array}$} & \multicolumn{2}{|c|}{$\begin{array}{l}\text { RMP2/6-31++G**// } \\
\text { RMP2/6-31++G** }\end{array}$} & \multicolumn{2}{|c|}{$\begin{array}{l}\text { RMP4/6-31++G**// } \\
\text { RMP2/6-31++G** }\end{array}$} \\
\hline & a.u. & $\mathrm{eV}$ & a.u. & $\mathrm{eV}$ & a.u. & eV \\
\hline $\begin{array}{l}\mathrm{CH}_{3} \mathrm{OHF}^{-} \\
\text {equilibrium }\end{array}$ & -214.45671 & 0.000 & -215.02240 & 0.000 & -215.04730 & 0.000 \\
\hline $\begin{array}{l}\mathrm{F}^{-}+\mathrm{HOCH}_{3} \\
\text { separated } \\
\text { fragments }\end{array}$ & -214.41611 & 1.104 & -214.97545 & 1.278 & -215.00124 & 1.253 \\
\hline $\begin{array}{c}\mathrm{CH}_{3} \mathrm{O}^{-}+\mathrm{HF} \\
\text { separated } \\
\text { fragments }\end{array}$ & -214.39208 & 1.758 & -214.95104 & 1.941 & -215.97621 & 1.934 \\
\hline
\end{tabular}

a) Zero Point energies calculated from force field evaluated at same level of theory as energy, except MP4 where MP2 zero point energies are used.

b) Notation " $\mathrm{a} / \mathrm{b}$ " means energy evaluated with theoretical model $a$ at the optimized geometry calculated with model $b$.

c) RMP4(SDTQ) frozen core energies. 
Table 6-VI: $A b$ initio Geometry and Frequencies for $\mathrm{OHF}^{-}$

\begin{tabular}{||c|c|c|c|c|c||}
\hline Method & $\begin{array}{c}\mathrm{R}_{\mathrm{e}}(\mathrm{O}-\mathrm{H})^{\mathrm{a}} \\
\AA\end{array}$ & $\begin{array}{c}\mathrm{R}_{\mathrm{e}}(\mathrm{H}-\mathrm{F}) \\
\AA\end{array}$ & $\angle \mathrm{OHF}$ & $\begin{array}{c}\text { Frequencies } \\
\left(\mathrm{cm}^{-1}\right)\end{array}$ & $\left\langle\mathrm{S}^{2}\right\rangle$ \\
\hline \hline UHF/6-31++G** & 1.031 & 1.400 & 180.0 & $\begin{array}{c}1070,1227 \\
2514\end{array}$ & 0.756 \\
\hline UMP2/6-31++G** & 1.078 & 1.346 & 180.0 & $\begin{array}{c}1064,1225 \\
2015\end{array}$ & 0.752 \\
\hline
\end{tabular}

a) $\quad R_{e}(\mathrm{O}-\mathrm{H})$ in the free hydroxyl radical is $0.955 \AA\left(\mathrm{UHF} / 6-31++\mathrm{G}^{* *}\right), 0.973 \AA$ (UMP2/6-31++G**) and $0.970 \AA$ (experimental, ref. 37).

Table 6-VII: $A b$ initio Energies for OHF-, including zero point energies.

\begin{tabular}{|c|c|c|c|c|c|c|}
\hline \multirow[t]{2}{*}{$\begin{array}{c}\mathbf{E}_{0} \\
\text { Total Energy" }\end{array}$} & \multicolumn{2}{|c|}{$\begin{array}{l}\text { UHF/6-31++G**// } \\
\text { UHF/6-31++G** }\end{array}$} & \multicolumn{2}{|c|}{$\begin{array}{l}\text { UMP2/6-31++G**// } \\
\text { UMP2/6-31++G** }\end{array}$} & \multicolumn{2}{|c|}{$\begin{array}{l}\text { UMP4/6-31++G**// } \\
\text { UMP2/6-31++G**b }\end{array}$} \\
\hline & a.u. & $\mathrm{eV}$ & a.u. & $\mathrm{eV}$ & a.u. & $\mathrm{eV}$ \\
\hline $\mathrm{OHF}^{-}$equilibrium & -174.84998 & 0.000 & -175.21214 & 0.000 & -175.22800 & 0.000 \\
\hline $\begin{array}{c}\left.\mathrm{F}^{-}+\mathrm{HO}{ }^{2} \Pi\right) \\
\text { separated } \\
\text { fragments }\end{array}$ & -174.80273 & 1.286 & -175.16113 & 1.388 & -175.17375 & 1.476 \\
\hline $\begin{array}{c}\mathrm{O}^{-}\left({ }^{2} \mathrm{P}\right)+\mathrm{HF} \\
\text { separated } \\
\text { fragments }\end{array}$ & -174.78075 & 1.884 & -175.13652 & 2.058 & -115.15280 & 2.046 \\
\hline
\end{tabular}
a) Zero point energies calculated from force field evaluated at same level of thenry
b) UMP4(SDTQ) frozen core energies. 
Table 6-VIII: Possible low-lying electronic states of [OHF] accessed in the photoelectron spectrum of $\mathrm{OHF}^{-}$

\begin{tabular}{||c|c|c|c||}
\hline & \multicolumn{2}{|c|}{$\begin{array}{c}\text { PUMP4(SDTQ-FC)/6-31G** } \\
\text { energy" at Anion Equilibrium } \\
\text { Geometry }\end{array}$} & $\begin{array}{c}\text { Electronic Configuration } \\
\text { (with reference to } \\
\text { Figure 6-6) }\end{array}$ \\
\hline${ }^{3} \Pi$ & a. u. & $\mathrm{eV}$ & \multicolumn{1}{|c|}{} \\
\hline${ }^{3} \Sigma$ & -175.033816 & 0.0 &. $.5 \sigma^{2} 1 \pi^{4} 2 \pi_{\mathrm{x}}{ }^{1} 2 \pi_{\mathrm{y}}{ }^{2} 6 \sigma^{1}$ \\
\hline${ }^{1} \Pi$ & -175.009523 & 0.6 &. $.5 \sigma^{2} 1 \pi^{4} 2 \pi_{\mathrm{x}}{ }^{1} 2 \pi_{\mathrm{y}}{ }^{1} 6 \sigma^{2}$ \\
\hline${ }^{1} \Delta$ & -174.931654 & 2.8 &. $.5 \sigma^{2} 1 \pi^{4} 2 \pi_{\mathrm{x}}{ }^{1} 2 \pi_{\mathrm{y}}{ }^{2} 6 \sigma^{1}$ \\
\hline
\end{tabular}

a) Spin projected UMP4 energies (see ref. 76)

b) MP2/6-31++G** anion geometry used, see Table 6-VI 
Figure Captions for Chapter 6.

Figure 6-1. Photoelectron Spectra of (top) $\mathrm{CH}_{3} \mathrm{OHF}-$ and (bottom) $\mathrm{C}_{2} \mathrm{H}_{5} \mathrm{OHF}^{-}$recorded at $213 \mathrm{~nm}$. Arrows against axis indicate energies corresponding to product and reactant asymptotes (see text). Step onsets are indicated by arrows above spectrum.

Figure 6-2. Photoelectron Spectrum of $\mathrm{CH}_{3} \mathrm{ODF}^{-}$recorded at $213 \mathrm{~nm}$. Arrows as for Figure 6-1.

Figure 6-3. Photoelectron Spectrum of $\mathrm{OHF}^{-}$recorded at $213 \mathrm{~nm}$. Arrows against the axis indicate energies corresponding to asymptotes for formation of $\mathrm{O}\left({ }^{3} \mathrm{P}\right)+\mathrm{HF}, \mathrm{F}+\mathrm{OH}$, and $\mathrm{O}\left({ }^{1} \mathrm{D}\right)+\mathrm{HF}$, in order of decreasing electron kinetic energy.

Figure 6-4. Calculated geometry for $\mathrm{CH}_{3} \mathrm{OHF}^{-}$(top) and $\mathrm{OHF}^{-}$(bottom). Bond lengths $(\AA)$, and angles, are those calculated by full geometry optimization at MP2/6-31++G**.

Figure 6-5. Potential energy profile along the hydroxyl hydrogen stretching coordinate in $\mathrm{CH}_{3} \mathrm{OHF}^{-}$. Cuts are calculated fixing geometry parameters at the MP2/6-31++G** equilibrium values and varying $\mathrm{R}_{\mathrm{OH}}$. To simplify calculations the F-H-O angle is treated as linear (this increases electronic energy by $2 \times 10^{-4}$ a.u.) and the $R_{O F}$ is fixed at the sum of MP2 equilibrium $R_{O H}$ and $R_{H F}$. $\mathrm{CH}_{3} \mathrm{OHF}^{-}$potential variation shown at $\mathrm{HF} / 6-31++\mathrm{G}^{* *}$ level (dashed - for absolute HF energy subtract 214.527 a.u.) and at MP2/6-31++G** (solid - for absolute MP2 energy subtract 215.077 a.u.) 
Figure 6-6. Schematic molecular orbital diagram for $\mathrm{OHF}^{-}$. Orbital occupancy shown is for ${ }^{2} \Pi$ ground state of anion.

Figure 6-7. Potential energy profile for ${ }^{2} \Pi$ and ${ }^{2} \Sigma$ electronic states of OHFalong hydrogen stretching coordinate at MP2/6-31++G** (solid lines). Cuts calculated with $R_{0 F}$ held at ${ }^{2} \Pi$ state equilibrium value. For absolute energies subtract 175 a.u. Dotted line shows Morse function used to approximate $\mathrm{OHF}^{-}$hydrogen stretching potential for construction of anion wavepacket.

Figure 6-8. Simulated $\mathrm{OHF}^{-}$photoelectron spectrum from two-dimensional wavepacket propagation on the ${ }^{3} \Pi$ RMOS surface (dashed) superimposed on experimental spectrum (solid). Also shown is the result of a one-dimensional simulation (sticks) described in text. Labels above sticks refer to $v_{3}$ quantum numbers (see Figure 6-11). Both simulations have been shifted by $0.22 \mathrm{eV}$ to higher electron kinetic energy (see text).

Figure 6-9. Wavepacket dynamics on the RMOS ${ }^{3} \Pi$ potential surface. Equally spaced contours of $|\Psi(t)|$ are superimposed on contours of the potential energy for (a) $t=0$, (b) $t=31$, (c) $t=62$, (d) $t=93$ femtoseconds. The potential contours drawn are for energies $0.25,0.75,1.25,1.75$ and $2.25 \mathrm{eV}$ above bottom of $\mathrm{O}{ }^{\prime 3} \mathrm{P}$ ) $+H F$ well, and the saddle point is marked by an $X$. The propagation is carried out in mass-scaled coordinates (defined in ref. 7), which are also the coordinates used in the plots, on a grid with 128 x 64 points along $x$ and $y$ respectively. A 10 point 
absorbing strip boundary is used to absorb flux leaving grid (see ref. 61). The initial wavepacket (see text for details) is propagated for 7680 time steps of 1 a.u. each.

Figure 6-10. Absolute value of the time autocorrelation function calculated in the two-dimensional propagation on the ${ }^{3} \Pi$ RMOS surface .

Figure 6-11. One-dimensional potentials for $v_{3}$ mode in $\mathrm{OHF}^{-}$and [OHF]. Anion potantial (bottom) is the Morse potential shown in Figure 6-7; neutral effective potential (upper) is a cut through ${ }^{3} \Pi$ RMOS surface at constant $x=x_{e}$ (see text). Calculated eigenstates are labelled by their $v_{3}$ quantum numbers.

Figure 6-12. Simulated $\mathrm{OHF}^{-}$- photoelectron spectrum showing contribution of ${ }^{3} \Pi$ and ${ }^{1} \Delta$ electronic surfaces. Component deriving from the ${ }^{2} \Delta$ surface is shown by short dashed line. The sum of two states' simulated photoelectron profiles, convoluted with the experimental resolution function, is shown by dashed line. Each state's profile simulated via independent wavepacket propagations; both have been shifted to higher electron kinetic energy by $0.22 \mathrm{eV}$ (see text). The initial wavepacket and propagation parameters are identical in two simuations, and are those given in caption of Figure 6-9. 
Figure 6-13. $\quad$ Simulated ${ }^{3} \Pi$ band (dashed) of $\mathrm{OHF}^{-}$photoelectron spectrum using the two scaled RMOS surfaces (see text), superimposed on the experimental spectrum (solid). (Top) The RMOS-A surface has the correct reaction exoergicity; the simulated spectrum has been shifted to higher electron kinetic energy by $0.23 \mathrm{eV}$. (Bottom) The RMOS-B surface has the correct exoergicity and a reduced barrier height of $0.29 \mathrm{eV}$. This simulated spectrum has not been shifted. The initial wavepacket and propagation parameters for both simulations are once again identical to those in Figure 6-9. 

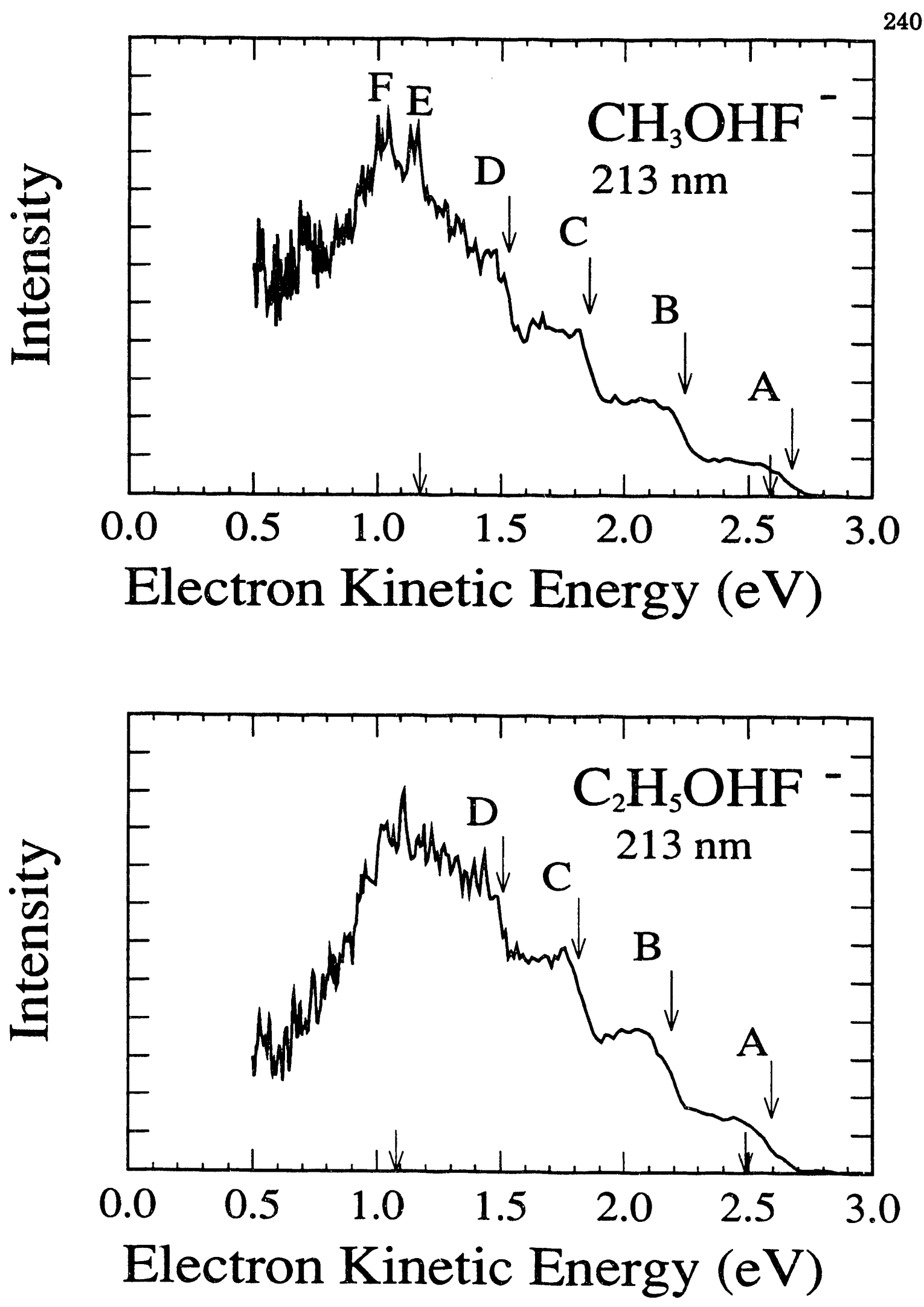

Figure 6-1 


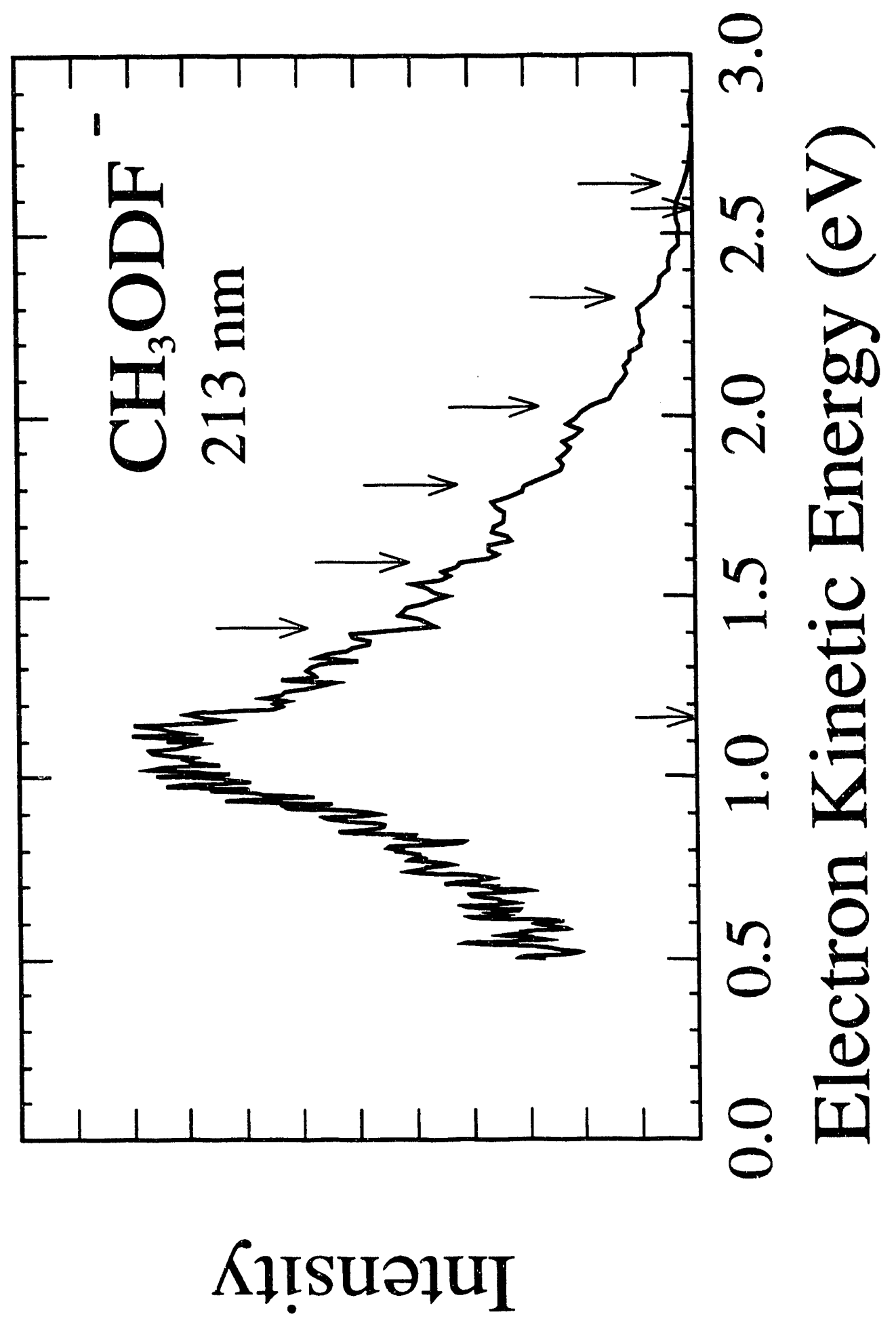

Figure 6-2 


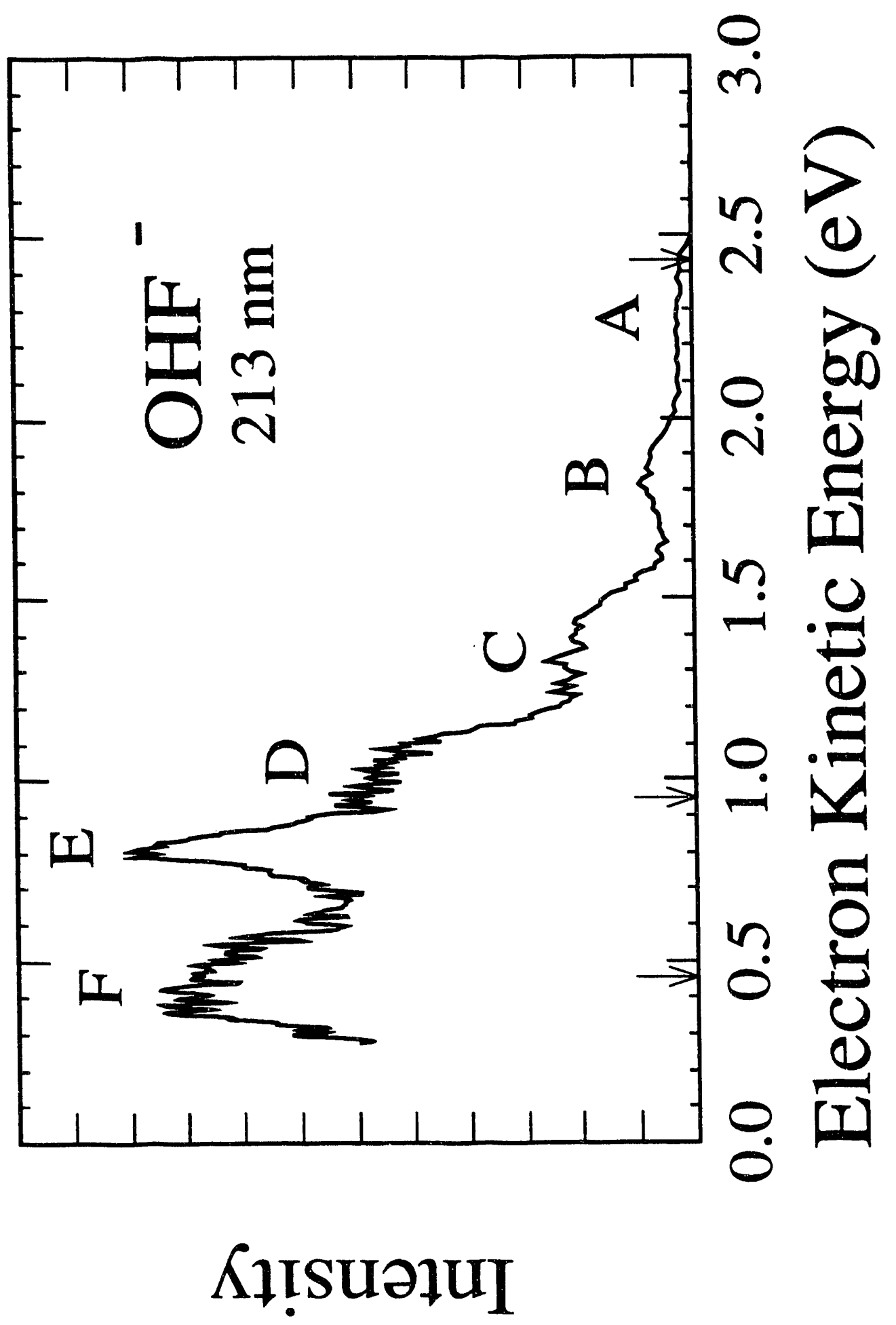

Figure 6-3 

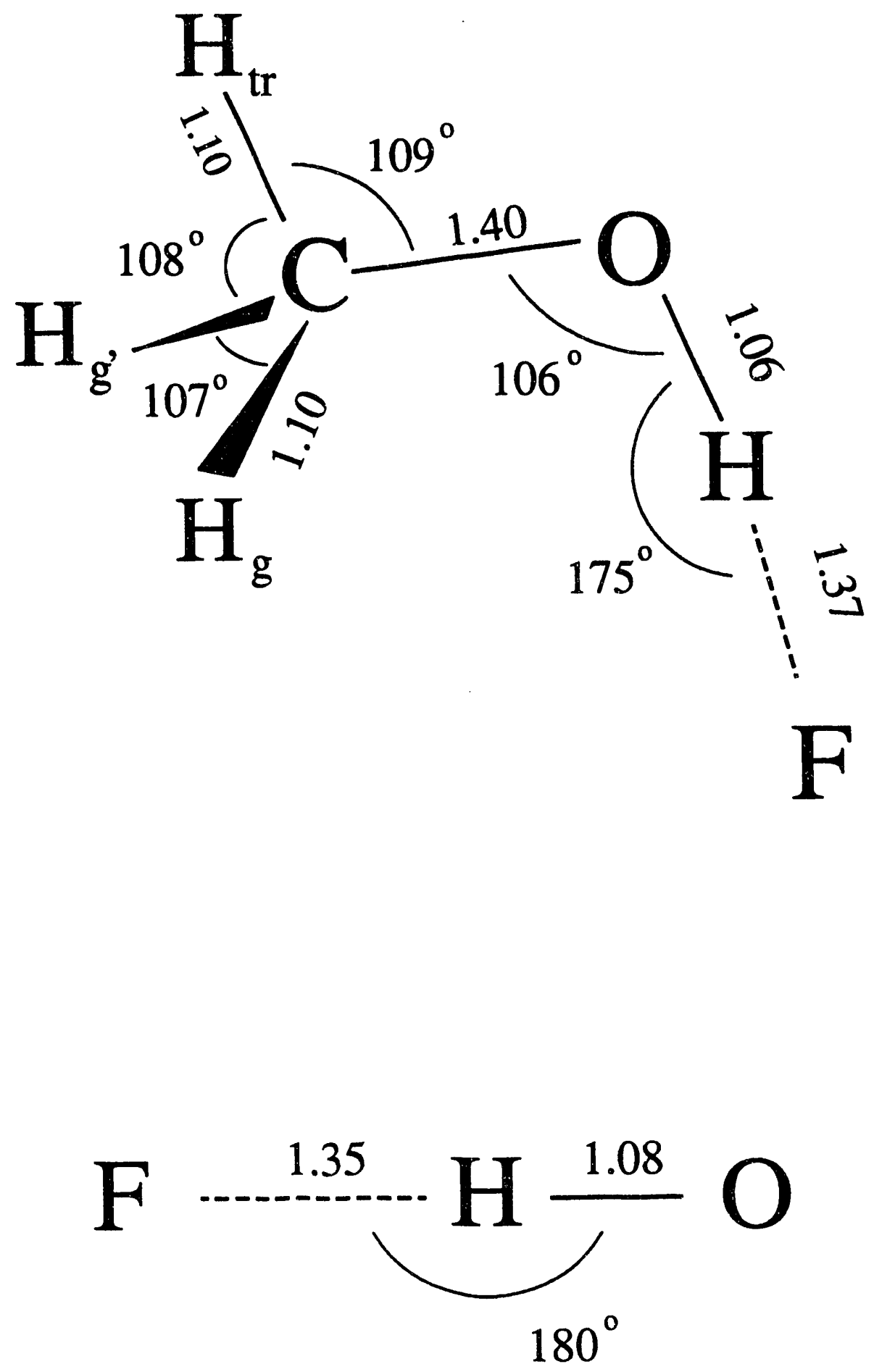

Figure 6-4 


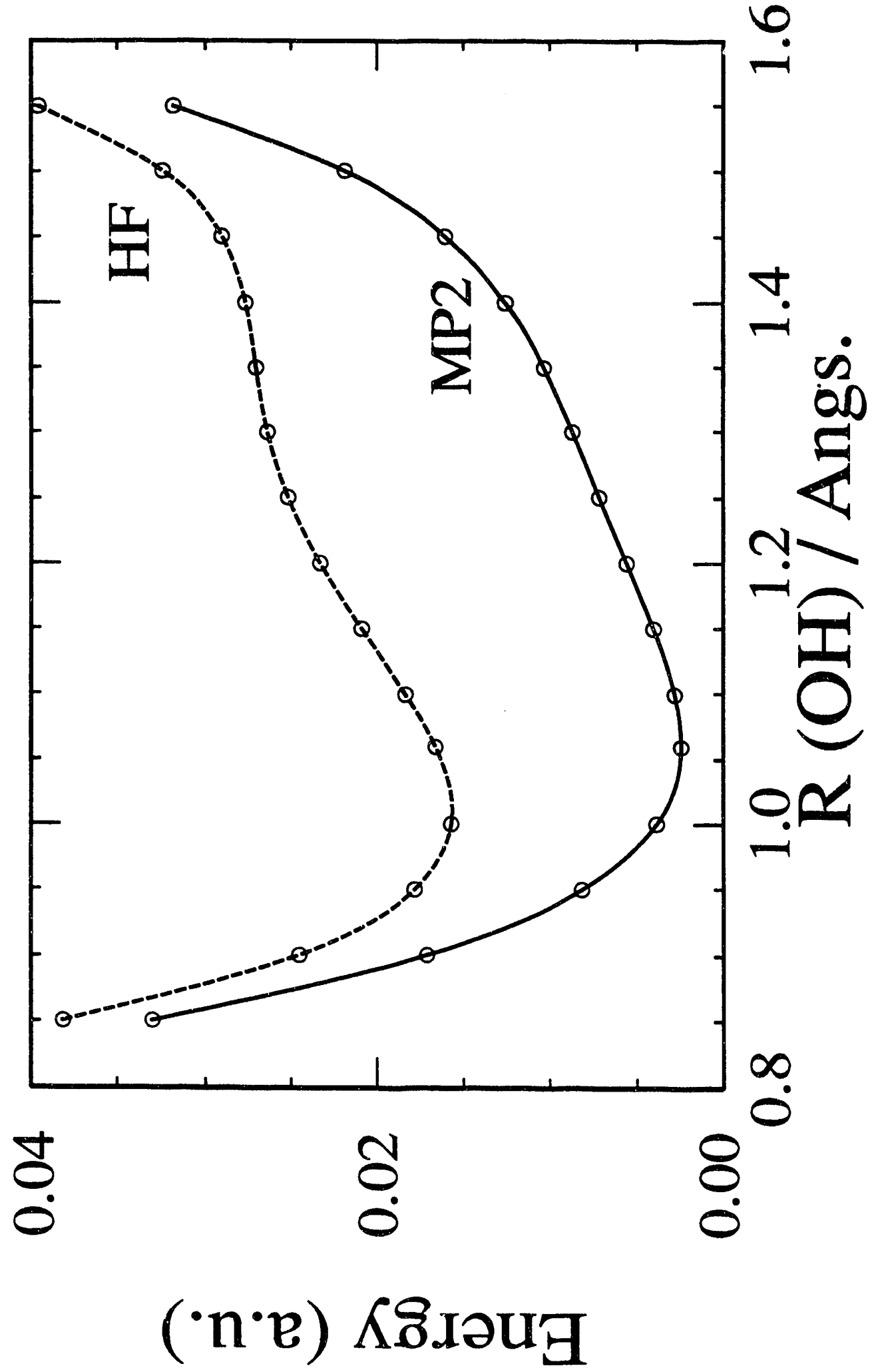

Figure 6-5 


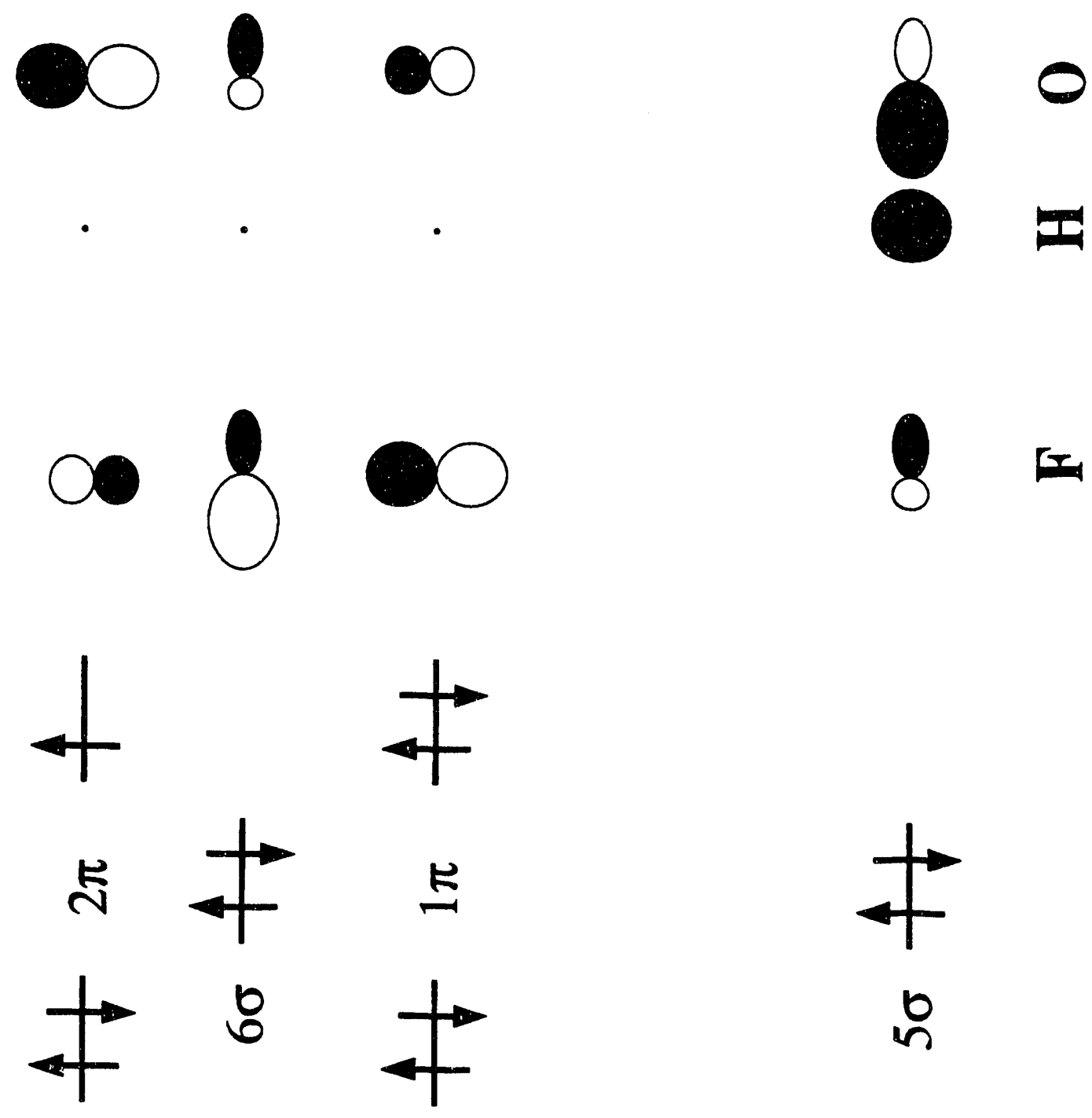

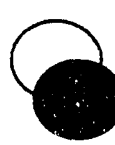

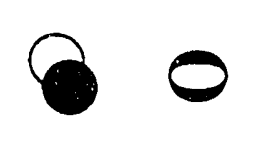

-

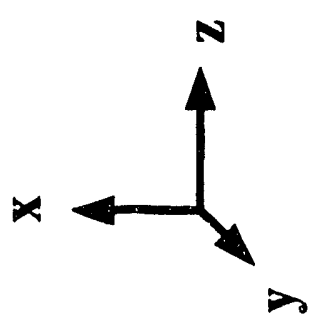

0

8

Figure 6-6 


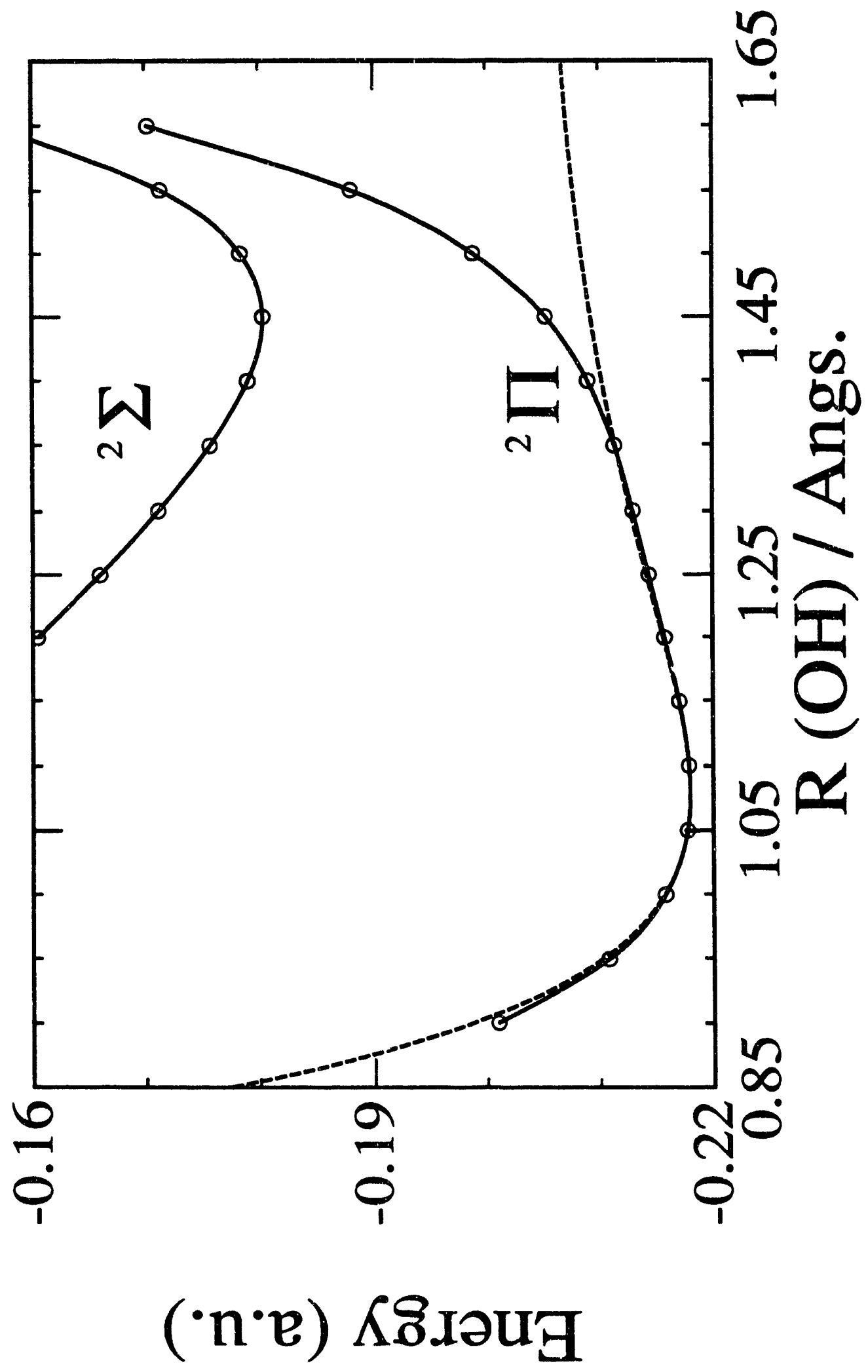

Figure 6-7 


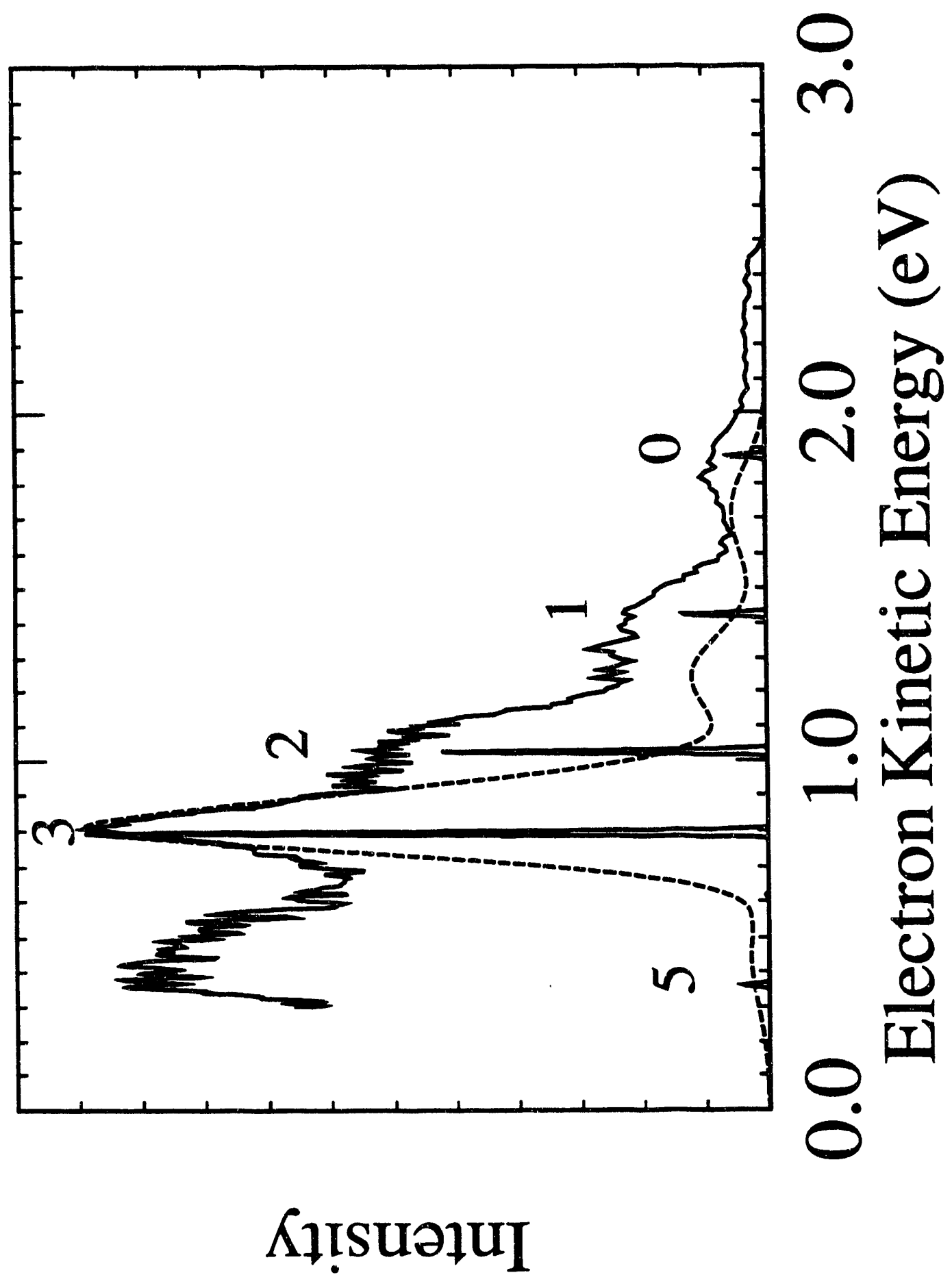

Figure 6-8 


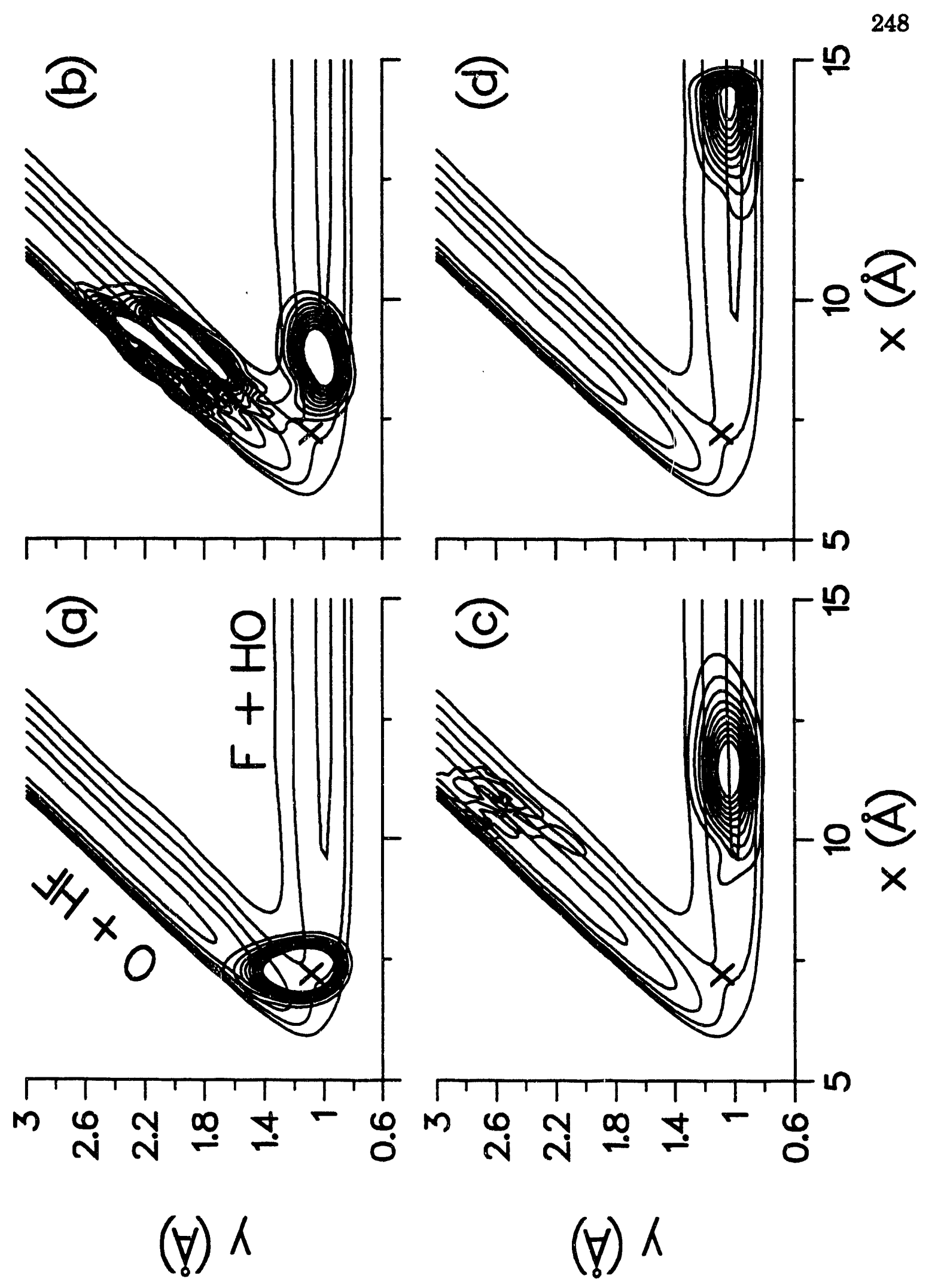

Figure 6-9 


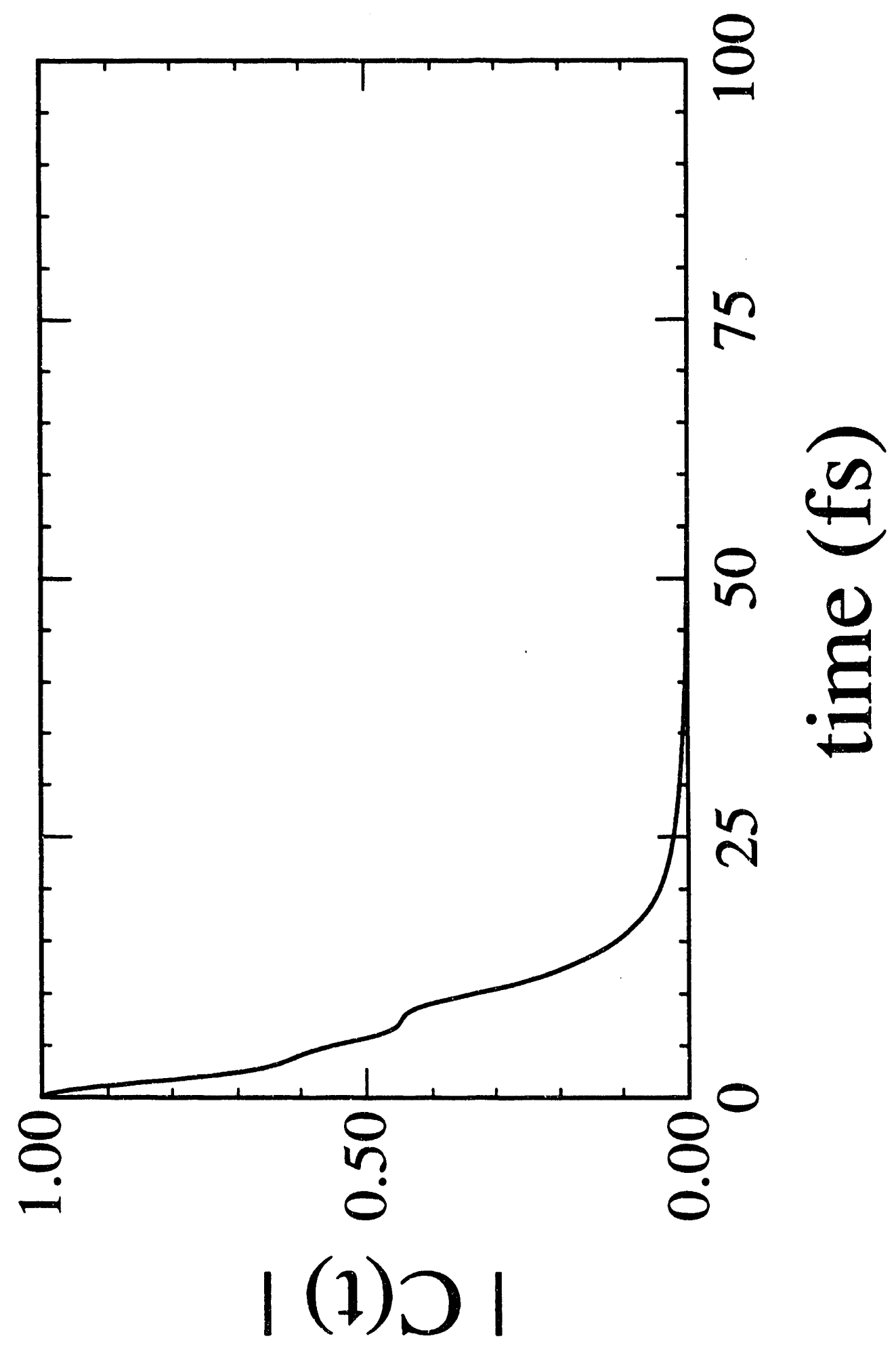

Figure 6-10 


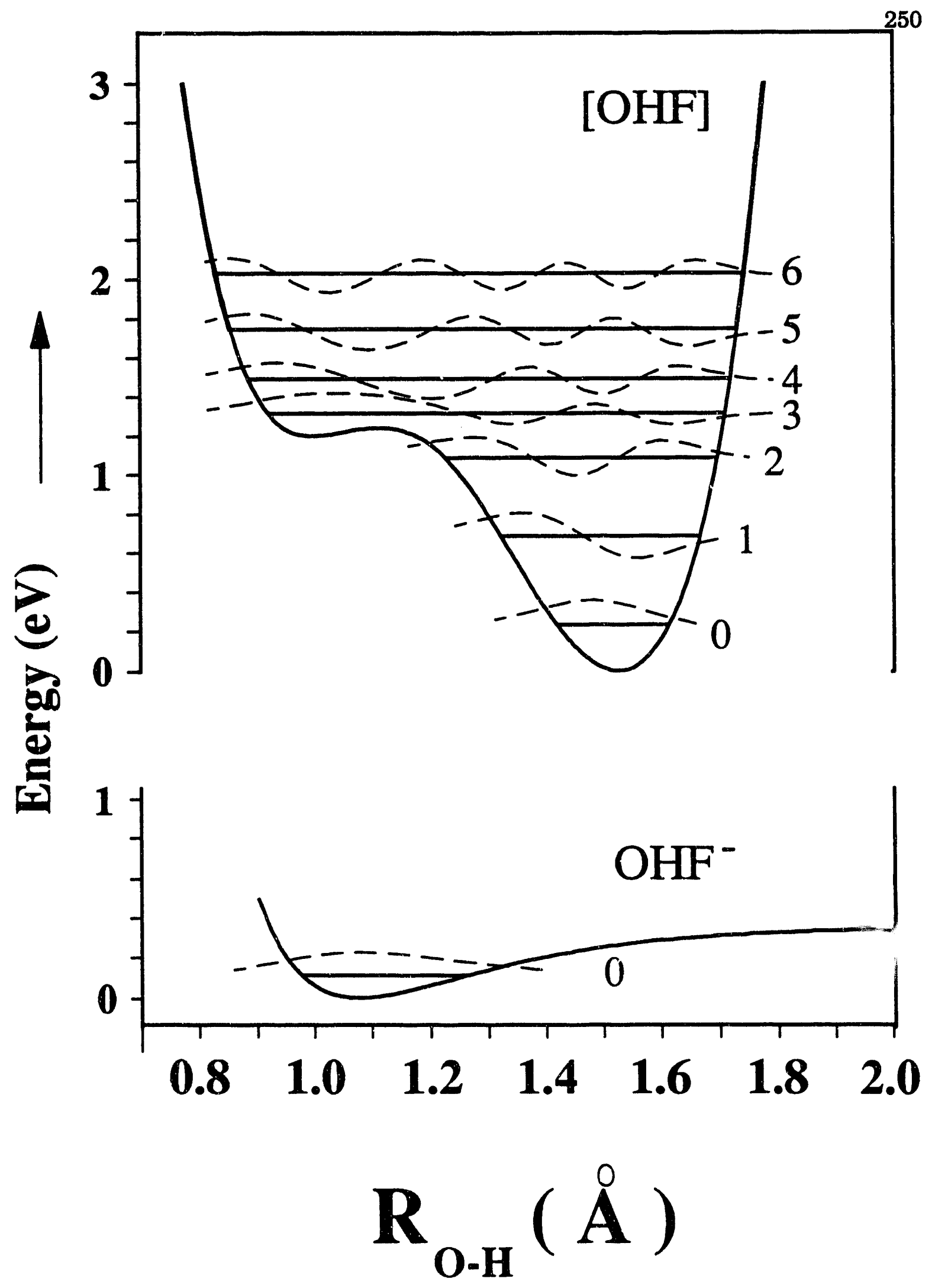

Figure 6-11 


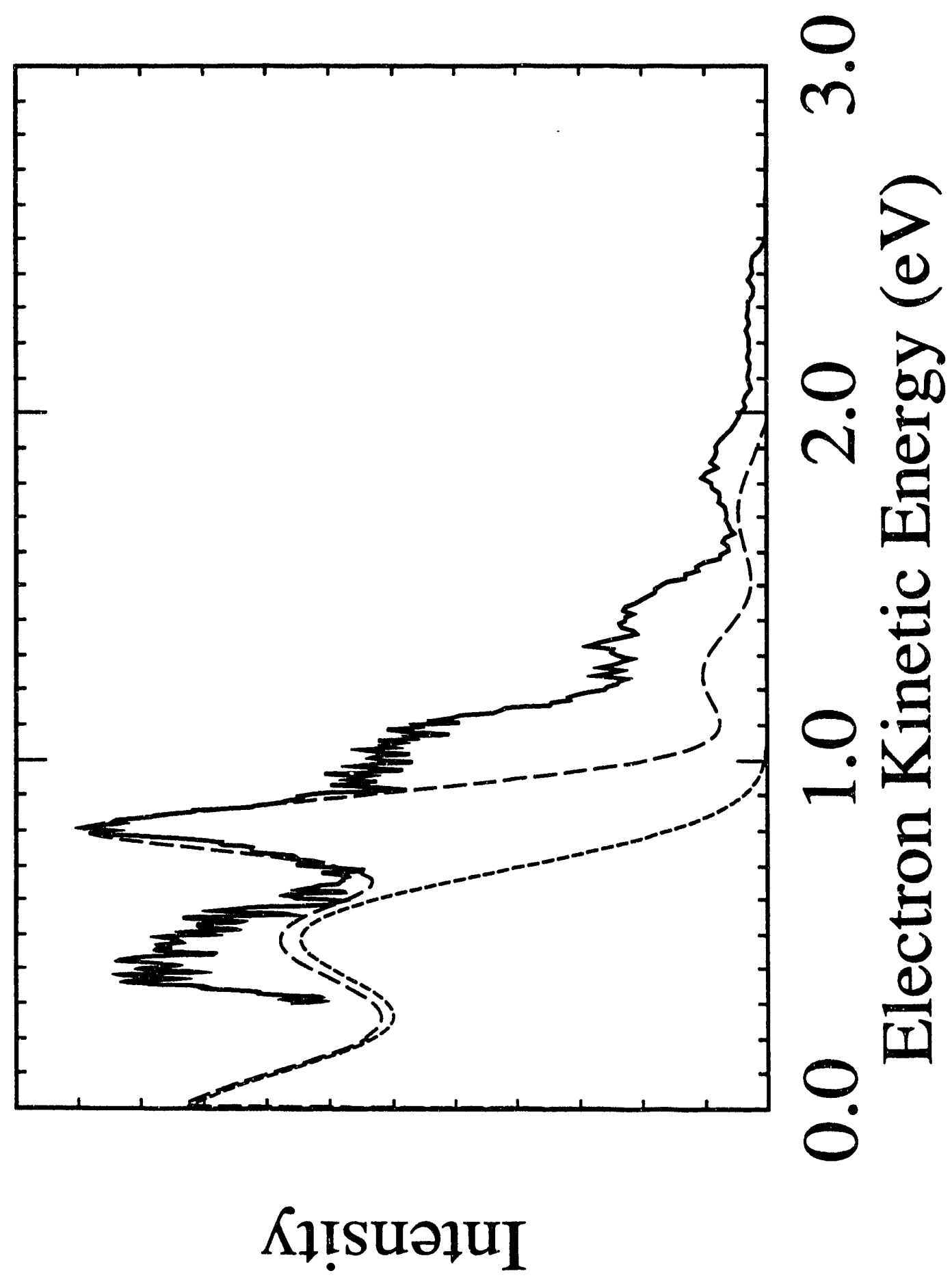

Figure 6-12 

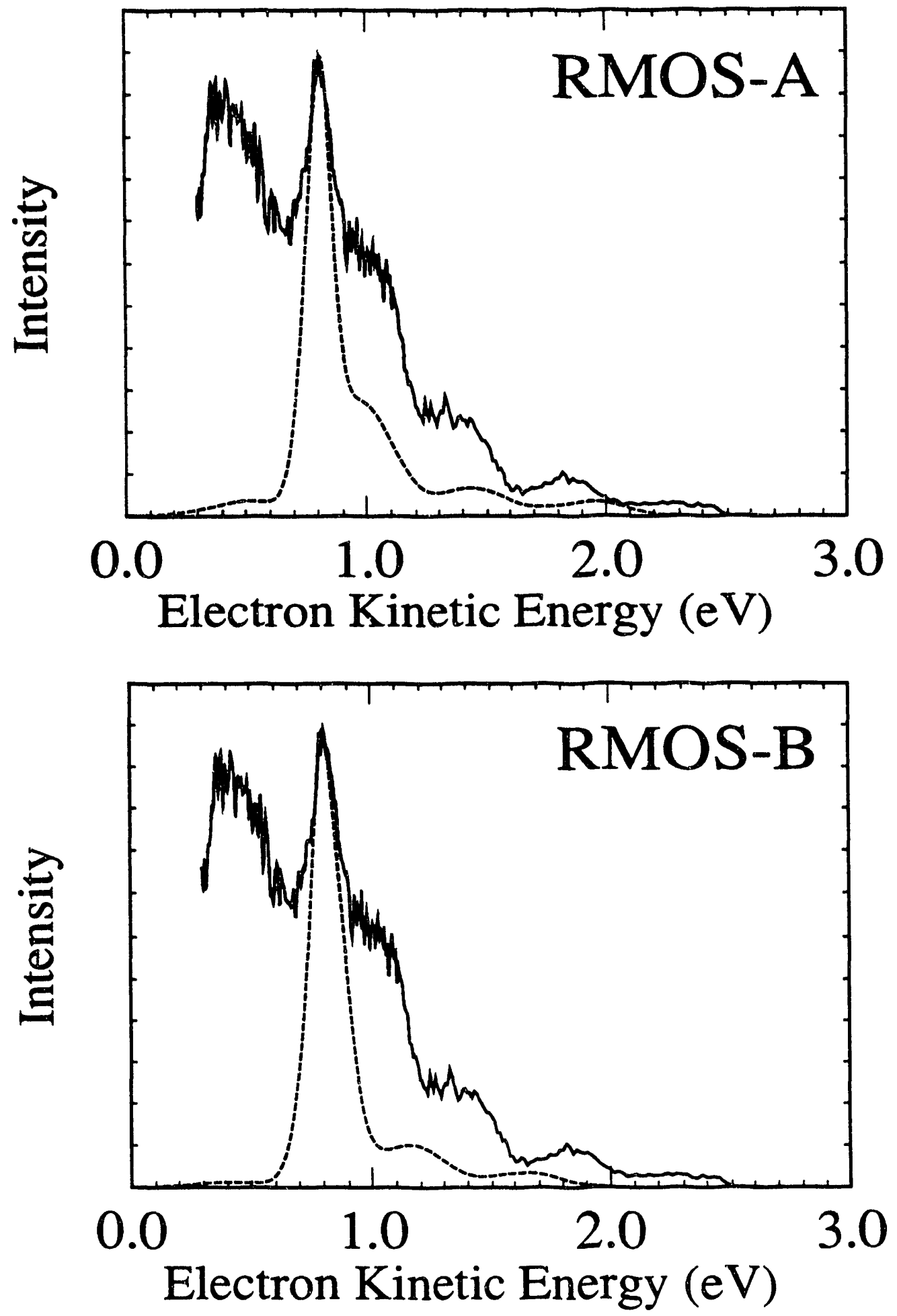

Figure 6-13 


\section{Postscript: new experimental results for OHF-}

Since publication of this work, we have recorded new spectra of $\mathrm{OHF}^{-}$in our laboratory. ${ }^{1}$ Our signal-to-noise has been much improved and the background due to stray electrons has been significantly reduced in the meantime. This has allowed to us to record spectra of $\mathrm{OHF}^{-}$with the lase. poiarized parallel $\left(\theta=0^{\circ}\right)$ to the direction of electron collection, where the signal is much lower. Figure 6-14 shows a comparison of the $\mathrm{OHF}^{-}$photoelectron spectrum at $\theta=90^{\circ}$ (as data shown earlier) and $\theta=0^{\circ}$. The feature at lowest electron kinetic energy (labelled $\mathrm{F}$ as in preceding text) is clearly accentuated relative to all other peaks in the $\theta=0^{\circ}$ spectra. As we have already discussed in Chapter 1 , this behavior is indicative of transitions occurring from the anion to different electronic states, and in these transitions the electron is probably being removed from molecular orbitals of different symmetry. This result confirms our tentative assignment, which was based only on the wave packet simulation analysis, that this single peak $\mathrm{F}$ in the photoelectron spectrum is due to a transition to an excited electronic surface of $[O H F]$, possibly the ${ }^{1} \Delta$. This is not only rather gratifying but also demonstrates, once again, the power of measuring the photoelectron angular distribution in assigning overlapping bands in these complicated transition state spectra.

1. E. H. Kim, unpublished work, 1992 
Figure caption for 6.7

Figure 6-14. $\mathrm{OHF}^{-}$photoelectron spectrum recorded at $213 \mathrm{~nm}$. (Top) $\theta=0^{\circ}$, and (Bottom) $\theta=90^{\circ}$. 
OHF$^{-} 213$ nm

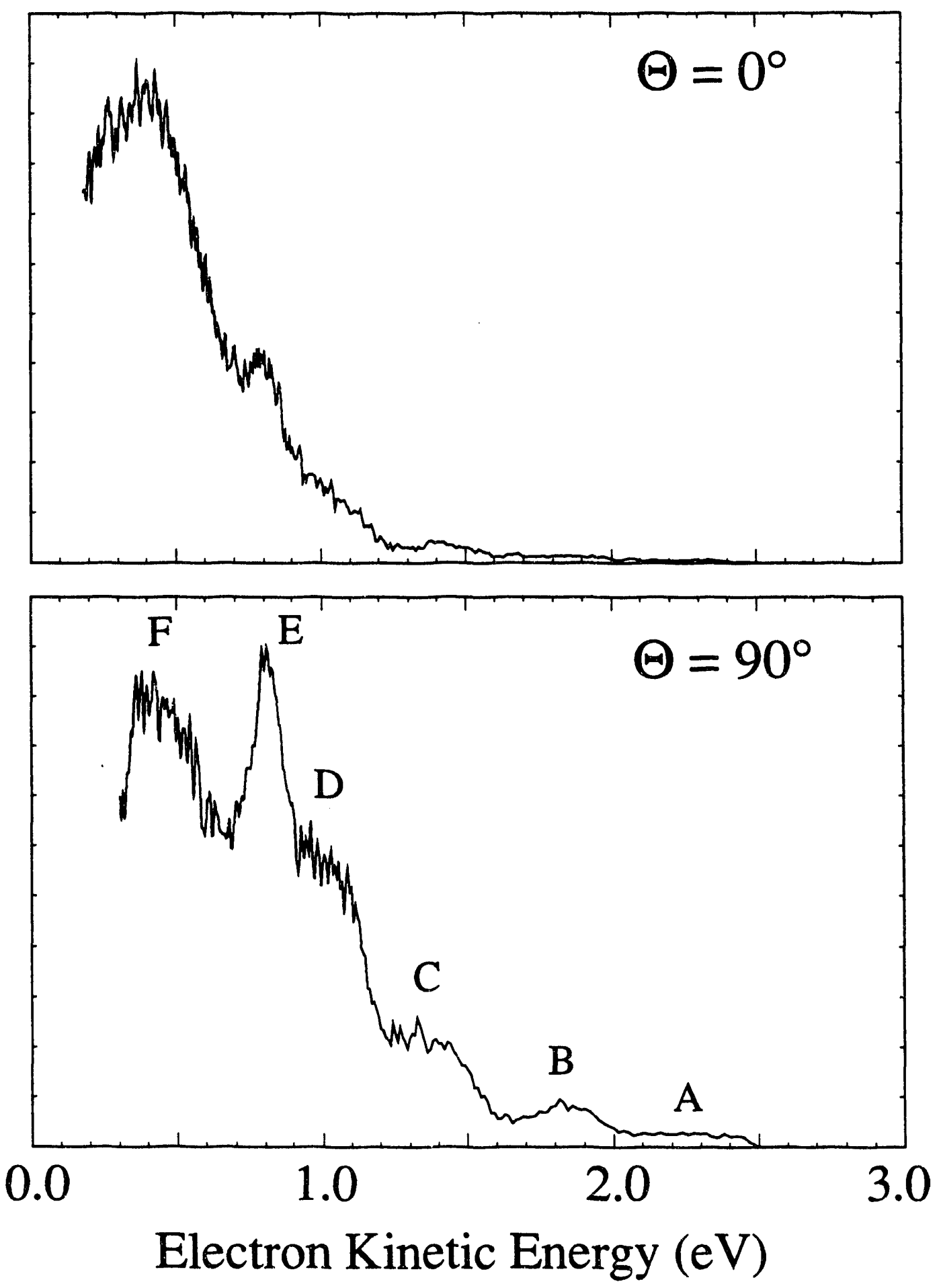

Figure 6-14 


\section{Chapter 7. Photoelectron Spectroscopy of $\mathrm{FH}_{2}^{-}$: results for the $\mathrm{F}+$ para $-\mathrm{H}_{2}$ reaction and analysis of the ${ }^{2} \Pi$ electronic bands.}

\section{Introduction}

In this chapter we describe some new results on the photoelectron spectroscopy of $\mathrm{FH}_{2}{ }^{-}$and extend our analysis of these spectra in the hope of characterizing the transition state of the prototype $\mathrm{F}+\mathrm{H}_{2}$ reaction. This reaction, along with its $\mathrm{D}$ atom isotopic variants, has been very extensively studied both experimentally and theoretically, particularly in the description of product and angularly resolved cross sections. It is not our purpose here to review that work; Alex Weaver has given an excellent historical review of work on this system in her $\mathrm{Ph}$. $\mathrm{D}$. thesis. ${ }^{1}$ Indeed our group has already reported a number of observations on the $\mathrm{F}+\mathrm{H}_{2}$ reaction, including previous photoelectron results, ${ }^{1,2,3,4}$ which are briefly summarized here.

Our earliest results, the $266 \mathrm{~nm}$ photoelectron spectrum of $\mathrm{FH}_{2}$ - recorded with the laser polarized perpendicular to the direction of electron collection, showed little detailed structure, ${ }^{2}$ but allowed comparison with some theoretical results of Zhang and Miller on $\mathrm{F}+\mathrm{H}_{2}{ }^{5}$ The $\mathrm{FH}_{2}{ }^{-}$photoelectron spectrum represented an attractive target for Zhang and Miller to simulate with their accurate $\mathrm{J}=0$ three-dimensional quantum calculation because, in contrast to the full collision experiments, the photodetachment spectrum has only small contributions from higher angular momentum states and is a particularly sensitive and local probe to the dynamics in the three atom interaction region. (See Figure 7-1; the anion equilibrium geometry calculated by Nichols $e t$. $a l^{6}$ is very close to the saddle point geometry on the T5a potential energy surface ${ }^{7}$. Subsequently, Weaver et al. reported far more extensive results for $\mathrm{FH}_{2}^{-}, \mathrm{FD}_{2}{ }^{-}$and 
$\mathrm{FDH}^{-}$at two different laser polarizations. ${ }^{3}$ The results with $\theta=0^{\circ}$, i.e. with the laser polarized parallel to the direction of electron detection, showed very powerfully how transitions from the negative ion to excited electronic states could be virtually eliminated. The excited state bands overlap and obscure transitions to the ground reaction surface in the $\theta=90^{\circ}$ spectra. The $\mathrm{FH}_{2}^{-} \theta=0^{\circ}$ spectra showed a wealth more information than the earlier spectrum, and the isotopically substituted spectra provided valuable clues on the origin of the observed peaks. Zhang and Miller extended $d^{4}$ their scattering calculations on the T5a potential energy surface, so as to simulate the photoelectron spectrum of $\mathrm{FH}_{2}{ }^{-}$over the entire energy range covered in the experiment and additionally simulated the $\mathrm{FD}_{2}{ }^{-}$spectrum. In most respects, the results agreed very satisfactorily with experiment. The experimental work up until August 1991, and comparisons with theory, have been reviewed in dietail in Weaver's thesis. $^{1}$

The purpose of this chapter is to describe new work in our laboratory, notably the synthesis of $\mathrm{FH}_{2}-$ from para $-\mathrm{H}_{2}$ and the photoelectron spectra of this species, and to report improved spectra of $\mathrm{FH}_{2}$ - recorded with normal- $\mathrm{H}_{2}$. The new normal- $\mathrm{H}_{2}$ spectra are superior for two experimental reasons: (a) our signal-to-noise for electron detection is improved by about a factor of three, and (b) we have constructed a new pulsed nozzle source for these experiments which should allow increased cooling of the rotations and internal modes of the $\mathrm{FH}_{2}$ - anion. Additionally, photoelectron spectra of $\mathrm{FH}_{2}-\left(\right.$ normal- $\left.\mathrm{H}_{2}\right)$ have been taken at a higher laser photon energy $(5.82 \mathrm{eV}, 213$ $\mathrm{nm}$ ) to complete the search for features due to excited electronic surfaces. Along with this new experimental work, we have examined several theoretical issues, notably the impact of nuclear spin statistics in the anion, and its effect on the photoelectron 
spectra. We will comment on the treatment of the anion in the theoretical work of Zhang and Miller. Finally, quantitative analysis of the contribution of the electronically excited states to the $\theta=90^{\circ}$ spectra are presented for the first time, including simulations of these bands. Prospects for new experiments on this interesting system are discussed.

\section{Experimental}

The instrument employed in this study is the same time-of-flight photoelectron spectrometer described in our earlier work on $\mathrm{FH}_{2}{ }^{-} ;{ }^{2,3}$ therefore here we will describe only modifications to the experimental apparatus and the particular details of the experiments carried out. $\mathrm{FH}_{2}-$ ions are made in the source region by clustering of $F^{-}$, produced from dissociative electron attachment to $\mathrm{NF}_{3}$, with $\mathrm{H}_{2}$. This is achieved by crossing a $1 \mathrm{keV}$ electron beam with a pulsed free jet expansion of reagent gases. The reagent gases, in the ratio $8 \% \mathrm{NF}_{3}, 32 \% \mathrm{H}_{2}$ and $60 \% \mathrm{~N}_{2}$, are allowed to mix thoroughly in a stainless steel cylinder before use; at run time the stagnation pressure of the mixed gases behind the pulsed valve is $80 \mathrm{psig}$.

Ions are made from both normal and para hydrogen. Recall that normal hydrogen is a 3:1 mixture of ortho and para hydrogen. The normal- $\mathrm{H}_{2}$ used in these experiments was obtained commercially and is $99.99 \%$ purity. Para $-\mathrm{H}_{2}$ was prepared by the U. C. Berkeley Department of Chemistry Low Temperature Laboratory. It was stored in standard aluminum gas cylinders, so as to reduce the para - ortho interconversion, and to reduce any isotopic exchange processes of impurities. The concentration of the para $-\mathrm{H}_{2}$ on preparation is $99.7 \%$. It has been observed that the half life for conversion of para $-\mathrm{H}_{2}$ to normal $-\mathrm{H}_{2}$ when stored in this way is on the 
order of three to four weeks. ${ }^{8.9}$ In these experiments the para $-\mathrm{H}_{2}$ was used within a few days of preparation to make a gas mixture suitable for the photoelectron experiment. The $\mathrm{NF}_{3} /$ para hydrogen/nitrogen gas mixture was only temporarily (few hours) held in the stainless steel cylinder for premixing of the gases prior to use. The stainless steel surfaces of this vessel were certainly poisoned, as far as their properties for catalysis of para - ortho hydrogen conversion, because of prior use of the mixing cylinder with other gas mixtures. It is not known, therefore, how much para - ortho conversion occurred at this stage, but clearly at least $50 \%$ para $-\mathrm{H}_{2}$ remained, else it would seem unlikely for us to observe any differences in the photoelectron spectra of $\mathrm{FH}_{2}-$ made from normal- $\mathrm{H}_{2}$ and para $-\mathrm{H}_{2}$.

The ion source has been modified from previous experiments to incorporate a new higher intensity pulsed valve, which is also more stable in its operation. The 213 $\mathrm{nm}$ spectra reported here, as well as previously published spectra, were recorded with the original pulsed valve, a General Valve Series 9, which is of a spring/ solenoid design. The new source incorporated a piezo-electric valve, of the design of Proch and Trickl. ${ }^{10}$ This valve accomplishes larger gas throughput in a shorter pulse, and has better shot-to-shot reproducibility. Superior cooling of the $\mathrm{H}_{2}$ is expected with this valve. The electron beam crosses the free jet at $90^{\circ}$. The position the two intersect is controlled by deflection of the electron beam. It is found that there is a strong variation in the temperature of the ions formed in the source depending on how far from the orifice the electron beam interacts with the jet. The ions appear to be created colder, i.e. the photoelectron spectrum is less congested and better resolved, if the electron beam intersects the jet some $25 \mathrm{~mm}$ from the orifice. This is considerably further away from the nozzle orifice than we normally operate the source, 
but the characteristics of hydrogen cooling in a free jet expansion are somewhat unusual. The degree of cooling of the $\mathrm{FH}_{2}-$ ions was similarly improved when nitrogen was included in the expansion.

The negative ions are extracted, mass selected and photodetached in the usual way. ${ }^{11}$ Spectra reported here were recorded with laser wavelengths of $213 \mathrm{~nm}$ (5.82 $\mathrm{eV})$ as well as $266 \mathrm{~nm}(4.66 \mathrm{eV})$. The pulsed laser light is plane polarized; as before we can adjust the angle $\theta$ between the electric vector of the laser radiation and the direction of electron detection by rotation of a half-wave plate. The second major change to our apparatus has been the upgrade of the electron detector. The electrons photodetached by the laser are detected at the end of a 1 meter flight tube, and their energy is analyzed by time-of-flight. For this detector we now use a pair of $75 \mathrm{~mm}$ diameter microchannel plates, rather than a pair of $40 \mathrm{~mm}$ plates. ${ }^{12}$ This increases the electron collection efficiency by a factor of 3.5 , with a similar improvement in the signal-to-noise. There is a slight loss in electron energy resolution; typically the instrumental resolution is $12 \mathrm{meV}$ at $0.65 \mathrm{eV}$, and, as before, degrades for higher electron energies as $E^{3 / 2}$.

\section{Results}

Photoelectron spectra were recorded for the $\mathrm{FH}_{2}-$ ion at both parallel and perpendicular polarizations of the laser. Both normal and para hydrogen was used to make the ions. The spectra are presented in Figure 7-2. The form of the two normal $-\mathrm{H}_{2}$ spectra are very similar to those reported earlier, ${ }^{1,3}$ except there is a noticeable improvement in signal-to-noise in the $\theta=90^{\circ}$ spectrum. The detailed structure in peaks $A, A^{\prime}$ and $B$ in the $\theta=0^{\circ}$ is a little different from that observed 
earlier; peak $\mathrm{C}$ is also a little better resolved in the new $\theta=0^{\circ}$ spectrum." We believe these differences are due to differences in source temperature. This will be examined in more detail below. The striking feature in Figure 7-2, however, is the pronounced differences between the spectra recorded with para hydrogen and those with normal hydrogen, particularly between 0.85 and $1.1 \mathrm{eV}$ in the $\theta=0^{\circ}$ spectra. Peak positions are listed in Table 7-1. There appears to be an extra peak $\left(A^{\prime}\right)$ at 0.97 $\mathrm{eV}$ electron kinetic energy that, because of its unimpressive nature in the normal $-\mathrm{H}_{2}$ spectra, was not previously thought significant. However, it is the dominant peak in the para $-\mathrm{H}_{2}$ spectra. It appears that the peaks $A$ and $B$ do also appear in the para $-\mathrm{H}_{2}$ spectra but as shoulders to the central peak $A^{\prime}$.

The two peaks observed in all four spectra at 1.26 and $1.21 \mathrm{eV}$ are due to a two photon process and correspond to the photoelectron spectrum of $\mathrm{F}^{-}$; the first photon dissociates $\mathrm{FH}_{2}-$ to $\mathrm{F}^{-}+\mathrm{H}_{2}$, the second photon detaches $\mathrm{F}^{-3}$

Table 7-1: Peak positions (electron kinetic energies) in the $\theta=0^{\circ}, 266 \mathrm{~nm}$ photoelectron spectra of $\mathrm{FH}_{2}{ }^{-}$.

\begin{tabular}{cccccc}
\hline & \multicolumn{5}{c}{ peak position $/ \mathrm{eV}^{\mathbf{3}}$} \\
\cline { 2 - 6 } $\mathrm{FH}_{2}$ - from & $\mathrm{A}$ & $\mathrm{A}^{\prime}$ & $\mathrm{B}$ & $\mathrm{C}$ & $\mathrm{D}$ \\
\hline normal $-\mathrm{H}_{2}$ & 1.000 & 0.972 & 0.941 & 0.815 & 0.54 \\
para $-\mathrm{H}_{2}$ & 0.996 & 0.970 & 0.942 & 0.81 & 0.51 \\
\hline \hline
\end{tabular}

a) Peak positions in Table 7-1 and the spectra shown in Figure 7-2 have been corrected for the small space charge shift $(<5 \mathrm{meV})$ in the electron kinetic energies. Uncertainties in peak positions are $0.005 \mathrm{eV}$, except for peaks $\mathrm{C}$ and $\mathrm{D}$ where the uncertainty is approx. $0.015 \mathrm{eV}$.

1 The peak labelling scheme of Refs. 1, 3 and 4 is also used here to prevent confusion. 
The $213 \mathrm{~nm}$ photoelectron spectra of $\mathrm{FH}_{2}^{-}$, where the ion has been synthesized only from normal- $\mathrm{H}_{2}$, are shown in Figure 7-3. The photon energy is $1.17 \mathrm{eV}$ higher. The spectra are essentially identical to their respective $266 \mathrm{~nm}$ counterparts, except for the $1.17 \mathrm{eV}$ shift to higher electron kinetic energy (eKE) and the diminished spectral resolution. The polarization dependence of the signal is the same. The important result is that no additional bands are observed for $\mathrm{FH}_{2}-$ photodetachment that are not present in the $266 \mathrm{~nm}$ spectra.

\section{Analysis and Discussion.}

\subsection{Nuclear spin statistics in the anion and its effect on the photoelectron} spectrum.

The results for the photoelectron spectra of $\mathrm{FH}_{2}-$ from para $-\mathrm{H}_{2}$ are somewhat surprising. As we will show, the differences in the $\mathrm{FH}_{2}^{-}$spectra with the ion prepared from normal and para hydrogen are due to differences in the nuclear spin statistics in the anion. The nuclear spin states of hydrogen, ortho and para, are carried through to the anion $\mathrm{FH}_{2}-$. The two forms of $\mathrm{FH}_{2}^{-}$(para and ortho), which we will loosely call para- $\mathrm{FH}_{2}{ }^{-}$and ortho- $\mathrm{FH}_{2}^{-}$, overlap two distinct sets of scattering states in the neutral, namely the states whose scattering wavefunction is symmetric to exchange of the $\mathrm{H}$ atoms (para states) and those that are antisymmetric (ortho). For example, scattering states that are symmetric with respect to hydrogen permutation are those that correlate to $\mathrm{F}+\mathrm{H}_{2}(\mathrm{~J}=$ even $)$.

Let us examine the anion in more detail. The restriction on the anion rovibrational wavefunction imposed by the nuclear spin symmetry appears in the bending / hindered rotor mode. This is well known for molecules of type $A_{2} B$ 
belonging to the $\mathrm{C}_{-v}$ point group, such as $\mathrm{N}_{2} \mathrm{O} .{ }^{13}$ The nature of the bending energy levels of $\mathrm{FH}_{2}$-is shown in Figure 7-4. Free rotation of $\mathrm{H}_{2}$ correlates into the bending states of the linear ${ }^{\prime 2}$ triatomic ion as shown in the Figure. This correlation diagram derives from work by Henderson and Ewing on Ar- $\mathrm{O}_{2}$ and Ar- $-\mathrm{N}_{2}$ complexes. ${ }^{14}$ In the free rotor limit, as for free $\mathrm{H}_{2}$, the para form exists only in even $\mathrm{J}$ states, where $\mathrm{J}$ is the internal rotor quantum number, and the ortho form of the triatomic only in odd $\mathrm{J}$ states. The nuclear spins are not scrambled in the clustering collision of $\mathrm{F}^{-}$with $\mathrm{H}_{2}$ to form the weak van der Waals complex, as the $\mathrm{H}-\mathrm{H}$ bond is not broken. The correlation diagram shows that, in the limit of strong anisotropy in the angular potential, i.e. a large barrier to internal rotation of $\mathrm{H}_{2}$ in the complex, the energy levels become identical to those of a degenerate harmonic oscillator. However, there is a doubling of each state due to the two equivalent positions of the $\mathrm{H}$ nuclei. For each state there is a pair of wavefunctions: one is symmetric and the other antisymmetric with respect to $\mathrm{H}$ permutation. In the rigid bender limit, the two ground state levels are degenerate but have wavefunctions of opposite permutation symmetry. In either limit, the relative proportions of symmetric to antisymmetric states reflects the ratio of para to ortho hydrogen used in the clustering process. Therefore para $-\mathrm{H}_{2}$ will form only even symmetry states of $\mathrm{FH}_{2}-$ shown in Fig. 7-4; normal $\cdot \mathrm{H}_{2}$ will form 1:3 symmetric to antisymmetric states.

*2 The equilibrium structure of $\mathrm{FH}_{2}$ - is assumed to be linear on the basis of $a b$ initio calculations of Simons ${ }^{6}$. A linear structure is consistent with the electrostatic forces of a charge interacting with the $\mathrm{H}_{2}$ quadrupole moment. 
We shall assume the barrier to internal rotation is large, ${ }^{, 3}$ that the bend can be approximately treated as a degenerate harmonic oscillator, and that the splitting between the two ground state wave functions ( $a, s$ symmetry) is negligible in the following discussion. This maybe a poor approximation if the bend is very strongly coupled to the van der Waals stretching mode. In that case, Figure 7-4 gives us some idea of the states formed in the intermediate anisotropy regime.

In Zhang and Miller's calculation, ${ }^{4,5}$ in order to compute the Franck Condon overlap of the anion with the $\mathrm{F}+\mathrm{H}_{2}$ scattering states, these authors assumed the degenerate harmonic oscillator limit for the bend and used the geometry $\left(\mathrm{R}_{\mathrm{P}, \mathrm{H}_{2}}=\right.$ $\left.2.138 \AA ; R_{\mathrm{H}-\mathrm{H}}=0.796 \AA\right)$ and harmonic frequencies $\left(\omega_{1}=302 \mathrm{~cm}^{-1}, \omega_{2}=693 \mathrm{~cm}^{-1}\right.$ and $\omega_{3}=3816 \mathrm{~cm}^{-1}$ ) from earlier results of Nichols et al..$^{6}$ However, Zhang chose the anion ground state wavefunction to be symmetric with respect to nuclear exchange, ${ }^{5}$ therefore the Frank Condon overlaps are computed with only the even set of scattering states. In fact Zhang's calculation employs a separation of the scattering matrix by the nuclear inversion symmetry, ${ }^{15}$ only one block, the para block, is being used in the Franck-Condon calculation. Thus, the appropriate comparison with

*3 Our $a b$ initio calculations on $\mathrm{FH}_{2}$ - suggest that the barrier to internal rotation of $\mathrm{H}_{2}$ is about $3000 \mathrm{~cm}^{-1}$. The barrier is relatively large compared to the $\mathrm{H}_{2}$ rotational constant, $60 \mathrm{~cm}^{-1}$. The calculations compare the energy at the MP2/6-31++G** optimized linear geometry, which is close to Nichols' CCSD geometry, ${ }^{6}$ and the energy for the rotated configuration $\left(C_{2 v}\right)$ with $R_{F, H_{2}}$ and $R_{\mathrm{H} \cdot \mathrm{H}}$ held constant. The calculated barrier is approximately invariant to the level of correlation correction to the energy. RHF, RMP2 and RMP4(SDQ) all give about the same barrier to internal rotation. 
experiment is with the para- $\mathrm{FH}_{2}^{-}$spectrum, and not with the normal- $\mathrm{FH}_{2}-$ spectrum as previously done., 4 Figure 7-5(a) shows the correct comparison of Zhang's 3D simulation of the $\mathrm{FH}_{2}^{-}$photoelectron spectrum with our para- $\mathrm{FH}_{2}^{-}$ results. The comparison is noticeably poorer with the para experimental spectrum than with the normal spectrum, and thus agreement between theory and experiment is not nearly as good as had been previously been thought the case., 4

The normal- $\mathrm{FH}_{2}{ }^{-}$spectrum, in contrast, contains transitions that are $75 \%$ due to antisymmetric states. Therefore to simulate this photoelectron spectrum a computation of the anion overlap with ortho scattering wavefunctions should be made and then added to the para simulation shown in Figure 7-5(a) in the correct ratio. Very recently, Manolopoulos has carried out exactly this calculation. Using a threedimensional scattering $\operatorname{code}^{16}$ that employs a methodology similar to that of Schatz, ${ }^{1 "}$ he has repeated (and reproduced) Zhang's result for para- $\mathrm{FH}_{2}{ }^{-}$, and has gone onto compute the ortho scattering states and their Franck Condon overlap with the antisymmetrized anion wave function. Together these simulations yield the theoretical photoelectron spectrum of normal- $\mathrm{FH}_{2}-$ shown in Figure $7-5(\mathrm{~b}),{ }^{18}$ where it is compared to the experimental normal- $\mathrm{FH}_{2}-$ spectrum. Taken together, the agreement of the para- $\mathrm{FH}_{2}-$ and normal- $\mathrm{FH}_{2}-$ simulations on the $\mathrm{T} 5 \mathrm{a}$ surface with our respective experimental spectra is quite disappointing.

However, there is one further effect of the nuclear spin statistics relevant to the anion formed in our experiment we should consider. It relates to the populations of excited anion bending states, and hence the appearance of hot bands in our spectra. Let us consider the distribution of she $\mathrm{H}_{2}$ rotational states that we expect in the free jet. Para $-\mathrm{H}_{2}$ has approximately $53 \% \mathrm{~J}=0$ and $47 \% \mathrm{~J}=2$ at room temperature, compared 
to $10 \%, 67 \%$, and $11 \% \mathrm{~J}=0,1,2$ for normal $-\mathrm{H}_{2}$ respectively. Because relaxation of the rotational energy in $\mathrm{H}_{2}$ may only occur by $\Delta J=2$ inelastic collisions, normal $-\mathrm{H}_{2}$ is not cooled well by a free jet expansion, however para $-\mathrm{H}_{2}$ is cooled much more effectively. Typical rotational distributions for pure $\mathrm{H}_{2}$ in collimated continuous molecular beams have been given by Pollard et al. as a function of $\mathrm{P}_{0} \mathrm{~d}$, the stagnation pressureaperture diameter product. ${ }^{19}$ These distributions are measured by the rotationally resolved photoelectron spectra of $\mathrm{H}_{2}$. In our work with pulsed valves, the calculated $P_{0} d$ would be of the order of 2500 Torr॰mm. However, the molecular beam is not skimmed in our apparatus and the effective nozzle diameter of the pulsed valve may be somewhat less than the physical orifice size. It seems reasonable, therefore, to assume a lower effective $P_{0} d$ for the pulsed expansion. In the $P_{0} d-100$ Torr $\bullet m m$ regime, which may be considered a worst case limit, the rotational distribution of $\mathrm{H}_{2}$ may be estimated from the work of Pollard as $18 \% \mathrm{~J}=0,75 \% \mathrm{~J}=1$ and $7 \% \mathrm{~J}=2$ for normal $-\mathrm{H}_{2}$ and $70 \% \mathrm{~J}=0,30 \% \mathrm{~J}=2$ for para $-\mathrm{H}_{2}$.

If we assume that the $J, M$ state distribution in $\mathrm{d}$ tree $\mathrm{H}_{2}$ is mapped onto the anion quantum state distribution, i.e there is no further clustering, then we may use the above $\mathrm{H}_{2}$ rotational distributions and the correlation diagram Fig. 7-4 to yield a conservativf estimate of the bend state population in the $\mathrm{FH}_{2}$ - complexes formed. Even if additicutat enoling does take place after clustering, the nuclear interchange symmetry restrictions sidl restrict scrambling of states with opposite permutation symmetry.

Therefore, $\mathrm{FH}_{2}{ }^{-}$made from normal $-\mathrm{H}_{2}$ may have a large number of excited anion states populated, maybe as high as $50 \% v_{2}=1$ if the $M$ states are statistically distributed among the $v_{2}, \ell$ states (see Figure 7-4). This possibility suggests that the 
normal- $\mathrm{FH}_{2}-$ photoelectron spectrum may have major contributions from anion hot bands. In the para- $\mathrm{FH}_{2}{ }^{-}$spectra, we suspect the contribution due to hot bands is smaller but may still be significant; hot bands in this case are derived only from $\mathrm{H}_{2}$ $\mathrm{J}=2$ states $(<30 \%)$.

\subsection{New work on the $\mathrm{F}+\mathrm{H}_{2}$ reaction using the 5SEC surface.}

Truhlar and coworkers have proposed another surface for the $\mathrm{F}+\mathrm{H}_{2}$ reaction, the 5SEC, that improves the description of the entrance channel and saddle point. The saddle point is earlier and lower than on the T5a surface and the bending potential is also flatter in the saddle point region. ${ }^{20}$ The product valleys for the two surfaces are essentially the same.

Kress and Hayes have recently performed three-dimensional scattering calculations for $\mathrm{F}+\mathrm{H}_{2}$ and made a correspondence between peaks and thresholds in their calculated cumulative reaction probability (CRP) with expected Franck Condon factors from the anion. ${ }^{21}$ These calculations were performed on both the T5a and 5SEC surfaces, and the results on the former were in good agreement with Zhang's Franck Condon simulation. ${ }^{4}$ This would seem to support using the CRP to predict the photoelectron spectrum. Kress's CRP results for the 5SEC surface are qualitatively different from the results on the T5a. The authors show that the resonance structure in the cumulative reaction probability calculated for the two surfaces is very different. In the same way as Zhang's calculations comment only on the $\mathrm{F}+$ para $-\mathrm{H}_{2}$ reaction, Kress' cumulative reaction probability is an even permutation sum (i.e. $\mathrm{H}_{2}(\mathrm{j}=$ even) only). Thus the energies and appearances of dynamical features in this calculation should also only be compared with our para- $\mathrm{FH}_{2}{ }^{-}$spectra. 
Interestingly, Kress predicts a trapped state resonance, labelled in their paper ' $a$ ', between the features that are assigned to the peaks $A$ and $B$ of our photoelectron spectra. Could this indeed be the dominant feature in the para $-\mathrm{FH}_{2}-$ photoelectron spectrum we have called $A^{\prime}$ ? The spacing between $A, a$, and $B$ in their calculation are 15 and $28 \mathrm{meV}$, which compare favorably with the spacings in Table 7-1. The fact that peak $\mathrm{A}^{\prime}$ is relatively diminished in our normal- $\mathrm{FH}_{2}^{-}$photoelectron spectrum is also consistent with the 5SEC assignment of this middle peak to a trapped-state resonance, $\mathrm{a}$, in contrast to $\mathrm{A}$ and $\mathrm{B}$ being quantized-bottleneck states. ${ }^{* 4}$ However, preliminary calculations of the Franck Condon factors from the ion (wave function symmetric with respect to $H$ permutation, i.e. para) with each of these states does not support peak 'a' having large intensity in the photoelectron spectrum. ${ }^{18}$

Full simulations for both para and normal- $\mathrm{FH}_{2}-$ will be shortly available and will allow a more quantitative discussion of the merits of this potential surface. ${ }^{18,22}$ Clearly there is a great deal more work to be done before we can fully interpret the photoelectron spectra of $\mathrm{FH}_{2}-$ and its isotopic variants!

"4 There is some variation in the terminology used to describe reactive resonances in the literature. We have typically used the term resonance only for states that are trapped, or quasi-bound, along the reaction coordinate. These are what Kress calls "trapped-state" resonances. The other type of peaks observed in our photoelectron spectra, which we call "direct scattering" states, are called "entrance channel" or "in-channel" resonances by some authors and "quantized bottlenecks" by Kress. 


\subsection{Electronic effects}

The approach of an $\mathrm{F}$ atom with a ground state $\mathrm{H}_{2}$ molecule may occur on three potential energy surfaces, $1^{2} \mathrm{~A}^{\prime},{ }^{2} \mathrm{~A}^{\prime \prime}$ and $2^{2} \mathrm{~A}^{\prime}$ in the most general symmetry of collision, $\mathrm{C}_{\mathrm{s}}$; the upper ${ }^{2} \mathrm{~A}^{\prime \prime}$ and $2^{2} \mathrm{~A}^{\prime}$ surfaces become degenerate in $\mathrm{C}_{m v}$ (collinear approach) so that there are two surfaces ${ }^{2} \Sigma$ and ${ }^{2} \Pi$. Figure $7-6$ shows the highest occupied molecular orbitals for the anion, where the $C_{m v}$ point group is appropriate. Photodetachment of an electron from the filled $1 \pi$ and $4 \sigma$ orbitals leads to the ${ }^{2} \Sigma$ and ${ }^{2} \Pi$ states, respectively, in the neutral. Only the lowest surface, the ${ }^{2} \Sigma$, where the fluorine atom approaches with the p orbital containing the unpaired electron along the $\mathrm{H}_{2}$ bond, adiabatically leads to reaction. The introduction of spin orbit coupling in the $\mathrm{F}$ atom splits the degeneracy of the upper ${ }^{2} \Pi$ surfaces in $\mathrm{C}_{\mathrm{\alpha v}}$ and the correct state labels are ${ }^{2} \Sigma_{1 / 2},{ }^{2} \Pi_{3 / 2}$ and ${ }^{2} \Pi_{1 / 2}$. The ${ }^{2} \mathrm{P}_{3 / 2}-{ }^{2} \mathrm{P}_{1 / 2}$ splitting in the fluorine atom is 0.0501 $\mathrm{eV} .^{23} \mathrm{~A}$ correlation diagram is shown in Figure 7-7(a).

A number of theoretical studies were made in the 1970's on the role of the two excited surfaces in $\mathrm{F}+\mathrm{H}_{2}$ collisions. Initially Truhlar and Muckerman considered how much the calculated reaction rate constant should be reduced because only one of the three orientations of fluorine approach would lead to reaction at thermal energies. ${ }^{24}$ Blais and Truhlar constructed semi-empirical valence bond surfaces for the ${ }^{2} \Sigma$ and ${ }^{2} \Pi$ states, but did not use the upper state surface in their classical calculations. ${ }^{25}$ The effects of spin orbit term in the Hamiltonian and non-adiabatic coupling between surfaces were next treated theoretically to assess the contribution of $\mathrm{F}\left({ }^{2} \mathrm{P}_{1 / 2}\right)$ on the reactive cross section. Two early $a b$ initio studies were made on the potential variation along the collinear $\mathrm{F}$ to $\mathrm{H}_{2}$ center of mass coordinate, $\mathbf{R}_{\mathrm{F}, \mathrm{H}_{2}}$, for both ${ }^{2} \Sigma$ and ${ }^{2} \Pi$ states. ${ }^{26,27}$ Spin-orbit coupling was included semi-empirically into 
one of these calculations, ${ }^{26}$ and both studies calculated the non-adiabatic coupling strengths between the surfaces. Tully applied the diatomics-in-molecules (DIM) method to construct potential curves for all three states, as a function of $R_{\mathrm{F}, \mathrm{H}_{2}}$, in both collinear and side-on geometries. ${ }^{28}$ In this work the spin-orbit interaction was included, and non-adiabatic coupling strengths were once again evaluated. Faist and Muckerman reformulated the DIM method and constructed a complete semiquantitative correlation diagram for reaction between several states of the fragment atoms and diatoms. ${ }^{29}$

Both Tully and Muckerman demonstrated that the $\mathrm{F}\left({ }^{2} \mathrm{P}_{1 / 2}\right)+\mathrm{H}_{2}$ may contribute significantly to the overall reaction rate constant, via non-adiabatic interactions, even at thermal temperatures. ${ }^{28,29}$ Quantum calculations by Zimmerman et al. and LePetit et al. extended this work and showed varying result? for the behavior of the multi-

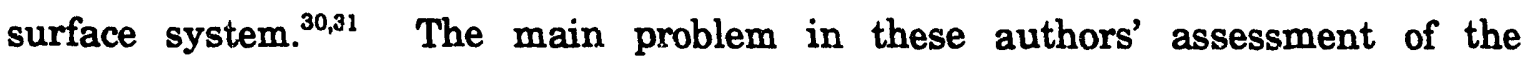
importance of the ${ }^{2} \Pi$ surfaces to the reaction dynamics was the barely semiquantitative knowledge of the shapes of these potential energy surfaces and their separation from the ground state. The non-adiabatic couplings are strongly dependent on the energy separations as a function of nuclear coordinates. Most theoretical effort subsequently concentrated on dramatically improving the quality of the ${ }^{2} \Sigma_{1 / 2}$ surface so as to reproduce newer experimental results, while assuming that the upper surfaces were not significant in the reaction. A recent study, using low energy scattering of magnetically analyzed $F$ atoms with $D_{2}$, reports experimentally determined potentials for all three states. ${ }^{32}$ However, only the long range part of each potential is characterized in these experiments. To our knowledge only Wright and coworkers have considered the upper state surface at short range recently. ${ }^{33}$ 
Our photoelectron results may finally address some of the questions about the shape of the excited state surfaces and their separations from the ground state surface in the latter's transition state region.

Presumably the reason for the absence of theoretical work on the upper state surfaces was because experimental work provided little data in this area. Experiments either did not explicitly look at the reaction of $F\left({ }^{2} P_{1 / 2}\right)+H_{2}$, or found, in contrast to some of the theoretical predictions, ${ }^{29,30}$ it to be insignificant compared to the reaction of ground state fluorine atoms. Neumark et al. concluded that $\mathrm{F}\left({ }^{2} \mathrm{P}_{1 / 2}\right)$ was unreactive in their cross beam studies of $\mathrm{F}+\mathrm{H}_{2}, \mathrm{HD}$ and $\mathrm{D}_{2} \cdot{ }^{34}$ This implies that non-adiabatic effects in the entrance channel are not large enough, at least at the collision energies employed in their study $(0.68-3.42 \mathrm{kcal} / \mathrm{mol}){ }^{34}$ to make this pathway competitive with the electronically adiabatic ground state reaction. Hepburn et al. made a crossed molecular beam study of a related system, $\mathrm{F}+\mathrm{HBr}$, and although they observed a significant exit channel non-adiabatic process forming $\mathrm{Br} *\left({ }^{2} \mathrm{P}_{1 / 2}\right)$ product from ground state $\mathrm{F}\left({ }^{2} \mathrm{P}_{3 / 2}\right)+\mathrm{HBr}$ rather than from $\mathrm{F}\left({ }^{2} \mathrm{P}_{1 / 2}\right)$, they concluded again that $F\left({ }^{2} \mathrm{P}_{1 / 2}\right)$ was unreactive compared to $F\left({ }^{2} \mathrm{P}_{3 / 2}\right) .^{35}$

What do our photoelectron spectra tell us about these excited state surfaces? The $213 \mathrm{~nm}$ results (Figure 7-3) show no additional electronic bands within $2 \mathrm{eV}$ of the ${ }^{2} \Sigma$ band except those seen in the $266 \mathrm{~nm}$ spectrum appearing at polarization $\theta=90^{\circ}$. Muckerman's semi-quantitative correlation diagram predicts that only the two ${ }^{2} \Pi$ states are expected within $6 \mathrm{eV}$ of the ground state. Hence we may confidently assume that the allowed transitions from the anion to the ${ }^{2} \Pi_{i}$ states are those in the $266 \mathrm{~nm}$ spectrum between $\mathrm{eKE}=0.5$ and $0.9 \mathrm{eV}$. Now it has been shown that, by setting the polarization of the laser parallel to the electron collection direction, the 
contribution of the ${ }^{2} \Pi$ states may be all but eliminated. ${ }^{3}$ This spectrum may then be used as a reference spectrum for the ${ }^{2} \Sigma$ band, and we may subtract this spectrum, appropriately scaled, from the spectrum collected with the perpendicular laser polarization geometry. Let us assume that feature A (at highest L $E$ ) in the normal$\mathrm{FH}_{2}-$ spectrum has no contribution from the excited electronic states, and so this peak is a marker of the contribution of the ${ }^{2} \Sigma_{1 / 2}$ surface to the photoelectron spectrum for the scaling procedure in the subtraction.

Figure 7-8 shows the subtracted result which we will assume represents the spectrum of transitions from the anion to the ${ }^{2} \Pi_{3 / 2,1 / 2}$ states only. The two photon $\mathrm{F}^{-}$ peaks at 1.21 and $1.26 \mathrm{eV}$ appear with relatively large intensity in the difference plot because the $\mathbf{F}\left({ }^{2} \mathrm{P}_{3 / 2,1 / 2}\right) \leftarrow \mathrm{F}^{-}$transitions also have electron angular distributions peaked at $\theta=90^{\circ}$. The noise in the region $0.9-1.1 \mathrm{eV}$ we assume is due to slight differences in the ${ }^{2} \Sigma$ band shape due to incomplete signal averaging, which is amplified in the subtraction process. The structure we are interested in lies between 0.5 and $0.9 \mathrm{eV}$. The band rises fairly sharply at $\mathrm{eKE}=0.9 \mathrm{eV}$, peaks at approximately $0.8 \mathrm{eV}$ and has a full width half maximum (FWHM) of ca. $0.3 \mathrm{eV}$. We note that the band is quite asymmetrical.

As described above, potential energy curves have been calculated for the ${ }^{2} \Pi$ state. We may therefore attempt to simulate this photoelectron band using these potentials. Tully calculated $V_{\Pi}$ as a function of $R_{F, H_{2}}$ with $R_{H-H}$ fixed at the equilibrium distance in $\mathrm{H}_{2}$ for both spin orbit components. Blais and Truhlar, ignoring the spin orbit interaction, calculated a valence-bond potential energy surface for the ${ }^{2} \Pi$ state and show a potential map as a function of $\mathrm{R}_{\mathrm{F}, \mathrm{H}_{2}}$ and $\mathrm{P}_{\mathrm{H} \cdot \mathrm{H}}$ for $\mathrm{C}_{-\mathrm{v}}$. Subsequent collinear dynamical calcul ${ }^{1}$ tions have used a modified form of the Blais-Truhlar surface or DIM 
surfaces. The $V_{n}$ potentials are essentially non-reactive as they correlate to high lying, repulsive, states of HF (see Fig. 7-7a). They may be approximated by the separable function

$$
V_{\text {II }}=V_{\text {bound }}\left(R_{H-H}\right)+V_{\text {rep }}\left(R_{F, H_{2}}\right)
$$

where $\mathrm{V}_{\text {bound }}$ is the bound ${ }^{1} \Sigma_{\mathrm{g}}^{+}$potential curve of $\mathrm{H}_{2}$, modelled by, say, a Morse function, and $V_{\text {rep }}$ is the repulsive interaction of the $F$ atom with $\mathrm{H}_{2}$ in a $\Pi$ configuration. The repulsive potential can be modelled by an exponential curve fit to each of Tully's ${ }^{2} \Pi$ DIM curves, ${ }^{28 b}$

$$
\begin{gathered}
V_{\text {rep }}=A_{3 / 2} \exp \left(-\beta_{3 / 2} R_{F, H_{2}}\right) \\
V_{\text {rep }}=A_{1 / 2} \exp \left(-\beta_{1 / 2} R_{F, H_{2}}\right)+\Delta
\end{gathered}
$$

where $\Delta=0.0501 \mathrm{eV}$, the spin orbit splitting in fluorine, ${ }^{23}$ and $\mathrm{V}$ is in units of $\mathrm{eV}$ and $\mathbf{R}_{\mathrm{F}, \mathrm{H}_{2}}$ in $\AA$.

To simulate the photoelectron band we need to calculate the overlap of the anion ground state wavefunction with the scattering states supported by each ${ }^{2} \Pi$ surface. If we assume that electronic and nuclear motions are uncoupled, i.e. ignore non-adiabatic effects, a relatively simple quantum mechanical calculation using the potential function given by Eqn. 1 will yield the scattering states for each spin-orbit surface; a fully coupled collinear calculation would resemble the formalism used by LePetit. $^{31} \mathrm{~A}$ wavepacket propagation in the time domain is formally equivalent to a time-independent calculation of scattering states, and so, as before ${ }^{36}$ we adopt this methodology to perform the simulation of the ${ }^{2} \Pi$ band. The contribution of the ${ }^{2} \Pi$ states, and the model we are using to describe it, is very similar to the contribution 
of the $\left.\mathrm{F}+\mathrm{HO} \rightarrow \mathrm{HF}+\mathrm{O}^{2} \mathrm{D}\right)$ reaction to $\mathrm{OHF}^{-}$spectrum. ${ }^{37}$ In the Franck-Condon region the $V_{n}$ potentials are fairly repulsive, compared to the ${ }^{2} \Sigma$ potential, so the wavepacket moves quickly out of this region, and only a short propagation time is necessary. The simulated bands due to the ${ }^{2} \Pi_{3 / 2}$ and ${ }^{2} \Pi_{1 / 2}$ are shown in Fig. 7-8. In the simulation we have assumed Simon's best ab initio geometry, a coupled cluster $\operatorname{CCSD}(T)$ optimized structure $\left(R_{\mathrm{F}, \mathrm{H}_{2}}=2.075 \AA, R_{\mathrm{H} \cdot \mathrm{H}}=0.770 \AA\right)$, and his MCSCF harmonic frequencies for the anion. ${ }^{6}$ For $V_{n}$, the Morse parameters for $V_{b o u n d}$ are derived from the constants in Huber and Herzberg. ${ }^{38}$

The first simulation assumes $\beta_{3 / 2}=5.53 \dot{A}_{-1}, A_{3 / 2}=3022 \mathrm{eV}, \beta_{1 / 2}=5.60 \dot{\AA}^{-1}$ and $A_{1 / 2}=2950 \mathrm{eV}$ in Eqn. 2; these parameters give the best fit to the curves shown in Figure 2 of Tully's paper. ${ }^{28 b}$ Each has similar shape and the shape reproduces the experimental band shape in that it rises fairly rapidly at lower scattering energies (high eKE) and has a longer tail at low eKE. However, it is immediately apparent that $V_{\text {rep }}$ is not repulsive enough to reproduce the FWHM of the band. The FWHM in the simulation, for each ${ }^{2} \Pi$ component, is only $0.065 \mathrm{eV}$. Further, the onset of the band (the high eKE edge) is very close to the $\mathrm{F}+\mathrm{H}_{2}$ asymptote, and in comparison to the experimental band is at too high electron kinetic energy. The collision energy scale, i.e. the energy, $\mathrm{E}^{(0)}$, above $\mathrm{F}+\mathrm{H}_{2}(\mathrm{v}=0)$, of the calculation is anchored to the electron energy scale by the formula ${ }^{3}$

$$
e K E(e V)=0.999-E^{(0)}(e V)
$$

which assumes a value for the dissociation energy of $\mathrm{FH}_{2}-$ of $0.260 \mathrm{eV}^{3}$

The second simulation uses a $V_{\text {rep }}$ that mimics the much more repulsive BlaisTruhlar potential; ${ }^{25}$ here we have considered just one ${ }^{2} \Pi$ surface and found $A=68.2$ $\mathrm{eV}$ and $\beta=2.40 \dot{A}^{-1}$ by comparison to the contour plot, Figure 2, of Ref. 30. This 
simulated band's onset is at much lower eKE $(0.75 \mathrm{eV})$ and the band has a much wider FWHM, $0.41 \mathrm{eV}$, than the simulations of the DIM-like potentials. This is clearly at the opposite extreme; the surface is now too repulsive at the anion geometrical configuration. The third simulation shown is a compromise "fit". It has simulated bands due to both ${ }^{2} \Pi_{3 / 2}$ and ${ }^{2} \Pi_{1 / 2}$ surfaces, $V_{\text {rep }}$ has the same form as in Tully's plot, but has the correct slope and potential energy at the Franck-Condon, i.e. anion, $F$ to $\mathrm{H}_{2}$ separation. The $\mathrm{V}_{\text {rep }}$ curves for this fit potential are shown in Figure 7-7(b) along with the ground state potential. The vertical energy differences from the ${ }^{2} \Sigma_{1 / 2}$ to the ${ }^{2} \Pi_{3 / 2}$ and ${ }^{2} \Pi_{1 / 2}$ surfaces at the anion geometry are $0.18 \mathrm{eV}$ and $0.21 \mathrm{eV}$, using the T5a potential ${ }^{7}$ for the ${ }^{2} \Sigma$ and the above "fitted" for ${ }^{2} \Pi$. In comparison, Simons' $a b$ initio calculation suggested $0.25 \mathrm{eV}$ for the ${ }^{2} \Sigma-{ }^{2} \Pi$ splitting at this geometry, ${ }^{6}$ whereas Tully's DIM curves suggest $0.01 \mathrm{eV}$ and $0.06 \mathrm{eV}$ separation from the ${ }^{2} \Sigma_{1 / 2}$ to the ${ }^{2} \Pi_{3 / 2}$ and ${ }^{2} \Pi_{1 / 2}$ respectively. ${ }^{28}$ Wright gives the ${ }^{2} \Sigma-{ }^{2} \Pi$ separation at the lower's saddle point geometry is $c a .0 .78 \mathrm{eV}^{33}$ The simulated bands in Figure 7-8(c) are separated by $0.03 \mathrm{eV}$ at the band maximum and the band FWHM for the ${ }^{2} \Pi_{3 / 2}$ and ${ }^{2} \Pi_{1 / 2}$ are 0.23 and $0.21 \mathrm{eV}$ respectively. The sum of the two simulated bands approximately reproduces the whole unresolved band in our experimental spectrum.

All simulations show a small bump at lowest eKE's in both spin orbit components. The bump is due to overlap with states correlating to $\mathrm{H}_{2}(\mathrm{v}=1)$; there is overlap to these vibrationally excited states because the anion has a slightly elongated $H \cdot H$ bond, and the valleys in the $V_{\Pi}$ surfaces have $R_{H \cdot H}$ set at equilibrium $\mathrm{H}_{2}$. The intensity of this band depends on two factors, the degree of $\mathrm{H}-\mathrm{H}$ elongation in the anion and on the anharmonicity assumed along the $\mathrm{H}-\mathrm{H}$ stretch in the anion. It seems reasonable to expect $\omega_{\mathrm{e}} \mathrm{x}_{\mathrm{e}}$ for this mode to be at least as large in the anion 
as it is for free $\mathrm{H}_{2}$, which we have assumed in the simulation, and it is most likely larger (which would yield more intensity in the $v=1$ bump). This may account for some of the signal extending out to low eKE's in the experimental difference plot.

The important result here is that the ${ }^{2} \Pi$ surfaces rise more steeply in the interaction region than predicted by the diatomics-in-molecules (DIM) approach, and we have determined a more realistic form for the potentials. It may now be possible to estimate, with somewhat more certainty, the non-adiabatic coupling between the three surfaces in the entrance valley, and once again assess the reactivity of $F\left({ }^{2} P_{1 / 2}\right)$ with $\mathrm{H}_{2}$.

\section{Summary}

In this work we have shown that there are pronounced differences in the ground state photoelectron band of $\mathrm{FH}_{2}{ }^{-}$when synthesized from norral and para hydrogen. This has been rationalized in terms of the nuclear spin restrictions on the anion wavefunction. Three dimensional quantum scattering results employing the T5a surface are compared to the experimental spectra. There are likewise strong differences in the theoretical Franck Condon overlaps to ortho and para parity states. It should be restated that the previous comparisons of quantum scattering calculations (which used only the para symmetry states) with the normal- $\mathrm{FH}_{2}{ }^{-}$spectrum were erroneous. When we make the correct comparison, the agreement between the theory and experiment is not as good as we originally had thought. The same considerations should be taken into account for the published comparison of the three-dimensional Franck Condon overlaps and the experimental spectrum for normal- $\mathrm{FD}_{2}{ }^{-4}$. New simulations for the photoelectron spectrum of normal- $\mathrm{FD}_{2}-(1: 2$ para: ortho $)$, as well 
as a calculation on $\mathrm{FDH}^{-}$, where there have been none to date, would be useful in determining where the discrepancies between theory and experiment lie.

Quantitative consideration of the excited state bands, assigned to the ${ }^{2} \Pi_{3 / 2}$ and ${ }^{2} \Pi_{1 / 2}$ states, has been made here, and collinear simulations have allowed the determination of the shape of these potential curves along the $R_{F, H_{2}}$ coordinate in the interaction region. We hope this will stimulate some $a b$ initio work on characterizing these surfaces, and their non-adiabatic coupling to the ground state surface.

There is clearly much still to learn about the $\mathrm{F}+\mathrm{H}_{2}$ reaction, and our photoelectron experiments have brought a new dimension into the fitting of the potential surfaces, both in the transition state region for the ground reaction surface and in the inner regions of the upper non-reactive surfaces. Further experiments to extract even more detail are possible. Zero electron kinetic energy (ZEKE) photodetachment spectra, with an attainable resolution of $5 \mathrm{~cm}^{-1}$, would be particularly useful for this transition state system. As the feature $A$ in the normal$\mathrm{FH}_{2}{ }^{-}$spectrum is quite narrow, a more concrete assignment of the $\mathrm{FH}_{2}{ }^{-}$internal states giving rise to the peaks in this region should be possible in a ZEKE spectrum.

One of the most serious limitations in deriving hard information about the neutral potential energy surfaces from our photoelectron spectra is the absence of high quality data on the anion precursor, notably the cluster dissociation energy, the equilibrium structure and the anion vibrational levels. Ab initio calculations are used extensively in the place of high resolution spectroscopic data. However, various 'consequence', or 'action', spectroscopies could be applied on a mass-selected ion beam to perform, for example, vibrational spectroscopy. One final experimental approach, that is being pursued in our laboratory, is to attempt photoelectron spectroscopy of 
selectively-prepared vibrationally excited negative ions. This idea has already been outlined in Chapter 1. In Chapter 5 we saw that the photoelectron spectra of AHBions in the $v_{3}=1$ quantum state reveals very different information about the neutral reaction surface. For $\mathrm{FH}_{2}^{-}$, pumping one quantum in the $\mathrm{H}_{2}$ stretching mode, e.g. via a stimulated Raman process, ${ }^{39}$ would allow overlap with a very different part of the ${ }^{2} \Sigma$ reaction surface. Although a quantum of vibrational energy in the $\mathrm{H}_{2}$ stretch exceeds the calculated ion dissociation energy, the vibrational predissociation lifetime may be longer than the time for the excited molecule to interact with the photodetachment photon.

\section{Acknowledgements.}

I would like to thank Dr. David Manolopoulos for providing results of his three dimensional calculations prior to publication, and for very useful discussions. I thank Prof. J. Zhang for communicating new Franck Condon results on the 5SEC surface. I am also grateful to Prof. Rich Saykally for useful discussions on nuclear spin statistics considerations in the $\mathrm{FH}_{2}{ }^{-}$anion. 


\section{References for Chapter 7}

1. A. Weaver, $\mathrm{Ph}$. D. thesis, University of California, Berkeley (1991)

2. A. Weaver, R. B. Metz, S. E. Bradforth and D. M. Neumark, J. Chem. Phys. 93, $5352(1990)$

3. A. Weaver and D. M. Neumark, Faraday Discuss. Chem. Soc. 91, 5 (1991)

4. J. Z. H. Zhang, W. H. Miller, A. Weaver and D. M. Neumark, Chem. Phys. Lett. 182, 283 (1991)

5. J. Z. H. Zhang and W. H. Miller, J. Chem. Phys. 92, 1811 (1990)

6. Nichols and Simons, J. Phys. Chem. 95, 1074 (1991)

7. R. Steckler, D. G. Truhlar, and B. C. Garrett, J. Chem. Phys. 82, 5499 (1985)

8. J. E. Pollard, D. J. Trevor, J. E. Reutt, Y. T. Lee and D. A. Shirley, J. Chem. Phys. 77, 34 (1982)

9. R. E. Continetti, Ph. D. thesis, University of California, Berkeley (1989)

10. D. Proch and T. Trickl, Rev. Sci. Instrum. 60, 713 (1989)

11. See chapters 3,5 , and 6

12. R. B. Metz, A. Weaver, S. E. Bradforth, T. N. Kitsopoulos and D. M. Neumark, J. Phys. Chem. 94, 1377 (1990)

13. G. Herzberg, Molecular Spectra and Molecular Structure Vol. II, Van Nostrand Rheinhold Co., New York (1955), pp. 220-221; P. J. Bunker, Molecular Symmetry and Spectroscopy, Academic Press, New York (1979)

14. G. Henderson and G. E. Ewing, J. Chem. Phys. 59, 2280 (1973); G. Henderson and G. E. Ewing, Mol. Phys. 27, 903 (1974)

15. J. Z. H. Zhang and W. H. Miller, J. Chem. Phys. 88, 4549 (1988)

16. D. E. Manolopoulos, unpublished work

17. G. C. Schatz, J. Chem. Phys. 90, 3582 (1989)

18. D. E. Manolopoulos, private communication

19. J. E. Pollard, D. J. Trevor, Y. T. Lee and D. A. Shirley, J. Chem. Phys. 77, 4818 (1982) 
20. G. C. Lynch, R. Steckler, D. W. Schwenke, D. G. Truhlar, A. J. C. Varandas and B. C. Garrett, J. Chem. Phys. 94, 7136 (1991)

21. J. D. Kress and E. F. Hayes, J. Chem. Phys. 97, 4881 (1992)

22. J. Z. H. Zhang, private communication

23. S. Baskin, J. O. Stoner, Jr., Atomic Energy Level and Grotian Diagrams Vol. 1, North Holland Publishing Company, New York (1978)

24. D. G. Truhlar, J. Chem. Phys. 56, 3189 (1972); J. T. Muckerman and M. D. Newton, J. Chem. Phys. 56, 3191 (1972)

25. N. C. Blais and D. G. Truhlar, J. Chem. Phys. 58, 1090 (1973)

26. R. L. Jaffe, K. Morokuma and T. F. George, J. Chem. Phys. 63, 3417 (1975)

27. F. Rebentrost and W. A. Lester, Jr., J. Chem. Phys. 63, 3884 (1976)

28. J. C. Tully, J. Chem. Phys. 59, 5122 (1973); J. C. Tully, J. Chem. Phys. 60, 3042 (1974)

29. M. B. Faist and J. T. Muckerman, J. Chem. Phys. 71, 233 (1978)

30. I. H. Zimmerman, M. Baer and T. F. George, J. Chem. Phys. 71, 4132 (1979)

31. B. LePetit, J. M. Launay and M. Le Dourneuf, Chem. Phys. 106, 111 (1986)

32. V. Aquilanti, R. Candori, D. Cappelletti, E. Luzzatti and F. Pirani, Chem. Phys. 145, 293 (1990)

33. M. Kolbuszewski and J. S. Wright, J. Chem. Phys. 96, 5548 (1992)

34. D. M. Neumark, A. M. Wodtke, G. N. Robinson, C. C. Hayden and Y. T. Lee, J. Chem. Phys. 82, 3045 (1985); D. M. Neumark, A. M. Wodtke, G. N. Robinson, C. C. Hayden, K. Shobatake, R. K. Sparks, T. P. Schafer and Y. T. Lee, J. Chem. Phys. 82, 3067 (1985)

35. J. W. Hepburn, K. Liu, R. G. MacDonald, F. J. Northrup and J. C. Polanyi, J. Chem. Phys. 75, 3353 (1981)

36. S. E. Bradforth, D. W. Arnold, R. B. Metz and A. Weaver, J. Chem. Phys. 92, 7205 (1990)

37. S. E. Bradforth, D. W. Arnold, R. B. Metz, A. Weaver and D. M. Neumark, J. Phys. Chem. 95, 8066 (1991)

38. K. P. Huber and G. Herzberg, Spectra of Diatomic Molecules Vol. IV, Van Nostrand Rheinhold Company, New York, 1979 
39. B. F. Henson, G. V. Hartland, V. A. Venturo and P. M. Felker, J. Chem. Phys. 97, 2189 (1992) 


\section{Figure Captions for Chapter 7}

Figure 7-1. Plot of $\mathrm{F}+\mathrm{H}_{2} \rightarrow \mathrm{HF}+\mathrm{H}$ ground state reaction surface with the $\mathrm{FH}_{2}{ }^{-}$ ground vibrational state wavefunction shown shaded. The contours for the neutral potential surface are determined from the T5a potential function of ref. 7. The saddle point $\left(R_{F, \mathrm{H}_{2}}=1.953 \AA, R_{\mathrm{H} \cdot \mathrm{H}}=0.762 \AA\right)$ is marked with a cross. The anion wavefunction assumes the $a b$ initio $\operatorname{CCSD}(T)$ equilibrium geometry $\left(R_{\mathrm{F}, \mathrm{H}_{2}}=2.075 \AA\right.$ and $\left.\mathrm{R}_{\mathrm{H} \cdot \mathrm{H}}=0.770 \AA\right)$ and MCSCF harmonic frequencies of Nichols et al. (ref. 6); the ellipse represents the $90 \%$ probability limits of the wavefunction. The axes for the plot are massed scaled Jacobi coordinates: $x=\left(\mu_{F, H_{2}} / \mu_{H_{2}}\right)^{1 / 2} R_{F, H_{2}}$ and $y=R_{H-H}$. The skew angle for $\mathrm{FH}_{2}$ is $46^{\circ}$.

Figure 7-2. Photoelectron spectra of $\mathrm{FH}_{2}^{-}$at $266 \mathrm{~nm}$. (Top) Ions made from normal $-\mathrm{H}_{2}$ (3:1 ortho/ para), and (Bottom) ions made from para $-\mathrm{H}_{2}$. Spectra recorded at two polarizations of the photodetachment laser: (Left) parallel $\left[\theta=0^{\circ}\right]$ and (Right) perpendicular $\left[\theta=90^{\circ}\right]$ to direction of electron collection.

Figure 7-3. Photoelectron spectra of $\mathrm{FH}_{2}{ }^{-}$, made from normal $-\mathrm{H}_{2}$, at $213 \mathrm{~nm}$. (Top) polarization parallel $\left(\theta=0^{\circ}\right)$ and (Bottom) perpendicular $\left(\theta=90^{\circ}\right)$ to direction of electron collection.

Figure 7-4. Correlation diagram for bend/ hindered rotor energy levels of $\mathrm{FH}_{2}$ Labels $\mathrm{J}$ and $\mathrm{M}$ correspond to the free rotor total angular momentum and its projection on the body fixed axis; $\mathrm{v}_{2}$ and $\ell$ are the vibrational quantum number and the vibrational angular momentum for the degenerate linear bend. The solid lines indicate vibrational states that 
are symmetric with respect to $\mathrm{H}$ nuclei permutation, the dashed lines for antisymmetric states. Figure adapted from that for $\mathrm{Ar} . . \mathrm{O}_{2}$ from Ref. 14.

Figure 7-5. (a) Three dimensional simulation (thin line) of $\mathrm{FH}_{2}{ }^{-}$photoelectron spectrum, considering only symmetric permutation states, of Zhang and Miller (Ref. 4) compared to $\theta=0^{\circ} \mathrm{FH}_{2}{ }^{-}\left(\right.$para $\left.-\mathrm{H}_{2}\right)$ experimental spectra (solid line). (b) Three dimensional simulation (thin line) of $\mathrm{FH}_{2}^{-}$ photoelectron spectrum, considering both symmetric and antisymmetric permutation states, of Manolopoulos (Ref. 18) compared to the $\theta=0^{\circ}$ $\mathrm{FH}_{2}{ }^{-}\left(\right.$normal $\left.-\mathrm{H}_{2}\right)$ experimental spectrum. The simulation is a weighted sum of transitions to ortho and para states. Both calculations assume the same anion and neutral parameters.

Figure 7-6. Highest inolecular orbitals for $\mathrm{FH}_{2}{ }^{-}$, showing the $3 \sigma$, the $1 \pi$ and the $4 \sigma$, all of which are fully occupied in the anion. Detachment (removal) of an electron from the $4 \sigma$ accesses the ${ }^{2} \Sigma$ reaction surface of $F+H_{2}$, whereas detachment from the $1 \pi$ accesses the upper ${ }^{2} \Pi$ surfaces. The molecular orbitals are the optimized MP2/6-31++G** orbitals evaluated at the computed equilibrium structure for the $\mathrm{FH}_{2}{ }^{-}$ion at the same level of theory.

Figure 7-7. (a) Electronic correlation diagram for $\mathrm{F}+\mathrm{H}_{2}$. (b) The variation of potential energy for the three lowest lying electronic states of $\mathrm{FH}_{2}$ as a function of the $\mathrm{F}$ to $\mathrm{H}_{2}$ distance. Lowest curve $\left({ }^{2} \Sigma_{1 / 2}\right)$ is calculated from the T5a surface (Ref. 7), upper ${ }^{2} \Pi_{3 / 2,1 / 2}$ surfaces are those calculated to best fit difference spectrum Figure 7-8 (see text). 
Figure 7.8. (a) Difference plot of the $266 \mathrm{~nm}$ normal $-\mathrm{H}_{2}$ spectra. Here the $\theta=0^{\circ}$ spectrum has been scaled and subtracted from the $\theta=90^{\circ}$ spectrum to yield the band due to transitions from the anion to the ${ }^{2} \Pi_{3 / 2}$ and ${ }^{2} \Pi_{1 / 2}$ $\mathrm{FH}_{2}$ states. (b)-(d) Collinear simulations of the ${ }^{2} \Pi$ bands described in text. Parameters in $V_{\text {rep }}$ used for simulations are chosen to (b) approximate DIM curves of Ref. 28, (c) approximate Blais-Truhlar surface, Ref. 25 and 30, and (d) yield a fit to photoelectron band (a). In simulations (b) and (d) transitions to both spin orbit components of the ${ }^{2} \Pi$ state have been considered, and are assumed to have equal transition probability (dashed and dot-dashed lines). The sum of the two sub-bands are shown by the solid line. 


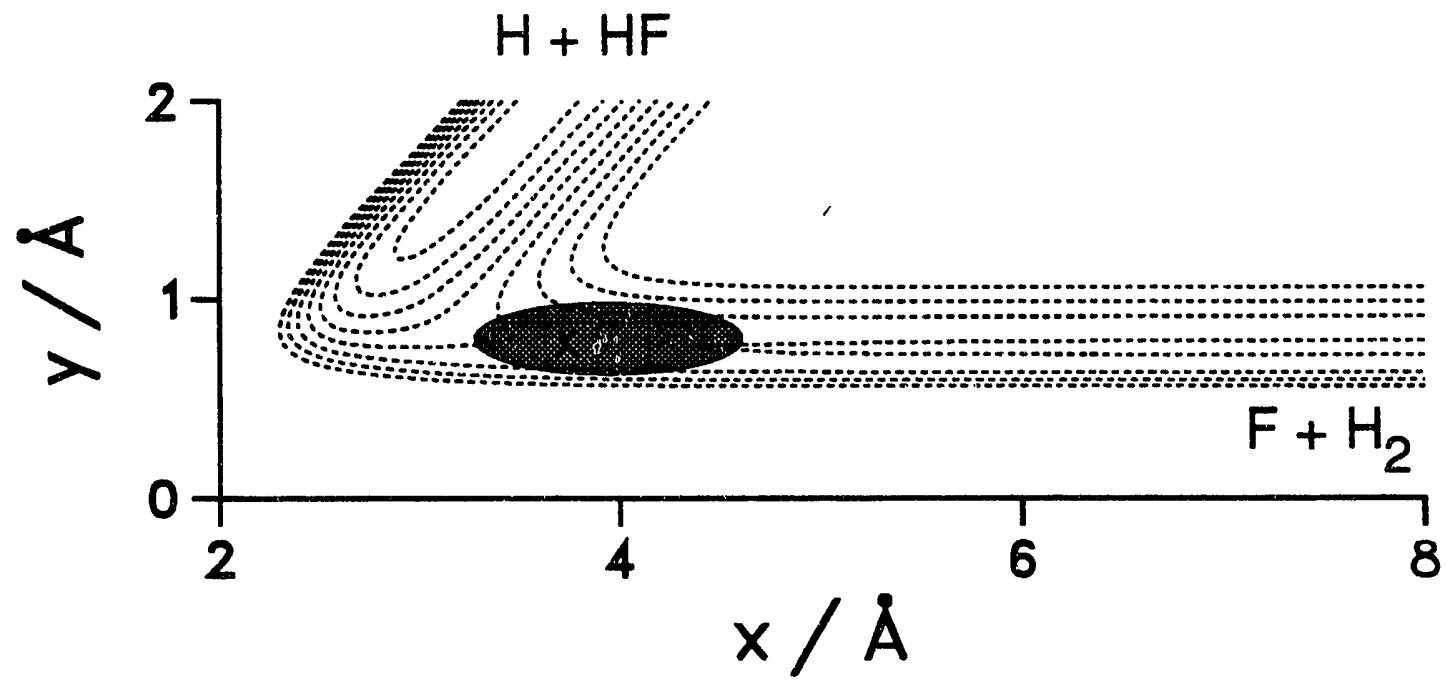

Figure 7-1 


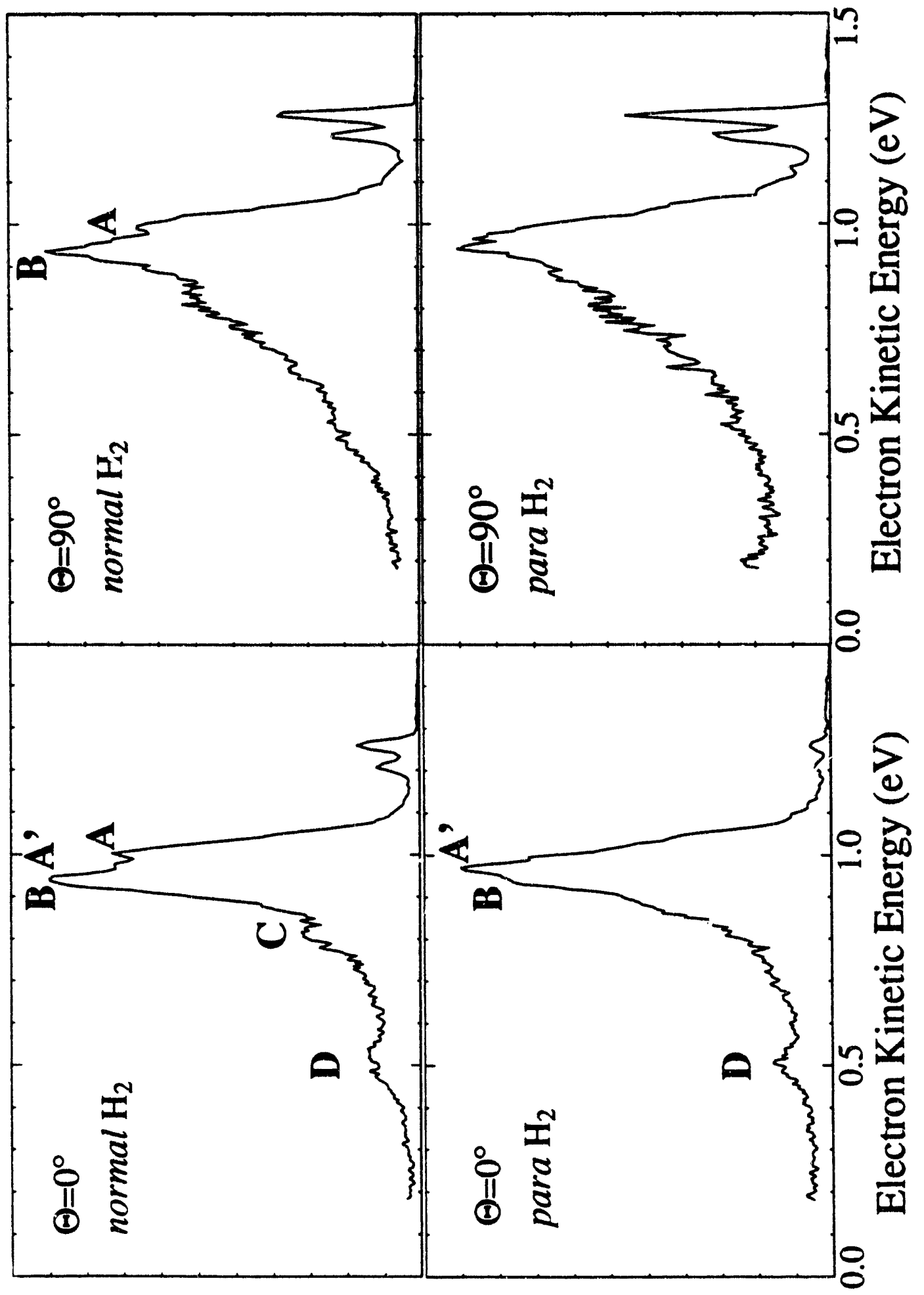

Figure 7-2 
$\mathrm{FH}_{2}^{-} 213 \mathrm{~nm}$
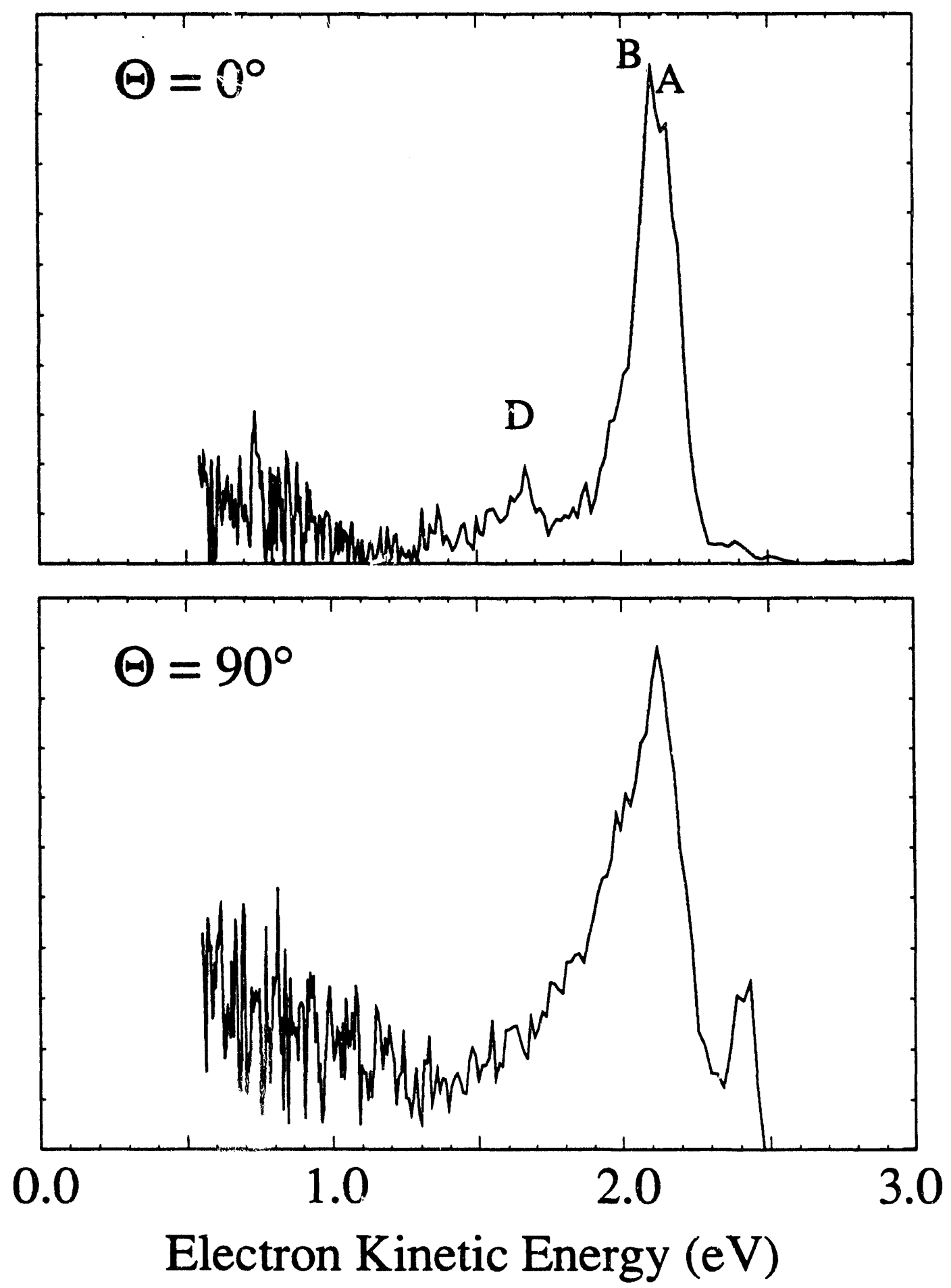

Figure 7-3 


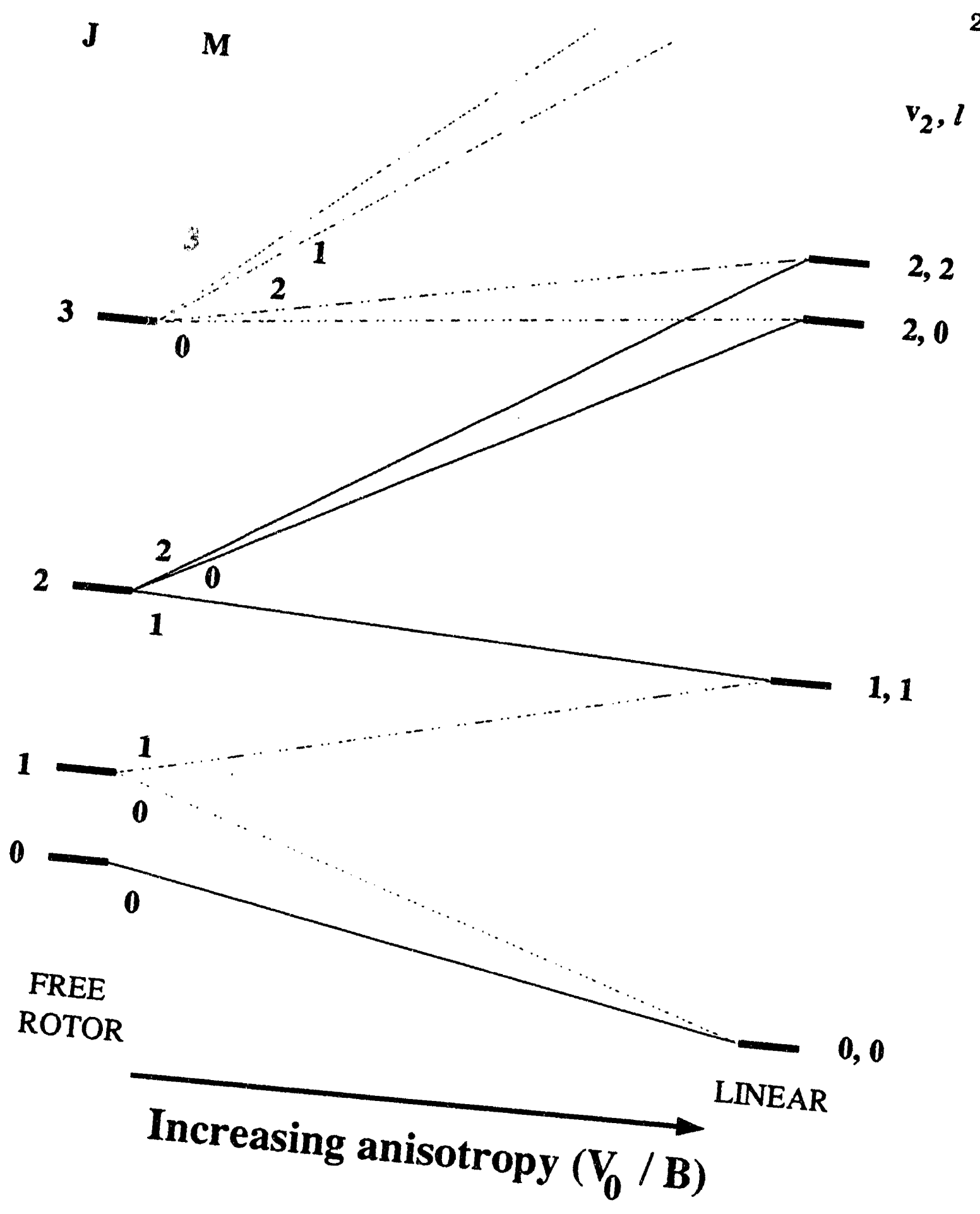

Figure 7.4 


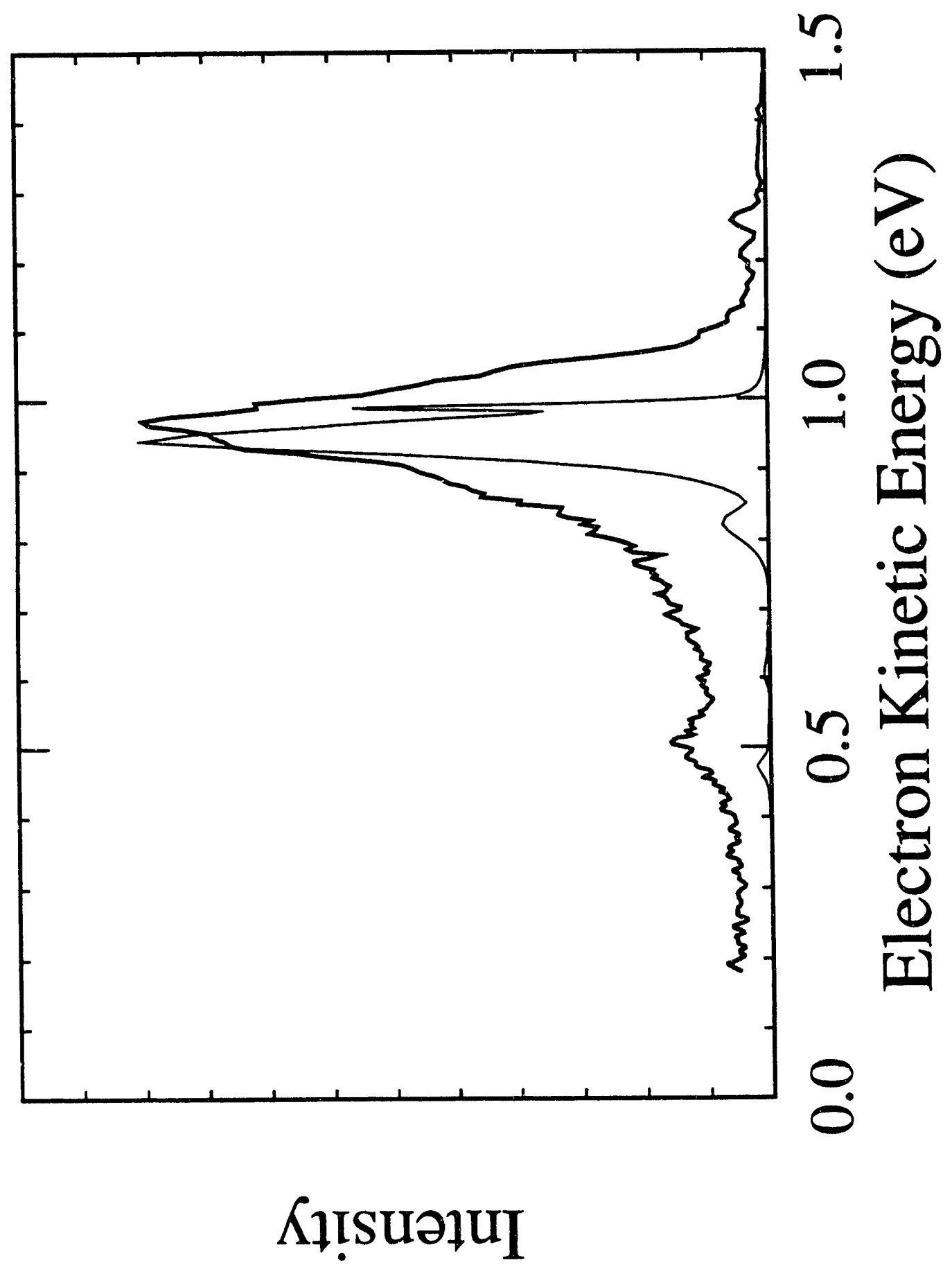

Figure 7-5(a) 


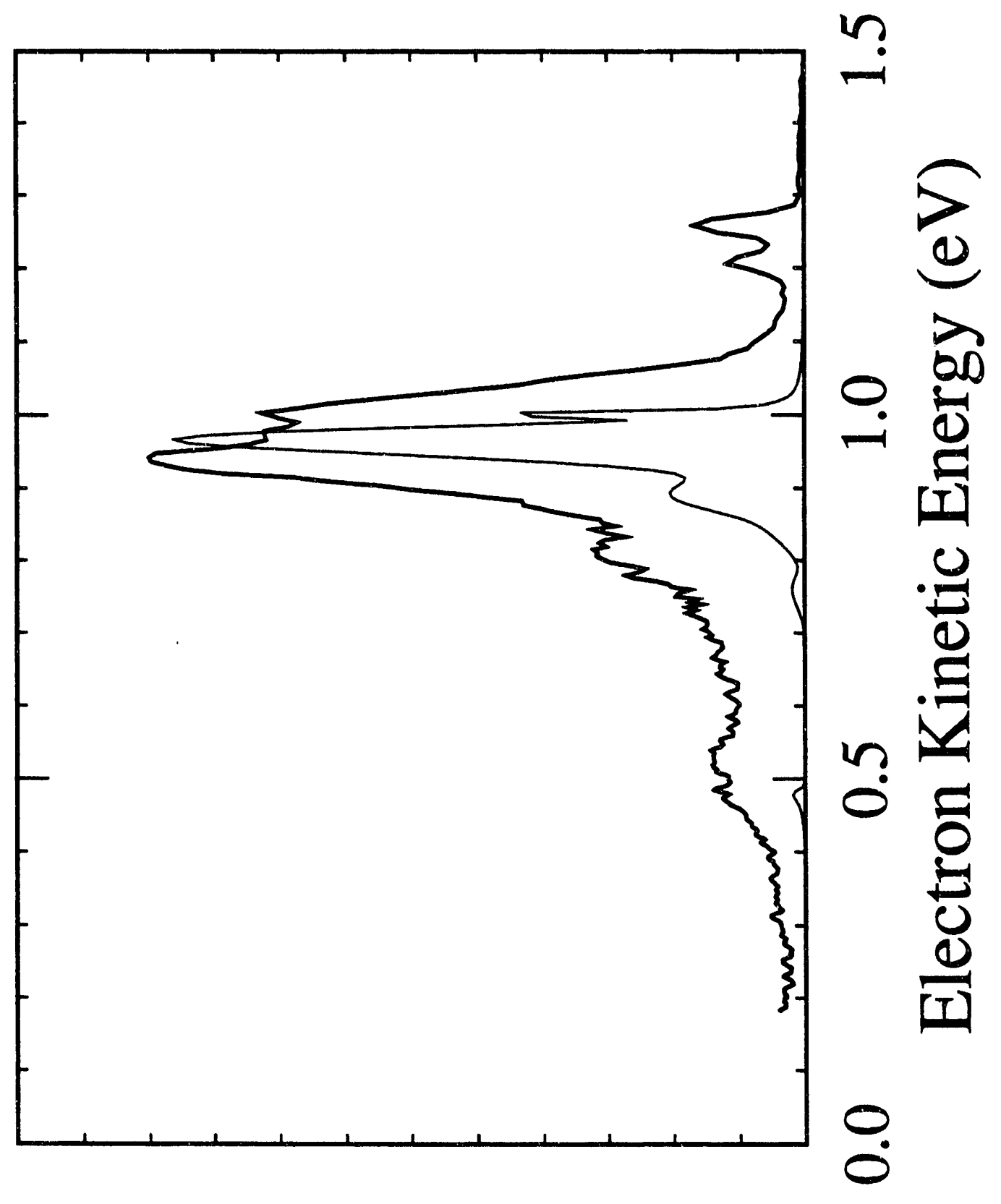

Kł!ฺ่งนวฺนI

Figure 7-5(b) 


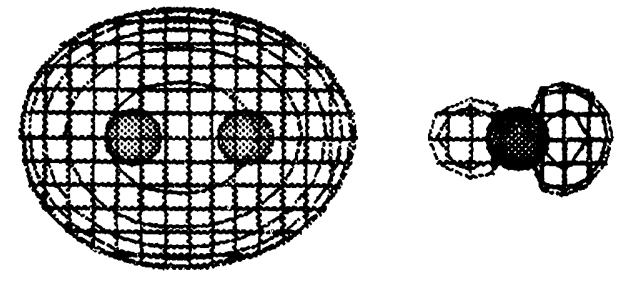

$3 \sigma$
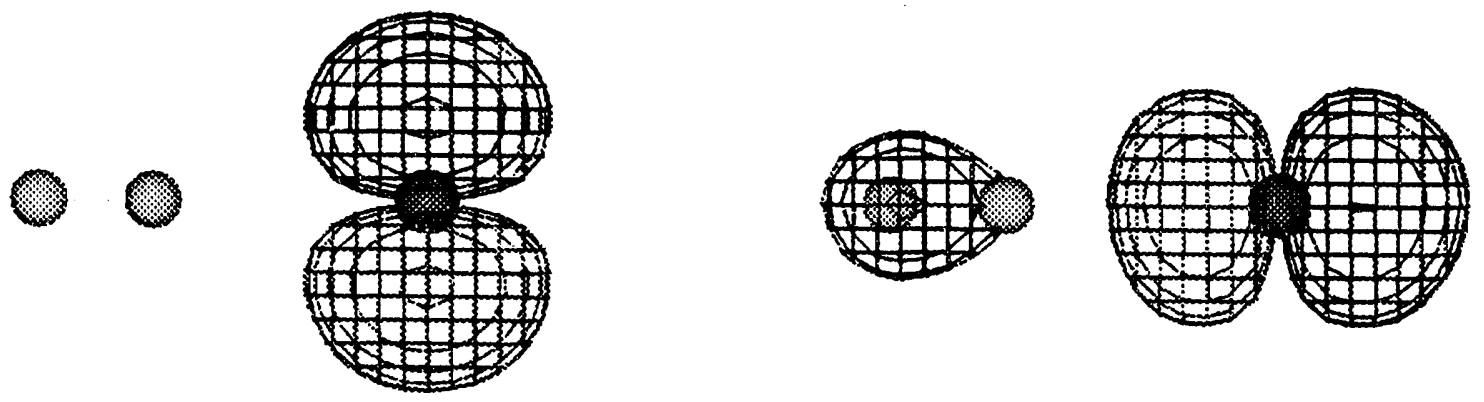

$1 \pi$

$4 \sigma$

Figure 7-6 


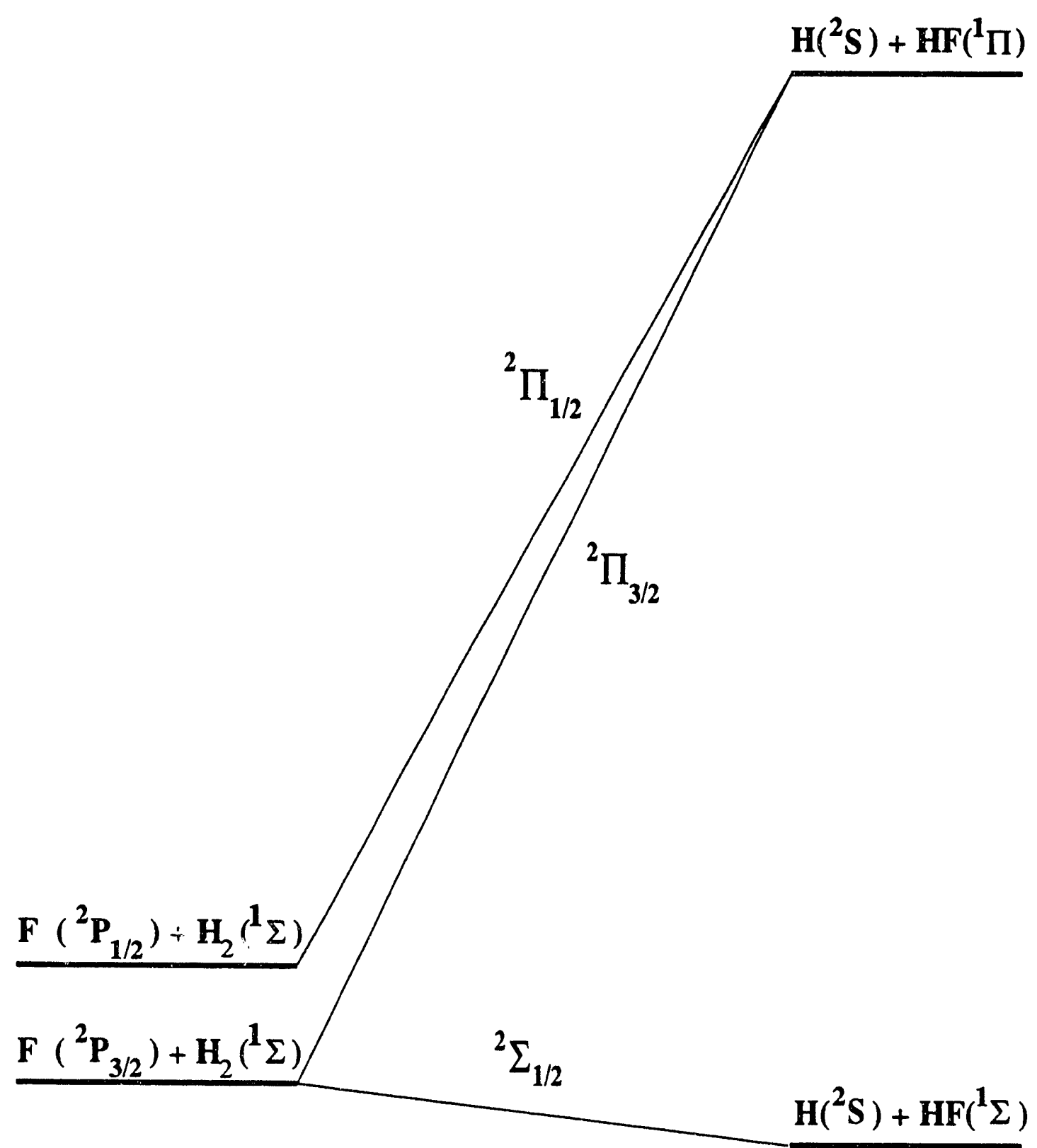

Figure 7-7(a) 


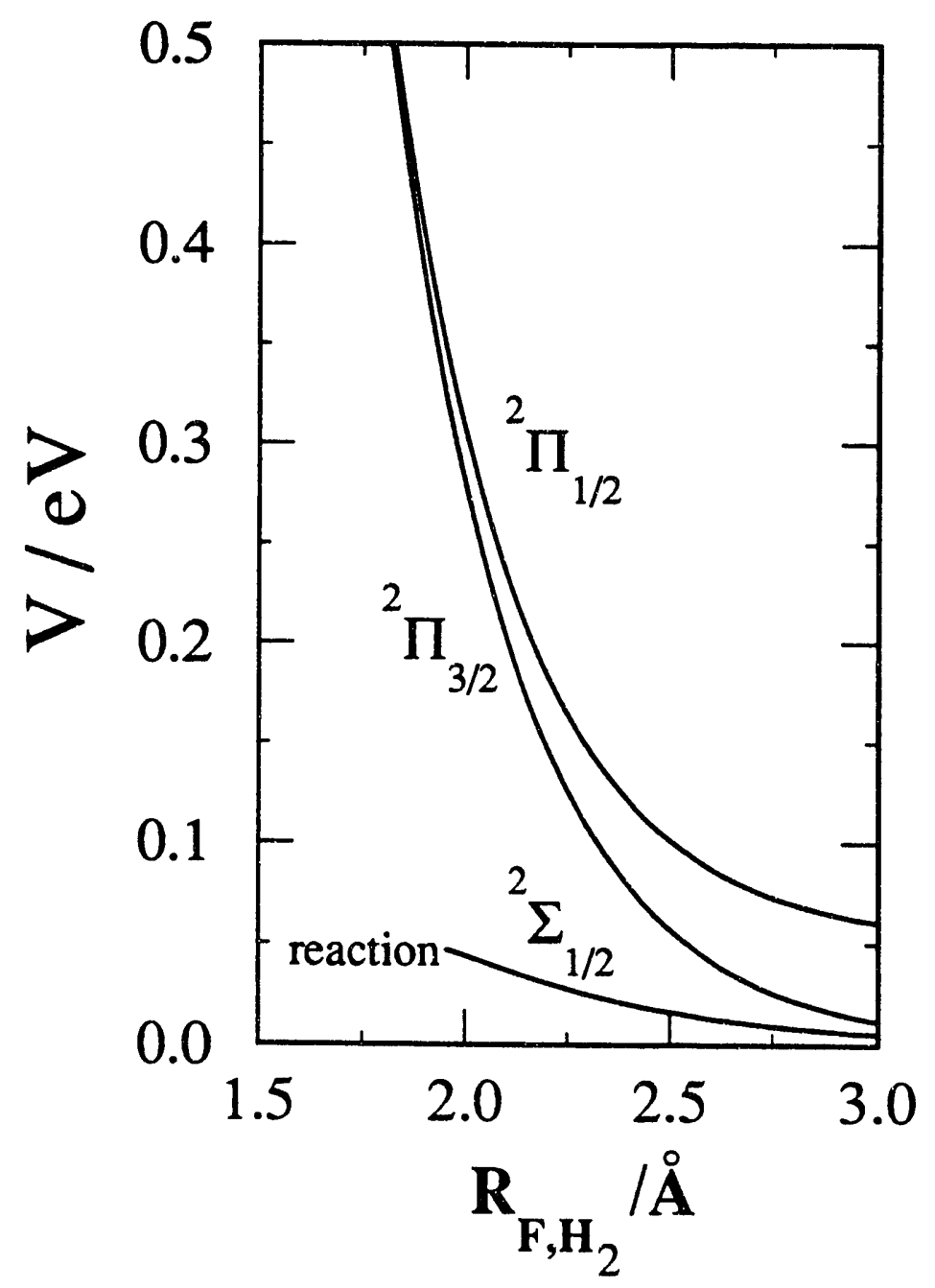

Figure 7-7(b) 


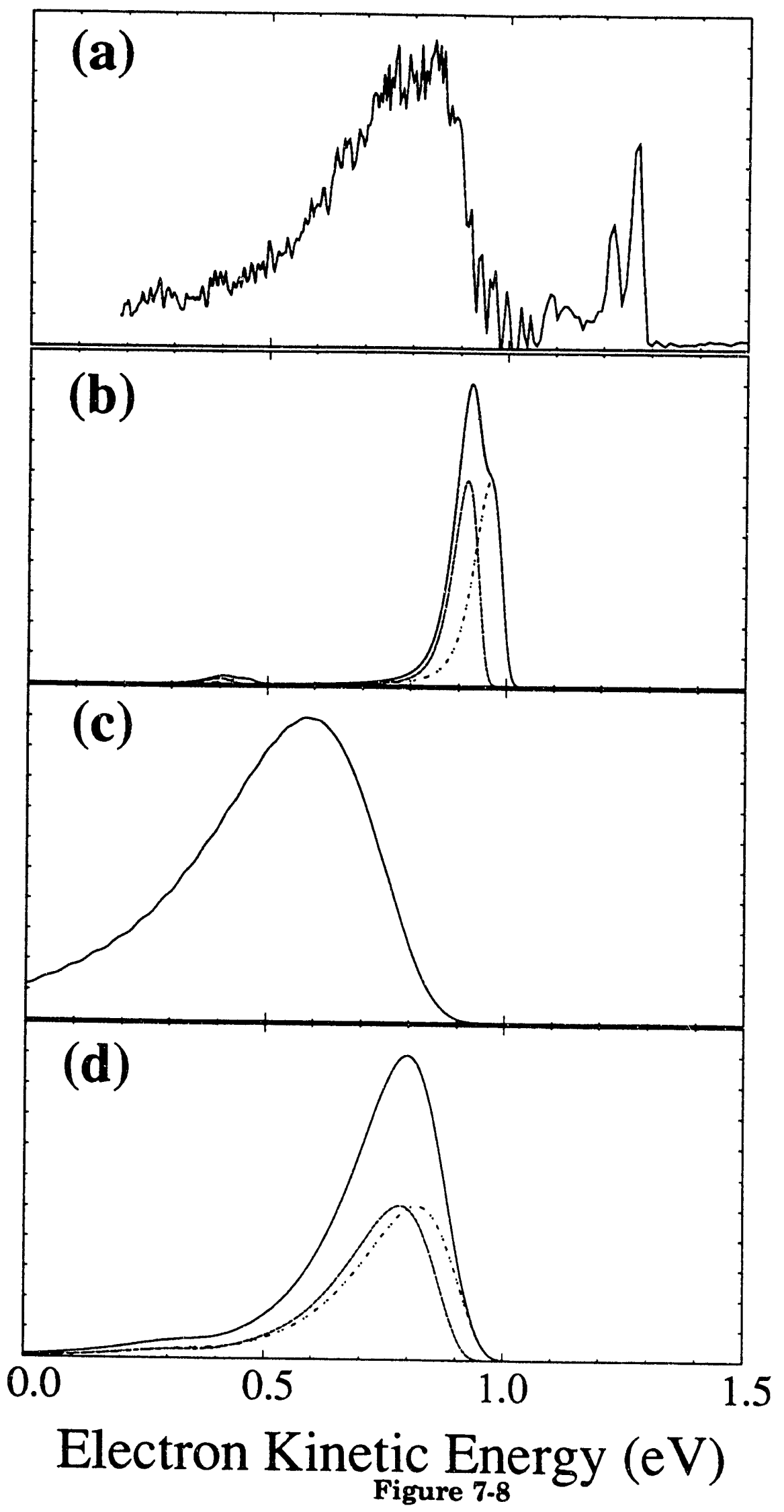

294 
Appendix A. Propensities in photoelectron angular distributions for linear molecular anions

The following table indicates whether the intensity in the photoelectron bands of the following negative ions favors $a \sin ^{2} \theta$ (intensity peaked around $\theta_{\max }=0^{\circ}, \beta<0$ ) or $\cos ^{2} \theta\left(\theta_{\max }=90^{\circ}, \beta>0\right)$ distribution.

Linear negative ions listed by molecular point group.

\begin{tabular}{lllll} 
Ion & $\begin{array}{l}\text { Anion } \\
\text { Electronic } \\
\text { State }\end{array}$ & $\begin{array}{l}\text { MO } \\
\text { removed }\end{array}$ & $\theta_{\text {max }}$ & $\begin{array}{l}\text { Neutral electronic } \\
\text { state }\end{array}$ \\
\hline $\mathrm{C}_{\text {ov }}$ & & & & \\
$\mathrm{FH}_{2}{ }^{-}$ & ${ }^{1} \Sigma^{+}$ & $\sigma$ & 0 & $\mathrm{X}\left({ }^{2} \Sigma^{+}\right)$ \\
& & $\pi$ & 90 & $\mathrm{~A}\left({ }^{2} \Pi\right)$ \\
$\mathrm{BrHI}^{-}$ & ${ }^{1} \Sigma^{+}$ & $\sigma$ & 0 & $\mathrm{X}\left({ }^{2} \Sigma^{+}\right){ }^{1}$ \\
& & $\pi$ & 90 & $\mathrm{~A}\left({ }^{2} \Pi\right)$ \\
$\mathrm{OHF}^{-}$ & ${ }^{2} \Pi$ & $\sigma$ & 90 & $\mathrm{X}\left({ }^{3} \Pi\right)$ \\
& & $\pi$ & 0 & $\mathrm{a}\left({ }^{1} \Delta\right)$ \\
$\mathrm{CN}^{-}$ & ${ }^{1} \Sigma^{+}$ & $\sigma$ & 0 & $\mathrm{X}\left({ }^{2} \Sigma^{+}\right)$ \\
& & $\pi$ & 90 & $\mathrm{~A}\left({ }^{2} \Pi\right)$ \\
$\mathrm{NCS}^{-}$ & ${ }^{1} \Sigma^{+}$ & $\pi$ & 90 & $\mathrm{X}\left({ }^{2} \Pi\right)$ \\
$\mathrm{NCO}^{-}$ & ${ }^{1} \Sigma^{+}$ & $\pi$ & 90 & $\mathrm{X}\left({ }^{2} \Pi\right)$ \\
$\mathrm{NO}^{-}$ & ${ }^{3} \Sigma^{-}$ & $\pi$ & 90 & $\mathrm{X}\left({ }^{2} \Pi\right)$ \\
& & $\pi$ & 90 & $\mathrm{a}\left({ }^{4} \Pi\right)$
\end{tabular}

1 At $213 \mathrm{~nm}$ the $X$ state intensity is approx. same at both $\theta=0^{\circ}$ and $\theta=90^{\circ}$. 


\begin{tabular}{lllll} 
Ion & $\begin{array}{l}\text { Anion } \\
\text { Electronic } \\
\text { State }\end{array}$ & $\begin{array}{l}\text { MO } \\
\text { removed }\end{array}$ & $\theta_{\max }$ & $\begin{array}{l}\text { Neutral electronic } \\
\text { state }\end{array}$ \\
\hline $\mathbf{D}_{\boldsymbol{c h}}$ & & & & \\
$\mathrm{IHI}^{-}$ & ${ }^{1} \Sigma_{\mathrm{g}}^{+}$ & $\sigma_{\mathrm{u}}$ & 0 & $\mathrm{X}\left({ }^{2} \Sigma_{\mathrm{u}}^{+}\right)^{2}$ \\
& $\pi_{\mathrm{g}}$ & 90 & $\mathrm{~A}\left({ }^{2} \Pi_{\mathrm{g}}\right)$ \\
& & & & $\begin{array}{l}\mathrm{X}\left({ }^{3} \Sigma_{\mathrm{g}}{ }^{-}\right) \\
\mathrm{a}\left({ }^{1} \Delta_{\mathrm{g}}\right) \\
\mathrm{b}\left({ }^{1} \Sigma_{\mathrm{g}}^{+}\right)\end{array}$
\end{tabular}

N.B. All transitions in $D_{o h}$ that remove a $\pi_{g}$ electron are forbidden in ZEKE.

$2213 \mathrm{~nm}$; at $266 \mathrm{~nm}$ the absolute counts are higher for $\theta=90^{\circ}$ to the ground ${ }^{2} \Sigma_{u}{ }^{+}$state than at $\theta=0^{\circ}$. 


\section{Appendix B. Calibration and Background Subroutines for experimental code TENURE.}

\section{Introduction}

These subroutines represent the part of the data acquisition code, TENURE, which deal with energy scale caibration and the fitting and subtraction of background spectra. The TENURE program is used to control data acquisition on the Neumark group photoelectron spectrometer. The calib2.pas routine is a completely revised version of the calibration routine found, along with the remainder of the TENURE source code, in the thesis of Dr. R. B. Metz. Fourier transformation and Wiener filtering is implemented in the subroutines ftshort.pas and four1.pas to smooth background spectra. Subtraction of the smoothed background is performed by the scale.pas subroutine. The source is written entirely in PASCAL and is suitable for compilation in the Borland Turbo Pascal 3.0 environment.

The use of, and principles behind, these two procedures are described in Chapter 2. Calibration is invoked with the <ALT $>-C$ combination from witin the TENURE program. The user is prompted for the detachment laser wavelength he o: she wishes to calibrate. The time-of-flight, uncertainty and ion beam energy information is entered for each calibration line. A linear or quadratic fit to these calibration points is performed; the fitting parameters and indicators of the fit quality are output. The user is then asked whether he/ she accepts the fit. If so the entered calibrant information is saved to disk and the fitted parameters become the current ones used for time-of-flight to electron kinetic energy conversion in the main program.

The fitting of a background spectrum with the Fourier/ Wiener filtering 
routines is activated by the <ALT $>$-F combination. The time-of-flight dataset currently active is fast Fourier transformed and saved to disk (FOURTR.DAT). The user then selects a Lorentzian filter function for the Wiener filtering by entering a half-width in number of channels: typically 15 gives good filtering. If this filter results in too much smoothing of the background, the Lorentzian filter width should be increased. After multiplication by the filter the Fourier dataset is back transformed into time-of-flight; the filtered dataset may then be stored at this point. The subsequent scaling and subtraction of the now smoothed background spectrum from one (or many) time-of-flight photoelectron dataset(s) is achieved with the <AIT >$\mathrm{N}$ combination.

\section{Source Listing}

\section{calib2.pas}

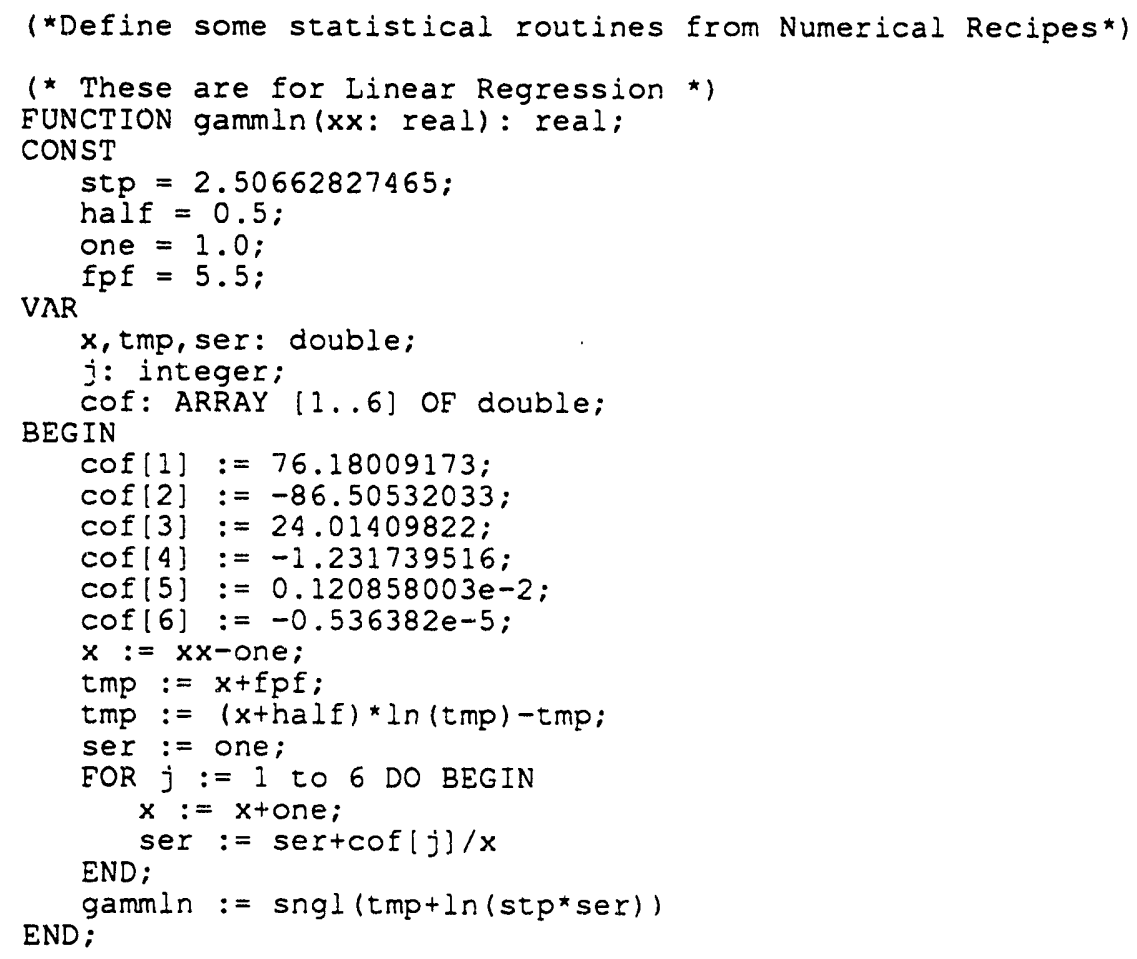




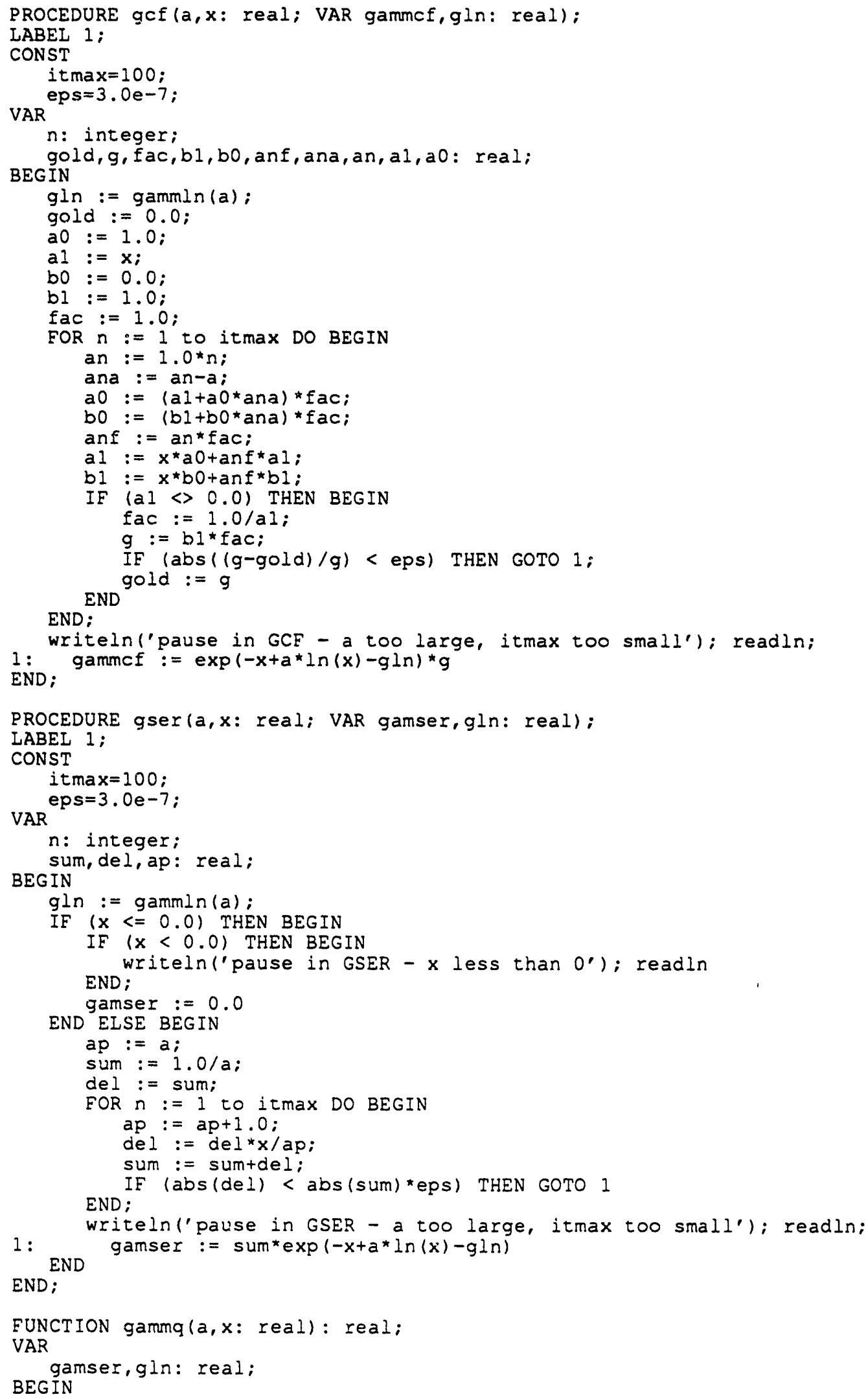


IF $((x<0.0)$ OR $(a<=0.0))$ THEN BEGIN END: writeln ('pause in GAMMQ - invalid arguments'): readin

IF $(x<a+1.0)$ THEN BEGIN

gser $(a, x$, gamser, gln):

gammq := 1.0-gamser

END ELSE BEGIN

gcf $(a, x, g a m s e r, g l n)$;

END

gammq := gamser

END;

PROCEDURE $f$ it $(x, y$ : glndata; ndata: integer; sig: glndata; mwt: integer; VAR $a, b$, siga,sigb, chi2, $q$ : real):

(* IINEAR REGRESSION FIT - Numerical Recipes *)

(* Programs using routine FIT must define the type TYPE

glndata = ARRAY $[1 \ldots$ ndata $]$ OF real;

in the main routine. *1

VAR

i: integer;

$w t, t, s y$, sxoss, sx, st 2 , ss, sigdat: real;

BEGIN

sx $:=0.0$;

sy $:=0.0$

st $2:=0.0$;

b $:=0.0$

IF (mWt $<$ O) THEN BEGIN

ss $:=0.0$;

FOR $i:=1$ to ndata DO BEGIN

wt $:=1.0 / \operatorname{sgr}(\operatorname{sig}(i))$;

ss : = ss+wt;

$s x:=s x+x[i] * w t$;

END

sy $:=s y+y(i) * w *$

END ELSE BEGIN

FOR $i:=1$ to ndata DO BEGIN

$s x:=s x+x[i]$;

END;

sy $:=s y+y[i]$

END:

Ss $:=$ ndata

sxoss := sx/ss;

IF (mWt $>$ O) THEN BEGIN

FOR $i:=1$ to ndata DO BEGIN

$t:=(x[i]-s \times 0 s s) / s i g[i]$;

st2 $:=s t 2+t * t$

END

$b:=b+t * y(i) / s i g(i)$

END ELSE BEGIN

FOR $i:=1$ to ndata DO BEGIN

$t:=x[i]-$ sxoss:

st $2:=s t 2+t * t$

END

$b:=b+t * y[i]$

END:

$b:=b / s t 2 ;$

a $:=(s y-s x * b i / s s$;

siga $:=\operatorname{sqrt}\left(\left(1.0+s x^{*} s x /\left(s s^{\star} s t 2\right)\right) / s s\right) ;$

sigb : = sqrt $(1.0 /$ st 2$)$ :

chi2 : $=0.0$ :

IF (mwt = 0) THEN BEGIl:

FOR $i:=1$ to ndata DO BEGIN

END:

chi2 : $=\operatorname{ch} 22+\operatorname{sqr}(y[i]-a-b * x[i])$ 


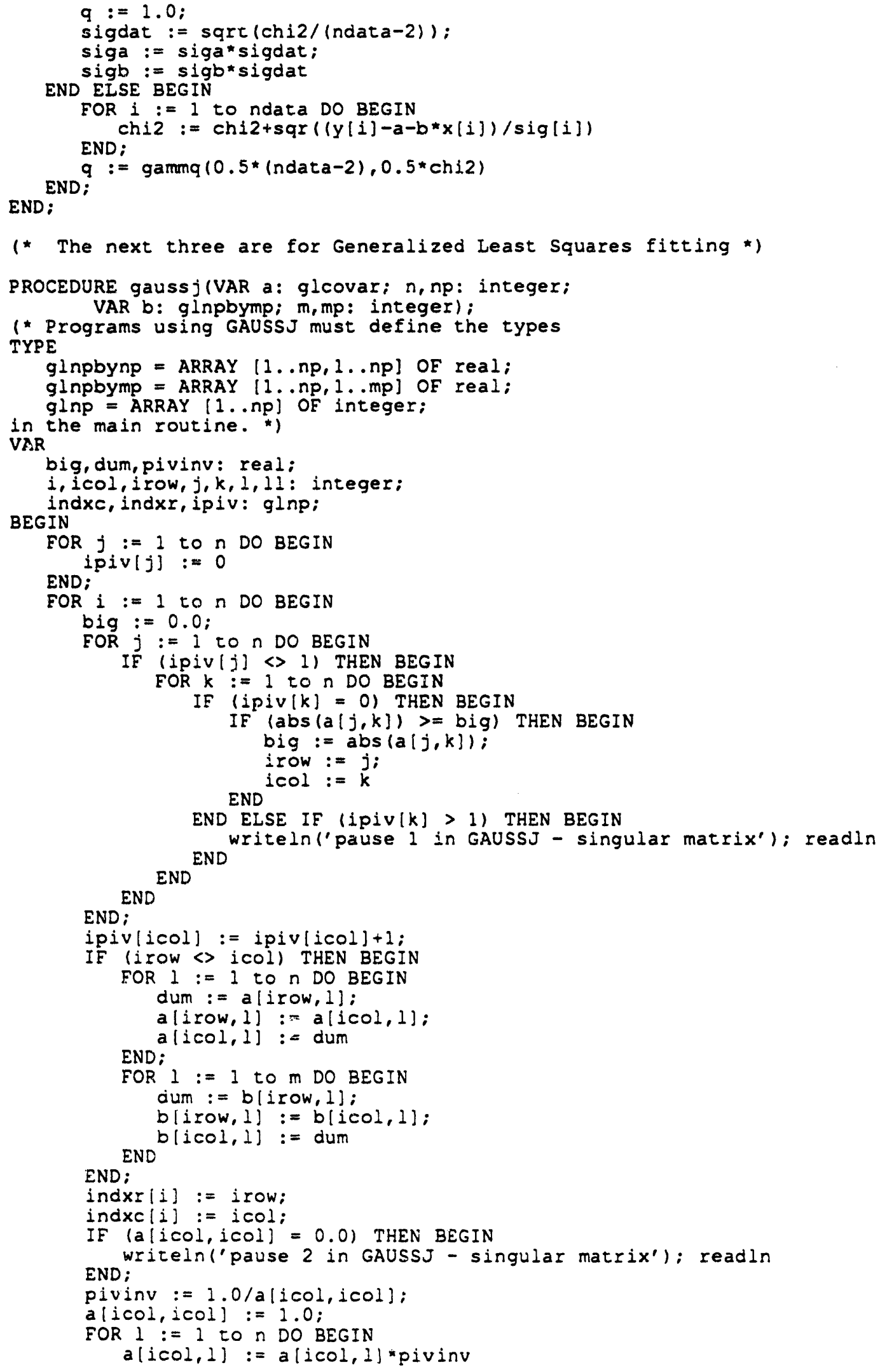


END;

FOR $1:=1$ to $m$ DO BEGIN

END;

$b[i c o l, 1]:=b[i c o l, 1] * p i v i n v$

FOR $11:=1$ to $n$ DO BEGIN

IF (II $<$ iCOl) THEN BEGIN

dum := a[1],icol];

a [1], icol] $:=0.0$;

FOR $i:=1$ to $n$ DO BEGIN

END;

$a[11,1]:=a[11,1]-a[i c o l, 1] \star d u m$

FOR $1:=1$ to $m$ DO BEGIN

END

$b[11,1]:=b[11,1]-b[i c o l, 1] * d u m$

END

END

END;

FOR $1:=n$ DOWNTO 1 DO BEGIN

IF (indxr[1] $<$ indxc[1]) THEN BEGIN

FOR $k:=1$ to $n$ DO BEGIN

dum $:=a[k$, indxr[1]];

$a[k$, indxr $[1]\}:=a[k$, indxcll $[1]]$;

END

END

$a[k$, ind $x \in\{l\}]:=$ dum

END

END;

PROCEDURE covsrt(VAR covar: glcovar; ncum: integer; ma: integer;

lista: gllista; mfit: integer);

(* Programs using routine covSRT must define the types TYPE

glcovar = ARRAY $[1 \ldots$ ncvm, $1 \ldots$ ncum $]$ OF real;

gliista = ARRAY [1..mfit] OF integer;

in the calling program. *)

VAR

$j, i:$ integer;

swap: real;

BEGIN

FOR $j:=1$ to ma-1 DO BEGIN

FOR $i:=j+1$ to ma DO BEGIN END $\operatorname{covar}[i, j]:=0.0$

END;

FOR $i:=1$ to mfit-1 DO BEGIN

FOR $j:=i+1$ to mfit DO BEGIN

IF (listalj] > Iista[i]) THEN BEGIN covarllista[j],lista[i]] := covar[i,j]

END ELSE BEGIN END

covar [lista[i],lista $[j]\}:=\operatorname{covar}[i, j]$

END

END;

swap := covar $[1,1]$;

FOR $j:=1$ to ma DO BEGIN

$\operatorname{covar}[1, j]:=\operatorname{covar}[j, j]$;

END:

covar[1ista[1], lista[1]]:= swap:

FOR $j:=2$ to mfit DO BEGIN

$\operatorname{covar}[1$ ista $[j], l i \operatorname{sta}[j]]:=\operatorname{covar}[1, j]$

END;

FOR $j:=2$ to ma DO BEGIN

FOR $i:=1$ to $j-1$ DO BEGIN

END

$\operatorname{covar}[i, j]:=\operatorname{covar}[j, i]$

END

END; 


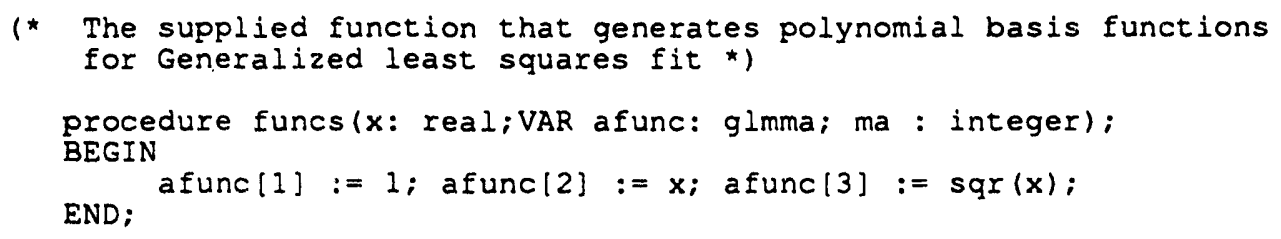




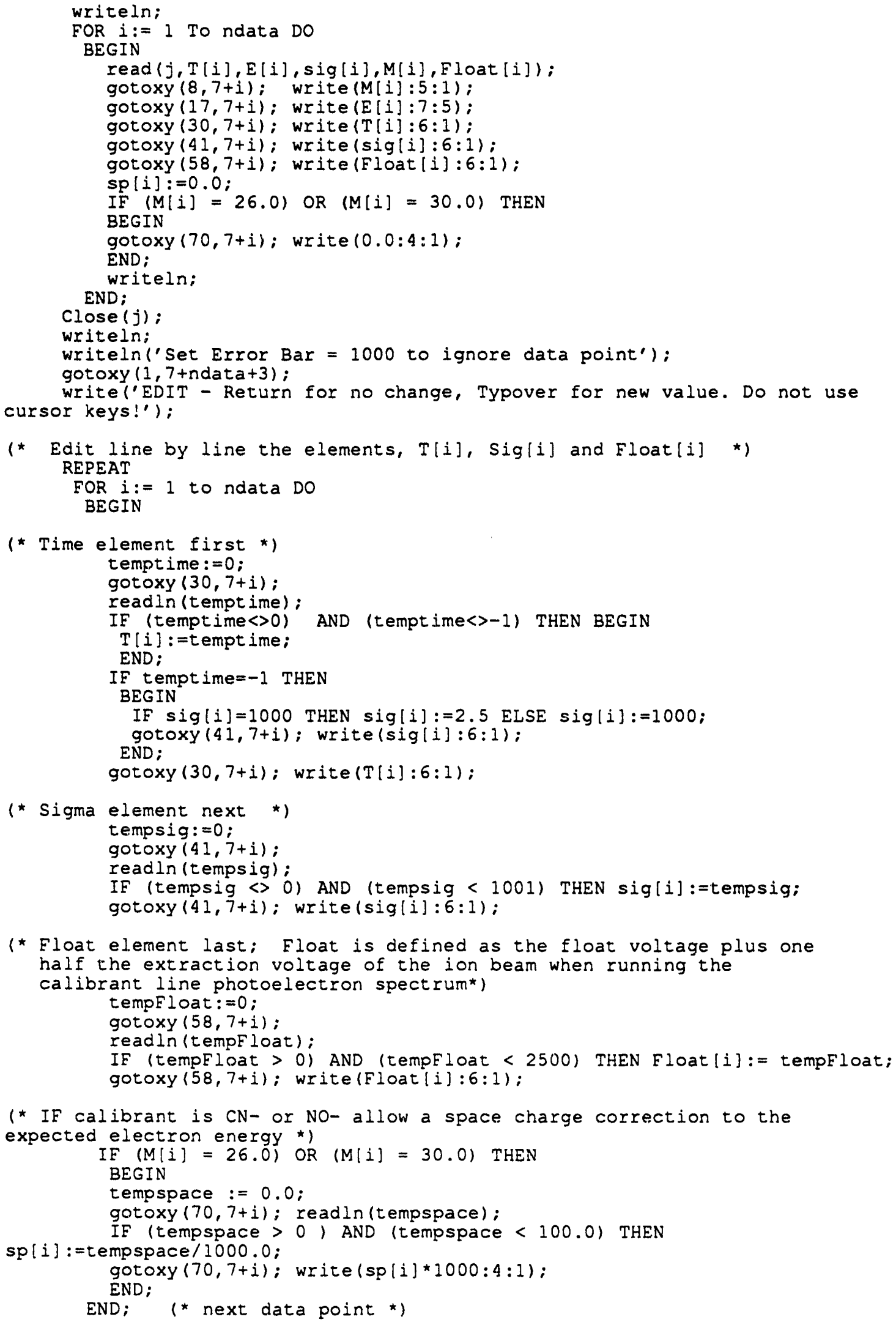




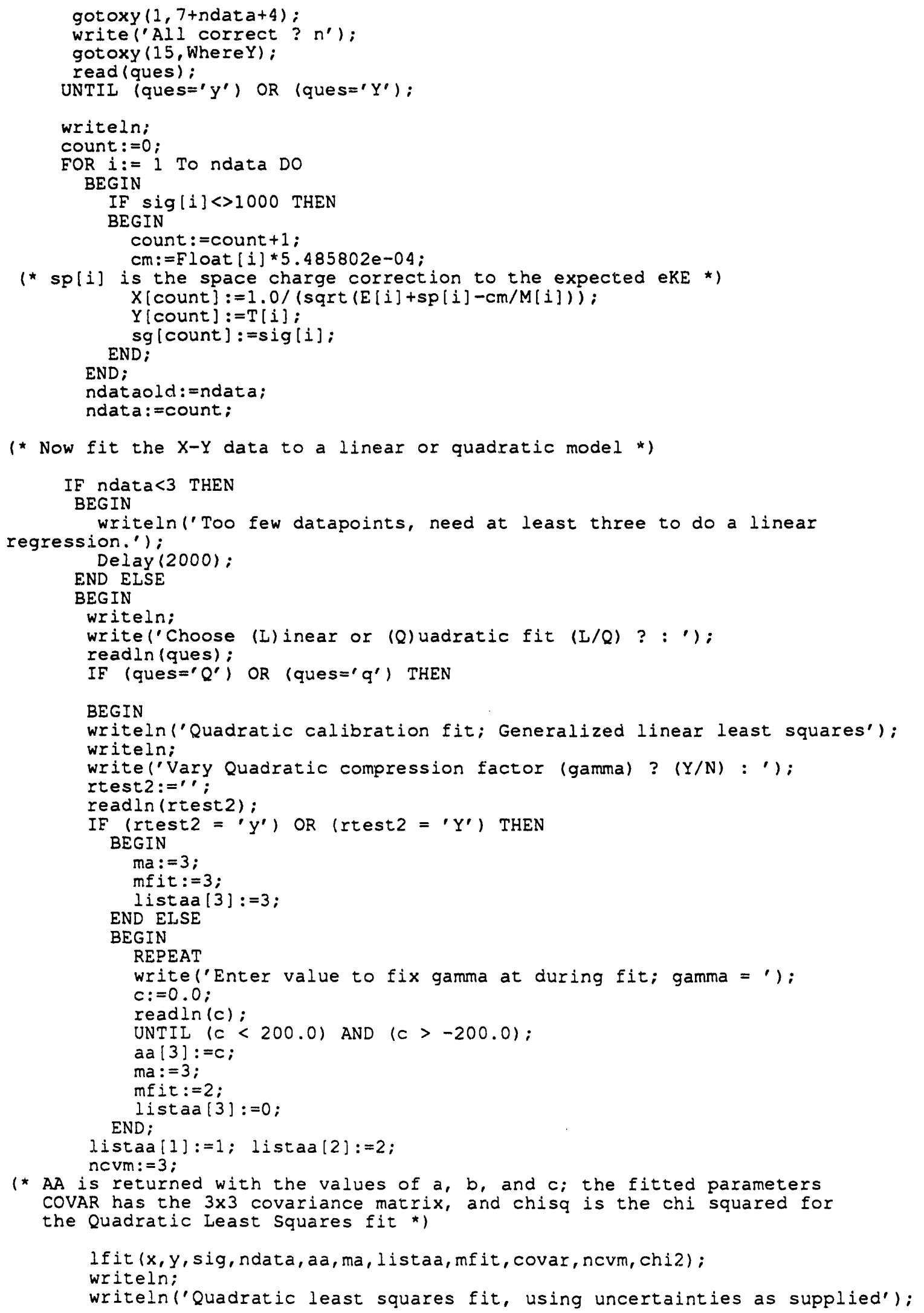




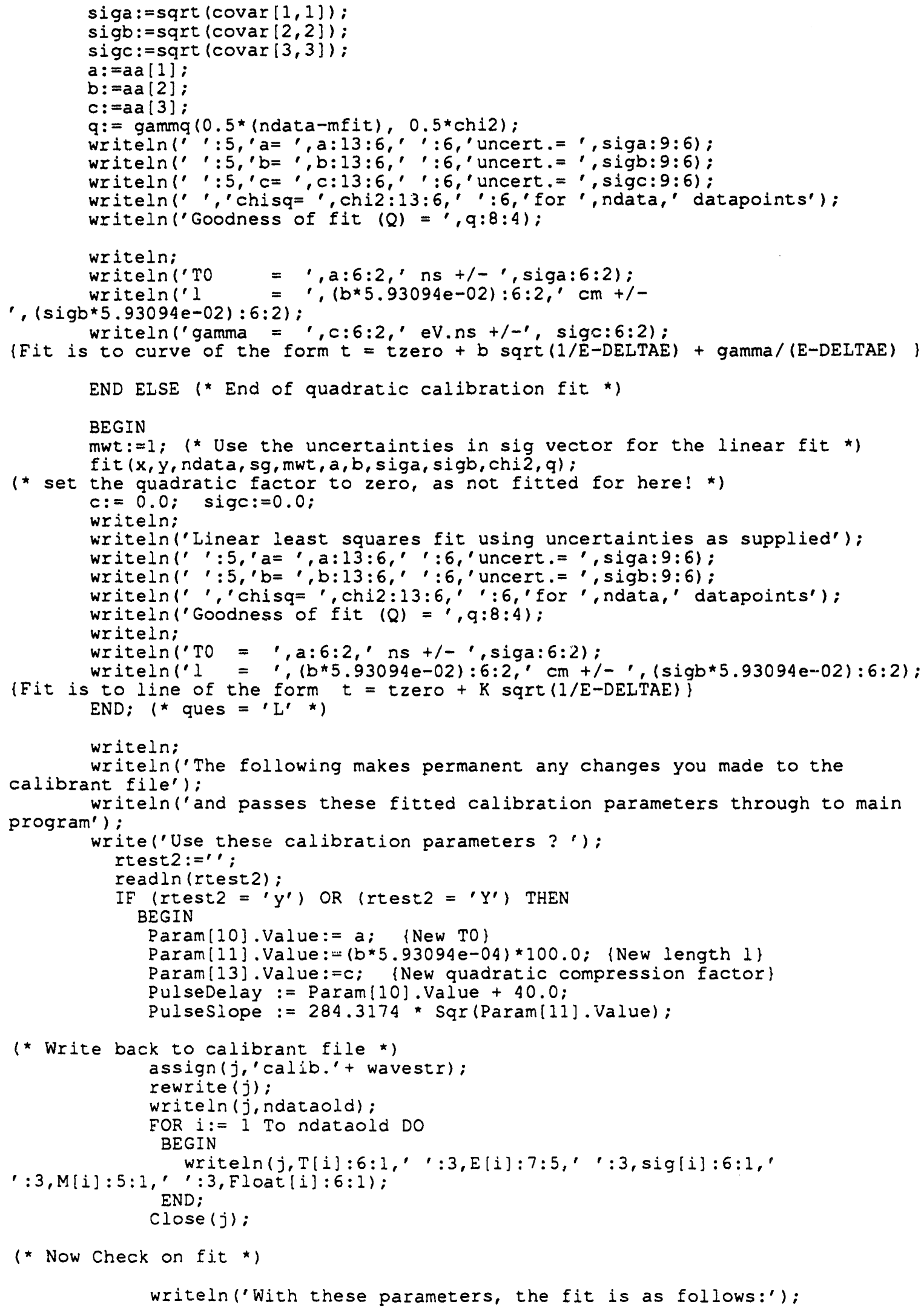




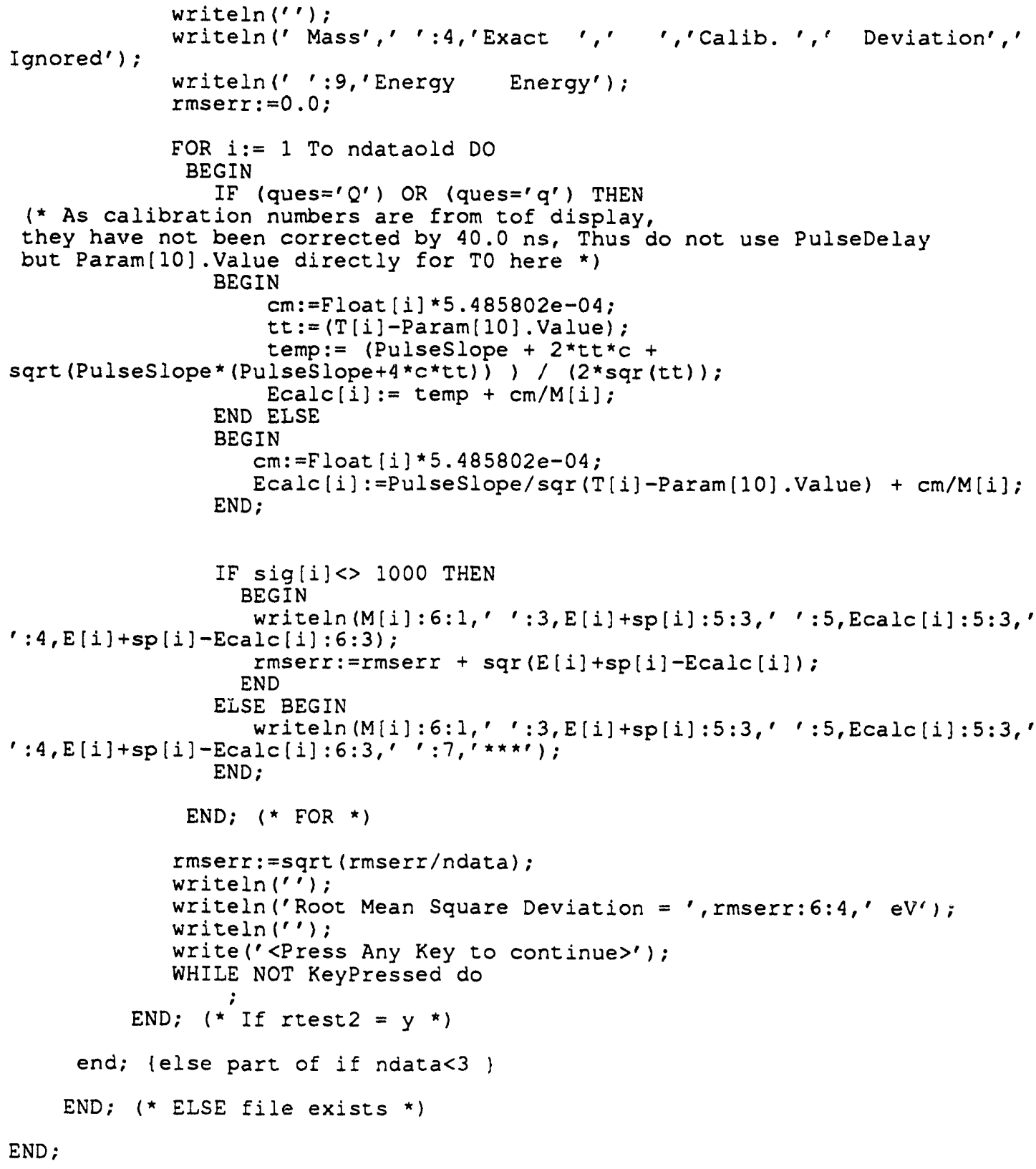

\section{ftshort.pas}

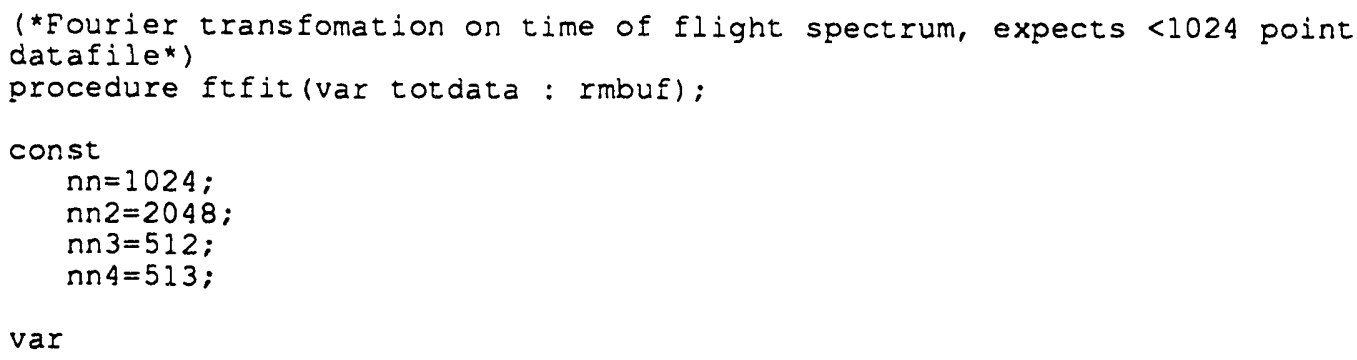




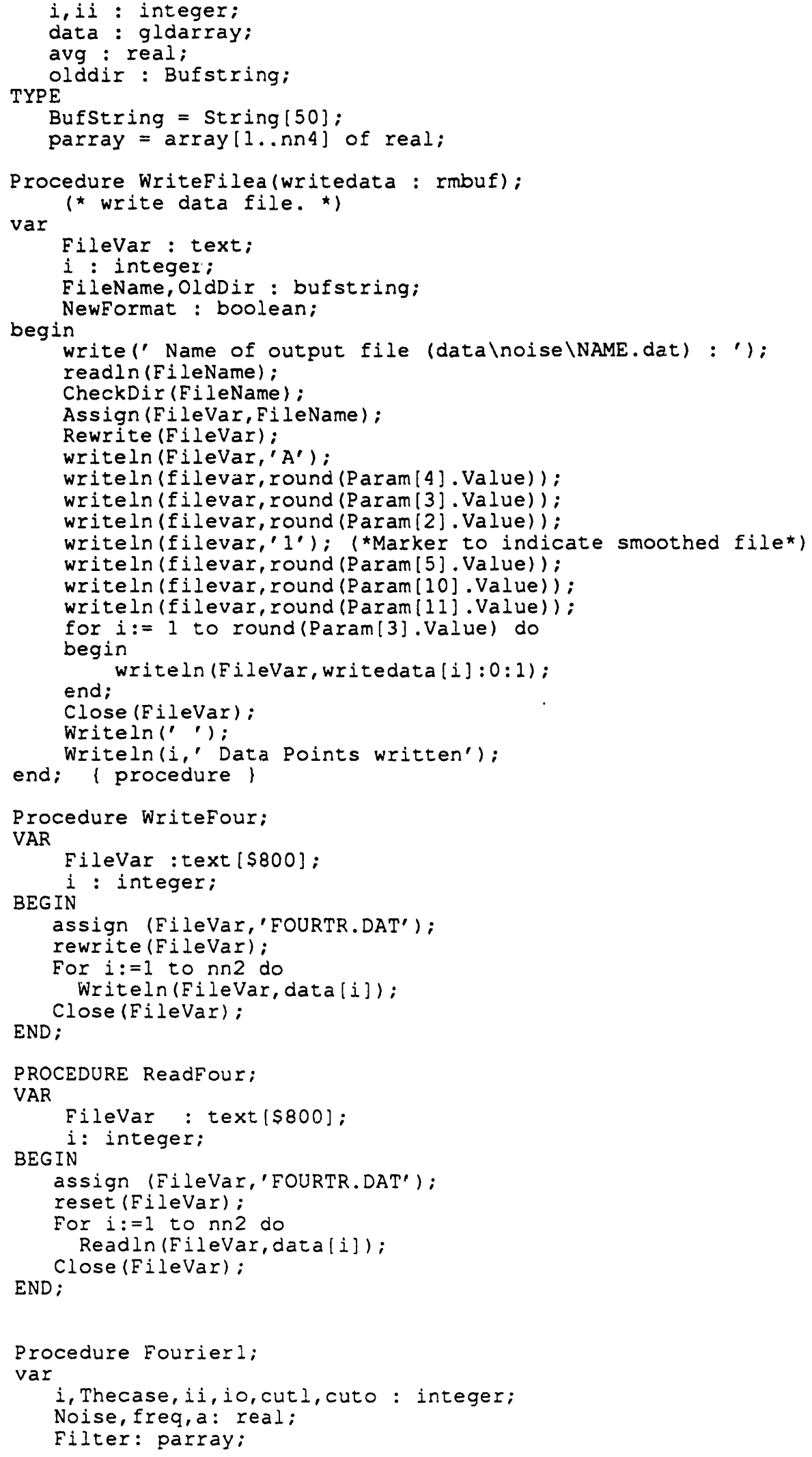




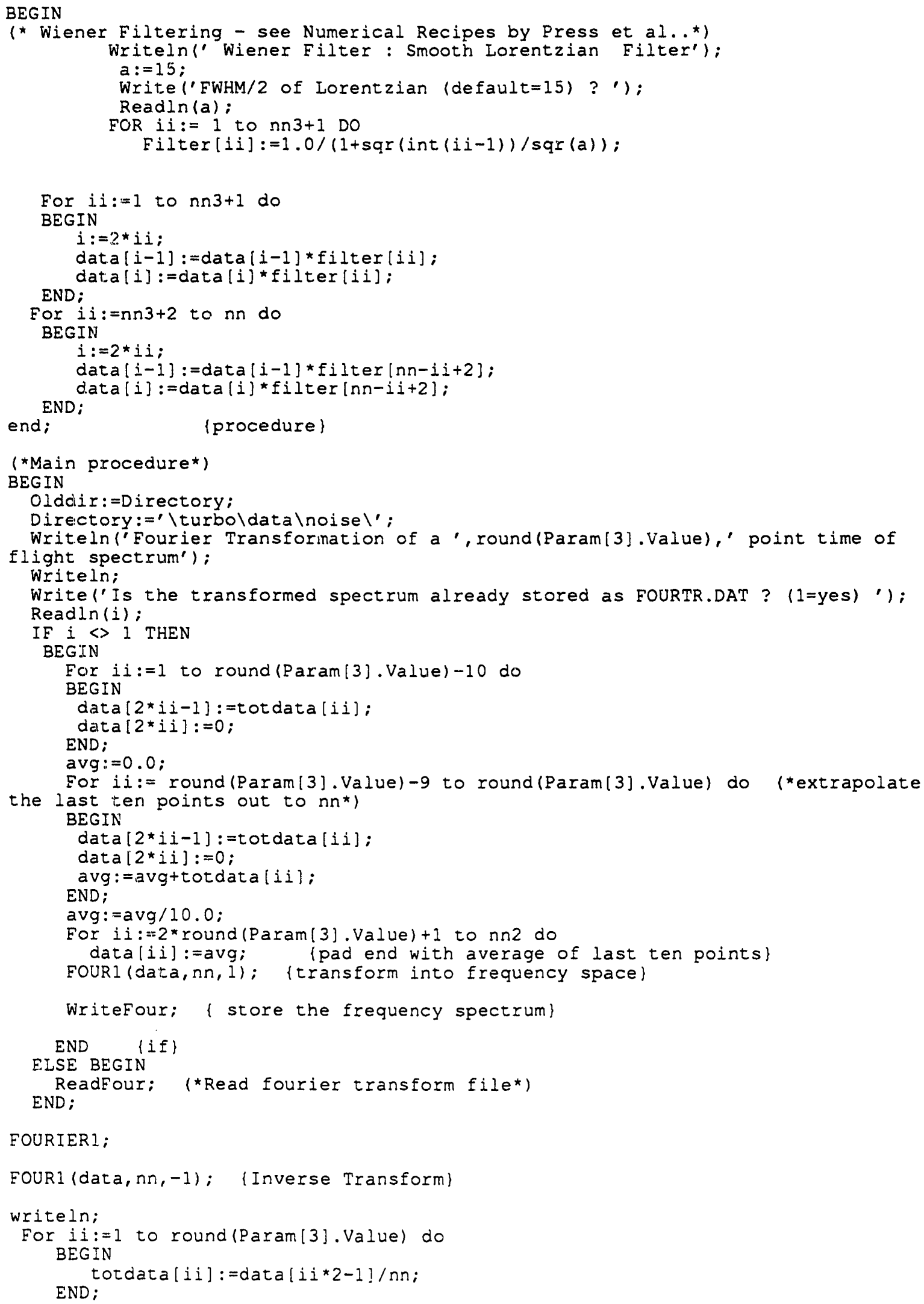


(* Set first two points to $\mathrm{min}$ and max of input data for comparison*)

totdata [1] :=maxval;

totdata $[2]:=0$ :

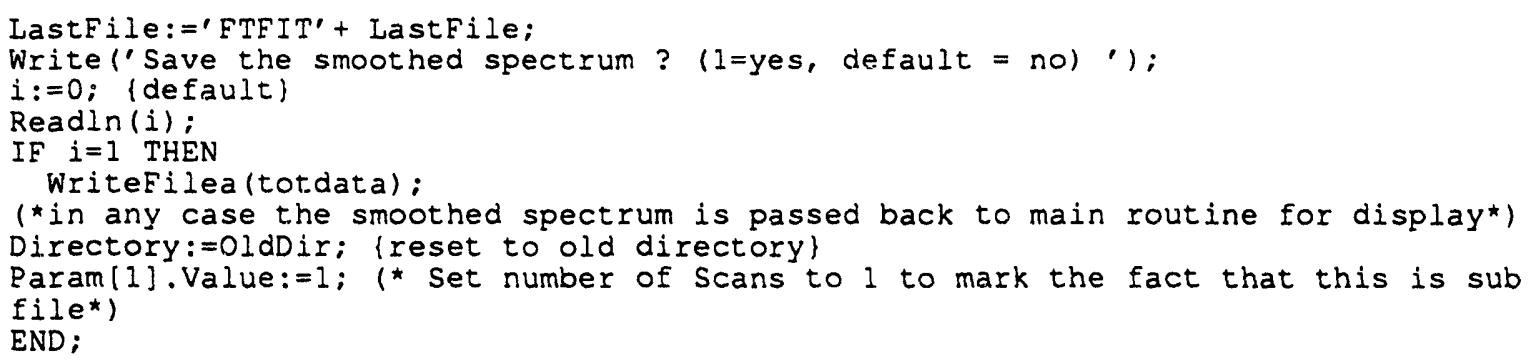

\section{four1.pas}

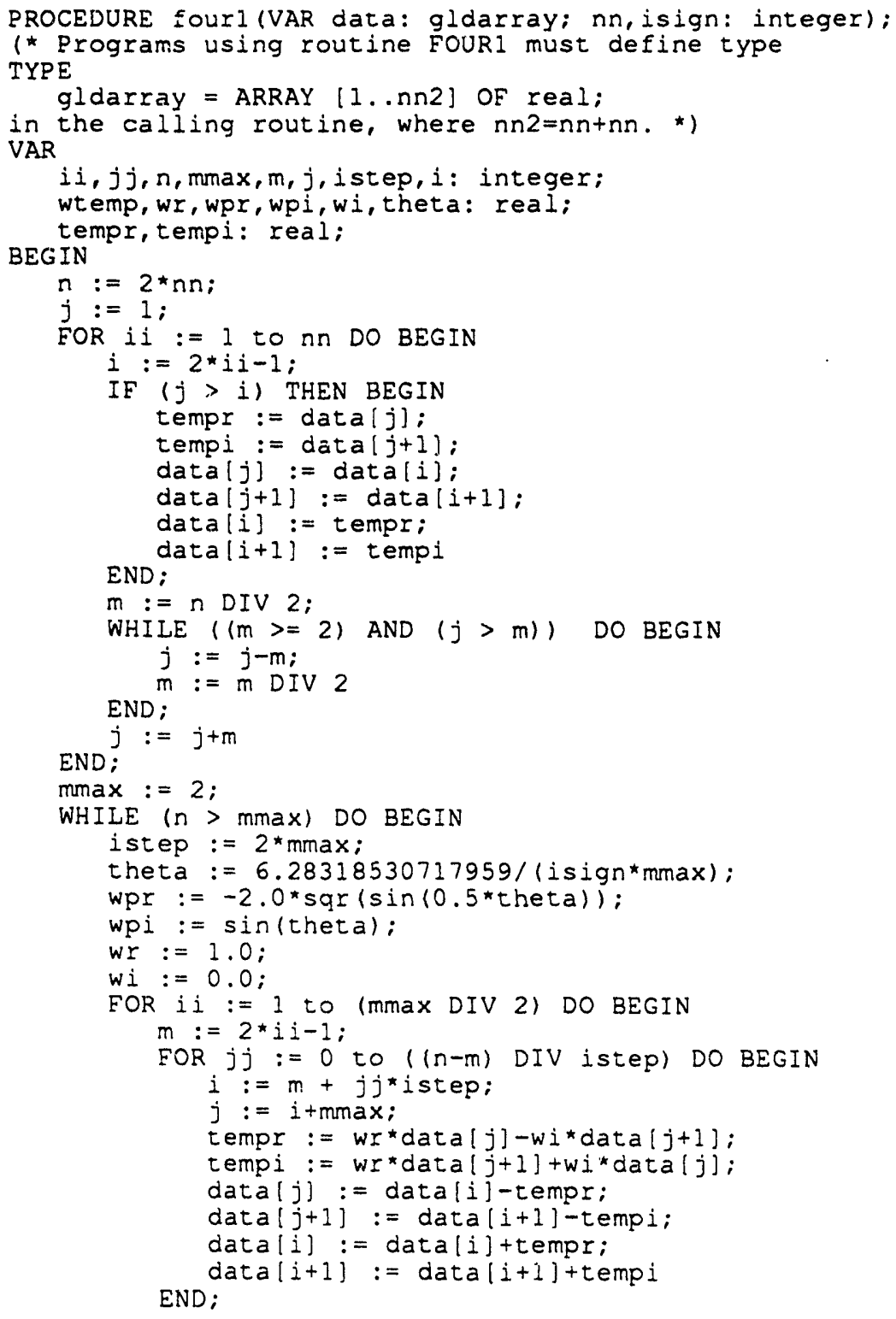


END;

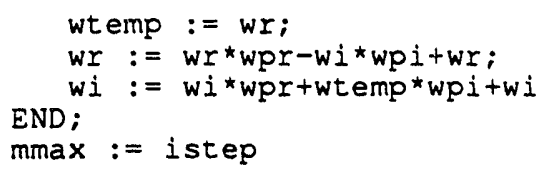

\section{scale.pas}

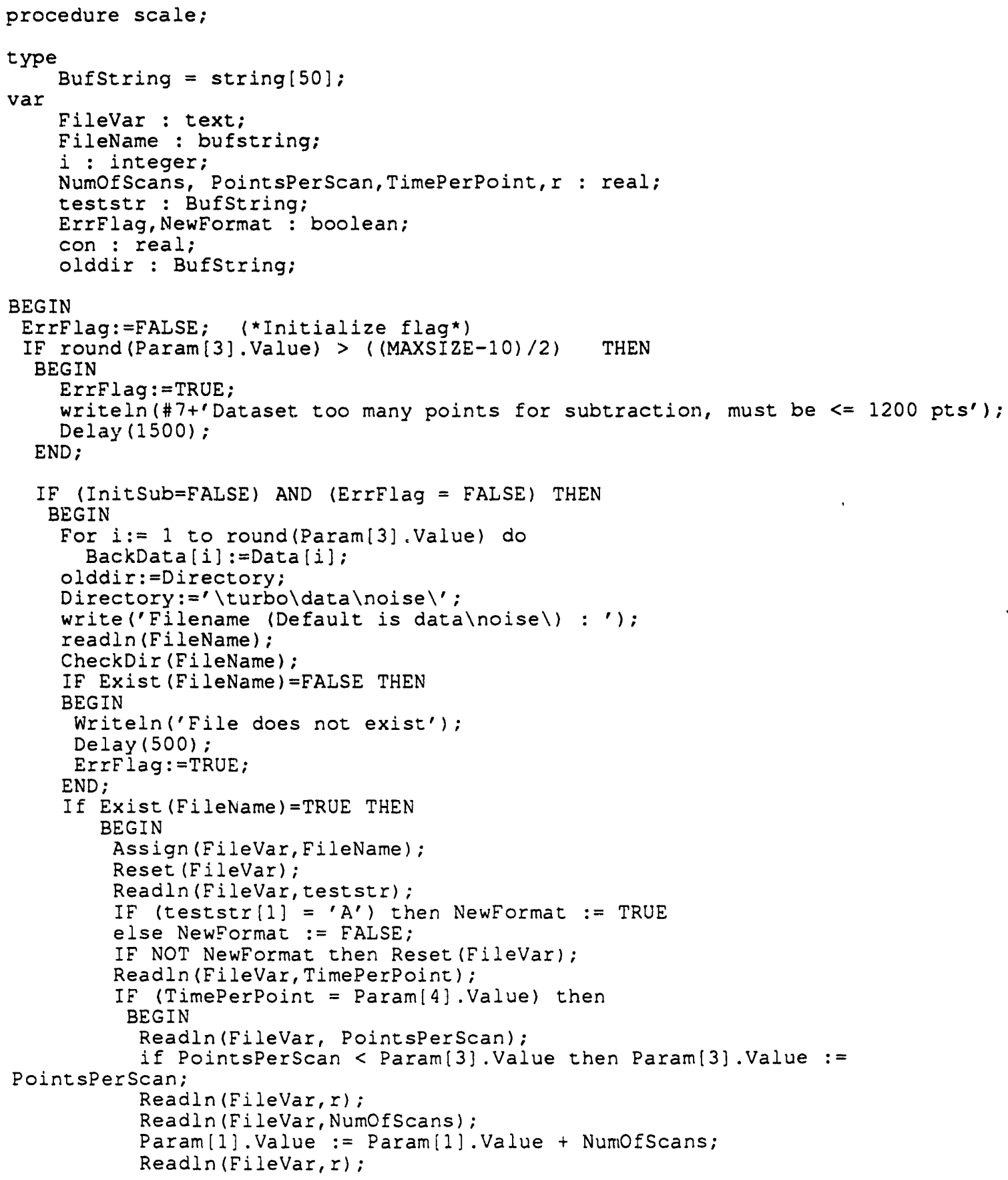




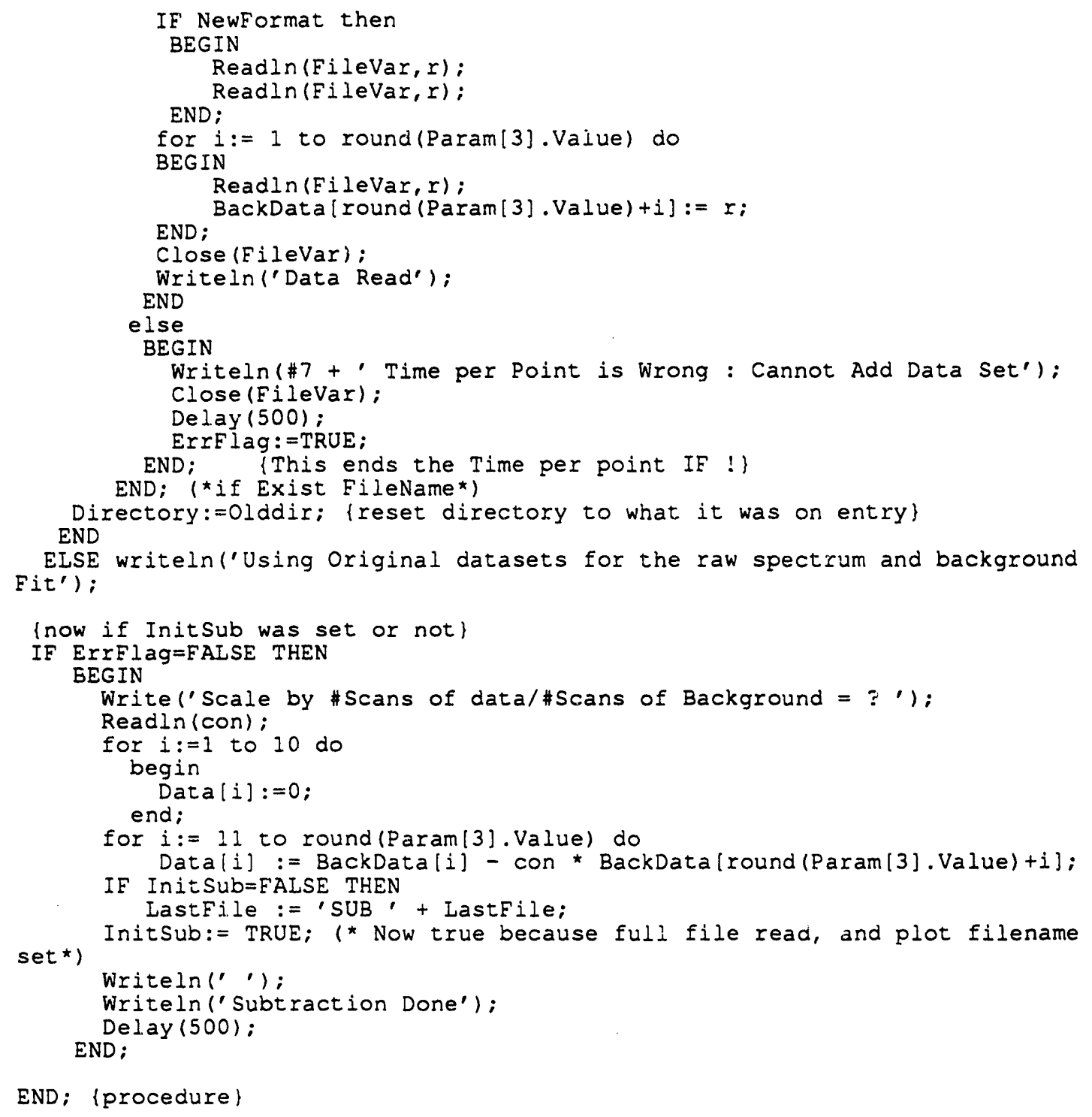




\section{Appendix C. Time Dependent Wave packet Propagation Codes}

\section{Introduction}

Codes have been developed to calculate the time-dependent dynamics of a wave packet in both one- and two-dimensional space. The resulting wave packet dynamics is used to compute the autocorrelation function and, in turn, the simulated photoelectron spectrum. The number of dimensions corresponds to the number of independent coordinates explicitly represented by the wave packet. A wave packet propagation is merely a solution of the discretized time-dependent Schrodinger Equation. Assuming a two-dimensional space, this is written

$$
i \frac{\partial \phi^{n}\left(i_{x}, i_{y}\right)}{\partial}=H \phi^{n}\left(i_{x}, i_{y}\right)
$$

where $\phi^{n}\left(i_{x}, i_{y}\right)$ is the $n$-th wave packet in time and is a complex function expressed in space at each grid point $\left(i_{x}, i_{y}\right)$. The method we adopt for solving (1) and the relevant references are detailed in Chapter 4. Here we consentrate on how to use the code, and how the code is constructed.

The Hamiltonian is time independent and, as usual, made up of a kinetic and potential energy term. The kinetic energy is evaluated by the Fourier method see Chapter 4) - this is generalizable into $\mathrm{N}$-dimensions. In one-dimension, the potential energy can be a bound or repulsive function of the single coordinate, $x$. In two dimensions, the potential, $\mathrm{V}(x, y)$, may be bound (i.e. possess a well) or be of a more general form, e.g. unbound or containing a saddle point. The propagation codes can handle any potential function. It is useful for the potential to be an analytic function of the coordinates. 
We will be concentrating on potential energy surfaces for direct bimolecular reactions, i.e. those with no wells and a single saddle point, for the neutral in the following discussion. For reaction surfaces, simple analytic forms, such as the London-Eyring-Polanyi-Sato (LEPS) function, are very often used. More recently $a b$ initio surfaces have been computed for light atom reactions (e.g. $\mathrm{H}+\mathrm{H}_{2}, \mathrm{~F}+\mathrm{H}_{2}, \mathrm{O}+$ $\mathrm{HF}, \mathrm{O}+\mathrm{Hcl}$, and $\mathrm{Cl}+\mathrm{Hcl}$ ); these are usually only evaluated at a set of points. In some cases analytic functions have been fit to the points, or a spline interpolation for the full surface has been given. In principle, as our method of solution of Equation (1) 1 zquires the potential only at a set of gridpoints, it is conceivable that the potential could be evaluated at each point on the grid by ab initio methods.

The two-dimensional code (prop2d22) grew out of the one-dimensional code (poop10), but the two-dimensional code is a lot more flexible in terms of potentials allowed for the anion and neutral and the controls the user may implement. The hasic usage of the codes is very similar. The one-dimensional code is useful mainly for preliminary calculations and for instructional use. Other more efficient techniques are available to solve the Schrodinger equation in one dimension on a grid for bound or purely repulsive potentials. Actually, the wave packet propagation does prove useful (and eficient) for an intermediate case where the dynamics involves a region of the upper state potential that can trap the wave packet and another region where the wave packet moves to dissociation. An example of this is a wave packet that is launched fairly high up on a Morse or Lennard-Jones potential (see Figure 4-1(iii)). One important use of the one-dimensional code is to examine the form of the autocorrelation function that derives from motion in just one dimension when comparing to a full two-dimensional propagation (see Chapter 5). 


\section{One-dimensional Propagation Code - prop10}

The executable code, prop10, is compiled by using the UNIX make utility and the makefile makeprop10. The code depends on four files of FORTRAN source, kosloff7.f, leps1.f, anionmorsewf1.f and wave2.f. If any of the source files to prop10 are modified, the prop10 program should be recompiled; to do so use the make utility as follows:

$$
\text { make -f makeprop10 }
$$

The code can be compiled and run on a Sun SPARCstation as the numerical demand is small enough that this machine is fast enough to cope with a reasonable length propagation; a 400 femtosecond propagation (4 a.u. time steps, 4096 steps) on a 64 point spatial grid consumes only 14 CPU seconds on a SPARCstation ELC.

The code is used as follows. An input deck (called koss.dat) is constructed that controls not only the way the code proceeds but also contains all physical information such as the potential parameters. The input deck is not free format, but comments may be left to the right of the input line to assist the user in making changes to a template file. A typical input deck is shown below:

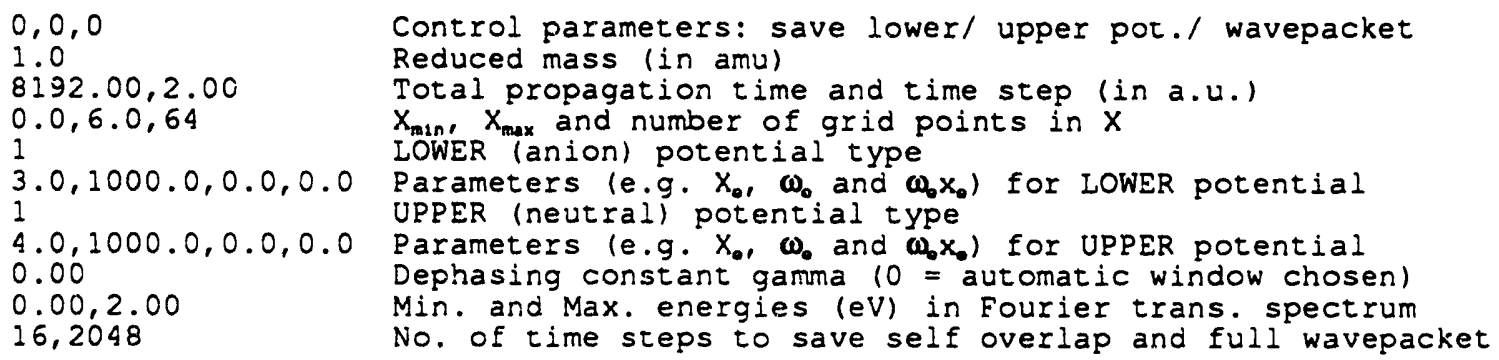

This is the only input to the program; no entry is expected from the keyboard. Most lines of the input deck are self explanatory. Line 1 sets the parameters $(0=$ no, $1=$ 
yes) that control whether the potential evaluated either for the lower or upper surfaces is to be saved to disk (in files called potlA.out and potlB.out) and whether the wave packet, or the wave packet and its derivatives, are to be saved to disk (in files called wavepkt.out and wave.drw). Wave packets, with our without derivatives, are saved at intervals determined by the second number in the final line of the input deck. The wave packet derivatives are useful only for debugging; if derivatives are selected, $\phi\left(k_{x}, k_{y}\right)$, the momentum space representation of the wave packet, and the result of the operators $T, V$, and $H$ acting on the wave packet $(T \phi, V \phi$ and $\mathbf{H} \phi)$ are saved into the files as well as the wave packet. This consumes disk space fairly rapidly, so save only a few wave packets! The wave.drw file, which is unformatted binary, can be read into a 1D wave packet graphing program draw3. If the control parameter for wave packet derivative storage is set to a value of -1 , then no information on the wave packets or its derivatives are saved to disk.

Total propagation times and the time step are entered on line 3 in atomic units. Atomic time units are the natural unit for a propagation; 1 femtosecond is approximately 41 a.t.u. The time step is chosen by consideration of criterion (25) in Chapter 4; if a too large time step is selected, the program will terminate with a message indicating that the propagation would be unstable. Next the grid range and grid size is determined on line 4. Remember that the range in coordinate space and the grid size determines the grid spacing $\Delta x$, which in turn determines the maximum kinetic energy that is possible on the grid (and therefore the largest energy eigenvalue). The selection of these parameters is the trickiest part of the whole operation. However, some very simple guidelines should ensure success: (i) The number of grid points must be a power of two, and should be at least 32 and never 
need exceed 256; usually 64 is adequate. Using a higher number of points than required will force the time step to be very small. (ii) Choose a range of coordinates that covers all the important parts of the anion and neutral potential surfaces physical intuition is all that is required here. For example, if there is a big change in geometry between anion and neutral then one knows that there will be high amplitude motion involving large kinetic energies. (iii) Start the calculation going and look at the value output for the maximum kinetic energy that the program calculates from your $\Delta x$. If it is reasonable then continue; if it is huge, then you can reduce the number of grid points and achieve a much faster propagation. If it is smaller than physically desirable, you need to reduce the grid range or increase the number of grid points.

The next few lines contain information on the lower and upper potentials. Information on the lower (anion) potential is required to compute an initial wave packet for the propagation; the ground state wave function for a harmonic potential or a Morse potential is constructed analytically for this purpose. For each potential the first control line indicates the potential type; this choice determines the form of the input expected on the following line(s). The potential types the program accepts are the following (the number indicates the potential type parameter to be entered on the control line):

(0) Morse function (data required on next line is $R_{e}$ in $\dot{A}, D_{e}$ in eV, $\beta$ in $\AA^{-1}$ and $\mathrm{V}_{0}$, the potential offset constant, in $\mathrm{eV}$ ).

(1) Harmonic/ Morse function (data required on next line is $R_{e}$ in $\AA, \omega_{e}$ and $\omega_{e} x_{e}$ in $\mathrm{cm}^{-1}$ and $\mathrm{V}_{0}$ in $\mathrm{eV}$ ). 
(2) LEPS potential function cut (data required on next six lines: line $1-V_{0}$, line 2 - masses of atoms A, B, and C in a.m.u., lines 3 - 5 contain the data $D_{e}$ in $\mathrm{eV}, \beta$ in $\AA^{-1}, R_{e}$ and the Sato parameter for each fragment $A B, B C$ and $A C$ respectively and finally line 6 - the value of $R_{A C}$ for which the 1D cut will be made).

(3) read potential from potB.in file (data required on next line $\mathrm{V}_{0}$ only).

Once information on both potentials has been entered, the final three lines of the input deck supply the windowing function parameter $\gamma$ (defined in Equation (29) of Chapter 4), the range of energies to be written to the output file absspec.out (which contains the final simulated energy spectrum $\sigma(E))$, and the step intervals for the program to calculate the autocorrelation $\Delta \tau_{\text {ovip }}$ and to save the wave packet. If $\gamma$ is chosen to be zero, the program calculates a default (conservative) window function that eliminates any spurious ringing in the energy spectrum.

The output is generated as follows: a summary of the input parameters, the potential function and the grid as well as the consistency checks on the norm and the average energy of the wave packet during the course of the propagation are sent to the file out.dat as well as to the screen. The norm should stay close to unity and the energy should remain constant. If the norm starts increasing wildly, this means the time step is too large, or perhaps the potential needs shelving (see Section 3.3.1 of Chapter 4). The autocorrelation is written to a file auto.dat (real part, imaginary part and modulus of $C(t))$ and the final energy spectrum to absspec.out suitable for plotting with a simple X-Y plotting package. The wave packet output has already been described. Some simple checks that can be performed on the calculation to make sure that everything proceeded smoothly are graphing the wave packet file with the 
draw3 program, plotting the autocorrelation and checking for smooth behavior and convergence checking by repeating the calculation with a reduced time step and grid spacing. 


\section{Two-dimensional Propagation code - prop2d22}

The executable code, prop2d22, can be compiled by using the UNIX make utility and the makefile makeprop2d22 (make -f makeprop2d22). If using the San Diego Cray Y/MP a convenient script file called prop2d that takes care of retrieving the source from the DataTree, as well as compiling and executing the code is available. To retrieve this execute the commands

$$
\begin{aligned}
& \text { dti get time/prop2d } \\
& \text { prop2d }
\end{aligned}
$$

on logging in. The prop2d script can also be used subsequently as a convenient interface for executing the code.

The two-dimensional propagation code depends on three files of FORTRAN source, koss2d22.f, potread2.f and graphicsy.f and the NAG numerical library and local graphical libraries. The dimensioning of arrays that determine grid size are controlled by an include file, param.inc. This file contains a single line dimension statement and should be edited prior to compiling the code to match the grid size that the user has specified in his/ her input deck. The graphicsy.f file is machine dependent; all graphics routines have been bundled into this source file; the file shown here uses the Computer Associates DISSPLA graphics library with a GKS/NCAR graphics interface available on the San Diego Supercomputer Center's Cray Y/MP. The prop2d22 code is generally run on this supercomputer as it makes heavy use of a vectorized two-dimensional fast Fourier transform routine. However it is possible to use the code on a fast scalar workstation, and one envisiges that it could be readily be ported to a RISC based scalar machine with suitable libraries 
installed. If the NAG library routine used for the two-dimensional FFTs is not available, the code may be compiled with an extra source file (twodfft.f) however the speed will be significantly reduced. To do this, the koss2d22.f code should be edited (as indicated in comments in the code) to remove reference to the NAG routine. The twodfft.f file contains subroutines based on code in Section 12.11 of Numerical Recipes - The Art of Scientific Computing by W. H. Press, B. P. Flannery, S. A. Teukolsky and W. T. Vetterling, Cambridge University Press, Cambridge (1989).

Typical run times are 20 Cray CPU seconds for a 320 femtosecond propagation (1.3 a.u. time steps, 10240 steps) on a $64 \times 32$ point spatial grid. Memory requirements are modest; for such a typical job $350 \mathrm{kwords}$ (2.8 Mbytes) are required. Propagations of 2 psecs on a $128 \times 64$ grid are the largest jobs that have been attempted. These large jobs yield excellent energy resolution, and the limitation at this point is less a question of CPU time but more expected errors accumulating in the phase of the wave packet (see Chapter 4).

The two-dimensional code is used in a very similar way to the one-dimensional code, however this code can treat a completely general wave packet propagation on a bimolecular reaction surface, suitable for two-coordinate photodissociation or photodetachment to a continuum. The quantum dynamics is exact, in contrast to the 2D adiabatic approaches employed by Metz (Ph. D. thesis, U.C. Berkeley (1991)), which are strictly applicable only in limiting heavy-light-heavy cases. However, because the calculation treats the two coordinates generally (fully coupled), it is computationally more demanding for a given dimensionality. At present we have not extended this method to three-dimensions, although other authors have achieved such 
three-dimensional wave packet calculations, and the code presented here should be easily generalizable. (See chapter 4 , section 4 for references).

The code is used as follows. An input deck (called koss2d.dat) is constructed, in a very similar way to the one-dimensional code. Once again, no input from the keyboard is required. This file controls the way the code proceeds and contains all physical information such as the masses of the atoms and potential parameters. The input deck is not free format, but comments may be left to the right of the input line, or at the end of the file, to assist the user in making changes to a template file. The reader is directed to remarks in Section 2 above concerning the meaning of many of the input lines which are common to both codes. A typical input deck is shown below:

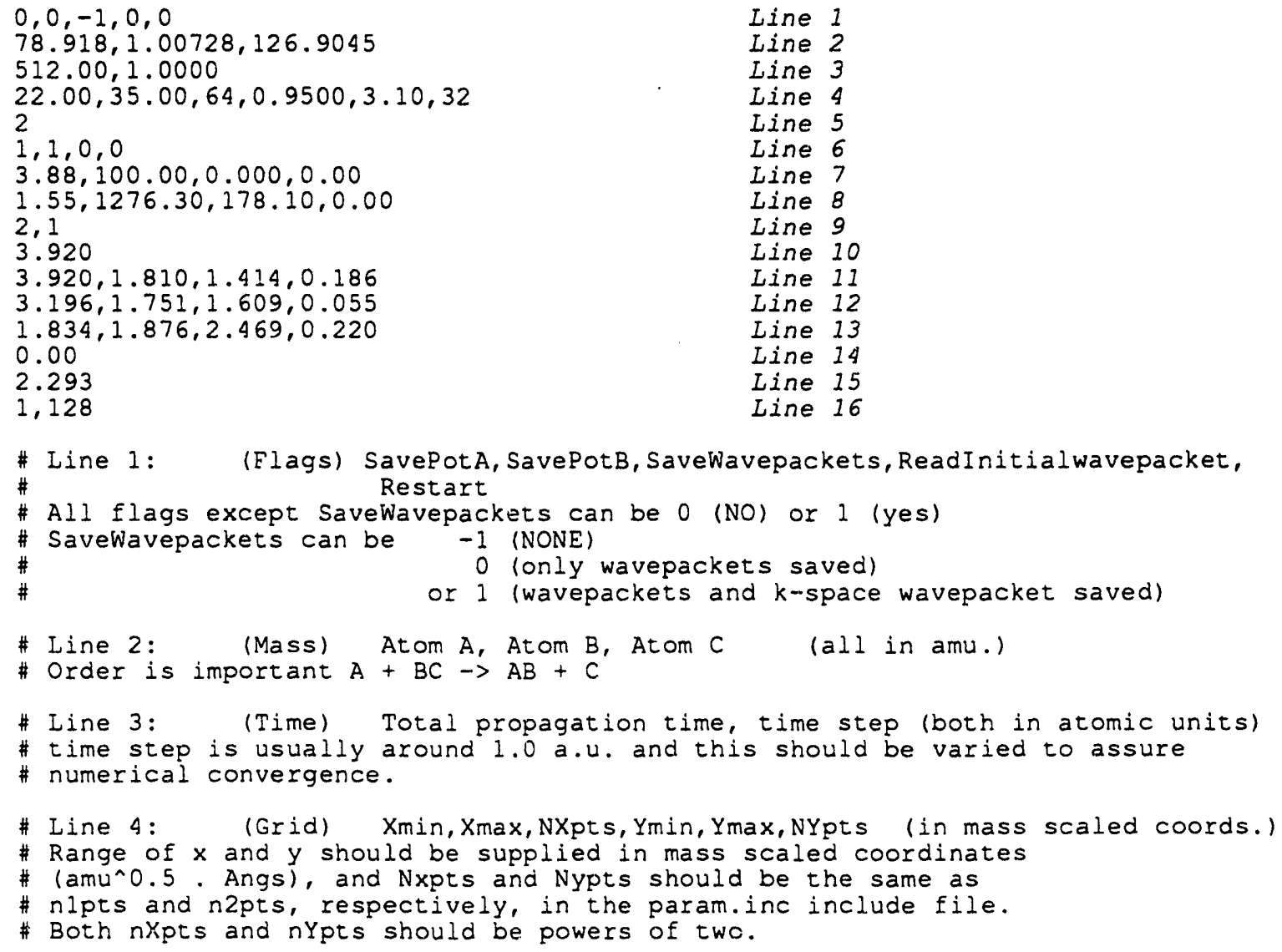




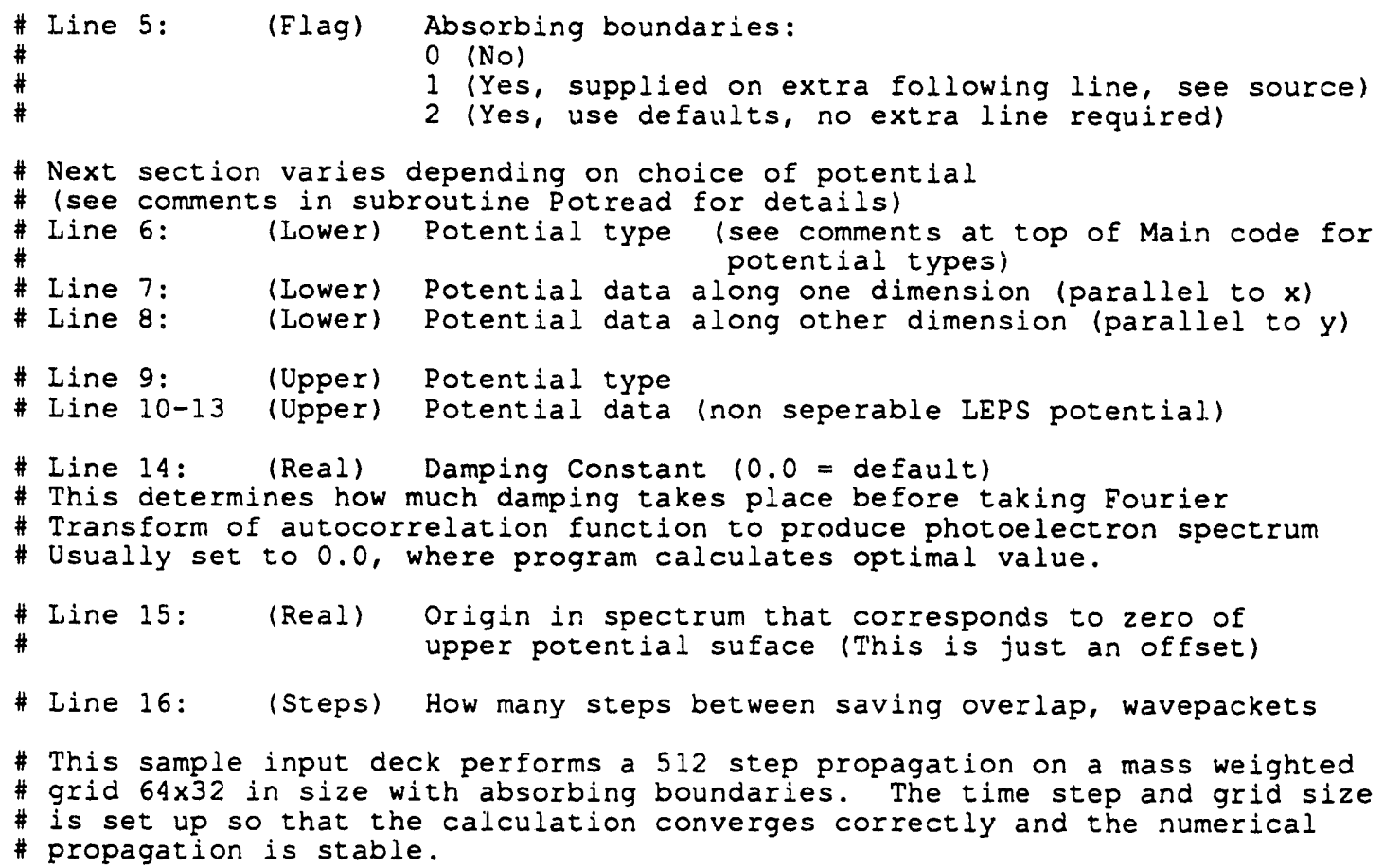

This template file has a set of detailed comments describing the purpose of each entry and line. This should be used in consultation with the comments below and in the header to the main code. Criteria related to the grid and time step are common to those described for 1D propagations. All lines marked "(flags)" should contain only integers; further nXpts, $\mathrm{nYpts,} \mathrm{both} \mathrm{step} \mathrm{intervals} \mathrm{indicating} \mathrm{when} \mathrm{to} \mathrm{save} \mathrm{the} \mathrm{overlap}$ and the wave packet, and the flags indicating potential type should be integers. All remaining entries should be real numbers.

Let us describe the input parameters that are new. The purpose of the extra flags ReadInitialwavepacket and Restart are fairly self-explanatory. If either flag value is set to one, the program attempts to read the a wave packet from the wave.dump file either for use as the $t=0$ wave packet or to continue the propagation from a previous propagation. Note ReadInitialwavepacket and Restart cannot both be one. See comments in subroutine initB source for more information on this 
advanced option. The mass of the three atoms is required to determine the appropriate reduced masses for the mass-scaled Jacobi coordinate grid. (See Chapter 4, section 3.1 for definitions). The range of $x$ and $y$ are expected in this mass-scaled coordinate system, however the equilibrium geometry data in the potential parameter input is expected in the relevant internal coordinates without mass-scaling.

The absorbing boundary information required in line 5 is again fairly selfexplanatory. Use of the default boundaries for a dissociative systern is recommended for initial calculations; for bound problems the absorbing boundaries should be turned off. Refer to section 3.3.2 of chapter 4 for details on absorbing boundaries.

Most of the potential types are common to the $1 \mathrm{D}$ code and have been described above. Notice that for the lower potential, four integers are expected on Line 6: two to describe the potential type in each spatial direction and two to describe the quantum state of the initial wave function. If 0,0 is chosen for the latter pair then the ground state wave function is computed for the potential type requ d. Note that the potential can be Morse oscillator along only one spatial direction; that direction must be along the $y$ coordinate. The upper potential requires only two (but it must have two) integers describing the potential type. In addition to harmonicl Morse (0 and 1), LEPS (2) and read-from-file (3), two new potential types have been added, and the LEPS potential extended. The LEPS function data is input exactly as before except the mass data has already been entered in Line 2 and no cut $R_{A C}$ is relevant or required. The other integer flag on the potential type line allows the zeropoint bend correction to be added to the LEPS potential energy at each point $(0=$ no correction, 1 = ZPB correction). The zero-point bend correction was suggested in the reduced dimensionality work of Prof. J. M. Bowman (Emory Univ.). The new 
functions available are (4) user-defined subroutine upotfn (compiled into code) and (5) rotated-Morse-oscillator spline (RMOS) function. (4) is useful for specific potentials, e.g. Lennay-Jones, quartic anharmonic oscillator or elaborate reaction potentials such as the T5a and 5SEC surfaces (of Truhlar and coworkers) for the $\mathrm{F}+\mathrm{H}_{2}$ reaction. The RMOS potential type is very useful for fitting $a b$ initio reaction surfaces; references for this potential type appear in Chapter 6.

If either a potential or an initial wave function is read from disk, this must be constructed in a rectangular array of the same size as the propagation grid with the same grid points. The imposition of a shelving criterion to the potential energy helps reduce computation time by allowing a larger propagation time step (see section 3.3.1 in Chapter 4). This is hard-coded in the source for all potential types. To modify the shelf value the user must edit the code and recompile. See section 4 below.

Completing the description of input parameters, Line 15 is a constant offset for calculation of the origin in the final photoelectron simulation. Unlike the $1 D$ code, this code transforms the simulation from the neutral internal energy scale to a true electron kinetic energy scale by use of formula (2) in Chapter $4 . \Delta$, of formula (2), is the offset supplied on this line.

The program, if run so as to produce graphical output (this is carried out by keywords on the prop2d22 command line or via the prop2d script), will generate all of the important computed information in graphical form. Craphical output is possible to any device the graphics software supports; typically this is to a Textronix 4013 emulator or to a graphics metafile. The latter may be converted into many other formats such as X11 bitmap or PostScript. The graphics capability of the code makes error checking and general use particularly easy. The values of the wave packet norm 
and energy (which are conserved only if there is no flux absorbed by absorbing boundaries) is displayed along with the contour plots of the wave packet. On these plots, contours of the upper potential surface are also shown. Plots are displayed at time intervals specified by wave packet step interval on the last line of the input deck. Viewing eight or so wave packets as a function of time is usually sufficient to interpret the wave packet dynamics.

Inadequacies in the choice of grid range and absorbing boundary are manifest in this moving image of the wave packet dynamics. To check the performance of the grid in the momentum domain (i.e. whether the full range of momenta in the wave packet fits on the Fourier space grid), the SaveWavepackets flag may be set to 1, and $\phi\left(k_{x}, k_{y}\right)$ will also be graphed as well as saved to the wavepkt.out file. rhe use of graphics is extremely important in giving the user a feel for the calculation he or she is attempting, and suggesting possible remedies if problems with the propagation arise.

Output to files out.dat and auto.dat is as for the $1 \mathrm{D}$ code. The potlA.out and potlB.out files containing the lower and upper potentials, and the wavepkt.out file containing the wave packet, are now two dimensional arrays of potential/ wave packet values; the wavepkt.out file gets very large, very quickly. The file absspec.out is in the same format as for the $1 D$ code but gives the spectrum as a function of the electron kinetic energy rather than of the neutral internal energy. There is no wave.drw file. Finally there is a dump file (wave.dump) written for the final wave packet of the propagation for restarting purposes. 


\section{How the prop10 and prop2d22 programs work}

This section is really only intended for users who wish to make changes to the codes. The methodology behind both codes is described in Chapter 4 of this thesis and the simple use of each code is described in sections 2 and 3 above. Both codes have very similar layout and the names of subroutines and functions are in may cases identical. The basic organization is as follows (subroutine names given in italics).

The main routine initializes the graphics device (if applicable) and calls subroutine const which defines some fundamental constants and then reads the input deck (file koss.dat or koss2d.dat). The necess ary input is described in detail in section 2 and 3 above. This subroutine establishes the parameters for the propagation, converting them into atomic units where appropriate, and (if applicable) the absorbing function. The potential parameters are read, and the lower and upper potentials are stored in the potential array xypot in subroutine potread. All the necessary conversion of coordinates for the $2 \mathrm{D}$ code are performed by statement functions like AMStoRab. Potread calls one of an assortment of subroutines to evaluate the potential depending on the potential type - lepstore (for LEPS potentials), upotfin (for user defined potentials) and rmos (for the RMOS spline functions) are the starting subroutines for calculation of each of these non-separable potentials.

For the upper state potential, where the propagation will proceed, the potread subroutine imposes a shelf on the highest numeric value the potential may take. The purpose of this is described in Chapter 4, section 3.3.1. This shelf value is hard-wired in the code for each potential type. If the value is unsuitable for the user's application, he/ she should change the value in this routine and recompile the code. One way to estimate an appropriate value for the potential shelf is to consider the 
classical turning points for motion on the upper surface, and the energy at the turning point. One classical turning point is $x_{\text {initial }}$, the center of the initial wave packet, and the energy is given approximately by the $\langle\mathrm{H}\rangle$ evaluated by the program at $t=0$. This energy must be considerably less than the chosen shelf value. Errors from choosing a value for the shelf that is too low will be obvious when the wave packet propagation is graphed; parts of the wave packet will spill into regions of configuration space that they should not be in! If in doubt raise the value of the potential shelf, and reduce the time step as necessary, and check for convergence.

Returning to the main routine, the input data is written to the output stream and to the file out.dat. The maximum kinetic energy supported by the grid is calculated and the program aborts if it finds the chosen tiven step is too large to maintain a stable propagation. The potentials are saved (potlsave) to disk, if the user has so requested, and the wave packet disk file(s) are initialized (initpkt). In the $2 \mathrm{D}$ code, if the NAG 2D-FFT routine is used, it is initialized now. The initial wave packet is now set up by a call to initB.

Init $B$ determines if there is to be a restarted propagation or if the wave packet is to be read from disk (readwave) or calculated. If the latter, the routine determines whether an analytic form for the initial wave packet exists (i.e. if the lower surface is harmonic or Morse), or whether a initial wave packet must be calculated numerically (relax). The 1D code is much more primitive in this subroutine - it will not read from the disk or restart a propagation, and it allows only an analytic initial wave packet. For calculation of the analytic initial wave packet, initWF or morsewf is called. In the $2 \mathrm{D}$ code, these two routines will calculate a $v=1$ wave function as well as $v=0$, allowing some experimentation with "hot-band" photoelectron spectra. 
InitB finally propagates the initial wave packet for its first time step, to calculate $\phi^{2}$, with second order Runge-Kutta. The initial wave packet, $\phi^{1}$, along with $\phi^{2}$ are passed back to the main routine where the initial packet is saved to disk (pktsav). The norm and average energy of the initial wave packet on the upper surface is evaluated (chk, chknrm, chken).

Finally we enter the main loop; the propagation now begins! This is the loop that gives the iterative solution of the time-dependent Schrodinger equation by second order differencing (SOD). In each pass through the loop, psi2 (or psi2ab, if absorbing boundaries are being used) is called. This routine contains the SOD formula. If absorbing boundaries are being used, it is at this point where the wave packet is multiplied by the absorbing function. In each operation of the SOD formula, $\mathbf{H} \phi$ must be calculated. This is also required each time the average energy is calculated (chken). The operation of $\mathbf{H}$ on $\phi$ is performed in the routines Hpsi, KEmat and PEmat; this is the core of the Fourier method.

KEmat evaluates the operation of the kinetic energy operator on the wave packet. It does this by performing a pair of discrete fast Fourier transforms on the data array. For the 2D code we use either c06fuf, the vectorized NAG library routine, or twodfft, the (slow) Numerical Recipes routine. In the 1D code the Numerical Recipes FFT routine is employed. Between the two FFTs, the wave packet (now represented in $k$ space) is multiplied by $\left(\mathbf{k}_{x}{ }^{2}+\mathrm{k}_{\mathrm{y}}{ }^{2}\right)$, or just $\mathrm{k}_{\mathrm{x}}{ }^{2}$ in one dimension. The KEmat routine is where both prop10 and prop2d22 spend most of their CPU time. In contrast, PEmat performs a simple multiplication of the wave packet by the potential at each grid point; each is already stored in memory. 
Inside the main loop the self overlap of the wave packet with the initial wave packet is computed (ovlp) and stored at regular intervals; the overlap is calculated by Simpson's rule (zsimpint, ztrapint). At more infrequent, but still regular, intervals the wave packet is checked for norm and average energy, and stored and graphed. These regular intervals are determined by the last two parameters set in the input deck. After the main propagation loop is complete some tidying up is done: the autocorrelation function is saved to disk, the energy spectrum is calculated by a onedimensional FFT of the autocorrelation function and saved (savabs), and files and graphics devices are closed up.

\subsection{Source Code Listing}

\subsubsection{One-dimensional Code - prop10}

\section{makeprop10}

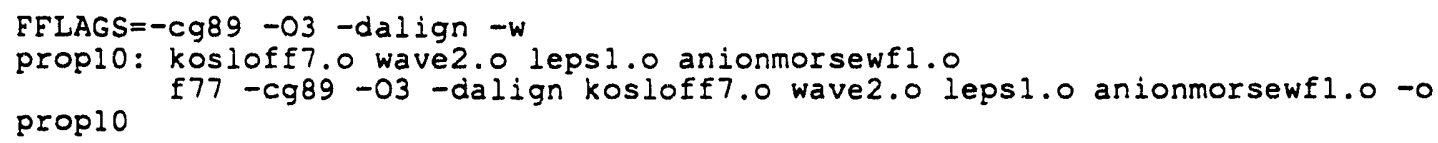

\section{kosloff7.f}

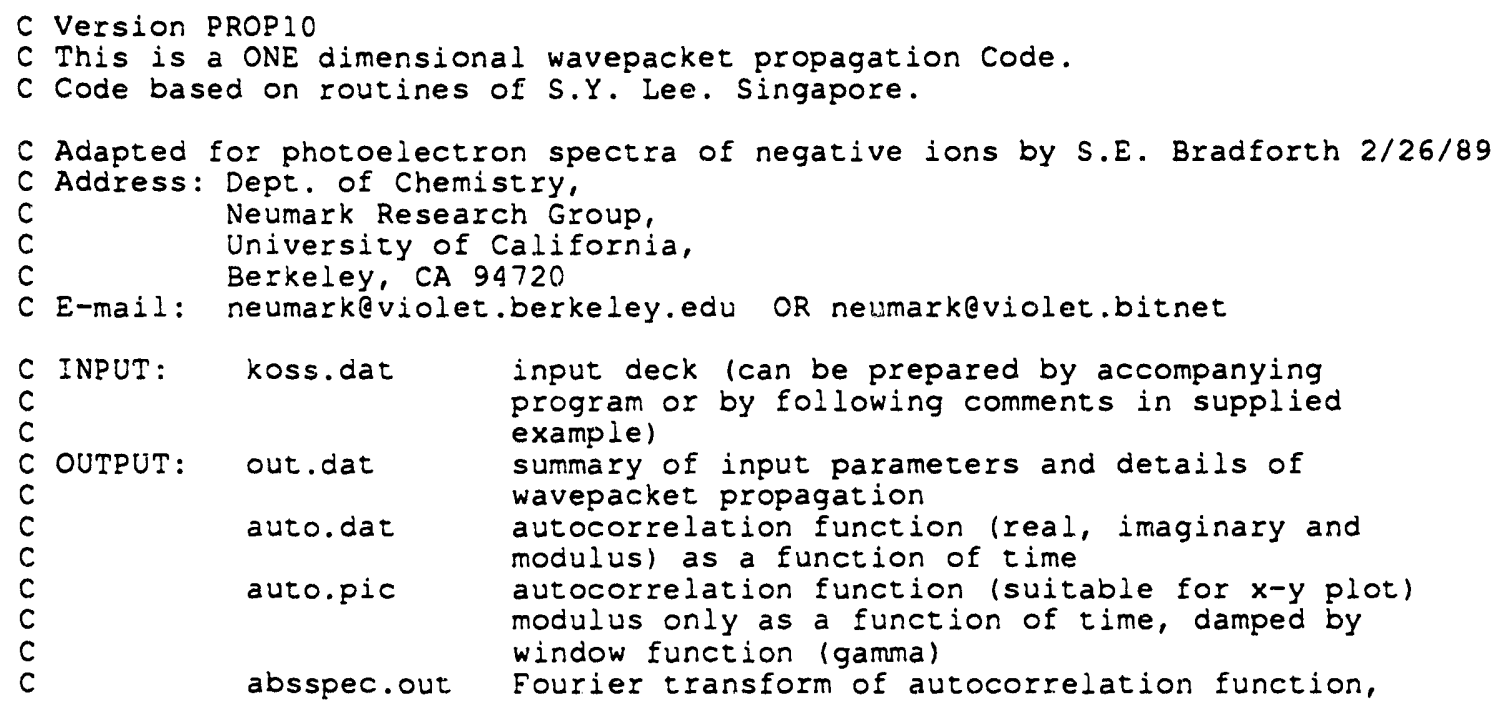




$$
\begin{gathered}
\complement \\
\vdots \\
\vdots \\
\vdots \\
\vdots \\
\vdots
\end{gathered}
$$

wavepkt.out

potlA. out

potiB.out

wave. out the absorption/photoelectron spectrum

real, imaginary and absolute value of wavepacket

as a function of time

(can include wavepacket derivatives)

lower potential

upper potential

formatted direct access wavepacket output

suitable for reading by

wave $1 D$ tektronix plotting program

C

C It is helpful to be able to graph wavepacket evolution in time, for

$C$ checking usefulness of run and that all criteria for successful propagation

$C$ have been satisfied. The file wave.out can be

C plotted with the "draw3" (seperate) program.

C The files potlA.out and potlB.out, (the lower and upper

C potential energy surfaces), are provided for for graphing and checking.

C Potential types included are:

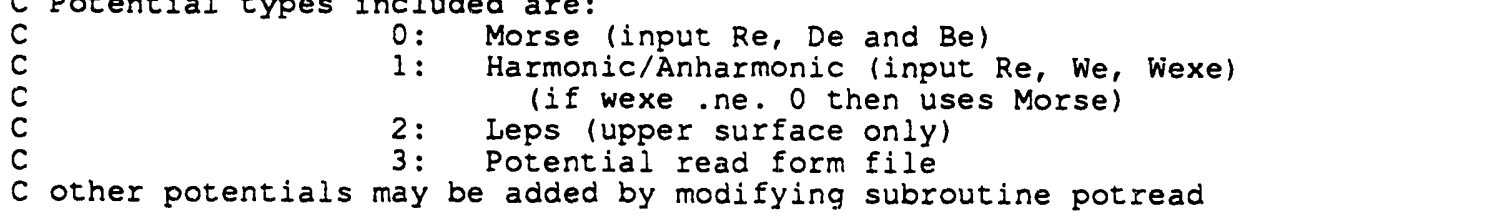

C There is no provision for absorbing boundaries in this code,

C Also restarting from arbritary time point is not coded for.

$C$ Notes for this version:

C $8 / 11 / 92$ Input xmin, xmax range in Angstroms.

C Include shelf for potentials in potread.

c Include conversion constants common block.

C Remove Time step printout except every npktsav steps.

C Co ie does not stop to query for whether potentials are to be saved or

C Whether to save derivatives in wavepkt.out.

C Instead this now comes from input deck as in $2 \mathrm{D}$ code.

C 1989 Change format for input of potentials.

C All potentials stored as arrays. 5/10/89

C Note all quantities in main routine are in Atomic units,

C conversions performed in $1 / 0$ routines (const(), savabs, pktsav, savovlp)

C Note all variables beginning with $z$ are complex

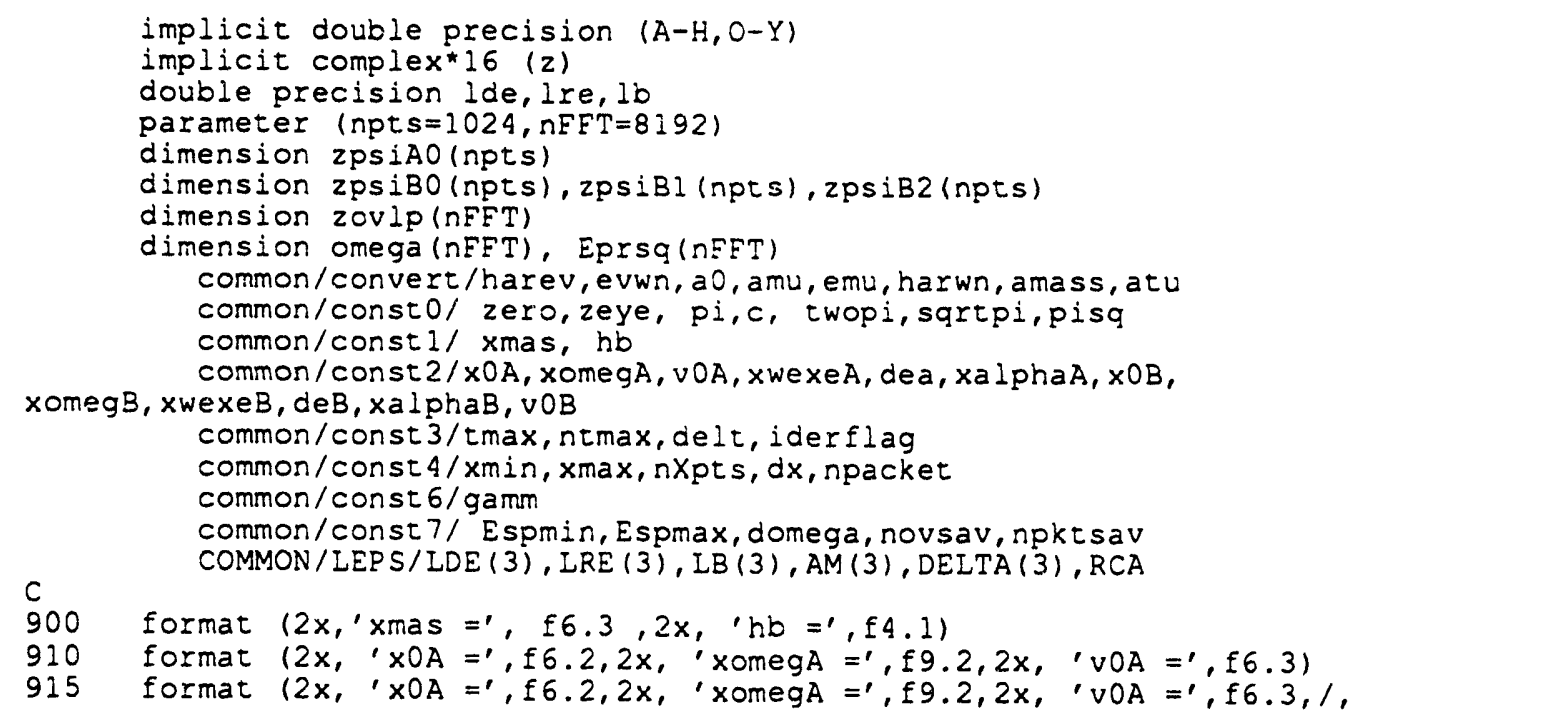




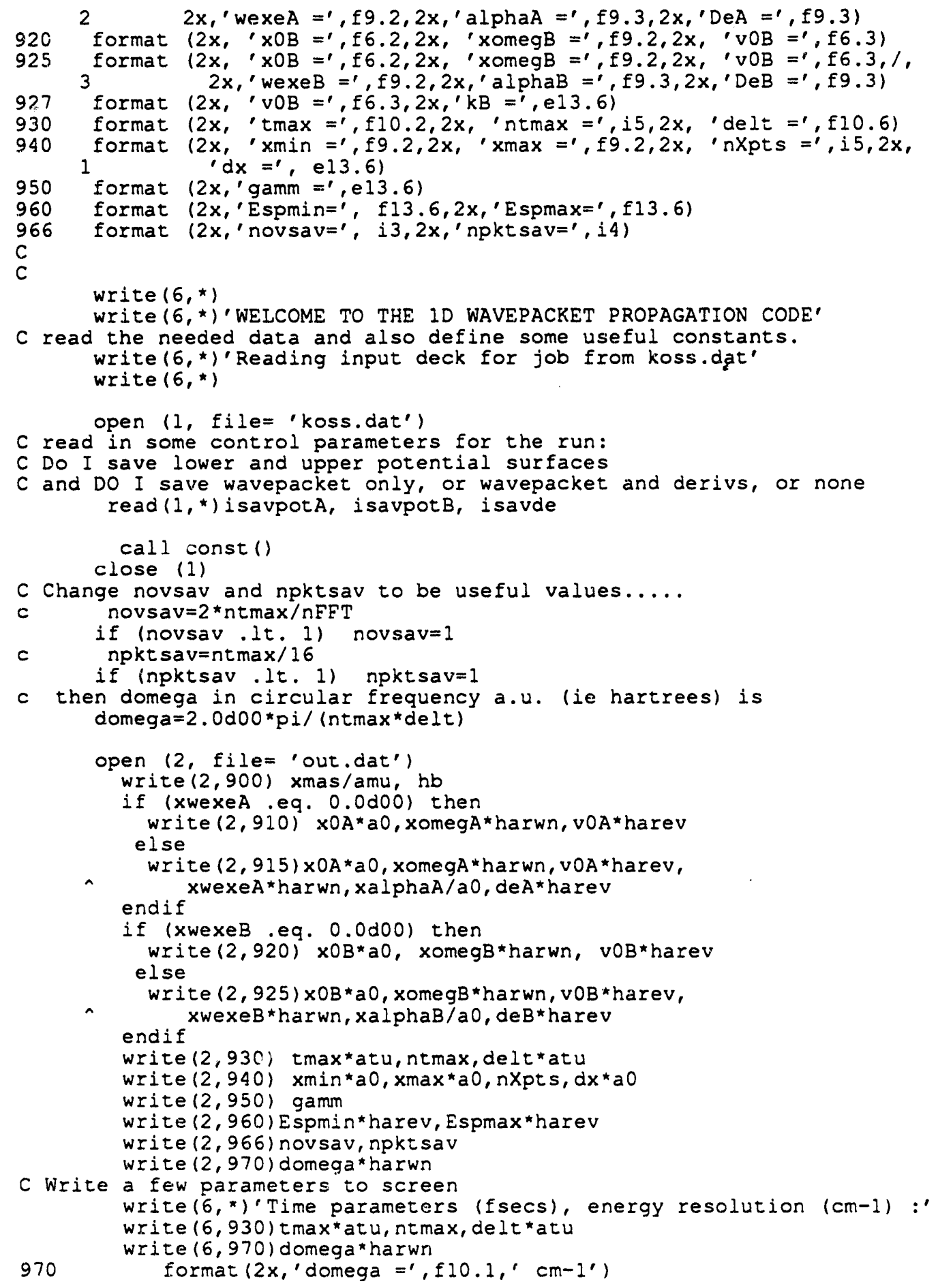

C criteria of succesful propagation given in Kosloff, $J$. Comput. Phys. 52, 35

C (1983); essentially the max kinetic energy representable on a grid with

C spacing $d x$ is given (in au) by $p^{\star}{ }^{*} p /\left(2^{*} \times \operatorname{mas} s^{*} d x^{*} d x\right.$ ) and the stability

c criterion is $\left\{\right.$ delt* $\left.\left.\left(i p i{ }^{*} p i\right) /\left(2 * x m a s^{\star} d x^{\star} d x\right)+V\right)<=1.0\right\}$

sqkmax $=4.9348 d 00 /\left(x \operatorname{mas}{ }^{*} d x^{*} d x\right)$

write $(6,912)$ sqkmax*27.2116 


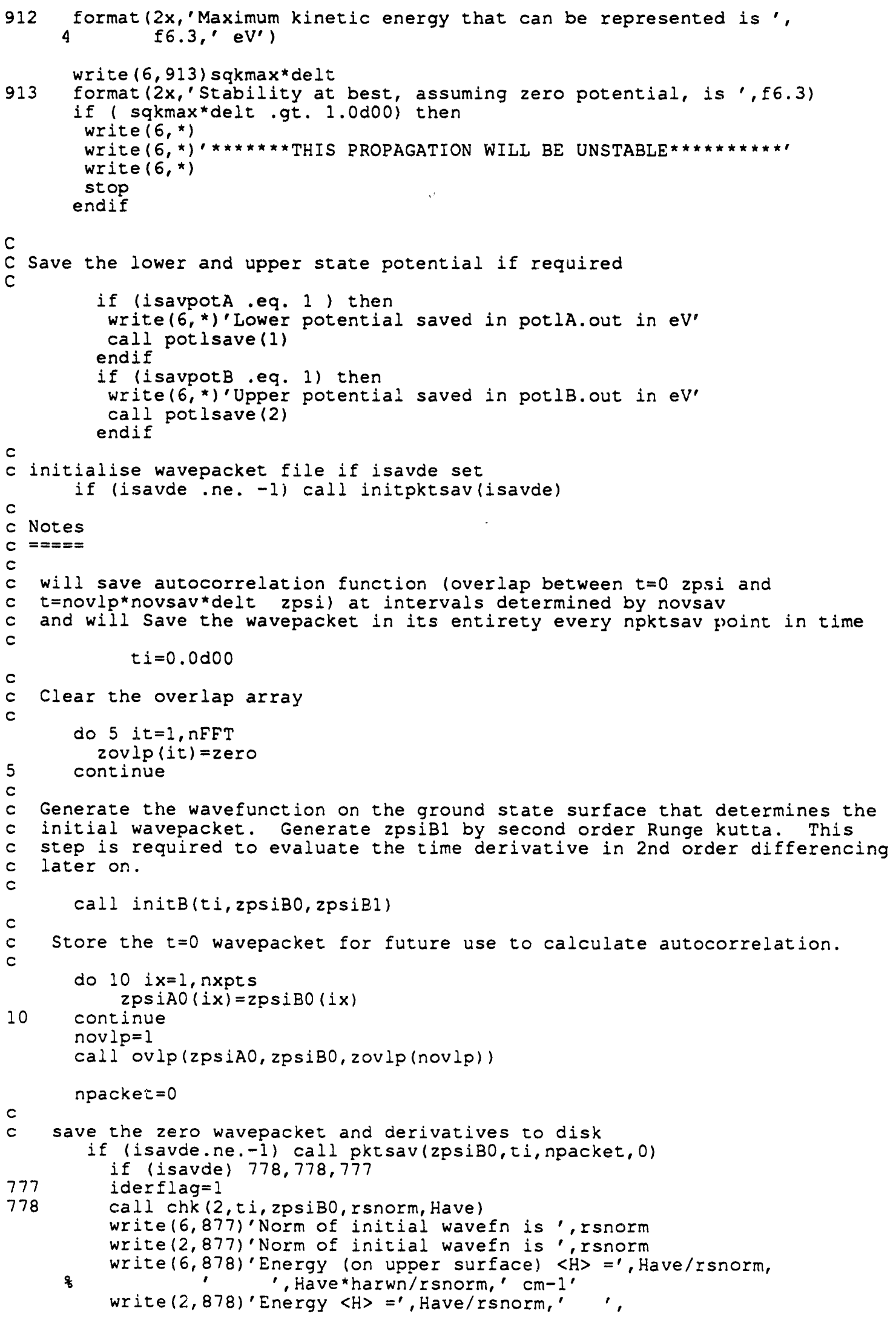




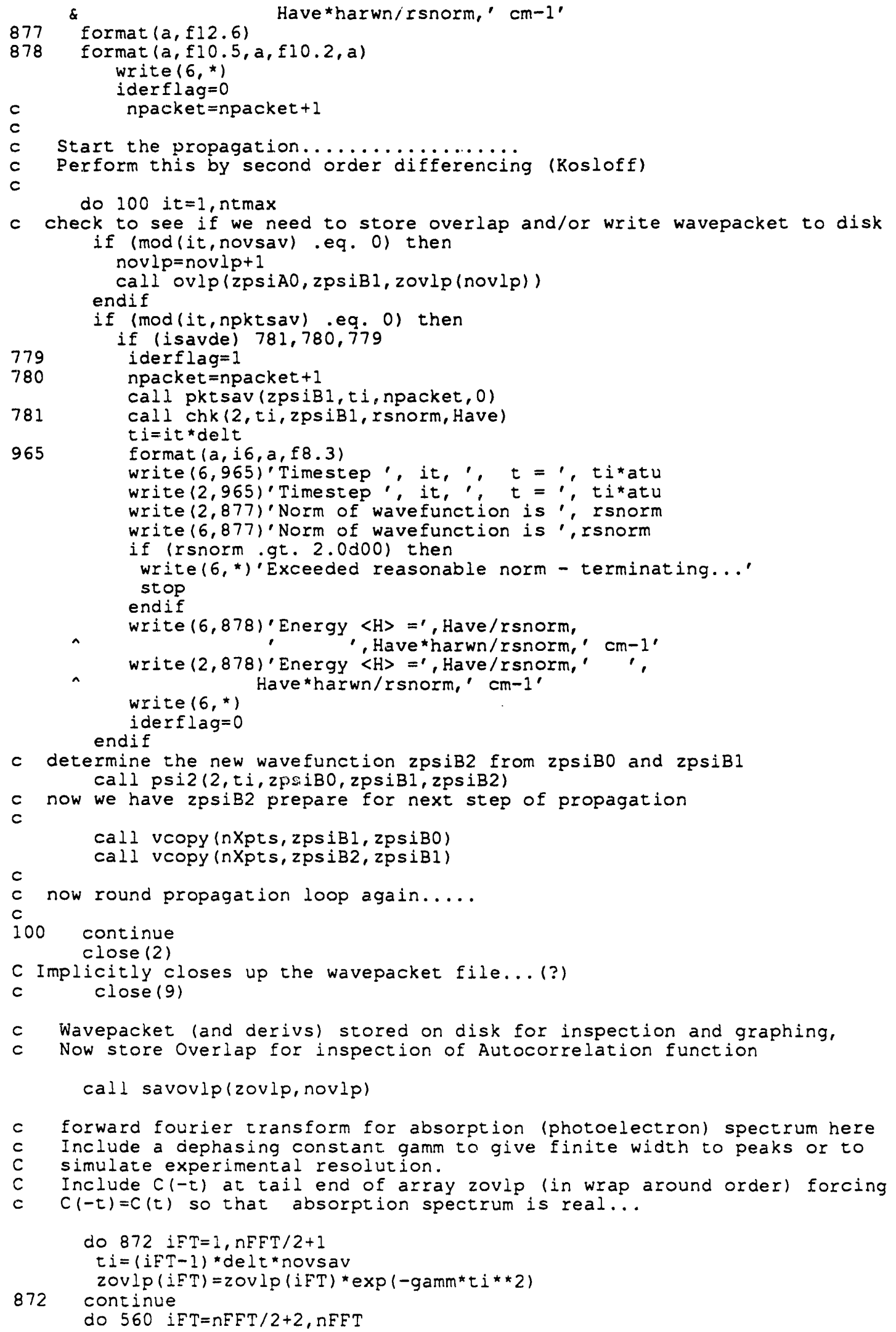


$j F T=n F F T-i F T+2$

zovlp (iFT) $=\operatorname{dconjg}($ zovlp $(j F T))$

560

cont inue

write $(6, *)$ 'Performing final fast Fourier Transform'

call FFT (zovlp, nFFT, I)

c

C

20

At the moment are using a two sided $C(t)$ and checking $I(w)$ to be real

domega $=2.0 d 0 * \mathrm{pi} /\left(\mathrm{nFFT}{ }^{\star}\right.$ delt*novsav)

absmax $=0.0$

$j F T=0$

do 120 iFT $=?, \mathrm{nFFT}$

$\mathrm{v} 1=(\mathrm{iFT}-1) *$ domega

if (vl.ge. Espmin and. vl .le. Espmax) then

$j F t=j F T+1$

omega $(j F T)=v 1$

Eprsq (jFT) =dreal (zovlp (iFT))

if $(\operatorname{Eprsq}(j F T) . I t .0 .0)$ Eprsq $(j F T)=0.0 d 0$

if (Eprsq(jFT).gt. absmax) absmax $=\operatorname{Eprsq}(j F T)$

endif

continue

ninit $=1$

$n f i n=j F T$

c Save spectrum(onega is in circular wavenumbers)

call savabs (omega, Eprsq, ninit, nfin, absmax)

All Done

$c$

stop

end

$c$

c

subroutine const ()

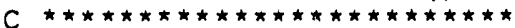

$C * * *$ read the needed data and also define some useful constants.

implicit double precision $(\mathrm{A}-\mathrm{H}, \mathrm{O}-\mathrm{Y})$

implicit complex*16 (z)

parameter (npts $=1024, \mathrm{nFFT}=8192)$

common/convert/harev, evwn, 20 , amu, emu, harwn, amass, atu

common/consto/ zero,zeye, pi,c, twopi, sqrtpi,pisq

common/const l/ xmas, hb

common/const 3/tmax, ntmax, delt, iderflag

common/const $4 / x \min , x \max , \mathrm{nxpts}$, dx, npacket

common/const $6 /$ gamm

common/const7/Espmin, Espmax, domega, novsav, npktsav

C

$C$ set conversion factors

harev $=27.211608 \mathrm{do}$

evwn $=8065.479 \mathrm{do}$

$\mathrm{aO}=0.52917706 \mathrm{dO}$

$\mathrm{amu}=1822.882 \mathrm{~d} 0$

emu $=9.109534 d-31$

harwn = harev*evwn

amass $=1.66056 \mathrm{~d}-27$

at $u=0.024199 \mathrm{do}$

$C$ set consto

zero $=\mathrm{dcmp} l \times(0.0 \mathrm{~d} 00,0.0 \mathrm{~d} 00)$

zeye $=\mathrm{dcmp} l \times(0.0 \mathrm{~d} 00,1.0 \mathrm{~d} 00)$

$p i=d a c o s(-1.0 d 00)$

twopi $=2{ }^{*} \mathrm{pi}$

sqrtpi= dsqrt $(p i)$

pisq $=p i * p i$

$c=2.99792458 d 10$

$\mathrm{hb}=1.0 \mathrm{do}$

c speed of light in $\mathrm{cm} / \mathrm{s}$ and hbar in atomic units

c 


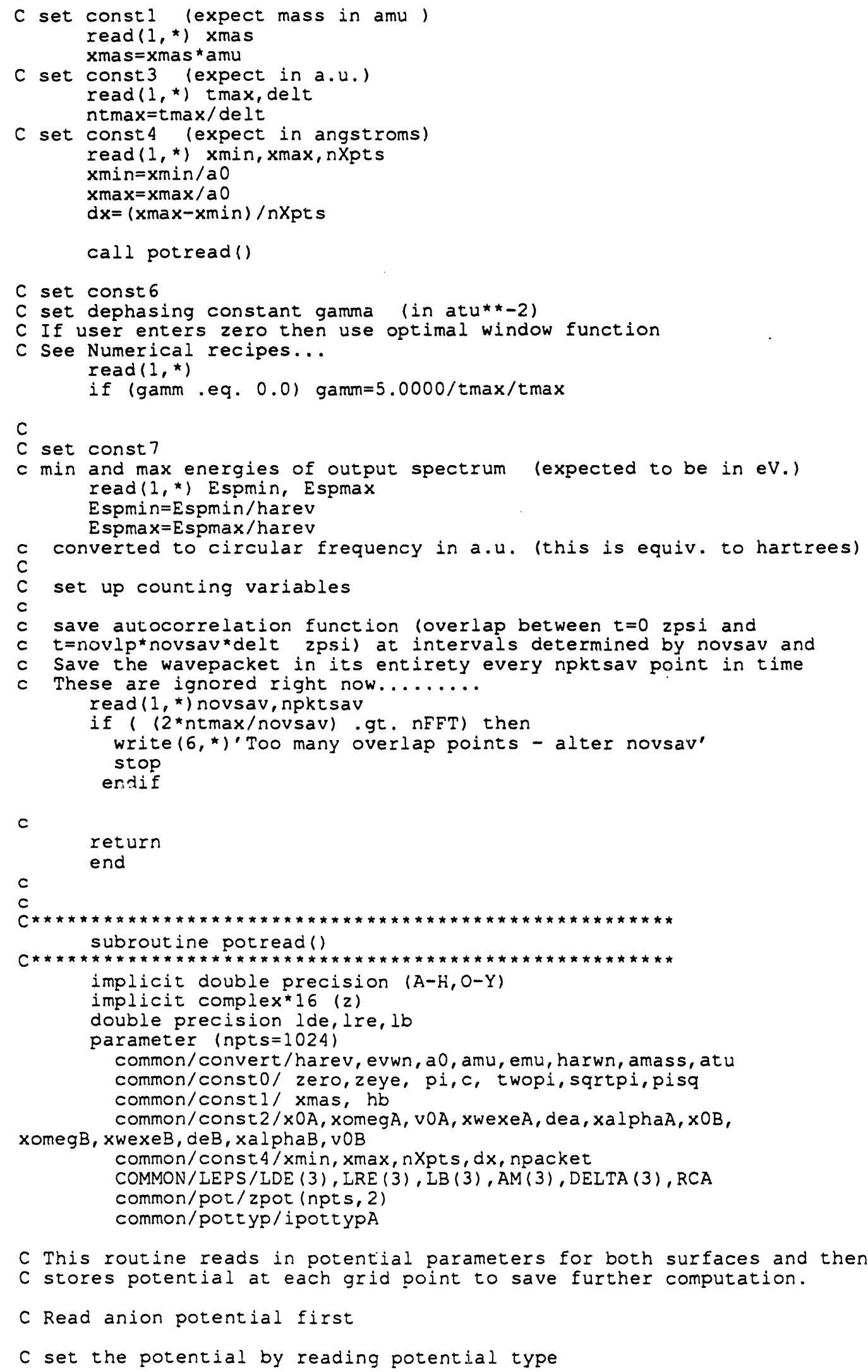




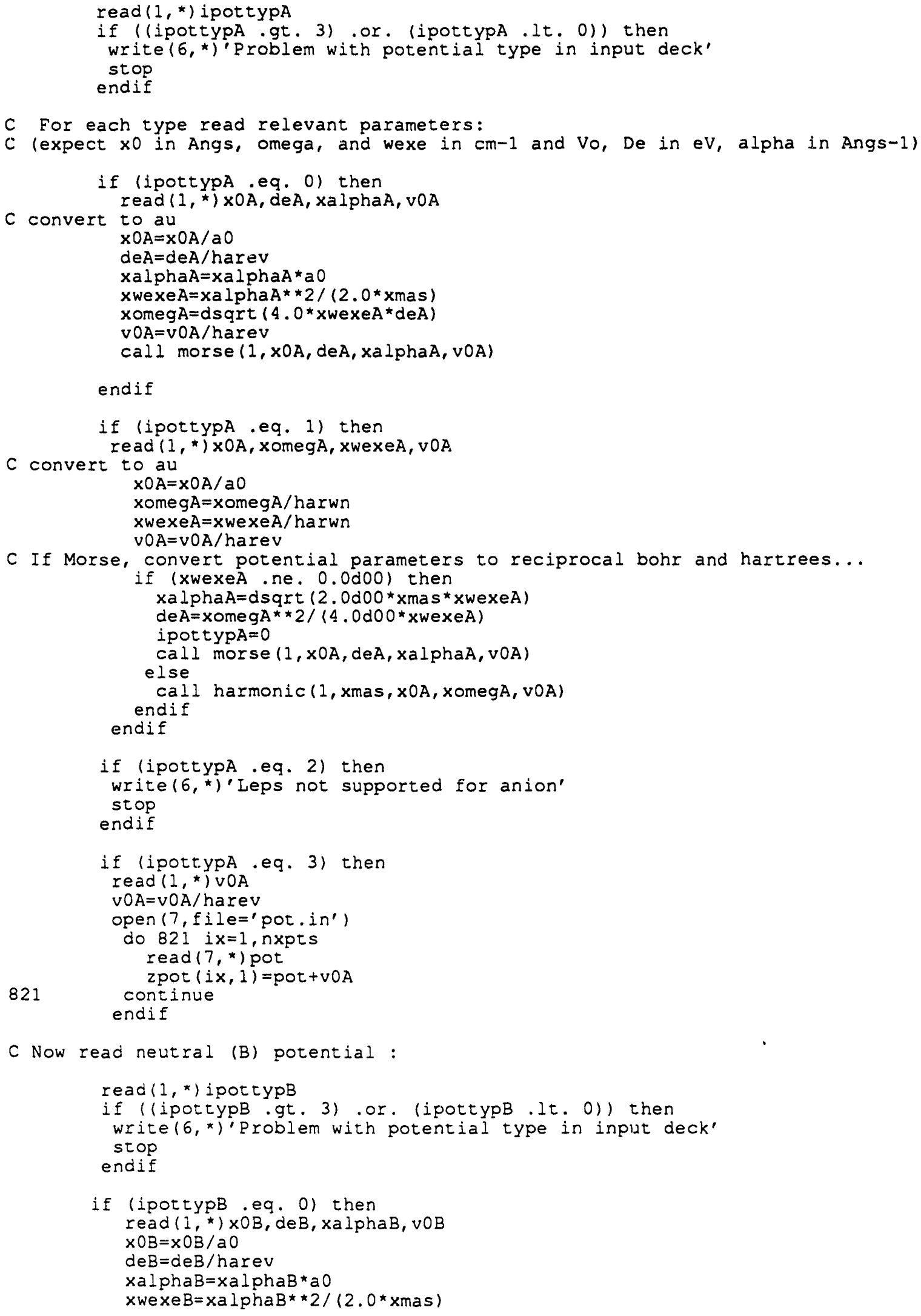

C If Morse, convert potential parameters to reciprocal bohr and hartrees... if (xwexeA ne. $0.0 \mathrm{~d} 00$ ) then 


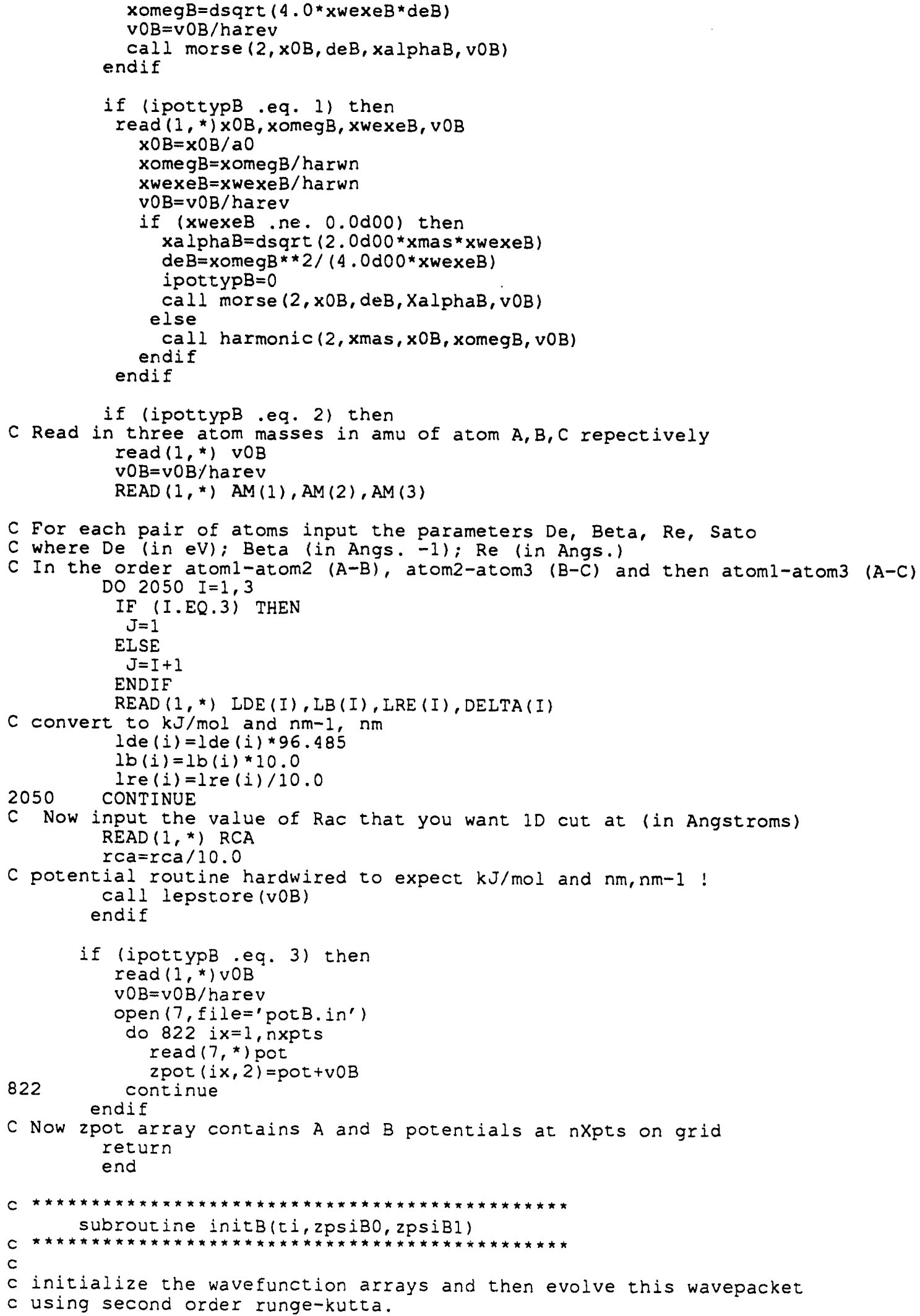




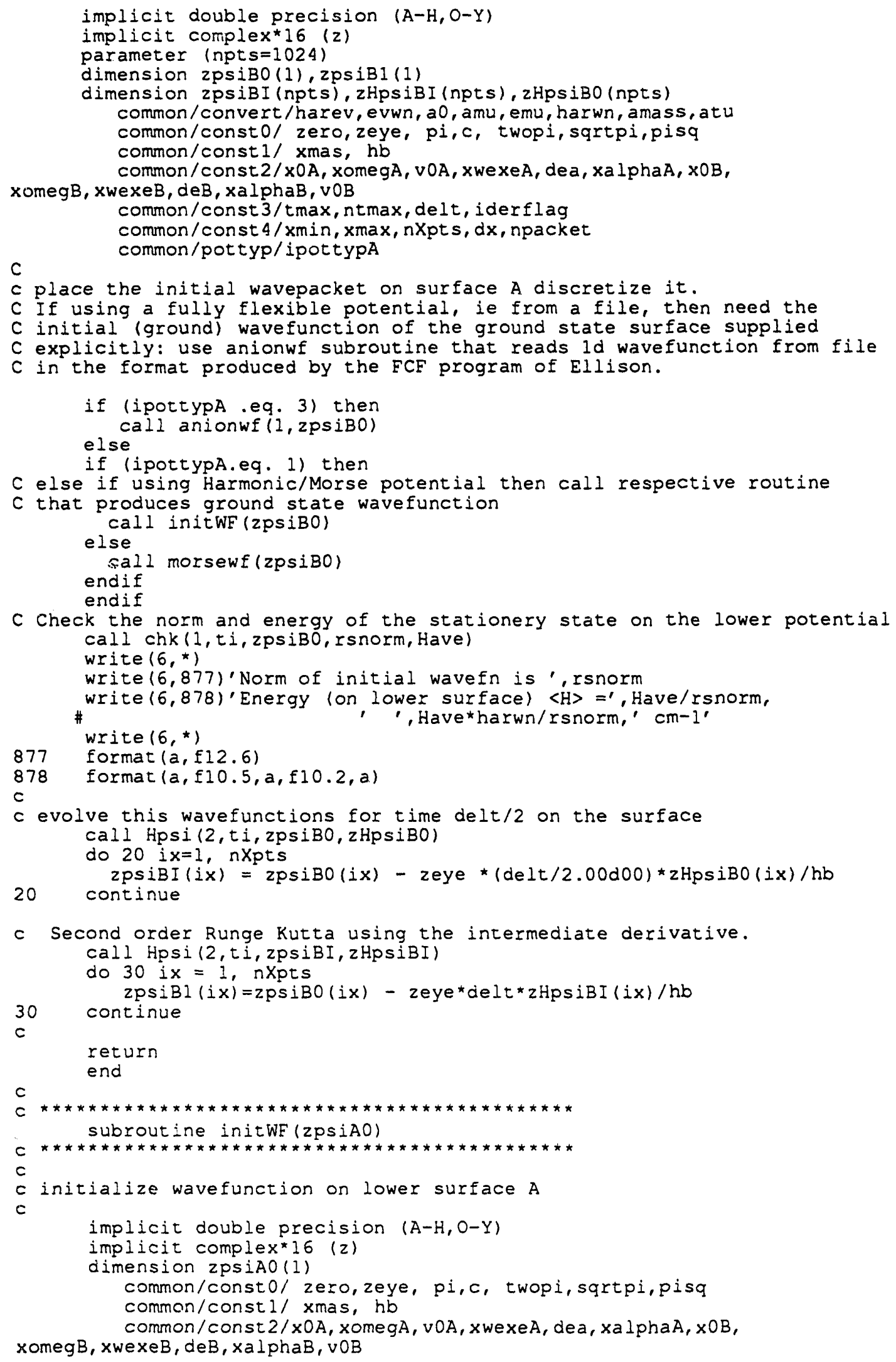


C

common/const $4 / x \min , \mathrm{xmax}, \mathrm{nXpts}, \mathrm{dx}$, npacket

C Initial wavefn on surface is ground harmonic oscillator

$C$ Only does the ground state wavefunction (lowest quantum

C state); for higher vibrational wavefunctions see the $2 d$ code

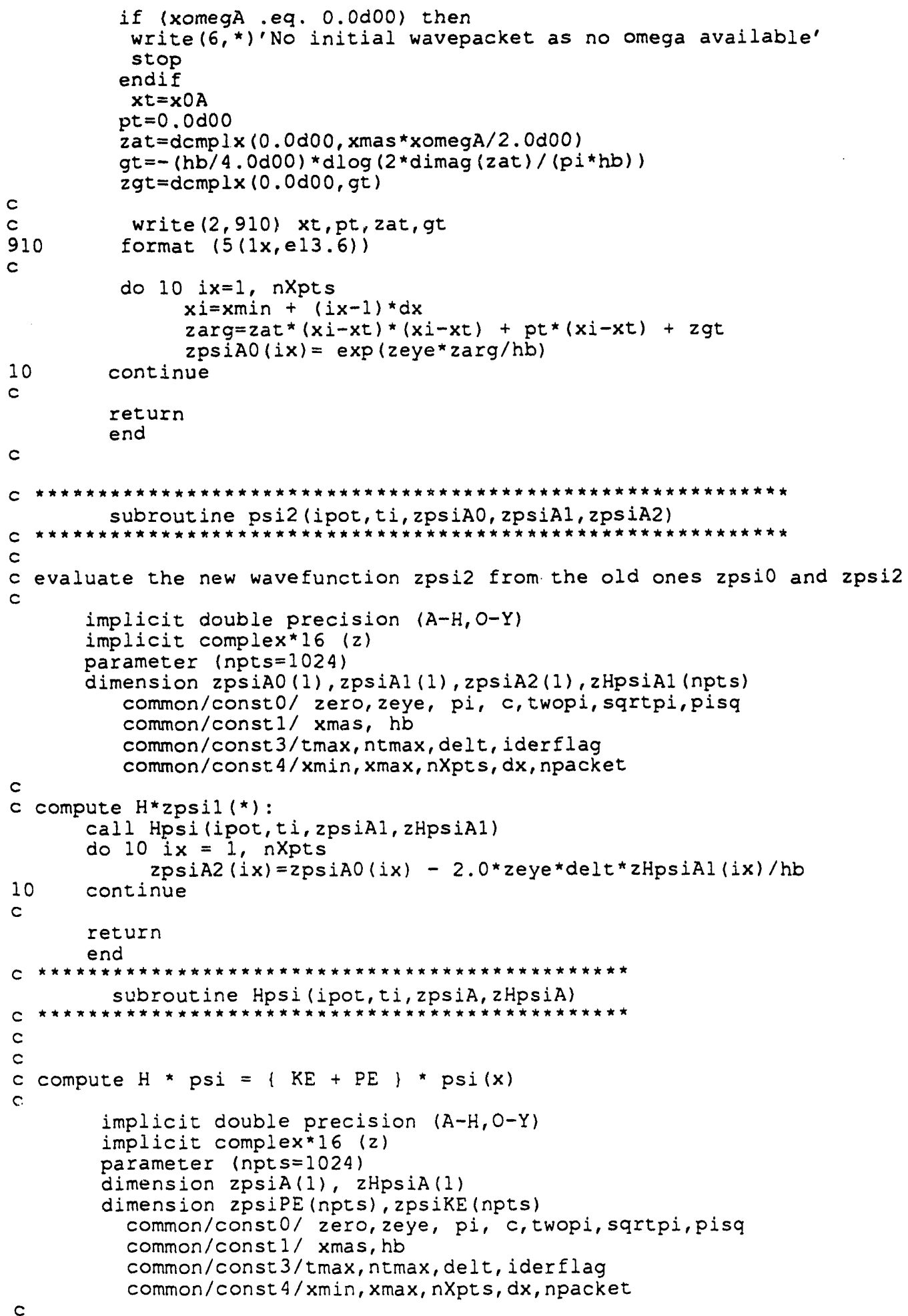


call KEmat (zpsiA, zpsiKE, ti)

call PEmat (ipot, zpsiA, zpsiPE)

do 10 ix $=1$, nXpts

$z H p s i A(i x)=z p s i P E(i x)+z p s i K E(i x)$

10

continue

if (iderflag .eq. i) then

write $(9, *)$ 'This is the second derivative....'

npacket $=$ npacket +1

call pktsav (zpsike, ti, npacket, -1)

write $\left(9,{ }^{*}\right)$ 'This is the Vpsi....' npacket $=$ npacket +1

call pktsav (zpsipe, ti, npacket, -2 )

write $\left(9,{ }^{*}\right)$ 'This is the Hpsi.....'

npacket $=$ npacket +1

call pktsav (zHpsiA, ti., npacket, -3 )

endif

$c$

return

end

subroutine KEmat (zpsiX, zpsiK,t)

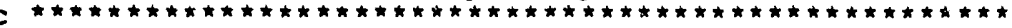

computes $(-h b * \star 2) /(2 * x$ mas $)) *(d / d x) \star \star 2[2 p s i x]=2 p s i K$

note zpsix(1) $->$ zpsi(x0), zpsi(nxpts) $\longleftrightarrow->$ zpsix(xf), etc.

uses forward and backward FFT to evaluate 2 nd derivative

implicit double precision $(A-H, O-Y)$

implicit complex*16 (z)

dimension zpsix(1), zpsiK(1)

common/consto/ zero, zeye, pi,c,twopi,sqrtpi,pisq

common/constl/ xmas, hb

common/const $3 /$ tmax, ntmax, delt, iverflag

$c$

common/const 4/xmin, xmax, nxpts, dx, npacket

backward fourier transform : $z p s i x(x) \Rightarrow z p s i k(k)$

10

is ign $=-1$

do 10 ix $=1$, nxpts

$\operatorname{zpsiK}(i x)=2 p s i x(i x)$

call FFT (zpsik, nXpts, isign

if (iderflag .eq. 1) then

npacket $=$ npacket +1

call pktsav(zpsik, $t$, npacket, -4)

$c$ endif

c compute the second derivative in the momentum domain.

$\mathrm{I}=\mathrm{nXpts} / 2$

do $20 \mathrm{k}=0$, nXpts -1

if $(k$.le. L) then else

$2 \operatorname{psik}(k+1)=-k k^{\star} \approx \operatorname{psik}(k+1) / n x p t s$

zpsiK $(k+1)=-(n X p t s-k) *(n X p t s-k) * z p s j K(k+1) / n X p t s$

20 continue

c forward transform: $z p s i k(k) \Rightarrow z p s i x(x)$

isign $=1$

call FFT (zpsik, nXpts, isign)

c scale results

$x I=x \max -x \min$

$\mathrm{cl}=-0.5 \mathrm{~d} 00 * \mathrm{hb} * \mathrm{hb} / \mathrm{xmas}$ 


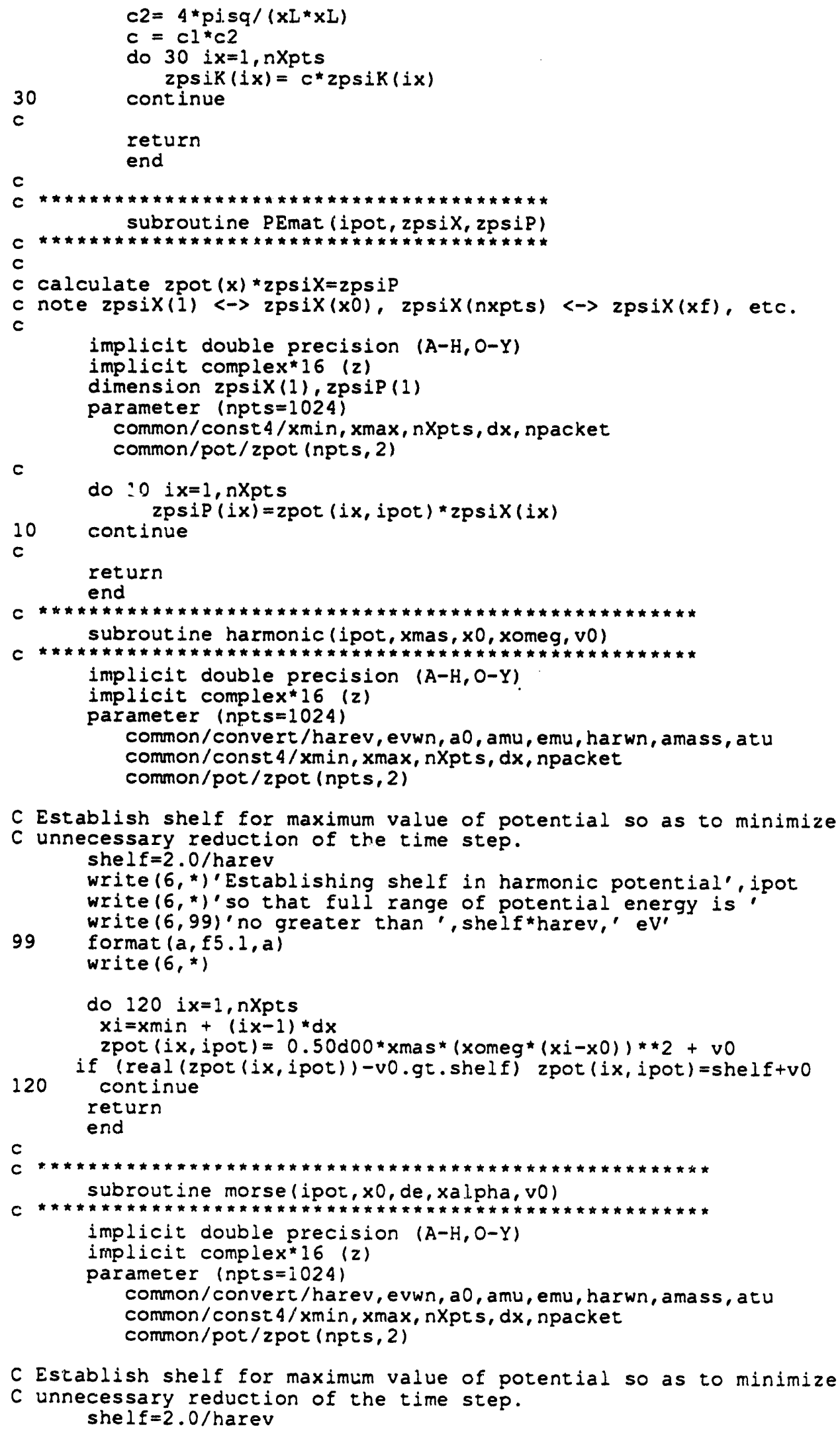

C Establish shelf for maximum value of potential so as to minimize

C unnecessary reduction of the time step. shelf $=2.0 /$ harev

write $\left(6,{ }^{*}\right)$ 'Establishing shelf in harmonic potential', ipot write $(6, *)$ 'so that full range of potential energy is'?

C Establish shelf for maximlm value of potential so as to minimize C unnecessary reduction of the time step. shelf $=2.0 /$ harev 
write $(6, *)$ 'Establishing shelf in Morse potential', ipot write $(6, *)$ ' so that full range of potential energy" write $(6,99)$ ' is no greater than' ', shelf karev,' ev' format $(a, f 5.1, a)$ write $(6, *)$

do 120 ix $=1, n x p t s$ $x i=x \min +(i x-1) * d x$ zpot (ix, ipot) $=\operatorname{de}(1.0 d 00-\operatorname{dexp}(-x a l p h a *(x i-x 0))) * \star 2+v 0$

120

if (real (zpot (ix, ipot))-vo.gt. shelf) zpot (ix, ipot) =shelf $+v 0$ continue return end

$c$

$c$

$c$

subroutine potlsave (ipot)

c

implicit double precision $(A-H, O-Y)$

implicit complex*16 (z)

parameter (npts=1024)

common/const 4 / xmin, xmax, nxpts, $d x$, npacket

c common/pot/zpot (npts, 2)

if (ipot .eq.1) open $(3$, file $=$ 'pot lA.out')

if (ipot. eq. 2 ) open $(3$, file='pot lB.out')

do 10 ix $=1$, nxpts

$x i=0.529177 *(x \min +(i x-1) * d x)$

$a=\operatorname{dreal}(z \operatorname{pot}(i x, i p o t)) * 27.2116000$

write $(3,930) \times i, a$

930 format $(2 x, f 8.3,2 x, f 20.10)$

10 continue

close (3)

return

end

$c$

$c$

$c$

subrout ine Vcopy $(n, 2 A, z B)$

subroutine veopy $(n, 2 A, z B)$

c copy a vector of length $\mathrm{N}$ from $\mathrm{ZA}$ to $z \mathrm{~B}$

$c$

implicit complex*16(z)

dimension $z A(1), z B(1)$

c

10

do 10 ix $=1, N$

$z B(i x)=2 A(i x)$

c continue
return

end

$c$

$c$

subroutine FFT $(x, n$, isign)

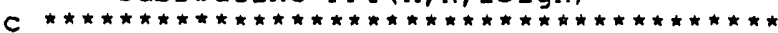

$c$

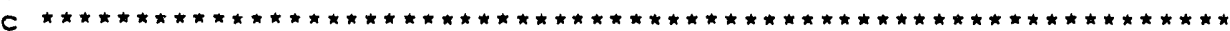

$c *$ The fft computes the discrete fast Fourier transform of a

$c$ * sequence of $n$ terms.

$c$ * The forward EFT computes

$c * \quad y(j)=\operatorname{sum}($ from $k=0$ to $n-1) \quad x(k) * \exp (2 * p i * i * j * k / n)$

$c$ * the backward FFT computes

$c * \quad y(j)=\operatorname{sum}($ from $k=0$ to $n-1) \times(k) * \exp (-2 * p i * i * j * k / n)$ *

c*

$c * x$ is a complex array of lengtin $n$.

$c * n$ is a power of 2 . $n<=16384$

$c$ * isign is the direction of the transform. If isign $>=0$ then

$c$ * the fft is forward, otherwise backward. 


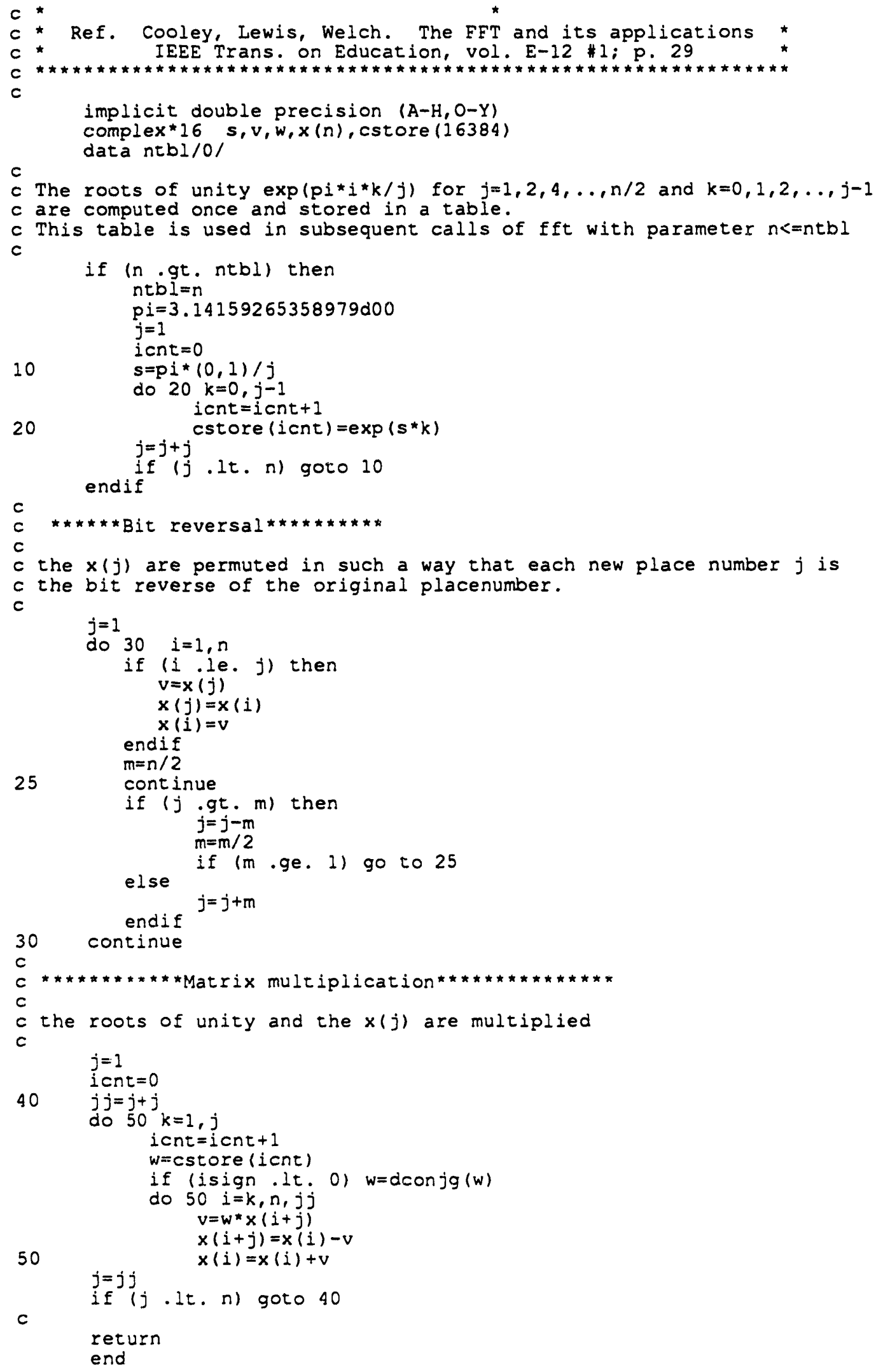




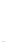
c

c Check that norm and energy are conserved implicit double precision $(A-H, O-Y)$

implicit complex*16 (z)

dimension zpsiA(1)

call chknrm (zpsiA, rnorm)

call chken (ipot, $t i$, zpsiA, Hav)

return

end

c

c

$c$

c

that the norm is conserved during numerical integration of TDSE. implicit complex*16 (2)

implicit double precision $(A-\mathrm{H}, \mathrm{O}-\mathrm{Y})$

parameter (npts=1024)

dimension zpsi(1),psisq(npts)

c common/const $4 / x m i n, x m a x, n x p t s, d x$, npacket

do 10 ix $=1$, nxpts

rpsi=dreal(zpsi $(i x)$ )

a ipsi=dimag $(z p s i(i x))$

psisq $(i x)=$ rpsi ${ }^{\star}$ rpsi+aipsi*aipsi

10

continue

call simpint (nXpts,psisq, dx, rnorm)

return

end

$c$

c

c check that energy is conserved during numerical intergration of the TDSE implicit double precision $(\mathrm{A}-\mathrm{H}, \mathrm{O}-\mathrm{Y})$

implicit complex*16 (z)

parameter (npts=1024)

dimension zpsiA(1), zHpsiA(npts), psiHpsi (npts)

c common/const $4 / x m i n, x m a x, n x p t s, d x$, npacket

call Hpsi(ipot, ti, zpsiA, zHpsiA)

do 10 ix=1, nxpts

psiHpsi $(i x)=\operatorname{dreal}\left(\operatorname{dconjg}(z \operatorname{psiA}(i x)){ }^{*} \operatorname{HpsiA}(i x)\right)$

10

continue

call simpint (nXpts,psiHpsi, dx, Hav)

c

return

end

$c$

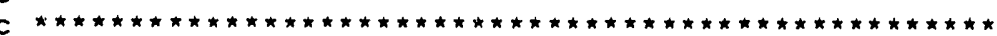

subroutine simpint ( $n x, f l, d x$, fint)

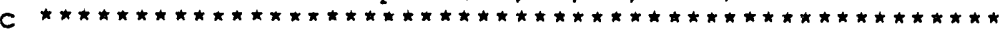

Simpson Rule integrator. This subprog im calls the trapezoidal

c integrator twice. Because of cancellat on of errors the result is

c accurare to the the order of $\left(1 / n x^{*} \star 4\right)$

c Rule valid only when $n x$ odd. Hence for even $n x$ the last piece of area 


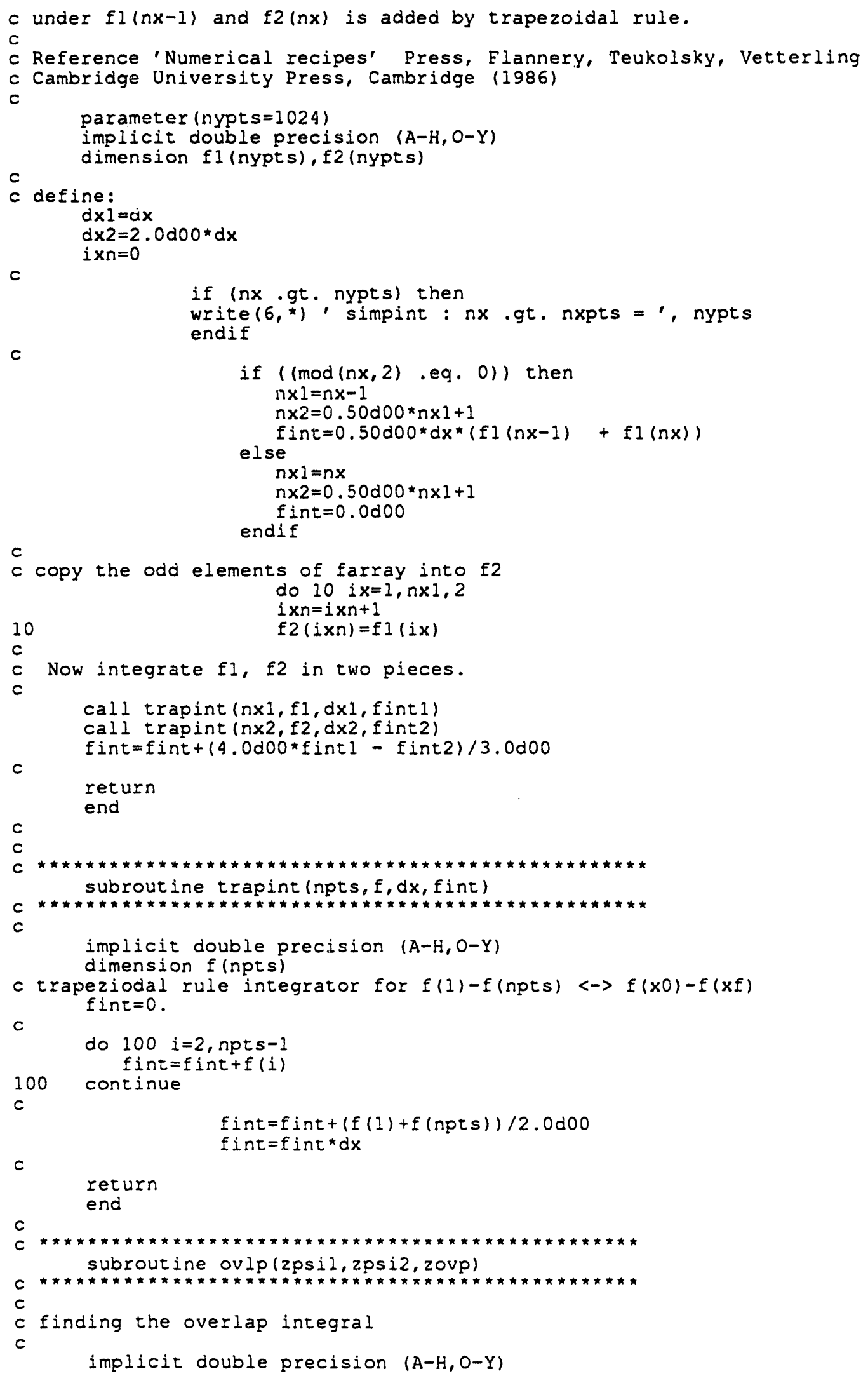




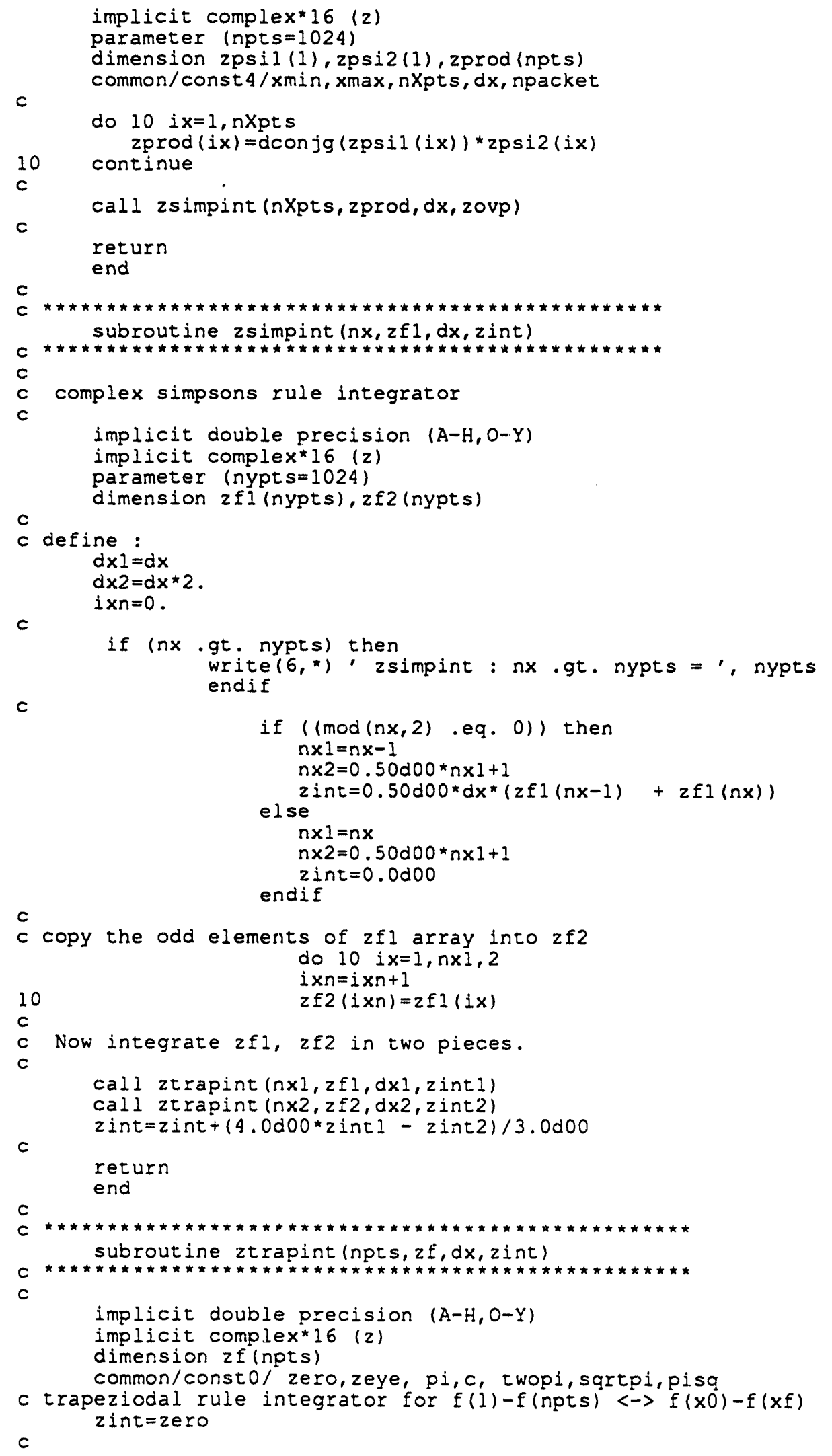




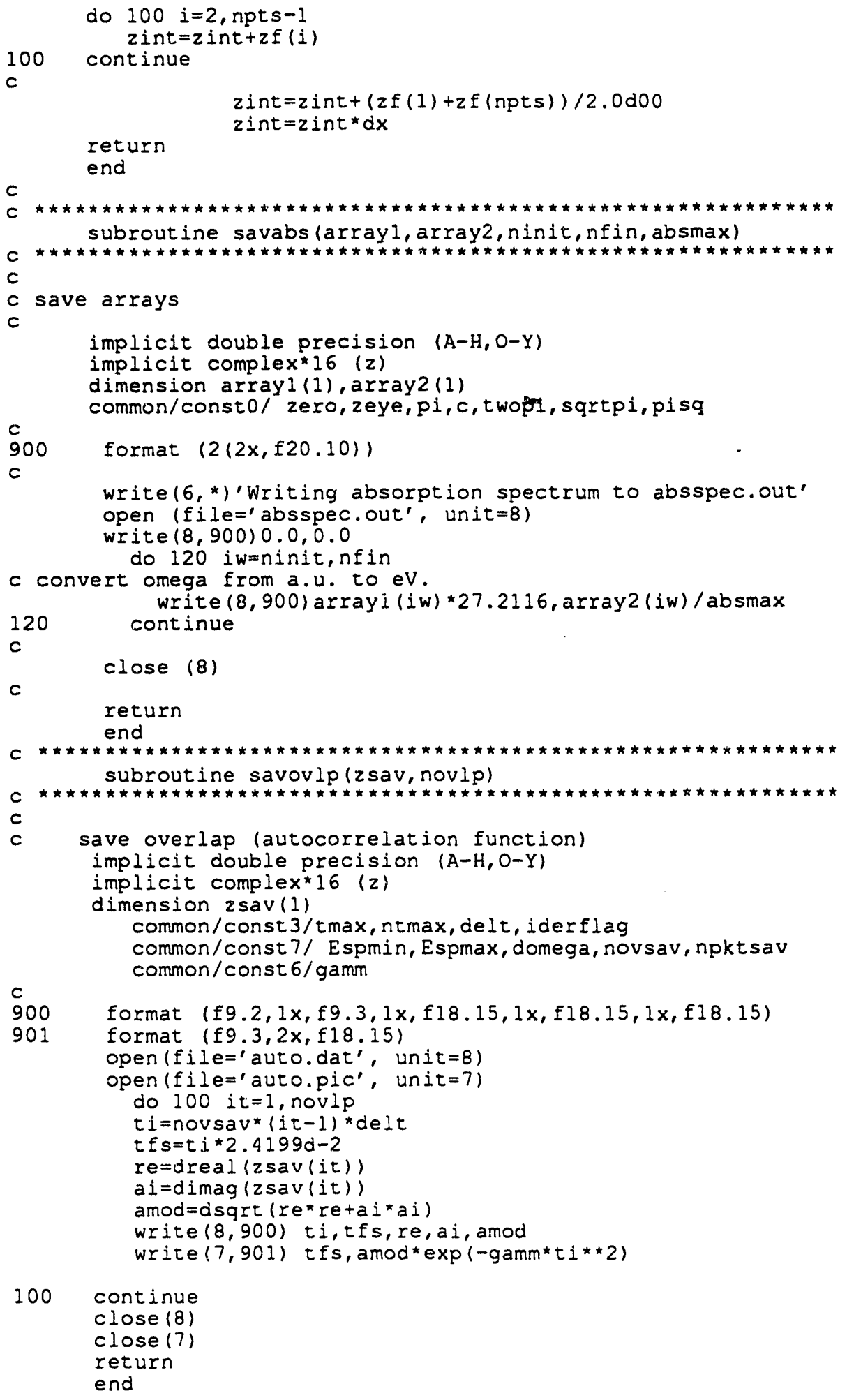

\section{wave2.f}




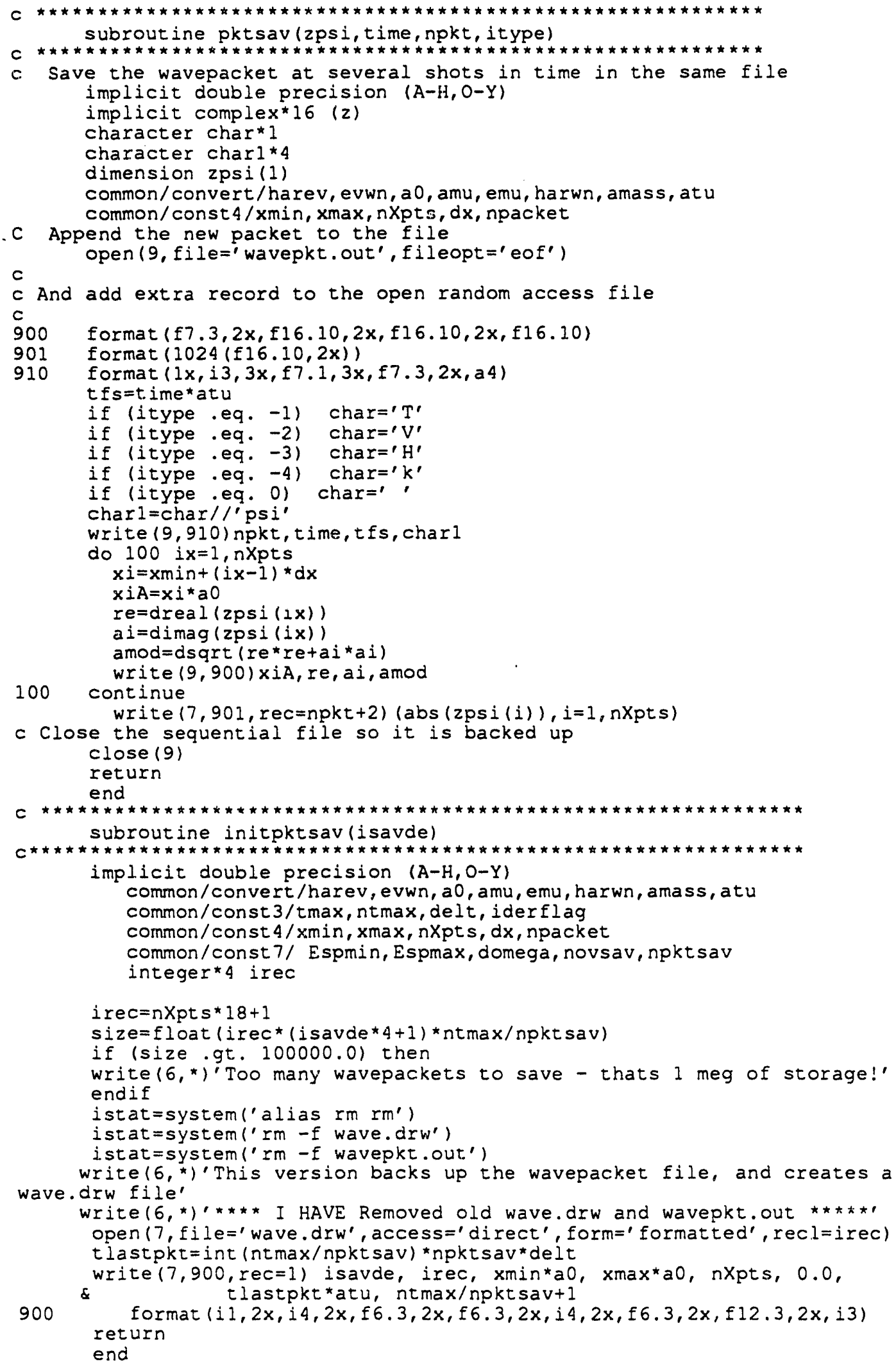




\section{leps1.f}

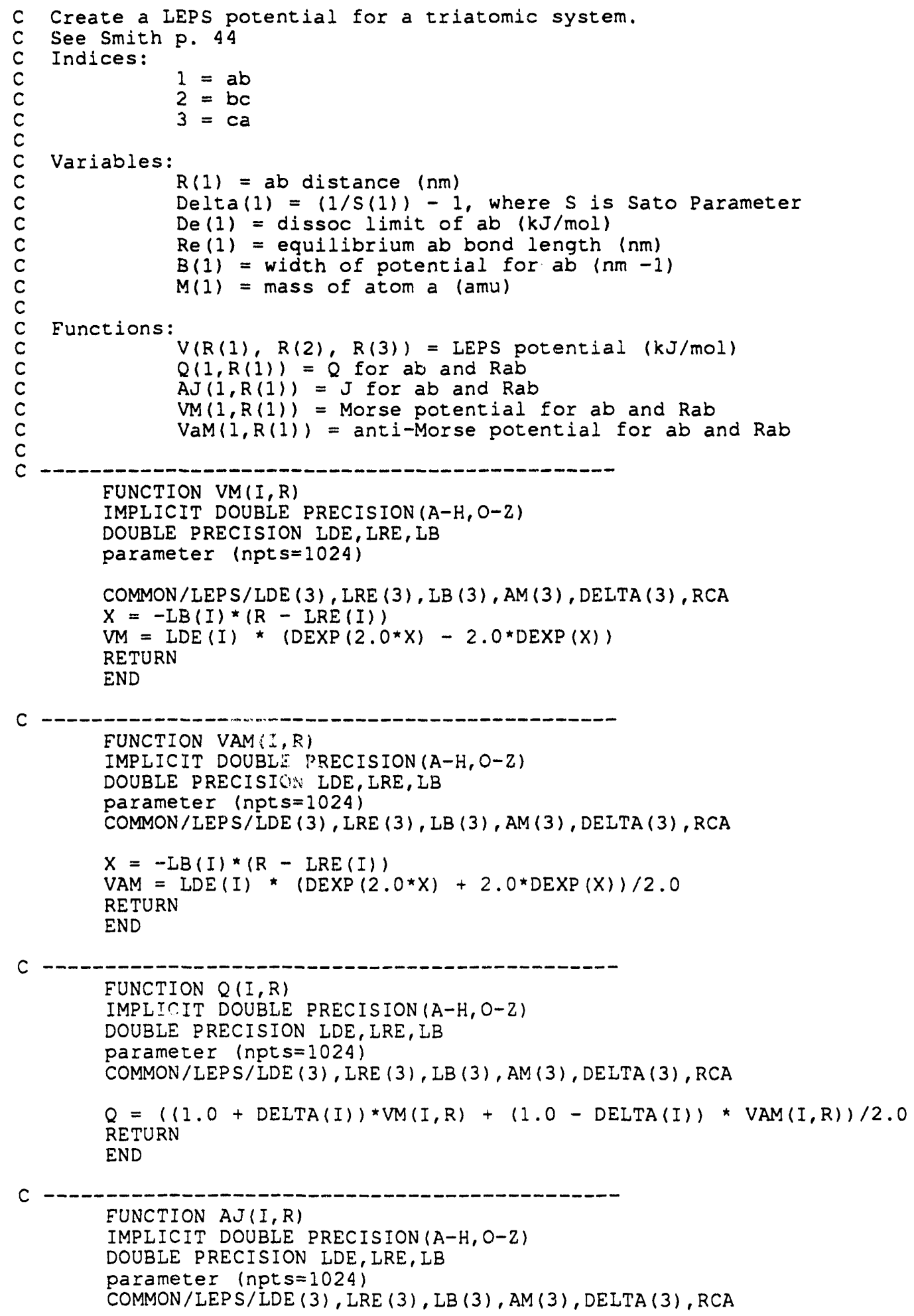




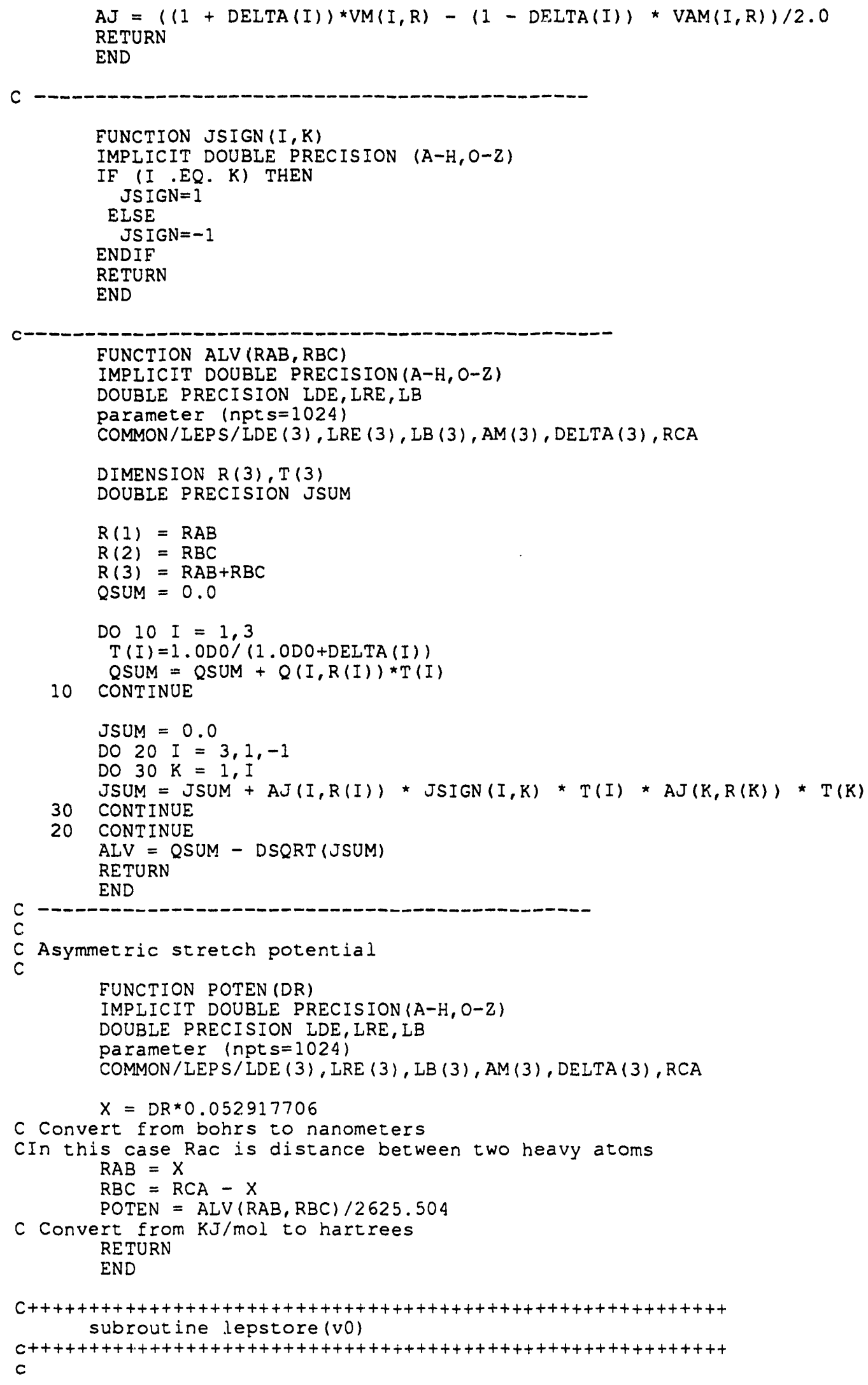


C Save the Leps potential in an array for later use...

C

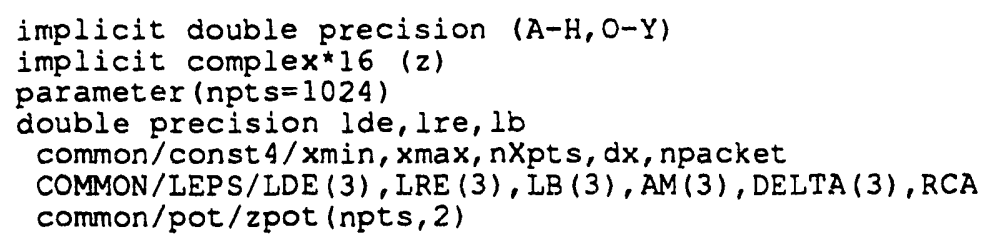

C Establish shelf for maximum value of potential so as to minimize

C unnecessary reduction of the time step.

$C$ For IEPS set this at the three atom dissociation limit $(0.0 \mathrm{eV})$ shelf $=0.0 /$ harev

\section{anionmorsewf1.f}

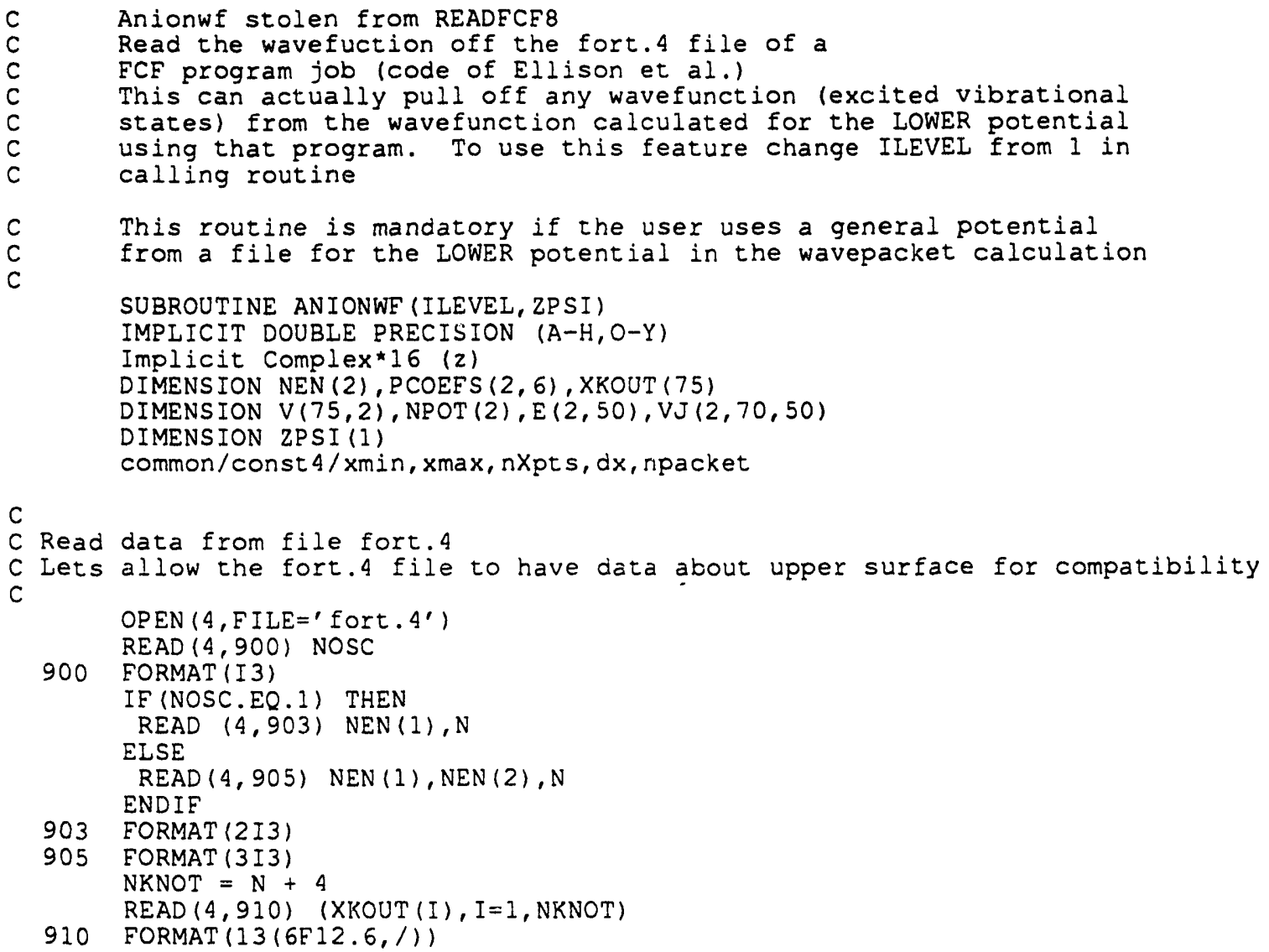




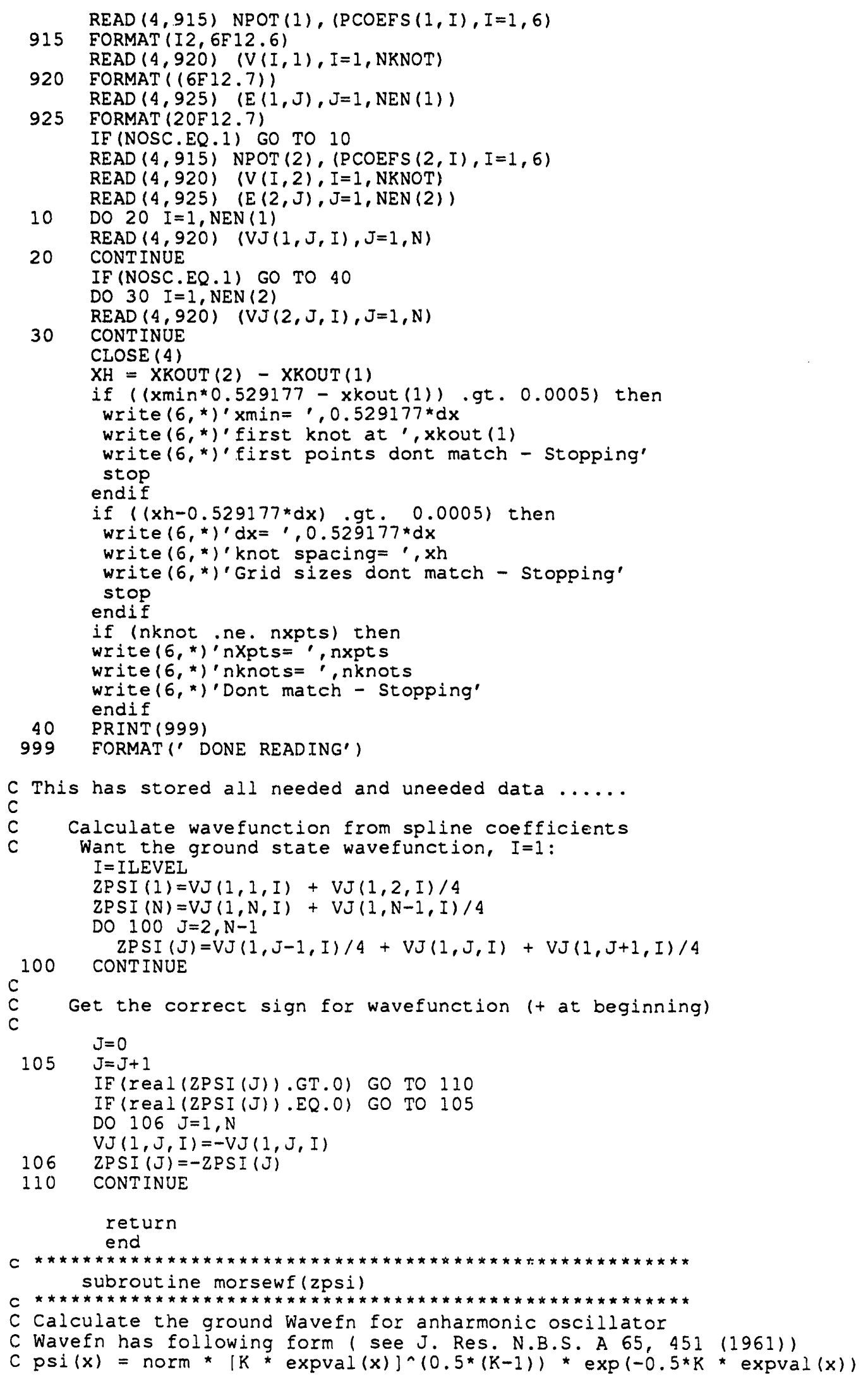




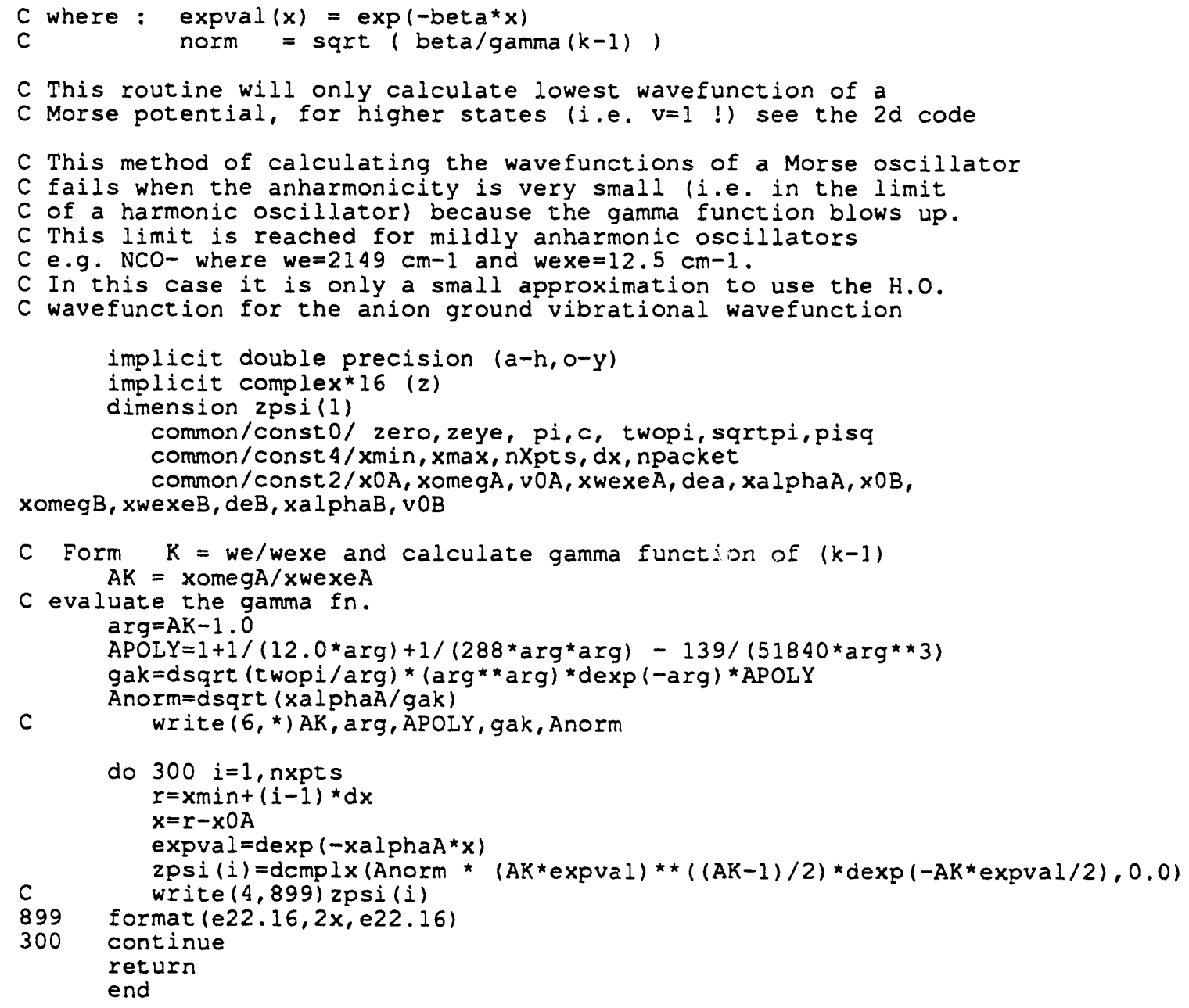

\subsubsection{Two-dimensional Code - prop2d22}

\section{makeprop2d22}

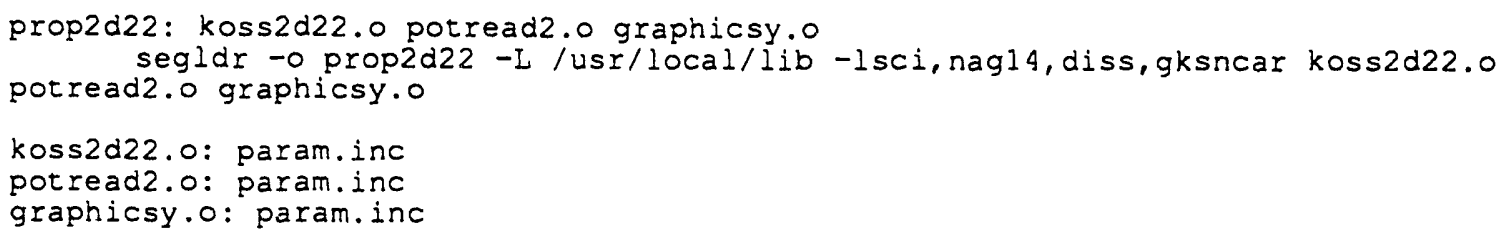

\section{param.inc}




\section{koss2d22.f}

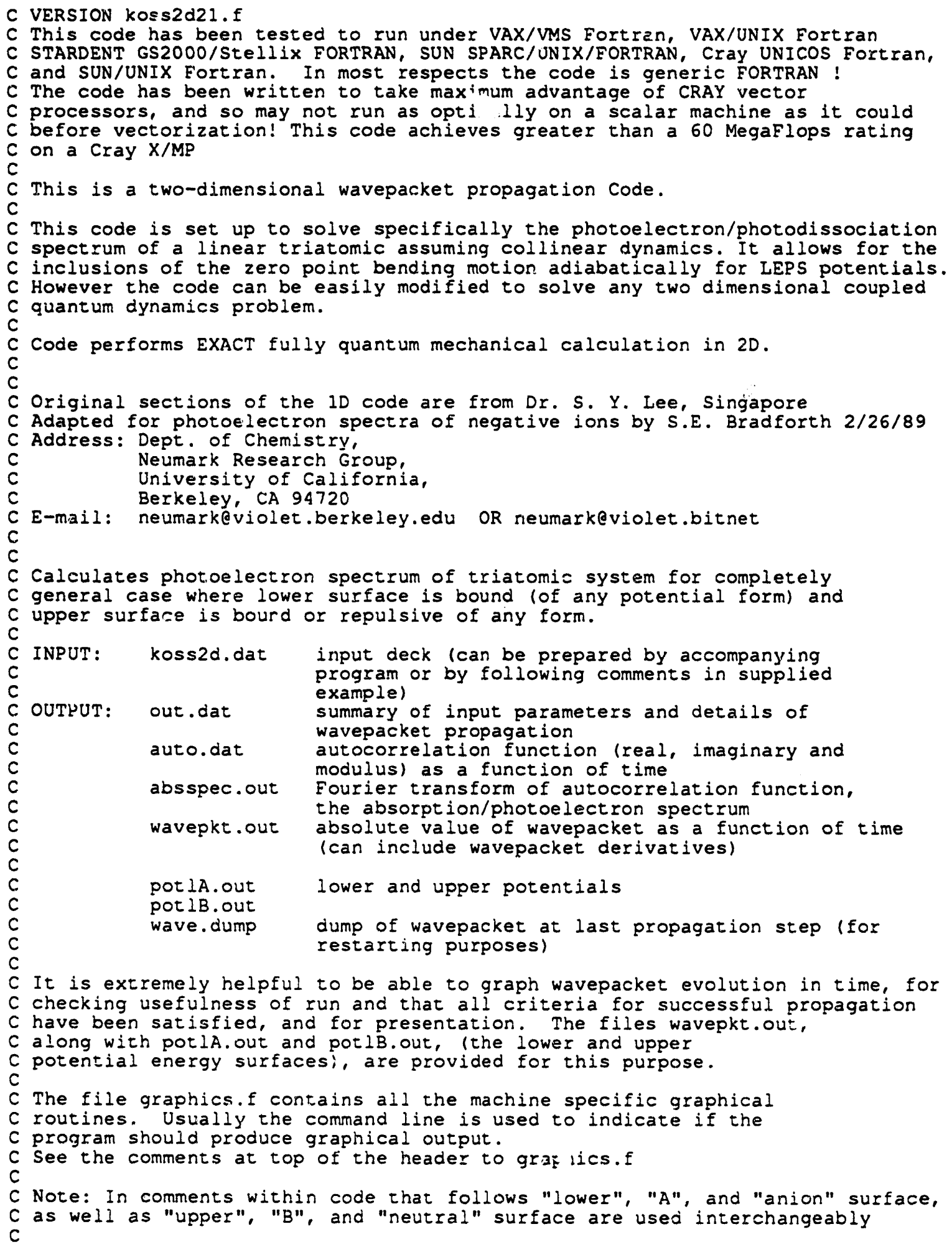


C Algorithm assumes no separation of variables or symmetry

$C$ so general coupled potentials, or reaction surfaces are allowed

$C$ for either anion or neutral.

C

$C$ Note flexibility of potential is determined by subroutine potdef, at the

$C$ present allows: (Number refers to potential type requested in input deck)

O SHO or Morse (expressed with we and wexe)

1 Morse (expressed with De and Be)

2 LEPS (only for neutral) with/without, z.p. bend correction

3 general supplied $2 d$ potentials from a file (on ms grid)

4 general functional form for $2 d$ potential supllied in function upotfn within this code

5 Rotated Morse Oscillator Spline function

Revised to perform symmetric/asymmetric systems; to include absorbing

$C$ boundary conditions and to start with a excited initial wavepacket.

C Will restart from any point and allows any form of starting wavepacket.

C Time dependent relaxation algorithm to find ground wavefunction of lower

C surface added 1990

C

C Code can use the Mark 13 NAG library for extremely efficient vectorised

C 2D FFT routine (10x faster). This routine also increases program speed (208)

$C$ on scalar machines. The NAG version is strongly recommended!

C THE PROGRAM SPENDS OVER $75 \%$ OF ITS CPU TIME INSIDE the 2D FFT

C ROUTINE SO IT IS ADVISABLE TO USE A VERY EFEICIENT ROUTINE, IIKE

C THE NAG ROUTINE COGfUF. IF SUCH A IIBRARY ROUTINE IS NOT

C AVAILABLE USE MUST BE MADE OF twodfft (from Numerical Recipes).

C

$C$ Note all quantities in main routine are in Atomic units,

$C$ conversions performed in $I / O$ routines.

C

C Note all variables beginning with $z$ are complex

PROGRAM PROP2D

implicit real*8 $(A-H, O-Y)$

implicit complex*16 (z)

real* 8 lde, Ire, lb

character arg*70

include "param.inc"

parameter (nFFT $=8192)$

dimension zpsiAO (nlpts, n2pts), zpsiBO (n1pts,n2pts)

dimension zpsiBl (n1pts, n2pts), zpsiB2 (nlpts, n2pts)

dimension zovlp (nFFT), omega (nFFT), Eprsq (nFFT)

common/convert/harev, evwn, a0, amu, emu, harwn, amass, atu

common/consto/ zero, zeye, pi, $c$, twopi, sqrtpi,pisq

common/const 1/ xmas, ymas, redmas, hb

common/const $2 \times A / \times O A$, xomegA, $v O A$, xwexeA, $x$ dea, xalphaA

common/const $2 \times B / x O B$, xomeg $B$, xwexe $B, x$ de $B, x a$ lpha $B, v 0 B$

common/const $2 y A / y O A$, yomegA, fcupA, ywexeA, ydeA, yalphaA

common/const $2 y B / y O B$, yomegB, ywexeb, ydeB, yalphaB, fcup

common/const3/tmax, ntmax, delt, iderflag

common/const $4 / x m i n, x \max , n x p t s, d x, y m i n, y m a x, n Y p t s, d y, n p a c k e t$

common/const 6/gamm

common/const 7/Espmin, domega, novsav, npktsav

C workspace defined in fftwork common block is required for NAG routine only common/fftwork/trigm (2*nlpts), trign (2*n2pts)

COMMON/LEPS/LDE (3), LRE (3), LB (3), DELTA (3)

common/mass/AM1, AM2, AM3, AMab, AMcxab, AJAcob

commoniflags/isavpot $A$, isavpot $B$, isavde, i readwav, irestart, iabs

common/absparam/xabs, yabs, cxabs, cyabs

common/wdraw/iwdrw, arg

C Check for argument on command line for graphics options

call argchk (igraph, iwdrw, arg) 
$C$ nlpts and nzpts define the grid size and are defined as constants

$C$ within program. They should be powers of two.

C They can be changed by replacing every parameter statement within code or

$c$ by using an include file if allowed in user's version of FORTRAN

c Initialize........

C Check that nipts is larger or equal to n2pts else problems

C with array construction.......

if (nlpts. It. n2pts) then

write $\left(6,{ }^{*}\right)$ 'nipts . It. n2pts - Change source code!!!'

stop

endif

C Start reading the needed input data and also define some useful constants. open (1, $\mathrm{file}=$ 'koss2d.dat')

call const (nFFT)

close (1)

if (iwdrw.eq.1) call inidrw()

C Program arrays set so nxpts=n1pts $>=$ nypts=n2pts -- Program checks for this. if ((nxpts.ne.nlpts).or. (nypts.ne.n2pts)) then write $\left(6,{ }^{*}\right)$ 'Illegal nxpts or nypts - check and remedy !' endif stop

$C$ novsav should be a factor of the total number of time steps ntmax and should $c$ be such that 2*ntmax/novsav $=<$ nFFT

C Change novsav and npktsav to be useful values.....

$c$ novsav $=2{ }^{*} n t \max / n F F$

if (novsav . It. 1) novsav=1

if $\left(22^{*}\right.$ tmaxinovsav. gt. nFFT) then write $(6, *)$ 'novsav is too large - Exiting' stop

endif

if $(\bmod (n t \max$, novsav) .ne.0) then

write $(\overline{6}, *)$ 'ntmax $=$ ', ntmax

write $(6, *)$ 'novsav is not a factor of ntmax - Please fix' stop

endif

if (isavde .eq. 1) then

if ((npktobe.1t. 2) .or. (npktobe.gt. 6)) npktsav=ntmax/4

else

if ( (npktobe .1t. 4) .or. (npktobe .gt. 30)) npktsav=ntmax/8 endif

if (npktsav, It. 1) npktsav=1

if (isavde .eq. -1) then npktobe $=0$ write $(6, *)$ 'There will be ', npktobe,' wavepackets stored'

Calculate Delta Omega (domega) in a.u. domega $=2.0 * \mathrm{pi} /($ ntmax*delt)

c

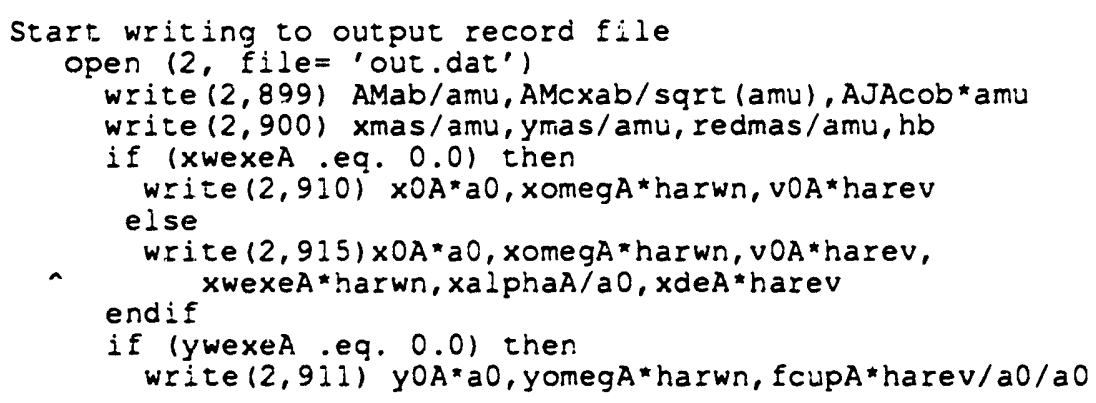




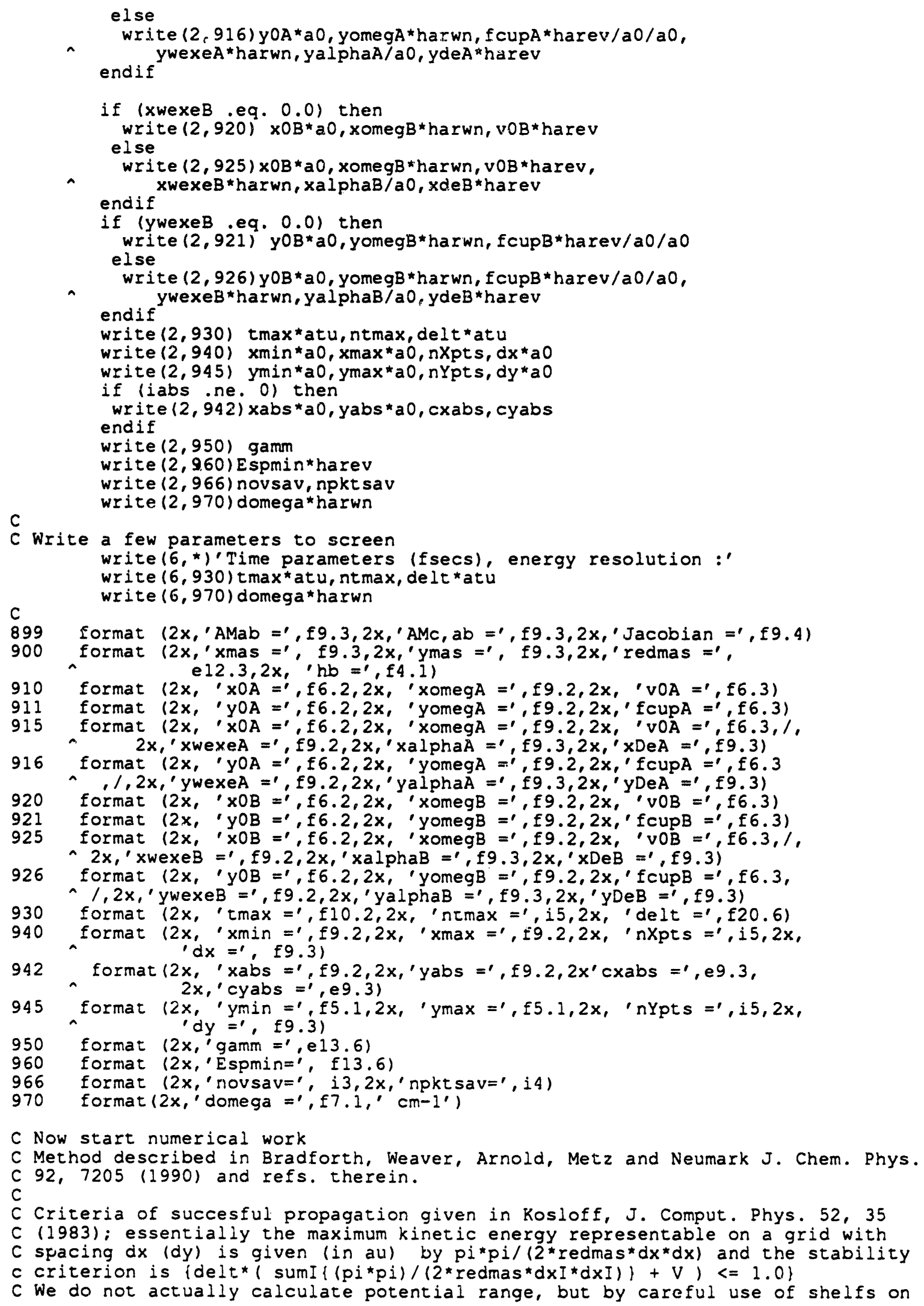


C potential values, the maximum kinetic enrgy should dictate the convergence c criterion

c

sqkmax $=4.9348 *\left(1.0 /\left(\right.\right.$ redmas $\left.{ }^{\star} d x^{\star} d x\right)+1.0 /\left(\right.$ redmas $\left.\left.{ }^{\star} d y y^{\star} d y\right)\right)$

write $(6,912)$ sqkmax harev

write $(2,912)$ sqkmax ${ }^{\star}$ harev

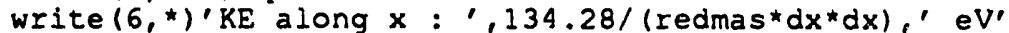

write $\left(6,{ }^{*}\right) ' \mathrm{KE}$ along $\mathrm{y}: \mathrm{x}^{\prime}, 134.28 /$ (redmas*dy*dy),' eV'

912

format $(2 x$, 'Maximum kinetic energy that can be represented is ',

4

f9.4,' $\mathrm{eV}^{\prime}$ )

write $(6,913)$ sqkmax ${ }^{\star}$ delt

write $(2,913)$ sqkmax*delt

913 format(2x,'Stability at best, assuming zero potential, is ',f6.3)

C

$C$ make crude check on whether propagation will be stable

if $($ sqkmax*delt .gt. 1.0) then

write $(6, *)$

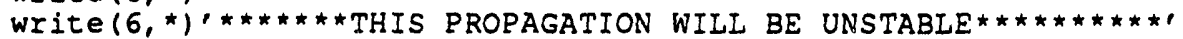

write $(6, *)$

stop

endif

C Save the lower/upper state potential surfaces

c.

if (isavpotA .eq. 1 ) then

write $(6, *)$ 'Potential saved in potlA.out in $\mathrm{eV}^{\prime}$

call potisave(1)

endif

if (isavpotB.eq. 1 ) then

write $(6, \star)$ 'Potential saved in potlB. out in $\mathrm{eV}^{\prime}$

call potlsave (2)

endif

c initialise wavepacket file if wavepackets are to stored

if (isavde .ne. -1) call initpkt)

c will store autocorrelation function (overlap between $t=0$ zpsi and

c $t=n o v l p^{\star}$ novsav*delt zpsi) at intervals determined by novsav

$c$ and will write the wavepacket to disk in its entirety every npktsav

$C$ point in time if required.

$c$

c Initialise $t i=0$ but routine initB may change this...

$t i=0.0$

C Initialize the 2D FFT routine in NAG library - needs to set up trig table in

C KEmat for more efficient further calls....

C INCIUDE if using NAG library, twodfft routine does not need to be initialized call KEmat (zpsiBO, zpsiBl, $t i, 1$ )

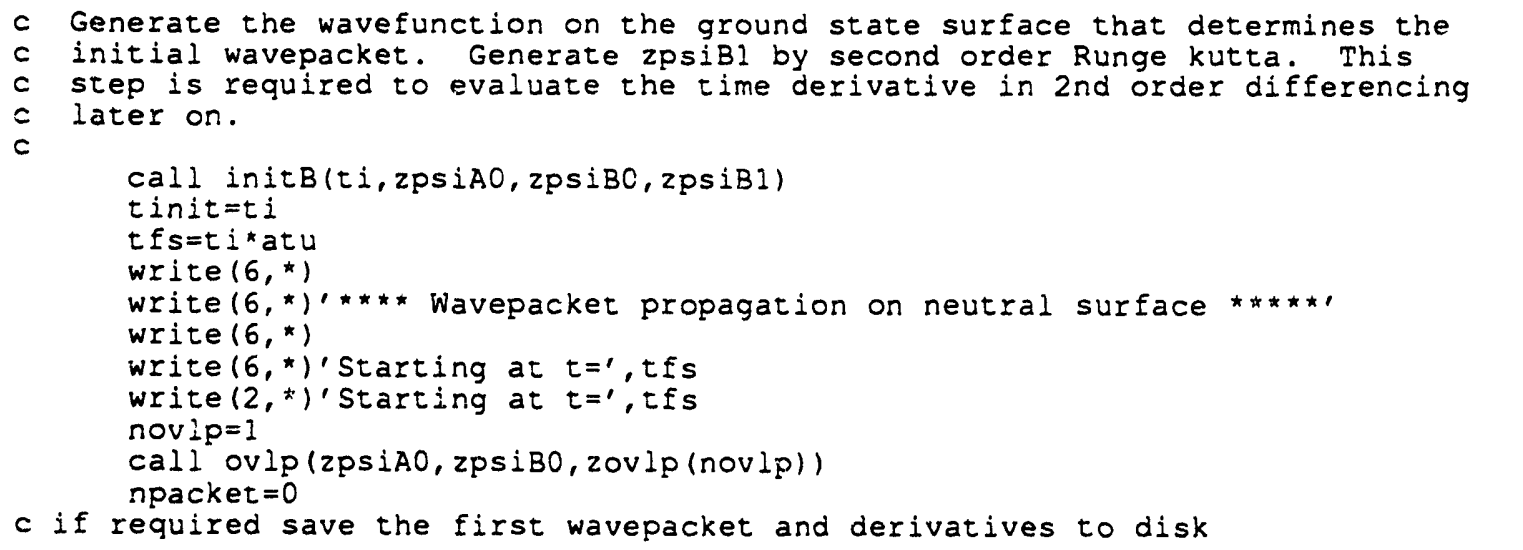


if ((irestart ne. 1).and. (isavde ne. -1)) then endif call pktsav(zpsiBo, ti, npacket, 0 )

(ive) $778,778,777$

if (irestart.ne.1) iderflag=1

call chk $(2, t i, z$ psiBO, rsnorm, Have)

write $(6, *)$ 'Norm of wavefn is', rsnorm

write $(2, *)$ 'Norm of wavefn is ', rsnorm

write $(6, *)$ 'Energy Hav =',

\& Have/rsnorm,' ',Have*harwn/rsnorm,' cm-1'

Have*harwn/rsnorm, cm-1'

iderflag $=0$

C First Drawing to screen if graphics required

if (iwdrw.eq.1) then

call iniplt $(\arg , 2)$

call wavdrw (zpsiBo, tfs, rsnorm, Have)

endif

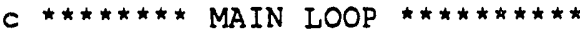

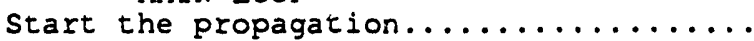

Perform this by second order differencing (Kosloff algorithm)

c

do 100 it $=1$, ntmax

$t i=t i+d e l t$

tfs=ti*atu

965 format ( $1 x$, 'Time step $, 16,1, t=1, f 10.4$ )

$c$ check to see if we need to store overlap and/or write wavepacket to disk if (mod(it, novsav) .eq. 0$)$ then novlp=novlp+l

call ovlp(zpsiAo, zpsiBl, zovlp (novlp)) endif

if (mod(it, npktsav) .eq. 0$)$ then

if (isavde) 781,780,779

779

iderflag=1

npacket $=$ npacket +1

call pktsav(zpsiBl, ti, npacket, 0)

781

call chk $(2, t i, z p s i B l$, rsnorm, Have

if (iwdrw .eq. 1) call wavdrw(zpsiBl, tfs, rsnorm, Have) write $(2,965)$ it, tfs

if (iwdrw.ne.1) write $(6,965)$ it, tfs

if (iwdrw.ne.1) "rite $\left(6,{ }^{*}\right)$ 'Norm of wavefn is', rsnorm

write $(2, *)$ 'Norm of wavefn is ', rsnorm

if (rsnorm.gt. 2.0) then

write $\left(6,{ }^{*}\right)$ 'Exceeded reasonable norm - terminating...'

stop

endif

if (iwdrw.ne.1) write $\left(6,{ }^{*}\right)$ 'Energy Hav $='$, Have/rsnorm,

e ', Have*harwn/rsnorm,' cm-1'

write $\left(2,{ }^{*}\right)$ 'Energy Hav $='$ 'Have/rsnor
Have*harwn/rsnorm,' cm-1'

iderflag $=0$

endif

$c$ determine the new wavefunction zpsiB2 from zpsiBo and zpsiB1

if (iabs ne. 0 ) then

else

call psi2ab(2,ti,zpsiBo, zpsiBl, zpsiB2)

call psi2(2,ti,zpsiBO, zpsiBl, zpsiB2)

endif

c now we have zpsiB2 prepare for next step of propagation

call vcopy (nXpts, nYpts, zpsiBl, zpsiBO)

call vcopy (nXpts, nYpts, zpsiB2, zpsiB1)

c now round propagation loop again.....

100 continue

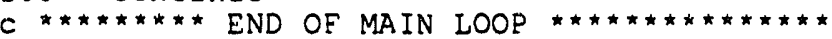

C closes up the wavepacket file, autocorrelation and output files 


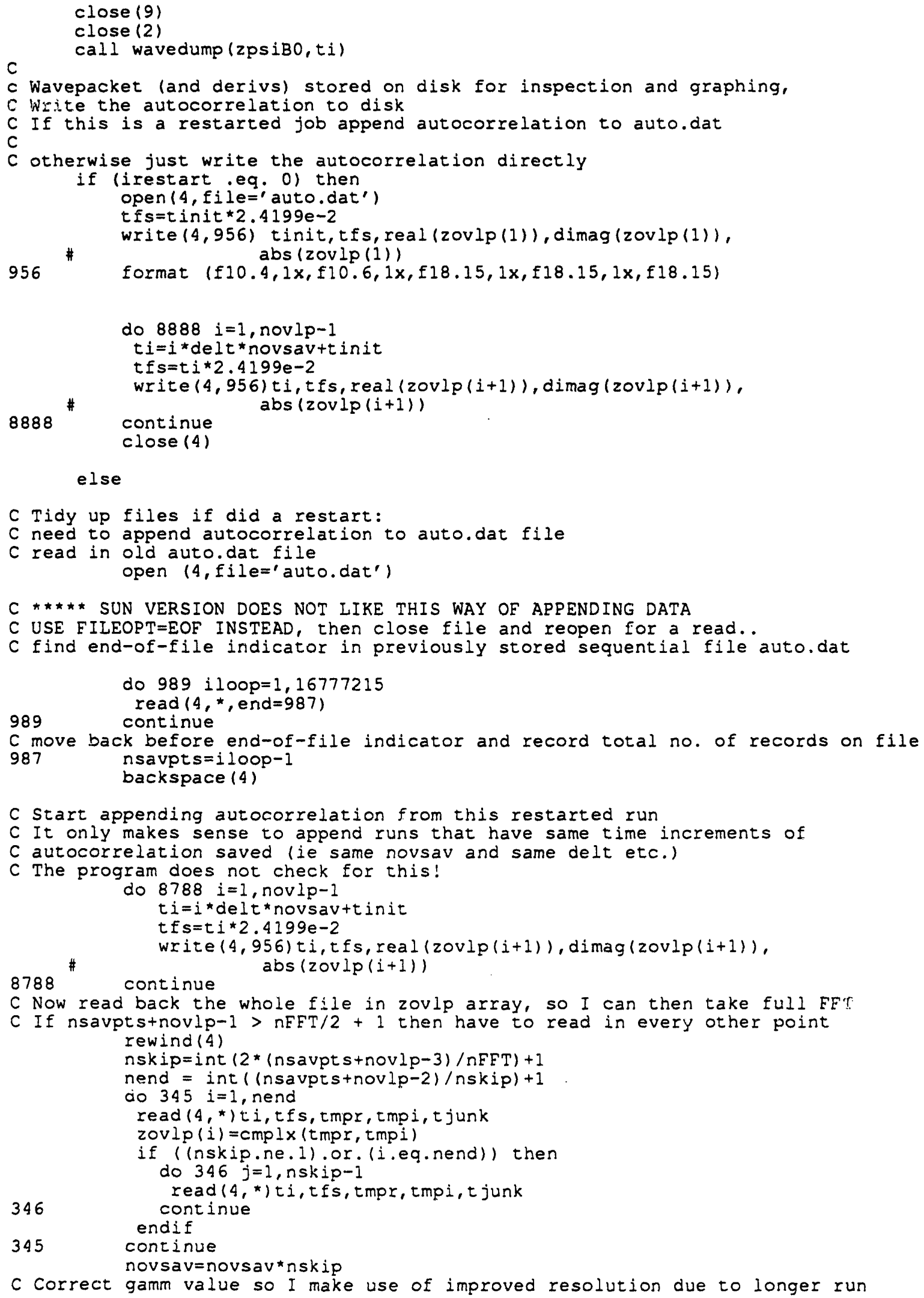


$C$ new maximum time is $\operatorname{tmax}=t i$ read in last line of auto.dat file $t \max =t i$

C Close up appended file endif close (4)

C calculate absorption spectrum now that we have complete autocorrelation

C function (ie. from $t=0$ ) in memory

$C$ if using default value of gamma $(0.0)$ then set to optimal value

C This value is somewhat conservative (to eliminate ringing on baseline)

$C$ Higher resolution can be pulled out of a given run by reducing gamma if (gamm .eq.0.0) gamm=5/tmax/tmax

call savabs (zovlp, nFFT, gamm, omega, Eprsq, jFT)

if (igraph.eq.I) then

if (iwarw .eq.0) call iniplt2 (arg)

call absdrw (omega, Eprsq, jFT) endif

C

if (igraph.eq.1) call pltfin

c ..... All Done

c

stop

end

$c$

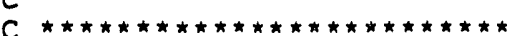

subroutine const (nFFT)

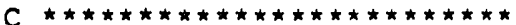

$C * * \star$ read the needed data and also define some useful constants.

implicit real*8 $(A-H, O-Y)$

implicit complex*16 (z)

include "param.inc"

common/convert/harev, evwn, a0, amu, emu, harwn, amass, atu

common/consto/ zero, zeye, pi,c, twopi,sqrtpi,pisq

common/constl/ xmas, ymas, redmas, hb

common/const $3 / t \max$, ntmax, delt, iderflag

common/const $4 / x \min , x \max , n x p t s, d x, y m i n, y m a x, n Y p t s, d y, n p a c k e t$

common/const 6/gamm

common/const 7/ Espmin, domega, novsav, npktsav

common/mass/AM1, AM2, AM3, AMab, AMcxab, AJAcob

common/flags/isavpot $A$, isavpot $B$, isavde, ireadwav, irestart, iabs

common/absparam/xabs, yabs, cxabs, cyabs

C

common/absvec/fabsx (nipts), fabsy (n2pts)

$C$ set conversion factors

harev $=27.211608$

evwn $=8065.479$

$\mathrm{aO}=0.52917706$

$\mathrm{amu}=1822.882$

emu $=9.109534 \mathrm{e}-31$

harwn = harev*evwn

amass $=1.66056 \mathrm{e}-27$

$C$ set consto

atu $=0.024199$

zero $=\mathrm{cmpl} \times(0.0,0.0)$

zeye $=\mathrm{cmpl} \times(0.0,1.0)$

$p i=\operatorname{acos}(-1.0)$

twopi $=2$ *pi

sqrtpi $=\operatorname{sqrt}(p i)$

pisq=pi *pi

C

$c=2.99792458 \mathrm{e} 10$

C Take instructions to save potentials wavepackets and derivatives from input

$C$ deck. Also see whether to read initial wavepacket from wave.dump and whether

$C$ job is a restart. These are stored as flags.

read $\left(1,{ }^{\star}\right)$ isavpot $A$, isavpot $B$, isavde, ireadwav, irestart 


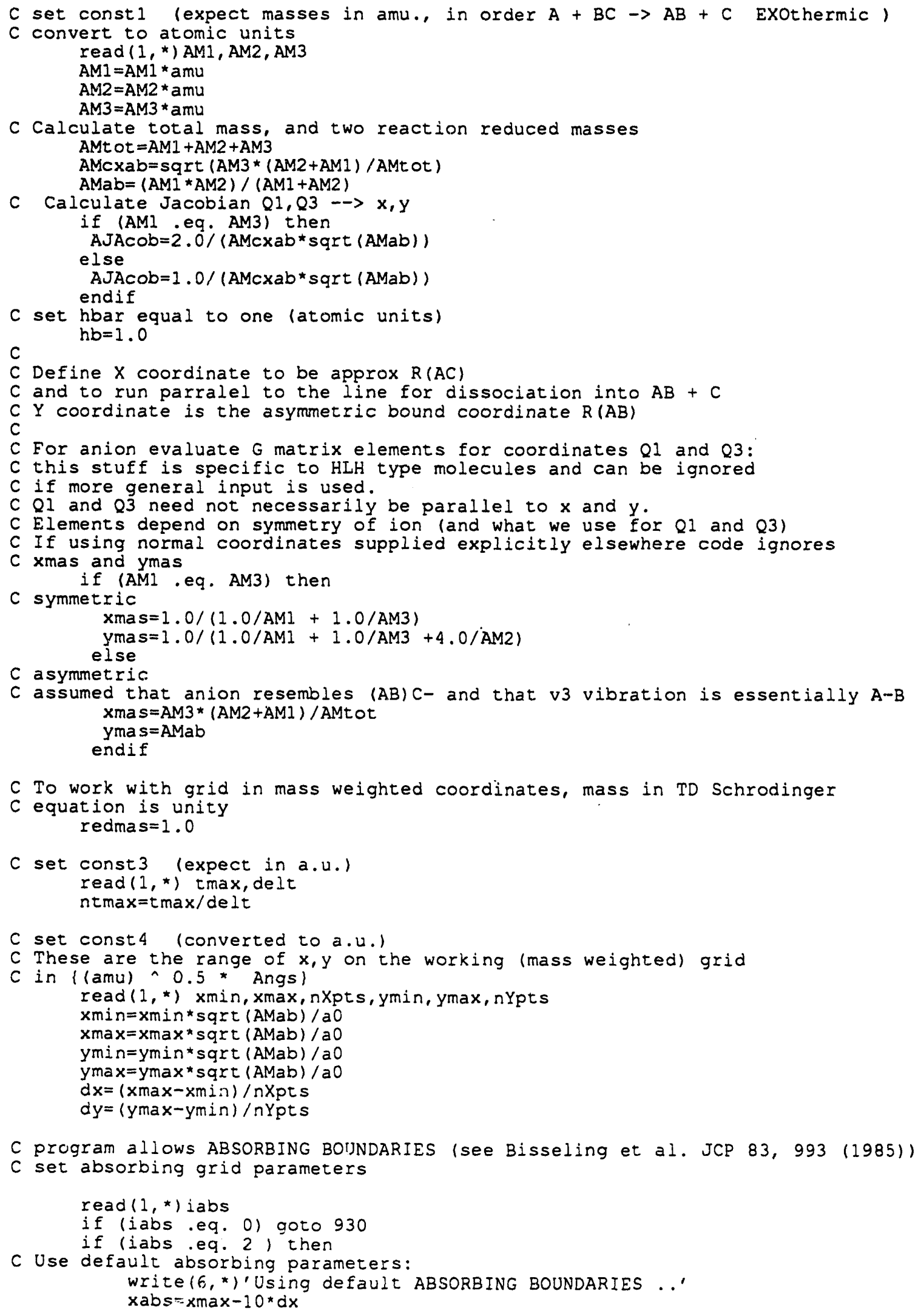


$y a b s=y \max -10 * d y$

C These are empirically derived best parameters : Try as first guess!

cxabs $=(\mathrm{delt} / 1.3) * 0.0005 / \mathrm{dx} / \mathrm{dx}$

cyabs $=($ delt $/ 1.3) * 0.0005 / d y / d y$

else if (iabs .eq. 1) then

write $\left(6,{ }^{*}\right)$ 'Using supplied ABSORBING BOUNDARIES ..'

read $\left(1,{ }^{*}\right)$ xabs, yabs, cxabs, cyabs

$x a b s=x a b s * s q r t(A M a b) / a 0$

yabs=yabs*sqrt $(\mathrm{AMab}) / \mathrm{aO}$

cxabs $=c x a b s / d x / d x$ endif

cyabs $=c y a b s / d y / d y$

C Calculate the absorbing vaues across the grid and store them (faster to store

C than to recalculate every time).

$C$ This calculates the absorbing boundary function

do $433 i x=1$, nxpts

$x=x \min +(i x-1) * d x$

if $(x$. Ie. $x a b s)$ then

$f a b s x(i x)=1.00000000$

else

fabsx $(i x)=\exp (-c x a b s *(x-x a b s) \star(x-x a b s))$

endif

433 continue

do 434 iy $=1$, nypts

$y=y \min +(i y-1) * d y$

if (y.le. yabs) then

fabsy $(i y)=1.000000000$

else

fabsy $(i y)=\exp (-c y a b s *(y-y a b s) *(y-y a b s))$

434 continue

$C$ Read in and calculate both potentials (store in memory)

930 call potread()

C set const 6

C dephasing constant gamma (in atu**-2)

read $(1, *)$ gamm

C Default value of gamma (this is chosen to give optimal spectrum - best window

C function - see Numerical Recipes FFT chapter for details) is set in main

$C$ routine if gamma is set to zero here (default)

C N.B. gamma can also be used to simulate a constant lifetime depletion of

$C$ the wavefunction as a function of time (eg. fluorescence, curve crossing)

C

C set const 7

C Espmin is the threshold energy for photoelectron spectrum

$c$ (expected to be in $\mathrm{eV}$ and coverted to a.u.)

C This just defines the spectral origin with refence to potential zero. read (1,*) Espmin

Espmin=Espmin/harev

if (Espmin.le. 0.0$)$ then

write $\left(6,{ }^{*}\right)$ 'This is not a valid threshold energy',

(a) 'for photoelectron spectrum'

write $\left(6,{ }^{*}\right)$ 'Espmin must be $>0.0$ or no P.E.S. !!'

stop

endif

c

C set up counting variables

save autocorrelation function loverlap between $t=0$ zpsi and

$t=n o v l p * n o v s a v * d e l t$ zpsi) at intervals determined by novsav and

Save the wavepacket in its entirety every npktsav point in time read $\left(1,{ }^{*}\right)$ novsav, npktsav

if $((2 * n[\max /$ novsav $)$.gt. nFFT) then write $(6,)^{*}$ 'Too many overlap points to store - alter novsav' stop 


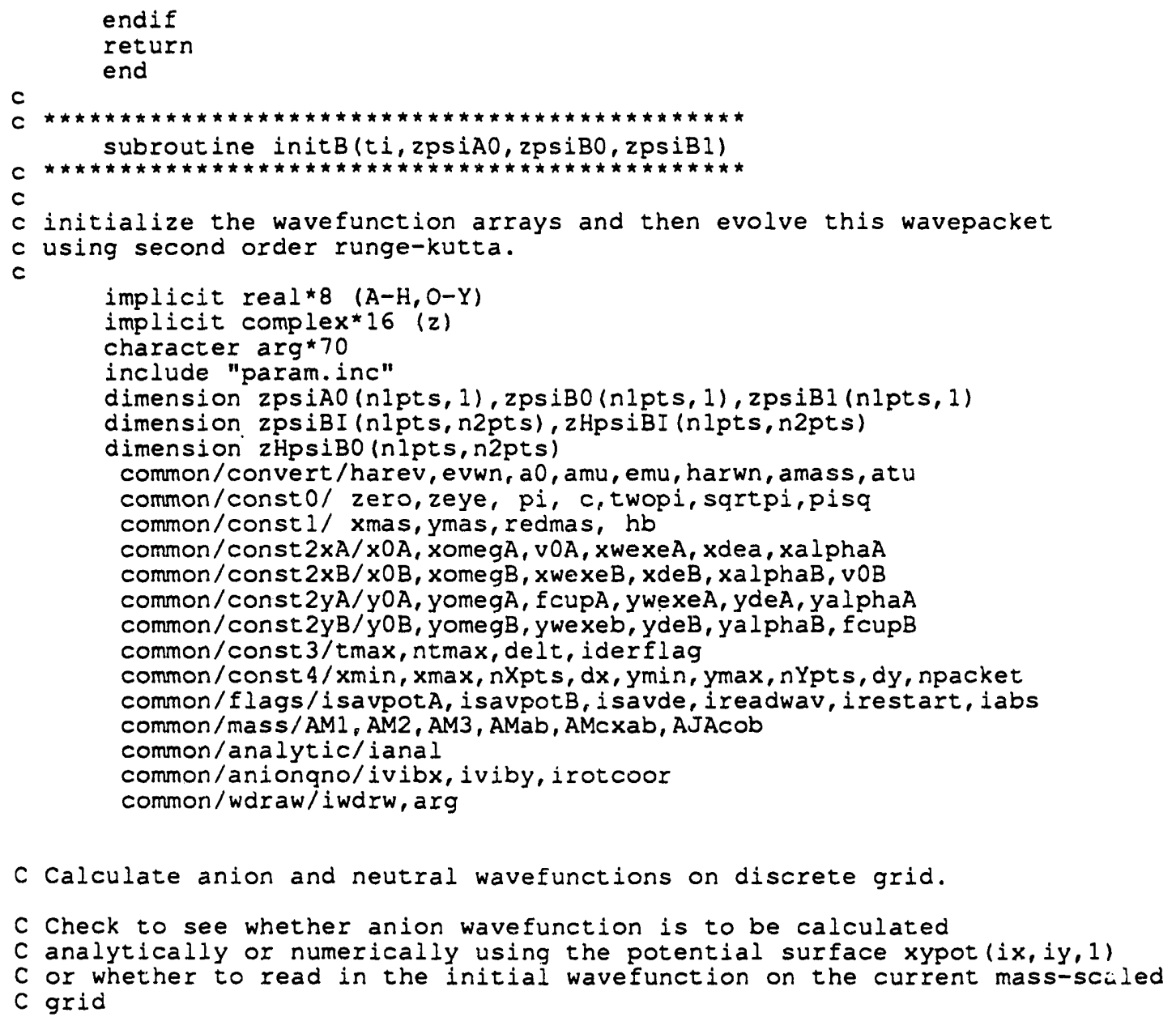

$t i=0.0$

rtJacobian=sqrt (AJAcob)

if ((irestart.eq.1).and. (ireadwav.eq.1)) then

write $\left(6,{ }^{*}\right)$ 'cannot read anion wavefunction as well as',

'starting neutral wavepacket from disk'

write $\left(6,{ }^{*}\right)$ 'Exiting....'

endif

if (ireadwav.ne.1) then

if (ianal.eq.1) then

C Analytic solution available

if (ywexeA .eq. 0.0 ) then

C Harmonic Oscillator separable in mass scaled coordinates after transformation

$C$ of coordinates else

call initWF (zpsiAo, tstart)

C Potential separable in mass scaled coordinates:

$C$ Morse Oscillator along $y$ and harmonic along $x$ endif

C Normalise call morsewf (zpsiAo, tstart)

do 47 iy $=1$, nypts

do 48 ix=1, nxpts

zpsiAO (ix, iy) =rtJacobian*zpsiAO(ix, iy)

48

continue

47 continue 
C No analytic solution for wavefunction available, calculate numerisally.

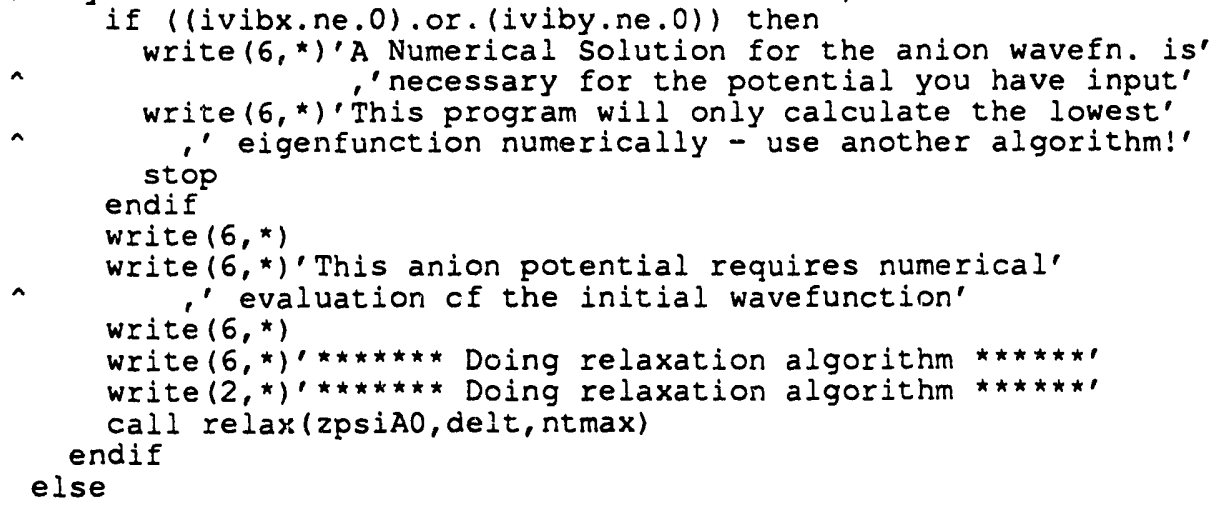

C Do a readwave: get anion wavefunction from disk (ie as solved for by C another program.) The wavefunction must be on same grid as used here.

C

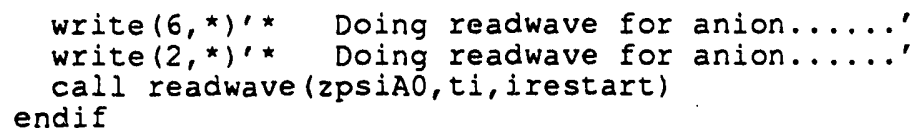

C Plot Anion wavepacket on anion potential

if (iwdrw.eq.1) then 
else

C Do a readwave for the neutral wavepacket, and get the start time for

C the propagation

call readwave (zpsiBo, ti, irestart)

^ write $\left(6,{ }^{*}\right) *$ start time $=$ ', ti,

endif ' $\mathrm{fs}$

a.u. ', ti*2.4177e-2,

C The neutral wavefunction is correctly normalised and ready for propagation

c Evolve neutral wavefunction for time delt/2 on the surface call Hpsi $(2, t i, z p s i B O, z$ HpsiBO)

do 22 iy $=1$, nYpts

do 20 ix $=1$, nXpts

zpsiBI $(i x, i y)=\operatorname{zpsiBO}(i x, i y)-$

20 continue

zeye *(delt/2.00)*zHpsiBO(ix, iy)/hb

22 continue

c Second order Runge Kutta using the intermediate derivative.

call Hpsi $(2, t i$, zpsiBI, zHpsiBI)

do 32 iy=1, nYpts

do 30 ix $=1$, nxpts

zpsiBl $(i x, i y)=z p s i B O(i x, i y)$

$\&$ zeye ${ }^{\text {delt }}$ * $z$ HpsiBI $(i x, i y) / h b$

30
32

continue

continue

c

return

end

$c$

$c$

subroutine initWF (zpsiA, tinit)

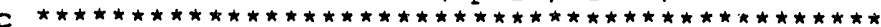

$c$

c initialize wavefunction on lower surface $A$

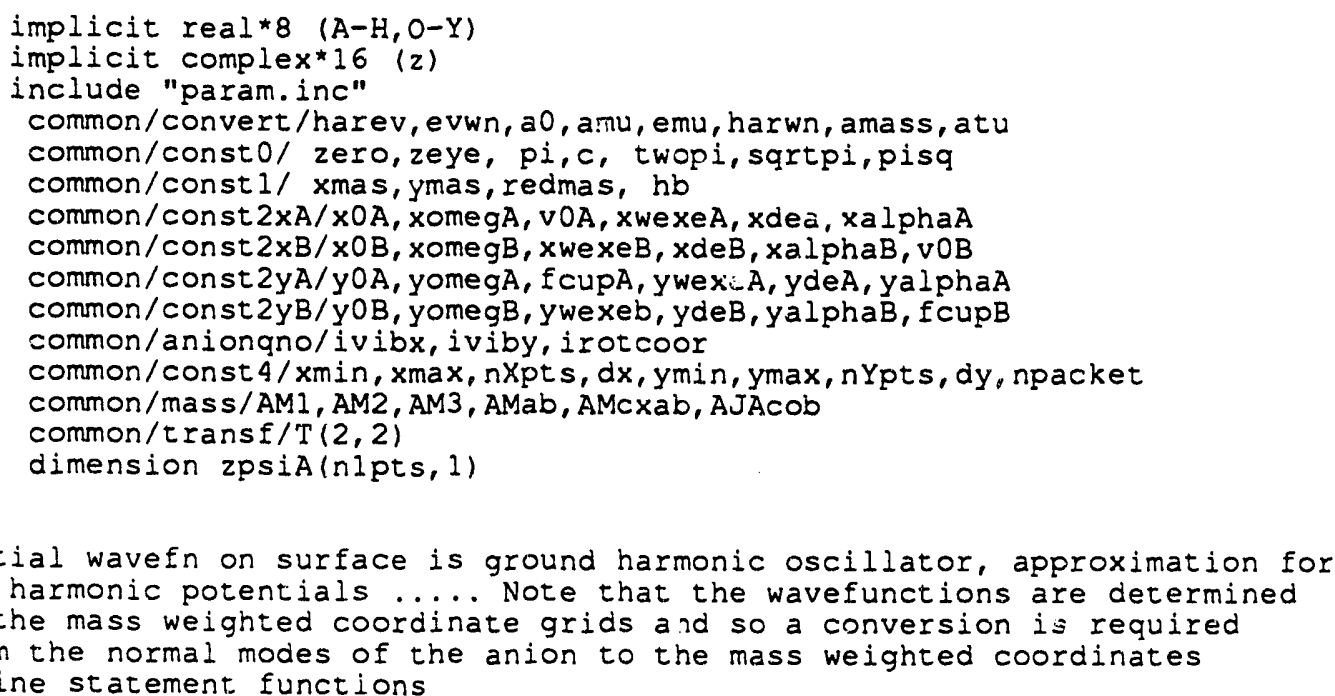

AmstoRab $(x x, y y)=y y / \operatorname{sqrt}(A M a b)$

AmstoRbc $(x x, y y)=-y y^{\star} \operatorname{sgrt}(A M a b) / A M 2+x x / A M c x a b$

AmstoQ1 $(x x, y y)=x x /$ AMc $x a b$

AmstoQ3 $(x x, y y)=$ AmstoRab $(x x, y y)$

AmstoS $1(x x, y y)=$ AmstoRab $(x x, y y)+$ AmstoRbc $(x x, y y)$

AmstoS3 $(x x, y y)=$ AmstoRab $(x x, y y)$-AmstoRbc $(x x, y y)$

AmstoNCl $(x x, y y$, rabeq, rbceq $)=T(1,1) *($ AmstoRab $(x x, y y)-r a b e q)$

$+\mathrm{T}(1,2) \times$ (AmstoRbc $(x x, y y)-$ rbceq)

Amstonc3 $(x x, y y$, rabeq, rbceq $)=T(2,1)$ * (AmstoRab $(x x, y y)-r a b e q)$ 
A $\quad T(2,2) *($ AmstoRbc $(x x, y y)-r b c e q)$

if (xomegA .eq. 0.0 ) then write $(6,)^{\prime}$ ' No initial wavepacket as no xomega available' stop

endif

if (yomegA .eq. 0.0$)$ then

write $(6,)^{\prime}$ ' No initial wavepacket as no yomega available'

stop

endif

if (ivibx.gt.1) then

write $(6, *)$ ' Illegal SHO $x$ vibrational quantum number'

stop

endif

if (iviby.gt.1) then

write $(6, *)$ ' Illegal sHO y vibrational quantum number'

stop

endif

$C$ perform conversion of coordinates : ***user supplied normal coordinates if (irotcoor.eq. 1) then

write $(6, *)$ 'Rotated Wavepacket'

do 13 iy $=1$, nYpts

$y i=y \min +(i y-1) * d y$

do 14 ix $=1$, nXpts

$x i=x \min +(i x-1) \star d x$

$x=A m s t O N C 1(x i, y i, x .0 A, y O A)$

$y=A m s t o N C 3(x i, y i, x O A, y O A)$

temp $=$ gauss $(x, 0.0, x$ omegA, amu, ivibx $)$

14

zpsiA $(i x, i y)=$ temp*gauss $(y, 0.0$, yomegA, amu, iviby)

13 continue

continue

else

$C$ perform conversion of coordinates : $\star \star \star$ symmetric

if (AM1 .eq. AM3) then

do 23 iy $=1$, nYpts

$y i=y \min +(i y-1) * d y$

do 24 ix $=1$, nxpts

$x i=x \min +(i x-1) \star d x$

$x=$ Amstosi $1(x i, y i)$

$y=\operatorname{Amstos} 3(x i, y i)$

temp $=$ gauss $(x, x 0 A$, xomegA, xmas, ivibx

zpsiA $(i x, i y)=$ temp*gauss $(y, y 0 A$, yomegA, ymas, iviby)

24

23

continue

continue

else

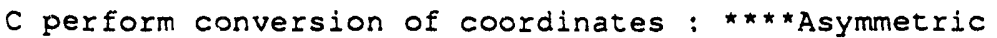

do 230 iy $=1$, nYpts

$y i=y \min +(i y-1) \star d y$

do 240 ix $=1$, nxpts

$x i=x \min +(i x-1) * d x$

$x=A m s t o Q 1(x i, y i)$

$y=$ AmstoQ $3(x i, y i)$

temp $=$ gauss $(x, x O A, x o m e g A, x m a s, i v i b x)$

240

zpsiA $(i x, i y)=$ temp ${ }^{\star} g a u s s(y, y O A$, yomegA, ymas, $i v i b y)$

230

continue

continue

endif

endif

tinit $=0.0$

return

end

C 
function gauss (Oi, OOA, omega, omas, ivibo)

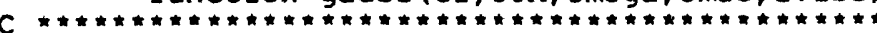

implicit real*8 $(A-H, O-Y)$

implicit complex 16 (z)

common/consto/ zero, zeye, pi,c, twopi,sqrtpi,pisq

common/const I/ xmas, ymas, redmas, hb

c gaussl includes the normalisation factor of $1 /$ sqrt (2)

if (ivibo.eq.1) then

gauss $1=\operatorname{sqrt}(2 *$ omega*omas $/ \mathrm{hb}) *(0 i-00 A)$

else

gauss $1=1.0$

endif

Anorm=sqre (sqre (omas*omega/ $(p i * h b))$ )

arg $=-(0 i-00 A) *(0 i-00 A) *$ omas*omega/(2.0*hb)

gauss $=\exp (a r g) * A n o r m * g a u s s l$

return

end

$c$

C

subroutine psi2(ipot, ti, zpsiAO, zpsiAl, zpsiA2)

$c$

evaluate the new wavefunction zpsiz from the old ones zpsio and zpsiz

c

implicit real* $B(A-H, O-Y)$

implicit complex*16 (z)

include "param.inc"

dimension zpsiAo (nlpts, 1), zpsiAl (nlpts, 1)

dimension zpsiA2 (nlpts, 1), zHpsiAl (nlpts, n2pts)

common/consto/ zero, zeye, pi, c,twopi, sqrtpi,pisq

common/const I/ xmas, ymas, redmas, hb

common/const $3 /$ tmax, ntmax, delt, iderflag

$c$ common/const $4 / x \ln$, xmax, nXpts, dx, ymin, ymax, nYpts, dy, npacket

c compute $H * z p s i l(1,1)$ :

call Hpsi (ipot, $t i, z p s i A l, z H p s i A l)$

do 10 iy $=1$, nYots

do 20 ix $=1$, nxpts

zpsiA2(ix, iy) =zpsiAO (ix,iy) - 2.0*zeye*delt*zHpsiAl(ix,iy)/hb continue

continue

c

return

end

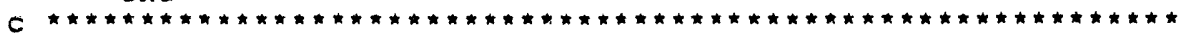

subroutine psi2ab(ipot,ti, zpsiAO, zpsiAl, zpsiA2)

$c$

C

e evaluate the new wavefunction zpsiz from the old ones zpsio and zpsi2 c

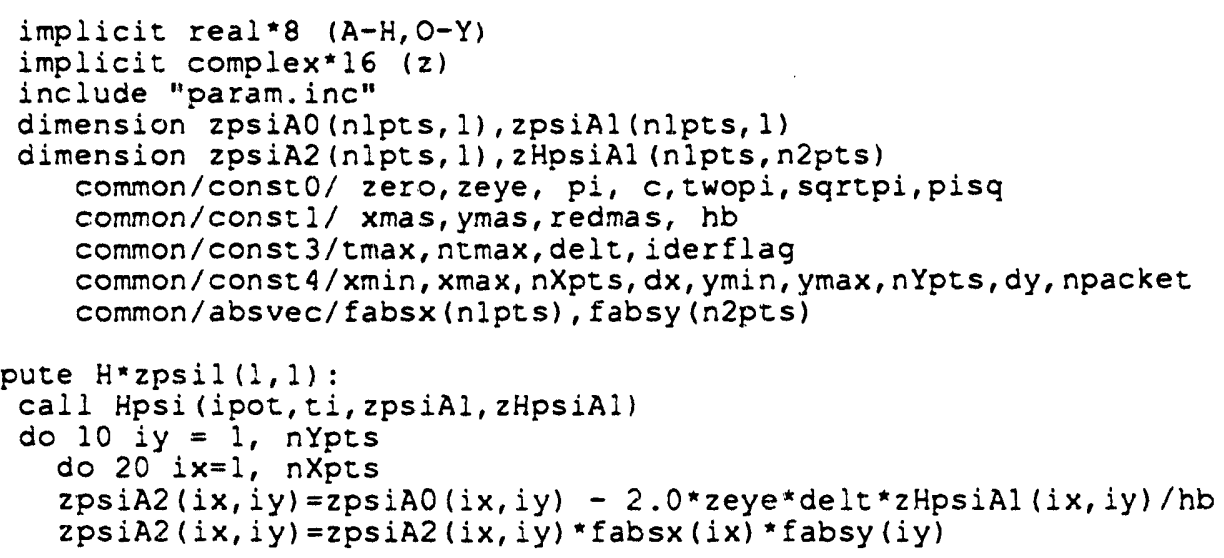




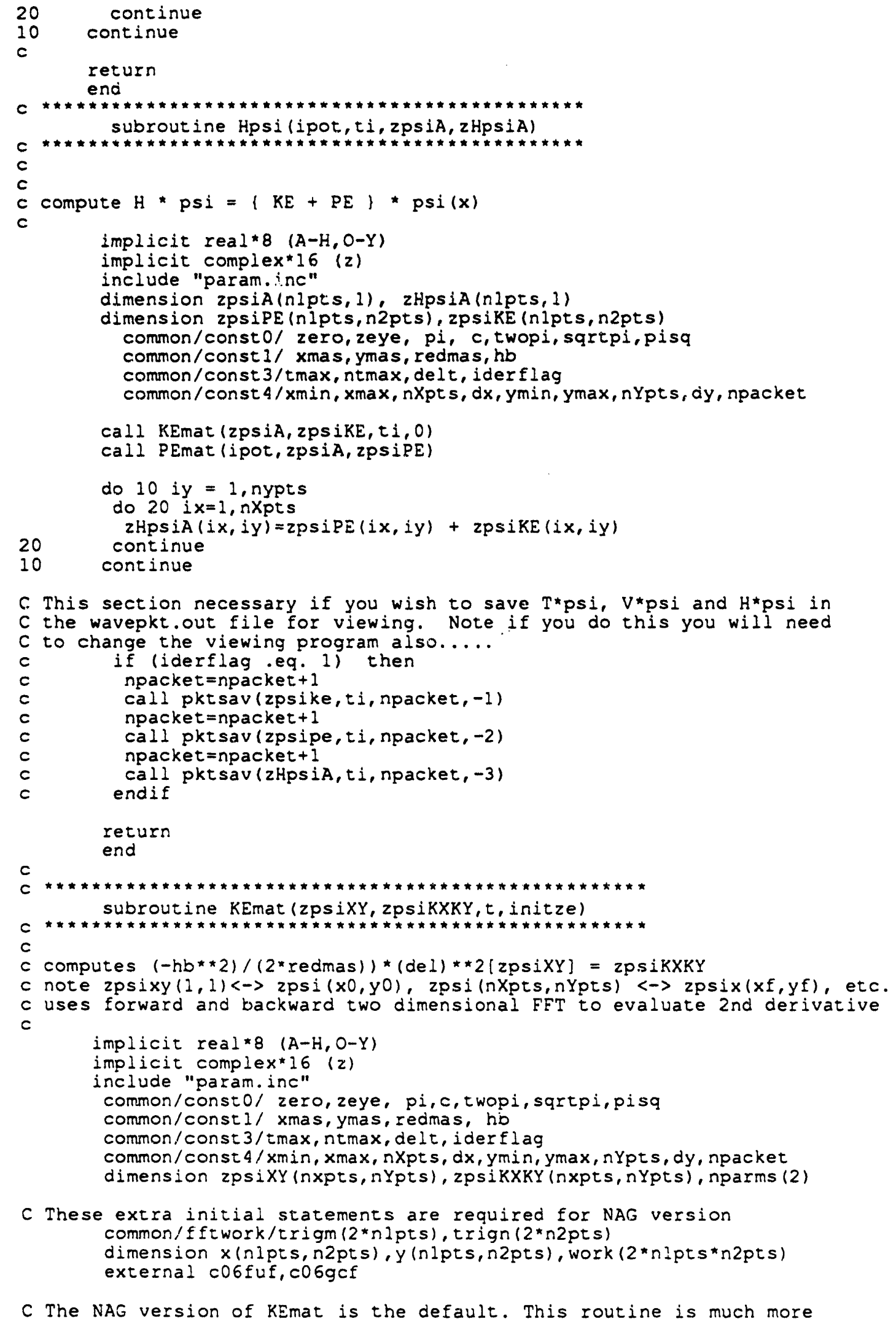


C efficient, particularly on a vector machine, and can give significant savings

$C$ in speed. The routine also allows non-powers of 2 as sizes for spatial grid.

C The co6fuf routine in the NAG 13 library requires the complex matrix

$c$ to be set up in two matrices, the real and imaginary parts, $x$ and $y$.

$C$ Hence semi-redundancy of the array zpsikXKY in this modified routine.

C However if this routine is not available, then the twodfft routine supplied C can serve as a replacement. To implement this version all the NAG using $C$ parts of this routine should be commented out, and the lower part should be C reinstated.

C S. E. Bradforth/ Cray-NAG version/ September 1989

C ONLY FOR NAG VERSION

C. check for the initializing call to set up TRIG workspaces.. if (initze .eq. 1) then

ifail $=0$ do 178 iy $=1$, nypts do 179 ix $=1$, nxpts $x(i x, i y)=r e a l(z p s i x y(i x, i y))$ $179 \quad y(i x, i y)=\operatorname{dimag}(z p s i X Y(i x, i y))$

178 continue

write $(6,)^{\prime}$ 'Initializing 2DFFT....'

$\&$

call co6fuf (nxpts, nypts, $x, y$, 'Initial', trigm,

if (ifail ne. O) then

write $(6, *)$ 'IFAIL $<0$ on initialization of $2 \mathrm{DFFT}$ ' stop

endif

return

endif

$c$

c backward fourier transform : $z \operatorname{psiXY}(x, y) \Rightarrow z \operatorname{psiKXKY}(k x, k y)$

c

ifail $=0$

do 1788 iy $=1$, nypts

do 1789 ix=1, nxpts

$x(i x, i y)=\operatorname{real}(z p s i x Y(i x, i y))$

1789 continue
1788 continue

$y(i x, i y)=\operatorname{dimag}(z \operatorname{ps} i X Y(i x, i y))$

continue

\&

call cogfuf (nxpts, nypts, $x, y$, 'Subsequent', trigm,

c

if (ifail ne. 0 ) then write $\left.(6,)^{\prime}\right)$ IFAIL $\ll 0$ in $\operatorname{KEmat}(1)$ - investigate ...' stop endif

$C$ only transfer the real, imaginary psi(k) matrices to complex matrix

$C$ if the user needs to see the psi(k) - otherwise skip this stage until after

C reverse transform to $x$ space ....

if (iderflag.eq. 1) then

npacket $=$ npacket +1

do 189 iy $=1$, nypts

do 188 ix=1, nxpts

zpsikXKY $(i x, i y)=\operatorname{cmplx}(x(i x, i y), y(i x, i y))$

188 continue

189 continue

call pktsav(zpsikxky,t, npacket, -4$)$

endif

$c$

$c$ compute the second derivative in the momentum domain.

$\mathrm{LX}=\mathrm{nXpts} / 2$

$L Y=n Y$ pts $/ 2$

$x L 2=(x \max x-x \min ) *(x \max -x \min )$

$y L 2=(y \max -y \min ) *(y \max -y \min )$

nprsq=nXpt $s^{\star} n Y p t s$ 


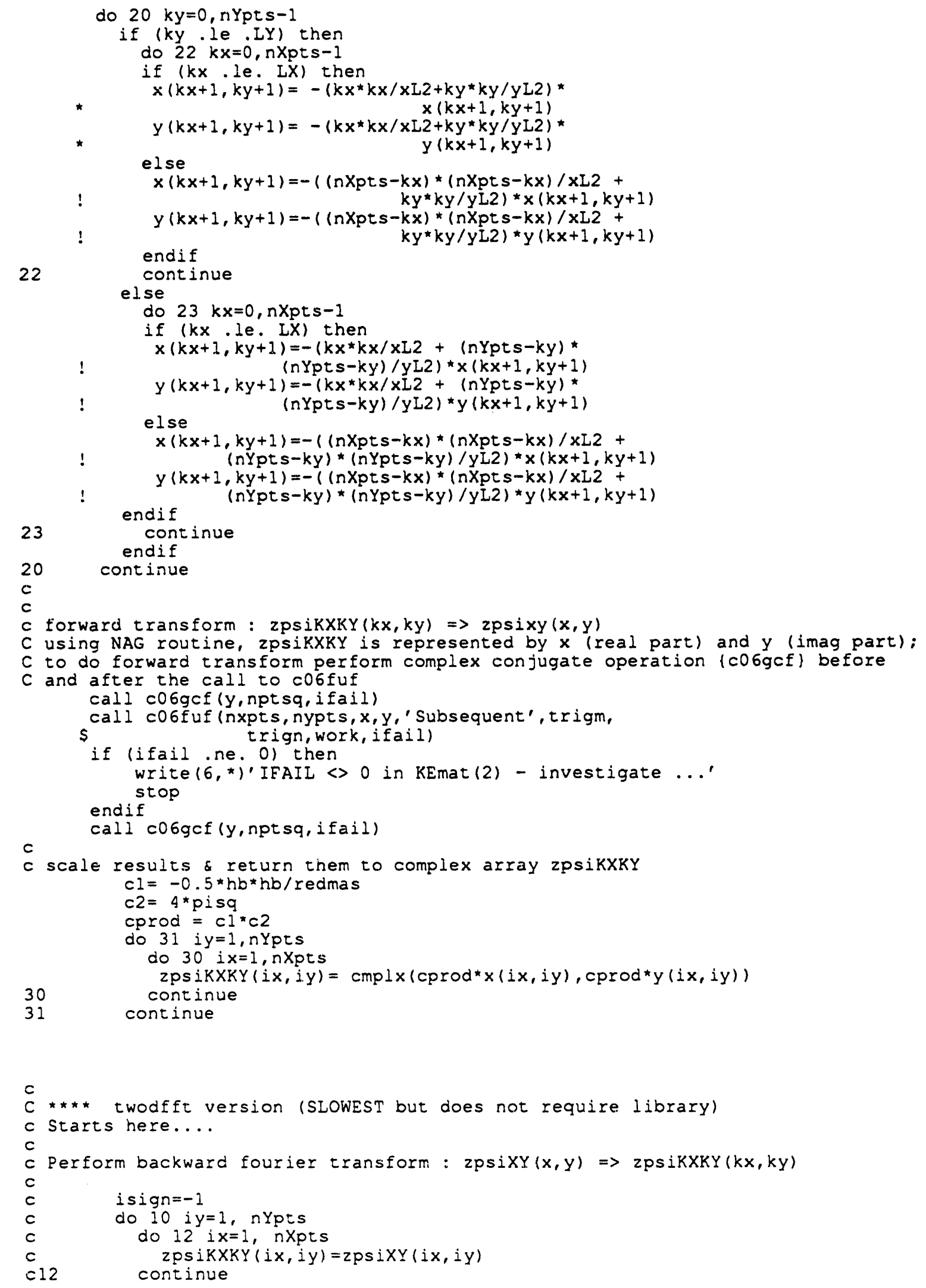




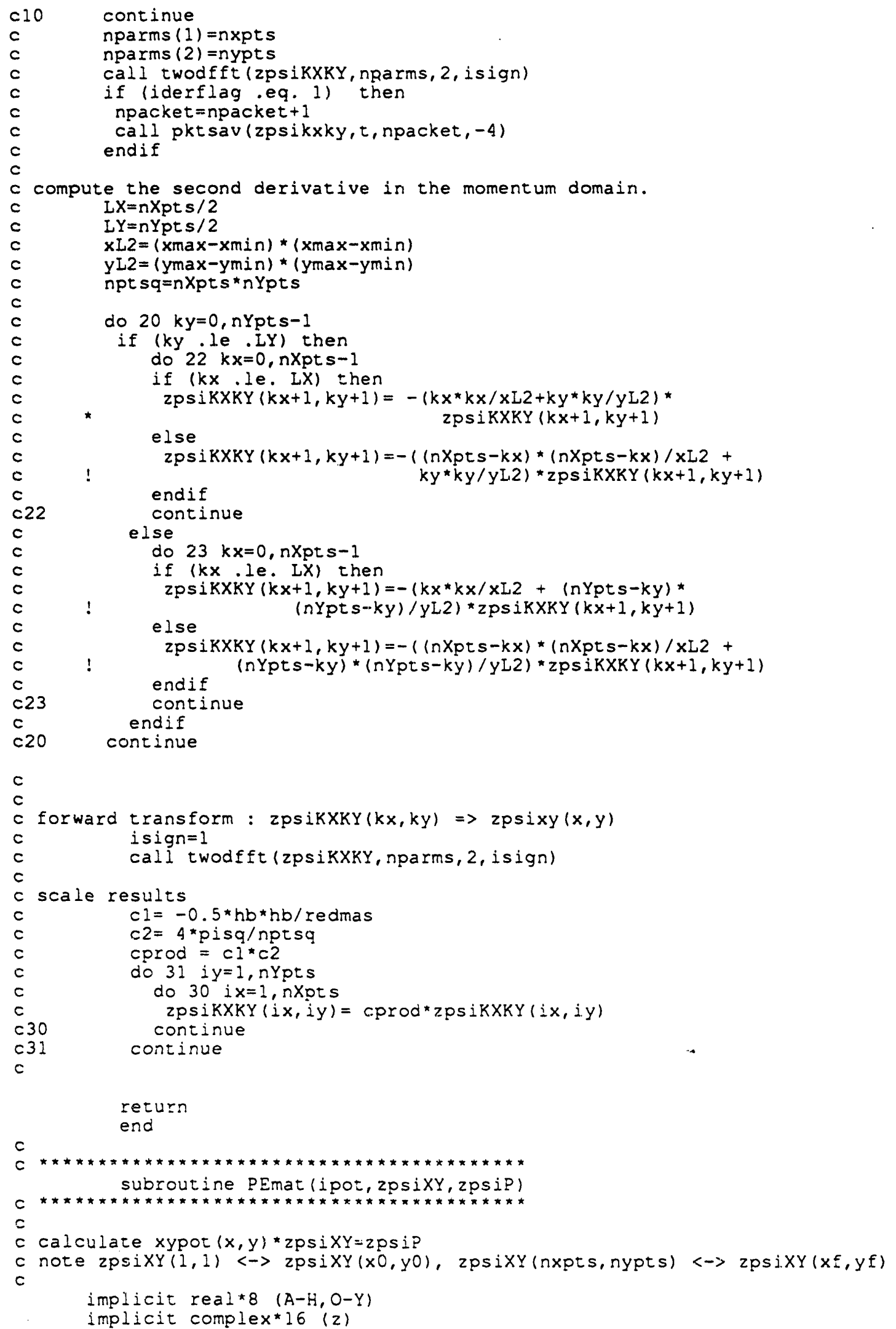




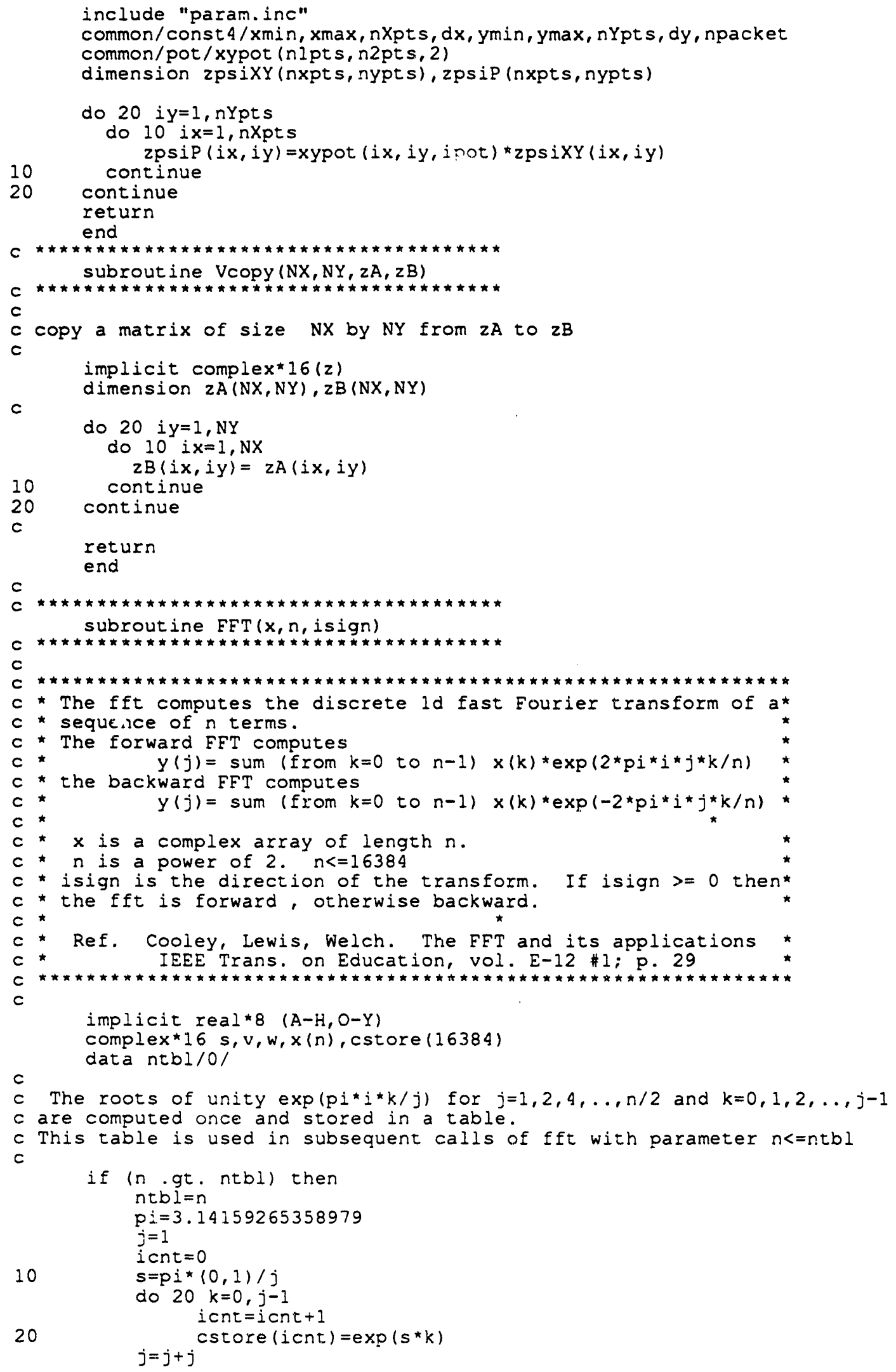




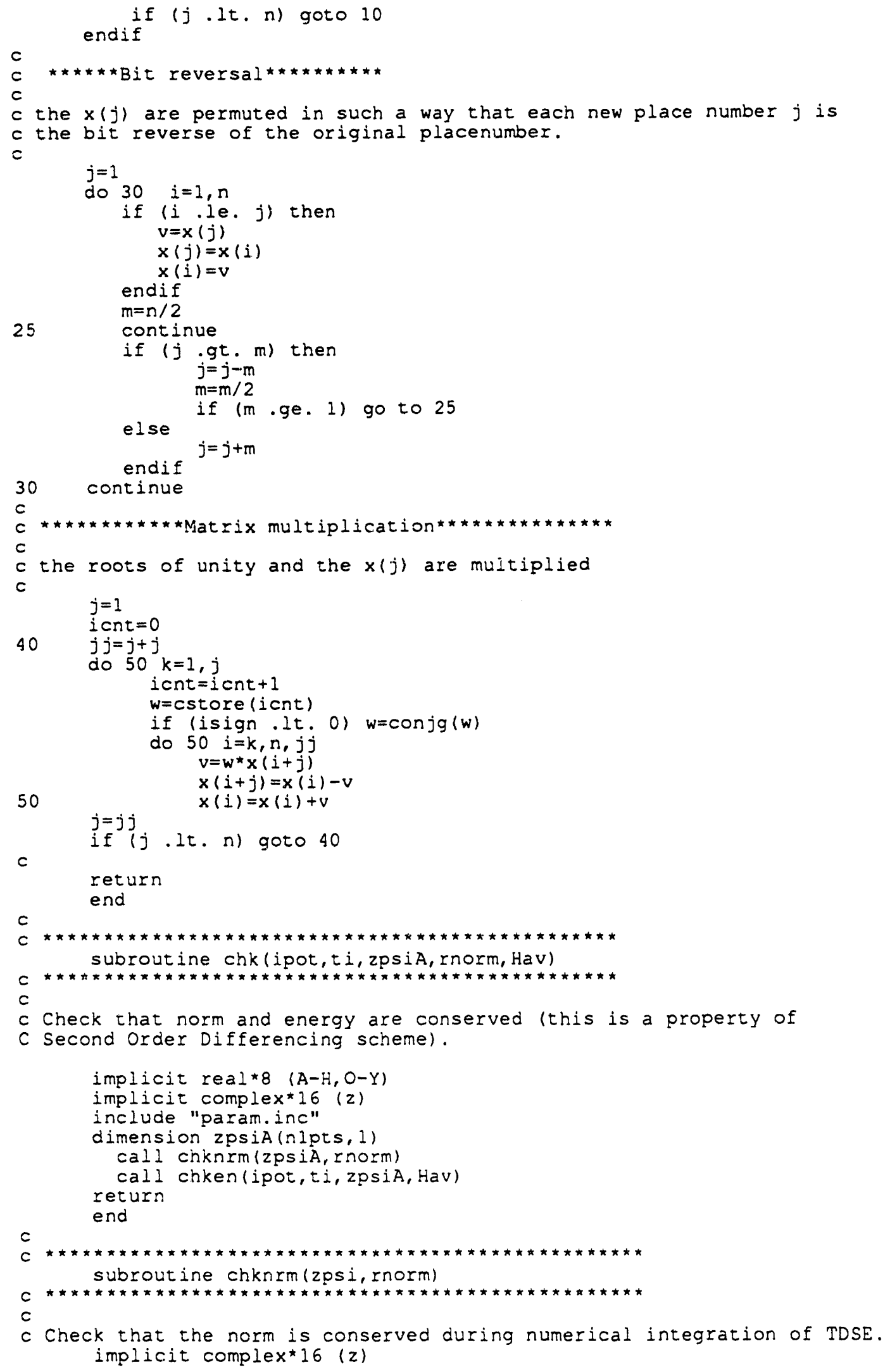




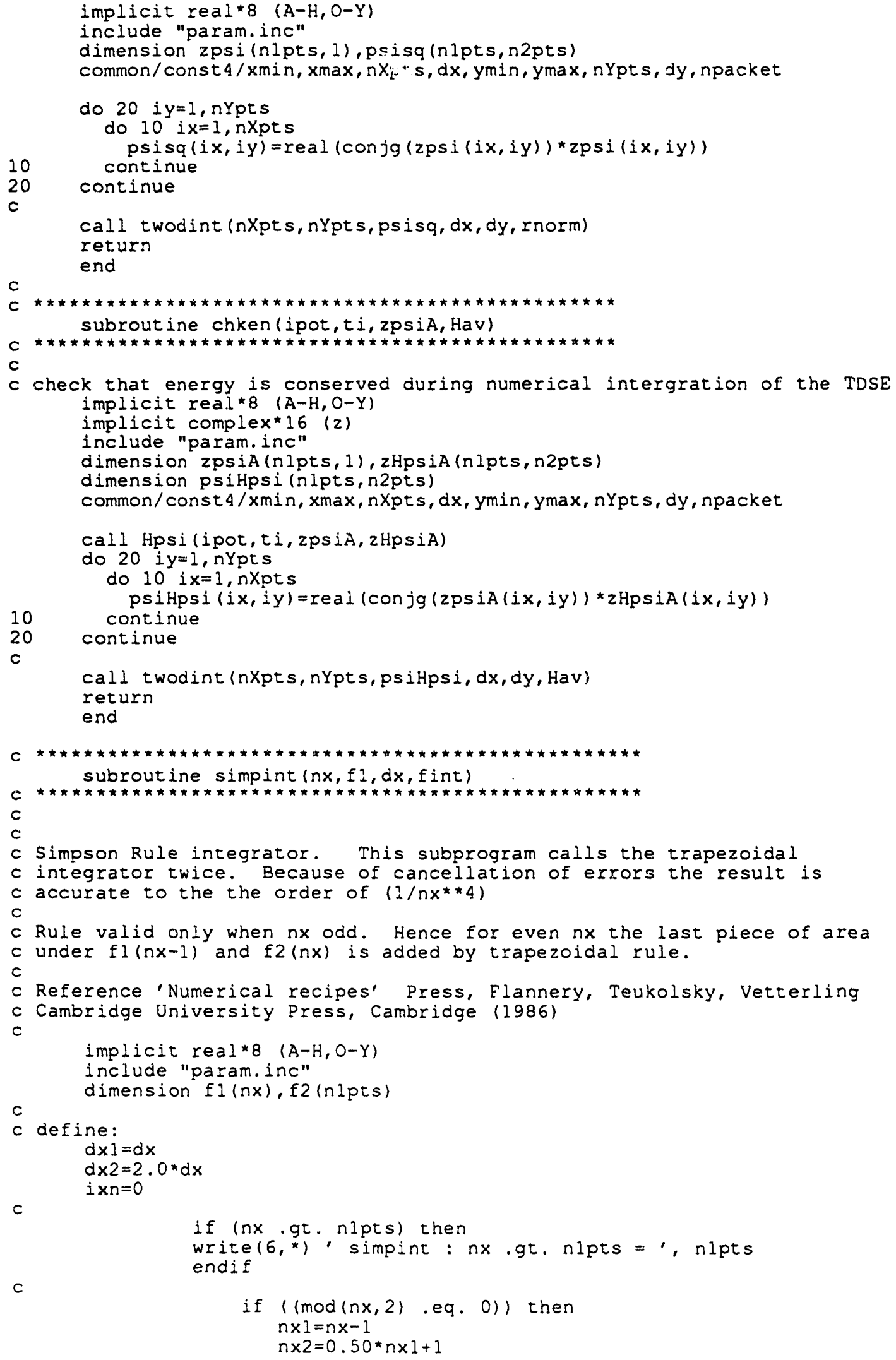




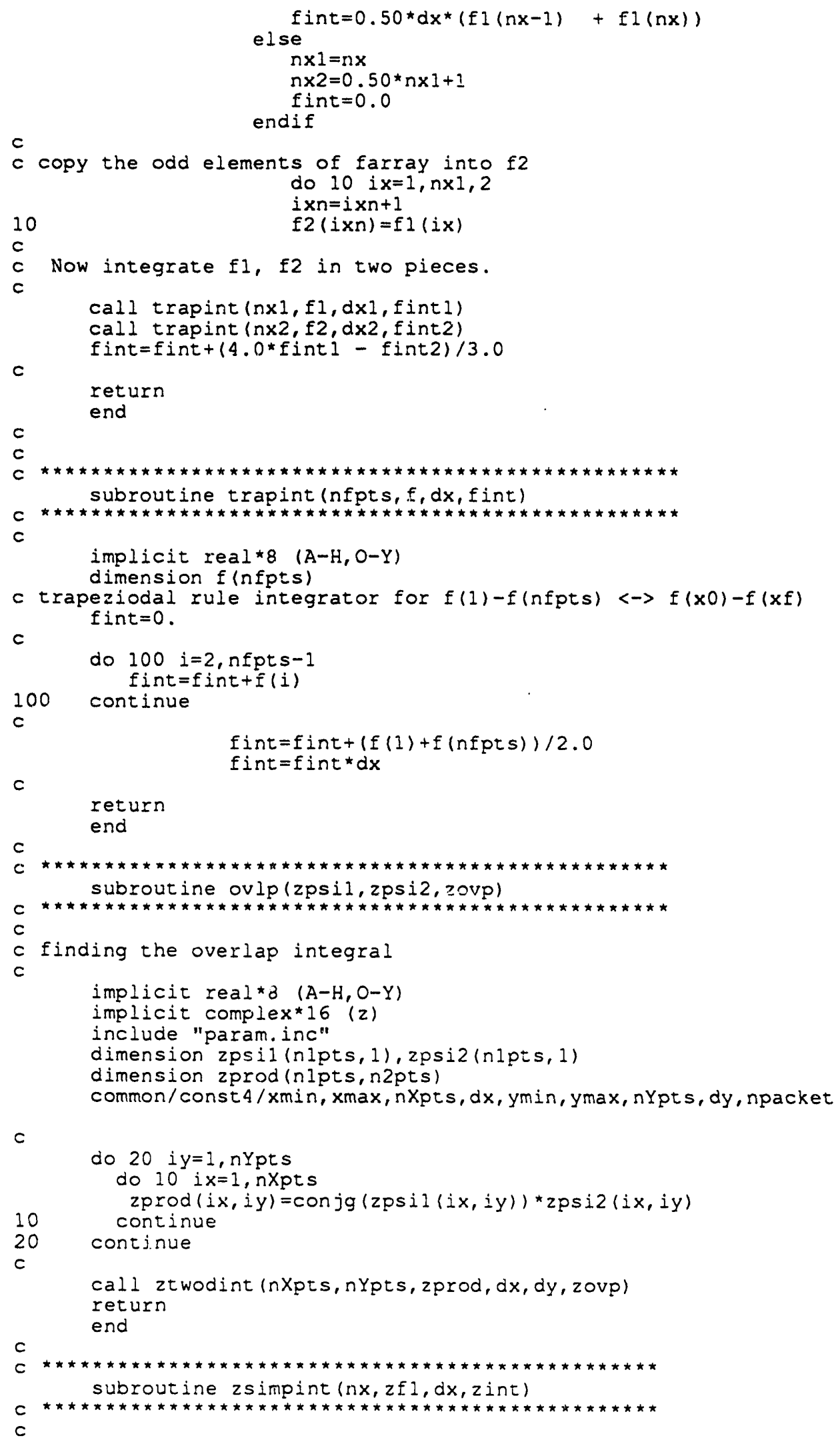

20 


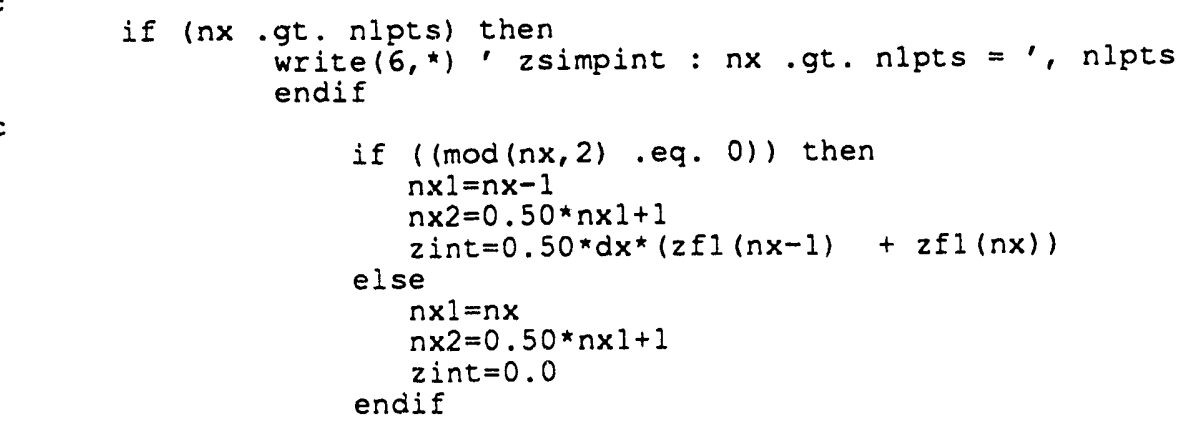


common/convert/harev, evwn, a 0, amu, emu, harwn, amass, atu

common/consto/ zero, zeye,pi,c,twopi, sqrtpi,pisq

common/const $3 / t \max$, ntmax, delt, iderflag

common/const7/ Espmin, domega, novsav, npktsav

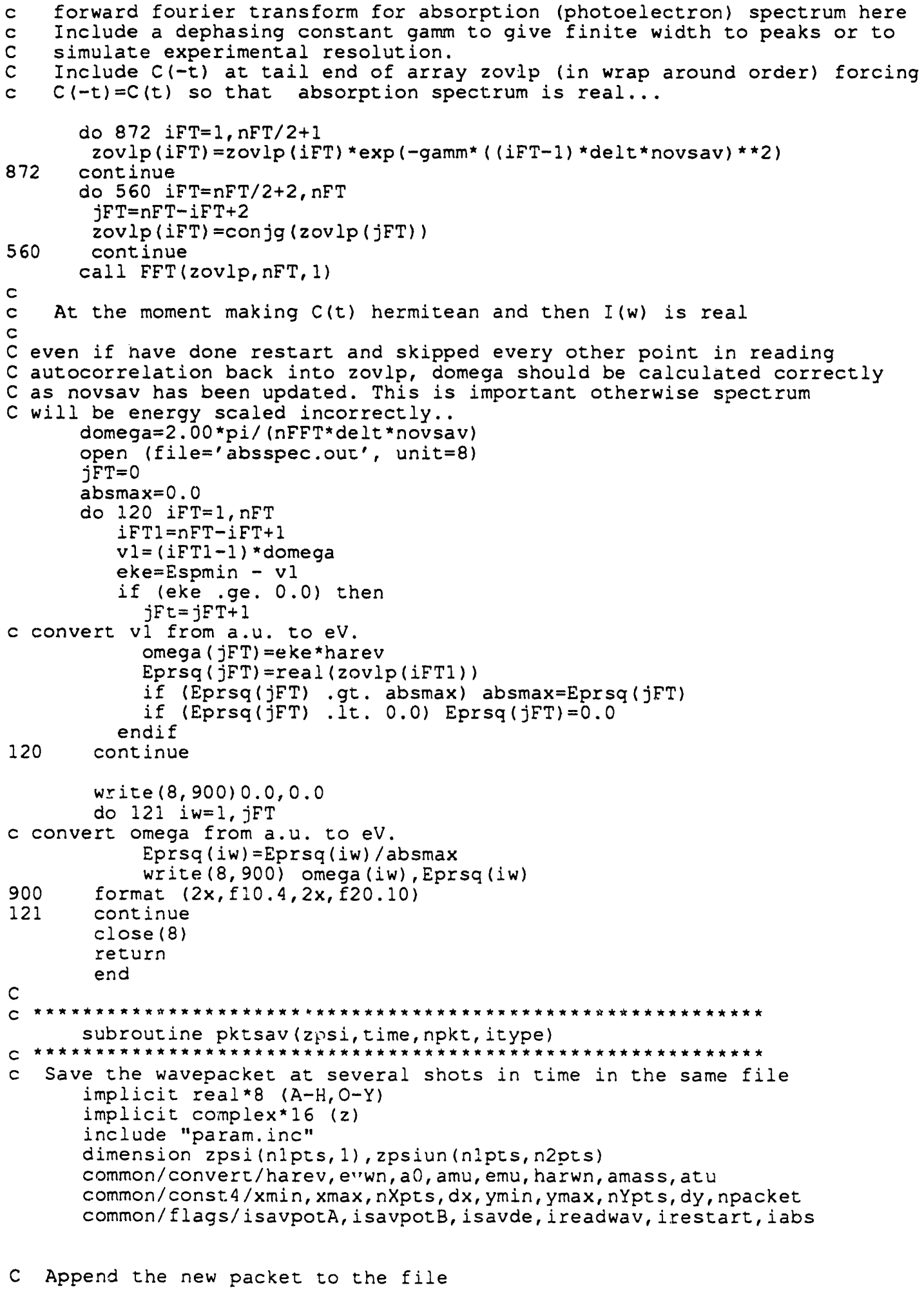




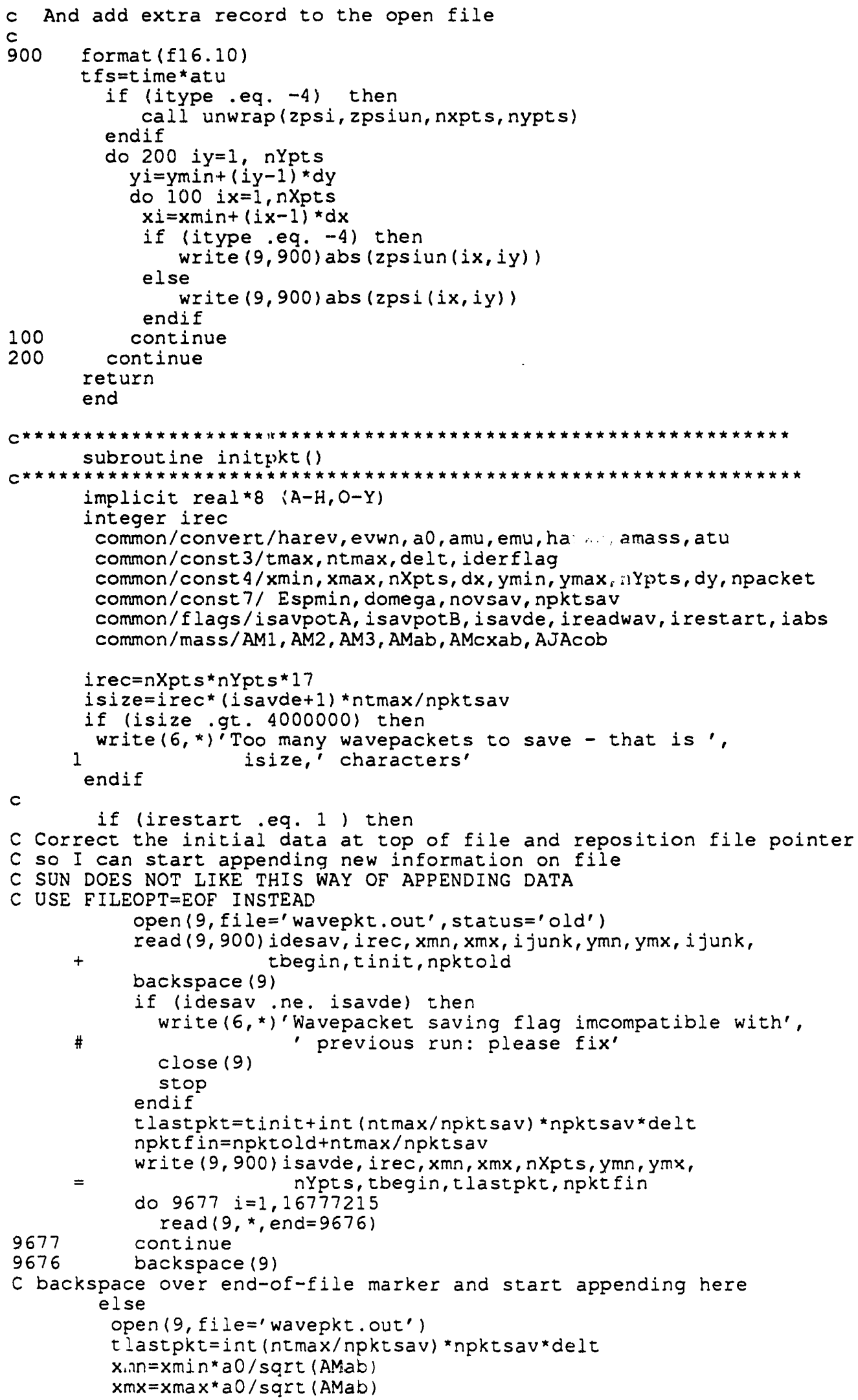




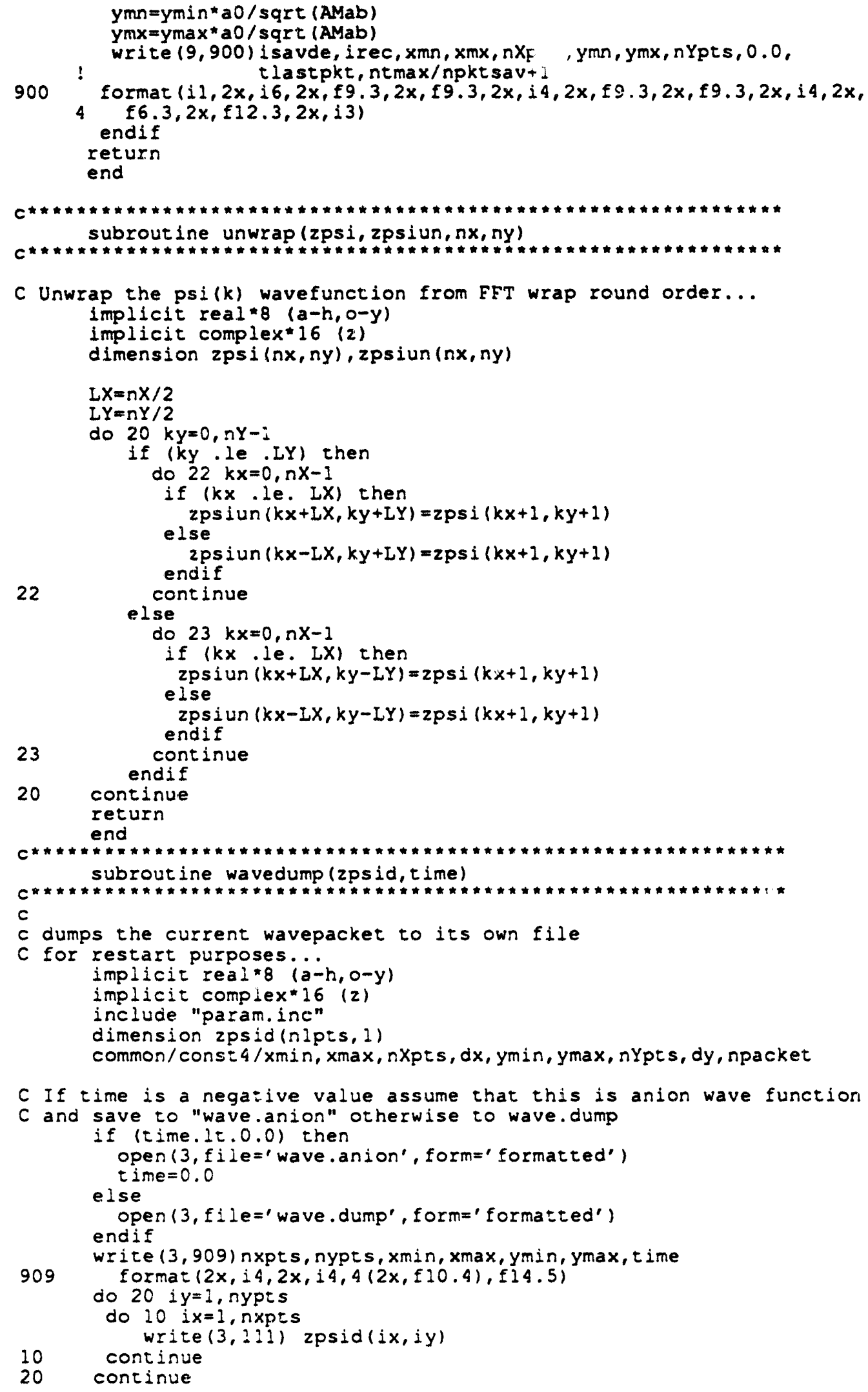


$C$ perform conversion of coordinates

if (irotcoor.eq.1) then

$x=$ AnstoNCl $(x i, y i, x O A, y O A)$

$y=A m s t o N C 3(x i, y i, x O A, y O A)$

temp=gauss $(x, 0.0, x o m e g A, a m u, i v i b x)$

else

$y=A m s t o Q 3(x . i, y i)-y O A$

$x=A m s$ toQ $1(x i, y i)$

temp $=$ gauss $(x, x O A, x o m e g A, x m a s, i v i b x)$

endilis

expval 1 wo (-yalphaA*y)

1

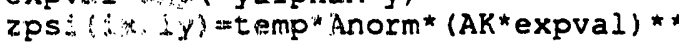

$((A K-1) / 2) * \exp (-A K * \operatorname{expval} / 2)$

$C$ Set up to check for $v=1$ Morse wavefunction using recurrence

C relationship - higher values - refer to above reference...

if (iviby .eq. 1) then

zpsi $(i x, i y)=z p s i(i x, i y){ }^{*} \operatorname{sqrt}(A K-3.0) *$

(AK*expval-AK+2.0)/(AK*expval)

24 continue

23 continue

endif

tinit $=0.0$

return

end

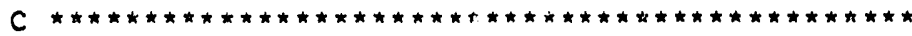

subroutine readwave (zpsi, time, irestart)

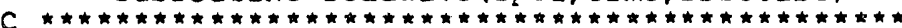

$C$ This is designed to either read in anion wavefunction calculated elsewhere

$C$ or to capture the last wavepacket from a previous run so

$C$ if a less ambitious job finishes, and user wishes to

$c$ restart, then user can load in this last position.

$C$ Note that the full wavepacket is normally only dumped at end of the job

C This can be altered if user worried that computer may crash over period of job

implicit real*8 $(A-H, O-Y)$

implicit complex*16 (z)

common/const $4 / x \min , x \max , n X p t s, d x, y m i n, y m a x, n Y p t s, d y, n p a c k e t$

common/const $2 y A / Y O A$, yomegA, fcupA, ywexeA, ydeA, yalphaA

dimension zpsi (nxpts, nypts)

open ( 3, file=' wave. dump', form=' formatted')

read $(3,909) n x, n y, x m n, x m x, y m n, y m x, t i m e$

909

format $(2 x, i 4,2 x, i 4,4(2 x, f 10.4)$, f14.5)

C Check the wave.dump file for compatibility

if ((nx.ne.nxpts).or. (ny.ne.nypts)) then

write $(6, *)^{\prime *}$ Incompatible read wavepacket,',

! 'nxpts/nypts in wave. dump'

stop
endif

if ( $(a b s(x m n-x m i n)$.gt. 0.001$)$.or.

!. (abs $(x \max -x \mathrm{mx}) . \mathrm{gt} .0 .001)$ ) then

write $\left(6,{ }^{*}\right)^{\prime}$ Incompatible read wavepacket,',

! ' $x \mathrm{~min} / \mathrm{xmax}$ in wave.dump'

write $\left(6,{ }^{*}\right) x m n, x m i n, x m x, x \max$

stop

endif

if $($ (abs $(y m n-y m i n) . g t .0 .001)$.or

: $\quad(a b s(y m x-y m a x) . g t .0 .001)$ ) then write $\left(6,{ }^{*}\right)^{*}$ Incompatible read wavepacket,',

! 'ymin/ymax in wave.dump' write $(6, *)$ ymn, ymin, ymx, ymax

endif stop 
C File ok for job, continue

if (irestart.ne.1) then

$C$ if not restarting then wavefunction should be real

do 10 iy $=1$, nypts

do 20 ix $=1$, nxpts

$\operatorname{read}(3, *)$ aps

$20 \quad \begin{aligned} & \text { zpsi }(i x, i y)=\operatorname{cmplx}(\text { aps, } 0.0) \\ & \text { continue }\end{aligned}$

10 continue

else

$C$ if restarting the wavefunction is complex \& already normalised

do 100 iy $=1$, nypts

do 200 ix=1, nxpts

read $(3,111)$ zpsi (ix, iy)

111 format $(\mathrm{e} 22.16,2 \mathrm{x}, \mathrm{e} 22.16)$

200 continue

100 continue

endif

close (3)

return

end

C

subroutine twodint (nXpts, nYpts, ff, dx, dy, xyint)

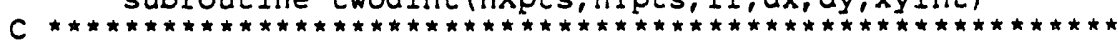

implicit real*8 $(A-H, O-Y)$

include "param.inc"

dimension ff (nxpts, nypts), temp (n2pts), ffcol (nlpts)

$C$ for every $y$ value calculate the 1 -d integral over $x$

$C$ note limits of integration are independent of $y$ do $12 j=1$, nYpts

do $13 i=1$, nxpts

13 continue

12 continue

C Now calculate the integral over all y values call simpint (nYpts, temp, dy, xyint)

return

end

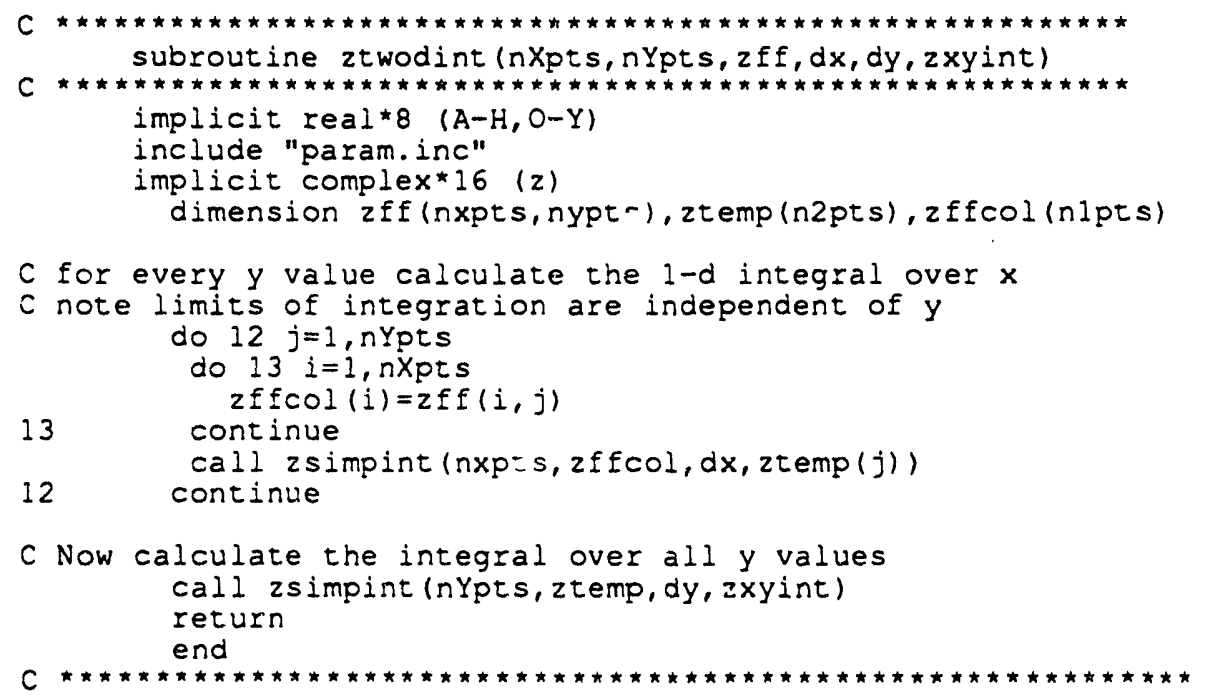


subroutine relax (zpsiAo, delt, ntmax)

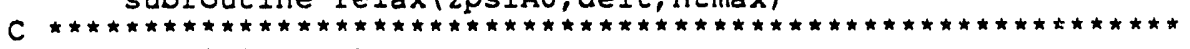

implicit real $\star 8(a-h, o-y)$

implicit integer $(i-n)$

implicit complex*16(z)

include "param.inc"

dimension zpsiAO (nlpts, 1), zpsiAl (nlpts, n2pts)

dimension zpsiAI (nlpts, n2pts), zHpsiAI (nlpts, n2pts)

dimension zHpsiaO (nlpts, n2pts)

common/convert/harev, evwn, a0, amu, emu, harwn, amass, atu

common/consto/ zero, zeye, pi,c, twopi, sqrtpi,pisq

common/const $2 \times A / x O A, x o m e g A, v O A, x w e x e A, x d e a, x a l p h a A$

common/const $2 \times B / x O B, x o m e g B, x w e x e B, x d e B, x a l$ phaB, $v O B$

common/const 1/ xmas, ymas, redmas, hb

common/const $4 / x \min , x \max , \mathrm{nXpts,} \mathrm{dx}$, ymin, ymax, nYpts, dy, npacket

common/mass/AM1, AM2, AM3, AMab, AMcxab, AJAcob

C This method of finding the lowest eigenstate of a bound potential surface

$C$ is described in R. Kosloff and H. Tal-Ezer, Chem. Phys. Lett. 127, 223 (1986)

$C$ It uses propagation of the initial guess wavefunction in IMAGINARY time to

$C$ let the wavefunction relax to the lowest eigenfunction of the Hamiltonian.

C The advantage of the method is it uses nearly all the same routines as the

$C$ time dependent propagation method used in this program for the neutral

C surface. However this wavefunction is everywhere real, unlike neutral psi.

C Generate initial guess:

C If the potential is morse/harmonic such that analytic solutions were not

$C$ implemented or the potential had linear coupling then uncoupled SHO $x$ SHO

$C$ centered at $x O A$, YOA with frequencies xomegA and xomegB will suffice as good

$C$ initial guesses to the ground state wavefunction.

$C$ If the potential was read $i$ or is a user supplied functional the form,

$C$ user is required to give the same information to generate an initial guess.

C Produce this initial guess

call initWF (zpsiAo, tinit)

C Normalise

It Jacobian=sqrt (AJAcob)

do 47 iy $=1$, nypts

do 48 ix=1, nxpts

zpsiAO $(i x, i y)=r t J a c o b i a n * z p s i A O(i x, i y)$

48

continue

47 continue

call chk ( $1, t i, z$ psiAo, rsnorm, Have)

write $\left(6,{ }^{*}\right)$ ' Norm of inital guess anion $W F='$, rsnorm

write $(6, *)^{\prime}\langle H\rangle$ of inital guess anion $W E='$

'Have kharwn/rsnorm,' cm-1'

C for the anion imaginary time propagation need delt and tmax,

$C$ at present use the same as for the neutral propagation

$C$ evolve the initial guess for time delt/2 on the anion surface

call Hpsi(l, ti, zpsiAO, zHpsiAO)

do 22 iy=1, nYpts

do 20 ix=1, nXpts

zpsiAI (ix, iy $)=z p s i A O(i x, i y)-(\operatorname{delt} / 2) * z H p s i A O.(i x, i y) / h b$

20 continue

22 continue

c Second order Runge kutta using the intermediate derivative. call $\operatorname{Hpsi}(1, t i, z p s i A I, z H p s i A I)$

do 32 iy=1, nYpts

do 30 ix $=1$, nXpts

30 continue

zpsiAl $(i x, i y)=z p s i A O(i x, i y)-d e l t \star z H p s i A I(i x, i y) / h b$

32 continue

call Vcopy (nxpts, nypts, zpsiAl, zpsiAO) 
C Now perform First Order Differencing in imaginary time for ntmax steps do 1000 it $=1$, ntmax

$t i=t i+d e l t$

call Hpsi( $1, t i, z p s i A O, z$ HpsiAO)

do 100 iy $=1$, nYpts

do 200 ix $=1$, nXpts

zpsiAl $(i x, i y)=z p s i A O(i x, i y)-d e l t \star z H p s i A O(i x, i y) / h b$

200 zpsiAl $(i x, i y)=\operatorname{cmplx}(\operatorname{real}(2 p s i A l(i x, i y)), 0.0)$

100 continue

continue

call Vcopy (nXpts, nYpts, zpsiAl, zpsiAO)

if $(\bmod (i t, 500)$.eq.0) then

C Check the norm, and nearly always renormalise psi

$C$ before continuing relaxation

$C$ even ground state component will relax at rate $\exp (-E . t)$

$C$ where $E$ is the energy in $a . u$. above the absolute energy zero.

call chknrm(zpsiAO, rsnorm)

rtrsnorm $=$ sqrt (rsnorm)

if $($ rsnorm.lt.0.5).or. (rsnorm.gt.2.00)) then

do 456 iy $=1$, nYpts

do 457 ix $=1$, nxpts

457

zpsiAO (ix, iy) =2psiAO (ix, iy)/rtrsnorm

456

$$
\text { continue }
$$

continue

endif

endif

if $(\bmod (i t,(n t \max / 4))$.eq.0) then

call chk $(1, t i, z p s i A 0$, rsnorm, Have $)$

write $\left(6,{ }^{*}\right)$ ' Time step $=$ ', it,' $\langle H\rangle=$ ',

1000 continue Have *harwn/rsnorm,' $\mathrm{cm}-1$

C Hopefully have converged ground state wavefunction

C Renormalisation will be carried out in initB

write $(6, *)$ ntmax,' propagation in imaginary time to find'

write $(6, *)$ ' lowest eigenstate of anion potential completed' write $\left(6,{ }^{\star}\right)$

return

end

\section{potread2.f}

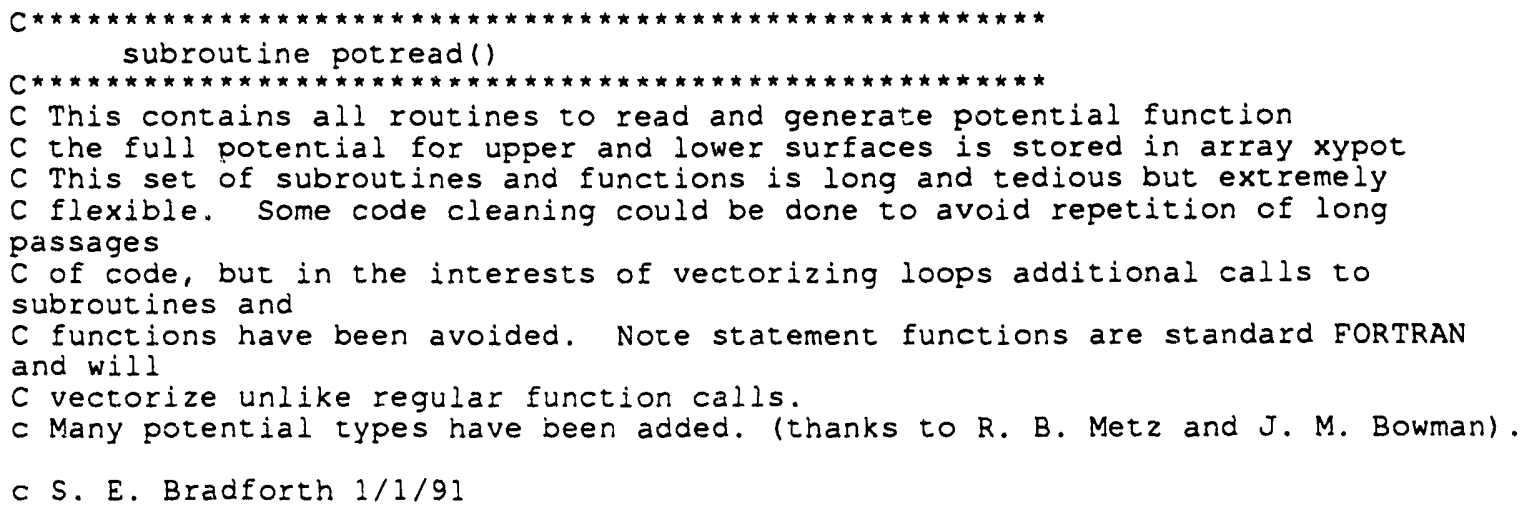


common/const $2 \times A / x O A, x o m e g A, v 0 A, x w e x e A, x d e a, x a l p h a A$ common/const $2 \times B / x O B, x o m e g B, x w e x e B, x d e B, x a l p h a B, v 0 B$ common/const2yA/YOA, yomegA, fcupA, ywexeA, ydeA, yalphaA common/const $2 y B / y O B$, yomegB, ywexeb, ydeB, yalphaB, fcup $B$ common/const $4 / x m i n, x m a x, n x p t s, d x, y m i n, y m a x, n Y p t s, d y, n p a c k e t$ common/flags/isavpotA, isavpot $B$, isavde, ireadwav, irestart, iabs common/pot/xypot (nlpts, n2pts, 2)

common/anionqno/ivibx, iviby, irotcoor common/mass/AM1, AM2, AM3, AMab, AMcxab, AJAcob common/analytic/ianal common/transf/T $(2,2)$

C This routine reads in potential parameters for both surfaces and then $C$ stores potential at each grid point to save further computation.

$C$ Define functions as statement functions

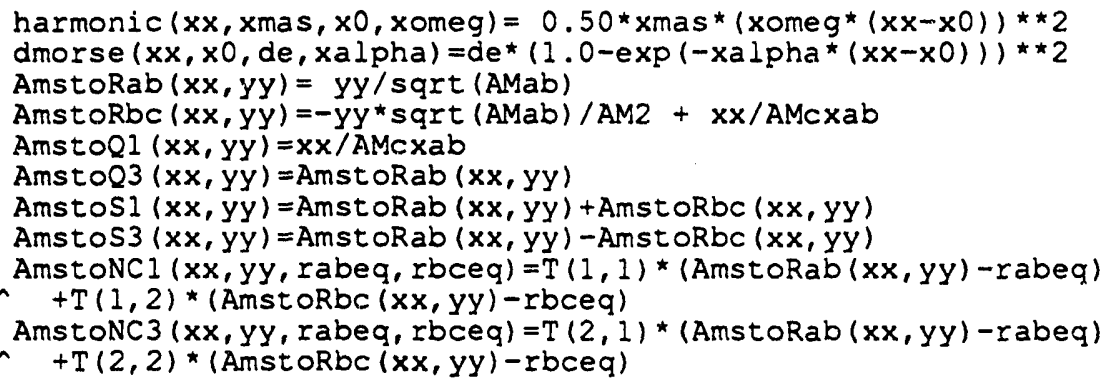

C Read anion potential first

$C$ set the potential by reading potential type read $\left(1,{ }^{\star}\right)$ ipot typA, ipottypAy, ivibx, iviby if ((ipottypA.gt. 4) .or. (ipottypA.it. -1)) then write $\left(6,{ }^{*}\right)$ 'problem with potential Ax type in input deck' stop endif

if ((ipottypay .gt. 4) .or. (ipottypay.1t. 0)) then write $\left(6,{ }^{*}\right)$ 'Problem with potential Ay type in input deck' stop endif write $\left(6,{ }^{\star}\right)$ 'setting up $2 d$ potentials in memory........'

$C$ set the flag desci.jing the potential as having analytic solutions or not iana $i=0$

C check for a seperable potential or one with linear coupling

if ((ipottypA.eq.1).or. (ipottypA.eq.0).or. (ipottypA.eq. -1)) then

$C$ proceed to calculate potential

C For each type read relevant parameters:

$C$ (expect $x 0$ in Angs, omega, wexe and $v 0$ in $\mathrm{cm}-1$ and De in $\mathrm{eV}$, alpha in Angs-1)

$C x$ coordinate (Q1 like) first, where reduced mass is xmas

C If ipottypA=-1 then expect potential parameters like those above, but

$C$ the coordinates along which the axes for potential and wavefunction are

$C$ defined are now not $x, y$ or $S 1,53$ but NCl, NC3 defined in statement function

$C$ above. This allows use of ab initio normal coordinates.

$C$ Mass is assumed to be included in the transfromation coefficients,

C IE a reduced mass of 1 amu will be assumed for both NCI And NC3

$C \times O A$ and $Y O A$ should be the equilibrium values of Rab and Rbc rather than

$C$ of $r(a c)$ and $R(a b)$

$$
\begin{aligned}
& \text { if }(\text { ipottypA.eq. }-1 \text { ) then } \\
& \text { irotcoor }=1
\end{aligned}
$$




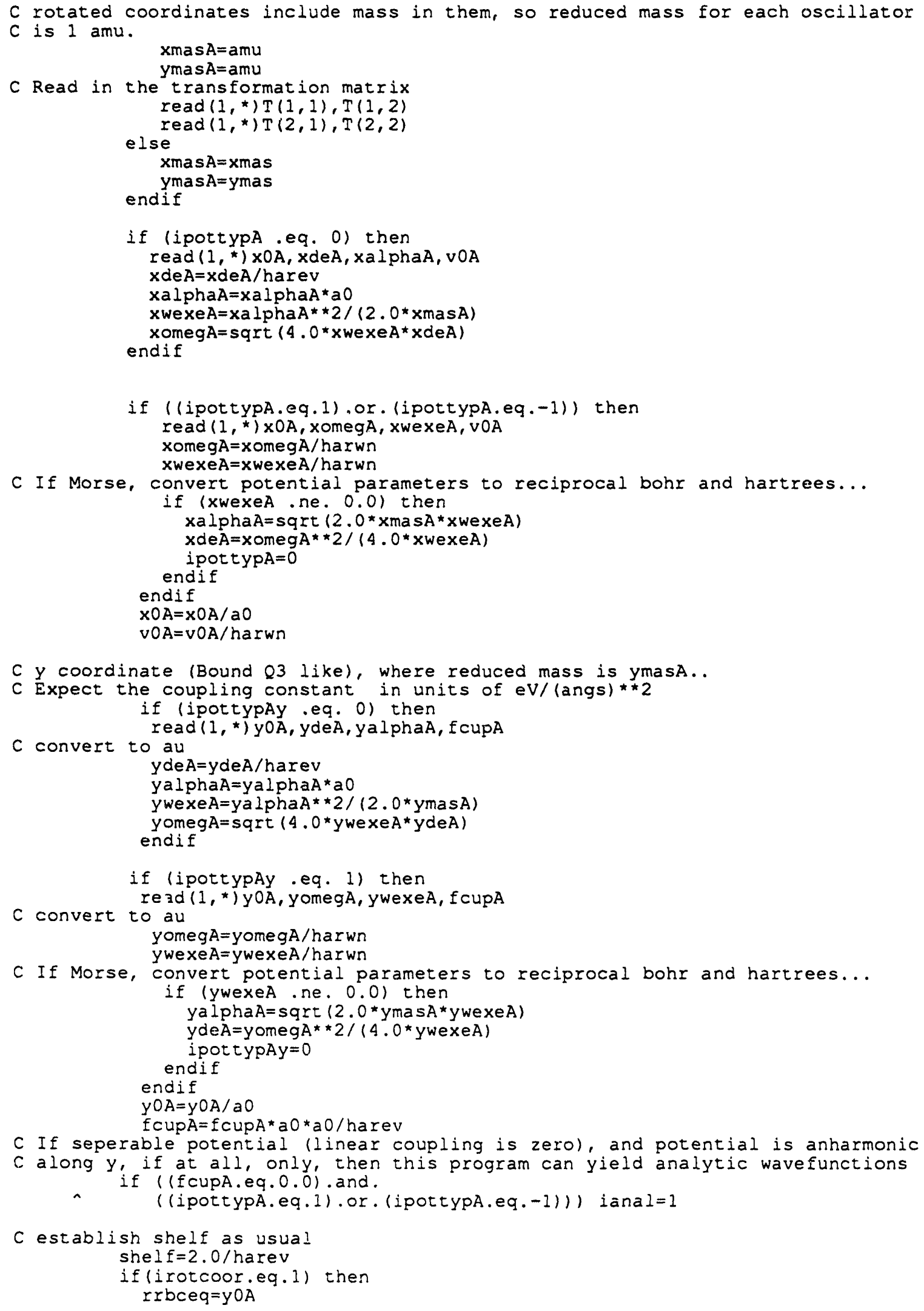

C If seperable potential (Iinear coupling is zero), and potential is anharmonic

$C$ along $y$, if at all, only, then this program can yield analytic wavefunctions if ( (fcupA.eq.0.0) and. 


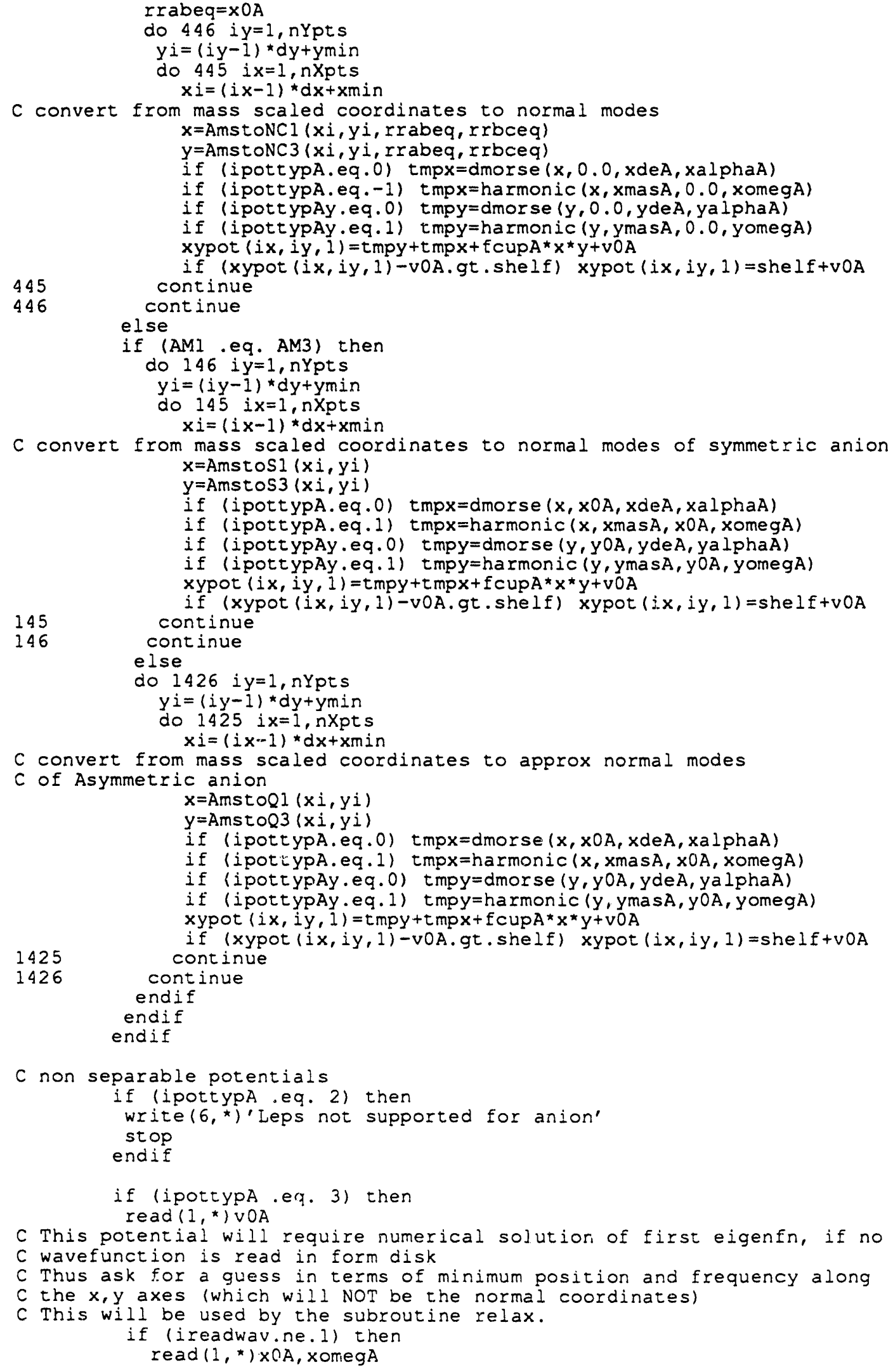




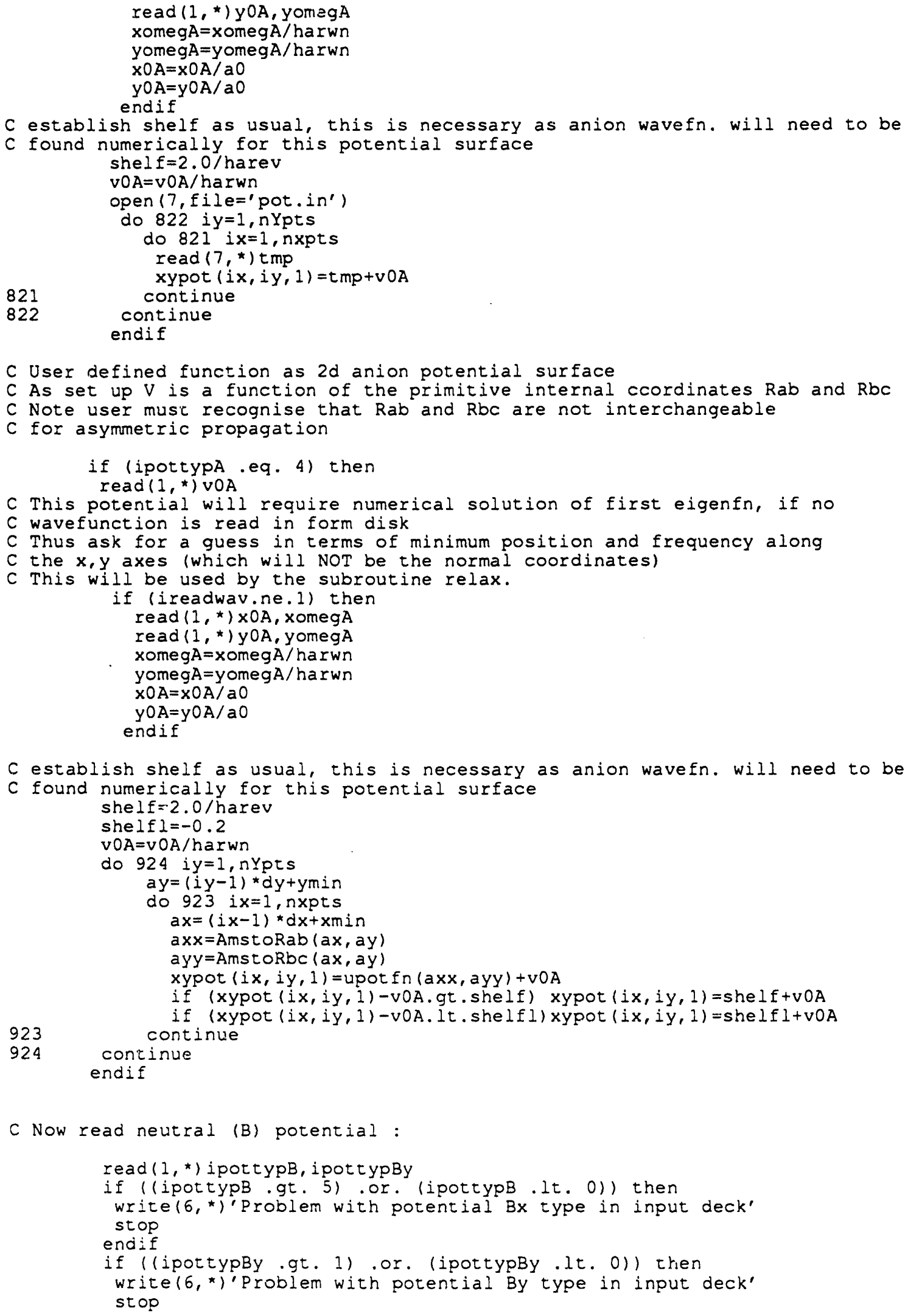




$$
\text { endif }
$$

$C$ as for anion potential construct pot. from seperable parts if appropriate.. if ((ipottypB.eq. 1) .or. (ipottypB.eq. 0)) then

if (ipottypB .eq. 0) then read $(1, \star) \times O B, x d e B, x a l p h a B, v O B$ $x$ de $B=x d e B /$ harev $x a l p h a B=x a l p h a B * a 0$ xwe xe $B=X a$ lpha $B * \star 2 /(2.0 * x m a s)$ $x$ omeg $B=\operatorname{sqrt}(4.0 * x$ wexe $B * x \operatorname{de} B)$ endif

if (ipottypB .eq. 1) then $\operatorname{read}\left(1,{ }^{*}\right) \times O B, x o m e g B, x w e x e B, v O B$ $x$ omeg $B=x$ omeg $B /$ harwn $x$ wexe $B=x$ wexe $B /$ harwn

if (xwexeB .ne. 0.0 ) then $x a l p h a B=\operatorname{sqrt}\left(2.0 * x \operatorname{mas}{ }^{*} x\right.$ wexeB $)$ $x \operatorname{de} B=x$ omeg $B \star \star 2 /(4.0 * x w e x e B)$ endif ipott $y \mathrm{p} B=0$

endif

$x O B=x O B / a O$

$v O B=v O B /$ harwn

C y coordinate, where reduced mass is ymas

if (ipottypBy .eq. 0) then read $(1, *)$ yOB, yde $B, y a i p h a B, f c u p B$

C convert to au yde $B=y d e B /$ harev yalphaB=yalphaB*a0 ywexe $B=y a$ lpha $B \star 22 /(2.0 *$ ymas $)$ yomeg $B=\operatorname{sqrt}(4.0 * y$ wexe $B \star y d e B)$ endif

$C$ convert to au

if (ipottypBy .eq. 1) then read $\left(1,{ }^{*}\right)$ yOB, yomegB, ywexe $B, f c u p B$

yomeg $B=$ yomegB/harwn $y$ wexe $B=y$ wexe $B /$ harwn

if (ywexeB . ne. 0.0 ) then yalpha $B=\operatorname{sqrt}\left(2.0^{*} y\right.$ mas*ywexe $\left.B\right)$ yde $B=y$ omeg $B \star \star 2 /(4.0 *$ ywexe $B)$ ipot typBy $=0$

endif $\mathrm{YOB}=\mathrm{yOB} / \mathrm{aO}$

fcup $B=f \operatorname{cup} B * a O * a 0 /$ harev

$C$ Note include shelf here for $B$ potential, partic. for Morse, $(6 \mathrm{eV})$ shelf $=6.0 /$ harev

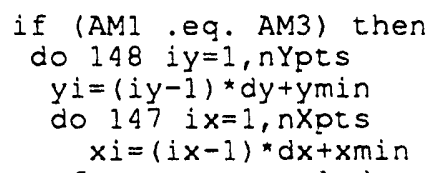




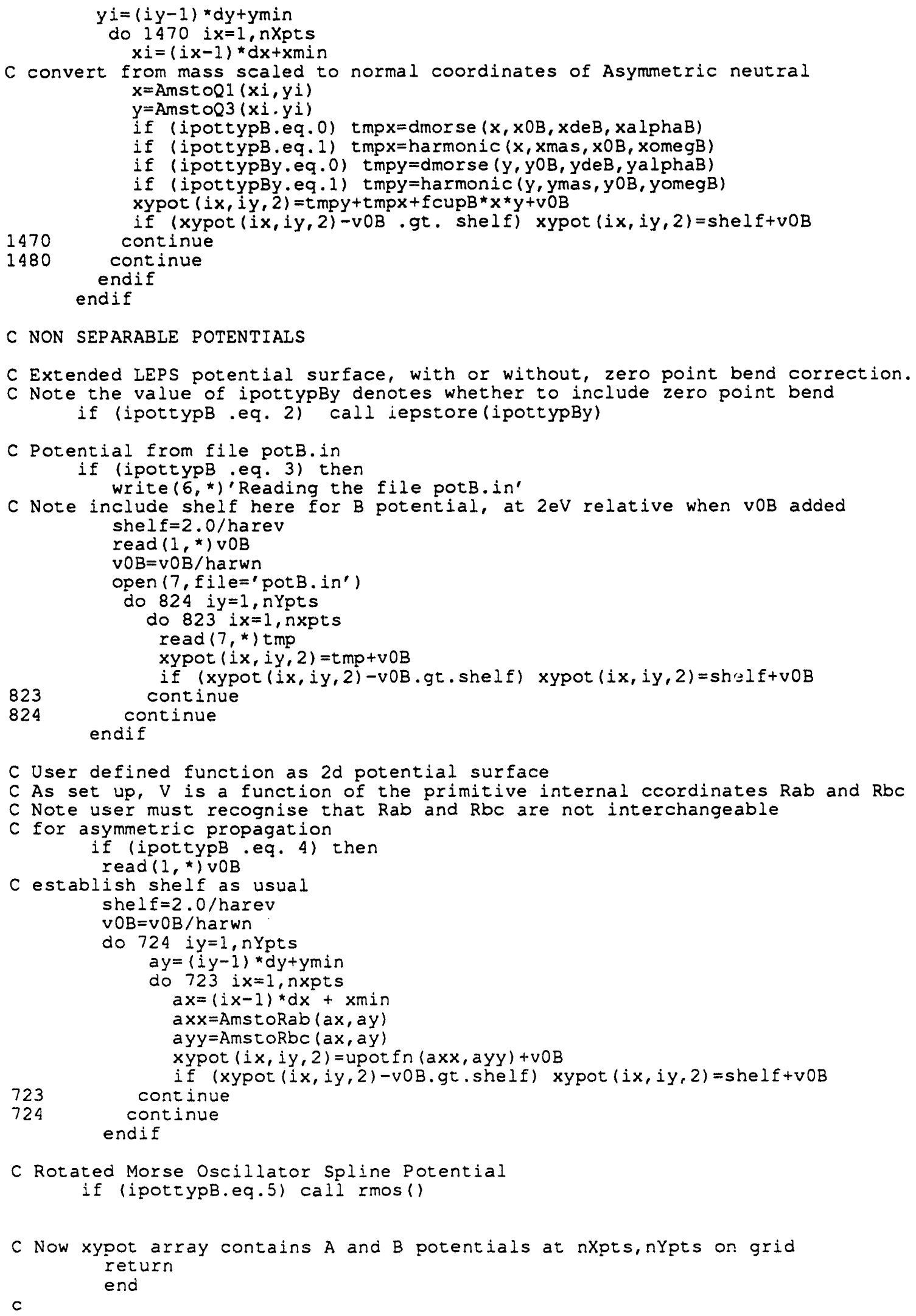




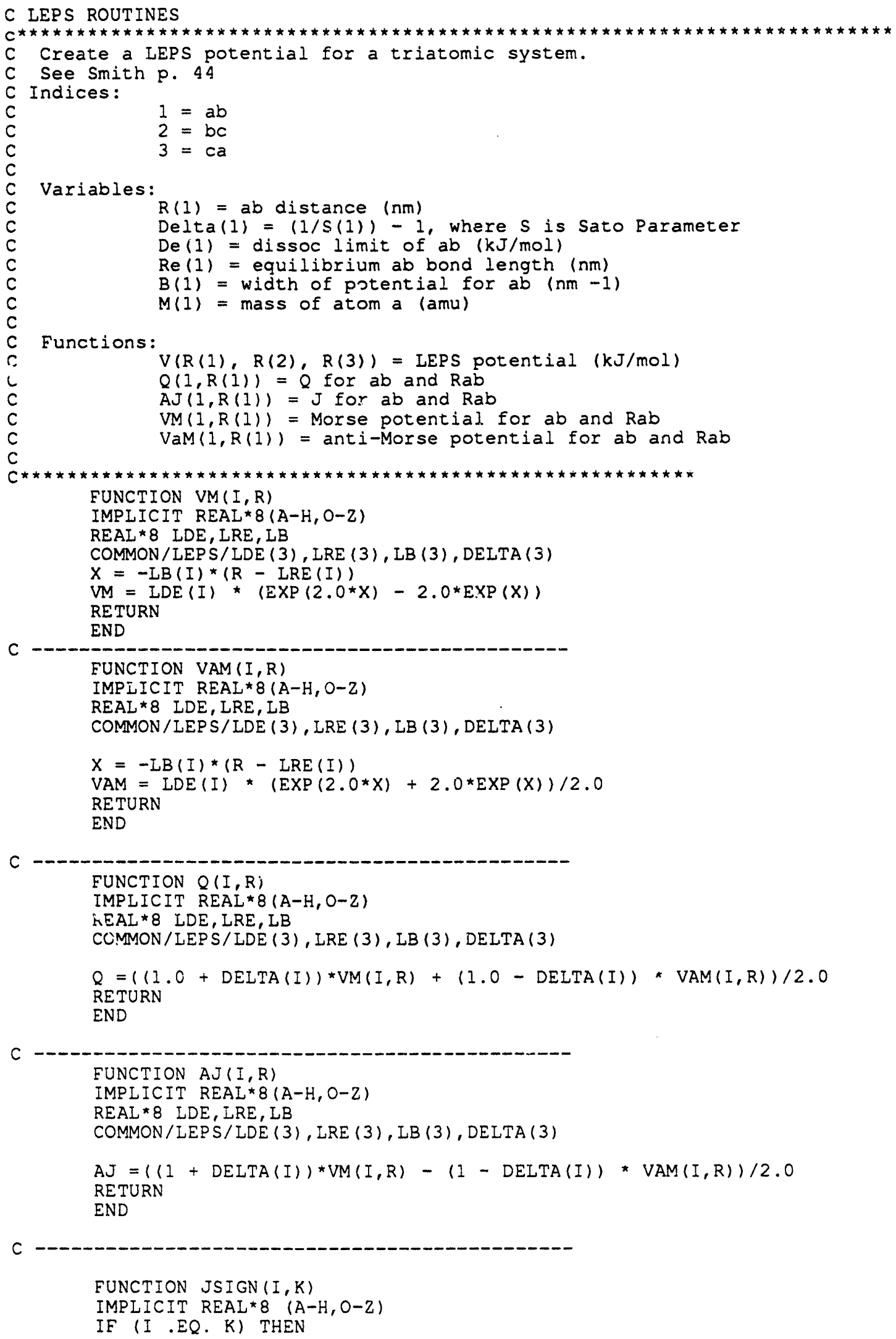




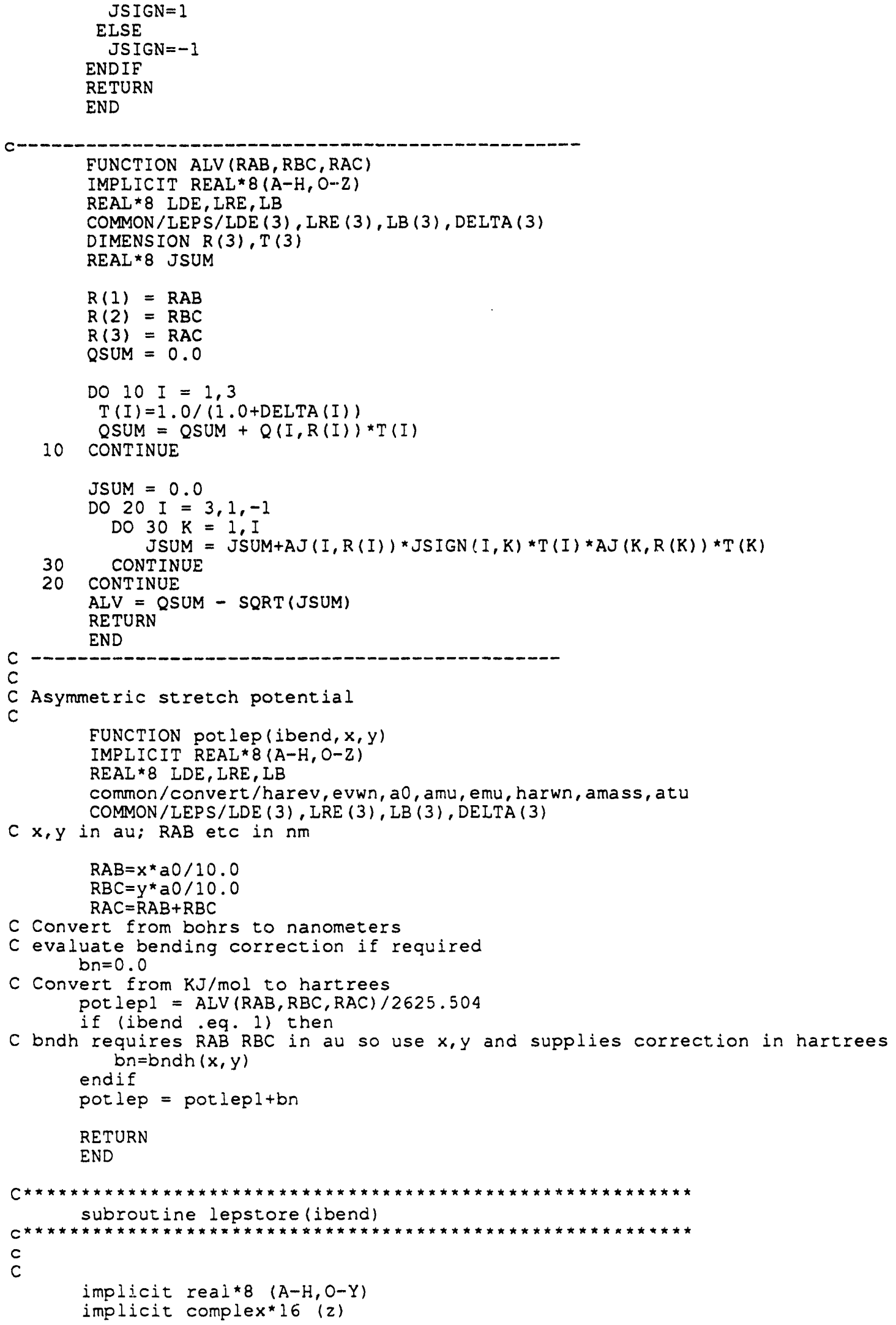




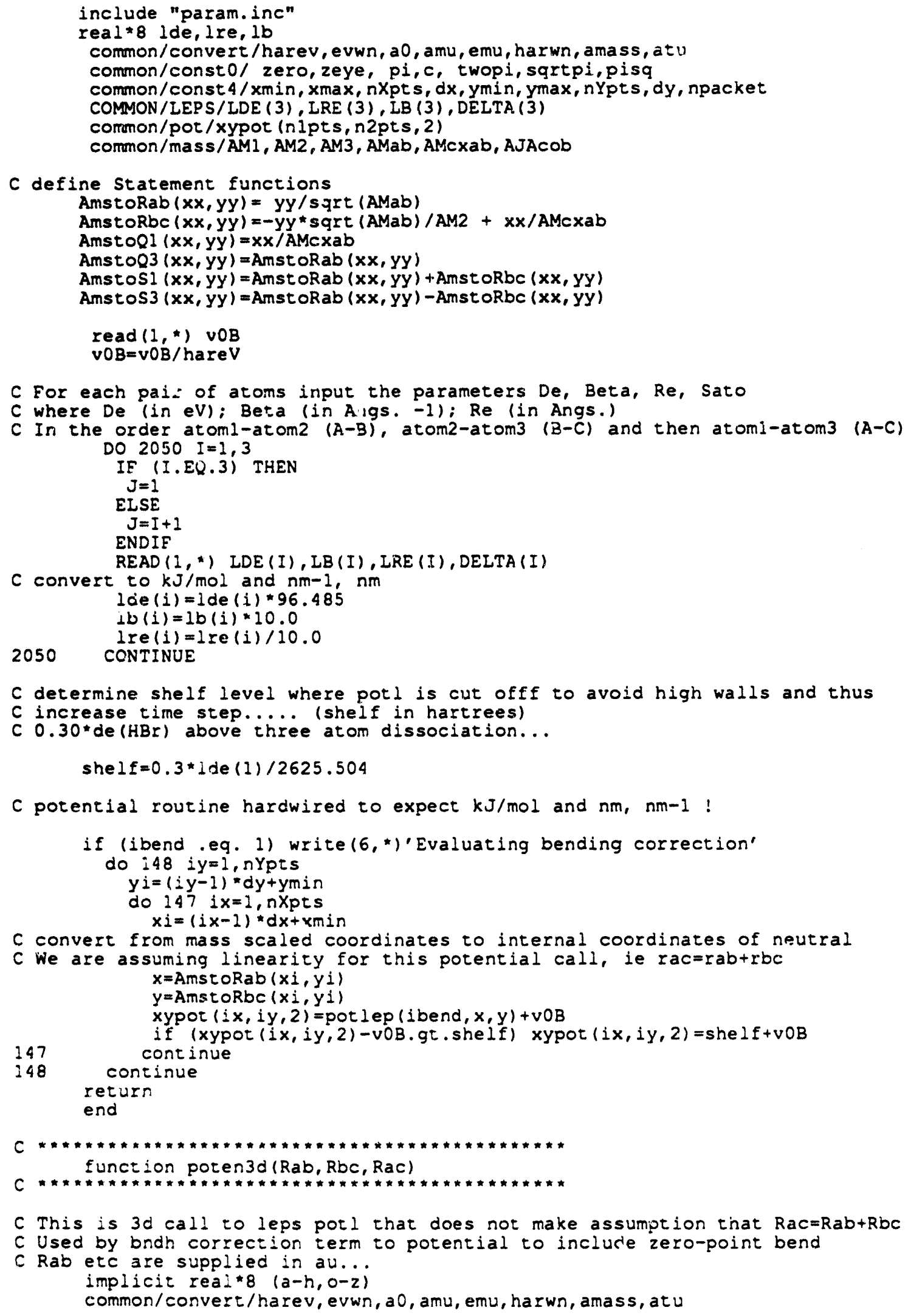




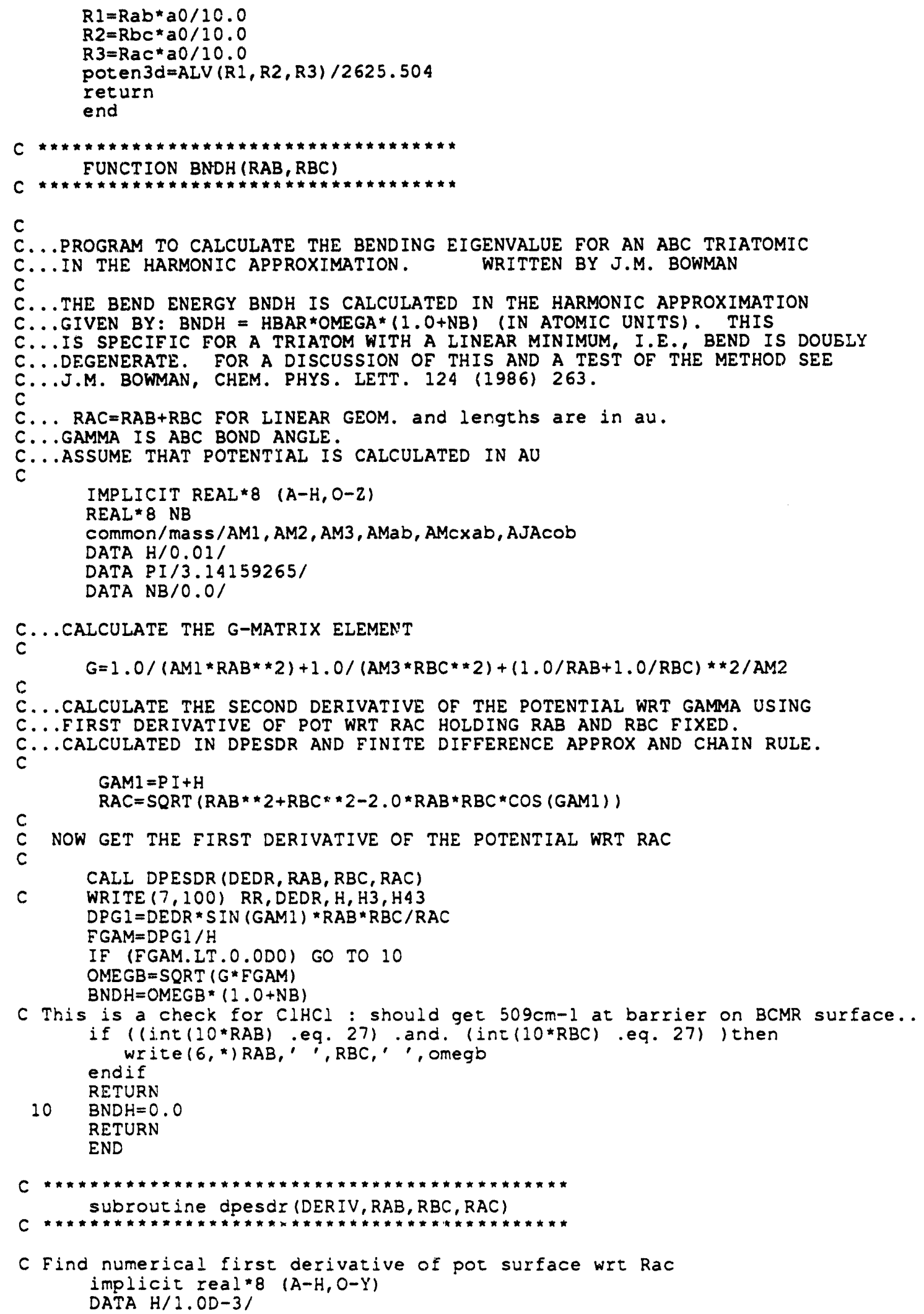




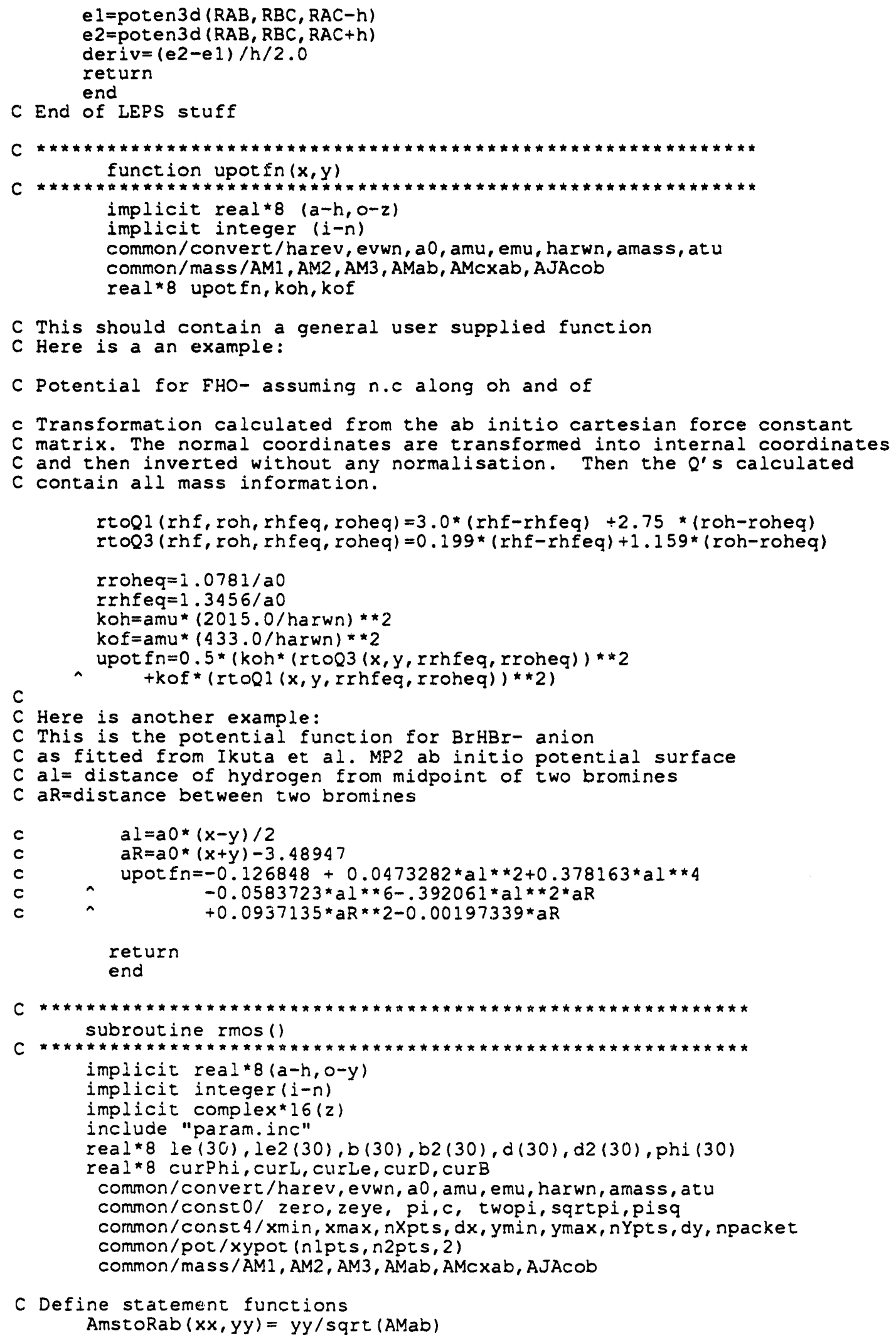

C Define statement functions

AmstoRab $(x x, y y)=y y / \operatorname{sqrt}(A M a b)$ 
AmstoRbc $(x x, y y)=-y y^{\star} \operatorname{sqrt}(A M a b) / A M 2+x x / A M c x a b$

C Rotated Morse Oscillator Spline Potential (RMOS):

C See for example Wagner et al. JCP 74, 4960 (1981) or

C Schatz Rev. Mod. Phys. 61, 669 (1989)

C S. E. Bradforth, August 1990

C Read in the RMOS parameters; expect distances and beta in a.u.

$C$ De is in eV. There will be ncut defined Morse curves and natural spline

$C$ interpolation between them

$$
\begin{aligned}
& \text { read }\left(1,{ }^{*}\right) \cup O B \\
& \text { vOB=vOB/harwn } \\
& \text { read }\left(1,{ }^{*}\right) \text { rabsw, rbcsw, Vab }
\end{aligned}
$$

$C \mathrm{Vab}$ is the dissociation energy of $A-B$ and should be positive

$C$ and in units of $\mathrm{eV}$. This sets the absolute energy before adding $v O B$ and

$C$ thus sets energies with respect to bottom of the well $A B$

C In usual circumstances then $V O B$ should be set to zero for this potential type

$C$ shelf set $0.3^{*} \mathrm{Vab}$ above three atom dissociation

$\mathrm{Vab}=\mathrm{Vab} / \mathrm{harev}$

shelf $=0.3 * V a b+V a b$

read $(1, *)$ ncut

do $10 i=1$, ncut

$\operatorname{read}(1, \star$ ) phi (i), $a(i), l e(i), b(i)$

$C$ set phi in radians

$\operatorname{phi}(i)=\operatorname{phi}(i) * \operatorname{acos}(-1.0) / 180.0$

$d(i)=d(i) /$ harev

10 continue

py $1=1.0$ e 37

pyn=py 1

call splin (phi, d, ncut, pyl, pyn, d2)

call splin(phi, le, ncut, pyl, pyn, le2)

call splin (phi,b, ncut, pyl, pyn,b2)

C Now calculate the potential on the mass weighted grid points do 20 iy $=1$, nypts

$y i=(i y-1) \star d y+y m i n$

do 30 ix $=1$, nxpts

$x i=(i x-1) \star d x+x m i n$

$r a b=A m s$ toRab $(x i, y i)$

$r b c=$ AmstoRbc $(x i, y i)$

cur $L=\operatorname{sqrt}($ (rab-rabsw $) * \star 2+($ rbc-rbcsw $) * \star 2)$

curPhi=atan ( (rbcsw-rbc) / (rabsw-rab))

if ((rab.lt.rabsw). and. (rbc.lt.rbcsw)) then

call splint (phi, d, d2, ncut, curphi, curD)

call splint (phi, le, le2, ncut, curPhi, curle)

call splint (phi,b, b2, ncut, curphi, curB)

xypot $(i x, i y, 2)=\operatorname{curD} *((1-\exp (\operatorname{curB} *(\operatorname{curL}-c u r L e))) * 2-1)$

$+\mathrm{Vab}$

if (xypot $(i x, i y, 2) . g t$. shelf) $x y p o t(i x, i y, 2)=$ shelf

xypot $(i x, i y, 2)=x y p o t(i x, i y, 2)+v O B$

else

xypot $(i x, i y, 2)=$ shelf $+v O B$

endif

continue

20 continue

return

end

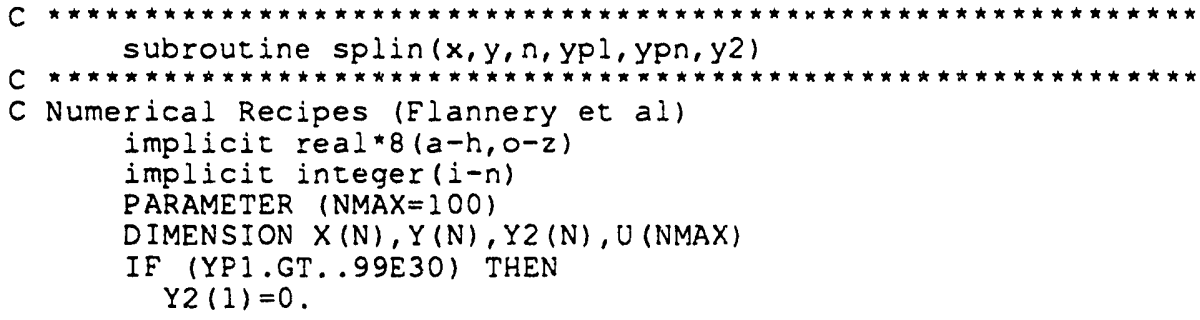


$U(1)=0$.

ELSE

$Y 2(1)=-0.5$

$U(1)=(3 . /(X(2)-X(1))) *((Y(2)-Y(1)) /(X(2)-X(1))-Y P 1)$ ENDIF

DO $11 \mathrm{I}=2, \mathrm{~N}-1$

$S I G=(X(I)-X(I-1)) /(X(I+1)-X(I-1))$

$P=S I G \star Y 2(I-1)+2$.

$Y 2(I)=(S I G-1) /$.

$U(I)=(6 . *((Y(I+1)-Y(I)) /(X(I+I)-X(I))-(Y(I)-Y(I-I))$

* CONTINUE

IF (YPN.GT..99E30) THEN

$\mathrm{QN}=0$.

$\mathrm{UN}=0$.

ELSE

$\mathrm{QN}=0.5$

$U N=(3 . /(X(N)-X(N-1))) *(Y P N-(Y(N)-Y(N-1)) /(X(N)-X(N-1)))$

ENDIF

$Y 2(N)=(U N-Q N * U(N-1)) /(Q N * Y 2(N-1)+1$.

DO $12 \mathrm{~K}=\mathrm{N}-1,1,-1$

12

$Y 2(K)=Y 2(K) \star Y 2(K+1)+U(K)$

return

end

C

subroutine splint ( $x a, y a, y 2 a, n, X, Y)$

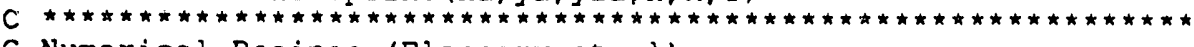

C Numerical Recipes (Flannery et al)

implicit real*8 $(a-h, 0-z)$

implicit integer $(i-n)$

DIMENSION XA(N), YA(N),Y2A(N)

$K L O=1$

$\mathrm{KHI}=\mathrm{N}$

1 IF (KHI-KLO.GT.1) THEN

$K=(K H I+K L O) / 2$

IF (XA (K) . GT . X) THEN $\mathrm{KHI}=\mathrm{K}$

ELSE

$\mathrm{KLO}=\mathrm{K}$

ENDIF

GOTO 1

ENDIF

$H=X A(K H I)-X A(K L O)$

IF (H.EQ.O.) PAUSE 'Bad $X A$ '

$A=(X A(K H I)-X) / H$

$B=(X-X A(K I O)) / H$

$Y=A \star Y A(K L O)+B \star Y A(K H I)+$

$\star \quad((A \star \star 3-A) \star Y 2 A(K L O)+(B \star \star 3-B) \star Y 2 A(K H I)) \star(H * \star 2) / 6$.

return

end

$c$

subroutine porlsave (ipot)

c

c

implicit real*8 $(A-H, O-Y)$

implicit complex*16 (z)

include "param. inc"

common/convert/harev, evwn, a0, amu, emu, harwn, amass, atu

common/const $4 / x m i n, x m a x, n X p t s, d x, y m i n, y m a x, n Y p t s, d y, n p a c k e t$

common/pot/xypot (nlpts, n2pts, 2)

c

common/mass/AM1, AM2, AM3, AMab, AMcxab, AJAcob

if (ipot.eq. I) open(1,file='potlA.out')

if (ipot eq. 2) open(1,file='potlB.out')

do 20 iy $=1$, nYpts 
930

10

20 do 10 ix $=1$, nxpts

$x i=(x \min +(i x-1) * d x) * a 0 / \operatorname{sqrt}(A M a b)$

$y i=(y m i n+(i y-1) * d y) * a 0 / \operatorname{sqrt}(A M a b)$

$a=x y p o t(i x, i y, i p o t)$ *harev

write $(1,930) \times i, y i, a$

format (f $9.3,2 x, f 9.3,2 x, f 15.6)$

continue

continue

close (1)

return

end

\section{graphicsy.f}

This file suitable for compilation on computer system that has DISSPLA, and GKS/NCAR graphics library, e.g. San Diego Cray Y/MP

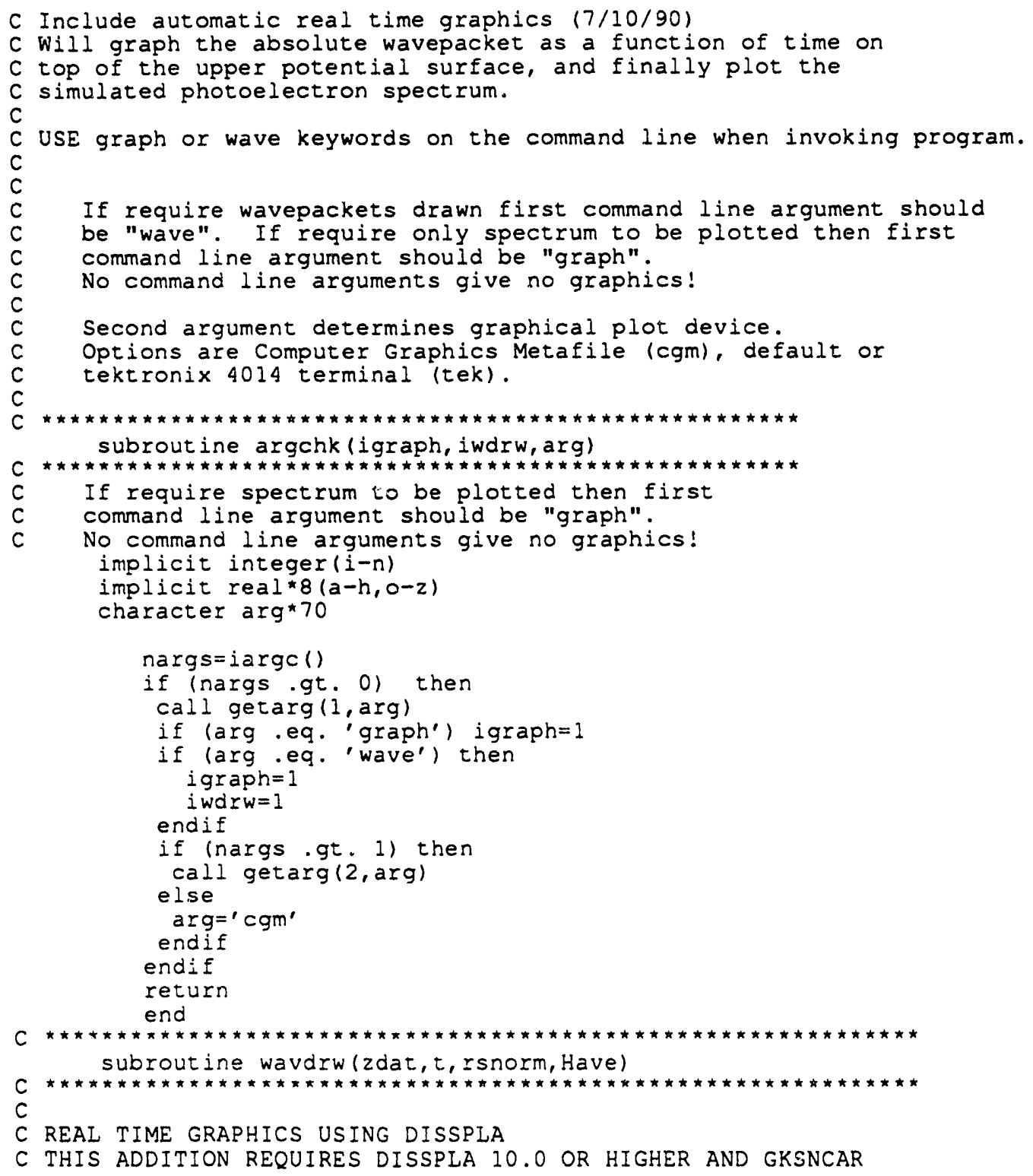


C LIBRARY AS ON SDSC Y/MP RUNNING UNICOS

C

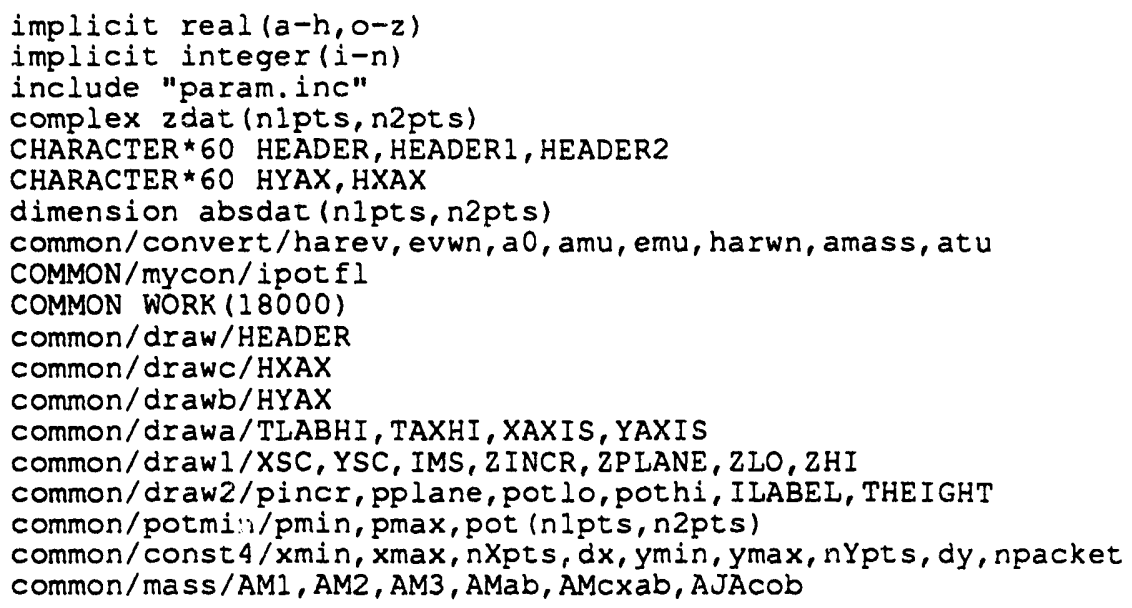

C SET CONTUR PARAMETERS

CALL BCOMON $(18000)$

if (pplane.eg.0) then

CALL 2BASE (PMIN)

else

CALI ZBASE (PPLANE)

endif

ipot $f l=0$

if (PINCR eq. 0.0 ) then

CALI CONMAK (POT, nlpts, n2pts, 'SCAIE')

else

CALL CONMAK (POT, nlpts, n2pts,pincr)

endif

CALL CONLIN $(0, '$ MYCON','NOLABELS', 1, 10)

CALL CONMIN (6.0)

CALI CONANG $(90$.

CALL HEIGHT (THEIGHT)

CALI CONTUR $(1$, 'LABELS', 'DRAW') 


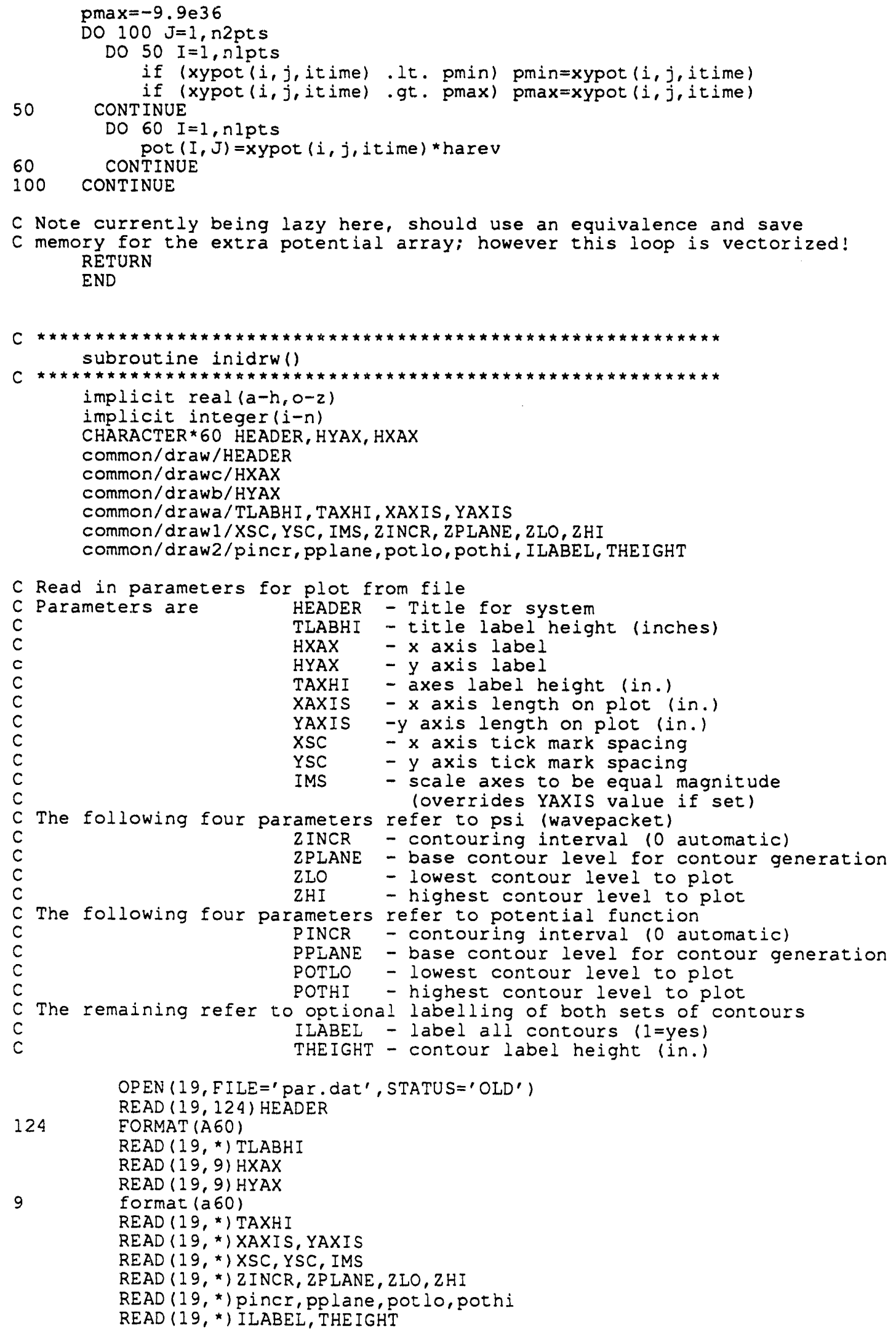




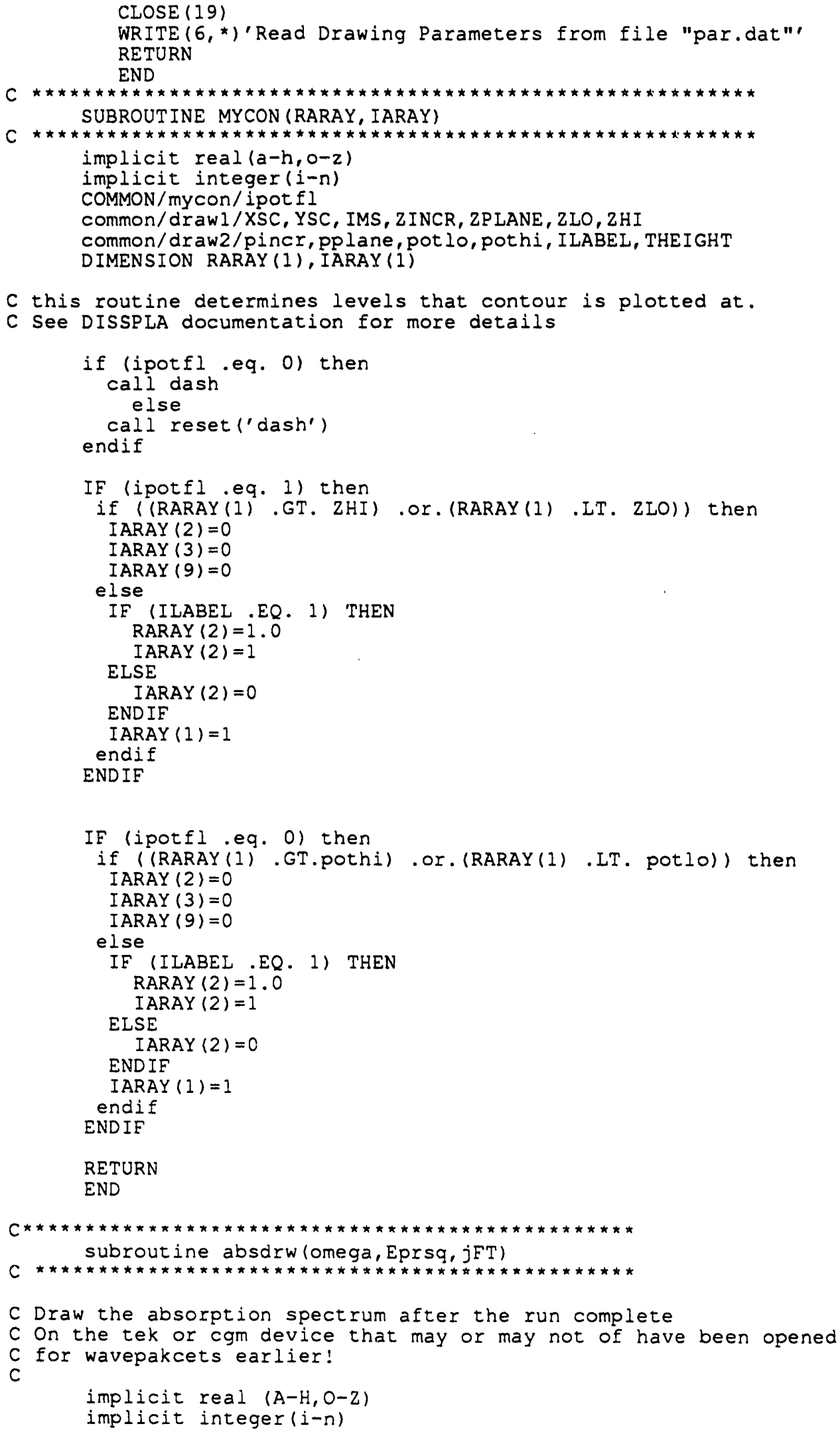


parameter ( $\mathrm{nFFT}=8192$ )

dimension Eprsq (nFFT), omega (nFFT)

common/convert/harev, evwn, a0, amu, emu, harwn, amass, atu

common/const7/ Espmin, domega, novsav, npktsav

C upper point on P.E. spectrum plotted is Espmin (in eV)

Espev $=$ Espmin *harev

C use simple plot $x-y$ primitive from DISSPLA

call HEIGHT $(0.20)$

CaIl AREA2D $(12.0,10.0)$

call XNAME (' (E) lectron (E) nergy / (eV) S',100)

call YNAME ('(I) ntensitys',100)

call HEADIN (' (S) imulated (P) hotoelectron (S) pectrums', 100,1.1, 1)

call graf $(0.0,0.2, E s p \in V, 0.0,0.2,1.0)$

call curve (omega, Eprsa, jFT, 0 )

call endpl (0)

return

end

C

C

subroutine iniplt 2 (arg)

implicit real ( $A-\mathrm{H}, \mathrm{O}-\mathrm{Z})$

implicit integer $(i-n)$

character arg*70

C Initialise device

if (arg.eq.'tek') then

call tk4014 $(960,1)$

else

if (arg.eq.' cgm') then

call opngks

else

write $(6,)^{\prime}$ 'Not valid graphics device. Exiting...'

stop

endif

endif

C SET ALPHABETS

CALL RESET ('ALI')

C SCRAP SUMMARY MESSAGES BUT DIRECT ERROR MESSAGES TO SCREEN

CALL SETDEV $(6,0)$

CALL PAGE $(16 ., 14$.

CALL INTAXS

CALL BASALF ('L/CST')

CALI MIXALF ('STAND')

CALL COMPLX

return

end

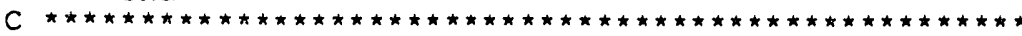

subroutine pltfin

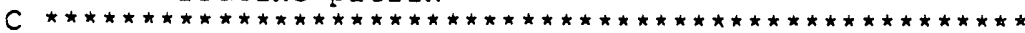

C Close up plotting device

Call DONEPI ()

$r \in i u r n$

end 


\section{Appendix D. Publications from Graduate Work}

S. E. Bradforth, D. W. Arnold, E. H. Kim and D. M. Neumark.

"Photoelectron Spectroscopy of $\mathrm{CN}^{-}, \mathrm{NCO}^{-}$and $\mathrm{NCS}^{-}$" J. Chem. Phys. in press (1993)

D. W. Arnold, S. E. Bradforth, E. H. Kim and D. M. Neumark.

"Anion Photoelectron Spectroscopy of Iodine-Carbon Dioxide Clusters" J. Chem. Phys. in press (1992)

D. W. Arnold, S. E. Bradforth, T. N. Kitsopoulos and D. M. Neumark.

"Vibrationally Resolved Spectra of $\mathrm{C}_{2}-\mathrm{C}_{11}$ by Anion Photoelectron

Spectroscopy" J. Chem. Phys. 95, 8753 (1991)

S. E. Bradforth, D. W. Arnold, R. B. Metz, A. Weaver and D. M. Neumark. "Spectroscopy of the Transition State: The Hydrogen Abstraction Reactions of Fluorine" J. Phys. Chem. 95, 8066 (1991)

R. B. Metz, S. E. Bradforth and D. M. Neumark.

"Transition State Spectroscopy of Bimolecular Reactions using Negative Ion Photodetachment" Adv. Chem. Phys. 81, 1 (1992)

A. Weaver, D. W. Arnold, S. E. Bradforth and D. M. Neumark.

"Examination of the ${ }^{2} \mathrm{~A}_{2}$ and ${ }^{2} \mathrm{E}$ " states of $\mathrm{NO}_{3}$ by negative ion photoelectron spectroscopy of $\mathrm{NO}_{3}{ }^{-"}$ J. Chem. Phys. 94, 1740 (1991)

B. E. Wurfel, N. Pugliano, S. E. Bradforth, R. J. Saykally and G. C. Pimentel.

"Broadband Transient Infrared Laser Spectroscopy of the Trifluorovinyl radical, $\mathrm{C}_{2} \mathrm{~F}_{3}$ - experimental and ab initio results" J. Phys. Chem. 95, 2932 (1991) 
A. Weaver, R. B. Metz, S. E. Bradforth and D. M. Neumark.

"Investigation of the $\mathrm{F}+\mathrm{H}_{2}$ transition state region via Photoelectron Spectroscopy of the $\mathrm{FH}_{2}^{-}$anion" J. Chem. Phys. 93, $5352(1990)$

S. E. Bradforth, A. Weaver, D. W. Arnold, R. B. Metz and D. M. Neumark

"Examination of the $\mathrm{Br}+\mathrm{HI}, \mathrm{Cl}+\mathrm{HI}$, and $\mathrm{F}+\mathrm{HI}$ hydrogen transfer reactions by Photoelectron Spectroscopy of $\mathrm{BrHI}^{-}, \mathrm{ClHI}^{-}$and $\mathrm{FHI}^{-}$" J. Chem. Phys. 92, 7205 (1990)

R. B. Metz, A. Weaver, S. E. Bradforth, T. N. Kitsopoulos and D. M. Neumark. "Probing the Transition State with Negative Ion Photodetachment: The $\mathrm{Cl}+$ $\mathrm{HCl}$ and $\mathrm{Br}+\mathrm{HBr}$ reactions" J. Phys. Chem. 94, 1377 (1990)

A. Weaver, R. B. Metz, S. E. Bradforth and D. M. Neumark.

"Observation of the $A\left({ }^{2} \mathrm{~B}_{2}\right)$ and $\mathrm{C}\left({ }^{2} \mathrm{~A}_{2}\right)$ states of $\mathrm{NO}_{2}$ by negative ion photoelectron spectroscopy of $\mathrm{NO}_{2}^{-"}$ J. Chem. Phys. 90, 2070 (1989)

S. E. Bradforth, A. Weaver, R. B. Metz and D. M. Neumark.

"Study of Hydrogen Transfer reactions by Negative Ion Photoelectron Spectroscopy" Advances in Laser Science - IV Proceedings of the 1988 International Laser Science Conference 1989, 657 (American Institute of Physics, New York)

A. Weaver, R. B. Metz, S. E. Bradforth and D. M. Neumark.

"Spectroscopy of the I + HI Transition State Region by Photodetachment of $\mathrm{IHI}^{-"}$, J. Phys. Chem. 92, 5558 (1988) 

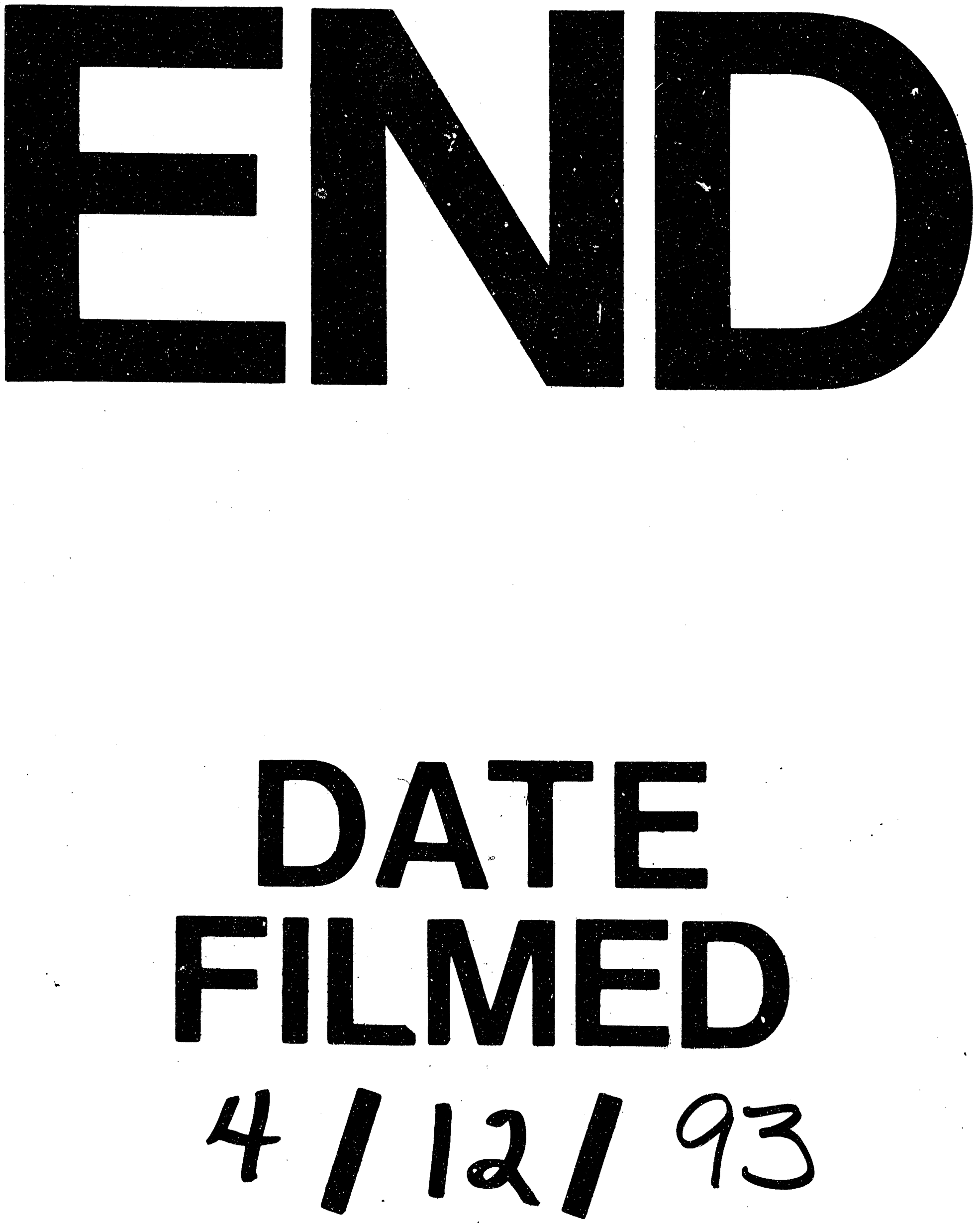